


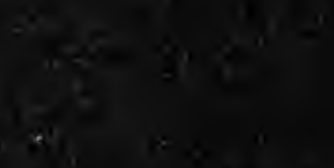




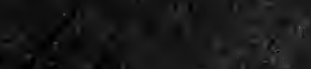

$5=98$ 
Cllive let arke 
Digitized by the internet Archive in 2007 with funding from Microsoft Corporation 


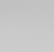




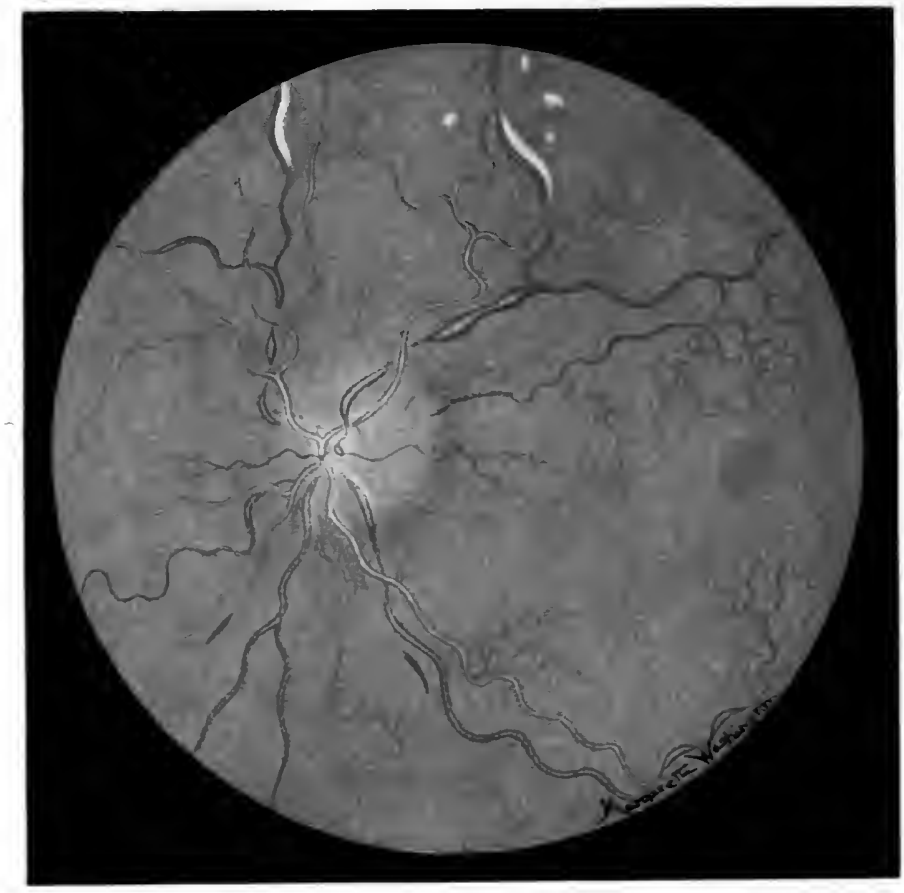

Eye-ground in Arteriosclerosis

(After de Sehweinutz) 


\section{BLOOD-PRESSURE,}

\section{ITS CLINICAL APPLICA'TIONS}

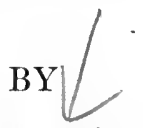

GEORGE WILLIAM NORRIS, A.B., M.D.

ASSISTANT PROFESSOR OF MEDICINE IN THE UNIVERSITY OF PENNSYLVANIA; VISITING PHYSICIAN

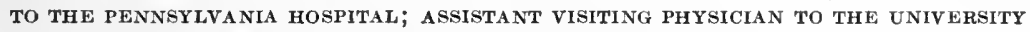
HOSPITAL; FELLOW OF THE COLLEGE OF PHYSICIANS OF PHILADELPHIA; MEMBER OF THE ASSOCIATION OF AMERICAN PHYSICIANS, ETC.

SECOND EDITION, REVISED AND ENLARGED

ILLUSTRATED WITH 102 ENGRAVINGS AND 1 COLORED PLATE

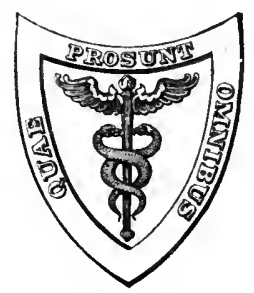

LEA \& FEBIGER

PHILADELPHIA AND NEW YORK 


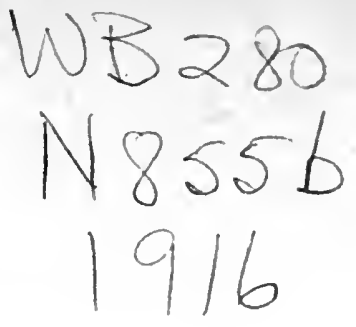

Entered according to the Act of Congress, in the year 1916, by LEA \& FEBIGER,

In the Office of the Librarian of Congress. All rights reserved. 


\section{DEDICATED}

TO THE MEMORY OF

\section{FREDERICK A. PACKARD, M.D.}

WIOSE MEDICAL TALENTS AND KINDLY SPIRIT WERE UNTIMELY LOST TO THE COMMUNITY OF PHILADELPIIA, IN WHICI IIE LIVED AT WHOSE SUGGESTION AND UNDER WHOSE GUIDANCE TIIE AUTIOR FIRST UNDERTOOK BLOOD-PRESSURE INVESTIGATIONS AND TO

WIIOSE SKILL AND SELF-SACRIFICING PROFESSIONAL CARE HE IN A LARGE MEASURE OWES IIIS LIFE. 



\section{PREFACE TO THE SECOND EDITION.}

Tre exhaustion of the first edition of this work in considerably less than two years after publieation is a source of gratification and pleasure to the author. In the process of revision for the second edition an increase in size has been necessary in order to include a survey of the constantly growing literature on blood-pressure.

The author has continued his endeavor to present the subject of blood-pressure in a condensed and practical form as definitely as the present state of our knowledge permits. Wherever possible, a summary of the experimental as well as the clinical data available for the consideration of a given topic has been included, together with references to articles upon which statements are based.

As in the earlier edition the chapters upon Physiology and upon Venous Blood-pressure were written by Dr. J. Harold Austin, to whom the author is also indebted for assistance in the preparation of the chapter dealing with the Functional Effieieney of the Circulation. 'The author's thanks are also due to Miss Eleanor A. Cantner for valuable assistance in the preparation of the charts and drawings, as well as in proof reading.

1530 Locust St.,

G. IV. N.

Philadelphia, 1916. 



\section{CONTENTS.}

\section{CHAP'TER I.}

'The Piysiology of Blood-pressure

CHAPTER II.

The Instrumental Estimation of Blood-Pressure

CHAPTER III.

The Instrumental Estimation of Blood-pressure (Continued)

CHAPTER IV.

Venous BLOOD-PRESSURE

CHAPTER V.

The Functional Efficiency of the Circulation as Determinable by Blood-pressure Estimation and Allied Tests . . . . 145

CHAPTER VI.

Arterial Hypotension 185

CHAPTER VII.

Bloon-pressure in Acute Infectious Disease . . . . . . . 196

CHAPTER VIII.

Blood-pressure in Chronic Infectious Disease . . . . . . 213

CHAPTER IX.

Exogenous Intoxications

CHAPTER $\mathrm{X}$.

Blood-pressure in Cardiac Disease, Etc. 
CHAPTER XI.

Blood-pressure in Arteriosclerosis-Vascular Crises . . . 250

CHAPTER XII.

Arterial Hypertensive Cardovascular Disease, Nephritis, Etc. 266 CHAPTER XIII.

The Treatuent of Arterial Hypertension 299

CHAPTER XIV.

Effects of Drugs and Glandular Extracts on Blood-pressure

(Arranged Alphabetically) . . . . . . . . . . . 324

CHAPTER XV.

Metabolic Diseases and Miscellaneous Conditions . . . . 347 CHAPTER XVI.

Diseases of the Nervous System • . . . . . . . . . 369

CHAPTER XVII.

Blood-PRessure in SURgery and Obstetrics . . . . . . . 376

CHAPTER XVIII.

Oрнтh.LMOLOGY . . . . . . . . . . . . . . 402 


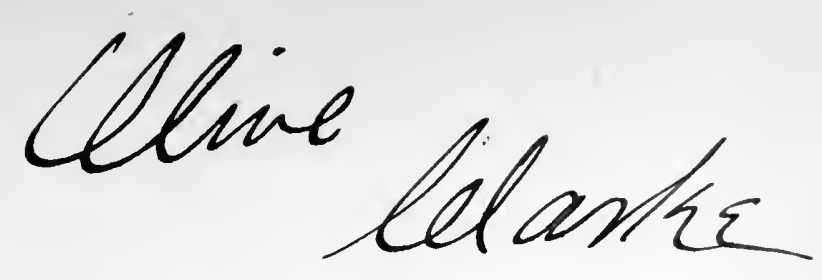

\title{
A CLINICAL STUDY OF BLOOD-PRESSURE.
}

\author{
CHAPTER I. \\ THE PHYSIOLOGY OF BLOOD-PRESSURE.
}

By J. HAROLD AUSTIN, M.D.

Definitions.-Blood-pressure is the term used to indicate the pressure exerted by the blood at a given point in the circulatory system at a given moment. Thus we may speak of intra-auricular, intraventricular, arterial, capillary, or venous blood-pressure. When not qualified, the term is used to refer to the arterial blood-pressure, and in man usually to the pressure in the brachial artery. The arterial blood-pressure is eonstantly undergoing rhythmic fluctuations due to the cardiac systoles. The highest pressure attained during one cardiac cycle is called the systolic pressure and is attained at the moment of the arrival of the crest of the pulse wave. The lowest pressure attained during a cardiac cycle is called the diastolic pressure. The difference between these two pressures is ealled the pulse pressure. Thus, if the systolic pressure be 120 and the diastolie 90 , then the pulse pressure would be 30 . By mean pressure is understood the average pressure at a given point. The mean pressure is not, however, the arithmetical mean between the systolic and diastolic pressures, because the pressure may remain for only a moment at the systolic level, falling very quickly to near the diastolic level and remaining near it throughout the greater part of the average cycle. This is illustrated in the accompanying diagram in which obviously the average pressure lies much nearer to the diastolic than to the systolic.

In addition to the pressure oseillations, dependent upon the cardiac cycle and ealled oscillations of the first order, there are slower oscillations in the arterial pressure dependent upon the respiratory movements. These are called blood-pressure oscillations of the second order. Finally, still slower oscillations, usually over ten to 
twenty cardiac cycles, occur. These are due to various factors, among which rhythmic variations in the activity of the vasoconstrictor centre or in the cardiac activity are the most important. These constitute the oscillations of the third order. All three types of oscillations are shown in the tracing (see Fig. 6).

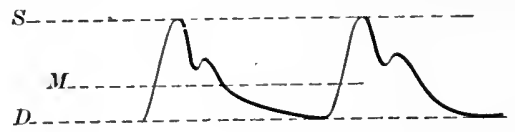

FIG. $1 .-S=$ systolic pressure $D=$ diastolie pressure; $M=$ mean or average pressure.

The pressure which is exerted by the blood against the vessel wall while the current flows unobstructed through the vessel is known as the lateral pressure. The pressure exerted by the blood against an obstruction in the lumen of the vessel is known as the end pressure. The end pressure exceeds the lateral pressure at any given point, and the difference is the pressure that is effective in producing the blood-flow at that point. The accompanying diagram

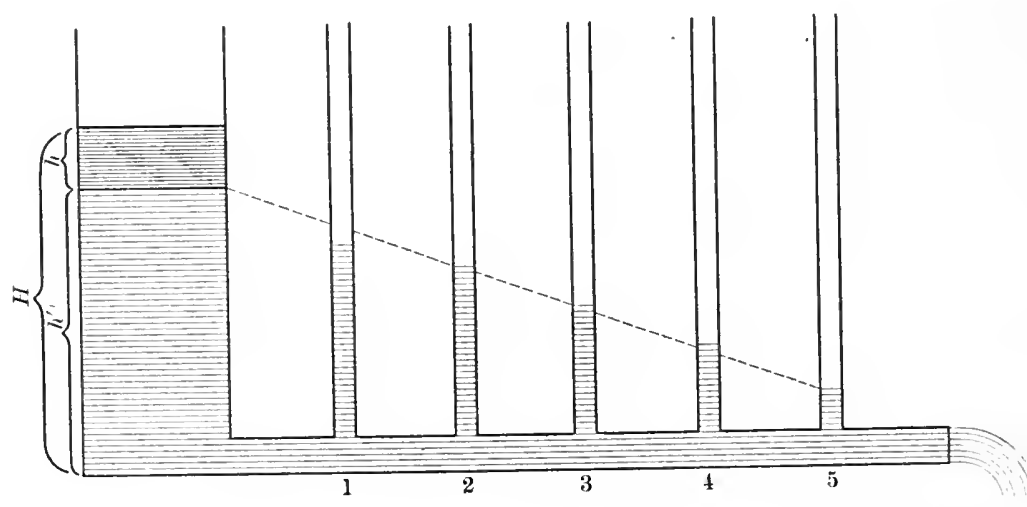

FIG. 2,-Illustrating lateral pressure: 1, 2, 3, 4, 5, lateral pressure at points along outflow tube; $h$, the difference between lateral pressure and end pressure at any point. (From Howell.)

(Fig. 2) illustrates the relation between lateral and end pressures. End pressure in a branch artery is shown to be approximately equal to the lateral pressure in the main trunk at the point at which the branch takes origin. The method used commonly for the measurement of blood-pressure in man shows the end systolic 
pressure in the brachial artery and the lateral diastolic pressure. ${ }^{1}$ Blood-pressure is usually expressed in millimeters (sometimes centimeters) of mercury above or below the atmospheric pressure at the same point. Thus blood-pressure of 120 is a pressure that will sustain in addition to the atmospheric pressure at the point, the pressure of a vertical column of mercury $120 \mathrm{~mm}$. high.

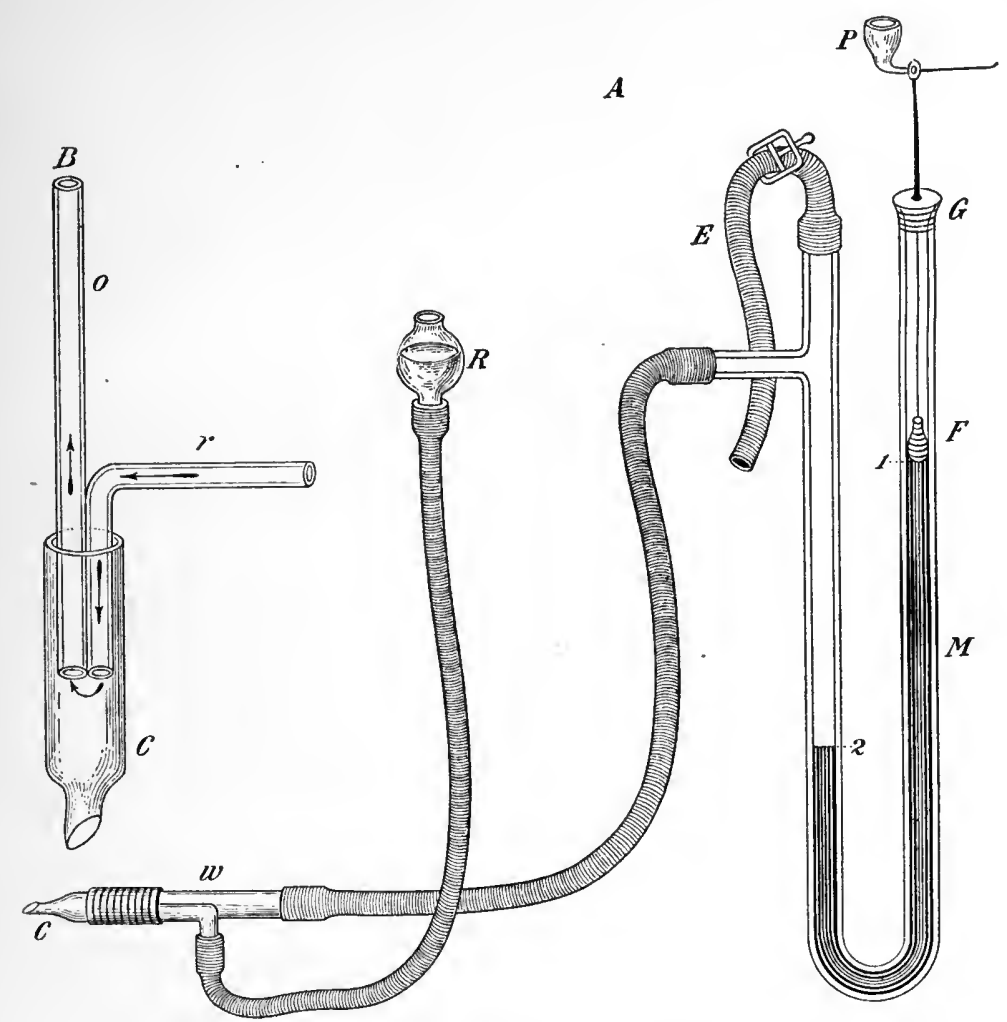

Frg. 3.- $M$, mereury manometer; $F$, float; $G$, perforated cap; $P$, recording pen; $C$, cannula for insertion into vessel; $w$, washout tube, shown in detail at $B ; R$ and $E$, for introduction and outflow of magnesium sulphate solution; both are clamped off while recording pressures. (From Howell.)

Methods of Measurement.- The first exact measurements of bloodpressure were published by the Rev. Dr. Stephen Hales, an Englishman, in a little book entitled Statical Essays, in 1733. He meas-

1 A. K. Cushny, Zentralbl. f. Physiol., Leipsic u. Wien, 1907-8, xxi, 77; N. D. Straschesto, Archiv f. d. ges. Physiol., Bonn, 1909, cxxviii, 1. 
ured the pressure in the femoral artery of a horse by connecting it with a glass tube and noting the height to which the blood rose in this tube held vertically. In 1828 Poiseuille applied the mercury manometer to the study of blood-pressure and in 1847 Ludwig invented the recording manometer with moving drum. Ludwig's method is essentially the one used today in physiological experiments. A manometer is connected by tubing filled with some fluid which will inhibit coagulation, such as saturated magnesium sulphate solution, with a cannula introduced into the artery. A manometer so connected gives a record of the end pressure in the artery.

In physiological work the mean pressure is obtained by constricting the communication between the manometer and the vessel

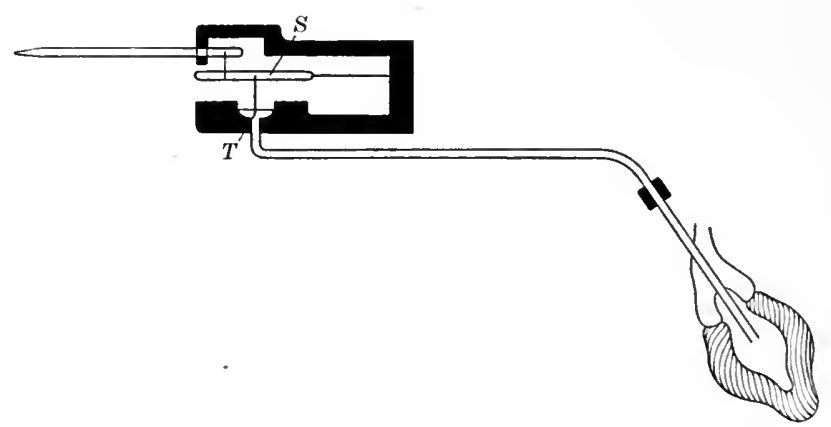

Fig. 4.-Diagram of Hürthle manometer. Tubing from heart or vessel to small tambour $T$, both filled with fluid. Movements of tambour magnified by compound lever $S$, and transmitted to writing pen. (From Howell.)

to a small opening. This prevents the transmission of the faster blood-pressure oscillation to the manometer, and leads to the production of a straight line representing the average or mean pressure. In studies upon man the mean pressure cannot thus be directly recorded and is, in fact, difficult to determine. All pressures determined upon human beings are therefore, as a rule, either systolic or diastolic pressures. It is the custom of some authors to speak of that pressure which is half-way between the systolic and diastolic pressures in man as the mean pressure. This seems to us misleading, inasmuch as it may in certain cases be considerably above the true mean pressure. Dawson ${ }^{1}$ has shown that the mean pressure in man is usually obtained by adding approximately one-third of the

I Brit. Med. Jour., 1906, ii, 996. 
pulse pressure to the diastolic pressure. For the measurement of the systolic and diastolic pressures in physiological work, Hürthle devised the membrane manometer which is shown in Fig. 4, since the inertia of the column of mercury in the mercury manometer is too great to give an accurate record of the rapid fluctuations.

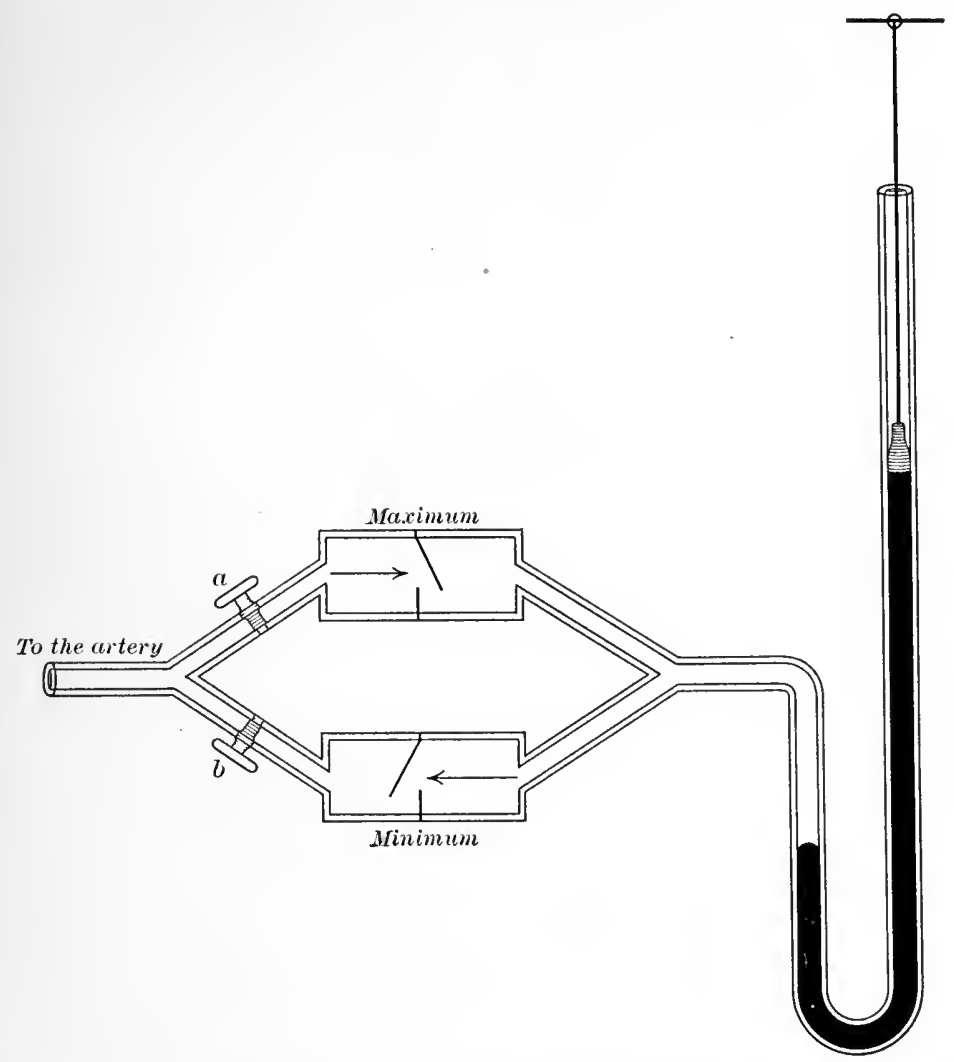

FIG. 5.-Maximum-minimum manometer. When cock $a$ is open and $b$ elosed, the highest pressure attained during the period of record is transmitted to the manometer and retained by the maximum valve. When $a$ is closed and $b$ opened, the mercury falls to the lowest pressure occurring in the period of record and is retained there by the minimum valve.

Another method in use in physiological work for the control of the membrane manometer is the so-called valve manometer, or maximalminimal manometer. This is illustrated in Fig. 5. By using one valve or the other, either the highest or the lowest pressure occurring within a given interval of time is recorded. 'The maximal press- 
ure recorded by such a manometer is not, however, identical with the systolic pressure, nor the minimal with the diastolic pressure. This may be seen by referring to Fig. 6 . The maximal-minimal manometer will record the highest or lowest pressure attained over a certain period of time, and this is usually long enough to admit the occurrence of all three orders of pressure oscillations. It would

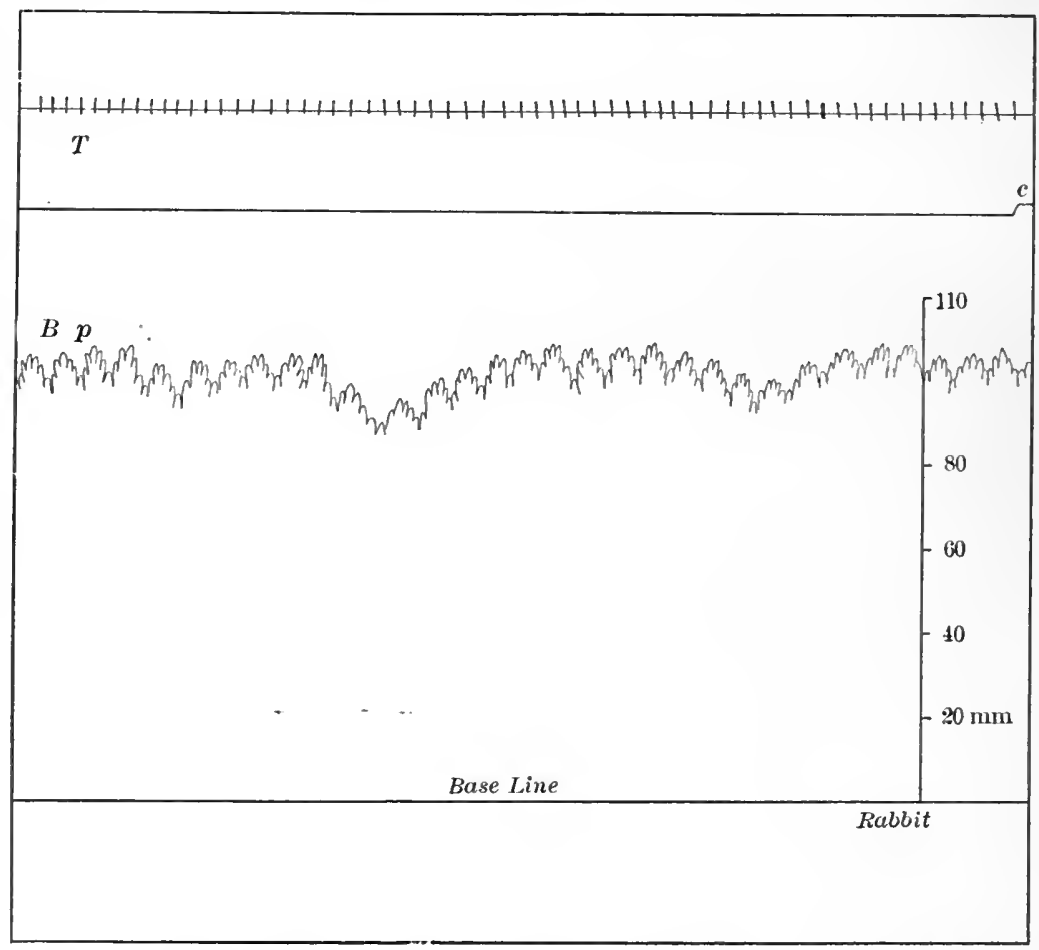

FIG. 6.-Diagram illustrating at $B p$ a typical blood-pressure curve dampened by constricting the communication between the artery and the manometer so as to reduce the amplitude of the shortest waves. Shortest waves, cardiac; longer waves, respiratory; longest waves (three in the tracing), waves of thitd order. $T$, time record in seconds. (From Howell.)

seem desirable, therefore, to use the terms maximal and minimal pressures in this sense. Certain authors, however, have used these terms as svnonymous with systolic and diastolic pressures respectively.

Measurements of the blood-pressure in the smaller arterioles and capillaries either in man or in animals must be made by other methods, and are described in a later chapter. 
Venous pressure is measured, in physiological work, in the same way as the arterial, but usually with a water manometer rather than with a mercury manometer. A pressure of $34 \mathrm{~cm} . \mathrm{H}_{2} \mathrm{O}$ is equal to a pressure of $25 \mathrm{~mm}$. Hg. Blood-pressure within the heart is measured by introducing an oiled cannula, either through one of the vessels or through a puncture wound, into the chamber within which the pressure is desired.

\section{BLOOD-PRESSURE THROUGHOUT THE VASCULAR TREE.}

The blood-pressure in an individual varies with the point of the cardiovascular system under consideration, its elevation with respect to the heart, and with the phase of the heart, whether systole or diastole. These variations are indicated in Table I, which gives approximate systolic and diastolic pressures in different parts of the cardiovascular system at the level of the heart in man. That the blood-pressure varies greatly in different parts of the vascular system has long been known, the marked difference in the force of the stream flowing from a eut artery and from a cut vein being a - striking illustration.

Table I.-Approximate Pressure in Cardovascular Tree of a Normad Man, Aged Twenty Years, at Level of Heart.

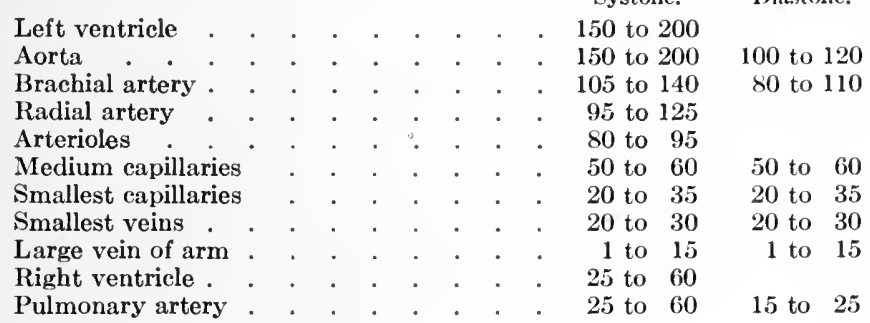

This table is extremely diagrammatic and is intended merely to emphasize certain facts. It will be noted that the chief fall in pressure occurs between the smaller arteries and the eapillaries, that is, in the arterioles; furthermore, that this affects the systolie pressure more than the diastolic, so that under normal conditions the pressure in the capillaries shows no pulse wave. When the arterioles are widely dilated this fall of pressure, especially of systolic pressure, in them is diminished and an appreciable pulse may penetrate into the eapillaries. Under these conditions the systolie 
pressure in the capillaries approaches that in the smaller arteries, and the diastolic pressure in the arteries approaches that of the capillaries. It will further be noted that any increase in venous pressure must promptly elevate the capillary pressure. Indeed, the capillary pressure is more readily raised by renous stasis than by increased arterial pressure.

In the recumbent position the lateral pressure throughout the entire length of the aorta is very nearly constant. In the upright position, however, the pressure at the lower end of the aorta is higher than the pressure at the arch, by the weight of the column of blood separating the two points; approximately $2 \mathrm{~mm}$. $\mathrm{Hg}$. per inch.

In the veins the pressure is largely dependent upon posture, i. e., upon the height of the point measured above or below the right auricle. At the right auricle the pressure is probably about 0 to 10 $\mathrm{mm}$. Hg., varying with the phase of respiration, and that in the trunk is about $2 \mathrm{~mm}$. higher for every inch below the heart; in the lower extremities, however, the venous pressure, while high, is not so high as this calculation would indicate (see p. 135). This relation leads to some distention of the reins in the lower and dependent portions of the body, while the veins of the upper portion of the body are but partially filled.

The Factors that Maintain and Regulate Blood-pressure.-The cardiovascular system may be considered from this point of view as consisting of six elements. (1) The heart, the source of energy. (2) The arteries acting as elastic ressels, dilating to receive each new ventricular delivery of blood, and contracting to maintain during the filling and resting periods of the heart an even flow through the capillaries. (3) The capillaries, the site of active functionation of the blood and with the smaller arterioles the chief source of the resistance in the circulation. (4) The veins which with the capillaries serve as a reservoir, maintaining a pressure just sufficient to fill promptly the relaxing heart for its next systolic contraction. (5) The blood itself, the incompressible, inelastic medium which fills the cardiovascular system. (6) The lymphatic system with its tissue spaces, serous cavities and lymphatic channels filled with lymph, acting as an additional but less promptly available reservoir for body fluids.

The Heart.-The heart is the chief source of the energy that maintains the blood-pressure. 'The factors that affect the heart's efficiency to this end are the factors that influence the output of blood per minute from the heart. They are as follows: (1) The 
diastolic filling of the ventricles; this is dependent upon $(a)$ the venous pressure, $(b)$ the length of diastole, and $(c)$ the cardiac tone. (2) The completeness of systolic emptying. (3) The number of beats per minute, or cardiac rate. (4) The efficiency of the valves of the heart. The cardiac cycle may be divided into two parts: (1) systole, or the period of cardiac contraction, (2) diastole, or the period of cardiac relaxation and rest. 'The ventricular systole and diastole are each further subdivided. The first one-third or onefourth of the ventricular systole is occupied in raising the pressure within the ventricle, from the pressure produced by the auricular systole to the diastolic pressure of the pulmonary artery or of the aorta. This is the so-called presphygmic period or "anspannungszeit." When this pressure is attained the semilunar valves open and, with the propulsion of the blood onward into the aorta, the pressure rises in both ventricle and aorta to the systolic pressure. This pressure is then maintained in the ventricle almost to the end of systole, driving the blood at an even rate out into the aorta and into the smaller vessels. With the relaxation at the close of systole the pressure in the ventricles falls to zero as the semilunar valves close. Diastole now commences. The venous pressure causes the tricuspid and mitral valves to open and the blood fills the ventricles at a rate dependent upon the venous pressure. The amount of distention produced in the ventricle by a given venous pressure is determined by the cardiac tone-the greater the tone, the less the distention. When this inflow is completed the diastole proper ends and the remainder of the cycle is occupied by a period of rest or diastasis.

With changes in cardiac rate, the duration of both systole and diastole are altered, but the former much less than the latter. The length of the systole in man, with a pulse rate of about 75 to 95 , is from 0.3 to 0.38 second. With slow rates that permit time for adequate filling of the ventricles Henderson, ${ }^{1}$ Bohr ${ }^{2}$ and Pütter $^{3}$ have found that providing an adequate venous pressure is maintained, the systolic discharge is under normal conditions practically a constant quantity for any given individual, and the changes in the rate influence only the duration of diastasis. Hence the cardiac output per minute under such conditions will be proportional to the cardiac rate. With a more accelerated pulse rate, however,

\footnotetext{
1 Am. Jour. Physiol., 1912-13, xxxi, 288, 352, 399.

${ }^{2}$ 'Skand. Arch. f. Physiol., 1909, xxii, 221.

3 Ztschr. f. kliti. Med., 1911, Ixxiii, 342.
} 
Henderson has shown that the ventricle has not sufficient time to receive its full quota of blood and the diastole is shortened. In consequence, the systolic discharge is diminished, and although the volume output per minute still increases as the pulse becomes more rapid, it falls farther and farther behind proportionally. Henderson holds to the view that the systolic output of the heart is determined entirely by two factors-the cardiac rate, altering the duration of the diastole, and the venous pressure. On the other hand, Zuntz ${ }^{1}$ and Plesch ${ }^{2}$ find evidence to support the view that, in addition, the heart is capable of varying its output per minute independently of the rate and of the venous pressure, as the result of direct changes in the cardiac tonus and in the amplitude of the cardiac stroke.

Henderson and Barringer have found that with normal cardiac tonus the venous pressure necessary to distend the right ventricle as rapidly as it relaxes is not more than $5 \mathrm{~mm}$. of water in excess of the intrathoracic pressure. This he calls the critical venous pressure. Studies upon the dog and man have led him to the view that while normally the pressure in the intrathoracic veins may during a very deep inspiration become less than atmospheric, it never normally becomes less than $45 \mathrm{~mm}$. of water above the intrathoracic pressure and therefore falls scarcely, if at all, below the critical pressure. In normal inspiration, the descent of the diaphragm, by compressing the abrlominal capillaries and veins, somewhat increases the venous flow into the thorax.

In an athlete, panting after a contest, not only the inspiratory descent of the diaphragm, but also the powerful expiratory contraction of the abdominal muscles must hasten the venous flow from the splanchnic area to the right auricle, and aid in maintaining the critical venous pressure in spite of the accelerated cardiac rate and hence increased cardiac output. It is probable that in extreme tachycardia, the shortening of diastole and consequent imperfect filling of the ventricle may actually lead to a diminished cardiac output per minute.

Henderson and Prince ${ }^{3}$ from a study of the oxygen pulse (the oxygen consumption by the body per minute divided by the pulse rate) with varying amounts of work, have concluded that the systolic discharge of a well-developed man, working hard and with a pulse rate of 140 per minute, is not less than 100 c.c. of blood.

\footnotetext{
${ }_{1}$ Ztschr. f. klin. Med., 1912, lxxiv, 347. ${ }^{2}$ Ztschr. f. Physiol., 1912, xxvi, 90.

${ }^{3}$ The Oxygen Pulse and the Systolic Discharge, Am. Jour. Physiol., 1914, xxxy, 106.
} 
Venous Pressure.-So long as an adequate venous pressure is maintained, the cardiac output per beat is probably dependent upon the duration of diastole. At slow and normal cardiac rates, the diastolic filling is complete, but at accelerated rates the filling becomes progressively diminished. Inadequate venous pressure reduces the cardiac output. On the other hand, in the presence of a dilated heart an abnormally high venous pressure probably tends to keep the ventricles overdistended, thus establishing a vicious cycle.

Cardiac Rate.-At slow and moderate rates the cardiac.output per minute is probably proportional to the cardiac rate. At more rapid rates the minute output increases with the pulse rate, but not proportionally. At extremely rapid rates imperfect ventricular filling may probably actually diminish the minute output.

We may sum up the effects of these factors as follows:

Cardiac Tone.-Either unduly diminished cardiac tone, by permitting dilatation of the ventricles, or unduly increased cardiac tone, by interfering with the diastolic filling, impairs the cardiac output.

Stenotic or incompetent valves tend to diminish the cardiac output, although this defect is at first compensated by increased amplitude of contraction and frequently by increased rate.

Of these various factors, changes in one may to some extent be compensated by changes in another. Elevation of venous pressure may be counteracted by increase of cardiac tone. On the other hand, no changes in cardiac activity can compensate a deficient venous return resulting from hemorrhage, from stagnation of the blood in the widely dilated peripheral vessels (vasomotor shock), or from some mechanical obstruction to the venous flow. The reflex acceleration of the cardiac rate that results is usually ineffective.

Marey noted that increased blood-pressure is followed by slowing of the pulse, and later Eyster and Hooker ${ }^{1}$ showed that this is due to vagus stimulation occurring reflexly when the bloodpressure in the carotids or the aorta is increased, even though changes in the cerebral circulation be excluded. The action of the vagus upon the heart is to diminish the cardiac rate by reducing the activity of the "pace-maker," and therefore to decrease the output per minute, and when extreme, to reduce the conductivity of the auriculoventricular bundle, producing partial or complete block.

1 Zentralbl. f. Physiol., Leipsic u. Wien, 1907, xxi, 615. 
Roy and Adami ${ }^{1}$ have shown by cardiometer studies that with increased aortic pressure, even though the output per minute may be increased, the residual blood (that remaining in the ventricles at the end of systole) is increased and the ventricles become more widely dilated during diastole. Excessive aortic pressure therefore leads to loss of ventricular tone, with dilatation and impairment of cardiac efficiency.

Arteries.-The elasticity of the arteries, by virtue of which they dilate during each systole to accommodate the ventricular output with only a moderate rise of the pressure within them, and then during diastole contract again, forcing a continuous stream through the capillaries and maintaining under normal conditions a fair pressure throughout diastole, is a property of the utmost importance. Without this elasticity the strain upon the cardiac muscle. would be enormously increased, as would be the strain during systole upon the vessel walls. Were the heart pumping into an absolutely rigid system, the cardiac systole would be prolonged, the systolic pressure enormously increased and the diastolic pressure would fall to zero, with a cessation of flow during diastole. The pulse pressure would equal the surstolic pressure. Such an extreme condition never actually exists, but some approach toward it may be seen in individuals with sclerotic arteries in whom often a systolic pressure of 200 may be associated with a diastolic pressure of 130 ; a pulse pressure, therefore, of 70 , as contrasted with the normal pulse pressure of about 30. Obviously this exaggeration of the pulse pressure by rigid vessels will be less marked if the cardiac rate be increased and the output per beat correspondingly diminished. The pulse pressure is not infrequently looked upon as a rough measure of the cardiac activity. This to some extent it is; yet we have just seen it is influenced also to a marked degree by the condition of the arteries. It has been commonly accepted that this elasticity of the arteries, while it results from the tonus of their muscular coats, is to be looked upon as a passive elasticity not unlike that of a rubber tube. Hasebroek $^{2}$ has, however, recently advanced the hypothesis that while the dilatation of the arteries is a passive result of the injected rolume of blood, their contraction is an active muscular act and, indeed, an important adjunct to the heart in the maintenance of blood-pressure and blood-flow. He believes this active arterial contraction to be the chief cause of the dicrotic wave of the sphygmogran.

'British Med. Jour., 1s\$\&, ii, 321.

${ }^{2}$ Deutsch. Archiv f. klin. Med., 1911, rii, 567. 
Arterioles, Capillaries, and Veins.-The smaller arterioles together with the capillaries constitute the main resistance in the circulation, and are chiefly responsible for the proper distribution of the bloodflow to various organs and tissues, as the relative demands of these for blood changes with altering activity. The blood which reaches the smaller arterioles at a mean pressure, but little below that in the aorta, leaves them at a very low pressure, varying with the portion of the body under consideration. Whether this fall of pressure occurs chiefly in the small arterioles or in the capillaries is a matter of dispute. The capillaries together with the veins of the body serve as a reservoir for the blood. Complete loss of tone in these vessels leads to the accumulation in them, particularly in the splanchnic region, of practically all the blood in the body. 'The capillary and venous tone is therefore responsible for maintaining the supply of blood to the right auricle and this, as we have already seen in considering the heart, is of the utmost importance for the maintenance of cardiac output and of blood-pressure.

The Vasomotor System.-In this relation it is the capillaries and veins of the splanchnic area that are of greatest significance. This was shown experimentally by Ludwig and Thiry ${ }^{1}$ in 1864 . These vessels alone are capable of containing all the blood of the body and were they to lose their tone the individual would promptly "bleed to death in his own vessels," as in vasomotor shock. In certain of the lower animals the tone of these vessels is relatively low. The hutch rabbit can be killed by simply holding it by the ears in the vertical position, the splanchnic vessels not having sufficient tone to overcome the force of gravity. In the dog manual pressure over the abdomen will cause a prompt rise of carotid blood-pressure of 20 or $30 \mathrm{~mm}$. of mercury due to the forcing of the blood from the vessels onward to the right auricle. The regulation of the tone of the arterioles, capillaries, and perhaps veins, is the most important factor toward this end. This is brought about by the vasomotor nervous system. The vasomotor system consists of two main parts, the vasoconstrictor system and the vasodilator system.

The Vasoconstrictor System.-The first important experimental studies upon this subject were those of Claude Bernard. ${ }^{2}$ In 1851 he noted the dilatation of the vessels in the ear of the rabbit after cutting its cervical sympathetic nerve on the same sille. Later he

1 Quoted by Howell, Text-book of Physiology, Philadelphia, 1912; Sitz. d. kais. Akad. d. Wiss. math. naturw. Cl., 1864, xlix, 2, p. 442.

${ }^{2}$ Leçons sur les liquides de l'Organisme, Paris, 1859. 
observed that electrical stimulation of the upper end of the cut nerve caused constriction of these dilated vessels. Thus was demonstrated the existence of vasoconstrictor nerves.

The vasoconstrictor system consists of a centre in the medulla which is connected with the ressels of the body by nerve paths, each consisting of three neurons. In the medulla beneath the middle of the fourth ventricle exists the bilateral nerve centre which is chiefly responsible for the maintenance of vasomotor tone. This centre is continuously in a state of tonic activity which at times undergoes rhy thmic fluctuations, giving rise to the Traube waves, to be described. Porter has recently found evidence against the view that both the arterial tonus and the vasomotor reflexes following stimulation of afferent nerves are controlled by the same master cells, the so-called vasomotor centre. He has been able by the use of curare in cats to more than double the sciatic and the depressor reflex change in blood-pressure, while the arterial tonus is left substantially unchanged. Possibly, therefore, we may be compelled to recognize two centres, a vasotonic and a vasoreflex centre, related but separable. ${ }^{1}$ The axons leading from these medullary cells pass down the cord to end around cells of the anterior horn, from the upper thoracic level to the upper lumbar, and constitute, with the medullary cell, the first neuron. The axons of the anterior horn cells are medullated and constitute the second neuron in the chain, the so-called preganglionic fibers. They pass from the cord in the white rami communicantes from the first thoracic to the second or fourth lumbar segments, and entering the sympathetic chain, have one of three destinations. Those destined for the extremities end around cells in the simpathetic chain. The non-medullated fibers of these sympathetic cells constitute the third neuron, the postganglionic fibers. They pass by the gray rami communicantes back to the spinal cord and thence accompany the appropriate spinal nerves to their destination. The preganglionic fibers destined for the deeper vessels of the head pass through the white rami of the first to sixth thoracic segments and up the sympathetic to the superior cervical ganglion. From this point the associated postganglionic fibers are distributed through the carotid and other vascular plexuses. The preganglionic fibers destined for the vessels of the abdominal and pelvic viscera, the most important of all in the regulation of blood-pressure, pass directly through the sympathetic 
chain and by way of the splanchnic nerves to the celiac, inferior mesenteric, or other large prevertebral ganglia. From these ganglia the postganglionic fibers are distributed to the plexuses around the abdominal vessels. Section of the first neuron in this chain, as by section of the cervical cord, leads to extreme loss of vascular tone with fall in blood-pressure, failure of the circulation, and death. If the circulation be, however, artificially maintained after section of the cervical cord, the spinal cells, the second neurons, gradually assume a moderate tonic activity and restore a very imperfect vascular tone. This may in turn be destroyed by destruction of the spinal cord. Finally, Goltz ${ }^{1}$ has shown that even after destruction of the cord, some vascular tone may be recovered which must be attributed to tonic activity either of the third postganglionic neuron or of the musculature of the vessel wall. Excitation of the vasoconstrictor centre with contraction of the peripheral vessels is called forth by the stimulation of pressor fibers, which may be demonstrated in almost any large nerve containing afferent fibers, but especially in the cutaneous nerves. These pressor fibers may be stimulated by electricity, by cold applied to the skin, and in other ways. Depression of the centre with diminished contraction of the vessels is called forth by stimulation of other afferent fibers, called depressor fibers. These likewise may be demonstrated in many of the large afferent nerves. Thus, application of warmth to the skin leads to local vascular dilatation, the result of stimulation of depressor fibers. Sensory stimuli from certain regions, notably the middle ear and the testis, give rise, as a rule, to depressor effects. Electric stimulation of the central end of the cut sciatic or of the splanchnic nerve may lead to either pressor or depressor effects.

Since mental work or especially mental interest leads to a constriction of the bloodvessels with a rise of blood-pressure, it seems likely that pressor and probably also depressor fibers pass from the cortical centres to the vasoconstrictor centre. Medullary anemia and increased $\mathrm{CO}_{2}$ concentration of the medullary blood are both potent stimulants to the vasoconstrictor centre. Nany drugs exert a direct effect upon this centre. Ludwig and $\mathrm{Cyon},{ }^{2}$ in 1866 , demonstrated in the rabbit the so-called depressor nerve of the heart. This is an afferent nerve rumning in the sheath with the vagus, or in other species forming a part of the vagus. Stimulation of its peripheral cut end is without effect, but of its central end, leads to

1 Arch. f. d. gesamt. Physiologie, 1896, lxiv, 397.

2 Bericht d. sächsisch. Gesellsch. d. Wissensch. math. Phys. Cl., 1866, p. 315. 
depression of the vasomotor centre and stimulation of the cardioinhibitory centre, tending to lower blood-pressure. It is supposed to exert a steadying effect upon blood-pressure and to be stimulated mechanically by increase of aortic pressure. The older view that it was stimulated chiefly by intraventricular pressure has been rendered improbable by the work of Eyster and Hooker. ${ }^{1}$ Einthoven ${ }^{2}$ has found an impulse passing up this nerve with every cardiac beat.

The Vasodilator System.-It was likewise Clande Bernard ${ }^{3}$ who first recognized the existence of vasodilator nerves. He noted that stimulation of the peripheral end of the cut chorda tympani nerve caused great dilatation of the bloodvessels of the submaxillary gland, with increased flow through the afferent vein; this vein in some cases actually pulsated. Dilator fibers have been demonstrated in certain of the cranial nerves and in the sympathetic chain. The chorda tympani conveys such fibers from the facial nerve for distribution to the submaxillary and sublingual glands and the anterior twothirds of the tongue. The glossopharyngeal nerve carries such fibers to the posterior third of the tongue, the tonsils, pharynx, and parotid gland.

From the cervical sympathetic dilator, fibers pass to the lips, gums, palate and skin of the face, passing through the Gasserian ganglion and thence with the fifth nerve. From the thoracic sympathetic dilator, fibers pass through the splanchnic nerves to the abdominal viscera. From the first to third sacral segments of the cord dilator, fibers pass to the hypogastric plexus, whence as the nervi erigentes they supply the penis, and when stimulated cause erection. The existence of dilator fibers to the limbs, first upheld by Goltz, has been renlered uncertain by the work of Bayliss. ${ }^{4}$ Both types of efferent fibers, vasoconstrictor and vasodilator, may exist in the same nerve, for example, in the splanchnic. As a rule these fibers react to different types of electrical stimulation, the dilators responding to weaker and to more slowly interrupted currents, responding less promptly, and once having done so, showing a more prolonged reaction than do the constrictor fibers. Moreover, a few days after section of such a nerve the irritability of the constrictor fibers is lost, while that of the dilators is still retained.

There is no evidence that there exists one general vasodilator centre associated with the dilator nerves, but rather that there are many centres through the cerebrospinal axis for the various areas

1 Loc. eit.

2 Quoted by Howell.

${ }^{3}$ Loc. cit.

${ }^{4}$ Jour. of Physiol., 1900, xxvi, 173; ibid., 1902, xxviii, 276. 
supplied. Probably the vasodilator system is of less significance in the regulation of the general blood-pressure, but is concerned in local augmentation of blood-flow in response to local needs as in increased glandular or muscular activity. So far as we know there is no tonic activity of the vasodilators. How the stimulation of the dilator fibers leads to dilatation of the vessels is a subject of speculation. Howell has contended that it is probably by direct inhibition of the muscles in the vessel walls. Vasomotor nerves have been shown to exist in all vessels except those of the heart, the lungs, and the brain; concerning these three organs the matter is still unsettled.

Chemical Regulation.-The possibility of a chemical regulation of vascular tone has attracted some attention. Gaskell has shown that acids in low concentration cause vascular dilatation, and in this connection the local action of lactic acid and carbon dioxide generated during muscular activity has been suggested.

The idea that a continued pouring of epinephrin from the adrenal glands into the circulation is a factor in the maintenance of normal vascular tone is now questioned. Hoskins and McClure ${ }^{1}$ have obtained no fall in blood-pressure after ligation of the adrenal vessels. They have also found that an amount of epinephrin in the circulation sufficient to affect the blood-pressure is enough to cause suppression of intestinal peristalsis. The various studies tending to show by one test or another that there is adrenalin demonstrable in the circulating blood have been shown by Stewart, O'Connor, Schultz, and Janeway and Park to be based on unreliable methods, and as Janeway and Park ${ }^{2}$ assert, at the present time there is no evidence that epinephrin, in amounts sufficient to produce its physiological effects upon any hitherto used test objects, exists in the circulating blood, with the exception of the blood from the suprarenal vein. Cannon's ${ }^{3}$ recent work, indicating that after excitement in cats there is an increased output of adrenal secretion, suggests that this may be a factor in the rise of general arterial pressure incident to excitement or exertion. As to the action of other organs of internal secretion upon the maintenance or regulation of blood-pressure the findings are inconclusive. Extracts of the pituitary gland produce on injection a brief fall in blood-pressure, followed by a rise. The depressor substance is related to cholin. ${ }^{4}$ The pressor

1 Amer. Jour. Physiol., 1911-12, xxx, 192; ibid., 1912-13, xxxi, 59.

${ }^{2}$ Jour. Exper. Med., Lancaster, 1912, xvi, 541.

${ }^{3}$ Amer. Jour. Physiol., 1913, xxxii, 44.

${ }^{4}$ C. J. Wiggers, Amer. Jour. Med. Sci., 1911, exli, 502. 
substance differs somewhat in its effects from that obtained from the adrenals. Extracts of various mammalian tissues, thyroid, liver, pancreas, thymus, testis, bone-marrow, the intestine from various levels, parathyroid, brain, prostate, ovary, in the hands of most observers produce on injection a fall of blood-pressure. ${ }^{1}$ This fall may be due in some instances to the presence of cholin. A pressor substance has been obtained from the kidney, and certain observers with some of the above-mentioned organs from certain mammals have obtained pressor instead of, or in addition to, depressor effects. This action of tissue extracts from such diverse sources is not, however, to be understood to mean that these organs are during life pouring into the blood-stream substances serving to influence and regulate blood-pressure. We have no proof that the substances extracted from these organs ever pass normally from them into the blood-stream, nor that they have any thing to do with the regulation of blood-pressure.

The Blood and the Lymph. - Within a wide range the tonus of the capillaries and veins compensates for changes in the total volume of blood. Thus, 2.8 per cent. of the body's weight, or about onethird of the entire volume of an animal's blood, may be withdrawn without any fall in the arterial pressure. Upon the withdrawal of a somewhat larger quantity, however, the capillaries and veins no longer can maintain an arlequate supply to the right auricle. Dimimution in the cardiac output results and the arterial pressure falls.

The effect on blood-pressure of the infusion of normal saline intravenously depends upon whether the pressure is normal or low at the time of the introduction. If the pressure be normal, Colnnheim showed that large quantities of normal saline may be introduced intravenously with only very slight and transitory elevation of blood-pressure. This is bccause the fluid introduced is rapidly removed by the kidney's and intestines. When, however, the bloodpressure is much below normal, either as the result of loss of vasomotor tone or of hemorrhage, the effect of introduction of normal saline is considerable and is persistent. The injection of defibrinated blood, however, as Mall has shown, always produces a marked rise of blood-pressure. It is possible by this means to raise and maintain the aortic pressure very considerably above normal. This is probably due to the content of colloidal substances in the blood which cannot be removed by kidney or intestine and which, 
being retained, retain the fluid also. Knowlton ${ }^{1}$ has shown that intravenous injection of colloidal solutions, such as gelatin, causes a similar persistent rise of blood-pressure without diuresis.

That the lymphatic system to some extent acts as a regulator of the amount of blood is unquestionable. Its action, however, is not very rapid and is limited in extent. It has been demonstrated that a rise in arterial blood-pressure is associated with increase in the proportion of erythrocytes, leukocytes, and dry substance of the blood, while a fall in arterial blood-pressure results in decrease in the proportion of these elements. This is probably largely to be explained by passage of the fluid elements of the blood into the lymphatic channels or vice versa-a transfer which has been supposed to take place chiefly in the lungs.

Viscosity of the Blood.-Increase in the viscosity of the blood increases the resistance to its passage through the vessels and in consequence tends to the elevation of blood-pressure, and vice versa. Clinically, thus far, no definite relationship has been established between the degree of hemic viscosity and the height of bloodpressure.

Blood-pressure and Blood-flow.-The purpose of blood-pressure is the maintenance of blood-flow. The rate of blood-flow between two points is determined solely by the difference of blood-pressure between these points and by the resistance to be overcome in the comnecting vessels. An increased difference of blood-pressure between two points with the same resistance must result in an increased blood-flow from the one to the other. Unaltered difference of blood-pressure between two points with diminished resistance will likewise result in an increased blood-flow from the one to the other. Increase in the physiological activity of any organ of the body demands an increased blood-flow through the organ. Practically all physiological changes in the resistance in the arterioles and capillaries, and in the arterial blood-pressure, are to be looked upon as the normal mechanism for securing the proper distribution of blood to the various organs and tissues of the body.

It is not to be supposed, however, that blood-flow is proportional to blood-pressure alone. Indeed, abnormally high blood-pressure is associated frequently, perhaps usually, with a diminished bloodflow due to an abnormal degree of resistance in the peripheral vessels. Nor is high blood-pressure to be considered an indication 


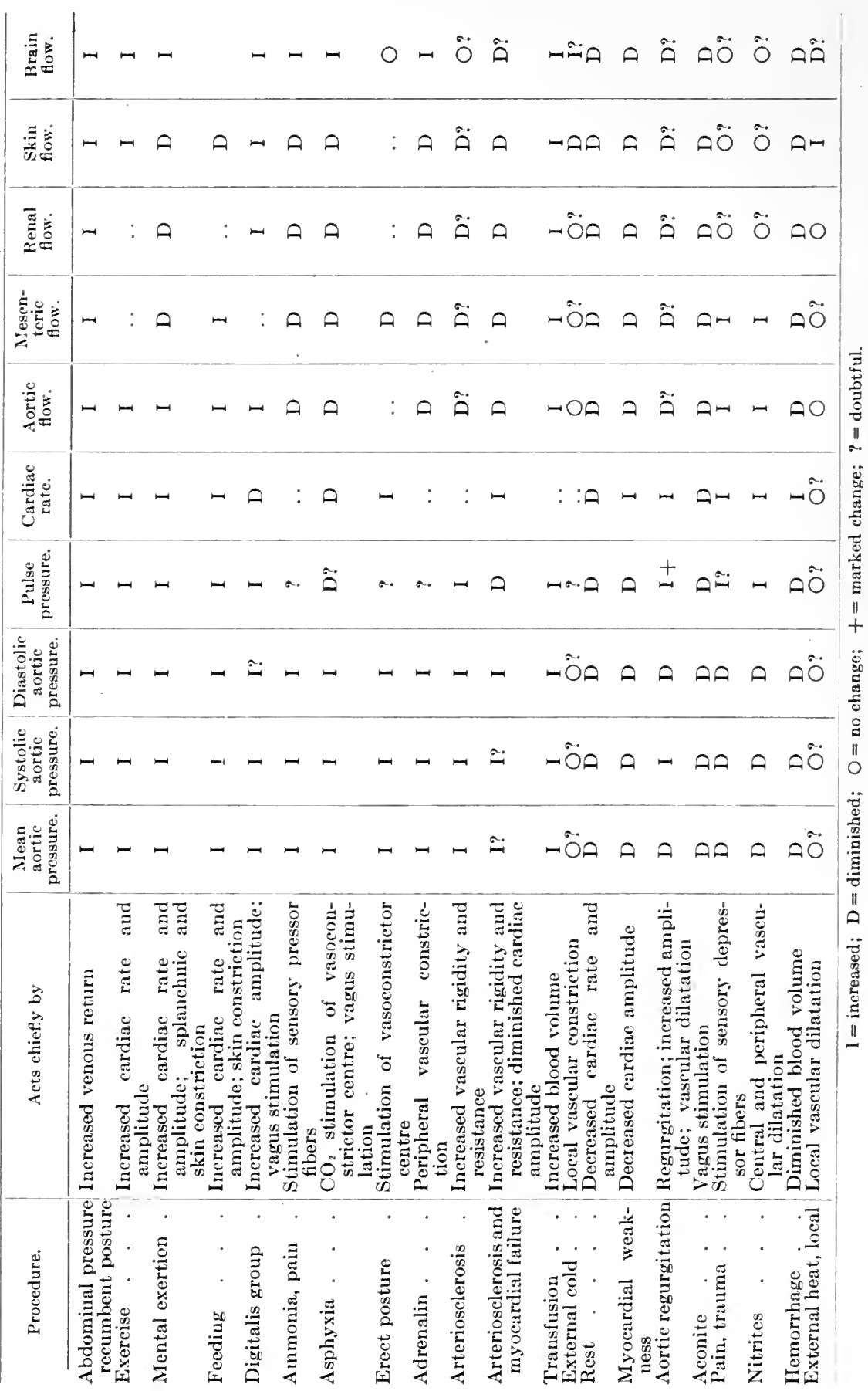


of normal cardiac power, since all the reserve power of the cardiac muscle may have been exhausted in maintaining this very pressure, and the heart may be at the point of failure with beginning dilatation and yet the pressure may still be maintained above normal.

These relations between blood-pressure and blood-flow are indicated somewhat crudely in the accompanying table of the typical effects of various procedures upon blood-pressure and upon bloodflow, both total and in certain vascular areas. It will be noted that there are conditions which alter the blood-flow through the skin and extremities in an opposite sense from that of the total blood-flow. This fact must be kept clearly in mind in employing those methods in clinical use for estimating blood-flow through the hand and arm.

Systolic, Diastolic, and Pulse Pressure.-In the discussion so far we have considered chiefly the effects of various factors upon mean blood-pressure and the relation of mean blood-pressure to blood-flow. Inasmuch, however, as in clinical work it is not the mean pressure but the systolic and diastolic pressure that we measure, the relation of these to the mean pressure under various circumstances must be considered. As already stated, there is no constant numerical relation between these three phases of blood-pressure that makes it possible from any two to calculate the third. It may be affirmed, however, that the mean pressure follows more closely in its fluctuations the diastolic pressure than it does the systolic, and the study of the diastolic pressure is therefore in many ways of greater value than is that of the systolic. Dawson's ${ }^{1}$ rule for roughly estimating the mean pressure in man is to add one-third of the pulse pressure to the diastolic pressure. The effect of various procedures upon these phases of blood-pressure, upon cardiac rate and upon bloodflow through various vascular areas, is shown in the accompanying table. It will be noted that the systolic, diastolic and mean pressures are altered in the same direction by most procedures-not, however, always to the same extent. Under some circumstances, notably in aortic regurgitation, this, as will be seen from the table, is not the case. In the average normal individual at rest the diastolic is 70 per cent. of the systolic pressure, but in not a few normal cases it may be as high as 85 per cent.

Pulse Pressure.-The pulse pressure is with qualifications an indication of the cardiac output per beat. As a rule increased ventricular output leads to increased pulse pressure and vice versa. 
The pulse pressure normally ranges between 35 and $50 \mathrm{~mm}$. Hg. With very few exceptions it should not fall below 20 per cent. of the diastolic pressure. The attempt has been made to establish formulas enabling one from the pulse pressure and the cardiac rate to estimate alterations in the cardiac output per minute-in other words, of the blood-flow from the heart. The accompanying table shows that, as a rule, changes in pulse pressure and aortic flow are in the same direction, but that this is not always true, for aortic regurgitation and so common a condition as arteriosclerosis tend to produce alterations of these factors in contrary directions. Moreover, only under very limited conditions is there any constant quantitative relation between the respective changes in these factors. Hence all attempts aiming to express in figures the bloodflow on the basis of the pulse pressure should be regarded with suspicion.

Effects of Respiration on Blood-pressure.-Ludwig, ${ }^{1}$ in 1847, first showed that respiration affects the aortic pressure. The following analysis of these effects is from Tigerstedt. ${ }^{2}$

When the vagi are cut and the lungs are being artificially ventilated, the capillaries of the lungs are compressed as the lungs are inflated, and dilated as the lungs are emptied. The first effect of inflation is therefore to drive the blood from the pulmonary capillaries on into the left ventricle $(A)$ with increased output and rise of aortic pressure. The capillary constriction then acts as a dam to further transmission of blood, the inflow to the left ventricle diminishes and aortic pressure falls $(B)$. This is still more marked when the lungs are first deflated and the eapacity of the pulmonary capillaries is increased $(C)$. Once they become filled, however, they readily transmit a liberal supply to the left heart and the aortic pressure rises $(D)$, to be still further increased as inflation again begins $(A)$. If respiratory movement be made very rapid, the phases $B$ and $D$ are eliminated and the highest pressure comes at the end of inspiration, the lowest at the end of expiration (Fig. 7).

In natural respiration the relations are altered. During inspiration the pulmonary capillaries are first distended and while filling with blood retard its onflow $(A)$, but when dilated permit a ready flow through to the left heart $(B)$; with the beginning of expiration they are compressed, emptied into the left heart with elevation of

${ }^{1}$ Arch. f. Anat. u. Physiol., 1847, vi, 242.

${ }^{2}$ Lehrbuch des Kreislaufes, 1893. 
the aortic pressure $(C)$, but during the remainder of expiration they offer considerable resistance to the onflow of the blood (D) (Fig. 8).

Erlanger and Festerling ${ }^{1}$ find the phases $A$ and $C$ so long as to almost do away with phases $B$ and $D$. They note toward the end of inspiration an acceleration of cardiac rate which tends to increase aortic pressure, and toward the end of expiration a retarding of cardiac rate.

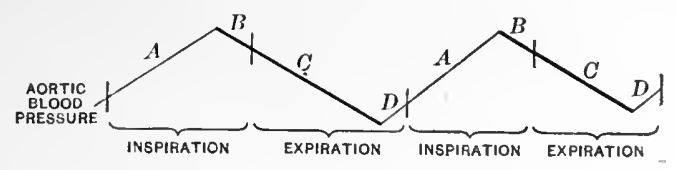

FIG. 7

These changes in cardiac rates, first noted by Ludwig, do not occur if the vagi have been cut; they are therefore reflex phenomena. Einhodt, Talma, Kronecker and Heinricius believe the aspiration of blood into the thorax during natural inspiration tends to increase diastolic filling, and diminish systolic emptying with a lowering of blood-pressure and, vice versa, in expiration. Fredericq has shown that if in an animal with artificial respiration and cut vagi and phrenics the artificial respiration be suspended, respiratory movements of the thoracic wall occur. These are, of course, without effect either on the pulmonary blood-flow or ventilation. However, during the inspiratory movement a fall of aortic pressure

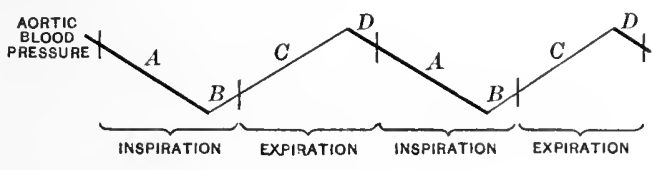

Fig. 8

occurs; during the expiratory movement, a rise. These changes can only be explained as effects arising from the vasomotor centre which induce alterations in peripheral resistance. There is therefore a coördination between the three centres, the respiratory, cardio-inhibitory and vasomotor as follows:

Respiratory.

Inspiration

Expiration
Vasoconstrictor.

Inhibition (dilatation)

Stimulation (constriction)
Cardio-inhibitory.

Cardiac acceleration

Cardiac retardation

1 Jour. Exper, Med., 1912, xv, 370. 
Obviously the changes in the cardio-inhibitory and vasomotor centres have opposite effects on the blood-pressure. Fredericq's ${ }^{1}$ experiment shows that these changes are not due, as Schiff supposed, to changes in the gases of the blood, the result of pulmonary ventilation.

The inspiratory fall of the diaphragm raises abdominal pressure, and serves as an adjunct to the aspirating action within the thorax in sending the blood from the abdomen into the thorax.

Henderson has concluded from recent experimental studies that the variations in arterial pressure are of the same character and bear the same relations to the phases of respiration in an animal which is breathing spontaneously after the chest has been opened, as in a subject with the thorax intact. This would argue against the importance of the changes in intrathoracic pressure as an explanation of the respiratory changes in arterial pressure. He concludes that with normal venous pressure, the respiratory variations of blood-pressure are always accompanied by and are due to associated changes in pulse rate, accelerated rate causing higher pressure. On the other hand, with a deficient venous pressure, such as exists frequently under experimental conditions, after hemorrhage or in shock, forcible expiratory contractions of the diaphragm may increase the venous pressure sufficiently to more than compensate for a slower pulse rate during expiration.

In animals under experimental conditions, the pulse as already noted, usually quickens during inspiration and slows during expiration. In man not only this relation but, according to Putzig, ${ }^{2}$ the exact opposite and many intermediate synchronisms likewise occur. Henderson and Barrington find, however, that in the large majority of their studies both from man and animals, no matter what the relation of pulse rate to respiratory phase may be, arterial pressure rises with the cardiac acceleration and falls with retardation.

Summing up the effects of respiration on the cardiovascular system under normal conditions we find:

Inspiration: First, fall of aortic pressure; second, cardiac acceleration, possibly slight rise of aortic pressure.

Expiration: First, rise of aortic pressure; second, cardiac retardation, possibly slight fall of aortic pressure.

These fluctuations of pressure, synchronous with respiration, con-

1 Arch. Ital. de Biologie, 1882, iii, 55.

${ }^{2}$ Ztschr. f. Exper. Path. u. Therap., 1912, xi, 115. 
stitute the second order of blood-pressure oscillations. In normal individuals these waves are a negligible factor in clinical observations, but in labored respiration they may amount to 8 to $10 \mathrm{~mm}$.

Traube-Herring Waves.-These were first noted by 'Traube. ${ }^{1} \mathrm{He}$ observed that in a curarized animal with artificial respiration, if the respiration was suspended, the aortic pressure rose and remained high for two or three minutes and that during that time, without any movements of the animal or any respiratory movement, the arterial pressure showed slow waves, about seven per minute, that might have an amplitude of $40 \mathrm{~mm}$. of mercury. The cardiac rate remained unaltered. After two or three minutes the arterial pressure began to fall and from this moment these waves ceased, or at most a few feeble ones were observed. If, now, artificial respiration were resumed, these slow waves, perhaps only two per minute, might again for a while be observed, the waves due to the artificial respiration and to the cardiac cycles being superimposed upon them. He considered these waves the result of the stimulus of $\mathrm{CO}_{2}$ upon the vasoconstrictor centre, and succeeded in producing them by maintaining respiration with an atmosphere containing 20 per cent. of $\mathrm{CO}_{2}$. The tracing in Fig. 6 illustrates these oscillations.

Intrapericardial Pressure.-If a cannula be introduced into the pericardium of an animal, the pericardium ligated around the cannula and normal saline introduced in. such a way that its pressure may be measured and controlled, it will be found that a very slight positive intrapericardial pressure will cause a fall of aortic bloodpressure with a diminished cardiac output. When the pressure rises to even so low a figure as 8 to $10 \mathrm{~mm}$. Hg. the cardiac output becomes practically nil and there is a rapid extreme fall of aortic blood-pressure. This phenomenon is due undoubtedly to occlusion of the great veins supplying the auricles, by an intrapericardial pressure exceeding the venous pressure.

Intra-abdominal Pressure.-If, while recording the carotid or femoral pressure of a dog, pressure be exerted over the abdomen with the hand or in any other way, the arterial blood-pressure will be seen to rise 10 to $20 \mathrm{~mm}$. Hg. and to remain at a somewhat elevated level while the pressure is maintained. This is due to the fact that, as already noted, the splanchnic vessels constitute the great reservoir for the blood. Increase of intra-abdominal pressure tends to empty these vessels onward into the right auricle,

1 Cent. f. d. med. Wiss., 1865, iii. 881 . 
and hence by increasing the venous supply to the heart to increase the cardiac output. At the same time the additional pressure increases somewhat the resistance offered to flow through the mesenteric capillaries, and hence resistance of the most important vascular area from the stand-point of the determination of bloodpressure. By both means increased intra-abdominal blood-pressure leads to increased arterial blood-pressure. Conversely, relaxation of the abdominal walls favors low arterial blood-pressure with stasis of the blood in the splanchnic area, but this tendency may be entirely counterbalanced by adequate vasomotor tone in these vessels.

Burton-Opitz ${ }^{1}$ has shown that an increased intra-abdominal pressure up to 20 to $30 \mathrm{~mm}$. $\mathrm{Hg}$. causes an increased blood-flow and bloorl-pressure in the carotid arteries and external jugular veins and also an increased blood-pressure in the femoral artery. There is evidently brought about, therefore, a shifting of the bloor from the splanchnic to the peripheral circulation and especially to the head under such conditions.

Extremely high intra-abdominal pressure, as from a very large ascites, may interfere with the return of blood to the heart, and lower arterial blood-pressure.

Blood-pressure and Intracranial Pressure.-The brain is enclosed in a bony encasement and is, so to speak, floating in the cerebrospinal fluid. This fluid fills the subarachnoid space, the ventricles and their communicating passages; all these spaces are normally in free communication.

Evidence indicates that the pressure of the cerebrospinal fluid is always equal to the venous pressure in the brain sinuses. This, pressure may vary from 0 in the upright position (Hill) ${ }^{2}$ to 50 or $60 \mathrm{~mm}$. Hg. in the convulsions of strychnine poisoning, or even more in cases of brain tumor. If the intracranial pressure exceeds the carotid pressure the circulation through the brain becomes entirely checked, due to compression of the capillaries. Complete occlusion of the larger cerebral arteries occurs, however, only when the intracranial pressure is raised (experimentally) to about 150 $\mathrm{mm}$. Hg. above the carotid pressure (Eyster, Burrows and Essick). ${ }^{3}$ The cerebral anemia produced by a rise of intracranial pressure

1 The Carotid Blood Flow in Relation to the Intra-abdominal Pressure, Amer. Jour. Physiol., 1914, xxxvi, 64.

${ }_{2}$ The Physiology and Pathology of the Cerebral Cireulation, London, 1896: Proc. Royal Soc., 1894, Iv, 52.

3 Jour. Exper. Med., 1909, xi, 489. 
to the level of the carotid pressure was shown by Cushing ${ }^{1}$ to produce two reflex effects: (1) stimulation of the vagus with slowing of the cardiac action; (2) stimulation of the vasomotor centre, with general vasoconstriction and rise of carotid pressure to the level of the intracranial pressure, and in consequence the reëstablishment of the cerebral circulation. If the increase of intracranial pressure be very gradual the vagus excitation may not be elicited. Cushing likewise studied the effects of localized pressure upon portions of the brain and obtained various results dependent upon the location selected. Pressure upon the medullary centres acts as does increase in general intracranial tension. The elevated blood-pressure associated with intracranial pressure secondary to neoplasms of the brain is to be considered a compensatory and beneficent process for the maintenance of blood-flow through the vital centres. If this compensatory rise does not occur, absolute cerebral anemia results. The time that different portions of the brain can endure anemia and recover varies greatly. Experiments by Crile and Dolley showed that the central nervous system of young animals endures anemia better than that of older ones. These parts of the brain, which preside over conscious life, the higher psychic centres, were in no instances resuscitated after total anemia of eight minutes, but always after only four minutes. The vasomotor centre was frequently resuscitated after. fifteen minutes, occasionally after eighteen, once after twenty, and once in a puppy after thirty minutes. The respiratory centre was more resistant, moderate reaction being obtained after as much as forty minutes of total anemia. We may say, therefore, that reduction of arterial pressure results in lessened blood supply and, as a rule, diminished activity within the brain; that if this reduction be great enough, complete anemia of the brain results with cessation of its activities, and unless the blood-supply be promptly restored, a permanent cessation; also that the arterial blood-pressure, so far as it concerns the brain, must be considered with relation not to the atmospheric, but to the intracranial pressure, hence a rise of intracranial pressure is equivalent to a fall of blood-pressure, and vice versa.

Blood-pressure and Intra-ocular Pressure.-The chambers of the eyeball are filled with fluids which distend the eyeball and maintain a pressure in these cavities normally of about 20 to $30 \mathrm{~mm}$. $\mathrm{Hg}$. in man. The intra-ocular pressure is measured by one of two methods:

${ }^{1}$ Mitteil. aus den Grenz. der Med. u. Chir., 1902, ix, 791: Amer. Jour. Med. Sci., 1903, exxv, 1017 . 
(1) Ophthalmomanometry, in which the pressure is measured directly by introducing a hollow needle into one of the chambers, preferably the anterior, and connecting it with a suitable manometer or system of manometers; (2) ophthalmotonometry, in which the degree of internal pressure is estimated by the amount of resistance to deformation offered by the walls of the eyeball. Obviously only the second method is, as a rule, applicable to man; it has, however, certain fallacies and objections among which are the error introduced by the inherent rigidity of the ocular walls, that due to the increase of intra-ocular pressure caused by the distortion of the eyeball, as well as the dangers and discomforts of the application of the instrument. These errors in the latest instruments are, however, largely eliminated. The pressures in the aqueous and vitreous chambers of the eve are practically identical, not differing by more than $1 \mathrm{~mm}$. Hg. The distensibility of the walls of the eyeball is so slight that under ordinary conditions any increase in the fluids within the eyeball, either in one of the chambers or in vessels of the choroid, must cause an increase in the intra-ocular pressure. This fact leads to almost complete suppression of the visible pulsation of the retinal arteries. Likewise the pressure in the bloodvessels, including capillaries and veins, can never fall below the intra-ocular pressure without collapse of these vessels and cessation of the flow through them. Since the outflow from the veins of the eve is usually a steady stream the venous pressure must be normally equal to the intra-ocular pressure. The capillary pressure within the eye is supposed by Parsons ${ }^{1}$ to be 40 to $50 \mathrm{~mm}$. $\mathrm{Hg}$., and that in the veins to equal the intra-ocular pressure.

The fluids distending the eyeball circulate along one of two courses: (1) That of the blood entering the ciliary arteries and leaving by the scleral veins, the venæ vorticosæ; (2) that of the lymph fluids filling the vitreous and aqueous chambers, entering the vitreous chamber from the vessels of the ciliary processes, passing into the aqueous chamber and leaving at the filtration angle by the spaces of Fontana for the canal of Schlemm and thence into the scleral veins. As long as the intra-ocular tension is to remain constant the combined inflow along these two courses must equal the combined outflow. All evidence indicates that the rate of secretion of lymph into the posterior chamber is proportional to intracapillary pressure in the eyeball. Therefore an increase of

'Diseases of the Eye, Philadelphia, 1912. 
intracapillary pressure not only tends to increase intra-ocular tension directly, but also indirectly by increasing lymph production. Rapid changes in intra-ocular pressure are probably due, as a rule, to changes in intravascular pressure and volume; slower changes may be due to alterations in the rate of lymph formation or removal. It is possible, as shown by ophthalmoscopic examination, to have distention of the retinal arteries and veins with normal intraocular tension due to compensatory adjustment in the quantity of lymph. As a rule, however, the intra-ocular pressure is closely related to the circulatory conditions in the eyeball.

The Intra-ocular Pressure Rises.-1. With increase in the arterial pressure in the arteries supplying the eyes, as from compression of the thoracic aorta, stimulation of the vasomotor centre or of the splanchnic nerves, asphyxia, general effects of adrenalin, injection of normal saline, etc. At times the intra-ocular tension may be shown to vary with the respiratory or the Traube-Herring bloodpressure waves.

2. With local dilatation of the arterioles of the eyes. The existence of vasomotor fibers for these vessels has not as yet been satisfactorily demonstrated, since changes in the intra-ocular pressure following stimulation or section of various nerves may in all cases be attributed either to effects upon the general blood-pressure, or to stimulation of the extra-ocular muscles ( $v$. infra).

3. From venous obstruction. Rise in general venous pressure is probably never sufficient to counterbalance the contrary effects of the associated fall of arterial pressure. Moreover, the relatively high venous pressure within the eyeball renders considerable changes in general venous pressure of but slight moment. On the other hand, local venous obstruction, as by ligation of the venæ vorticose, leads to marked rise of intra-ocular pressure (up to $90 \mathrm{~mm}$. Hg.), (Adamük, Leber, Koster, Gzn) with increased transudation of highly albuminous lymph. There follows a slow return to normal pressure in the course of a few weeks.

4. From increased lymph formation from any of the abovenamed causes of increased capillary pressure.

5. From decreased lymph drainage either from venous obstruction or from obstruction of the filtration angle, as with pupillary dilatation in glaucoma.

The intra-ocular tension falls as the result of the reverse of the above-mentioned factors:

1. From fall in the blood-pressure in the arteries supplying the eyes. 
2. From local arteriolar constriction such as may be obtained from adrenalin (Parsons) or nicotin (Henderson and Starling) by injection into the carotid, their local effects preceding their general systemic effects.

3. From diminished lymph formation secondary to any of the above-named causes of reduced blood-pressure.

4. From improved lymph drainage, as by freeing of the filtration angle by pupillary contraction.

The Relation of Blood-pressure to Secretion.-The activity of all glands is associated with increased blood-flow through the gland. This increased blood-flow that is associated with glandular activity was early noted in connection with chorda tympani stimulation and the resultant abundant flow of saliva. It was naturally suggested that the abundant flow of saliva might be entirely the result of the increased circulation and capillary blood-pressure in the gland, due to the vasodilator fibers in the chorda tympani. Ludwig showed, however, by measuring the pressure in the salivary duct by means of a manometer that following stimulation of the chorda tympani the pressure in the salivary duct might exceed the bloorpressure. Moreover, if blood-pressure be shut off from the gland, stimulation of the chorda still gives secretion for a short time, whereas if atropin be injected into the gland, chorda stimulation causes dilatation of the vessels and increased blood-flow but no secretion. Hydrochlorate of quinine injected into the gland causes vascular dilatation but no secretion. Therefore, while an abundant blood-flow is essential for the proper functioning of actively secreting glands, it is not in itself the cause of the increased secretion.

Effects of Exercise on Blood-pressure.-The effect of exercise on bood-pressure has been studied by many. One of the first investigations was that of Mare ${ }^{2}$ who measured the systolic pressure in the carotid of a horse before and after a run of ten minutes and noted a fall from $108 \mathrm{~mm}$. Hg. before to 102 after. Kaufmann ${ }^{3}$ likewise found after prolonged exertion in horses a fall of pressure. Almost uniformly, however, the other observers, investigating for the most part the dog and man, have noted a rise in pressure after exercise. Tangl and Zunst ${ }^{4}$ measured the maximal and minimal carotid pressures in the $\log$ and from them calculated the approximate mean pressure. After having the animal run up steps they

${ }^{1}$ L. Hill and M. Flack, Proc. Royal Soc., London, 1912, S. B. lxxxv, 312

2 Traveaux du Laboratoire, 1876.

${ }^{3}$ Arch. f. d. ges. Phrsiol., 1892, liii.

4 Ibid., $1895,1 \times x, 544$. 
found a rise of from 6 to $23 \mathrm{~mm}$. Hg. After very severe exertion the elevation was extreme, amounting to $120 \mathrm{~mm}$. Hg. Experimenting upon himself, von Basch $^{1}$ had, in 1887, noted after a quick tenminute climb up a hill a rise from $125 \mathrm{~mm}$. Hg. to $180 \mathrm{~mm}$. Voll Maximowisch and Rieder ${ }^{2}$ studied the effect on 27 individuals of $2500 \mathrm{~kg}$. of work performed on a Gärtner ergostat in three to five minutes and they obtained in 21 individuals a rise; in 5, no change; and in 1, a fall of systolic pressure; the greatest rise was $50 \mathrm{~mm}$. Hg.; the one fall was $20 \mathrm{~mm}$. Oertel ${ }^{3}$ in 8 individuals after mountain climbing noted a rise in all, varying from 3 to 43 $\mathrm{mm}$, the average being $17 \mathrm{~mm}$. A rise after exertion has likewise been noted by Zadek, ${ }^{4}$ Friedmann, Grebener ${ }^{5}$ and Grünbaun, ${ }^{6}$ Edgecomb and Bain, Eichberg,7 Russell, Williamson, ${ }^{7}$ Routhier and Boussaguet, ${ }^{9}$ and Graupner. ${ }^{10}$ Moritz ${ }^{11}$ obtained somewhat similar results. Bruck ${ }^{12}$ has noted in man with the Riva-Rocci and with the Hürthle apparatus an immediate rise upon commencing sudden severe exertion, up to 30 or $40 \mathrm{~mm}$. Hg., followed after a few pulse beats by a fall to subnormal and then a prompt return to from 18 to $38 \mathrm{~mm}$. above normal. This brief primary rise he attributes to increased intrathoracie pressure. He measured the intrathoracic pressure by introducing a rubber bag into the esophagus and found it at the time of the primary rise of bloodpressure to be from 50 to $130 \mathrm{~mm}$. Hg. This pressure forces the thoracic blood on into the left ventricle and hence into the aorta with rise of pressure. The following brief fall he attributes to interference with the entrance of more blood into the thorax.

Erlanger and Hooker, ${ }^{13}$ making graphic records of maximal and minimal pressure in man, find that very moderate muscular exertion as, for example, walking, may diminish the minimal pressure while increasing the pulse pressure, pulse rate, and presumably the blood-flow. More severe muscular exertion increases these and in addition the minimal pressure.

Probably the chief cause of the increased pressure accompanying exercise is increased cardiac activity. A factor in influencing

${ }^{1}$ Berl, klin. Woch., 1887, xxiv, 206. ${ }^{2}$ Deutsch. Arch. f. klin. Med., 1890, xlvi.

${ }^{3}$ Therapie d. kreislaufstörüngen, v. Ziemssen's Handbuch d. allg. Therapie, iv.

"Zeitsch. f. klin. Med., 1881, ii, 509. ‘ Wien. med. Jahrbuch., 1882, p. 197.

${ }^{6}$ Wien. med. Presse, 1899, xl, 49. T. Jour. Amer. Med. Assoc., 1908, li, 1000.

${ }^{8}$ British Med. Jour., 1909, i, 530.

${ }^{9}$ Compte rend. de la Soc. de Biol., Paris, 1910, Ixviii, 1037.

to Deutsch. med. Woch., 1906, xxxii, 1028.

$"$ Deutsch. Arch. f. klin. Med., 1903, Ixxvii, 339.

12 Ibid., 1907, xei, 171.

13 Johns Hopkins Hospital Reports, 1904, xii, 53. 
greatly the rise of pressure accompanying exertion is the degree of mental effort involved. An unusual form of exertion or one requiring close attention causes much more increase of pressure than the same amount of work performed in the course of habitual action. Thus, Karrenstein ${ }^{1}$ found in 172 observations on 72 soldiers making a $5.7 \mathrm{~km}$. mareh in seventy minutes, that 39.5 per cent showed a rise, 16.3 per cent. no change, and 44.2 per cent. a fall; the average pressure before and after the march was unchanged. Of 39 observations on 25 soldiers following a two to three hours' mareh, 12.8 per eent. showed a rise and 69.2 per cent. a fall, the average being lowered from 106.1 to $98.8 \mathrm{~mm}$. $\mathrm{Hg}$. In these eases little if any mental effort was involved. The effect of mental eflort has been shown by Putermann, ${ }^{2}$ who studied with the Gärtner tonometer the blood-pressure of 43 boys immediately before and after a school examination. A rise oceurred immediately before the examination in 37 , a fall in 4 . The rise was usually 10 to 20 $\mathrm{mm}$. Hg., but one rise of $50 \mathrm{~mm}$. was noted. Any excitement may lead to a similar rise; indeed, the mere taking of the blood-pressure in a susceptible subject. This psychie rise affeets the systolic pressure more than the diastolie. The acceleration of the pulse during exereise is due to depression of the eardio-inhibitory eentre. It seems that the secondary fall of pressure due to peripheral vasodilatation is the result of depression of the vasoconstrietor centre and not of active vasodilatation, and that a similar depression of the respiratory centre may oceur from cortical inhibition. ${ }^{3}$

Effects of Posture.-It has already been noted that the chief reservoir for the blood is the splanchnic area which of itself can eontain all the blood of the body. Normally, an adequate return of blood from this reservoir to the right auriele is seeured by means of the vasomotor tone of the splanehnic vessels. In the upright posture this return must occur against the force of gravity. In the recumbent posture, the antagonistie action of gravity is removed. Normally in man the reflex regulation of the splanchnie vasomotor tone is such that change from the recumbent to the upright posture is immediately compensated by an increased splanchnic vasomotor tone, and the aortic blood-pressure is maintained almost unaltered or there may be noted even a eonsiderable rise of the diastolic pressure. In subjects with defective vasomotor compensation,

${ }^{1}$ Ztschr. f. klin. Med., Berlin, 1903, 1, 322 (bibliography).

2 Wien. med. Woch., 1904, liv, 265.

${ }^{3}$ Martin and Gruber, The Influence of Muscular Exercise on the Activity of Bulbar Centres, Amer. Jour. Physiol., 1913, xxxii, 315. 
however, there may occur a fall of the aortic blood-pressure upon transition from the recumbent to the upright posture, and this may be of some diagnostic value. The common treatment of syncope by placing the patient recumbent has for its basis the effort to make gravity assist instead of resist the action of the splanchnic vasomotor tone, which in this condition as in surgical shock and collapse, is inadequate. Change from sitting posture to standing causes, normally, according to most observers, a rise of from 5 to $15 \mathrm{~mm}$. $\mathrm{Hg}$. of systolic brachial pressure. Erlanger and Hooker, studying the minimal and pulse pressures, found usually but not constantly on assuming the standing posture a rise in the minimal pressure, but a slight fall in pulse pressure. Karrenstein ${ }^{1}$ noted a little lower brachial systolic pressure in recumbent than in sitting posture. On stooping he found in 90.8 per cent. of 153 cases a rise of from 1 to $45 \mathrm{~mm}$. (average 10.8). The effect of posture upon vessels not at the level of the heart has been considered (p. 23). Barach and Marks ${ }^{2}$ upon changing the posture from the upright to the recumbent in an entirely passive manner in forty-eight subjects between fifteen and thirty years of age, have observed the following effects which differ from those of earlier observers: In changing from the erect to the horizontal the maximal pressure was usually increased, the eases varying from an increase of $28 \mathrm{~mm}$. $\mathrm{Hg}$. to a decrease of $20 \mathrm{~mm}$. Hg. The minimal pressure was almost always diminished, varying from an increase of $4 \mathrm{~mm}$. Hg. to a decrease of $44 \mathrm{~mm}$. Hg. When the erect posture is resumed after five minutes, the maximal pressure almost invariably falls and the minimal almost invariably rises. In the falling of the maximal pressure when the erect posture is resumed, it will nearly always fall considerably below the previous height of the first reading, while the minimal pressure may be higher or lower than the first reading with almost equal frequeney. The pulse pressure in its variations follows closely the maximal pressure. Persons with poor muscular development show a tendency to reversal of the pressure curve.

Effect of Feeding.-After eating a full meal Karrenstein ${ }^{3}$ noted usually a rise of systolic pressure of 10 to $20 \mathrm{~mm}$. Erlanger and Hooker ${ }^{4}$ obtained variable effects upon minimal pressure, but a constant inerease of pulse pressure. These authors noted a slow increase of pulse pressure throughout the day, it being smallest in the early morning before rising. They found immersion of the body in warm water to increase minimal pressure, pulse pressure

1 Loc. cit.

${ }^{3}$ Loc. cit.

4
2 Arch. Int. Med., 1913, xi, 485.

4 Loc. cit. 
and pulse rate; in cold water to increase minimal pressure, but to decrease pulse pressure and rate. Immediately after the ingestion of food the systolic pressure rises (about $8 \mathrm{~mm}$. Hg. in healthy young adults), then gradually falls until the beginning of the next meal. The diastolic pressure is much less and variably affected by feeding. Associated we find an increased pulse rate and pulse pressure. ${ }^{1}$

Sleep.-Tarehanoff ${ }^{2}$ noted a fall of aortic pressure in young dogs of 20 to $50 \mathrm{~mm}$. Hg. during the early stages of sleep. Leonard Hill, ${ }^{3}$ Brush and Fayerweather, ${ }^{4}$ Howell and Brooks, and Carroll ${ }^{5}$ have studied the phenomenon in man and note the greatest fall one or two hours after commencement of sleep; this may amount to $20 \mathrm{~mm}$. Then begins a gradual rise continuing to the maximum, usually reached about 5 P.M. the following day. If sleep is disturbed the usual drop is less profound. In day sleepers it is the day pressure which is lower than the night. The minimal blood-pressure is according to Weysse and Lutz very uniform throughout the day, but with a tendency to a slight lowering as the day progresses.

Altitude.-'The study of the effects of altitude on blood-pressure has been approached in two ways: (1) By the study of the bloodpressure of individuals remaining for longer or shorter periods at various altitudes, and (2) by the use of the pneumatic chamber to ehange the atmospheric pressure.

The blood-pressure may either be measured as so many $\mathrm{mm}$. of Hg. above the surrounding atmospheric pressure, or the absolute pressure of the blood may be measured. It may be stated at once that changes in atmospheric pressure are associated with approximately equal changes of the absolute blood-pressure in the same direction. Thus, if at $760 \mathrm{~mm}$. atmospheric pressure (ordinary atmospherie pressure at sea level) the absolute systolic bloodpressure is $895 \mathrm{~mm}$. (135 mm. above atmospheric pressure), then if the atmospheric pressure be reduced to $460 \mathrm{~mm}$. the absolute systolie pressure will be reduced to about $595 \mathrm{~mm}$. Since all the manometers ordinarily used measure the elevation of the bloodpressure above the surrounding atmospheric pressure, it is this difference of pressure that is ordinarily discussed. Moreover, it is this difference of pressure and not the absolute pressure which is

\footnotetext{
1 Weysse, H. W., and Brenton, R. L., Diurnal Variations in Arterial Blood Pressure, Amer. Jour. Physiol., 1915, xxxvii, 330.

2 Arch. ital. de Biol., 1894, xxi, 318.

¿ Lancet, 1898 , i, 282.
}

4 Amer. Jour. Physiol., 1901, v, 199.

5 Trans. Assn. Amer. Phys., Philadelphia, 1912, xxvii, 8. 
the measure of the functional activities of the circulatory system. It is to be understood, therefore, that whenever pressure is here referred to it is the pressure in excess of the surrounding atmospheric pressure, that is, the relative blood-pressure, that is meant.

The first to study the effects of changes of atmospheric pressure upon the blood-pressure was Paul Bert. ${ }^{1}$ Upon lowering the atmospheric pressure he obtained a slight diminution of the relative blood-pressure.

Lazarus and Schirmunski ${ }^{2}$ in 1884 studied a man in the pneumatic chamber with the following results:

Atmospherie pressure.
Mm. Hg.
760
670
440
380
460
660
760

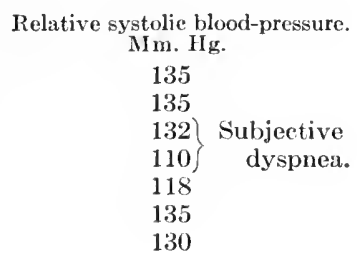

Camus $^{3}$ with rabbits in a pneumatic chamber reduced the pressure from 760 to $200 \mathrm{~mm}$. with, in some animals, little or no fall of the relative blood-pressure, but with other rabbits on reaching about $250 \mathrm{~mm}$. atmospheric pressure the relative blood-pressure showed a marked fall.

Mosso, ${ }^{4}$ with a $\operatorname{dog}$ in a pneumatic cabinet, with reduction of atmospheric pressure to $228 \mathrm{~mm}$. $\mathrm{Hg}$., obtained a slight fall of relative blood-pressure. On the other hand, Fränkel and Geppert ${ }^{5}$ and also Dietrick with lowered atmospheric pressure obtained a slight rise of relative bloorl-pressure, while G. Liebig ${ }^{6}$ obtained a rise in 2 men, but in 2 others a fall.

The approximate relation of atmospheric pressure to altitude is shown in the following table from Camus:

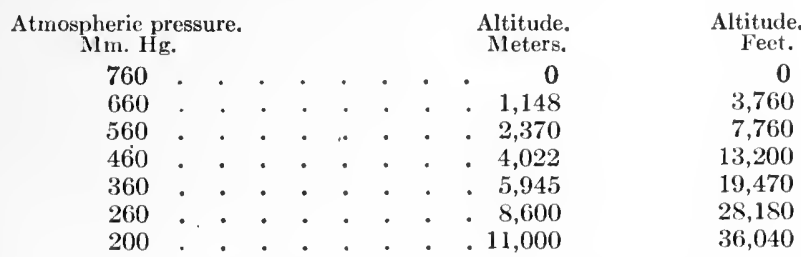

1 La Pressure harometrique, Paris, 1878. $\quad{ }^{2}$ Ztschr. f. klin. Med., 1884, vii, 299.

"Jour. de physiol. et de pathol. gén., Paris, 1903, v, 643.

${ }^{4}$ Arch. ital. de Biol., 1909, xliii.

5 Ueber die Wirkungen der verdünnte Luft, Berlin, 1883, p. 65.

${ }^{6}$ Sitzungsberichte d. gesellsch. f. Morphol. u. Physiol., Munich, 1896, xii, 37.

7 Loc. cit. 
Studies of individuals actually at various altitudes are not numerous.

Schneider and Hedblom ${ }^{1}$ studied the effect on a series of individuals passing from 1700 elevation to 6000 feet and again later from 6000 to 14,109 feet. In passing from 1700 to 6000 feet they observed falls of relative pressure of from 3 to $7 \mathrm{~mm}$. $\mathrm{Hg}$., with no changes in the average diastolic pressure. In passing from 6000 to 14,109 feet they found an average fall of both systolic and diastolic pressures of $7 \mathrm{~mm}$. Hg. and an average rise of pulse rate of 26 beats per minute. The effect upon the systolic pressure was more constant than that upon the diastolie. The effect of the altered altitude was most marked immediately following the arrival at the new level, and those individuals showed most changes who were most affected subjectively by the change of altitude. Small changes of elevation were without any effect.

Stachelin ${ }^{2}$ studied blood-pressure during balloon ascensions to a height with atmospheric pressure of $690 \mathrm{~mm}$. without noting any effect on either systolic or diastolic pressure. Staubli ${ }^{3}$ noted at St. Moritz (5800 feet) no constant change in the blood-pressure of healthy subjects.

Clough $^{4}$ has recently published studies of the effects of a rapid deseent of 1700 feet in a mine shaft. He found that the rapid change in altitude either up or down frequently caused a fall of systolic pressure of $5 \mathrm{~mm}$. He detected no significant difference between the average blood-pressure of individuals living at the altitude of 5000 feet and those at sea level.

Gardiner and Hoagland ${ }^{5}$ studied individuals living for a year at an altitude of 6000 feet and found their relative pressures only siightly lower than those observed in individuals of the same age at sea level. They studied the systolic pressures of 22 college men going by train from a level of 6000 feet to 14,109 . The average relative pressure at 6000 feet was $126 \mathrm{~mm}$. Hg.; on arrival at 14,109 feet, $121 \mathrm{~mm}$. Hg.; three and a half hours after arrival, $11 \mathrm{~S} \mathrm{~mm}$. $\mathrm{Hg}$. Smith $^{6}$ in a recent study has concluded that an altitude of 6230 feet does not produce any consistent alteration of blood-pressure in either normal or tubereulous individuals.

${ }^{1}$ Amer. Jour. Physiol., 1908-9, xxiii, 90 (bibliography).

2 Med. Klin., 1909, v, 361.

${ }^{3}$ Verhandl. d. Kong. f. inn. Med., 1910, p. 695.

${ }^{4}$ Arch. Int. Med., 1913, xi, 590.

5 Trans. Amer. Climat. Assn., 1905.

6 Effect of Altitude on Blood-pressure, Jour. Amer. Med. Assn., 1915, lxiv, 1812. 
Age.-In children the blood-pressure has been declared by Michae $^{1}$ to be more closely proportional to the height or weight than to the age of the child. Sex during childhood appears to be of no importance.

\begin{tabular}{|c|c|c|c|c|c|c|c|c|c|c|c|c|}
\hline Weight. & & & & & & & & & & & Systo & $\begin{array}{l}\text { e pressure (Michael). } \\
\text { Mm. Hg. }\end{array}$ \\
\hline Infancy & ? & . & . & . & . & . & . & . & - & . & . & 75 to 90 \\
\hline 30 to 40 & pounds & $\theta^{\circ}$ & . & . & & . & . & . & . & . & . & 95 \\
\hline 40 to 50 & “ & . & . & . & . & . & . & . & . & . & . & 100 \\
\hline 50 to 60 & “ & . & . & . & . & . & . & . & . & . & . & . 107 \\
\hline 60 to 70 & " & . & . & . & . & . & . & . & . & . & . & 112 \\
\hline 70 to 80 & “ & . & . & . & . & . & . & . & . & . & . & 116 \\
\hline 80 to 90 & “ & . & . & . & . & . & . & . & . & . & . & . 122 \\
\hline 90 to 100 & “ & . & . & . & . & . & . & . & • & . & . & 126 \\
\hline
\end{tabular}

Judson and Nicholson ${ }^{2}$ give the following table of averages at different ages based upon a series of 2300 observations made with a modified Erlanger method and by the auseultatory method reading the diastolic pressure at the beginning of the fourth phase.

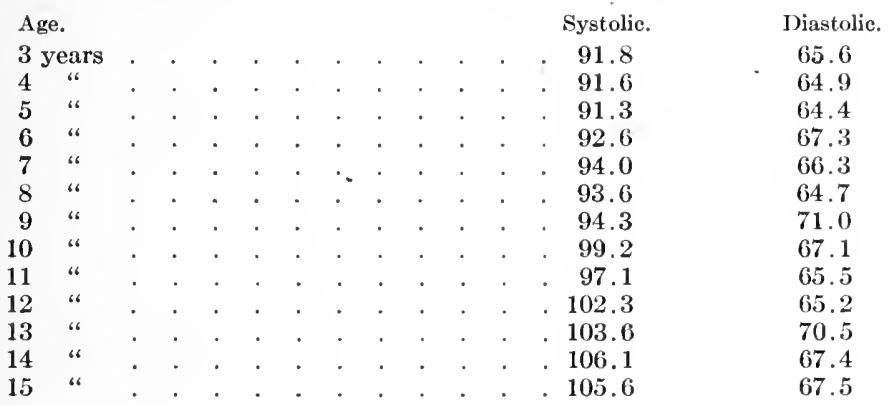

During adult life the normal systolic blood-pressure may be inferred from the following table from Woley: ${ }^{3}$

\section{Age.}

15 to 30 years

30 to 40

40 to 50 ."

50 to 60 "

60 to 65

\begin{tabular}{ccc}
\multicolumn{3}{c}{ Systolic blood-pressure. } \\
High. & Low. & Average. \\
141 & 103 & 122 \\
143 & 107 & 127 \\
146 & 113 & 130 \\
149 & 115 & 132 \\
153 & 120 & 138
\end{tabular}

Females during adult life average, according to Woley, about $8 \mathrm{~mm}$. lower in systolic pressure than males of the same age. The diastolic pressure is normally, at rest, about 70 per cent. of the systolic. (See p. 131.)

${ }^{1}$ A Study of Bloog-pressure in Normal Children, Amer. Jour. Dis. Child., 1911, i, 272 .

2 Amer. Jour. Dis. of Child., 1914, viii, 257.

3 Jour. Amer. Med. Assn., 1910, lv, 121. 
The average minimal pressure in healthy young males of twenty years of age was found by Weysse and Lutz to be $\$ 5 \mathrm{~mm}$. Hg. with a maximal pressure of $120 \mathrm{~mm}$.

Melvin and Murray, 1 in a series of normal cases using the auscultatory method and rearling the diastolic pressure at the beginning of the fourth phase, find the normal diastolic pressure to range from 50 to 82 with an average value of 66 , or 56 per cent., of the systolic. This is much lower, however, than the gradually recognized figure.

\section{THE EFFECTS OF ALTERATION OF BLOOD-PRESSURE UPON THE ORGANS OF THE BODY.}

I. Effects upon the Heart.- The first effect of a sudden elevation of aortic pressure through increase of peripheral resistance is, as we have seen (see p. 31), a reflex inhibition of the heart tending to reduce the cardiae output and restore the blood-pressure to normal. When a permanent increase in the peripheral resistance develops, be it the result of continued arteriolar spasm or of arteriolar fibrosis, although the cardiac output per minute may be, and probably often is, diminished, the force of the ventricular systole is increased to meet the higher aortic pressure and deliver its output against this, and if the nutrition of the heart be good there develops a cardiac hypertrophy chiefly of the left ventricle. It may readily be seen that this phenomenon is a necessary compensatory adjustment if an adequate blood-flow through the more resistant arterioles is to be maintained. It has been further suggested that the hypothetical substances which, circulating in the blood, have called forth the arteriolar spasm may also act as a direct stimulant to the cardiac muscle. This, for example, is true of adrenalin. In favor of this view is the fact that hypertrophy in hypertension cases in man can be observed, as a rule, not only in the left ventricle but in all four chambers of the heart; the hypertrophy of the left ventricle is usually greater, however, than that of the other chambers (Senator). ${ }^{2}$ It has also been suggested, but less favorably received, that the cardiac stimulation is the primary effect of these hypertensive substances, the arteriolar spasm and fibrosis being secondary. Experimental evidence bearing upon this point is difficult to obtain and to interpret, and the question remains unsettled.

${ }^{1}$ British Med. Jour., 1914, ii, 500 .

${ }^{2}$ Die Erkrankungeu der Nieren, Wien, 1902, p. 114. 
The final effect upon the heart of maintaining for a prolonged period a blood-pressure considerably above normal is cardiac exhaustion beginning, as a rule, with dilatation and weakening of the left ventricle. Then follow with falling blood-pressure, which may still, however, be considerably above the normal, the signs of circulatory failure throughout the body: edema, accumulation of fluid in the serous cavities, venous congestion, cyanosis, dyspnea, edema of the lungs, renal insufficiency, and the disturbances of the internal organs resulting from passive congestion. This outcome is hastened by coronary sclerosis, which impairs the cardiac nutrition. The effect of low blood-pressure upon the heart is, as a rule, to call forth a reflex acceleration of cardiac rate and aetivity. So far as the coronary circulation suffers from the lowered blood-pressure a detrimental influence is exerted on the cardiae nutrition.

II. Upon Arteries and Arterioles.-Associated with continued high blood-pressure are, in the great majority of cases, certain changes in the vessel walls. These consist of fibrous thickening of the inner and outer coats of the arteries and arterioles with increase of their elastic tissue. The muscular eoat shows sometimes a thinning, sometimes a thiekening, but the latter is due more often to increase of the fibrous tissue of the muscular coat than of the muscle tissue itself. That these fibrous ehanges in the vessel walls are the direct result of strains or minute tears consequent upon the high blood-pressure has been suggested. That they are the direct effect of those toxie substances which have produced the arteriolar spasm and high blood-pressure is possible. Finally, in some cases these arteriosclerotic ehanges in the vessel walls result from the toxins of acute infections or of syphilis and develop before any rise of blood-pressure has occurred; once developed, however, they increase the resistance to the flow of blood through these vessels. The effect of this in leading in turn to a rise of arterial blood-pressure and eventually, if the eoronaries be not too sclerosed, to cardiac hypertrophy, will be diseussed later.

III. Upon Capillary Pressure, Venous Pressure, and the Pulmonary Pressure.-On these high arterial pressure has little effect. The arterial pressure is confined between the left ventricle on the one hand and the "stopeock," the arterioles, on the other hand, and marked ehanges may occur in the arterial pressure with no change whatever in the pressure in the capillaries, veins, or pulmonary vessels. 
As we have noted, the capillary pressure is dependent more upon the venous than upon the arterial pressure.

IV. Upon the Kidneys. - Goll's experiments, performed under Ludwig, were the first to show conclusively the effect of changes in blood-pressure upon the activity of the kidneys. Goll cut the vagi of a dog, determined the blood-pressure by means of a cannula introduced into the carotid, then from the two ureters collected all the urine excreted for half an hour. He then stimulated continuously with a weak faradic current the peripheral end of one of the cut vagi, thereby diminishing the cardiac aetivity and causing a fall of blood-pressure. This procedure was continued for half an hour, during which time the urine from the ureters was again collected. It was found that the fall in blood-pressure had markedly reduced the amount of urine excreted in the half-hour and that it had reduced the water of the urine more than it had the solids, so that while the actual amount of solids was reduced, the percentage of solids and the specific gravity of the urine were increased.

He then took another dog and similarly recorded the carotid pressure and measured the urine excreted from the two ureters for half an hour. He then bled the dog freely; a fall in bloodpressure resulted and persisted. During the next half-hour, while the blood-pressure was low, the urine was again collected and was found as in the first experiment to be reduced in amount; again the water was more reduced than the solids. The blood which had been withdrawn from the animal was defibrinated and at the end of the half-hour was reinjected, restoring the animal's bloodpressure to about the original level. The urine was now again collected for half an hour and was found to have increased in quantity to about the original level of excretion and the water had increased more than the solids, so that while the elimination of solids had increased, the percentage of solids and the specific gravity were reduced to about normal.

Later Meyer performed the following experiment: In a rabbit he constricted the vena cava above the entrance of the renal veins to half its natural diameter. This, he found, led to the excretion of a diminished quantity of highly concentrated urine which contained albumin.

It will be observed that, although Meyer's experiment increases the blood-pressure in the capillaries of the kidney, it diminishes the output of urine. The activity of the kidney is not, therefore, proportional to the blood-pressure, as Goll's experiment might 
suggest, but to the rate of blood-flow through the renal vessels. In both Goll's experiments the fall of blood-pressure reduces the flow through the renal vessels, and in Goll's second experiment the reinjection of the blood with the resultant rise of pressure increases the flow through the kidney. In the same way digitalis, which increases the amplitude of the cardiac systole and thus augments the cardiac output, thereby raising the blood-pressure and thus increasing the blood-flow through the organs, is a drug which produces diuresis. On the other hand, adrenalin, when injected intravenously, inereases the arterial blood-pressure much more than does digitalis, but does so by constriction of the arterioles and therefore reduces the blood-flow through the organs. The effect of a large dose of adrenalin upon the excretion of urine is to greatly diminish or entirely stop it during the brief period of the drug's effectiveness. The comparison of the effects of these two drugs on renal activity is a good illustration of the impossibility of estimating the effectiveness of the circulation by the arterial blood-pressure.

Nitroglycerin and the nitrites act chiefly by dilating the arterioles and thus lowering blood-pressure. Did they influence all arterioles equally their effect would be to increase the blood-flow through all the organs, with a lowering of blood-pressure. In fact, however, they dilate the mesenteric vessels disproportionately. Very small doses may lead to increased blood-flow through the kidneys with diuresis. However, larger doses dilate the mesenteric arterioles so much more than the renal that the blood-flow is diverted through the former with a fall of aortic pressure, and the flow through the renal vessels diminishes, as does the urinary output.

We find, therefore: (1) A rise in the general arterial bloodpressure caused by increasing the bulk of the blood or by augmenting the cardiac output, increases the blood-flow through the kidneys and in consequence the renal activity. (2) A change in the general arterial blood-pressure (rise or fall), produced in such a way as to diminish the blood-flow through the kidneys, diminishes the renal activity. 
CHAPTER II.

\section{THE INSTRUMENTAL ESTIMATION OF BLOOD-PRESSLRE.}

No one who has had any experience with instruments of precision in controlling his tactile impressions, will flatter himself with the delusion that he can gauge endovascular tension with anything approaching accuracy with the finger. Extremes are easily recognized but the intervening moiety is a fertile source of error. The size of the vessel, the character of the surrounding tissues, the volume of the pulse and the state of the arterial wall itself, all contribute to one's confusion. The estimate of arterial tension has therefore been relegated to the more exact field of sphygmomanometric measurement, just as has the determination of alterations of bodily temperature to the thermometer.

With the older methods and with most of the early instruments only the systolic pressure could be gauged. This is no longer sufficient. It is now possible to estimate the diastolic pressure with approximate accuracy by a number of different methods. The systolic index alone tells but a part, and that but a small part, of the whole story.

A large number of instruments for the estimation of human bloodpressure have been devised and modified. This gradual development will not concern us here as the subject is mainly historic in its interest and has already been ably described by others. ${ }^{1}$ In many of the older instruments, either the principle was faulty or the technique unsatisfactory, hence we shall consider mainly those which are of practical utility today.

Nearly all of the modern instruments are constructed with an elastic cuff or arm band which, when inflated and encircling one of the extremities, compresses, and when the pressure is raised sufficiently, obliterates the arterial pulse below the cuff. This

1 Janeway, The Clinical Study of Blood-pressure, New York, 1904, p. 43; Tigerstedt, Lehrbuch d. Physiologie d. Kreislaufes, Leipsic, 1893, p. 321; Ergebniss der Physinlogie, 1907, vi, 265; Vachide and Lahy, Arch. gén. de Méd., 1902, pp. 349, $4 \times 0,602$. 
point corresponds to the systolic pressure. It indicates the point at which the external pressure is just sufficient to overcome the internal resistance (blood-pressure, velocity, the arterial wall, and the surrounding soft tissues). The velocity factor is negligible and the resistance of the arterial wall and soft tissues normally does not exceed 10 to $7 \mathrm{~mm}$. respectively. Hill has shown that the obliteration pressure in the femoral artery of the dog is the same (within 1 to $2 \mathrm{~mm}$. Hg.) as the systolic pressure taken in the opposite femoral with a cannula and the Hürthle manometer.

The amount of pressure required is measured by means of either (1) a mercury manometer graded in millimeters; (2) an aneroid; (3) a metallic spring manometer; or (4) a compressed-air manometer with an indicator; depending upon the particular instrument employed.

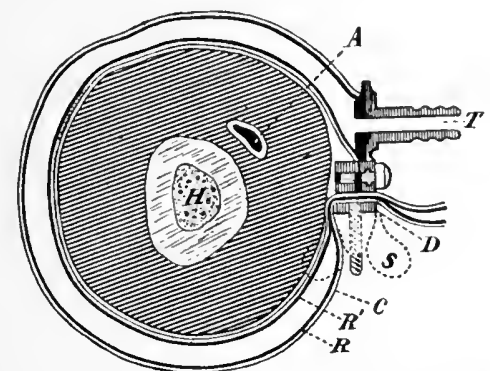

Fig. 9.-Arm in eross-section with Riva-Rocei cuff: $R$, outer wall of rubber tube; $R^{\prime}$, inner wall of rubber tube; $S$, screw fastening; $D$, clamp; $C$, silk cover; $A$, brachial artery; $H$, humerus; $T$, tube leading to manometer.

The Character of the Cuff.-The compression may be exerted upon the vessel in question either by (a) a limb-encircling, distensible elastic cuff (applied to a segment of the arm or leg), or $(b)$ by means of a solid or a fluid pad overlying the artery; in either case surrounded by an external inexpansile fabric. Of these the former is more satisfactory (see Fig. 9).

The accompanying figures illustrate the prineiples involved in the application of a cuff to the arm. In Fig. 9 we have a rubber cuff completely encircling the arm, but insufficiently inflated to occlude the artery. In Fig. 10 the rubber extends only part way around the arm, but the pressure is high and the arterial lumen therefore completely obliterated.

The width of the cuff, as was shown by von Recklinghausen, ${ }^{1}$ is

1 Ueber Blutdruckmessung heim Menschen, Arch. f. exper, Path. u. Pharmakol., 1901, xlvi, 78 . 
of vital importance. If it is too narrow, readings higher than the actual endarterial pressure may be obtained. The soft tissues of the arm offer considerable resistance to compression, which may,

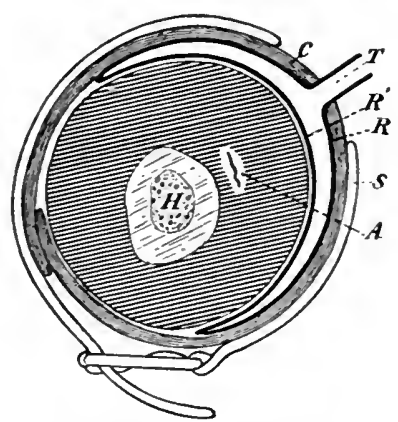

FIG. 10.-Arm in eross-section with Hill and Barnard cuff: $R$, outer wall of rubber bag; $R^{\prime}$, inner wall of rubber bag; $S$, strap, fastened by buckle; $C$, leather cuff; $A$, brachial artery; $H$, humerus; $T$, tube leading to manometer.

however, be largely overcome by lengthening the segment included in the cuff. The reason for this is shown in (Fig. 11).

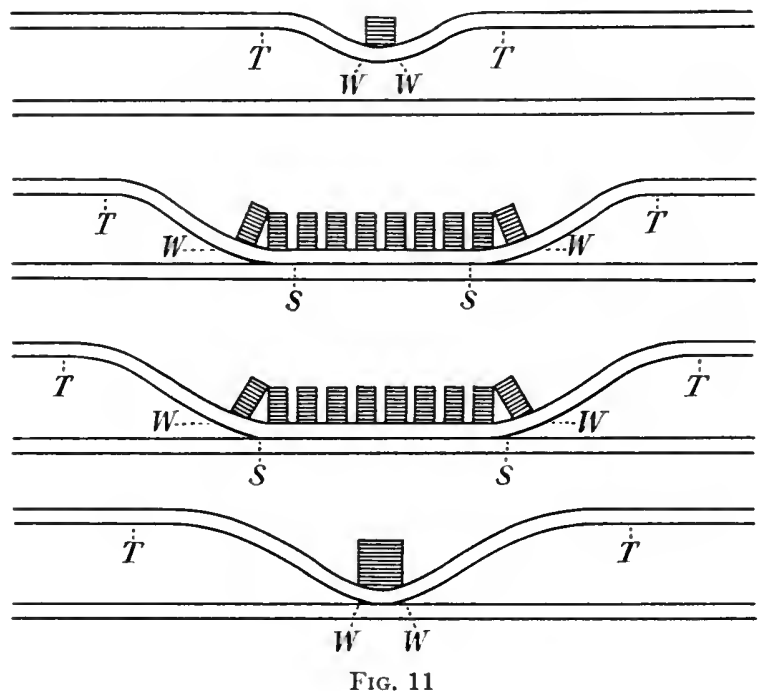

If ${ }^{-a}$ distended artery is partially obliterated by the pressure of a single block-as in the case of a narrow cuff-we not only' have to overcome the internal pressure of the vessel but also the 
oblique tension of the wall and surrounding tissues $(W-T)$, and as a result too high readings are obtained. With a series of blocks exerting a similar pressure to the square inch, only the outer blocks $(W)$ will be concerned in overcoming the pull of the artery and tissues at $W-T$, while the intervening ones $(S-S)$ will exert their full pressure.

With a broad cuff an error of +7 to +9 per cent. may be assumed. With a narrow cuff the figure may reach +40 per cent., while the Gärtner finger ring has a margin of error of +19 per cent.

There was for a time much discussion as to the accuracy of von Recklinghausen's contention, caused by the belief that a cuff of $12 \mathrm{~cm}$. interfered with the progression of the pulse wave and yielded subnormal values. But the correctness of his view has now been definitely established by experiments (1) on the cadaver with artificial circulation (Gumprecht), (2) on animals (Fellner, Rudinger, Schelling and others); (3) on the living human subject (O. Müller and Blauel, Janeway). A narrower cuff may be advantageously employed for children (3 inches, $7 \mathrm{~cm}$.).

'The outer covering of the elastic cuff, whether of canvas, leather, or cloth, must be of a non-distensible character and the rubber tubing connected therewith must be sufficiently inelastic to prevent any oscillation or stretching with increased pressure. If these facts are overlooked we introduce a new source of error for which we should have to allow with each increment of pressure. For this reason the outer covering must completely enclose the elastic cuff, which must not be allowed to slip out of place while in use. With a view to obviating this possibility, some cuffs are constructed in which the outer and inner cuffs are made as one piece (Vaquez, Uskoff, Oliver, Janeway).

The elastic cuff must of course be sufficiently thin and flexible to add but a negligible increment to the manometrical pressure. This desideratum, as shown by Gumprecht, is easily furnished.

The Location of the Cuff, etc.-The brachial artery is usually selected for blood-pressure estimation because of its accessibility, and because either in the erect, sitting, or recumbent postures it is at about the cardiac level. The presence of a thin shirt or waist between the arm and the cuff produces only a negligible error. In fact it is much better to have a shirt interposed but evenly distributed than to have a tight sleeve or an undershirt rolled up into an arm-encircling band above the cuff. In children the thigh is often the preferable site, on account of the smallness of the arm. 
In adults, especially for purposes of comparative study, either the thigh or the calf may be chosen. The brachial artery is considered the site of choice because "it gives us the systolic lateral pressure within the subclavian, since brachial and axillary are continuous in direction, and therefore a near approximation to systolic lateral pressure in the aorta. This, combined with estimation of diastolic lateral pressure in the brachial, which is practically the same as aortic diastolic pressure, gives the best insight into actual variations of sistemic blood-pressure" (Janeway). 'This statement has been questioned by some observers since, as was first shown by Bing, the pressure in the two brachial arteries of the same individual may vary $20 \mathrm{~mm}$. or more. The brachial pressure is generally, but by no means invariably, equal to that of the aorta.

The cuff's furnished with the Tech and the Mercer instruments are so constructed that the stethoscope used for the auscultatory method is applied at the level of the cuff and not below it, as is usually the case. This gives readings on the average $10 \mathrm{~mm}$. higher than those obtained in the usual manner and, in the opinion of the author, adds an unnecessary increment of error.

\section{PALPATORY METHOD.}

\section{A. Estimation of the Systolic Pressure.-Method of Application} for Instruments of the Riva-Rocci Type (Fig. 12).-The rubber cuff (D) (which should be $12 \mathrm{~cm}$. in width) is applied so as to encircle the arm or leg, secured by a non-distensible canvas or leather cuff, and strapped or tied snugly in place (generally above the elbow or knee). The tube connecting with the rubber cuff is attached to the manometer $(M)$ (the valve $C$ on the latter being opened to admit air), and is inflated by means of a syringe bulb or pump $(P)$ from the distal side of the manometer. The systolic pressure is read by placing the finger on the radial pulse and pumping the mercury well above the point at which the pulse at the wrist disappears. We then close the valve $(C)$, open the second escapement valve $(E)$, and as the mercury falls note the point at which the radial pulse again becomes perceptible. If the disappearance of the pulse during gradually increasing pressure is chosen as a criterion for the systolic level, instead of its reappearance after obliteration during a falling manometrical pressure, higher values ( 3 to $10 \mathrm{~mm}$. $\mathrm{Hg}$.) will be obtained. 'The return of the pulse after obliteration is also a much more sudden and well- 
marked phenomenon than the disappearance during an increasing pressure, and the exact moment at which it occurs is much easier to determine. It is essential, however, that the pressure should be allowed to drop slowly and steadily, otherwise the first few small beats will not be appreciated. A much more important reason lies in the fact that the lower reading is also somewhat nearer the

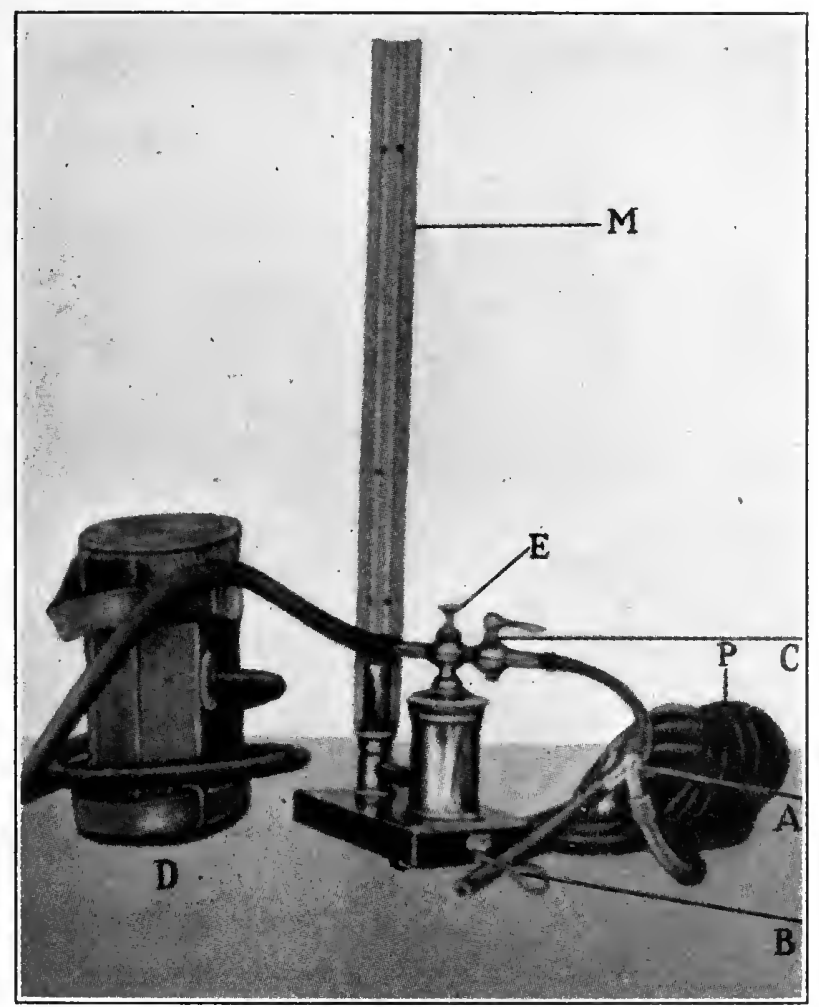

FIg. 12.-The Stanton sphygmomanometer.

actual intra-arterial pressure, because we more or less counterbalance the overestimation caused by the resistance of the arterial wall and the tissues of the arm. More accurate readings will be obtained if the radial artery is palpated with the ball instead of the tip of the finger. The latter should rest against the overhanging edge of the radius, thus permitting the application of a very uniform pressure (Hirschfelder) (Fig. 13). 
The reappearance of the pulse below the point of constriction may be recognized by other means. Thus, if instead of the palpating finger a sphygmograph is attached to the wrist, or if a second cuff communicating with some sort of recording device is substituted, the return of the pulse can be made manifest.

B. Estimation of the Diastolic Pressure.-The estimation of the diastolic pressure was, until the auscultatory method was discovered and generally adopted, a more difficult and uncertain procedure. By means of the last-named procedure, however, we are now able to make readings which from the stand-point of accuracy and celerity compare very favorably with those employed for the

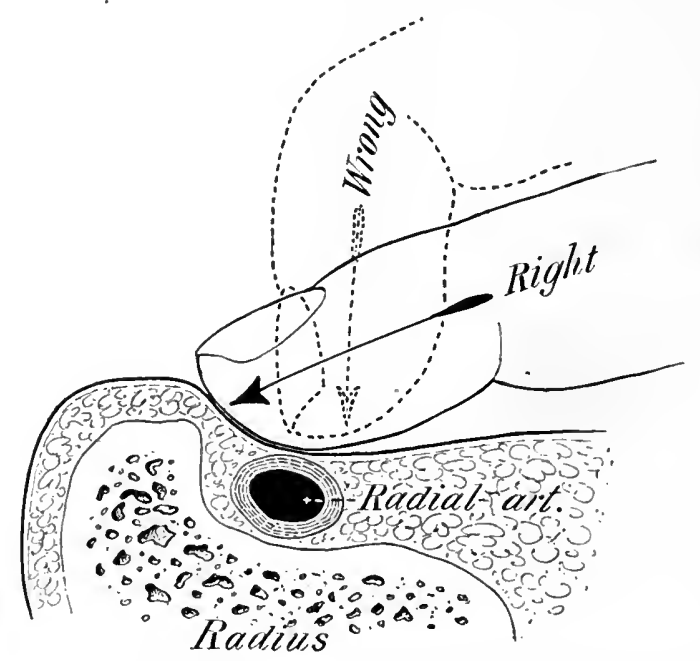

FIG. 13.-Position of the finger in palpating the radial artery.

systolic pressure. The estimation of the diastolic pressure is of the greatest importance and for general clinical purposes no cxamination can be considered complete without it.

The basis for all determinations of the diastolic pressure rests on the fact that when the pressure in the brachial cuff is at the diastolic level, the greatest oscillation in the caliber of that portion of the artery which lies beneath the cuff will occur. If the cuff pressure is higher, only the upper portion of the pulse wave will pass through; if lower, the artery will not completely collapse. Whichever the method of estimation of the minimal pressure employed, the end in view is always that of making manifest, in 


\section{celive lelaske}

one way or another, the exact point at which this alternate maximum filling and collapse occurs. Thus by:

1. Palpation (Strassburger).-The- radial artery below the cuff is palpated between the finger and the radius, while the manometrical pressure is slowly increased. It will be noted that at a certain point, usually quite well marked, the pulsations suddenly become throbbing (knocking or bounding, "klopfend"), owing to a complete alternate distention and collapse of the artery. This phenomenon occurs when the minimum pressure is reached. The first bounding pulse is to be chosen as the criterion (often this characteristic continues for some time during the rise of pressure). The method has been found very accurate when compared to graphic registration; the first bounding wave corresponding to the first full wave on the tracing (Gallavardin). Although this method has been highly recommended by Ehret, ${ }^{1}$ who palpates the brachial artery immediately below the cuff, we cannot commend it; it requires long practice, and even one who is skilled in its performance is hampered by too much of the personal equation.

2. Visualization.- With that pressure in the cuff at which the greatest arterial fluctuation occurs, the greatest oscillation will also be transmitted to the mercurial column. Hence observation of the lowermost point of the maximum mercurial oscillation was for a long time used as a criterion of the minimum pressure. Owing, however, to its great inertia, mercury is with difficulty set in motion by the compressed air, but once in motion its oscillation may be maintained or even increased by very slight pressure changes. Furthermore, many instruments were equipped with a ${ }^{\top}$-shaped manometer so that the actual mercurial fluctuation was diminished by half. It has been partly as the result of this that so many different instruments have been placed on the market, although, of course, compactness, portability, and cost have also entered into the problem.

'The Stanton instrument owes much of its well-deserved popularity to the fact that, being of the single tube type and well proportioned, it yielded large mercurial excursions.

The maximum oscillation in other forms of apparatus, spring manometers (v. Recklinghausen), aneroids (Pachon, Rogers), is also used to determine the point of maximum arterial fluctuation.

${ }^{1}$ Ueber eine einfache Bestimmungsmethode des diastolischen Blutdruckes, Münch. med. Woch., 1909, No. 12. 


\section{THE AUSCULTATORY METHOD.}

In 1905 Korotkow suggested the determination of blood-pressure by auscultation. Owing to its simplicity, celerity, and accuracy this method has supplanted all others for clinical work.

If, instead of palpating the artery below the compressing cuff, we apply the bowl of a stethoscope and listen, we will hear a variety of sounds during the fall of the mercurial column. Five distinct phases can generally be made out and quite clearly differentiated:

(1) A sound not unlike the first cardiac sound. (2) This same sound plus a hissing murmur. (3) The murmur disappears and only the sound is heard. (4) The sound suddenly becomes very much muffled. (5) The sound disappears. These phases occur while the pressure in the cuff is falling in the order given.

The first sound or phase is due to the sudden distention of the collapsed brachial artery caused by the return of the pulse wave, the sound waves being reënforced by the resonating quality of the cuff. (Compression with an Esmarch bandage usually fails to produce a sound. Gittings. ${ }^{1}$ ) The onset of this phase indicates the systolic pressure.

The Second Phase.- The addition of a murmur to the first sound is due to the formation of fluid veins, whirls, eddies, produced as the blood flows past the constriction into the relatively dilated artery below the cuff.

The Third Phase.-Disappearance of the murmur is caused by sudden vibrations of the vascular wall produced by the increased volume of blood which, although diminished in rapidity of flow, now reaches the artery at the point of auscultation. The constricting pressure being diminished, the fluid veins below the cuff gradually disappear. This phase is generally distinctly louder than the first. It corresponds to the stage of large oscillation in the graphic method, and its end occurs simultaneously with the Ehret phenomenon and the last of the maximal graphic waves (see Fig. 17).

The fourth phase ${ }^{2}$-muffling of the sound-arises when maximal arterial filling and collapse no longer occur. Furthermore, the

${ }^{1}$ Auscultatory Blood-pressure Determinations, Arch. Int. Med., August, 1910.

${ }^{2}$ This phase, not mentioned by Korotkow, was first described by Ettinger, Wien. klin. Woch., 1907, p. 992. 
blood-stream becomes slower and the cuff loses its resonating quality owing to a fall of tension. According to Macwilliam and Melvin, ${ }^{1}$ the fourth phase owes its characteristics to the fact that external pressure has become insufficient to cause a flattening of the circular arterial tube. They believe that maximum arterial oscillation occurs, not as stated by Marey and generally accepted, at the point at which external pressure in the cuff and internal pressure in the artery are exactly counterbalanced, but at the

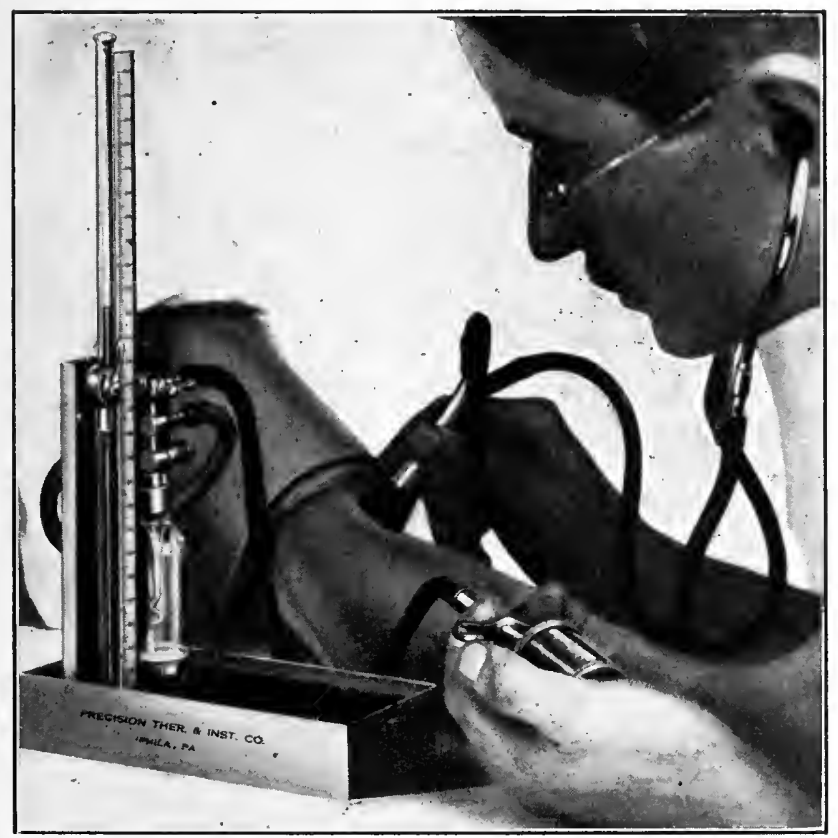

Fig. 14.-The auscultatory method.

point at which external pressure is sufficient to distort the arterial tube so as to produce a half-flattening of the artery. The onset of this phase indicates the diastolic pressure. ${ }^{2}$

The fifth phase-disappearance of all sound-oceurs when normal arterial relations are again established. The onset of this phase is accepted by some authorities as indicating the diastolic pressure,

1 The Estimation of the Diastolie Blood-pressure in Man, Heart, 1914, v, 153.

2 Macwilliam, J. A., and Melvin, G. S., The Estimation of the Diastolic Pressure, Heart, 1914, v, 153. 
although it seems well established that the beginning of the fourth phase should be chosen as the criterion. ${ }^{1}$

It occasionally happens that the change from the third to the fourth phase is not clearly defined and it is sometimes necessary to make several observations before the exact point of change can be established. This is, however, not of ten the case among those who are accustomed to the auscultatory procedure. The identification of the onset of the fifth phase is much less readily established, and even when accomplished is subject to a much greater element of personal error. Some recent observations indicate that when we are confronted with sudden changes in pressure due to psychic influences (observations on students), the fourth phase is more consistently established, whereas among hospital patients the fifth phase shows less variability. ${ }^{2}$

The actual average duration of the different phases as measured in millimeters of mercury was found by Goodman and Howell ${ }^{3}$ to be $14,20,5,6 \mathrm{~mm}$. in the order given. These last-named authors have opened up a very suggestive line of investigation in this field by measuring the actual duration of the different phases and their relation to each other under various pathological conditions. In cases of aortic regurgitation and hypertension, for instance, the fifth phase is persistent. (In aortic insufficiency a pistol-shot sound is often heard over the arteries when no pressure is being exerted.) A long third phase indicates a powerful systole. This phase is also lengthened in arteriosclerosis even in the presence of cardiac weakness. Atypical auscultatory findings are often associated with cardiac insufficiency (Krylow). In some apparently normal cases all the phases cannot be made out, but in the rast majority, although some may be brief, the five phases are distinctly remarked. Variations in the strength of successive systoles are often better appreciated by auscultation of the partially compressed artery than of the precordium "tonal arhỵthmia."

${ }^{1}$ Lang and Manswetowa, in comparison with the Hürthle spring manometer, obtained closely corresponding readings by taking the auseultatory readings at the beginning of the fourth phase, Deut. Arch. f. klin. Med., 1908, xciv, 441; Fischer also believes that the beginning of the fourth phase represents the diastolic pressure, Deut. med. Woch., 1908, p. 1141; Taussig, A. E., and Cook, J. E., Determination of the Diastolic Pressure in Aortic Regurgitation, Areh. Int. Med., 1913, xi, 542; Warfield, L. M., Studies in Auscultatory Blood-pressure Phenomena, Jour. Amer. Med. Assn., 1913, lxi, 1254.

2 Kilgore, Berkley, Rowe, and Stabler, A Quantitative Determination of the Personal Factor in Blood-pressure Measurements by the Auscultatory Method, Arch. Int. Med., 1915, xvi, 927,

${ }^{3}$ Trans. College of Physicians, Philadelphia, 1911; Amer. Jour. Med. Sci., September, 1911 . 
In estimating blood-pressure by the auscultatory method any type of manometer-mercurial, aneroid, compressed air-may be employed.

As a general rule a long and intense murmur phase, beginning at a high pressure, points to a vigorous heart action. If it inereases under exercise, we assume that the heart is using its reserve force. When the murmur phase disappears under light work cardiac

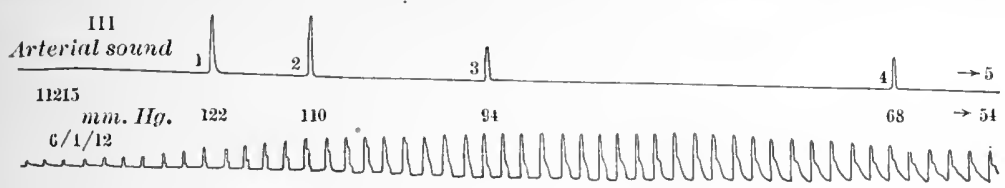

F1G. 15.-Fast drum. Sudden decrease in size of pulse wave at 4, marking the change from clear, sharp tone to dull tone.

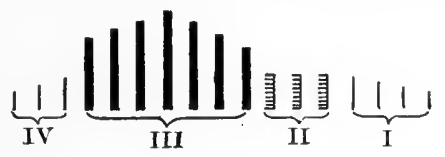

Fig.. 16.-Diagrammatie representation of the auscultatory phases (after Gallavardin). $I$, arterial tone (muffled); $I I$, tone and murmur; $I I I$, arterial tone (loud and without a murmur); $I V$, muffled sound.

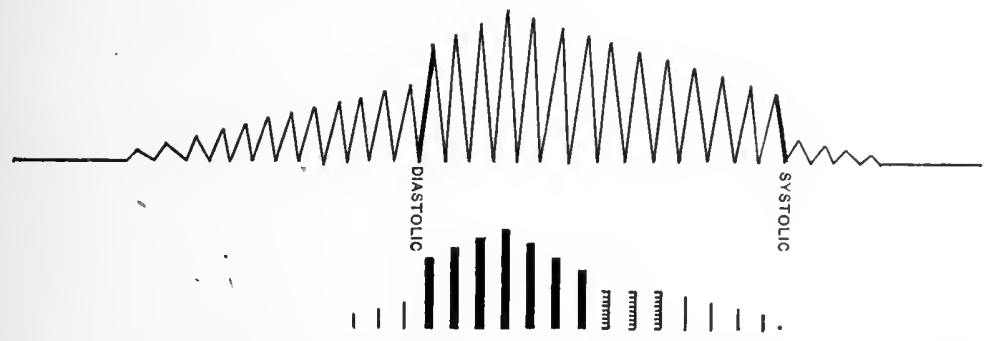

Fig. 17.- Showing the relationship between the oscillatory and the auscultatory phenomena (Gallavardin). Here the end of the third phase is drawn as corresponding to the last large oscillation.

weakness is implied, while the splitting of the tones of the first phase points to a dilated hrpertrophy of the ventricle. If the sound or the murmur is subject to variations in duration and in intensity, cardiac insufficiency is predicated. The non-appearance of the murmur phase has a similar significance. ${ }^{1}$

\footnotetext{
1 Tornai, J., Ueber d. diagnostisches Wert d. auskultatorischen Blutdruckmessumgen, in besondere vom Standpunkt der Funktions-pruefung d. Herzens, Zeitsr.hr. f. Phys. u. diät. Therap., 1909, xiji, 504 et seq.
} 


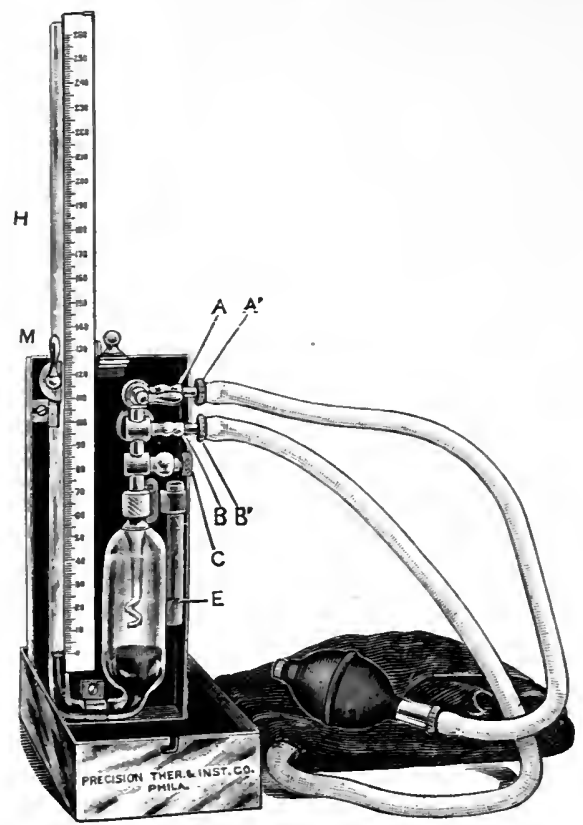

Fig. 18.-The new Nicholson sphygmomanometer. Showing the method of connecting the unions $A^{\prime}$ and $B^{\prime}$ with the stopcock $A$ and the connection $B$. The needle valve $C$ must be closed, and the stopcock $A$ open as shown in the cut. To close the instrument remove the glass tube $H$, sliding it into the metal holder $E$. Fold the seale down and then remove the metal unions $A^{\prime}$ and $B^{\prime}$. The lid can then be made to close by firm pressure on its upper end. The lid cannot be closed until the stopcock $M$ has been turned at right angles to the tube, and when so turned no mercury can escape. When closed the instrument fits into a moroceo case containing also the bulb and cuff, and can be carried in the pocket.

Accuracy is assured: (1) by a millimeter scale, especially compensated for the lowering of the level in the mercury reservoir: (2) by the zero (0) point on the scale being adjustable to the mercury level, so that the readings are not affected by climate and temperature changes; (3) by the scale reading directly in millimeters of mercury, the primary standard, it does not have to be checked up; (4) by the use of a large column of mercury; there is no separation of the mercury, and oxidation and capillary errors are avoided; (5) by the use of a steel stopcock and flint-glass no amalgam is formed with the mercury, so no friction; (6) by a steel needle valve there is a perfect air release. The mercury remains clean owing to a new method of preventing powder from the rubber tubes being sucked into the mercury reservoir.

Portability. (1) All parts are thoroughly protected by a metal case; (2) by a special stopcock no mercury can be lost; (3) by stopcock $A$ one has an additional safeguard should the rubber washer in the pump leak slightly, until it can be replaced by a new washer. The mercurial column can be absolutely maintained by closing the stopcock. This permits of using the arm band to produce Bier's hyperemia. By inflating the apparatus to 50 to $60 \mathrm{~mm}$. Hg. and then closing the stopcock $A$ one can maintain the pressure for any length of time desired. This is impossible of attainment on any apparatus not having this stopcock. This is the only pocket sphygmomanometer which has this special feature. In addition to the above the apparatus is simple to opcrate, using a wide, soft cuff, and has an automatic catch on the lid which holds the instrument in the upright position when in operation. (Manufactured by the Precision Thermometer and Instrument Co., Philadelphia.) 


\section{INSTRUMENTS FOR ESTIMATING BLOOD-PRESSURE.}

\section{A. RIVA-ROCCI TYPE.}

The new Nicholson sphygmomanometer is a readily portable mercurial column instrument enclosed in a metal case. The lid when raised automatically locks in the upright position and acts as a support for the instrument (Fig. 1r).

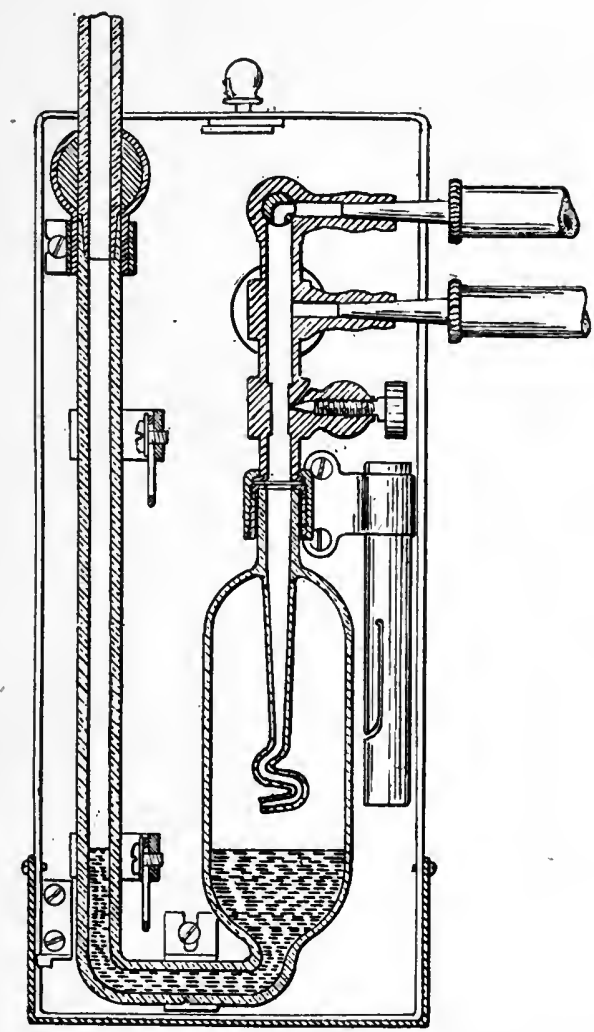

Fig. 19.-Vertical cross-section of Nicholson's new pocket sphygmomanometer.

We believe this instrument to be the best practical sphygmo. manometer yet devised. It is small, accurate, readily portable and well constructed. Nicholson has also devised an even smaller, more compact, cheaper, and yet accurate instrument-the Princo sphygmomanometer - which deserves an equal endorsement.

This instrument may also be obtained furnished witl a Feddé indicator (oscillating index), which consists of a vertical tube con- 
taining a pith ball, the excursions of which during alternations of pressure are both free and extensive, thus affording signal aid in determining the diastolic pressure by the visual method (Fig. 52).

Another instrument of the Riva-Rocei type, long familiar to American physicians, is that, of Janeway (Fig. 22). It is now supplied with a metal needle-valve pump instead of the rubber bulb depicted in the cut. ${ }^{1}$
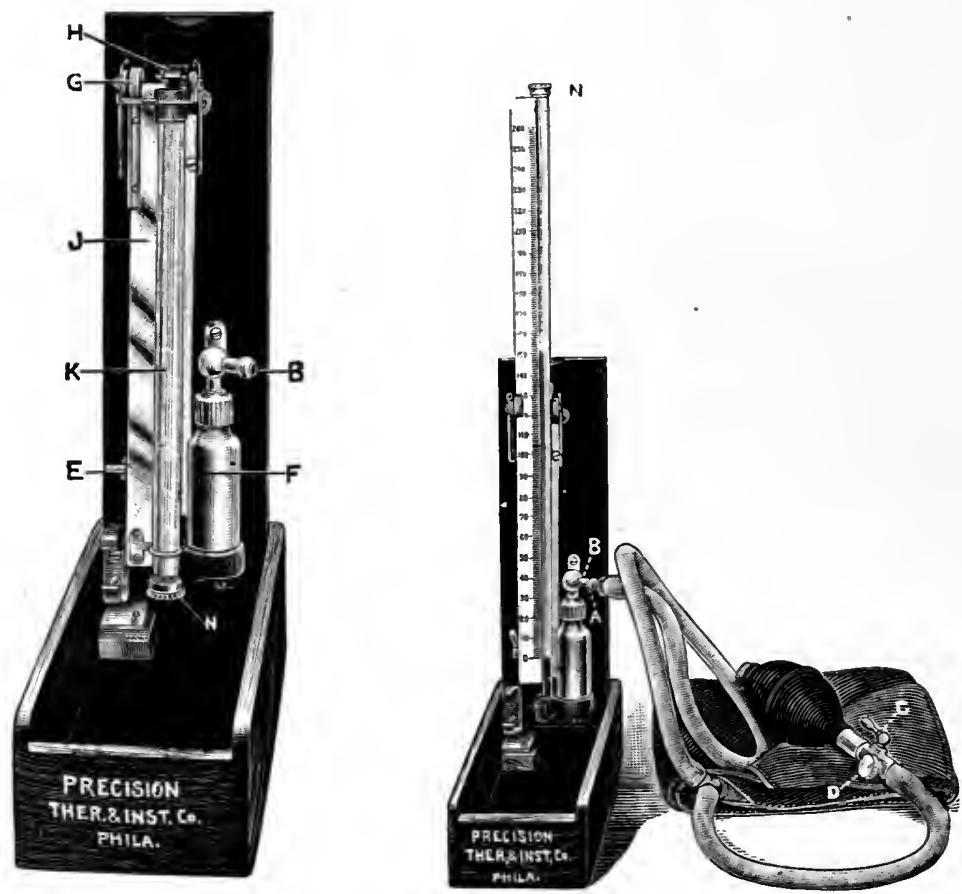

F1G. 20.-Nicholson's Princo sphygmomanometer. The instrument in a semifolded and open position. $A$, terminal of tubing; $B$, socket for the same; $C$, stopcock: $D$, needle valve; $E$, clamp level to set scale at proper mercurial level; $F$, mercurial reservoir; $G$, hinge which permits folding of calibrated standard; $H$, automatic valve which permits folding of manometer tube and retains mercury when in transit; $J$, scale folded; $K$, tubing folded; $N$, screw cap to permit removal of mercury.

The Mercer sphygmomanometer is a mereury instrument the form and construction of which are shown in Fig. 23. The instrument may be clamped to a table or held in the hand while readings are made. The cuff and bulb are carried separately.?

Leonard $\mathrm{Hill}^{3}$ has devised a compact and convenient form of

I Manufactured by Dressler-Beard Mfg. Co., 286 Second Avenue, New York.

2 Manufactured by A. Kuhlman \& Co., 203 Jefferson Avenue, Detroit, Mich.

${ }^{3}$ A New Form of Mereury Sphygmometer, British Med. Jour., February 19, 1910 
mercury sphygmometer. It consists of a single graduated tube the base of which is sealed into a small reservoir in the lower end

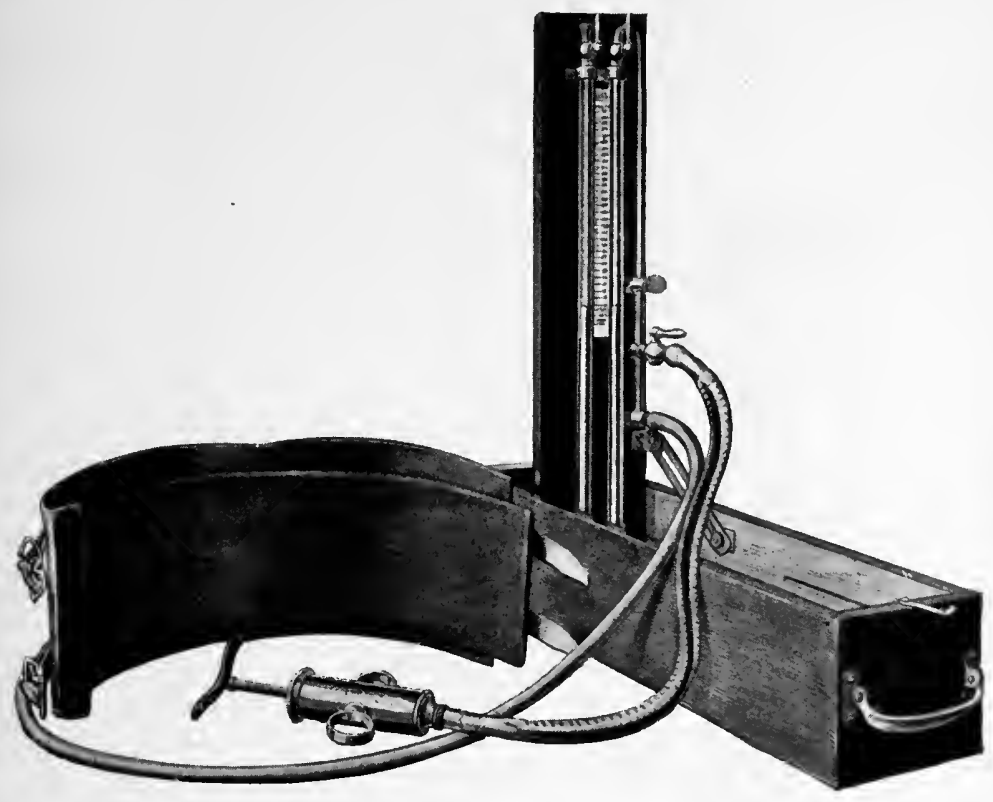

FIG. 21.-Faught's mercury sphygmomanometer showing relation of parts, metal pump, and special expansion tubing for inflator.

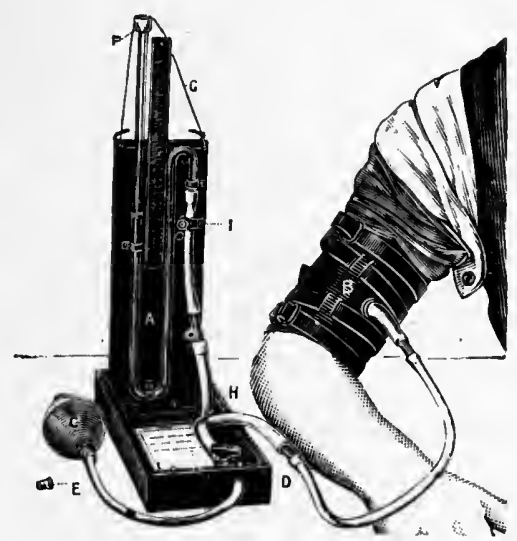

Fig. 22.-Janeway's sphygmomanometer.

of which it opens $(E)$. The tube leading from the cuff is attached to the other end of the reservoir, which contains the mercury. 'The 
chief advantages claimed for the instrument are: (1) The manometer being of the single tube variety halves the error of reading
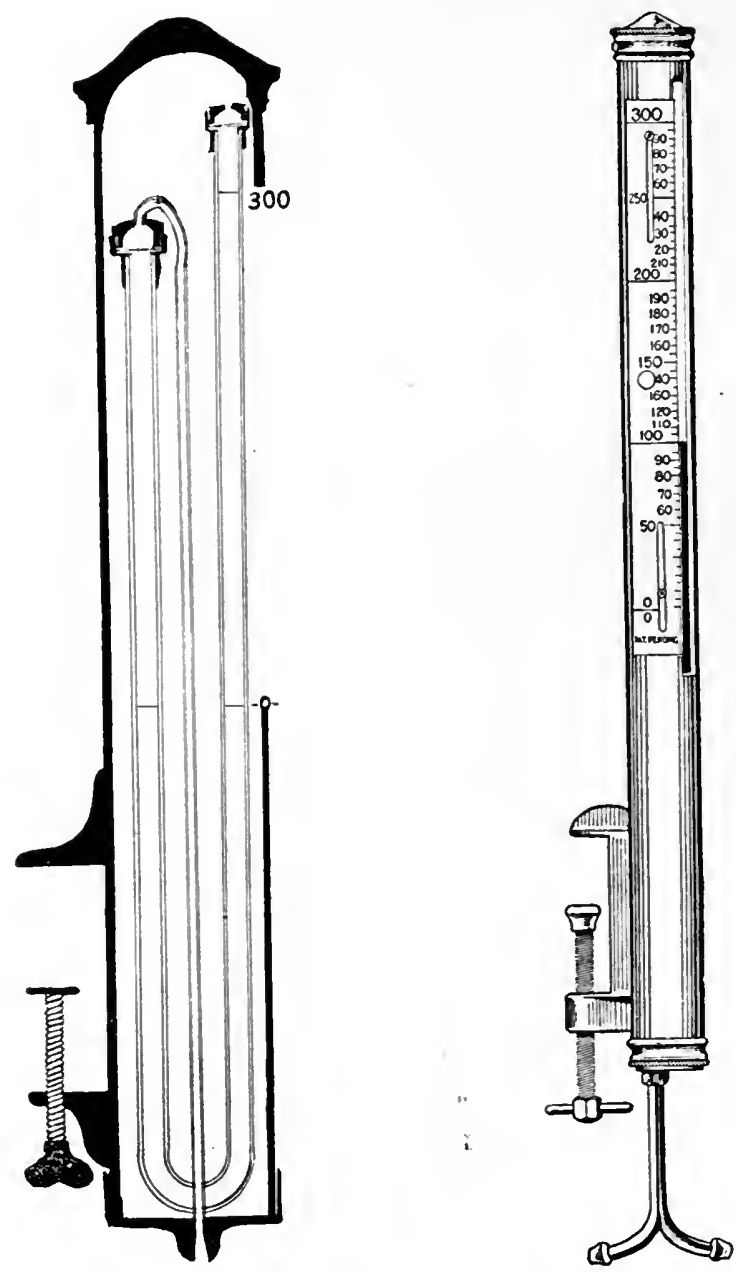

FIG. 23.- The construction of the manometer of the Mereer instrument. A U-tube manometer with the top of each limb of the $U$ closed with a barometer kid gasket held securely in a metal socket. The gasket allows the air to pass freely but retains the nercury. The manometer is enclosed in an outer metal casing tube which is slotted and provided with a direet reading scale. The shorter limb of the $U$ is connected with a slip socket in the bottom of the outer tube by a small netal tube as shown. Only glass and kid come in contact with the mercury.

as compared with the L-tube type. (2) Owing to the capillary opening the mercury cannot spill. It can be carried in any position, 
requires no rubber caps or stopcocks. (3) Ready portability; no box is required; the wooden case, cuff and pump can be carried in a handbag (Fig. 24).

Several inexpensive instruments of the Riva-Rocei type are on the market which, especially if the auscultatory method be employed, will be found entirely satisfactory for clinical work. Among

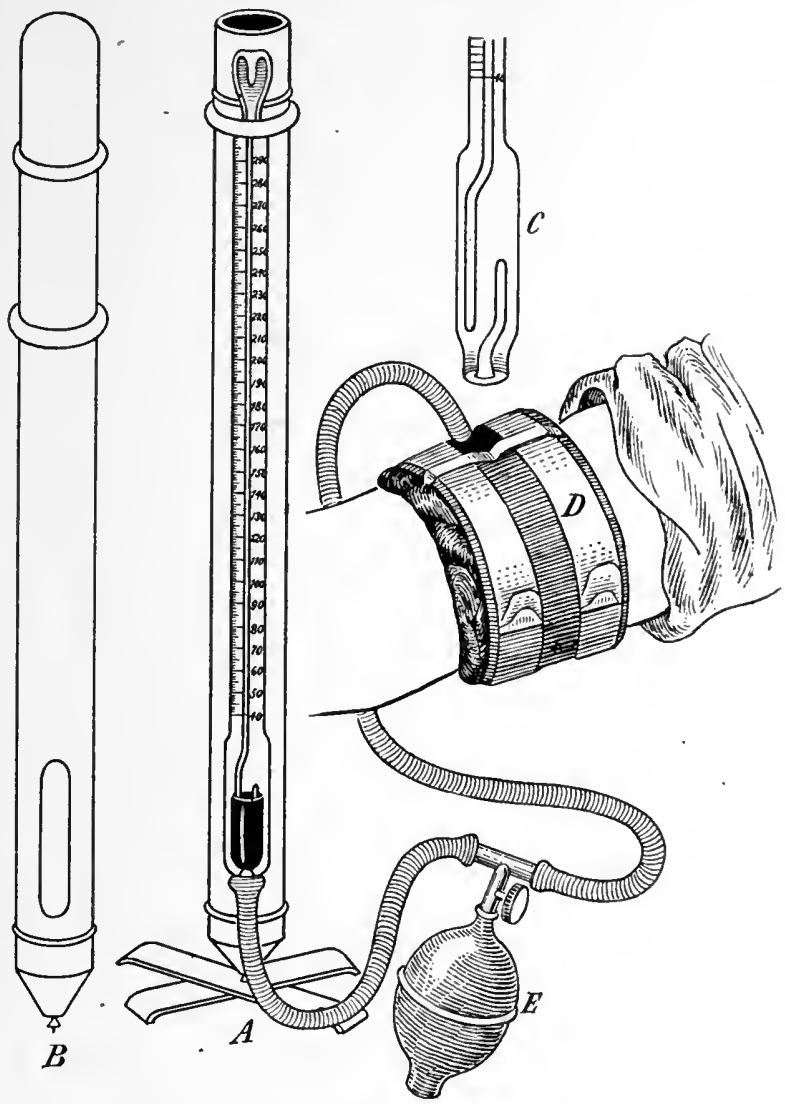

Fia. 24.-Hill's sphygmometer: $A$, sphygmometer; $B$, case; $C$, section of mercurial reservoir; $D$, brachial cuff; $E$, pump.

these the instruments of Linnell, ${ }^{1}$ Kercher, ${ }^{2}$ Cook, ${ }^{3}$ Sahli, ${ }^{4}$ BruhnsFahraeus ${ }^{5}$ may be mentioned. (See p. 133.)

i A Pocket Mercury Manometer, Jour. Amer. Med. Assn., October 12, 1912, lix.

2 Blood-pressure apparatus manufactured by Lander, Cleary \& Co., Philadelphia.

${ }^{3}$ Cook sphygmomanometer manufactured by the Kny-Scheeres Co., Now York.

${ }^{4}$ Manufactured by Büehi \& Sohn, Bern, Switzerland.

- Manufactured by Stein \& Werner, Stoekholm. 
The Gärtner tonometer ${ }^{1}$ consists of small distensible rubber rings which are applied to the finger, and connected with a manom-

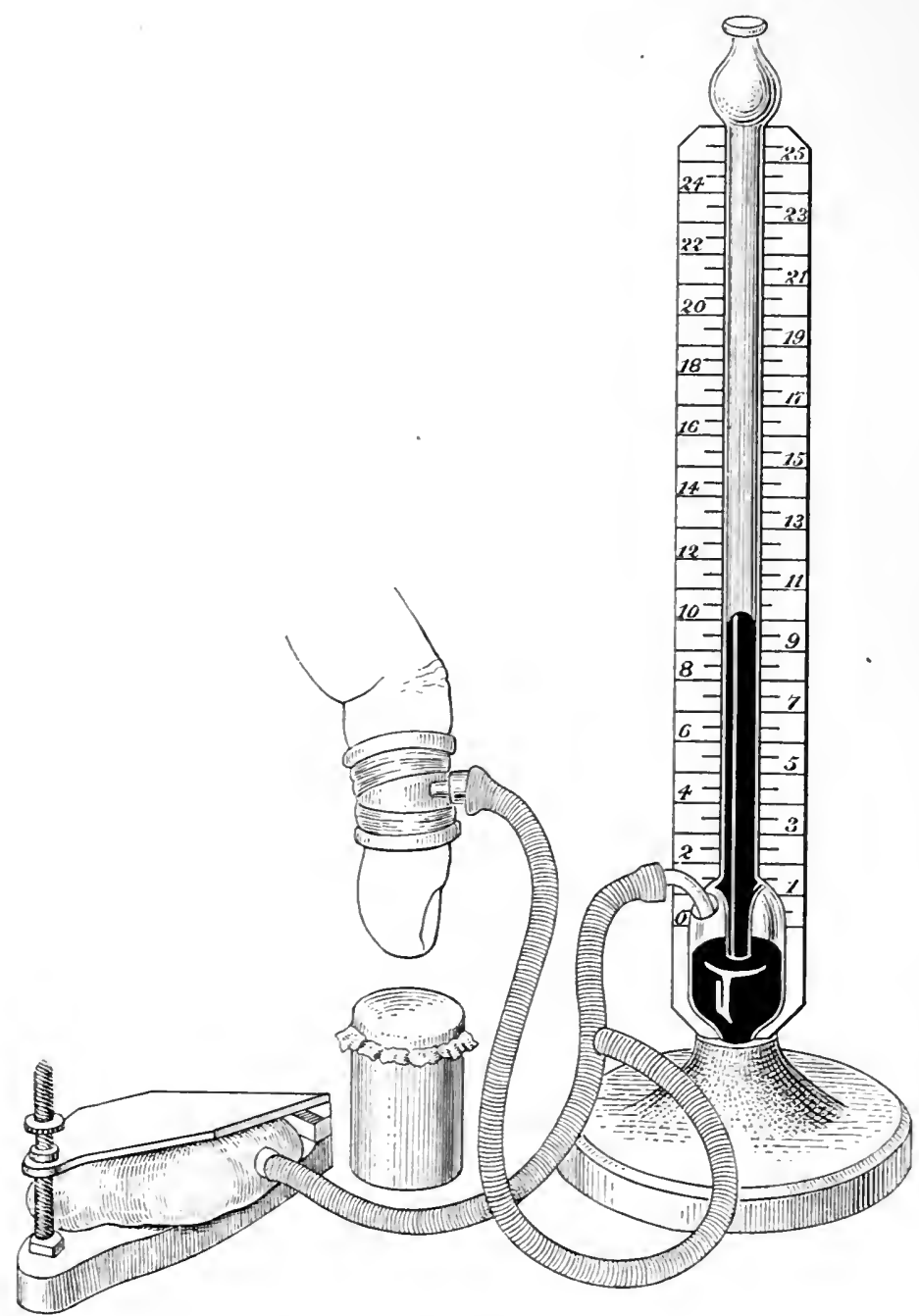

FıG. 25.-Gärtner's tonometer.

eter. The finger is rendered bloodless and the point at which the blanched skin becomes rosy is taken as the criterion of the

1 Wien. med. Woch., 1899, xlix, 1412. 
systolic pressure. This instrument yields variable amounts of error but is still occasionally employed in connection with brachial readings to determine the pressure ratio between large and small arteries, in the hope of thus throwing some light on the state of vascular tonus (Fig. 25).

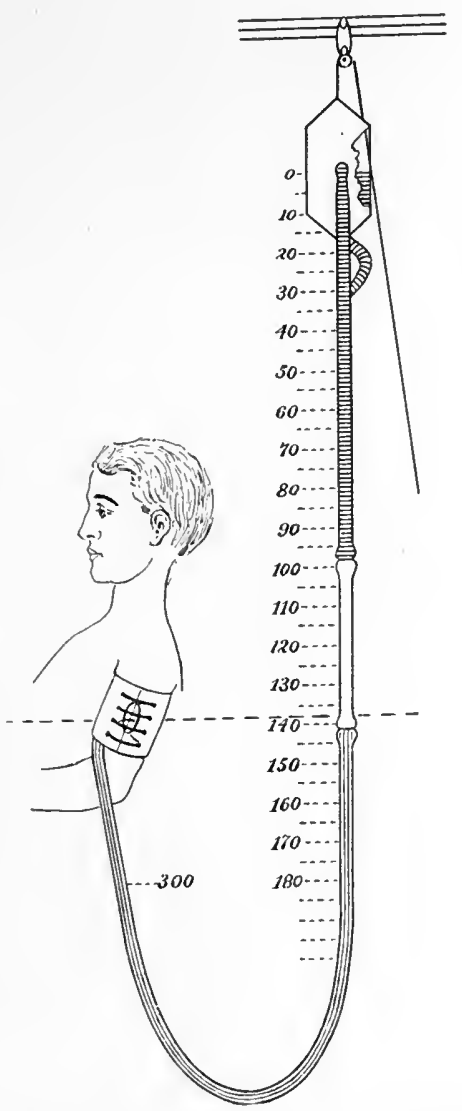

Fig. 26.-Bishop's blood-pressure apparatus.

"'The method of Gärtner is open to the objection that the arteries of the fingers are probably small enough to participate directly in vasomotor changes. 'Therefore a rise of the general blood-pressure, which in part is caused by constriction of the digital arteries, may be associated with a fall of pressure in the terminal phalanges. That this does occur is practically demonstrated by the fact that cold, which usually causes peripheral constriction with consequent 


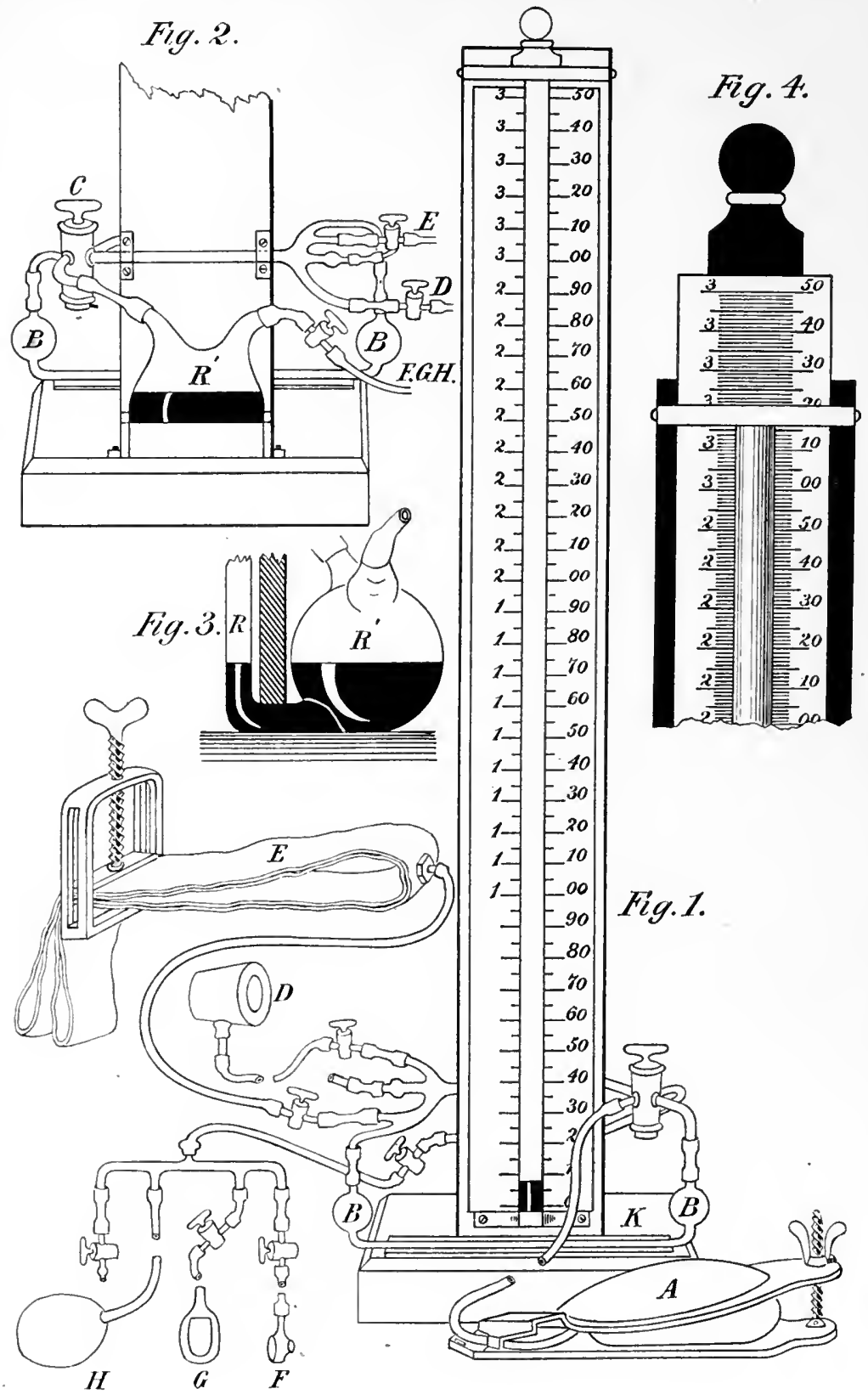

FIG. 27 
rise of the general blood-pressure, seems to cause a fall of pressure as estimated by the method of Gärtner."1

What may be described as a home-madeblood-pressure apparatus has been devised by Bishop. The accompanying diagram is sufficiently explanatory to indicate the general principle upon which the instrument is based ${ }^{2}$ (Fig. 26).

\section{A. COMPRESSED-AIR MANOMETERS.}

Compressed-air instruments are small, light and compact. A small quantity of mercury or colored liquid is kept in the bulbous end of the glass tube. When pressure is raised, a drop of this fluid is forced up into the glass tube. This is simply used as an index by means of which the height of the pressure can be read off.

The Oliver Instrument. ${ }^{3}$ - Method.-Lay the instrument on a flat surface and lift the upper end of the manometer by the brass ring,

${ }^{1}$ Erlanger, The Circulation, Jour. Amer. Med. Assn., October 27, 1906.

${ }^{2}$ For detailed description see L. F. Bishop, The Measurement of Human Bloodpressure, Arch. Diag., April, 1908.

${ }^{3}$ Manufactured by Dressler-Beard Mfg. Co., 386 Second Avenue, New York.

LegEND For Fig. 27.

Universal sphygmomanometroscope. At Janowsky's Clinic in Petrograd the blood-pressure in the upper extremities is determined by comparison of the findings of five different appasatus. These include the Riva-Rocci cuff, the tonometer, the two spring manometers of Basch and the water manometer of Zipliaef-Janowsky. N. van Westenrijk, of Petrograd, has suceeded in combining these various apparat us in a single one in which one mereury manometer answers for all. It is arranged so that the zero point can be adjusted, and it allows comparison of the findings with different technique and registration of the pressure up to $350 \mathrm{~mm}$. Hg. The mercury tube $R$ (Figs. 1 and 3 ) opens below into the reservoir $R^{\prime}$, the graduated scale (Figs. 1 and 4) being fastened within the mercury tube and the reservoir. The whole is mounted on a weighted wooden standard. The right-hand branching arm of the reservoir $R^{\prime}$ (Fig. 2) is connected with the tube which forks to the Riva-Roeci euff $E$ (Fig. 1), the Gärtner tonometer ring $D$, the Basch contrivance for determining the pressure in the capillaries $F$, the rubber bulb for determining the blood-pressure in the veins $G$, and the rubber bulb $H$ for increasing the pressure in the glass chamber $F$. All these are controlled with stopcocks in the tubes. The left-hand branching tube from the reservoir $R^{\prime}$ is connected with the rubber bulb $A$, which is eompressed by a screw lever to increase the pressure (Fig. 1) in the cuff and ring and also in the manometer. The tube $B$, with its two enlargements and capillary lumen (oscillometer) runs in the groove $K$, and is brought into connection separately with the cuff, ring, etc., on the other side of the reservoir $R^{\prime}$, by turning the stopcock $C$. The apparatus allows the Riva-Rocci cuff to be used for tonometry as well as in the usual manner. The illustrated description is published in the Mitteilungen der Gesellschaft für innere Med., 1908, vii, 202. Universal sphygmomanometroscope, manufactured by Eberhard, Petrograd, Russia. 
at the same time opening out the hinged prop at the back to the extreme right-hand corner of the box. This places the scale at an

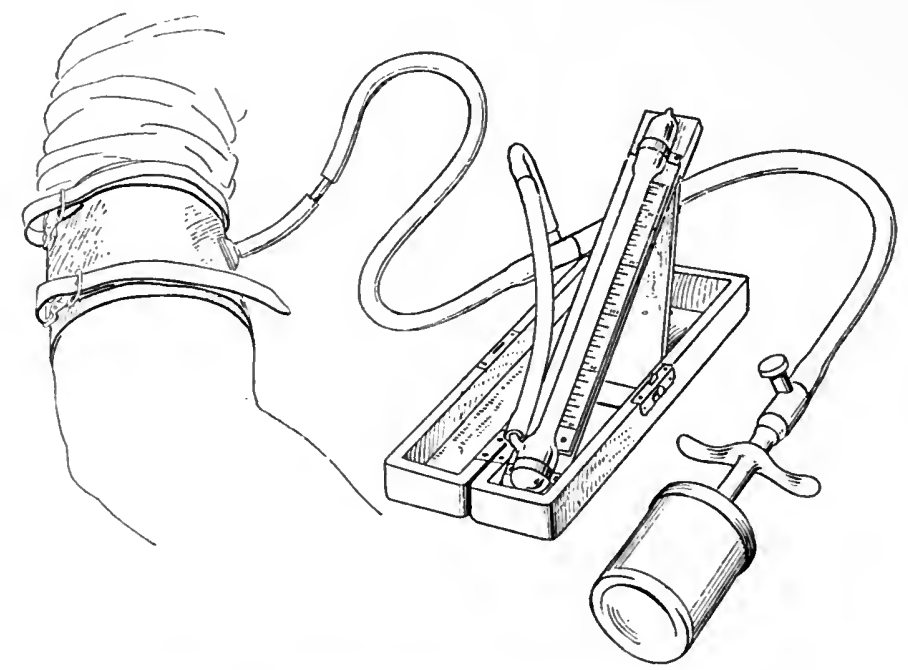

IIG. 28. - The Oliver mercurial compressed-air manometer.

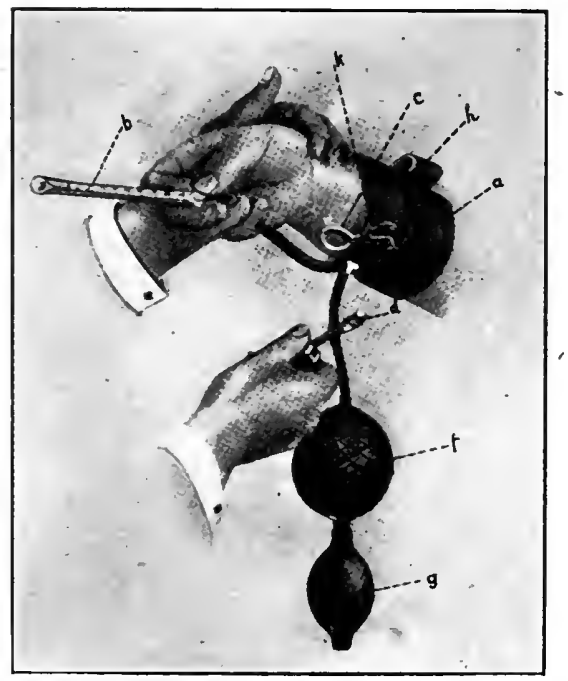

F1G. 29.- Hertz sphygmomanometer. ${ }^{1}$

${ }^{1}$ Das Neue Modell meines Blutdruckmessers, Wien. klin. Woch., 1911, No. 37, p. 1306. Kolomoitzew (Müneh. med. Woch., July 20, 1909, p. 1482), although obtaining figures within normal ranges with this instrument, found an accurate aceordance with Riva-Rocei reading in only 13 per eent. of the cases. 
angle of about 45 degrees and facilitates observation. 'Temperature variations are compensated for by the vacuum which surrounds the pressure chamber. The scale is in millimeters of mercury, having been standardized. Any mercury remaining in the tube may be shaken down, as in a thermometer, when the box is closed. A modification of this instrument is made by 'Tech, of New York. The instrument is simple and compact. It consists of the usual cuff and inflation bulb. The manometer is of the compressed-

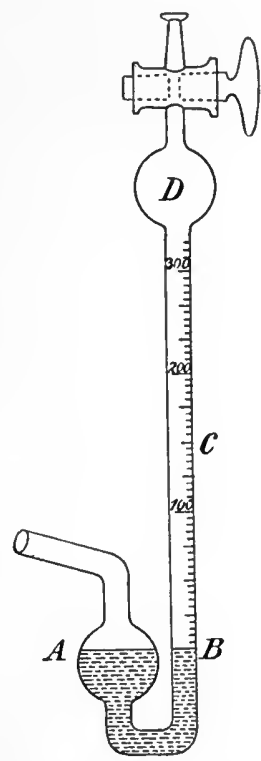

F1G. 30.-The Bendick Instrument. Diagram showing the glass tube of the water sphygmomanometer: $A$, bulb partially filled with water on which pressure is exerted by a column of air in the tube leading from the cuff; $B$, level of water in upright calibrated tube $C$, which contains air above; $D$, bulb in the tube $C$, to contain the air as the water rises. At the top of the tube is a cock for closing the tube after the water is put in below.

air type, a drop of mercury being used as an index. It is open to the criticism, however, that the small scale with widely spaced gradations militates against accuracy.

Hertz's Sphygmomanometer.-Method.-After application to the wrist as shown in the cut, the cautery bull $f$ is tensely inflated, the clip $d$ cutting off communication with the cuff. When this is accomplished the clip $d$ is opened and the droplet of mercury approaches the distal end of the manometer. The sy'stolic press- 
ure is read at the point at which the radial pulse disappears; the diastolic pressure, at that at which the pulse becomes diminished in volume.

Bendick's air-water sphygmomanometer ${ }^{1}$ is calibrated in millimeters of mercury. Water is poured into the reservoir until the points $A$ and $B$ are reached. The stopcock is then closed above $D$. The pressure in the cuff is exerted on the fluid at $A$ and transmitted to $B$ while the air in the column is being compressed. The bulb

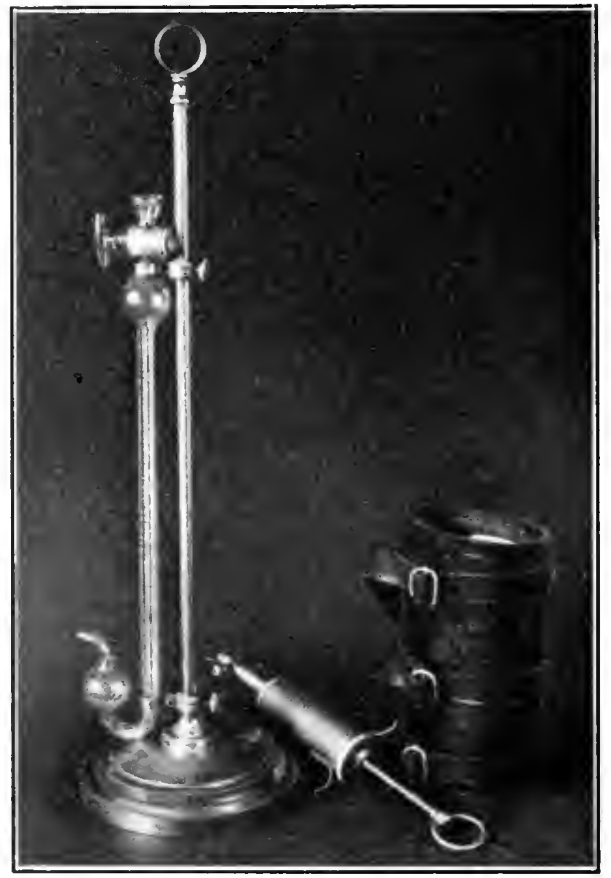

FIG. 31.-Bendick's air-water sphygmomanometer.

acts as a reservoir for the fluid, the bulb $B$ for the air, allowing the liquid to rise the entire length of the tube in recording pressures up to $300 \mathrm{~mm}$. Hg. The two bulbs allow for a larger and more accurate seale than is the case where straight tubes are used. The metal 'T-tube with a stopcock and needle-valve escapement allows the air pump to be disconnected and controls the fall of pressure.

1 A New Air-water Sphygmomanometer, Jour. Amer. Med. Assn., 1911, lxi, 1873. Manufactured by Eimer \& Amend, 205 Third Avenue, New York City. 


\section{CHAPTER III.}

\section{THE INSTRUMENTAL ESTIMATION OF BLOOD- PRESSURE (CONTINUED).}

III. The Graphic Method of Estimating Blood-pressure.-If instead of employing the finger below the cuff in order to determine the presence and character of the pulse wave, a sphygmograph is substituted, one obtains a graphic tracing of the appearance, increase, maximum oscillation, and final disappearance of the pulse, which occurs while the pressure in the cuff is falling from above the systolic to below the diastolic pressure. Such a procedure has the advantage of furnishing a permanent record, and also of allowing one to judge more accurately the exact point at which the maximum pulsation is established. The graphic method is therefore employed where the greatest accuracy is demanded, as in experimental work.

In the instruments of Erlanger, Uskoff, Muenzer, instead of a sphygmograph on the radial artery, the brachial pulsation of the cuff itself is transmitted indirectly to a kymograph. "Indirectly," because the pressure in the cuff, were it not "stopped down," would be too great for the delicate membranes connected with the tambour.

The systolic pressure is determined by noting with a decreasing pressure the point at which the first full pulse wave is transmitted to the drum. While the cuff pressure is still above this level a series of small elevations often appear which are due to the impaction of the pulse wave against the upper margin of the cuff, the central portion of which still occludes the blood-flow.

'The lower end of the maximum excursions is generally taken as an indication of the diastolic pressure because at this point there is just sufficient pressure in the cuff to cause a complete diastolic collapse of the artery, while at the same time permitting a complete distention during systole. Pressure on either side of the cuff being about equal, although alternate, the greatest oscillation of the interposed membrane occurs. Below this level the cxcursions grow progressively less because with the falling manometrical pressure less and less of the arterial pulsation is transmitted to 
the gradually loosening cuff. In some cases it is difficult to determine the exact point at which the inframaximal pulsations begin.

When the diastolic pressure is being estimated by the graphic method it sometimes happens that two regions of maximum oscillation occur. "The first diminution in amplitude occurs apparently at the moment the blood begins to reënter the arm with considerable relocity. The amplitude again increases when the vessels of the arm become distended with blood." The second maximum is undoubtedly the minimum pressure (Erlanger and Hooker).

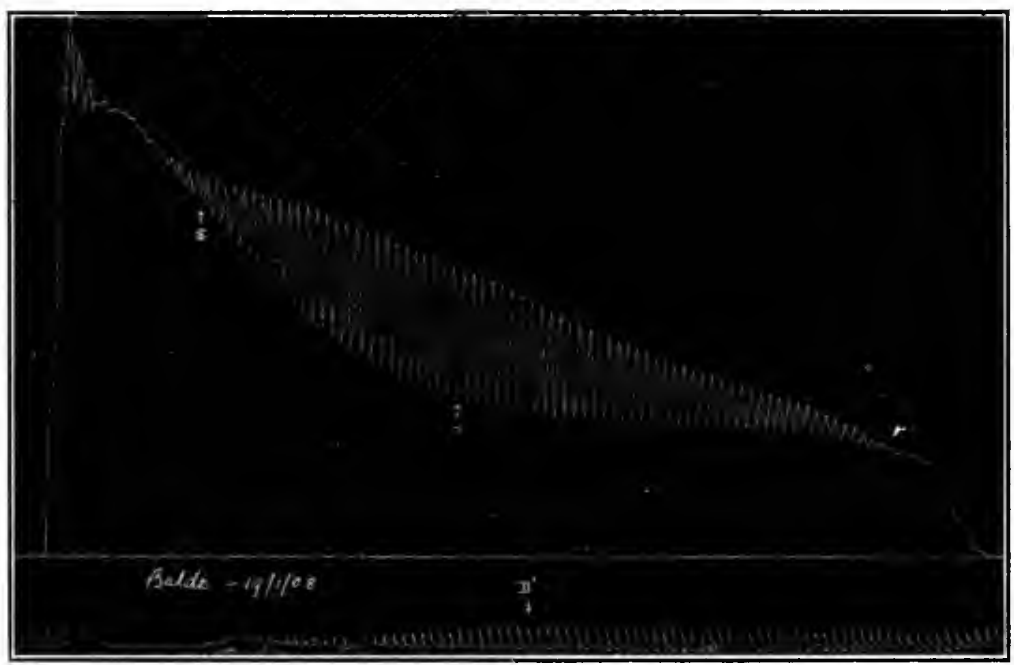

FIG. 32.-Pulse tracing made with the Gibson instrument. Here the first full pulse wave $S$ indicates the systolic pressure. It is the first wave which has overcome the pressure in the brachial cuff and reached the radial artery. Preceding the point $S$ a number of small waves are seen which are produced by the impact of the pulse waves against the upper portion of the brachial cuff, a segment of the artery being still obliterated. Tracing taken by rapid inflation and gradual escape with quickly revolving cylinder. It shows systolic pressure to be 170 ; diastolic pressure, according to Gibson, $106(D)$; and by Masing's method, $90\left(D^{\prime}\right)$. (Tracing after Gibson.)

Occasionally a successive series of maximum wares at about the diastolic level are observed. This is attributed by Sahli to two factors: (1) With increasing compression, when the minimal pressure is exceeded, the pulse wave undergoes a stasis underneath the (broad) cuff which increases the pulsatile excursions of the tonograph. (2) At the same time a continuously diminishing amount of the sphygmographic wave becomes arailable for transmission. These effects tend to counteract each other and 
the resulting waves will vary according to which of the two effects predominates. Another explanation is that the collapse of the artery being gradual, at first only that portion of the artery which underlies the centre of the cuff is compressed; some time is required before the whole segment is similarly affected (von Recklinghausen). According to this argument, with a rising pressure one should choose the beginning of the large oscillations as the diastolic criterion. This source of confusion may be obviated by the simultaneous employment of a brachial and a radial cuff, in which event the lower cuff will show a distinct oscillatory diminution the moment the diastolic level in the upper cuff is exceeded.

If the pressure "be allowed to fall more slowly than in von Recklinghausen's experiments the maximum oscillations will be followed immediately by the abrupt diminution in amplitudethe diastolic pressure corresponds with the abrupt diminution in amplitude" (Erlanger).

"There are obviously two processes at work tending to alter the amplitude of the peripheral pulse while the artery is being compressed: (1) some unknown factor which tends to enlarge the pulse amplitude; (2) the pressure from without must constantly tend to diminish the amplitude of the peripheral pulse by diminishing the caliber of the artery. It is true that the latter factor will not be great so long as the external pressure is less than the intra-arterial minimum; the great diminution in caliber will occur when the diastolic pressure is exceeded. There is no justification for Sahli's assumption that the arteries suffer no lateral expansion with the advent of the pulse wave and that therefore the caliber of the artery is not diminished by external pressure until that pressure exceeds the diastolic" (Erlanger).

Gibson advised selecting the middle point of the greatest amplitude of oscillation as the diastolic point (Fig. 36). Strassburger takes the point at which the previous maximal oscillation begins to diminish under increasing pressure. Macwilliam and Melvin, ${ }^{1}$ who have investigated the relation of the diastolic pressure to the maximum oscillation, believe that the latter occurs not at the point at which internal and external arterial pressure are equalized, but when a "half-flattening" of the arterial lumen is brought about.

The oscillation, they believe to be compounded of " $(a)$ the difference in capacity between the half-flattened and the circular

1 The Estimation of Diastolic Pressure in Man, Heart, 1914, v, 153. 
tube, $(b)$ the distention caused by the systolic wave and $(c)$ such volume change as may result from longitudinal expansion at each pulse beat." The point of maximum oscillation varies greatly owing not only to a disturbance in the relationship between the components just mentioned, but also owing to (1) distensibility and flexibility of the arterial wall, (2) the interval between systoles, (3) the form of the internal pressure curve, and (4) the degree of arterial elongation at each beat. Finding none of the previously mentioned criteria always accurate they suggest taking as the index of the diastolic pressure "the level of the external pressure just after the abrupt diminution has taken place," that is, the point at which pressure is just insufficient to cause flattening of the artery.

If a tracing is taken from the artery below the seat of compression a sphygmographic curve results. In such a curve the onset of the large waves is accompanied by a change in the form of the waves. At the upper systolic level of the large waves the pulse previously anacrotic, becomes katacrotic; at the lower level the diastolic wave falls away almost vertically instead of obliquely (von Recklinghausen). These facts furnish us with a further means of recognizing the diastolic pressure provided we are able to recognize the katacrotic notch.

The diastolic pressure by the graphic method, as shown by Müller and Blauel, may be as much as 28 per cent. too high. Although the artery at once increases its oscillations when the diastolic level is reached, it takes some time for a complete arterial collapse to be established.

The systolic pressure cannot always be absolutely determined because of the primary small subsystolic waves due to concussion of the upper border of the cuff. The diastolic pressure cannot always be absolutely measured because (1) there is still a difference of opinion as to which criterion should be used (beginning, middle, or end of the large waves or the onset of the anacrotic notch), and (2) because it is not always possible to determine the exact point at which these changes occur. But since it is admitted that clinical blood-pressure readings are approximate estimations only, and not exact mathematical calculations, the results obtained will be sufficiently accurate for practical purposes.

Since the foregoing lines were written, corroboration of the statements made has been furnished by the investigations of Kilgore, ${ }^{1}$

1 The Large Personal Factor in Blood-pressure Determinations by the Oscillatory Method, Areh. Int. Med., 1915, xvi, 893. 
who showed that identical tracings made with the Erlanger apparatus were very variously interpreted by different individuals, even by those who were familiar with the instrument and who were using the same criteria of the systolic and the diastolic pressures.

Erlanger states that these discrepancies can be minimized if not actually obviated by.(1) using as the systolic criterion the change in form, with the paper moving at sufficient speed for this purpose; (2) by using the first consistent decrease in amplitude as the diastolic index; and (3) in doubtful cases controlling the readings made by the method of continuous escapement by readings made by that of intermittent escapement. ${ }^{1}$ (See page 91.)

\section{INSTRUMENTS FOR GRAPHIC REGISTRATION.}

When using the graphic method, some sort of an automatic registering device should be attached to the instrument, for other-

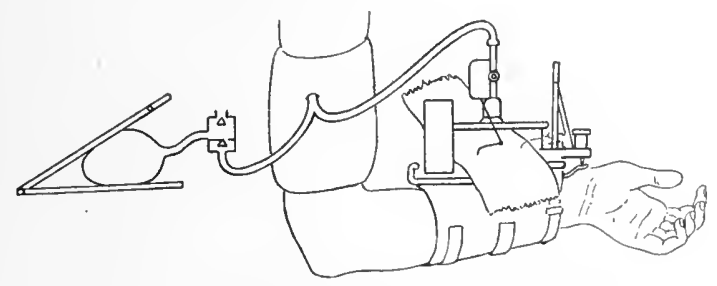

Fig. 33.-The Jacquet sphygmotonograph.

wise one is forced to mark by hand the various pressure levels on the moving paper while watching the fall of the manometer, a procedure which is always irksome and often inaccurate. Various devices have been employed to obviate this necessity by substituting an automatic indicator which marks the varying height of pressure upon the record. Thus in case of the sphygmotonograph of Jacquet ${ }^{2}$ (Fig. 33) the stylet of a small metallic manometer communicating with the brachial cuff registers directly on the tracing. The beginning of the curve represents $50 \mathrm{~mm}$. $\mathrm{Hg}$. and each elevation of $1 \mathrm{~mm}$. on the tracing is equivalent to a pressure increase of $10 \mathrm{~mm} . \mathrm{Hg}$. This device can be attached to the regular Jacquet cardiosphygmograph and has the endorsement of Gal-

\footnotetext{
1 Erlanger, J., An Analysis of Dr. Kilgore's Paper, ete, ibicl., p. 917.

${ }^{2}$ Manufactured by C. \& E. Streisguth, Strasburg, Germany. A. 1I. Thomas \& Co., Philadelphia agents.
} 
lavardin. It is open to the criticism which applies to all metallic manometers - the necessity of standardization. ${ }^{1}$

Bingel $^{2}$ has devised an instrument in which every pressure change of $10 \mathrm{~mm}$. is marked on the tracing by the interruptions of an electric current. 'This spacing is rather wide even for clinical purposes.

The sphygmomanometer devised by G. A. Gibson ${ }^{3}$ (Fig. 35) consists of "a mercurial manometer, the lumen of which is exactly that of the ordinary physiological kymograph." 'The air contained in the armlet can be increased, and the pressure on the limb therefore elevated, by means of a large syringe, and the pressure may be raised quickly or slowly, according to requirements. By means of a valve the pressure may also be lowered quickly or slowly.

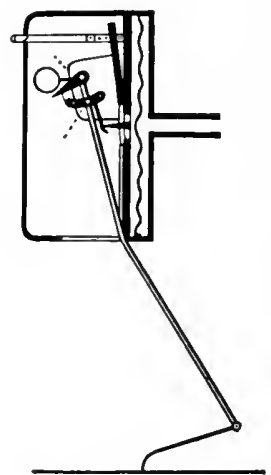

FIG. 34.-Cross-section of the registering metallic manometer of Jacquet.

A float rests upon the mercury, surrounded, as is usual in the physiological laboratory, by alcohol, and an upright rod of aluminum leads to a horizontal arm which writes on the revolving cylinder. In order to have the absolute zero, a fixed arm traces the abscissa upon the cylinder, which is driven by a clock-work placed horizontally, as in the instrument of Erlanger. The pulsations of the artery below the point of compression are recorded by means of a

\footnotetext{
1 Silbermann, who has studied the changes which occur in the diastolic portion of the sphygmogram during an increasing cuff pressure, believes that some deductions can thus be drawn concerning the amount of vascular tonus. Neue Untersuchungsergebnisse bei d. Blutdruckmessung mittels des Tonographen, Deut. Aerzte-Zeitung, May 15, 1909.

${ }_{2}$ Ueber Messung des diastolischen Blutdruckes beim Menschen. Münch. med, Woch., 1906, xxvi, 1246. Manufactured by Albrecht, Tübingen, Germany.

${ }^{3}$ Quart. Jour. Med., 1907, i, 103.
} 
transmission sphygmograph. This consists of a tambour brought into contact with the brachial or radial artery, as may be most convenient, by a pelotte resting upon the vessel. It is adjusted to the arm by means of a spring provided with a screw. 'This

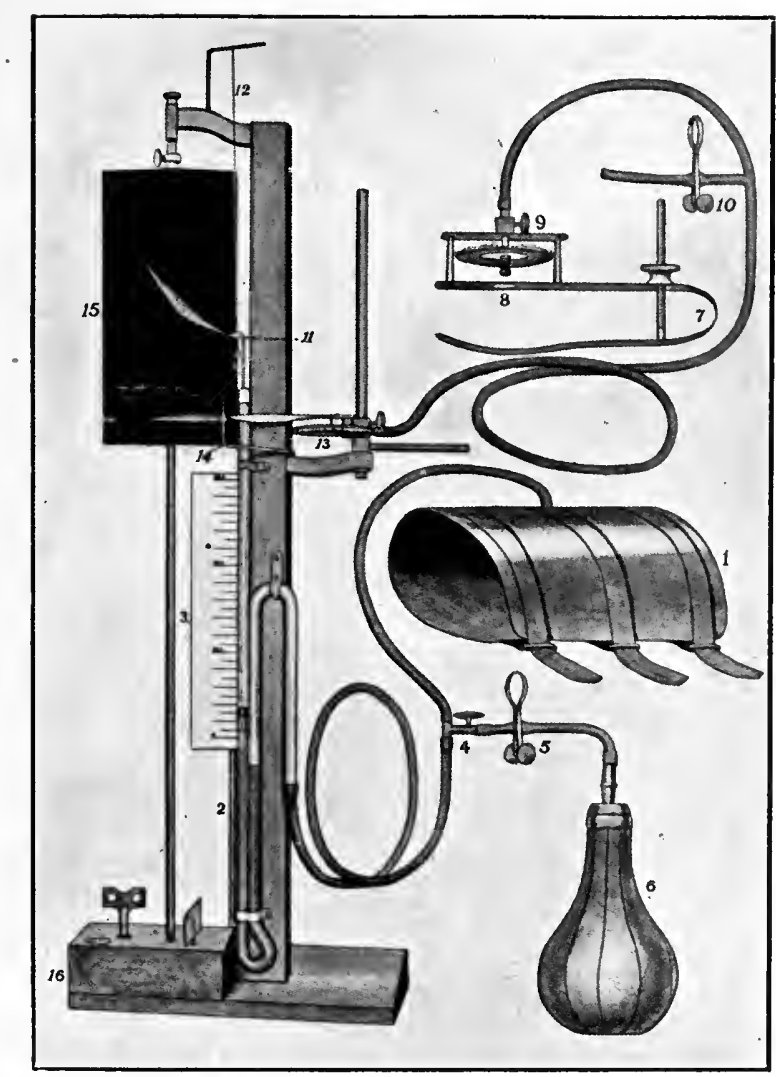

Fig. 35.-Gibson's sphygmomanometer. The recording sphygmomanometer deseribed in the text: 1 , armlet; 2 , manometer; 3 , seale; 4, valve for regulating pressure; 5 , elip, acting as a valve to syringe; 6 , inflation syringe; 7 , support for transmission sphygmograph; 8 , pelotte; 9 , serew for adjusting sphygmograph to artery; 10, elip for arranging pressure; 11, recording lever of manometer; 12, weighted thread for adjusting lever; 13, tambour reeording movements of transmission sphygmograph; 14, arn marking abscissa; 15, revolving eylinder; 16, elockwork.

tambour is brought into communication by rubber tubing with another tambour, the movements of which are recorded on the cylinder simultancously with the movements of the kymograph. 
The best tracing is obtained when the tambour in contact with the artery is larger than that connected with the recording lever by means of which the movements are amplified. The whole apparatus is shown in Fig. 35.

"In using the instrument the pressure within the cuff may be raised gradually or quickly, the latter being the more usual eourse. If it is slowly raised, the tracing of the kymograph shows at first a line of ascent with small oscillations, but as it rises the pulsations become more and more marked, and the excursion of the index more extensive, until a maximum point of amplitude is attained,

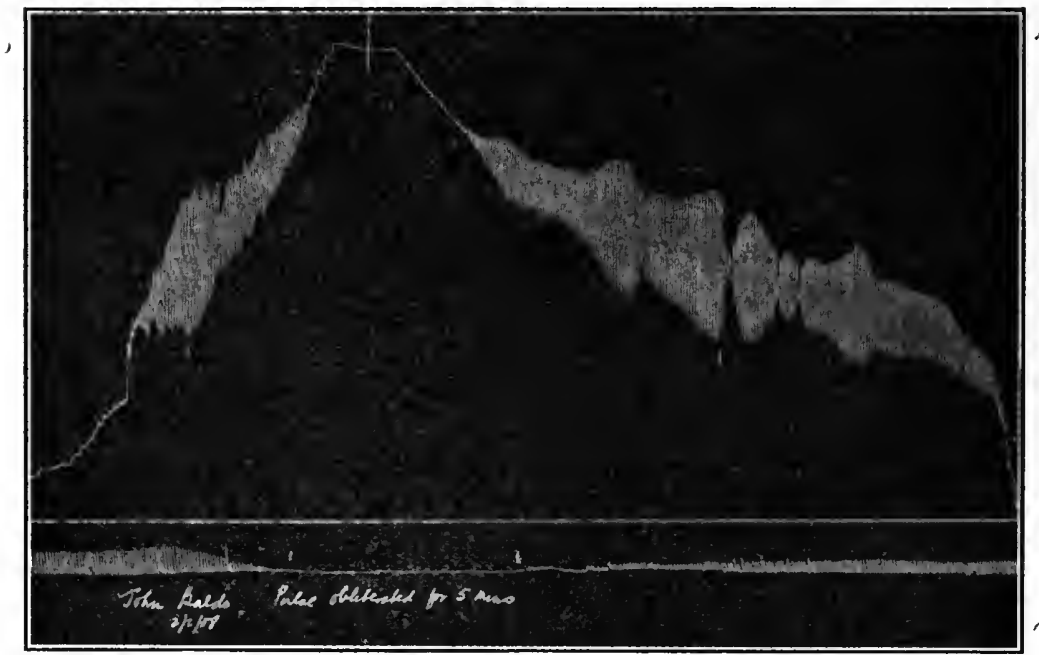

Fig. 36.-Tracing obtained by slow inflation and slow continuous escape. The ascending curve shows the pressure to be 180 systolic and 120 diastolic. The descending curve shows it to be 180 systolic and 118 diastolic.

when they begin to diminish and gradually disappear. Simultaneously the transmission sphygmograph records a gradual diminution in the amplitude of the pulsations, which finally cease. When all the movements of the kymograph, as well as of the sphygmograph, have come to an end, the pressure is allowed to fall by the escape of air from the valve, and the events which follow are the converse of those just described." Such a tracing is shown in Fig. 36.

The manometer is of the double column ( $\mathrm{U}$-shaped) variety and in reading the record the height of the abscissa must be doubled, e. g., actual reading $60 \mathrm{~mm}$., corrected reading $120 \mathrm{~mm}$. 
If the pressure in the cuff is allowed to fall continuously, the oscillating mercury acquires a progressively increasing momentum which tends to depress the lowest point of movement beyond its true level. This point is well illustrated in Fig. 37, a tracing taken by the method of intermittent escapement. In order to obviate this, Gibson advocates choosing the mean instead of the lowest point of the curve when reading the diastolic pressure.

The advantage of this instrument over that of Erlanger lies in the fact that the height of the mercurial column is automatically recorded, and that the personal equation is entirely eliminated.

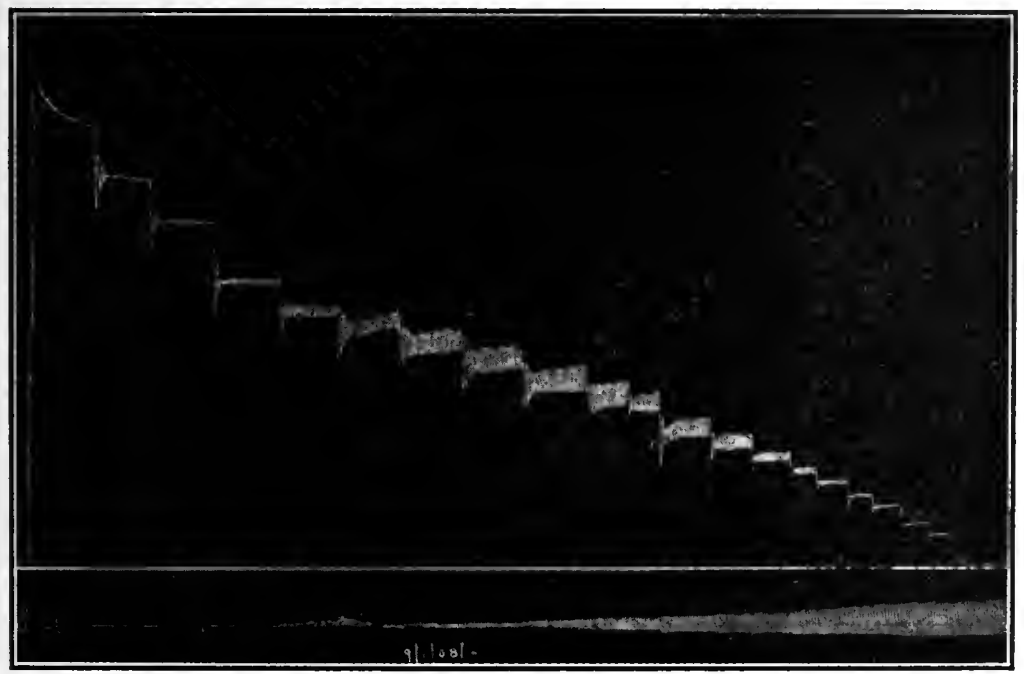

FIc. 37.- Tracing taken by rapid inflation and gradual intermittent escape, interrupted approximately after each descent of 5 to $10 \mathrm{~mm}$. $\mathrm{Hg}$. It shows a systolic pressure of 118, and a diastolic pressure of 74 . The varying effects of inertia are to be observed, as described in the text. (After Gibson.)

Its disadvantage in relation to the former is due to the inertia of the mercurial column which renders it less sensitive to delicate pressure ehanges. A closely similar instrument has been devised by $C$. Singer, ${ }^{1}$ which is better adapted to clinical purposes in being less bulky and heavy, without sacrificing aceuracy.

The Erlanger ${ }^{2}$ sphygmomanometer is an instrument somewhat

${ }^{1}$ A Clinieal Apparatus for Obtaining Graphie Records of Blood-pressure, Lancet, February 5, 1910, p. 365. Manufaetured by Hawksley \& Son, London. The instrument fits into a ease $14 \frac{1}{2} \times 5 \times 4$ inehes, the cuff is carried in the cavity of the eylinder. The tracings are made with ink pens upon white paper.

2.Johns Hopkins Hospital Report, 1904, xii, 62. 


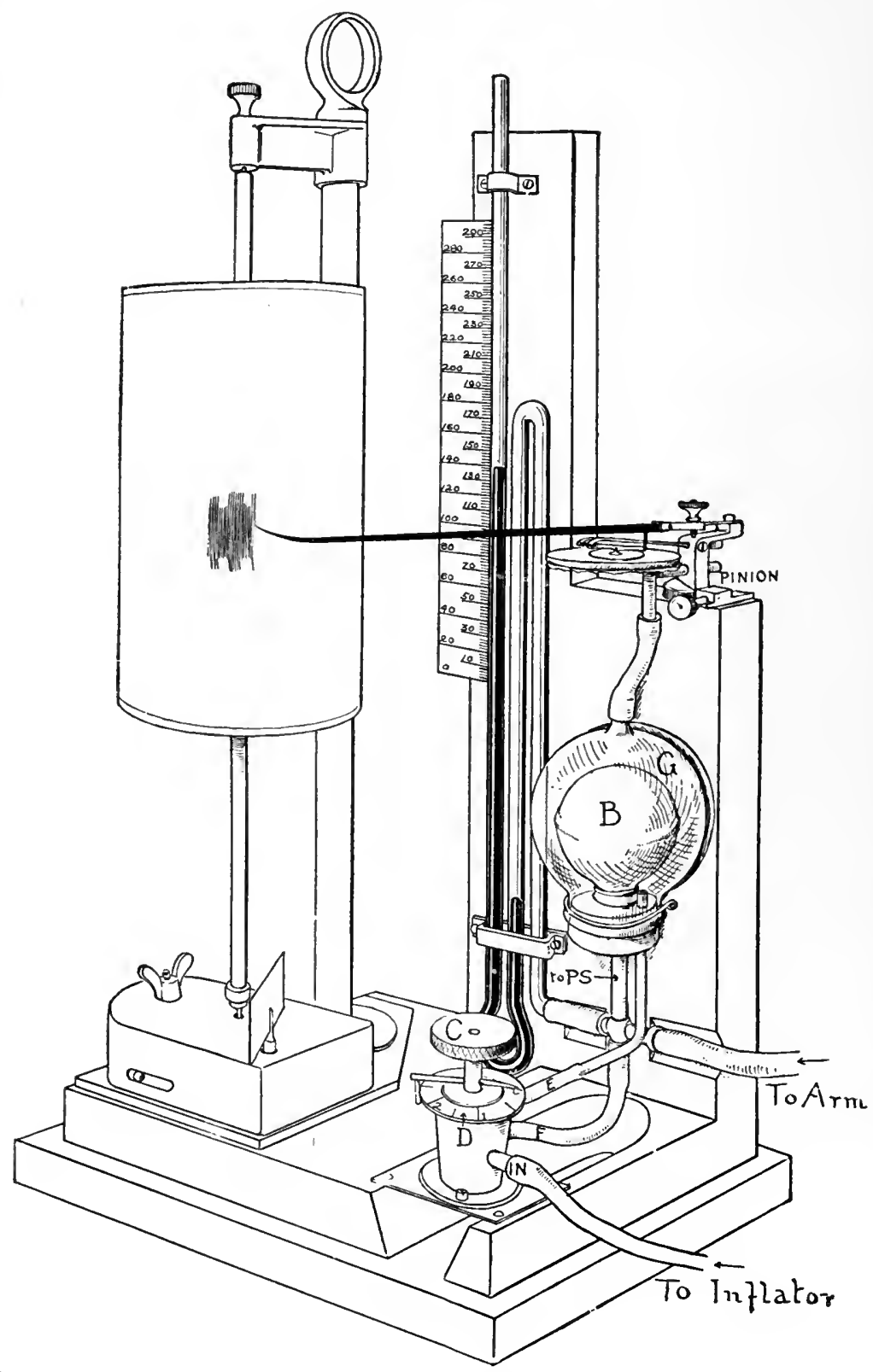

FiG. 38.-Erlanger's sphygmomanometer. 
similar to that last described. Owing to its size and construction it is better suited for the research laboratory than for the hospital. It has the advantage of yielding permanent graphic records, but necessitates considerable skill in its employment.

While somewhat complicated in construction it differs from other instruments of this type chiefly in its method of recording pulsations. A U-shaped manometer connects by means of a fourway tube with (1) the arm cuff; (2) a special stopcock; (3) a rubber bulb enclosed in an outer glass bulb. The latter is interposed in order to reduce the pressure between the cuff and the delicate tambour. Records are made on smoked paper on a kymographion by means of an aluminum needle (Fig. 38). The pump consists of a Politzer bag, and heavy, rigid tubing is employed.

Some little time is required to make the records and considerable familiarity with the instrument is necessary, but graphic records eliminate subjective errors. It can be obtained with polygraphic attachments and used to make pulse tracings, but is, so far as the blood-pressure is concerned, not automatically recording, $i . e$., the height of the mercurial column must be observed and recorded by the operator while the tracing is being made.

The systolic readings obtained with this instrument average $5 \mathrm{~mm}$. higher than with the Riva-Rocci method with a broad cuff. The diastolic readings correspond within a range of from 5 to $15 \mathrm{~mm}$.

The Uskoff 1 sphygmotonograph (Fig. 39) was devised with the intention of supplying a portable instrument which could be used both for blood-pressure and for pulse tracings. The latter function it excellently fulfils, furnishing good simultaneous tracings of the brachial artery and any one other desired pulsation, and requiring practically no adjustment.

Blood-pressure tracings taken with this instrument and automatically registered by an extremely ingenious escapement device are for two reasons not satisfactory. (1) Owing to the smallness of the opening which connects the interior of the glass bulb with the atmospheric air, large pulsations will transmit relatively more of their movement to the tambour than is the case with small pulsations. As a result of this, neither the sudden ehange from large to small pulsations (diastolic pressure), nor yet the change in the type of the individual waves which should appear at this point

${ }^{1}$ Der Sphygmotonograph, Zeit. f. klin. Med., 1908, Ixvi, 1 and 2. Manufactured by E. Zimmerman, Leipsic. 
(see p. 86) are demonstrable (Staehelin and Faustusi). (2) The rubber, glass-enclosed balloon exerts variable degrees of tension for increasing increments of pressure, largely owing to the effect of the silk netting by which it is surrounded (Erlanger $\left.{ }^{2}\right)$.

The author, who has used this instrument in practice for several years on account of its other excellent features, having long since become convineed of the inaccuracy of the blood-pressure tracings, has employed it with entire satisfaction as a simple manometer

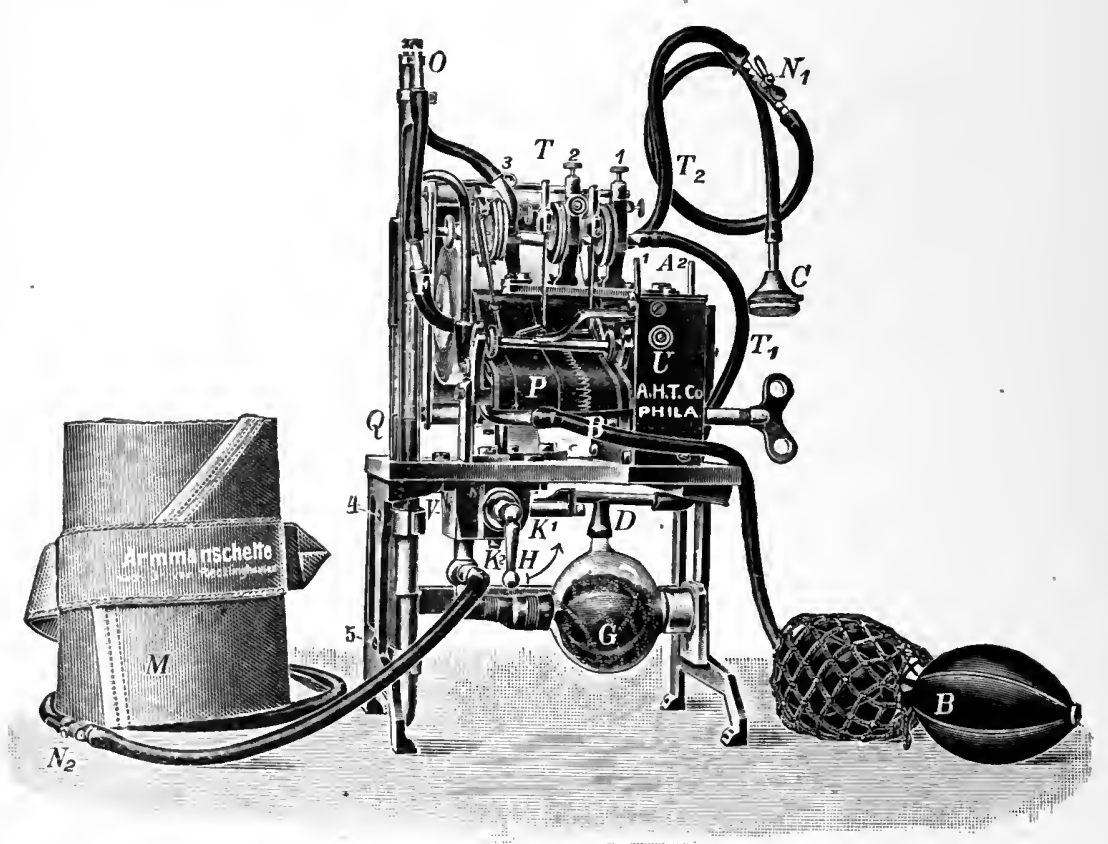

FIG. 39,-The Uskoff sphygmotonograph.

in comnection with the auscultatory method. The driving mechanism for the tracing is now furnished with two speeds, and a roll of smoked paper 25 meters in length, thus eliminating two of its early shortcomings.

The Uskoff sphygmotonograph simultaneously records the following:

a. Blood-pressure in millimeters of mercury.

${ }^{1}$ Das Verhalten des Blutdrucks beim Menschen wahrend der Erholung von Muskelarbeit, Zeit. f. klin. Med., Ixx, 444.

${ }^{2}$ Criticism of the Uskoff Sphygmotonograph, Arch. Int. Med., 1912, ix, 22. 
b. Carotid pulse (or jugular pulse or apex beat).

c. Arterial pulsation from the upper arm (brachial) at varying pressures (systolic and diastolic).

d. Time in one-fifth second.

In other words, there are four tracings upon the paper, three of which are fixed by the limits of the apparatus, $i$. e., blood-pressure in millimeters of mereury, arterial pulsations at various pressures and time tracing, while the fourth record may be taken at pleasure from either the jugular vein, carotid artery or apex beat.

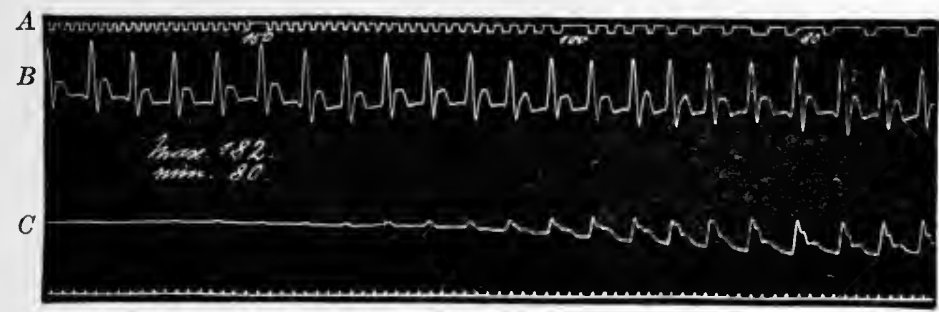

F1G. 40.-Tracing of blood-pressure made with the Uskoff apparatus. The upper tracing $A$ represents the pressure in millimeters of mercury, and the traeing is made while the pressure is reduced from $200 \mathrm{~mm}$. to $50 \mathrm{~mm}$., each vibration representing $2 \mathrm{~mm}$. of mercury column, with the one vibration, omitted every $50 \mathrm{~mm}$. The second tracing $B$ represents one taken from the carotid artery and recorded by indicator 2. This indicator may be used to record either the apex beat or the jugular or other vein pulsations at will. The third tracing $C$ is the sphygmotonogram or the tracing of the arterial (brachial) pulse under a falling pressure. With $200 \mathrm{~mm}$. of pressure this indicator traces a straight line because of the total obliteration of the pulse. At $162 \mathrm{~mm}$. of pressure the pulse reappears and at $92 \mathrm{~mm}$. of pressure has reached the lowest point of maximum oseillation. If the float on the mercury column continues to sink, the internal pressure is shown to be greater and the vibrations become smaller and smaller because of the elastie layer between the cuff and the artery, until it disappears entirely. This tracing taken under diminishing pressure constitutes, therefore, an accurate record of both the diastolic and systolic pressure.

Method of Operation.-The cuft $M$ is attached in the usual manner to the upper arm and pressure applied by means of the bulb $B$ until the pulse disappears. The pressure thus applied is first transmitted to the mercury manometer $Q$, lifting the perforated float as the mercurial column rises. At the same time it is transmitted to the rubber bulb enclosed in the glass bulb $G$ which is in air-tight connection with both the cuff and the manometer. With the handle of the cock $I I$ in the vertical position, the air in the glass bulb is expelled through the cock $K^{2}$ which is automatically opened at the same time as $K^{1}$.

When the pulse has, by application of sufficient pressure, disappeared, the cock $I I$ is turned in the direction of the arrow, 
which movement connects the glass bulb $G$ with the outside air through an exceedingly small opening, and the pulsations appear because air pressure in the cuff and manometer is thus slowly relieved through the valve $V$ and is divided between the rubber bulb and the air in the glass bulb $G$. The pulsations are at the same time transmitted from the air in the glass bulb $G$ by means of the third tabulature in connection with the indicator to the tracing paper.

The pressure arising from compression of the rubber bulb $B$ is also transmitted to two small outlets confronting each other on the top of the manometer, between which openings the perforated float passes as it rises and falls on the mercury column. The small holes in the float are arranged $1 \mathrm{~mm}$. apart with outstanding marks. The 50 millimeter gradations are indicated by the omission of $1 \mathrm{~mm}$. The passage of the air current through the small openings and through the corresponding perforations in the float operates indicator 3, and thus graphically records the actual pressure in millimeters of mercury at any moment throughout the operation.

Another instrument of the graphic type is that of Silbermann ${ }^{1}$ (Fig. 41). It is comprised of a radial and a brachial cuff, a mercury manometer, float, revolving drum for smoked paper, and an automatic registering device. The latter consists of a vertical arm from which comb-like projections extend. These projections are regularly spaced at intervals corresponding to $5 \mathrm{~mm}$. $\mathrm{Hg}$. in the manometer. When the kymographion revolves a series of parallel, horizontal lines-like a ruled page-are drawn on the smoked paper upon which the sphygmographic tracing is recorded. 'These parallel lines take the place of a scale on the manometer. The instrument is also supplied with a time marker, recording fifths of a second, and the driving mechanism with two speeds.

Method.-The brachial cuff $B$ is applied to the arm, inflated just enough to bring the 0 pressure level in the manometer on a level with the first tooth of the comb, and then clamped off. The heavy, double cautery bulb is now inflated to its maximum capacity so that when the clamp is released after the kymograph is set in motion, a steady and continuous rise in manometrical pressure will result. The radial cuff $D$, consisting of a small rubber capsule held in place by a strap, can be brought under different degrees

${ }^{1}$ Der Tonograph, Med. Klinik, August 30, 1908, No. 35, p. 1347. Manufactured by Oehmke, 21 Luisenstrasse, Berlin. 
of tension by means of a screw. When applied, this is so adjusted as to produce the maximum radial oscillation. It is used as an indicator of the levels at which with a rising pressure (1) the last pulse wave traverses the brachial cuff (systolic pressure);.(2) the first maximum oscillation occurs (diastolic pressure). Readings obtained with this instrument are from 4 to $5 \mathrm{~mm}$. Hg. lower than with the Riva-Rocei method, discrepancies which result from the weight of the float and the friction produced between the float

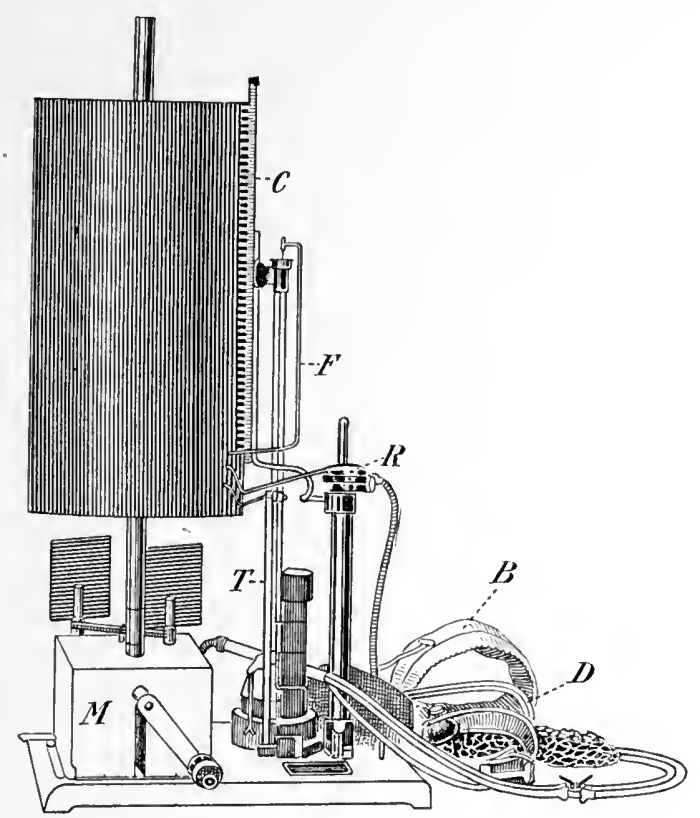

FIG. 41.-Silbermann's tonograph: $B$, brachial cuff; $D$, radial cuff; $R$, radial pulse recorder; $T$, time marker; $F$, float, indicating height of mercury in manometer; $C$, comb to mark $5 \mathrm{~mm}$. spacings on kymograph; $M$, driving mechanism.

recorder and the smoked paper. As this error is a constant one the author suggests setting the abscissa line on a level with the second tooth of the comb (Fig. 42).

Another graphic instrument has been devised by Brugsch. ${ }^{1}$ It consists of a U-shaped manometer and a revolving drum covered with white paper ruled in centimeter squares, which can be set at any level. A registering float superimposed on the mercurial

Zur Frage der Sphygmotonographie, nebst Beschreibung eines neuen Sphygmotonographen, Zeit. f. Exp. Path. u. Therap., May 30, 1912, xi. 
column transmits its height and pulsations directly to the drum. The latter is set at such a level that the abseissa of the centimeter

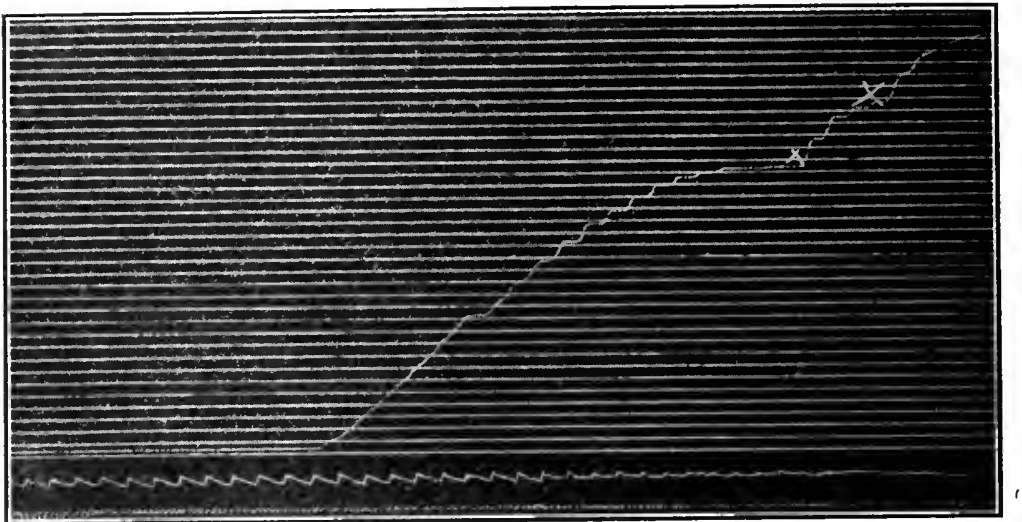

FIG. 42.--Tracing made with Silbermann's tonograph showing systolic pressure, $118 \mathrm{~mm}$; diastolic pressure, $65 \mathrm{~mm}$.

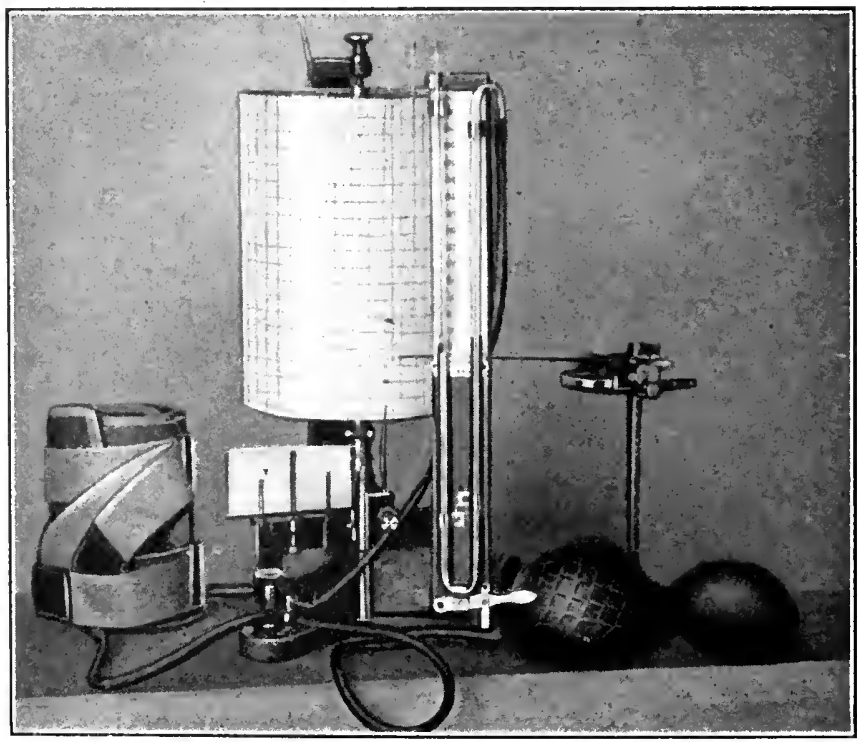

FIG. 43.-Brugsch's sphygmotonograph.

ruling and the zero point of the mercury correspond. Thus the actual height of the tracing above the base line, multiplied by 2 
(U-shaped manometer) gives us the correct reading. 'The brachial pulsations are transmitted by means of rubber tubing to a piston recorder and inscribed by means of ink pens upon the drum below the pressure curve (Fig. 43).

A more elaborate and complicated instrument, based on the Erlanger principle, is the sphygmoturgograph of Muenzer ${ }^{1}$ (Fig. 44). The tubing $S$ connects pump $P$ with cuff $M$ and is interrupted by stopcock $h$ to prevent communication with the glass-enclosed balloon $B$, until this is desired. 'The tubing $T$ comnects the balloon with the tambour. When air is pumped

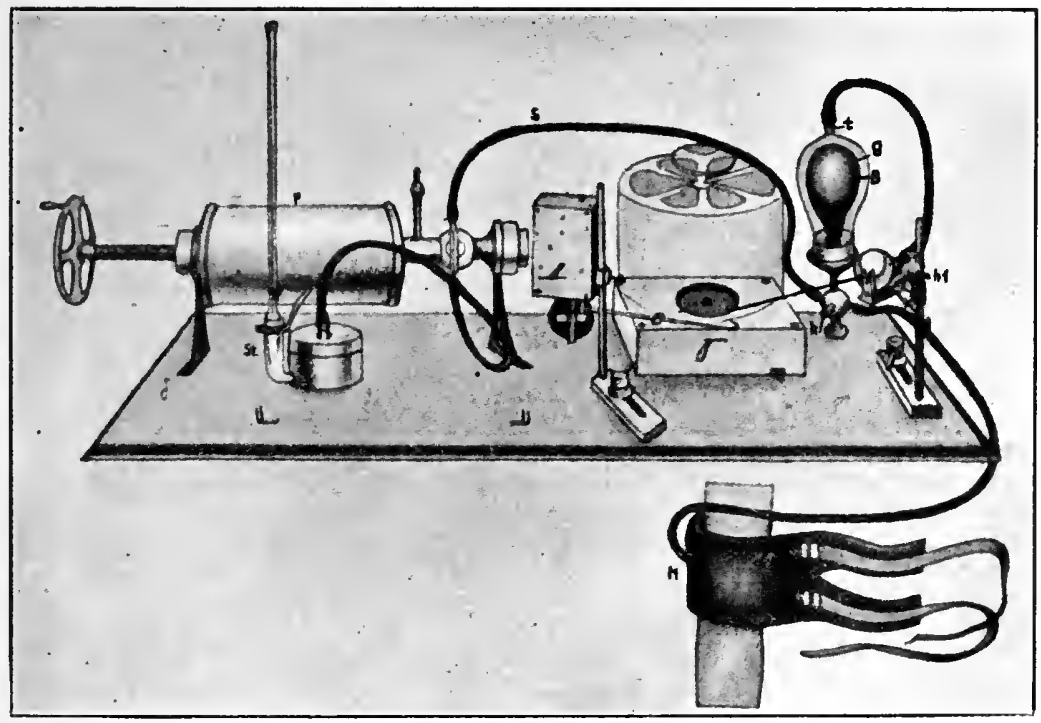

FiG. 44.-Muenzer's sphygmoturgograph.

into the system it reaches the manometer $S t$, the cuff $M$ and the balloon $B$. With the cuff applied to the arm and sufficiently inflated, the cock $h$ being closed, the pulsations are transmitted from the cuff through the balloon to the kymograph upon which they are recorded. The kymograph is supplied with two speedsa slow one for blood-pressure tracings, a rapid one for pulse tracings -and a time marker. In order to obtain the greatest and most accurate oscillations it is necessary to close both the cocks $h$ and

${ }^{1}$ Apparat. z. objektiver Blutdruckmessung, ete., Münch. med. Woch., 1907, xxxvii, 1809; also Ueber Blutdruckmessung, Zeit. f. exper. Path. u. Therap., 1907, iv. 


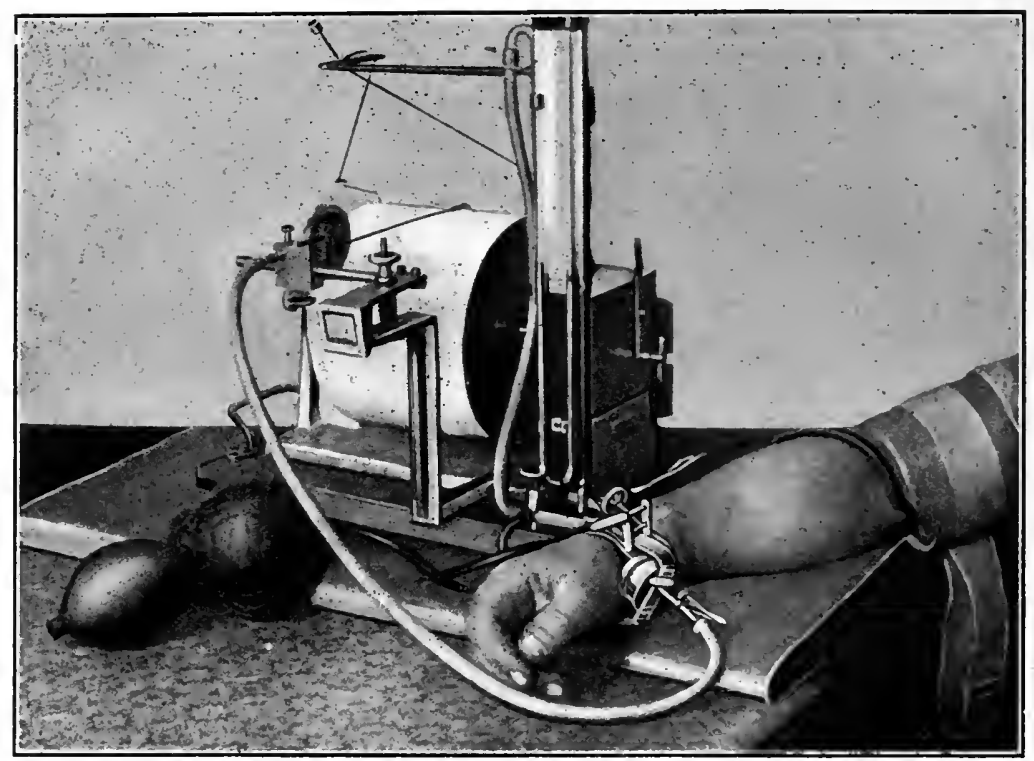

Fig. 45.-Turgotonograph. (Strauss-Fleischer.)

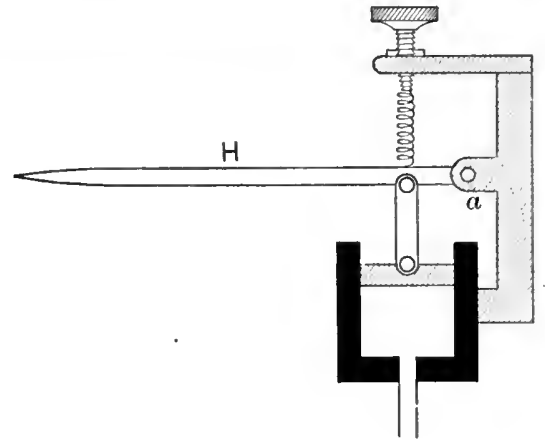

$A$

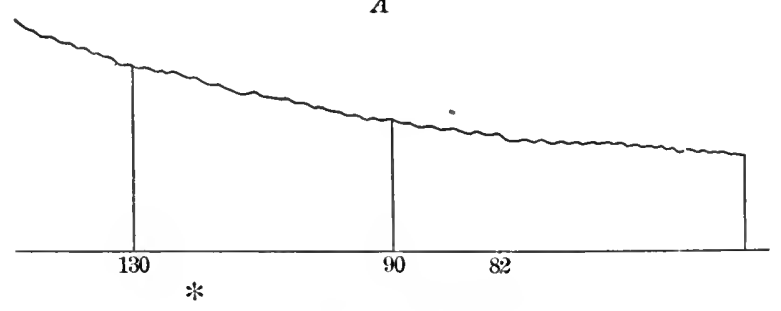

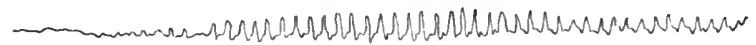

B

FIG. 46.- $A$, pen used for recording oscillations; $B$, tracing made with the Brugsch apparatus. (Brugsch.) 
$h^{\prime}$ in order to exclude the pump and the manometer, since in recording the pulsations only the cuff and the balloon are necessary.

A somewhat similar instrument has been designed by Fleischer, ${ }^{1}$ who, however, instead of using the glass-enclosed balloon to diminish the force of the pulsations between the cuff and the tambour, has constructed a device consisting of a metal cylinder $m$ enclosing a celluloid cylinder Sch floated on a layer of oil $P$. The air enters from the cuff through the tube $r$ and finding no means of escape imparts its pulsations to the counterweighted lever $G$ at the point $A$, whence a wire $D$ again transmits the motion to the aluminum recording needle $S$. (Figs. 47 and 48.)

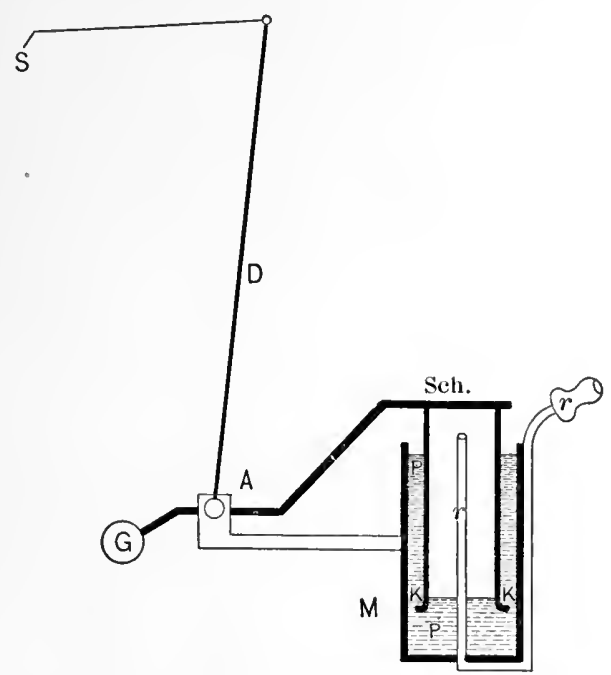

FIG. 47.-Strauss-Fleischer device for damping the force of the pulse wave.

The cuff shown in the accompanying Fig. 48 is used for the transmission of the pulse. It consists of a metallic cylinder $L$, the upper closed end of which is perforated by a tube which connects with the polygraphic tambour. Its lower end is closed by means of a "lense" consisting of a rubber capsule $G$ filled with glycerin. The cuff is held in place by the straps $R$ and its pressure can be adjusted by the thumb-screw $S p$. With this cuff tracings can be

1 Ueber turgotonographische Pulsdruckbestimmung, Berlin. klin. Woch., 1907, No. 35, 1108. 
made of most of the superficial arteries, including the digital and temporal vessels. ${ }^{1}$

Bussenius's instrument for the graphic registration of the bloodpressure ingeniously eliminates the necessity of a driving mechanism for the strip of smoked paper in the following manner: A narrow strip of paper supported in an upright slot, falls by gravity past the oseillating tambour. 'The descent of the strip is delayed by the displacement of glycerin around a metallic plunger, which is so graded that the strip will fall $1 \mathrm{~cm}$. per second. Both systolic and diastolic pressures can be estimated with this instrument; in fact, two tracings may be made consecutively on the same strip and used to control each other (see Fig. 49). It is merely essential that the begiming of the tracing corresponds with $0 \mathrm{~mm}$. pressure in the manometer. ${ }^{2}$

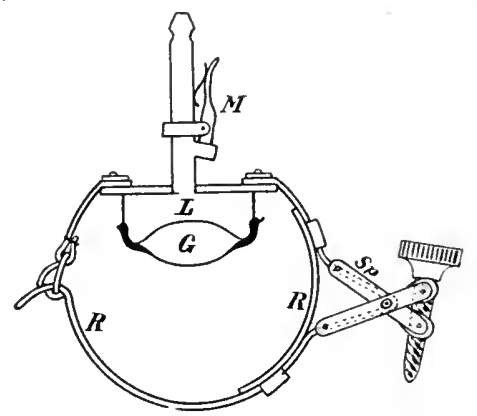

FIG. 48.-Cuff used with Fleiseher instrument.

"Wybauw's instrument ${ }^{3}$ is essentially a modification of Erlanger's. He, however, uses a divided cuff (4 and $9 \mathrm{~cm}$. broad) which yields a more aceurate systolic reading, since the impactions of the pulse wave against the upper part of the cuff are not transmitted to the tambour. The height of the pressure is not automatically recorded.

IV. Visual (Oscillatory) Methods.-Instruments of this type are constructed in association with different kinds of oscillating devices which indirectly magnify the fluctuations of the mercurial column, or otherwise render the pulsations of the cuff more readily perceptible. The oscillatory method is more satisfactory for the determination of the diastolic pressure than the method of palpation, but less so than that of auscultation.

${ }^{1}$ Zur Methodik der Pulschreibung, Berlin. klin. Woch., 1909, xlvi, 2141.

${ }^{2}$ Mauufactured by W. Oehmke, Berlin.

${ }^{3}$ Graphische Blutdruckbestimmungen. hei unrege]massiger Herzwirkung, Zaitschr. f. klin. Med., 1911, lxxiii, 204-220. 


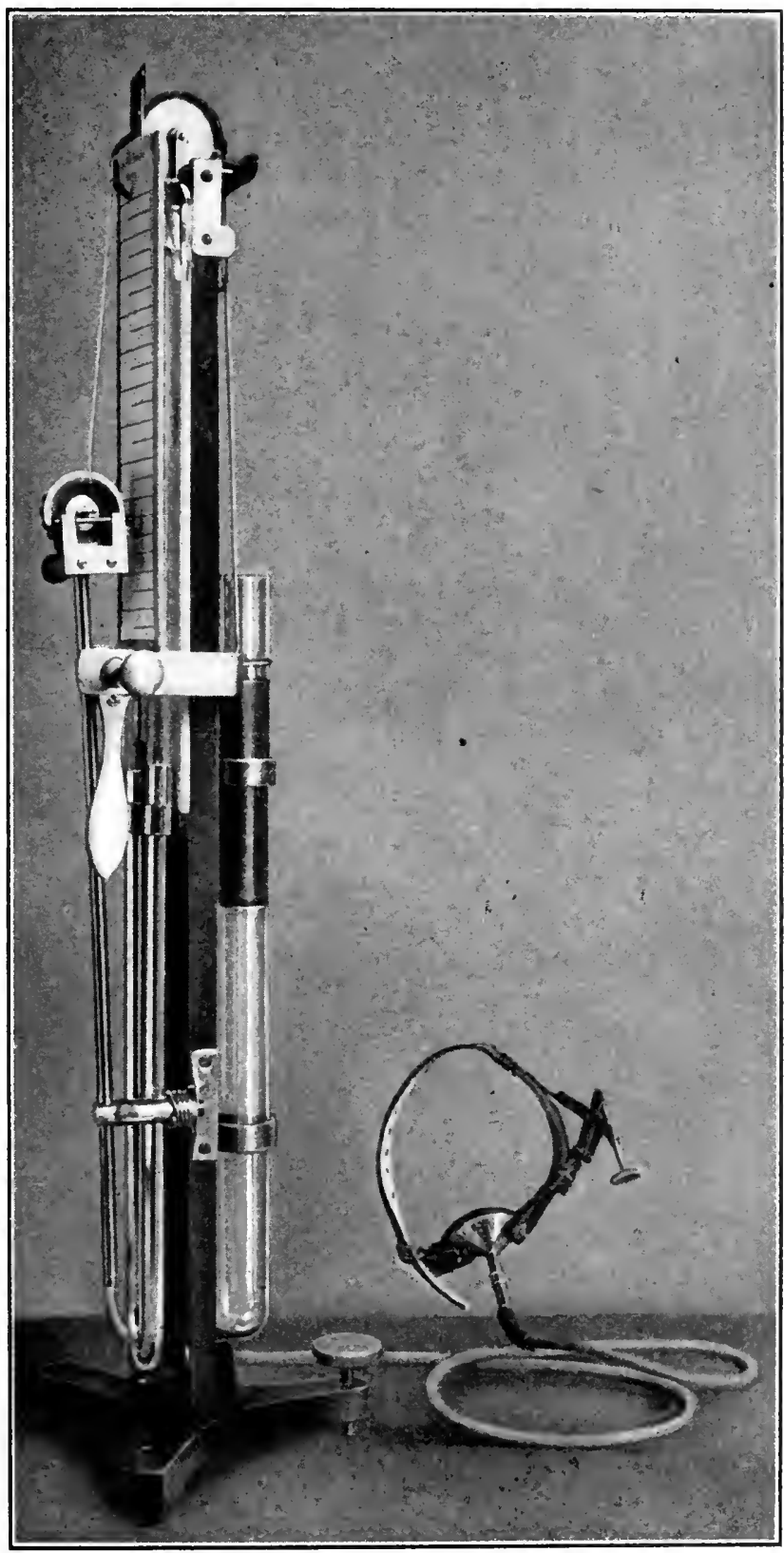

Fig. 49.-Bussenius's sphygmotonograph 
of excursion by different devices. (II) Those which indicate these two factors upon the same dial. The former usually render too high systolic readings. The diastolic pressure as indicated

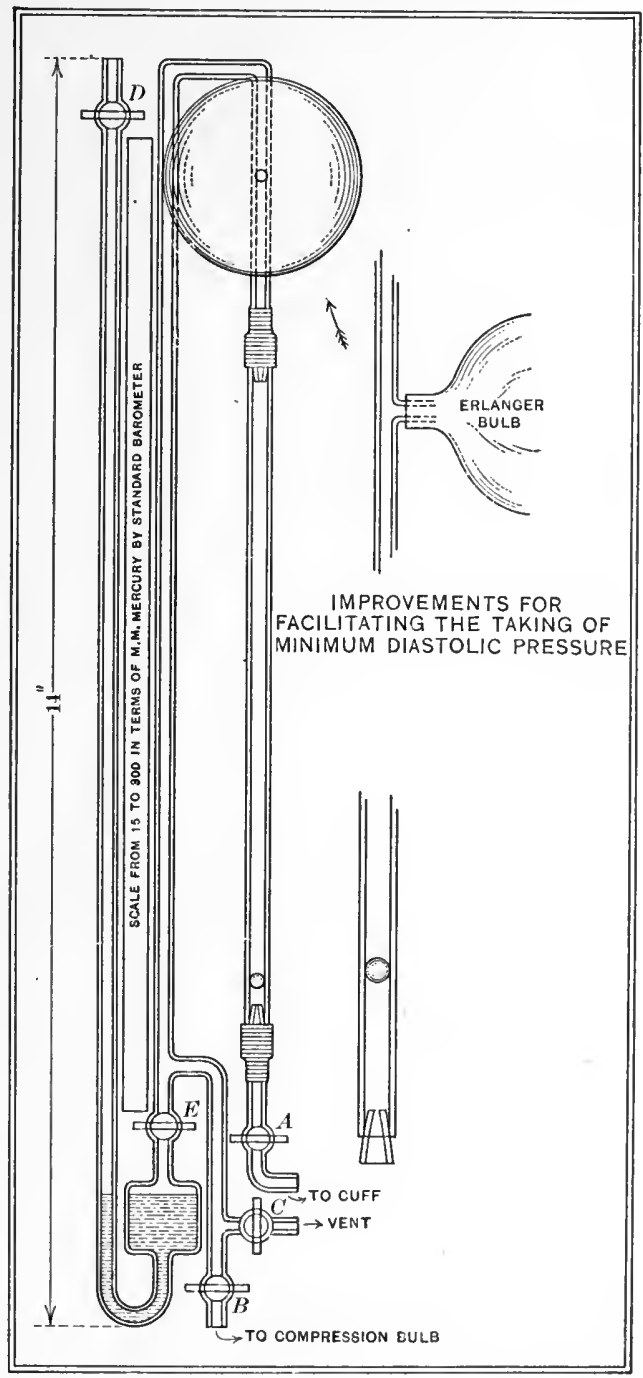

FIG. 52.-Feddé's oseillometer. This instrument has been modified by R. L. Hoobler by using two cuffs with a stopcock conneetion between them, in circuit with the pith-ball indicator. This permits the reading of the systolic as well as the diastolic pressure, and yields better results in infants (Nicholson). This oseillatory device may be used in conjunction with many types of instruments. It is furnished (at an extra cost) with the Nicholson and the Faught sphygmomanometers. 
by type II is praetically similar to that obtained by palpation. In type I the readings are somewhat higher. ${ }^{1}$

Class I.-The apparatus of $B$ ing ${ }^{2}$ (Fig. 50) consists of a manometer, pump, two euffs and a colored liquid indieator somewhat similar to that devised by $\mathrm{Pal}^{3}$ (Fig. 51). Here the lower cuff $b$,

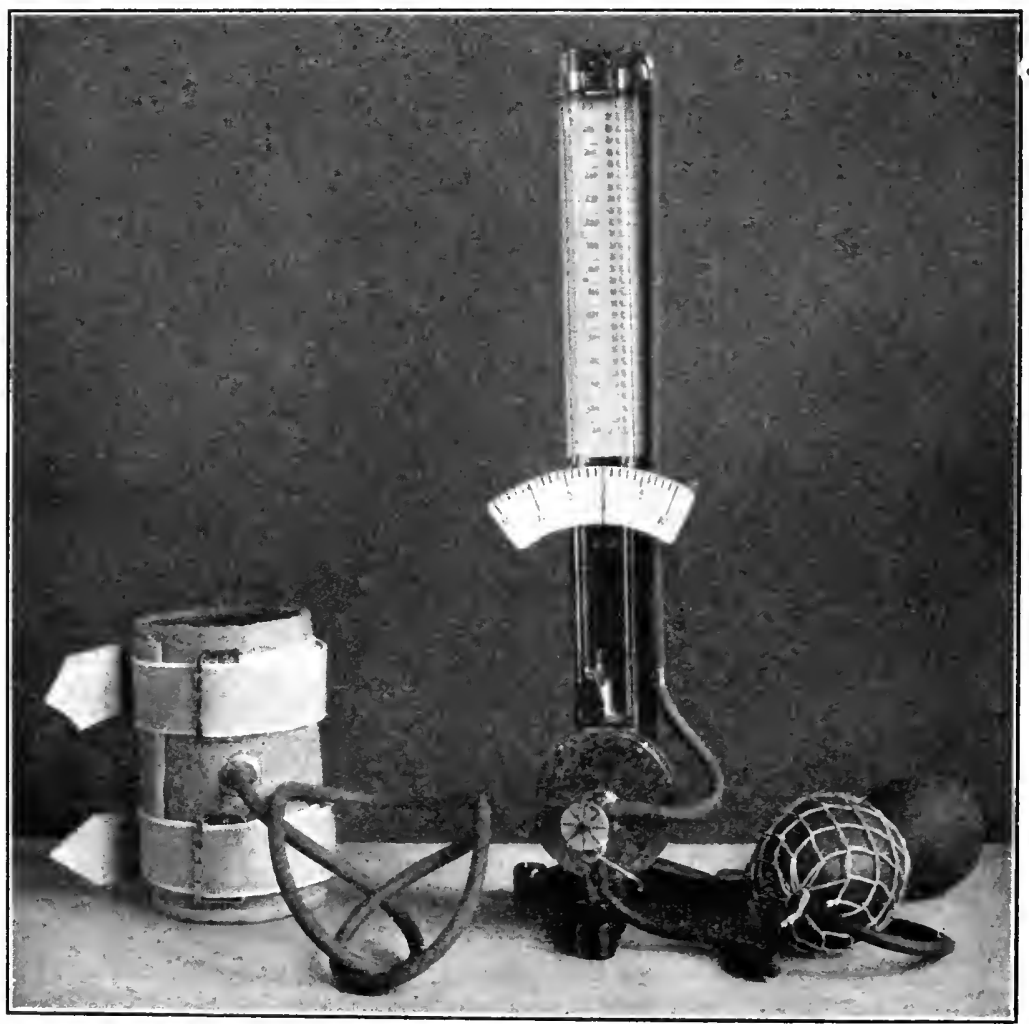

F1(i. j3.-Widmer's oscillometer.

substituting the finger in the palpatory method, transmits such pulsations as pass the upper cuff $a$ to the colored oil droplets in the indicator $r$. The pressure on either sile of the oil droplets

I Cordier and Relattu, Arch. d. Mal, du Corur, 1911, iv, 737.

${ }^{2}$ Ein Apparat. z. Messung des Blutdrucks bei Menschen, Berlin. klin. Woch., 1907, No. 22: Blutdruckmessungen bei Menschen, Berlin. klin. Woch., 1906, No. 52.

${ }^{3}$ Ein Sphyomoskop. z. Bestimmmng des Pulsdruckes, Zentralls, f. inn. Med., Fehmary, 1906, No. 15. 
being equalized, a very slight pulsation in the cuff produces an extensive oscillation in the sphygmoscope.

The values obtained average $10 \mathrm{~mm}$. higher than with the RivaRocei method, chiefly owing to the fact that in the last-named procedure peripheral contraction of the brachial artery may cause a local elevation in pressure without affecting the rest of the systemic circulation. This factor is said to be obviated in the Bing apparatus by the second cuff. ${ }^{1}$

The Sphygmoscope (Pal).-The capillary tube $C-C^{\prime}$, containing a few drops of colored petroleum, communicates with the tube $A-B$, the left arm of which $(A-I I)$ is smaller in caliber than the

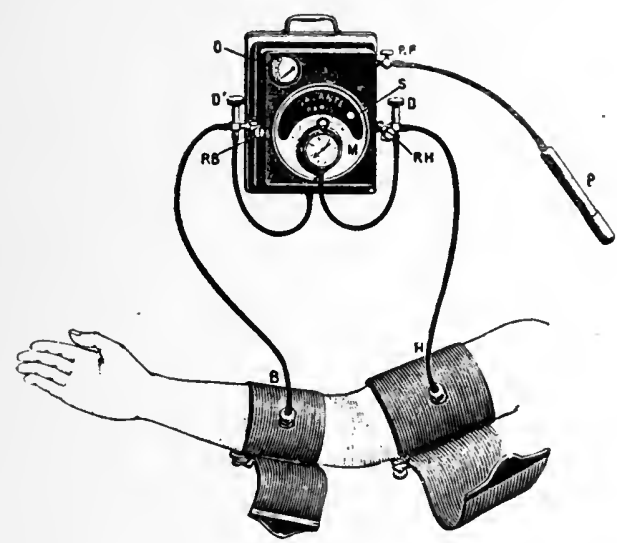

FIG. 54.-Vaquez's sphygmo-signal.

right $(I I-B) . \quad A$ is a two-way cock enabling communication to be established (through 3 and 4) with two manometrical cuffs, $e . g$. , one on the arm, another on the finger. $B$ is an escapement cock. The tube 2 communicates with a manometer, while 1 is comnected with the pump (Fig. 51).

Method.-After the pressure has been raised in the cuff, $I I$ is turned 45 clegrees, thus closing all communications throngh the four-way cock, whereupon oscillations of pressure are manifest in the capillary tube $C-C^{\prime}$.

${ }^{1} \mathrm{z}$. Werth has devised a somewhat similar instrument, equipped with an oscillator which transmits the radial pulsations from a rubber glycerin-containing pelotte which ean, however, only be used for the systolic oseillations. Uelber d. Messung d. Systolischen Blutdrucks auf Optischen Wege, Münch. med. Woch., 1910, Ivii, 12s6. Manufactured by Oehmke, Berlin, Luisenstrasse, 21, N. IV. 
Widmer's Oscillomanometer.-The first full oscillation (systolic pressure), and the maximum oscillation (diastolic pressure), are indicated upon the graduated scale, while the actual pressure is read from the mercury manometer ${ }^{1}$ (Fig. 53).

Another instrument based on the same principle is the "sphygmosignal" of Vaquez. ${ }^{2}$ Here again two cuffs are employed, but with a metallic manometer and a different form of indicator (Fig. 54).

\section{E. ANEROID INSTRUMENTS.}

CLASS II.-The most accurate aneroid instrument at present available is unquestionably that of Pachon ${ }^{3}$ It has recently been highly commended by Bachmann ${ }^{4}$ who has made extensive studies regarding its accuracy.

"In estimating the systolic pressure it is customary to observe the disappearance of the arterial pulsation at the radial (method of Riva-Rocci), occasionally at the brachial below the cuff, at any rate always at some distance from the point of compression. This method of applying Marey's principle is essentially faulty and the results obtained are necessarily erroneous. Indeed, it can be shown that while the pulse has disappeared at the radial there is a pronounced pulsation in that portion of the brachial artery under compression. Pachon, who demonstrated this fact by means of his instrument (to be described presently) and by the sphygmosignal of Vaquez, gives the following theoretic explanation of his experimental finding:

"The pulse wave has its origin in the contraction of the ventricle and represents a certain amount of kinetic energy. This wave of pressure spreads from the root of the aorta to the end of the arterial system and in so doing loses energy little by little, owing to friction against the sides of the vessels. As the resulting distention of the arterial walls is normally extremely slight (Poiseuille), very little external work is performed by the pulse wave. If, however, a segment of an artery is compressed so that a portion of its internal pressure is counterbalanced, that segment will yield

\footnotetext{
${ }^{1}$ Manufactured by W. Oehmke, Berlin.

${ }^{2}$ For further description see Vaquez, Soc. de Biologie, 1908.

${ }^{3}$ Oscillómetre sphygmanometrique à grande sensibilite et à sensibilite constante, Comptes rendu de Soc. de biolog., lxvi, 776; also Paris Médicale, 1911, xxxi, 122. Manufactured by C. Verdin, rue Linné 17, Paris. A. H. Thomas \& Co., Philadelphia agents.

${ }^{4}$ The Measurement of the Arterial Tension in Man, New York Med. Jour., February 4,1911 .
} 
to a greater extent to the distending force of the pulse wave and the vessel wall will begin to move. In other words, external work will be done. The compression may be such that the extent of the movement of the vessel wall (hence the amount of work done by the pulse wave) will exactly balance the amount of energy possessed by the wave. At this moment the pulse wave is entirely utilized in performing mechanical work; it is therefore absorbed at this point and no pulsation can be felt below it. The blood-flow, however, has not been interrupted, the intermittent flow has merely been changed into a continuous one. This explains why wide cuffs, with a larger area for the absorption of the pulse wave, give lower figures than narrow ones. It serves to explain also why the arterial

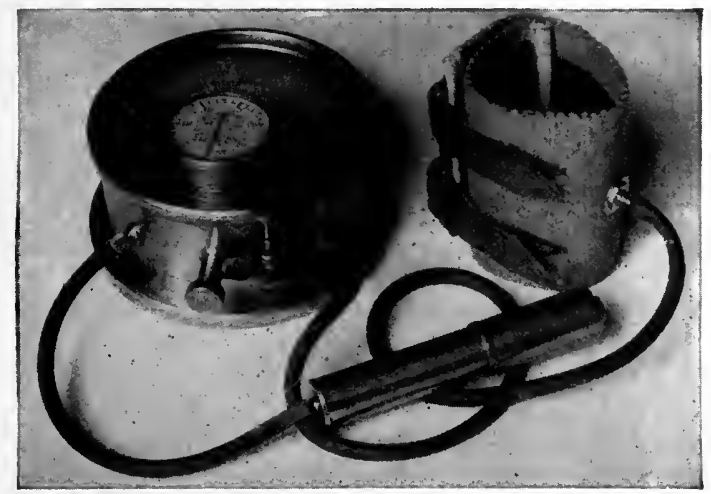

FIG. 55.-The Pachon sphygmometric oscillometer.

pressure in man appears so low in comparison to that observed in the other mammalia. Benezur reached practically the same conclusions by the combined use of the Recklinghausen and Gärtner instruments" (Bachmann).

Marey's principle for the determination of the minimum or diastolic pressure has been more correctly applied, in that the oscillations of the compressed artery are transmitted directly to the instrument and their amplitude noted. As stated by Pachon, however, the instrument which is to reproduce these pulsations must have both a great and a constant sensibility. The mercury manometer has naturally a constant sensibility, but owing to the inertia of the mercury this sensibility is but slight, surely not sufficient to enable one to compare differences in the amplitude 
of oscillations at various levels of pressure. Everyone who has used the mercury manometer for the determination of the diastolic pressure is impressed with its imperfections for this purpose. A few investigators have sought to overcome this defect by placing an elastic bag in connection with the cuff, through which combination the arterial pulsations are taken up and transmitted by appropriate means to a tambour to be magnified and graphically recorded (Erlanger, I'skoff'). The first objection to this method is that the bag camnot be made sensitive on account of the relatively high pressure to which it must be subjected. The second objection is that such a bag has not a constant sensibility at various pressure levels. The amplitude of its oscillations will be smaller the

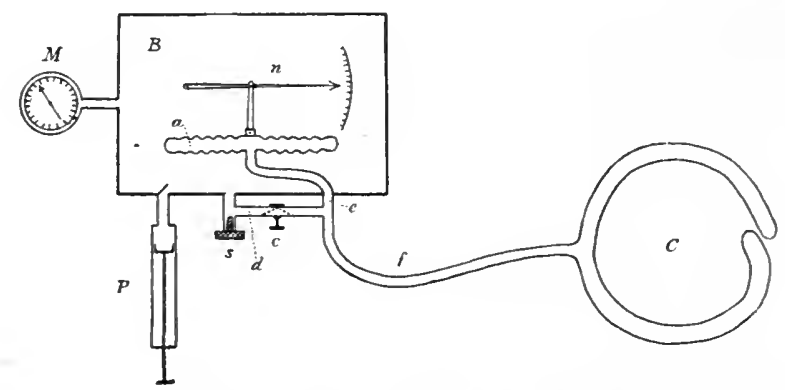

Fic. 56.-Diagram showing the relation of the essential parts of Paehon's sphygmumetrie oseillometer. (After Pachon.) $B$ is a rigid metallie box hermetically sealed and containing in its interior an aneroid drum $a$ with which is connected the lever $n$. $M$ is an aneroid manometer which indicates the level of the pressure in the entire system. The box, manometer, aneroid drum $a$, and the cuff are normally in free communication by means of the tubes $d, e, f$. The pressure ean be raised in the system by means of the pump $P$; it can be lowered by allowing the air to escape by unserewing the valves. The cuff and the aneroid $a$ can be cut off from the rest of the apparatus by compressing the rubber tube $d$ by means of the compressor $c$ (ealled separator by Pachon). (Bachmann.)

greater its state of tension (distention), the force of the oscillations remaining the same. It will therefore lead to error to compare the amplitule of oscillations obtained at various pressure levels.

All these objections have been removed in the simple and ingenious instrument devised recently by Pachon. The main features in the construction of this instrument, which he names a sphygmometric oscillometer, can best be understood by reference to Fig. 56.

The position of the aneroid $a$ is in the interior of the box, and 
the free communication of the interior of the same manometer with the box and the cuff have solved the problem of devising an instrument which would have both a great and a constant sensibility at any pressure level. By reason of this arrangement the same pressure is exerted at all times on the external as on the internal surfaces of the drum $a$; hence this drum is always in a position of rest or zero tension, except when by compressing $c$ the cuff is placed in direct communication with the interior of the drum and any change of pressure within the cuff may then act upon it. These changes of pressure due to the pulsations of the artery are normally very slight, so that the drum $a$ could be made of relatively thin metal, hence very sensitive. This sensibility will always be the same, no matter what the level of the pressure around the arm may be, since it always takes up the pressure changes in the cuff by starting from a state of no tension.

"In using the instrument the cuff is placed around the arm in the usual manner. Air is pumped into the system until the pressure is well above the normal arterial pressure. The amount of the pressure is read from manometer $M$. The observer now confines his attention to the manipulation of valve $s$ and compressor $c$, using preferably one hand only in order to insure free opening of the tube $d$ when valve $s$ is opened and closed. If on compressing $c$ the hand $n$ does not oseillate, valve $s$ is opened and the pressure allowed to fall one-half to one centimeter; $c$ is again pressed upon and the hand $n$ observed. This maneuver is repeated until oscillations of the hand $n$ to the extent of, say, one degree of the dial are shown. This moment indicates the return of the pulse at the point compressed, and therefore the maximum or systolic pressure there. This is read on manometer $\boldsymbol{M}$. To determine the minimum or diastolie pressure, the pressure in the system is lowered in the manner explained above and the compressor $c$ manipulated between each lowering of pressure; at the same time the hand $n$ is observed and at the moment its excursions are greatest the level of diastolic pressure has been reached, and can be read on manometer $M$. This is the most satisfactory instrument I have seen for the determination of the diastolic pressure; the oscillations are large and the moment of greatest amplitude is clear cut, for both immediately before and immediately after this moment the oscillations are noticeably smaller."

"The determination of the systolic pressure in such instruments is a vexed question. Erlanger obtains small pulsations with his 
instrument, as shown by the movements of the lever, before the true systolic level has been reached. This he attributes to the 'hydraulic ram action' of the pulsating proximal stump of the artery against the edge of the cuff. Pachon now uses a cuff adapted to the wrist, where, on account of the small size of the vessels, and the fairly thick edge of the cuff, the danger of this source of error should be greatly minimized. The writer observed that an oscilla-

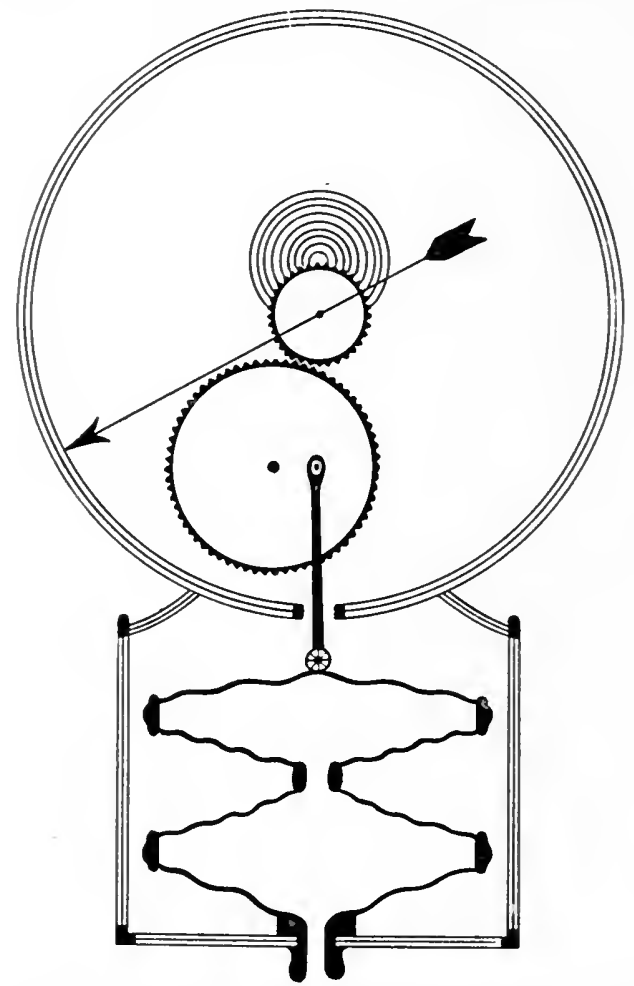

FIG. 57.-Diagram illustrating the construction of the Tycos aneroid.

tion of the hand $n$, equivalent to one degree of the scale, indicates the return of the pulse at the point compressed, as controlled by the palpation of the artery under similar conditions. To determine this point a cuff is placed around the upper arm and one around the wrist. The two cuffs are connected with the Pachon instrument by means of a Y-tube; a stopcock is placed in the course of each tube coming from the cuffs. A pressure is put around the wrist equal to the diastolic pressure at this point. When a pressure is 
applied around the brachial equal to the systolic pressure and the stopcocks and compressor suitably manipulated, no pulsation is seen at the wrist. The pressure must be lowered considerably around the brachial before a pulsation is indicated at the wrist. If now the radial cuff is removed and the brachial systolic pressure estimated by the ordinary method of palpation of the radial artery, it will be observed that the pulse is felt when the pressure around

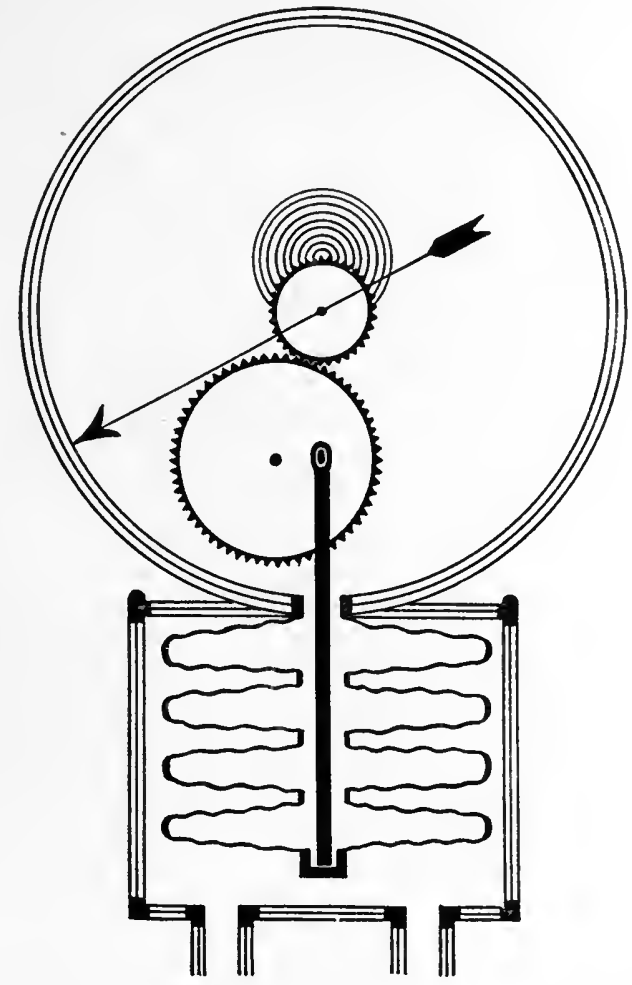

FIG. 58.- Showing the construction of the Faught aneroid.

the brachial is at the same level as when the indicator of the Pachon instrument showed an oscillation of approximately one degree, when it was in relation with the radial cuff. The following table will show the result of a few observations" (Bachmann):

$\begin{array}{ccccc}\text { Systolic pressure in } & \begin{array}{c}\text { Pressure in brachial cuff } \\ \text { at which pulse appears } \\ \text { at wrist. }\end{array} & \begin{array}{c}\text { Diastolic pressure at } \\ \text { wrist (Pachon). }\end{array} \\ \begin{array}{c}\text { Age. } \\ \text { brachial artery (Pachon). } \\ \text { In mm. Hg. }\end{array} & 130 & \begin{array}{c}\text { (Pachon.) } \\ \text { (Taetile.) }\end{array} & 80 \\ 26 & 125 & 105 & 105 & 90 \\ 33 & 130 & 105 & 105 & 90 \\ 8 & & 110 & 110 & \end{array}$


The systolic readings with the "oscillometer" are from 20 to $40 \mathrm{~mm}$. Hg. higher than with the Stanton apparatus (Riva-Rocci method). In the diastolic readings there is less discrepancy. This instrument is well suited to ward or office work. It is free from error due to personal equation, yields-especially for the diastolic pressure-satisfactory results, and is quickly manipulated; but its weight militates against its ready transportation, and it is open to the objection which applies to all aneroids, namely, the necessity of occasional standardization.

A number of compact little blood-pressure instruments fitted with aneroids are on the market. These instruments consist of a

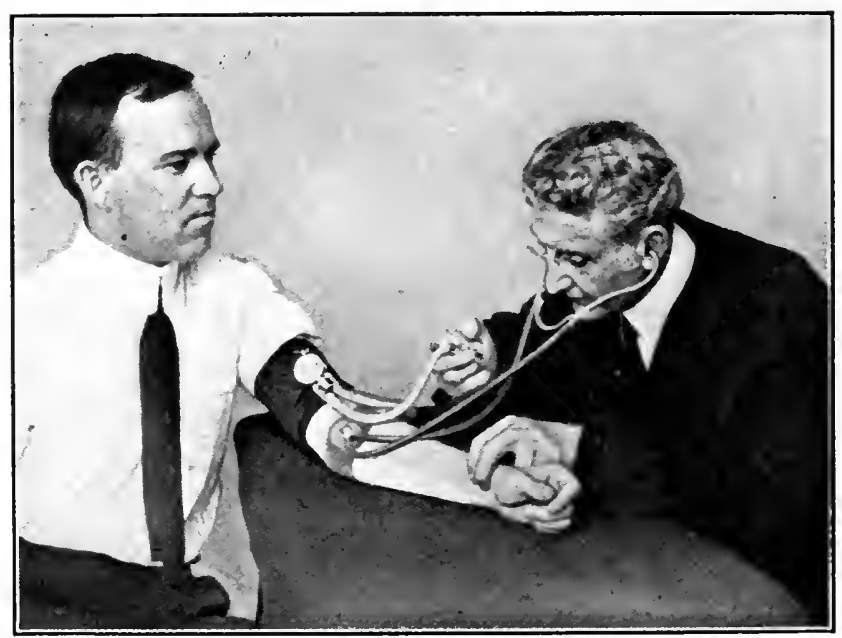

Fis. 59.-An aneroid instrument in use. (Auscultatory method.) ${ }^{1}$

chamber of corrugated metal which expands under pressure and imparts its movement to a calibrated dial. They can be carried in the pocket and are free from all danger of spilling the mercury, etc. Breakage is also less likely. They give reallings sufficiently accurate for clinical work, but they have to be standardized from time to time, as they are apt to get out of adjustment. Unless the readings obtained are occasionally checked up with an accurate manometer, one can never be sure that the results are not erroneous.

${ }^{1}$ Manufactured by G. P. Pilling \& Son Co., Philadelphia. A somewhat similar instrument-the Rogers "Tycos" sphygmomanometer-is manufactured by the Taylor Instrument Co., Rochester, New York. 
What has already been said regarding the size and character of the cuff of course applies equally to this type of apparatus.

Standardization of the Aneroid.-The accuracy of the aneroid can be readily tested with an ordinary mercury manometer, if a three-way stopcock is used to connect the two manometers with a pump. The pressure is raised $5 \mathrm{~mm}$. at a time and the readings indicated by the two instruments are compared, thus showing the amount of correction which must be applied to the aneroid at a given level.

The von Recklinghausen tonometer, ${ }^{1}$ an instrument which has found considerable favor in Germany, is based on the principle "first introduced into physical science by Bourdon and later incorporated into the kymograph of Fick, that if a very shallow, curved, elongated air chamber is fixed at one end of the arc with a pressure apparatus, while the other is closed, any increase of pressure will be made manifest by a flattening or elongation of the arc, thereby imparting a certain movement to the end that is free. By a very simple device this movement of the air chamber is communicated to an axis on which, guarded by a hair-spring, there is fastened a long needle whose tip is made to move along a graduated scale previously standardized, the final figures being read off from the dial." The pressure which is supplied by a long-stroke bicycle pump is transmitted from a standard cuff to the dial, the graduations of which are equivalent to centimeters of water (Figs. 60 and 61 ).

It is for several reasons desirable that water should be adopted as a standard for measurement rather than mercury, and possibly this will sooner or later come to pass. First of all, pressure values can be directly translated into grams; second, water and blood have nearly the same specific gravity; so that calculations can readily be made as to differences in pressure at different levels of the body by simply measuring the difference in height in centimeters, $e . g$., blood-pressure at cardiac level 140, at level of spleen $140-20=120$.

Water having less inertia than mercury, and being used as a unit in many other connections, would undoubtedly be more desirable as a standard for blood-pressure. Unfortunately, however, nearly all blood-pressure readings have been made with mercury, so that

${ }^{1}$ Unblutige Blutdruck Messung, Deut. Arch. f. exp. Path. u. Phar., 1906, lv. Manufactured by C. \& E. Streisguth, Strasburg. A. H. Thomas \& Co., Philadelphia agents. 
not only is nearly all the literature based on this standard, but we have come to think of blood-pressure in millimeters of mercury. A transposition of values from $\mathrm{mm}$. $\mathrm{Hg}$. to $\mathrm{cm}$. $\mathrm{H}_{2} \mathrm{O}$ is not readily

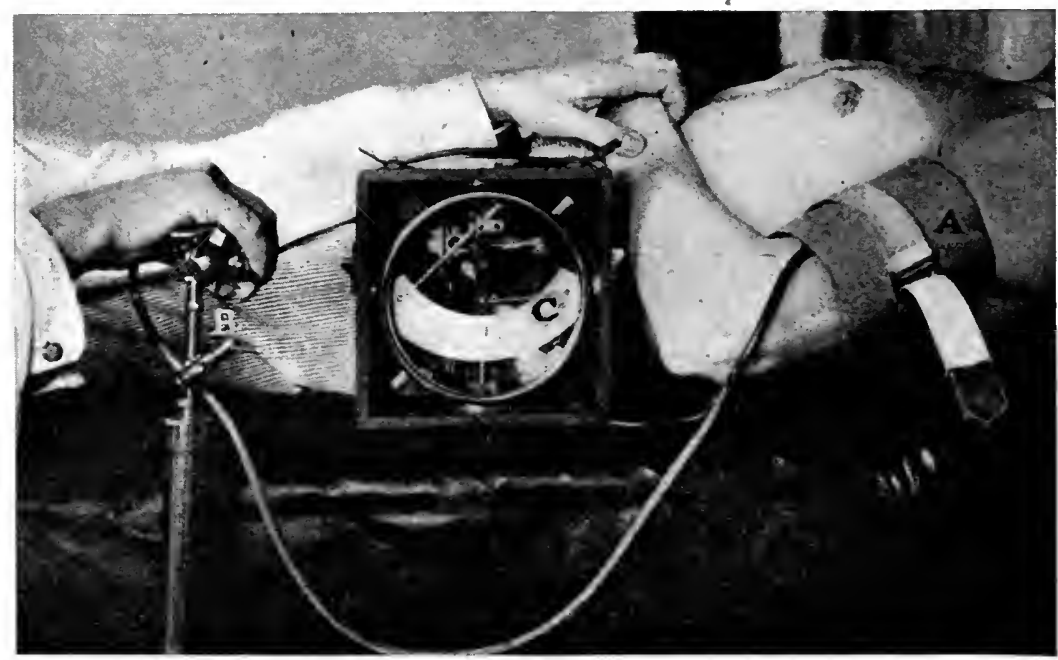

Fig. 60.-The von Recklinghausen tonometer; $A$, cuff; $B$, pump; $C$, tonometer dial.

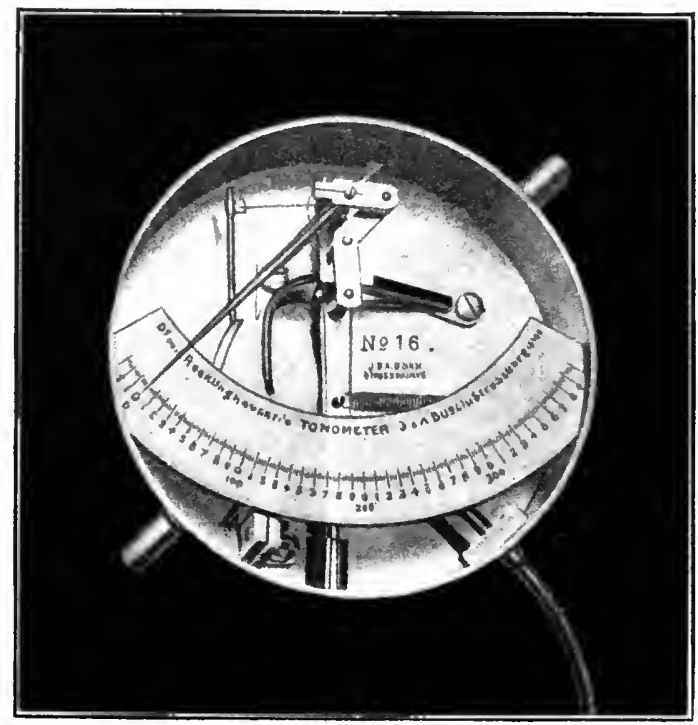

Fig. 61.-Tonometer. 
made without reference to a special table. This is one reason why the instrument has never found favor, in this country at least. It is perhaps a pity, but we are confronted by a fact. Another reason lies in the construction of the instrument. It measures tension by means of a metallic spring which perhaps even more often than in the case of aneroids requires "control" and occasional correction to insure accurate readings.

The systolic pressure as recorded with this instrument is slightly above the actual pressure. The diastolic pressure, especially if the pulse pressure be large, is too low. Strassburger ${ }^{1}$ suggests that observations be based both on oscillation and on palpation; and

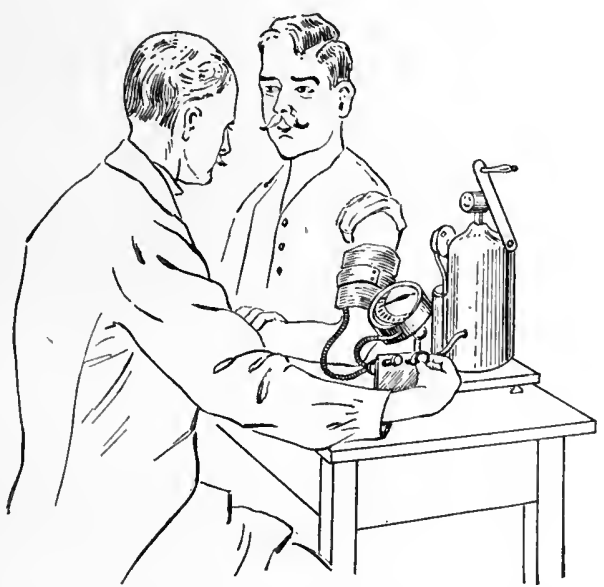

Fig. 62.-The new von Recklinghausen tonometer.

further, that not only the extent of the excursion be used as a criterion of the minimum pressure, but also the point at which the needle begins to "hesitate" and to "tremble." He believes that in this manner the error due to the arterial elasticity (which varies in individuals, and for different degrees of pressure), as well as that resulting from partial loss of pulsation, due to imperfect transmission from the cuff, may be mutually controlled.

The Recklinghausen instrument has recently been modified, making it smaller in bulk (21 by $9 \mathrm{~cm}$., weighing $\frac{3}{4} \mathrm{~kg}$.) and more readily transportable. It is also supplied in another modification

I Weitere Untersuchungen u. d. Messung d. Diastolischen Blutdruckes heim Menschen, Deut. med. Woch., 1909, xxxiv, 56 and 100. 
for office use, with a good-sized compressed-air tank, so that very rapid inflation of the cuff is made possible by the release of a screwvalve, and repeated estimations may be made without renewing the air supply in the tank. Care must of course be exercised when releasing so high a pressure lest the limits of the instrument be overstepped and the metallic spring be thus permanently damaged ${ }^{1}$ (Fig. 61).

\section{Tables of Relative Values.}

To convert centimeters water into millimeters $\mathrm{Hg}$.

$\begin{array}{cc}\text { Cm. } & \text { Mm. } \\ \text { w. } & \text { Hg. } \\ 1 & 1 \\ 2 & 1 \\ 3 & 2 \\ 4 & 3 \\ 5 & 4 \\ 6 & 4 \\ 7 & 5 \\ 8 & 6 \\ 9 & 7 \\ 10 & 7\end{array}$

$\begin{array}{rr}\text { Cm. } & \text { Mm. } \\ \text { w. } & \text { Hg. } \\ 10 & 7 \\ 20 & 15 \\ 30 & 22 \\ 40 & 30 \\ 50 & 37 \\ 60 & 44 \\ 70 & 52 \\ 80 & 59 \\ 90 & 66 \\ 100 & 74\end{array}$

$\begin{array}{rr}\text { Cm. } & \text { Mm. } \\ \text { w. } & \text { Hg. } \\ 110 & 81 \\ 120 & 89 \\ 130 & 96 \\ 140 & 103 \\ 150 & 111 \\ 160 & 118 \\ 170 & 125 \\ 180 & 133 \\ 190 & 140 \\ 200 & 148\end{array}$

$\begin{array}{cc}\text { Cm. } & \text { Mrm. } \\ \text { w. } & \text { Hg. } \\ 210 & 155 \\ 220 & 162 \\ 230 & 170 \\ 240 & 177 \\ 250 & 185 \\ 260 & 192 \\ 270 & 199 \\ 280 & 207 \\ 290 & 214 \\ 300 & 221\end{array}$

$\begin{array}{cc}\begin{array}{c}\mathrm{Cm} . \\ \mathrm{w} .\end{array} & \begin{array}{c}\mathrm{Mm} . \\ \mathrm{Hg} .\end{array} \\ 310 & 229 \\ 320 & 236 \\ 330 & 244 \\ 340 & 251 \\ 350 & 258 \\ 360 & 266 \\ 370 & 273 \\ 380 & 280 \\ 390 & 288 \\ 400 & 295\end{array}$

To convert millimeters $\mathrm{Hg}$. into centimeters water.

\begin{tabular}{|c|c|c|c|c|c|c|c|}
\hline$\underset{\mathrm{Hg} .}{\mathrm{Mm} .}$ & $\begin{array}{c}\mathrm{Cm} \text {. } \\
\mathrm{w} \text {. }\end{array}$ & $\begin{array}{c}\mathrm{Mlm} \text {. } \\
\mathrm{Hg} .\end{array}$ & $\underset{w}{\mathrm{Cm} .}$ & $\begin{array}{c}\mathrm{Mm} . \\
\mathrm{Hg} .\end{array}$ & $\underset{w}{\mathrm{Cm}}$. & $\begin{array}{l}\mathrm{Mm} . \\
\mathrm{Hg} .\end{array}$ & $\begin{array}{c}\mathrm{Cm} . \\
\mathrm{w} .\end{array}$ \\
\hline 1 & 1 & 10 & 14 & 110 & 149 & 210 & 285 \\
\hline 2 & 3 & 20 & 27 & 120 & 163 & 220 & 298 \\
\hline 3 & 4 & 30 & 41 & 130 & 176 & 230 & 312 \\
\hline 4 & 5 & 40 & 54 & 140 & 190 & 240 & 325 \\
\hline 5 & 7 & 50 & 68 & 150 & 203 & 250 & 339 \\
\hline 6 & 8 & 60 & 81 & 160 & 217 & 260 & 352 \\
\hline 7 & 9 & 70 & 95 & 170 & 230 & 270 & 366 \\
\hline 8 & 11 & 80 & 108 & 180 & 244 & 280 & 379 \\
\hline 9 & 12 & 90 & 122 & 190 & 257 & 290 & 393 \\
\hline 10 & 14 & 100 & 136 & 200 & 271 & 300 & 407 \\
\hline
\end{tabular}

The Potain instrument has never found favor in this country. It is generally and justly regarded as inaccurate, partly on account of its construction and largely on account of its application. It is still frequently used in France, however, with the idea of comparing the pressure in different arteries-radial, temporal, dorsalis pedis, etc. The pressure required to obliterate the pulse is applied directly over the artery, the compression being exerted upon the pelotte with the fingers. Thus it is very easy to exert the pressure mainly against the tissues and but slightly if at all upon the artery.

Francois Frank.$^{2}$ has designed an instrument (sphygmopalpeur) for the transmission of arterial or venous pulsations without circular

\footnotetext{
Iv. Recklinghausen, H., Neue Apparat. z. Messung des arteriellen Blutrlrucks beim Menschen, Münch. med. Woch., 1913, lxi, 869.

${ }^{2}$ Compt. Rend. Soc. Biol., 1909, Ixviii, 525; Soc. Biol., Paris, July 25, 190s, p. 226.
} 
fixation, and recommends its use in connection with the Potain sphygmomanometer. It consists of a Marey tambour which is attached to an upright stand through a series of levels and joints so that it can be set at any angle or direction, the pressure exerted by it varied at will. Contrary to the usual findings, he obtained higher readings with the Riva-Rocci instrument, a fact which he explains as follows.

"The Potain apparatus acts only on the radial artery, while the method of circular compression by producing venous stasis reduced the amplitude of the arterial pulse and has the effect of counterpressure, thus yielding too low a reading."

The sphygmomanometer of Bouloumie consists of a combination of the Potain and the Gärtner apparatus. It has been used in an effort to study the relationship of arterial and capillary pressure. ${ }^{1}$

V. Subjective Method.-The subjective sensations of the patient may also be used to determine the systolic and diastolic readings. With the cuff and manometer applied in the usual manner the patient is told to notice the onset of throbbing in the arm below the cuff-this indicating the systolic pressure. The disappearance of the pulsatory sensations corresponds to the diastolic pressure. Quite accurate readings may thus be sometimes attained, but the procedure introduces a considerable and quite unnecessary amount of the personal equation, and is therefore rarely employed.

Francois Frank $^{2}$ in contrasting the findings of the subjective method with those obtained by the Gärtner instrument, found that the two phenomena (recoloration and sense of pulsation), although identical as regards the height of manometric pressure, are not so as regards time when compared to the beginning of the elevation of a volumetric curve, there being a temporal difference of from 4 to 6 seconds between the two. This emphasizes the importance of allowing pressure to fall slowly. He suggests that the pulsatile phenomenon, which manifests itself later than the beginning of recoloration, indicates when at its maximum the minimum pressure in the collateral arteries of the fingers; while the recoloration and the initial swelling is the index of the maximum pressure of the arterioles. He finds that the maximal digital arterial

\footnotetext{
1 Bouloumie's instrument described: Amblard, These de Paris, 1907, No. 261, p. 51 .

${ }^{2}$ La sphygmomanometrie digitale par le procede de Gaertner avec et sans anemic prealable, ete., Compt. Rend. Soe. Biol., February 5, 1910, p. 234.
} 
pressure is often approximately two-thirds that of the radial pressure, although this relationship is by no means a constant one.

Methods of estimating pressure based on subjective sensations are entirely too inaceurate to be of much use. This applies no less to reeent refinements than to the method suggested by von Frey, which consisted in submersion of the arm in mercury and the measurement of its depth to the point at which the greatest pulsation was felt.

Comparative Values.--The palpatory (Riva-Rocei) method yields results which are on the average $7.5 \mathrm{~mm}$. Hg. higher for every 100 $\mathrm{mm}$. Hg. than the actual intra-arterial pressure, and 5 per eent. lower readings than are obtained by the graphic method, because a eertain amount of arterial distention must occur before the pulse can be palpated. If a sphygmograph or a second (radial) cuff is used to determine the return of the pulse instead of the finger, somewhat higher readings (10 to $15 \mathrm{~mm}$.) will generally be obtained. The end of the maximum oseillation (diastolic pressure) can be more readily determined by this means than by palpation, especially in case of the novice.

The auscultatory procedure yields results which are closely comparable to the graphie method, provided that the fourth phase (muffling) and the last of the large oscillations be chosen as the criteria of the diastolic pressure (Lang and Manswetowa, Bickel, Warfield, Schrumpf and Zabel, Engle and Allen). If the fifth phase is chosen Windle found that the results corresponded within $5 \mathrm{~mm}$. In the average case it will not make more than this degree of difference whether we ehoose the fourth or the fifth phase, but in cases with a long fourth phase the discrepancies may amount to 10 to $12 \mathrm{~mm}$. With a little training the fourth phase is quite as readily reeognized as the fifth. The vast majority of cases show readings $5 \mathrm{~mm}$. higher by auscultation than by palpation.

The oscillatory (visual) method tends to yield higher values (5. to $10 \mathrm{~mm}$.) than that of palpation, and figures practically identical with the graphic method (Schrumpf and Zabel). It has a distinct fiek of utility in taking the pressures of infants and individuals with very small pulse pressures. The greatest discrepaneies oecur in arteriosclerotie eases.

The graphic method is more time-consuming and troublesome than the other methods, but in the hands of those who are familiar with its technique is the most exact. It is generally accepted as a standard of clinical accuraey. The aetual readings are eon- 
siderably higher than the endo-arterial pressure. 'The margin of error may be as high as 25 per cent.

On close analysis Zabel found that when well-marked diserepancies existed between the findings of different methods, the phase of "large oscillations" was long, whereas in the class in which the results were closely in accord, this phase was brief. Careful observation, he believes, shows that the phase of large oscillations really consists of two distinct parts. In the beginning the first large oscillations exhibit a crescendo quality which bespeaks the increasing pressure in the edges of the cuff. Following this in some cases a second increase in the oscillation abruptly occurs, which corresponds to the Ehret phenomenon and to the beginning of Korotkow's

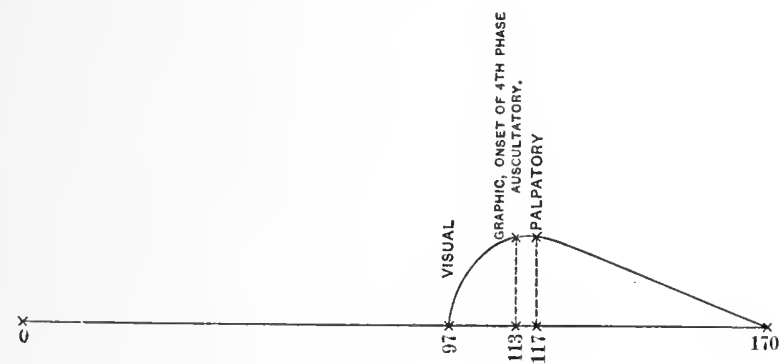

Fra. 63.-The diastolic pressure as determined by different methods. (Bickel.)

third phase. According to this conception the first of these two diastolic phenomena occurs when the central portion of the arterial lumen is collapsed; the second, when the entire lumen is obliterated. Thus the:

First critical point $=$ onset of large oscillations $=$ beginning of fourth auscultatory phase = collapse of central arterial wall.

Second critical point $=$ onset of maximum oscillations $=$ Ehret's phenomenon $=$ third auscultatory phase $=$ complete arterial occlusion.

In corroboration of this hypothesis are the facts that (1) in brachial arteriosclerosis Ehret's phenomenon is of gradual, not sudden, onset; (2) lacks in its brusque quality, and (3) is sometimes entirely absent. 


\section{RELATIVE ADVANTAGES OF THE DIFFERENT METHODS.}

In order to obtain the greatest accuracy (as in experimental work) and for the purpose of having permanent demonstrable records the graphic method excels.

For routine clinical work where simplicity and celerity, combined with reasonable accuracy, are the chief desiderata, the auscultatory method is to be preferred. The older palpatory method offers more chance for error, due. to the personal equation, and with it much more difficulty is experienced in determining the diastolic pressure. Furthermore, the auscultatory method has to a considerable extent eliminated the question, "Which sphygmomanometer shall I buy?" Because with the last-named method any accurate manometer, if supplied with a suitable cuff, etc., will answer the purpose.

The sphygmographic attachment at the wrist, aside from being troublesome to adjust, results in venous stasis, which elevates the curve and diminishes the excursions. The oscillatory devices have the disadvantage that the eye has to measure two different points at once. They are useful, however, when the pulse is small, and some of them can be used to register the pulsation in the smaller arteries. So far as clinical accuracy is concerned, it is not of great moment which method is employed. The results will generally not vary more than $10 \mathrm{~mm}$. Hg., figures well within the range of allowable error. These clinical methods are after all only relative estimations, and if the same instrument is used on all cases, and the examiner is conversant with the technique, the results will be sufficiently accurate for comparison and for practical purposes generally.

Sources of Error.-There is a more or less prevalent impression that marked errors in the clinical determination of blood-pressure may be brought about through the varying influence of the soft tissues surrounding the artery, such as the size and muscular development of the arm.

In the investigations of Müller and Blauel, ${ }^{1}$ conducted on arms about to be amputated and controlled by manometrical pressure, readings from the artery directly showed that the broad cuff gave a reading about $10 \mathrm{~mm}$. higher than the actual pressure, a margin

${ }^{1}$ Zur Kritik des Riva-Roccischen u. Gärtnerschen sphygmomanometers, Deut. Arch. f. klin. Med., $1907, \mathrm{xci}, 517$. 
of error which waxed larger as the width of the cuff was decreased. But both Hensen ${ }^{1}$ and Janeway ${ }^{2}$ lave reported cases with very great differences between the soft parts of the two arms (atrophy flaccidity against hypertrophy) which yielded identical readings on the two sides. Certainly if an error exists it lies well within the limits of daily physiological fluctuation, provided a cuff with a width of $12 \mathrm{~cm}$. is employed.

The extent of the role played by hypertonicity of the arterial wall in causing erroneous blood-pressure readings has been much discussed. It has been claimed that some of the excessively high readings occasionally obtained in man $(350 \mathrm{~mm}$. or over) could not represent the actual endovascular tension, but must be due to resistance of the arterial wall.

The subject has recently been reviewed and studied in a series of ingenious experiments by Janeway and Park, ${ }^{3}$ who found that although the arterial wall does offer definite resistance to compression, greater in large, thick-walled vessels, yet this resistance in a normal adult brachial is equivalent to only a few millimeters of mercury. In children it is an entirely negligible factor, and in adults with a normal blood-pressure the error thus introduced into clinical blood-pressure measurements is not greater than $10 \mathrm{~mm}$. Hg., "a figure less than the spontaneous variations in pressure from minute to minute."

"Atheroma, even of considerable degree, is without appreciable effect on the compressibility. Calcification of the arterial wall, when segments longer than $6 \mathrm{~cm}$. are examined, increases only moderately its resistance to compression. 'The overpressure dependent on this factor in our experiments did not exceed $17 \mathrm{~mm}$. Hg. In clinical blood-pressure determinations, if a wide arm-piece be used, and the return of the first fully developed pulse wave be taken as the index, as recommended by von Recklinghausen, even advanced arterial thickening and calcification probably do not introduce an error of any importance. The only factor altering the compressibility of an artery which seems capable of introducing an error of real importance in the clinical measurement of systolic blood-pressure is the state of contraction of the arterial walls.

${ }^{1}$ Beit. z. Physiol. u. Path. d. Blutdrucks, Deut. Areh. f. klin. Med., 1900, lxviii, 443.

2 Influence of the Soft Tissues of the Arm on Clinical Blood-pressure Determinations, Areh. Int. Med., 1907.

${ }^{3}$ An Experimental Study of the Resistance to Compression of the Arterial Wall, Arch. Int. Med., November, 1910. 
It is impossible from our experiments on surviving ox arteries to set definite numerical limits for this in man. From these experiments, however, combined with our study of human arteries after amputation and postmortem, we feel that a degree of hypertonic contraction of the brachial artery sufficient to cause an error of more than $30 \mathrm{~mm}$. Hg. seems improbable, and of more than $60 \mathrm{~mm}$. incredible, during life" (Janeway').

Practically all the evidence at hand supports the foregoing statements. The latest corroboration is furmished by Macwilliam and Kesson, ${ }^{1}$ who found that relaxed arteries (Ringer's solution, manipulation, sodium fluoride) with normal or thickened walls vielded only trivial degrees of resistance to compression. Thickened arteries from old animals, especially when contracted (barium chloride, epinephrin) below the body temperature, were capable of exerting considerable resistance. In the smaller arteries constriction was sometimes sufficient to resist a passage of fluid at 440 $\mathrm{mm}$. Hg. Arterial contraction in such arteries could be greatly diminished by repeated or long-continued compression and by massage of the artery. This procedure, they suggest, may be used to determine how great a percentage of a high pressure is due to vascular resistance, although it does not enable one to determine whether this vascular resistance is due to arterial contraction or to other causes.

They have further shown that soft spots in the course of an irregularly sclerosed artery are not bridged over by the brachial cuff but that the latter acts effectively eren in very limited soft areas, thus preventing the resistance offered by the rest of the artery from having any great effect upon the readings of the instrument.

Much stress has been laid by some investigators upon variability of the conduction of the pulse ware due to alteration in the arterial lumen and changes in the resiliency of the arterial wall. Thus Hill believes that systolic pressure readings are affected by (a) the actual maximum pressure of cardiac systole; $(b)$ the conduction of the pressure wave to the periphery. 'The resistance of the arterial wall to compression affects the systolic index of blood-pressure but little, but the relative hardness or softness of the vessel affects the conduction of the systolic wave considerably, especially if

\footnotetext{
1 The Estimation of Systolic Blond-pressure in Man with Special Reference to the Influence of the Arterial Wall, Heart, 1913, iv, 279.
} 
it is large. The force of the wave is damped down in soft arteries as sound waves are damped by velvet. ${ }^{1}$

Most of the experimental evidence, however, indicates that constrictions of the artery sufficient to cause any considerable diminution of conduction can hardly occur between such large vessels as the aorta and brachial artery; and further, that increased arterial rigidity is equally insufficient to cause an important source of error. The difference in pressure obtained in arm and leg, or two arms or two legs, is the result of pressure differences in the arteries in question, and not due to abnormal conduction of the aortic pressure.

The elasticity of the aorta and its branches is important in causing a lower systolic and a higher diastolie pressure in the systemic arteries than would a rigid tube, but in blood-pressure estimations by the obliteration method, the resiliency of the intervening arterial tube appears to be negligible. ${ }^{2}$

\section{PRECAUTIONS TO BE OBSERVED IN TAKING READINGS.}

Blood-pressure readings should, for purposes of comparison, always be taken in the same (preferably the horizontal) posture. At all events the position should be such that the reading is made at a point about on a level with the heart, thus eliminating the error due to the weight of the blood-column. When possible, observations should be made about the same time of day and in the same relation to meals. They should not be taken during excitement, after exercise, or shortly after the patient has partaken of hot food or drink, tea, coffee or alcohol, and not when the extremities are overheated or chilled. All muscular activity on the part of the patient must be prevented and complete relaxation, both general and local, obtained. Observations made under conditions of spasm-subsultus, tetanus, etc.-are absolutely unreliable. Edema of the tissues is also a fertile source of error, and readings obtained from anasarcous patients are of no value. Fear, excitement or psychic effort have a marked effect on the blood-pressure. In taking one's own pressure, higher results are often found than those obtained by another observer immediately before or after-

${ }^{1}$ Hill, L., Measurement of Systolic Blood-pressure in Man, Heart, 1910, i, 73; Hill and Rowlan ds, Heart, 1912, iii. 219.

2 Maewilliam, Kesson, and Meloin, The Conduction of the Pulse Wave and its Relation to the Estimation of Systolic Blood-pressure, Heart, 1913, iv, 393 
ward, simply as the result of psychic concentration. Schrumpf relates an instance in which the anticipation of an austere prognosis raised the pressure of a patient 33 per cent., to fall again promptly when reassurance was forthcoming, and only to rise once more when complaining of his insomnia and worries. Gibson found that his own pressure was increased $35-40 \mathrm{~mm}$. after delivering a lecture. Due allowance for variability of the systolic pressure must always be made in neurotic patients.

Von Recklinghausen observed in his own case a rise of $14 \mathrm{~cm}$. $\mathrm{H}_{2} \mathrm{O}$ caused by the entrance into his presence of a person whom he intended to berate (see Physiological Variations). Putermann ${ }^{1}$ found that the anticipation of an examination almost without exception raised both blood-pressure and pulse rate in schoolchildren. In extreme cases, psychic states may cause a-rise of 90 $\mathrm{mm}$. Hg., generally in association with marked pleasure, anger, or fright. The diastolic pressure in these cases is practically unaffected. ${ }^{2}$ The interposition of a thin undergarment between the cuff and the arm is unobjectionable, in fact, may be positively beneficial. In cold weather a chilly cuff applied to the arm, both by the displeasure it causes the patient, and by the stimulating effect it has upon the vasomotor nerves, may yield erroneous readings. In very high-strung individuals it is best to warm the cuff before applying it.

It sometimes happens that the initial systolic blood-pressure reading on a given subject yields distinctly higher figures than can be obtained in subsequent attempts. Gallavardin and Haour ${ }^{3}$ found, in the study of 100 cases, that this initial high pressure, which may amount to $35 \mathrm{~mm}$. in women and $25 \mathrm{~mm}$. in men, may last fifteen minutes, although in 50 per cent. of these cases the normal point was reached at the end of five minutes. Only the systolic pressure was affected, the diastolic level remaining constant. 'These figures are certainly high.

Such an increased pressure may be due to: (1) Psychic influences - excitement or fear, chiefly noted in high-strung individuals. (2) Pain-arising from the forcible inflation of the cuff on sensitive arms or in cases of marked hypertension. (3) Local stimulation

\footnotetext{
${ }^{1}$ Ueber d. Beeinflussung d. Zirkulationssystem durch d. Schulexamina, Wien. med. Woch., 1904, liv, 265.

${ }^{2}$ Schrumpf, P., Die psychogene Labilität des Blutdrucks u. ihre Bedeutung in d. Praxis, Deut. med. Woch., 1910, xxxvi, 2385.

${ }^{3}$ Baise systolique de la tension arterielle au moment de la mensuration, Arch. de Mal. du Cœur, etc., 1912, v, 81.
} 
of the vasomotor nerves from pressure. (4) Prolonged application of the cuff. 'This tends to relax the artery, and by reflexly diminishing the force of systole, possibly lessens the conduction of the pulse wave. It also produces marked increase in the venous pressure and congestion of the tissues below the cuff.

Attention has been called to the fact that in estimating the diastolic pressure by increasing the manometer pressure, different results are sometimes obtained than when the diastolic index is taken during a falling manometric pressure, especially if the pressures are gauged on both arms simultaneously. A comparison of the diastolic values with a rising pressure may thus indicate an abnormal tendency toward blood-pressure variation in a given individual. ${ }^{1}$

\section{THE PERSONAL EQUATION OF THE EXAMINER.}

Schultze ${ }^{2}$ found that ordinarily with a trained observer a series of ten successive measurements will yield a fair average of accuracy. Much closer correspondence in successive readings is obtained if the manometer is read and the pulse palpated by the same person, than if these duties are relegated to two individuals. This results from the strained mental attitude of the palpator who is almost certain to become "the dupe of expectant attention," knowing that his sense of touch is being "controlled" and fearing lest, after all, he fail to feel the first pulse wave. His sense of perception being therefore raised to its maximum, it often happens that he becomes subjectively conscious in his finger tips of his own pulse, especially if, as is necessary for accuracy, the manometric pressure is allowed to fall but slowly, and the procedure is therefore prolonged. Subjective appreciation of the pulse is more marked in the second finger, and in certain individuals. It is generally perceived from ten to thirty seconds after the finger tips of a dependent hand have been lightly placed upon a solid object (e.g., table). 'This factor is in our experience only rarely a cause of confusion.

The frequency with which the subjective pulse appears in "controlled" observers indicates how variable is the acuteness of our tactile perception. If the pressure is read and the pulse is palpated

\footnotetext{
${ }^{1}$ Macwilliams, J. A., and Melvin, G. S., The Significance of Blood-pressure Readings in Man, Brit. Med. Jour., 1914, p. 777.

${ }^{2}$ Ueber d. psychologischen Fehlerquellen bei d. palpatorischen Bludruckmessung nach Riva-Rocei u. v. Recklinghausen, Arch. f. d. ges Physiol., Bonn, 1908, exxiv, 392 .
} 
by the same individual the discrepancies of successive readings will be far smaller, owing to the subjective suggestible element (expectant attention). The first reading having yielded a definite result, we consciously or unconsciously "expect" the pulse to reappear at about the same level. This is illustrated by Schultze in Fig. 64, which shows how much more closely the results of ten consecutive readings corresponded when the suggestible element is not eliminated.

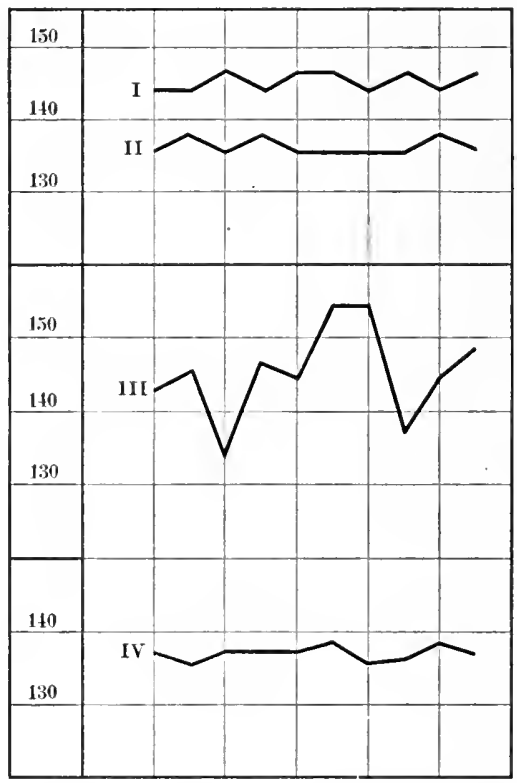

Fig. 64.-In the first two estimations, $I$ and $I I$, the manometer was olserved while feeling the pulse wave. In the last two, $I I I$ and $I V$, the manometer was not read until after the pulse wave had been perceived.

In the first two estimations, I and II (10 each), the examiner obscrved the manometer while feeling for the pulse wave; in the last two, III and IV, the manometer was not read until after the pulse wave was perceived. ${ }^{1}$ When the suggestible element was eliminated, successive readings tallied less closely.

Another possible source of error lies in the fact that the threshold

${ }^{1}$ Schultze, ueber d. psychologischen Fehlerquellen dei d. palpatorisehen Bludruckmessung nach Riva-Roeci u. v. Recklinghausen, Areh. f. d. ges Physiol., Bonn, 1908. cxxiv, 392. 
of perception for the appearing and disappearing pulse are not the same. In the former we judge of a subsensible stimulus which is being raised to, in the latter of a sensible stimulus which is being lowered beyond, the point of perception. The point of disappearance is definitely more difficult to determine. Finally, the mercury in the manometer rarely if ever rises or falls so gradually that the examiner has time to decide for each individual millimeter of pressure whether or not a pulsation is present. Therefore our measurements are for subjective reasons generally exact only within 3 or $5 \mathrm{~mm}$. Hg.

In order to minimize all these sources of error the following procedures should be adopted: (1) Discard the result of the first reading, using it simply to demonstrate the harmless and painless character of the procedure; and when possible make subsequent readings after some little time has elapsed. (2) Avoid making blood-pressure estimations when the subject is excited, anxious or worried, as a result of the examination, etc. (3) Make several consecutive readings and if they correspond more or less closely, take the arithmetic mean. (3) Make the observations as quickly as is consistent with accuracy; do not look at the manometer until the pulse is felt-at this point the air escapement should be tightly closed until the reading is made. (4) Allow the pressure to fall to zero between observations, and permit sufficient time to clapse between readings for the venous pressure (stasis) to fall to the normal level. Never make a reading with a broken mercurial column, and be sure that the mercury corresponds with the zero on the scale before inflation. Needless to say, most of these sources of error are eliminated by using the auscultatory method which has therefore come into merited favor.

In certain forms of cardiac arhythmia, especially auricular fibrillation and extrasystole, it becomes very difficult to gange the bloodpressure, as the pulse is never equal in volume or in tension for many consecutive beats. A rough estimate of the actual pressure may be obtained by measuring first the strongest and then the weakest pulsations and thus establishing an average. (See Auricular Fibrillation.)

Possible Accidents. - In certain dyscrasic states-purpura, hemophilia, ete-- too prolonged application of a highly inflated cuff may cause capillary hemorrhage. 'This is very rarely the case.

Mohr has reported thrombosis following repeated blood-pressure observations in a patient, suffering from tubereulous pneumonia. 
This is, we believe, the only case of the kind on record. Hill has reported edema in a paralytic arm following the application of the cuff in a case with a blood-pressure of $220 \mathrm{~mm}$.

\section{THE VALUE, ACCURACY AND SIGNIFICANCE OF BLOOD- PRESSURE ESTIMATIONS.}

Blood-pressure readings furnish us with important and valuable data, but they must be interpreted in relation to other physical signs. Such observations alone do not furnish us with a basis for treatment any more than they, unaided, permit us to make a diagnosis. In this respect a sphygmomanometer is like a thermometer -its readings must be construed in the light of other findings. We have seen cases exhibiting a practically normal systolic and diastolic pressure associated with dyspnea, cyanosis, and edema, showing that the blood-flow was not normal.

Observations of the systolic pressure alone are of relatively little value. They indicate, it is true, the strain to which the arteries are subjected, but the diastolic pressure, the variations of which correspond more or less closely to those of the mean pressure, is the real indieation of the work the heart has to do. Systolic pressure may suddenly vary greatly from many diverse causes-fright, excitement, etc.-but the diastolic pressure is a much less easily disturbed factor, and may hence be a valuable criterion in deciding whether organic, vascular or cardiac changes are present. Since the diastolic pressure can now be readily, quickly and accurately determined by observing the appearance of the fourth auscultatory phase, there can no longer be any excuse for neglecting this far more important of the two phases of arterial tension. We have already learned in most conditions to attribute more importance to the diastolic than to systolic readings.

Pulse Pressure.-The difference between the systolic and the diastolic pressure roughly indicates the rolume of the pulse. From a study of pulse pressure certain deductions can often be drawn, but it must be remembered that these are not absolute nor always trustworthy criteria. ${ }^{1}$

\begin{tabular}{|c|c|c|c|c|}
\hline Inereased peripheral resistanee & & $\begin{array}{c}\text { Systolic } \\
\text { pressure. } \\
+\end{array}$ & $\begin{array}{c}\text { Diastolic } \\
\text { pressure. } \\
++\end{array}$ & $\begin{array}{c}\text { Pulse } \\
\text { pressure. } \\
-\end{array}$ \\
\hline Deereased peripheral resistance & . & - & -- & + \\
\hline Inereased heart rate . . & . & + & ++ & - \\
\hline Decreased heart rate. . & . & - & -- & + \\
\hline Inereased systolic discharge & . & $+t$ & + & + \\
\hline Deereased systolic discharge. & . & -- & - & - \\
\hline
\end{tabular}

${ }^{1}$ Wiggers, C. J., Modern Aspects of the Circulation in Health and Disease, Phila., 1915, p. 68. 
The diastolic pressure is normally about three-fourths of the systolic pressure and the pulse pressure, one-quarter of the systolic pressure. A pulse pressure persistently as low as 20 or as high as $60 \mathrm{~mm}$. is definitely pathological.

A high systolic with a normal diastolic pressure may result from emotional disturbances and as a result of physical exertion.

A low systolic with a high diastolic pressure often indicates myocardial weakness in association with severe pressor factors such as nephritis or vascular spasm.

A low systolic and diastolic pressure occurring in a person who is up and about (essential hypotension), while it does not necessarily imply a poor circulation, is definitely associated with diminished reserve force-"staying power."

A high systolic with a low diastolic pressure is most characteristically seen in aortic insufficiency; it may, however, result from peripheral vascular dilatation associated with forcible heart action.

\section{THE EXTREMES OF PRESSURE COMPATIBLE WITH LIFE.}

Hypertension.-Systolic blood-pressures above $235 \mathrm{~mm}$. are unusual and above $250 \mathrm{~mm}$. are rare. Hirst has reported a recovery in puerperal eclampsia with a pressure of $420 \mathrm{~mm}$. Hg. Needless to say, such a pressure could not long be maintained without something giving out. Many patients live for years with pressures ranging about 200 systolic and 120 diastolic, and occasionally such pressures are compatible with hard manual labor, ${ }^{1}$ but a sudden termination in these cases is always a possibility. Strauss's patient lived for five years with a systolic pressure between 260 and $270 \mathrm{~mm}$. Cook and Briggs reported $400 \mathrm{~mm}$. in a case of cerebral hemorrhage.

Hypotension.-A systolic pressure of 105 is common among many essential hypotension cases which are up and about. Not many are able to do so, however, if the systolic pressure falls below 100 or the diastolic below 50. Rolleston has reported a remarkable case of a man with a lingual carcinoma, who lived for several weeks with systolic and diastolic pressures of 70 and 35 respectively. Agonal systolic pressures of $40 \mathrm{~mm}$. have been reported and 60 mm. probably represents the lowest figure at which the coronary and medullary circulation can be kept up, even for a short time. Some of the earlier figures, such as those of John, of 35 to 40 in

${ }^{1}$ McCurdy, S. M., Physical Examination and Regeneration of Employees, Jour. Amer. Med. Assn., 1915, lxv, 2050. 
a case of advanced tuberculosis, are open to question on account of faulty instrumentation. Janeway has seen systolic pressures of $40 \mathrm{~mm}$. as transient phenomena during surgical operations.

All clinical blood-pressure estimations must be accepted only as approximately correct. In addition to the fact that the determination of the diastolie pressure presents certain difficulties we are confronted with the fact that, even if this could be accurately measured, we would still be only measuring the blood-pressure of the brachial artery. Living vessels possess a distinct tonus-the ability to contract and to dilate-the amount of which in a given case we are thus far unable to determine. In addition to this we must bear in mind that the blood-pressure mechanism is an extremely labile one which is subject to frequent fluctuations, due to many different causes.

On the other hand, thousands of blood-pressure measurements on different individuals have shown that pressure relations are fairly constant under similar circumstances; that there are definite and distinct bounds beyond which normal variations do not extend, and again that there are certain abnormal values which, if constantly found in a given individual, point very definitely to well-established pathological conditions.

\section{THE CHOICE OF AN INSTRUMENT.}

For general office work some instrument of the Rira-Rocci type is unquestionably to be preferred. Of this type of instrument a great many different models have been placed on the market. Although varying in detail of construction they are all based on the same principle. Good results may be obtained with most of them. On the whole, the last model of Nicholson's sphygmomanometer is the most satisfactory instrument to be obtaincd, being compact, readily portable and yet possessing the advantages of the mercury manometer.

In the personal choice of an instrument we should be governed by a number of factors, $i$. e.: (1) Accurate standardization. (2 General workmanship. The instrument should be made, of flint glass, as the lead in American glass reacts with the mercury. In the same way the mercury will form an alloy if allowed to come in contact with any metals save steel and platinum, producing turbidity and increasing friction. It is also affected by rubber, owing to the sulphur which this substance contains. All joints must of 
course be tight. It is essential for accuracy that the cuff have a width of $12 \mathrm{~cm}$. and that the canvas or leather backing be sufficiently rigid to maintain the width of the aforesaid rubber portion. (3) Compactness and portability-including the ease and celerity with which the instrument ean be set up for use, the devices employed to prevent the spilling of the mercury when in transit, and the bulk of the apparatus when taken down. (4) The amplitude of the pulsation transmitted to the mercurial column. If the auscultatory method of blood-pressure reading be employed, the amount of visual oscillation obtainable loses its importance, and satisfactory results may be obtained with any aceurate manometer if fitted with a proper cuff.

Compressed-air manometers are small and accurate (since the stopeock when open equalizes barometric and temperature variations). The adjustment of the drop used as an indicator is sometimes troublesome. Aneroid instruments for bedside use, owing to their small size and ready transportability, have much to commend them. When well made they may not require adjustment for considerable intervals, although comparisons with a mereury manometer must from time to time be made.

Spring manometers require frequent standardization, without which procedure inaccuracies may result. The von Recklinghausen apparatus is somewhat bulky and is based on c.c. $\mathrm{H}_{2} \mathrm{O}$, which standard has not been widely adopted.

The kind of pump selected is of minor importance. The rubber bulb is light but less durable than the heavier metal variety. The von Recklinghausen bicycle-pump type is both heavy and bulky, but one or two strokes suffice for inflation. Compressed-air tanks, such as are currently used in rhinological work, may be used when rapid inflation of the cuff is desired. Errors lue to stasis may thus be minimized.

\section{CLASSIFICATION OF BLOOD-PRESSURE INSTRUMENTS.}

I. Mereury manometers:

1. Reservoir: Riva-Rocci, Cooke, Stanton, Nicholson, Hill, Kercher, Hollman, Gärtner, Westenrijk.

2. U-shaped; Janeway, Faught, Martin, Limnell, Fellner, Hamilton, Schneider, Beachler, Brown.

II. Compressed-air manometers: Oliver, Bendick, Herz.

III. Aneroid manometers: Rogers, Brunton, Tycos, Fanght, Pachon, Jacquet, McKeson, Bristol. 
IV. Spring manometers: von Recklinghausen.

V. Instruments for graphic registration: Erlanger, Gibson, Bingel, Singer, Uskoff, Silbermann, Brugsch, Stursberg, Muenzer, Strauss-Fleischer, Bussenius, Wybauw.

VI. Instruments consisting of or which may be fitted with special oscillating indices, such as the Feddé or Pal oscillometers, Bing, Nicholson, Vaquez, Faught, Widmer. 


\title{
CHAPTER IV.
}

\section{VENOUS BLOOD-PRESSURE.}

\author{
By J. HAROLD AUSTIN, M.D.
}

VARIOUS methods of determining venous blood-pressure in man have been devised. They may be grouped into five types:

I. Gärtner's Phenomenon.-Gärtner, ${ }^{1}$ in 1902 , contended that if the left arm is slowly raised, the veins at the elbow which become distended when the arm is dependent will suddenly collapse upon reaching a certain level. He assumed that the elevation at this point above the level of the right auricle is a measure in centimeters of blood of the pressure at the right auricle. It is.to be remembered that $10 \mathrm{~cm}$. of blood equals $10.6 \mathrm{~cm}$. of water. As the level of the heart Gärtner took the junction of the upper border of the left fifth costal cartilage with the sternum. Oliver ${ }^{2}$ used the same method but observed the veins on the dorsum of the hand. Prym, ${ }^{3}$ lowever, showed that Gärtner's phenomenon occurs at different levels when the arm is raised at different rates, and that, moreover, the veins have a tonus of their own which may be stimulated by cold or by stroking, and this tends to accelerate their collapse. Meinertz ${ }^{4}$ has noted that exercise of one arm frequently raises the level at which the veins collapse in that arm without affecting the level in the other arm. The state of the circulation and vascular tonus locally, as well as the pressure in the right auricle, therefore influence Gärtner's phenomenon.

II. Spring Manometers.-In 1898 Oliver $^{5}$ attempted to measure venous pressure by means of a spring-pressure manometer. A button attached to the spring of the manometer was pressed against one of the larger veins of the arm with sufficient force to obstruct the flow. The vein for an inch or so proximal to this point was then stripped by the finger. The pressure against the vein by the

1 Münch. med. Woch., 1904, Ixxiv, 2038.

${ }^{2}$ Quar. Jour. Med., Oxford, 1907-08, i, 59.

${ }^{3}$ Münch. med. Woch., 1904, li, 60.

${ }_{4}$ Ztschr. f. exper. Pathol. und Therap., 1908.

5 Jour. Physiol., 1898, xxii, li; 1898, xxiii, v. 
manometer was then gradually reduced and at the moment the emptied section of vein began to fill, the pressure recorded by the manometer was noted. This was believed to equal the venous pressure at that point. Subsequently both Frey ${ }^{1}$ and Sewall ${ }^{2}$ independently devised similar spring manometers.

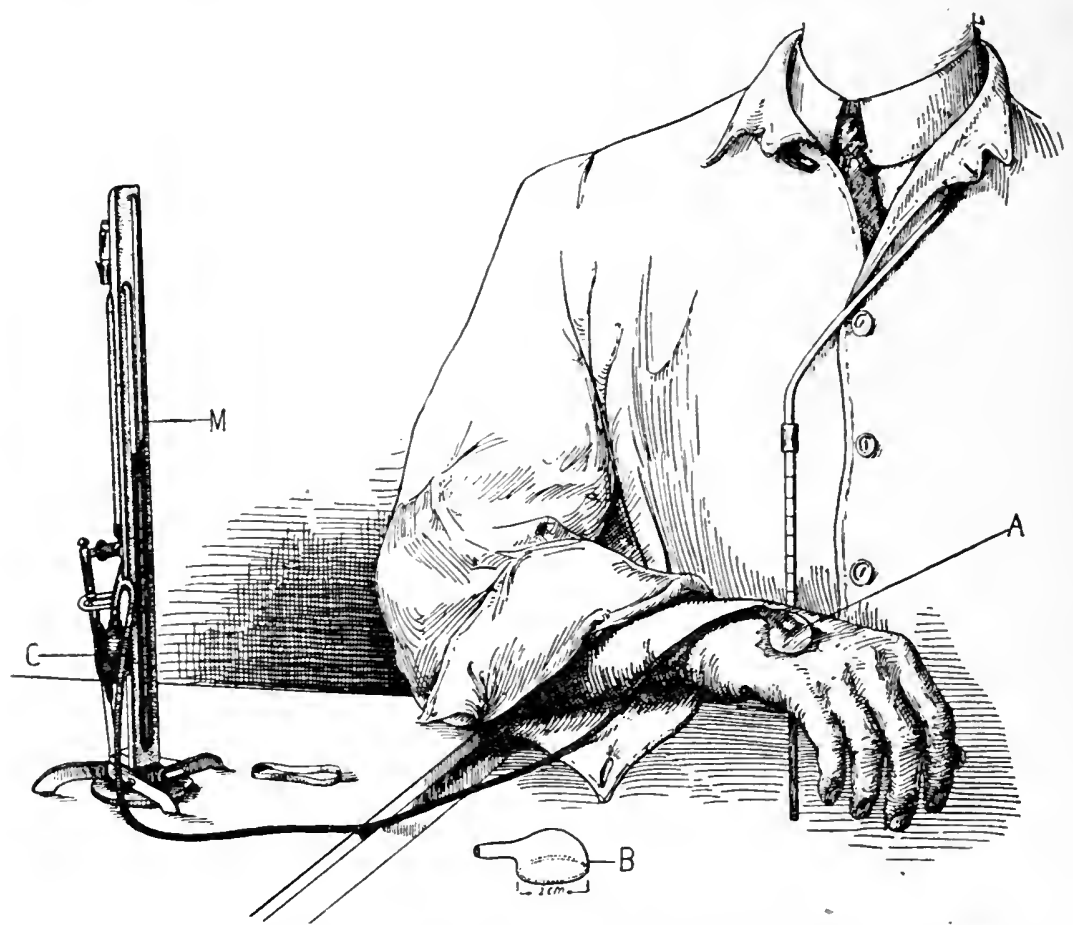

FiG. 65.-Hooker's venous pressure apparatus. A small glass chamber $(B)$ measuring 1 by $2 \mathrm{~cm}$. is held temporarily by a rubber band over a suitable vein on the back of the hand, as shown at $A$. A rim of collodion is applied and in drying it seals the chamber to the skin. The rubber band is then removed and the chamber connected by a rubber tube to the water manometer $M$. By pressing on the manometer bulb $C$ the air pressure in the chamber is raised and a reading is made "at the point where slight oscillations of pressure eause the vein shadow to come and go promptly just before the vessel is empletely collapsed." This pressure is recorded directly by the water manometer. The hand is held at the level of the midpoint of the anteroposterior diameter of the body at the costal angle.

\section{Pressure Chambers over the Vein (Fig. 65).- In 1904 von} Basch $^{3}$ attempted to measure venous pressure by placing a small

1 Deutsch. Archiv f. klin. Med., 1902, Jxxiii, 511.

2 Jour. Amer. Med. Assn., 1906, xlvii, 1279; Trans. Assoc. Amer. Phys., Philadelphia, 1906, xxi, 20.

${ }^{3}$ Wien. klin. Rundsrhau, 1900, pp. 549 and 572 . 
glass cylinder over the vein, and raising the air pressure in the cylinder until the vein collapsed or releasing the air until the vein refilled. 'This method was improved upon by von Recklinghausen,' and later by Hooker and Eyster, ${ }^{2}$ so as to reduce the error caused by pressure of the wall of the cylinder against the vein. The latter authors compared readings made with a manometer in the facial vein of a dog with those made with their instrument over the neighboring exposed external jugular. The pressure was artificially altered by tilting the animal's body. The results follow:

\begin{tabular}{|c|c|c|}
\hline Observa & ation. & Manometer in faeial vein. \\
\hline 1 & & $4.5 \mathrm{~cm} . \mathrm{H}_{2} \mathrm{O}$ \\
\hline 2 & & $10.0 \mathrm{~cm} . \mathrm{H}_{2} \mathrm{O}$ \\
\hline 3 & & $.13 .0 \mathrm{~cm} . \mathrm{H}_{2} \mathrm{O}$ \\
\hline 4 & . & $5.0 \mathrm{~cm} . \mathrm{H}_{2} \mathrm{O}$ \\
\hline
\end{tabular}

Pressure chamber over external jugular. $4.0 \mathrm{~cm}, \mathrm{H}_{2} \mathrm{O}$

$10.0 \mathrm{~cm} . \mathrm{H}_{2} \mathrm{O}$

$14.0 \mathrm{~cm} . \mathrm{H}_{2} \mathrm{O}$

$5.0 \mathrm{~cm} . \mathrm{H}_{2} \mathrm{O}$

A further improvement of this instrument has been made by Hooker, ${ }^{3}$ and in its present form the instrument has proved most satisfactory. Two conditions are necessary for its use: First, that the vein shall stand out sufficiently from the surrounding skin level to give a distinct shadow by oblique illumination and, second, that the vein wall must be collapsible. Hence "old patients with phlebosclerosis, patients with exceedingly edematous or fat hands, and patients with continuously small veins are not satisfactory for this method" (Clark).

IV. Double-cuff Manometers. (Fig. 66).-In 1912 two instruments upon the same principle were introduced independently, one by L. Frank and M. Reh ${ }^{4}$ and the other by A. A. Howell. ${ }^{5}$ The instruments consist of two cuffs, each attached to a water manometer. One cuff is applied to the upper arm, the other to the forcarm. The forearm cuff is inflated so as to fit against the arm suugly without exerting, however, more than $1 \mathrm{~cm}$. (Frank and Rehl) to $3 \mathrm{~cm}$. (Howell) $\mathrm{H}_{2} \mathrm{O}$ pressure. The upper cuff is then slowly inflated until the water in the forearm manometer is seen to be rising. This rise is assumed to be evidence that the venous flow beneath the upper euff has been obstructed and that the blood, being dammed back, has increased the volume of the forearm with consequent displacement of air from the lower cuff and a rise of water in its

${ }^{1}$ Arch. f. exper. Path. und Pharm., 1906, lv, 463.

2 Johns Hopkins Hosp. Bull., 190s, xix, 274.

${ }^{3}$ Amer. Jour. of Physiol., 1914, xxxv, 73.

'Zeitsch. f. exper. Path. und Therap., Berlin, 1912, x, 241.

'Arch. Int. Med., Chicago, 1912, ix, 148. 
attached manometer. The forearm cuff and its manometer are used, therefore, as a sort of plethysmograph. It is further assumed that the blood-flow beneath the upper euff is first obstructed when the pressure in this cuff equals the venous pressure. The instrument of Frank and Reh differs from Howell in that it provides for graphic registration of the readings. Reference to the table on p. 141 shows that Howell's figures are somewhat higher than those of Frank and Reh, which may be in part explained by the higher pressure used by Howell in his plethysmographic cuff.

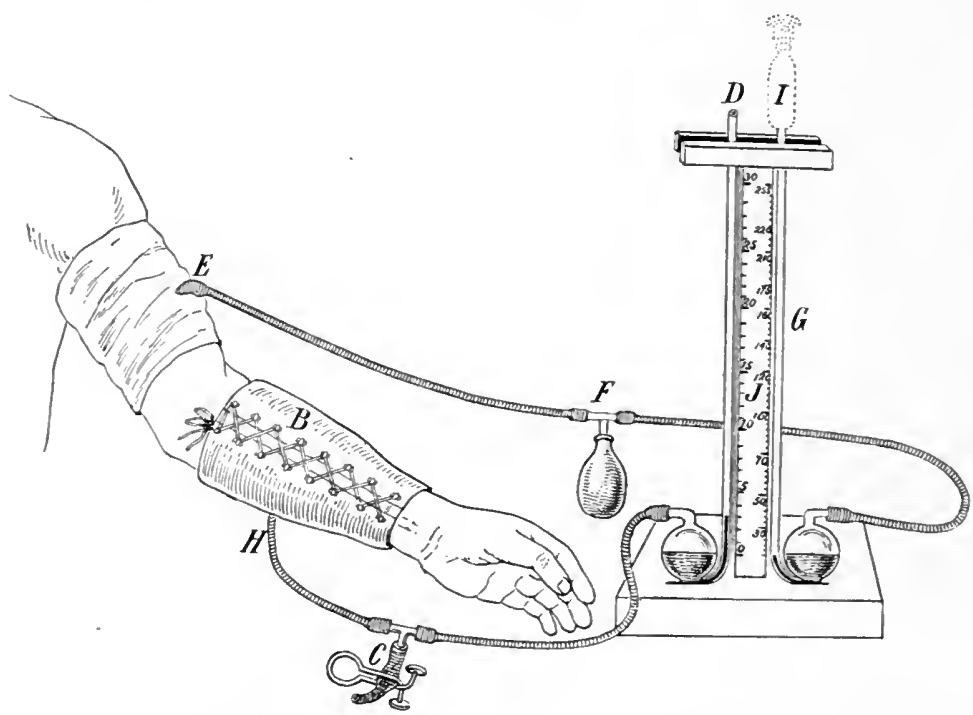

FIG. 66.-Howell's venous pressure apparatus. Upper and lower cuffs applied and attached to their respective water columns. $E$, light cuff for constricting upper arm and obstructing venous return; $F$, glass $\mathrm{T}$ and bulb, by means of which pressure is raised in $E ; G$, water column measuring pressure in $E ; B$, inelastic covering of light material encircling rubber bag; $C$, glass $\mathrm{T}$, side tube, and clip, by means of which pressure can be raised; $D$, water column measuring pressure.

v. Intravenous Needle.-In 1909-10 Moritz and von Tabora ${ }^{1}$ published studies of venous pressure measured by the pressure required to eause normal saline to enter the median vein at the level of the heart. The method was to place the patient in the recumbent position, and then introduce into the median vein with the usual precautions a needle connected with a burette of normal saline. The saline was allowed to enter the vein, its level in the burette falling until further flow ceased. The level of the saline above the

${ }^{1}$ Deutsch. Arch. f. klin. Med., 1909-10, xeviii, 475. 
heart was then read, and the resulting figure was assumed to be the venous pressure at the heart in centimeters normal saline, whieh since $10 \mathrm{~cm}$. normal saline equals $10.07 \mathrm{~cm} . \mathrm{H}_{2} \mathrm{O}$ may be reckoned as $\mathrm{cm} . \mathrm{H}_{2} \mathrm{O}$. In all the other methods described the patient is in sitting posture, and the level of the heart is by most observers

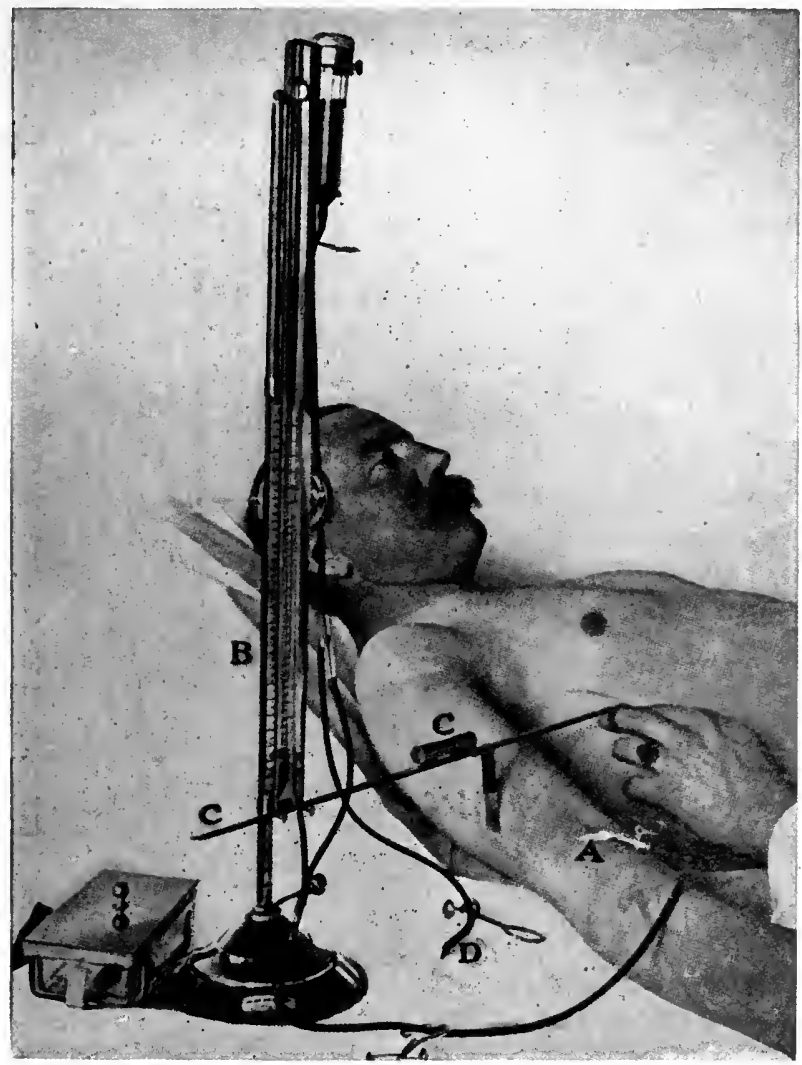

FIG. 67.-The Moritz-Tabora venous blood-pressure apparatus: $A$, cannula in vein ; $B$, manometer scale; $C$, level; $D$, escapement. (After Hoffmann.)

taken according to von Recklinghausen's rule as at the level of the apex of the subcostal angle. With the patient reeumbent, Moritz and von Tabora assume the heart level to be that of a point on the fourth rib $5 \mathrm{~cm}$. below the level of the anterior thoracie surface. This method of measuring venous pressure is the most accurate of those described for use in man. 
Capillary Pressure.-In 1875 the attempt to measure capillary pressure was first made by von Kries. ${ }^{1}$ A small glass plate of known area was laid upon the skin or mucous membrane and pressure was exerted evenly upon it by means of delicate weights. The amount of weight required to produce a just perceptible pallor of the skin or mucous membrane was taken to be the capillary pressure, and the weight and area over which it acted being known, the pressure per unit of area and hence the pressure in $\mathrm{mm}$. $\mathrm{Hg}$. $\left(1 \mathrm{~mm} . \mathrm{Hg}=0.0136 \mathrm{gm}\right.$. per sq. mm.) or in $\mathrm{cm} . \mathrm{H}_{2} \mathrm{O}\left(1 \mathrm{~cm} . \mathrm{H}_{2} \mathrm{O}\right.$ $=0.01 \mathrm{gm}$. per sq. mm.) can be calculated. Von Basch and von Recklinghausen measured capillary pressure with the same apparatus employed for measuring venous pressure by applying it over a skin area, the former noting the pressure required to just cause complete blanching, the latter raising the pressure above the point required to produce complete pallor, and on gradually lowering the pressure noting the moment when flushing first became apparent and also when flushing became complete.

Lombard ${ }^{2}$ has recently developed a refinement of these methods. He finds that if the skin be wet with glycerin or with a transparent oil, by the use of a low-power magnification (from 10 to 35 diameters), and either bright daylight or a Nernst lamp, the papillee of the skin with their superficial bloodvessels are rendered visible. He applies this method either with a weighted glass plate according to von Kries, or with a specially constructed chamber similar in general principle to von Recklinghausen's. Lombard's chamber consists of an inverted truncated cone. The smaller end, which fits against the skin, has a diameter of $24 \mathrm{~mm}$., its walls are $3 \mathrm{~mm}$. thick, and the pressure is therefore applied over a circular area of $18 \mathrm{~mm}$. in diameter. Over the periphery of this area the skin is covered by a rubber dam, which aids in preventing leakage between the chamber and skin. A central circular area $5 \mathrm{~mm}$. in diameter is left open for inspection through the microscope. In using the von Kries plate, Lombard has found the optimum dimension for his purposes to be $1 \mathrm{~mm}$. square. If larger plates be used, the weight is borne disproportionately at the edges. Lombard has shown, as may be seen in the following table, great variation in pressure in capillaries of different sizes. The measurement of capillary pressure in individuals or in pathological conditions can hardly be of value, therefore, while we lack means of discriminating

${ }^{1}$ Berichte ü. d. Verhandlung der königlich sä̈chsisch. Gesellschaft d. Wissenschaften z. Leipsic math. Phys., 1875, clxxvii, 147.

${ }^{2}$ Amer. Jour. Phys., 1912, xxix, 335. 
between the particular type of capillary under consideration. Inspection of the table suggests that the grosser methods of von Kries, von Basch, and von Recklinghausen measure the pressure in the medium-sized and larger capillaries on the arterial side.

TABLE I.

von Kries, finger, first pallor

\section{Capillary pressurc.}

von Basch, hand, complete pallor

von Recklinghausen, hand, first flus

von Recklinghausen, hand, complete flush

Lombard-Largest capillaries

" Medium capillaries

Smallest capillaries, back of hand

Smallest capillaries, finger nail

Most superficial and smallest veins

Subpapillary venous plexus

TABLE II.

Sewell

von Basch.

von Recklinghausen: filled empty

Hooker-Fyster

Frank and Reh

Howell

Moritz and Tabora

Summary
At level of heart. Normal

Cm. $\mathrm{H}_{2} \mathrm{O} \quad \mathrm{Mm}$. $\mathrm{Her}$.

4.6 to $5.2 \quad 3.4$ to 3.8

$8.8 \quad 6.5$

14 to 22

20 to 26

3 to 10

av. 8

1 to 6

4 to 13

av. 7.6

1.1 to 8.7

av. 5.2

1 to 13
2.2 to 7.3
av. 5.9
0.7 to 4.4
2.9 to 9.5
av. 5.6
0.8 to 6.4
av. 3.8
0.7 to 9.5

Cu. $\mathrm{H}_{2} \mathrm{O}$ Level of heart.

51.3

34 to 41

93 to 99

75

82 to 95

48 to 61

20.6 to 30.9

23.3 to 37.5

20.5 to 27

13.5 to 20.5 in. $1 \mathrm{Hg}$.

37.7

25 to 30

68 to 72.5

55.1

60 to 70

35 to 45

15.1 to 22.7

17.1 to 27.6

15 to 20

10 to 15

Cin. $\mathrm{H}_{2} \mathrm{O}$ Mathologic. $\mathrm{Mm}$. $\mathrm{Ig}$.

to 17

7 to 25

av. 13.9

to 12.5

up to 25

up to $18+$

\section{VARIOUS FACTORS INFLUENCING VENOUS PRESSURE.}

The position of a part with relation to the heart is probably the most important factor in determining venous pressure. The weight of the blood is such that the pressure of one inch of blood is approximately equal to $1.9 \mathrm{~mm}$. $\mathrm{Hg}$. or $2.6 \mathrm{~cm}$. $\mathrm{H}_{2} \mathrm{O}$. It has been shown, however, by von Recklinghausen that the veins of the feet do not actually exhibit the pressure demanded by calculation upon this basis. This is shown in the following table:

\section{Venous Pressure at Various Levels.}

(Data from vou Recklinghausen.)

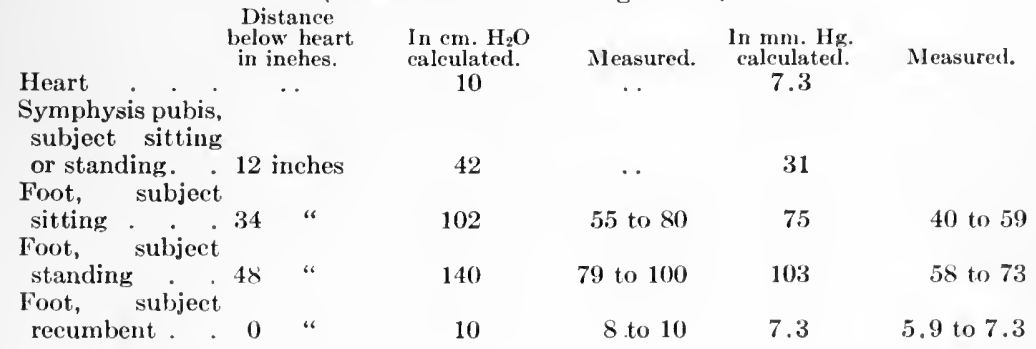


This discrepancy von Recklinghausen supposes to be due to the action of the muscle masses of the extremities which, by their intermittent pressure upon the veins, aided by the action of the valves of the veins, serve to propel the blood up into the vena cava, where he supposes the venous pressure actually exhibits its calculated value. This importance of muscular activity in promoting the return of venous blood from the extremities may explain in part the discomfort incident to prolonged standing in one position. The importance of position in the determination of venous pressure is further shown by the fact that raising one arm above the level of the head increases the venous pressure in the opposite arm.

Barach and Marks ${ }^{1}$ have noted that readings of venous pressure in the arm do not always show a constant relative difference proportional to the elevation or depression above or below the level of the right auricle. Hence venous pressure should be measured at the level of the right auricle rather than at another level followed by correction to the level of the auricle.

Changes in arterial pressure do not readily alter the venous pressure, and the capillary pressure, at least of the smaller capillaries, is much more dependent upon venous than upon arterial pressure. Hooker finds that there is no relation between venous pressure and changes in pulse rate, and that local changes in vascular tone (cold, heat, etc.) do not alter the venous pressure in the hand. BurtonOpitz ${ }^{2}$ was able to show only a very slight fall of venous pressure in the external jugular of a dog upon occluding a portion of the arterial supply to the head.

Normal venous pressure at the level of the right auricle was found by Barach and Marks to range in the erect posture between 8 and $18 \mathrm{~cm} . \mathrm{H}_{2} \mathrm{O}$ and in the recumbent posture between 3.5 and $11 \mathrm{~cm}$. $\mathrm{H}_{2} \mathrm{O}$. Muscular exertion either general or local was found by Hooker and Wolfsohn, ${ }^{3}$ Elfers ${ }^{4}$ and Schott, ${ }^{5}$ to increase the venous pressure from a slight rise up to $14 \mathrm{~cm} . \mathrm{H}_{2} \mathrm{O}$. Hooker ${ }^{6}$ has noted a distinct diurnal variation under normal conditions of health even in individuals confined to bed. He observes a gradual rise throughout the day from 10 to $20 \mathrm{~cm} . \mathrm{H}_{2} \mathrm{O}$ to an average diurnal pressure of $15 \mathrm{~cm}$. and a fall during sleep to as low as 7 to $8 \mathrm{~cm}$. During

\footnotetext{
1 Arch. Int. Med., 1913, xi, 485.

${ }^{2}$ Amer. Jour. Physiol., 1903, ix, 198.

${ }^{3}$ Amer. Jour. Physiol., 1909-10, xxv, 24; 1911, xxviii, 235.

${ }^{4}$ Inaug. Diss., Kiel, 1911.

${ }^{5}$ Deutsch. Arch. f. klin. Med., 1912, cviii, 537.

${ }^{6}$ Amer. Jour. Physiol., 1914, xxxv, 73.
} 
sleep he has noted a venous pulse of peripheral origin in the veins of the hands. Clark ${ }^{1}$ found in 4 bed patients without cardiac complications, a diurnal variation of from 8 to $10 \mathrm{~cm} . \mathrm{H}_{2} \mathrm{O}$, the maximum $(10-16 \mathrm{~cm}$.) being reached from 2 to 8 P.M., and the minimum $(5-8 \mathrm{~cm}$.) between 10 P.M. and 6 A.M. This author from repeated observation on 14 cases, 6 with decompensation, 8 with good compensation, concludes that a venous pressure above twenty is pathological. In cardiac cases, the diurnal variation was found by Clark to be reversed, the highest pressure occurring during the sleeping hours. Venous pressure continuously above $20 \mathrm{~cm}$. which was not lowered by digitalis, was an indication of grave cardiac involvement. On the other hand, with a venous pressure below $20 \mathrm{~cm}$. no definite change in the pressure was observed from digitalis therapy. The venous pressure and the urinary output generally showed a significant inverse variation in cases of cardiac decompensation. Aspiration of pleural fluid and venesection both lowered venous pressure as a rule in his series, but after the latter procedure the subsequent rise of venous pressure was rapid.

Respiration.-Burton-Opitz made careful studies of the effect of respiration upon the venous pressure in the external jugular of a $\mathrm{dog}$, and found that with expiration the jugular pressure rose and that during the pause following expiration it began to fall and continued to fall throughout the early part of inspiration, a curve similar, therefore, to that of the arterial pressure in the respiratory phases. Upon expiration the intrathoracic pressure rises and the venous blood does not readily enter the thorax, but being dammed back causes rise of peripheral venous pressure. Upon inspiration the intrathoracic pressure falls and the venous blood flows readily into the thorax, with fall in the peripheral venous pressure; near the thorax the venous pressure may be negative during inspiration. The respiratory variation found in the external jugular pressure is as much as $3.8 \mathrm{~cm} . \mathrm{H}_{2} \mathrm{O}$, and becomes greater as the thorax is approached. The opening of the thorax by increasing the intrathoracic pressure (which is normally less than the atmospheric) causes a prompt rise of venous pressure of from 1.5 to $3.5 \mathrm{~cm} . \mathrm{H}_{2} \mathrm{O}$.

Cardiac Action.-Impaired cardiac action by leading to venous stasis produces an increase of venous pressure. Experimentally, stimulation of the peripheral end of the sectioned vagus produces a slight rise of venous pressure. Clinically, any form of myocardial

${ }^{1}$ Arch. Int. Med., 1915, xvi, 587. 
decompensation leads to increase of venous pressure which may rise to $30 \mathrm{~cm} . \mathrm{H}_{2} \mathrm{O}$ in the veins of the arm at the cardiac level. Indeed, inereased venous pressure may, in the early stages, be the only sign of circulating stasis.

Intravenous Injection.-That intravenous injections increase the venous pressure more proportionately than they do the arterial was shown by Bayliss and Starling. ${ }^{1}$ Rise of venous pressure at the heart also results from abdominal pressure, bandaging or elevation of the extremities, or from elevation of the abdomen above the level of the heart. The importance of venous pressure in relation to cardiac output has already been diseussed. Experimentally as a result of therapeusis, a rise of venous pressure occurs after large doses of epinephrin, pituitrin, and alcohol, not after digitalis, strophanthin, strychnin or eaffein. This rise is proportionate to, and apparently the result of disturbed heart action. It is therefore apparently simply a stasis reaction and not the result of vasomotor influence. A fall of venous pressure may follow the administration of the nitrites or large doses of morphin, and since cardiac functionation remains unimpaired, may be attributed to a direct influence on the rasomotor mechanism. ${ }^{2}$

${ }^{1}$ Jour. Physiol., 1594, xvi, 159.

${ }^{2}$ Capps, J. A., and Mathews, S. A., Venous Blood Preesure as Influenced by the Drugs Used in Cardiovaseular Therapy, Jour. Am. Med. Assn., 1913, Ixi, 388. 


\section{THE FUNCTIONAL EFFICIENCY OF THE CIRCLLATION AS DETERMINABLE BY BLOOD-PRESSURE ESTIMATION AND ALLIED TESTS.}

Is the following chapter are described a number of methods which have been suggested in the hope of throwing more light on the question of the functional efficiency of the circulation. Most of them are based on sound theoretic principles, and would be invaluable if our instrumental measurements were sufficiently exact and comprehensive to insure an accurate basis for our deductions. Unfortunately, however, the data obtainable by sphygmomanometry, etc., are, even when collated' with the greatest care, subject to unavoidable sources of error, and hence our results are only approximate. 'To use such data as a basis for elaborate calculations, entailing the use of involved mathematic and algebraic formulæ for the elucidation of the complex factors included in the static and dynamic laws of the circulation, only magnifies our errors and often leads to a veritable reductio ad absurdum. 'Thus far it must be admitted no entirely satisfactory method has been perfected, mainly because we cannot measure vascular tonus, or estimate the role of psychic processes. Furthermore, to yield definite indications many of the tests have to be carried to a point at which concomitant symptoms and ordinary physical signs are of themselves sufficiently evident criteria of functional insufficiency. Unquestionably, however, some of the methods about to be described do throw a useful light on the problem under eonsideration, especially if the results thus obtained are accepted with due reserve.

Many are the attempts which have been made and numerous the methods suggested for determining the functional capacity of the heart. 'The importance of this subject from a diagnostic, prognostic, and therapentic stand-point camnot be overestimated. Such procedures as are based in part, at least, upon blood-pressure measurements will be here considered.

Generally speaking, such tests depend upon the way in which 
the pulse rate and blood-pressure both respond-quantitatively, qualitatively, and temporarily - to definite amounts of muscular exertion or to change of posture (see p. 48). This subject has been extensively studied by Masing, Buttermann, Christ, Staehelin, and others. ${ }^{1}$ The work performed was either definitely measured with various forms of apparatus, or roughly estimated by such tasks as lifting, stair-climbing, walking, etc.

"In blood-pressure and exercise we have an unfailing guide to the true condition. Whatever the abnormality the great question is the integrity of the heart muscle, the reserve force of the heart, for upon this chiefly depends the prognosis. The pulse should be counted, the heart examined, and the blood-pressure taken, and then with the cuff attached to the arm, the applicant should be exercised to the equivalent of climbing two flights of stairs and then reëxamined quickly. Exercise when not too severe will regulate the pulse if the condition is simple. If serious disease is present the irregularity will be greatly increased. If the heart muscle is in prime condition the systolic pressure will jump 15 to $40 \mathrm{~mm}$. and, tested every two minutes, will be found to resume its original place in six or eight minutes. If the condition is bad, in a powerful effort of the heart to respond, the rise may be even greater, but the return will be exceedingly slow, sometimes requiring 20 or 30 minutes. If the state of the muscle is extremely bad and the reserve force exhausted, the systolic pressure may fall instead of rising and the diastolic remain stationary, or rise a little, creating a very small pulse pressure. It will be a long time before graphic instrument tracings are required in life insurance, if ever, but we have in exercise and blood-pressure a thoroughly reliable test, and it is the duty of the examiner to be safe in his recommendation of every applicant."'2

Crampton's Test of Vasomotor Efficiency.-In rising from the recumbent to the erect posture blood-pressure tends to fall as a result of gravity. Unless this tendency were automatically regulated, suncope would occur as a result of cerebral anemia. In a normal vigorous man, however, such a change of position causes a rise of blood-pressure amounting to $S$ or $10 \mathrm{~mm}$. $\mathrm{Hg}$. This increase may result from increased vasomotor tone or from increased cardiac work, or as a result of both factors. Bearing these facts in mind

${ }^{1}$ For bibliography, see Norris, G. W., The Functional Capacity of the Heart, International Clinies, 1907,17 th series, vol. $i$.

'Lankford, J. S., The Heart in Life Insurance, Med. Rec., October 17, 1914, p. 687. 
Crampton ${ }^{1}$ has devised the following table to test vasomotor efficiency through the observation of pulse rate and blood-pressure responses to postural change. The tables while setting what is perhaps a high normal have shown that vasomotor tone in the same individual varies greatly as a result of mental or physical fatigue, infectious processes, etc. It may be used with especial edification in the study of essential hypotension cases.

The Technic.- "The sphygmomanometer is adjusted over the brachial artery and the patient is placed on a comfortable couch with a low pillow. The heart rate is counted by quarter-minutes and a gradually descreasing rate is usually observed. Counting should continue until two successive quarter-minutes are the same, this is multiplied by 4 and recorded. The systolic pressure is then taken preferably by auscultation. The patient stands, the heart rate is counted as before until it reaches the 'standing normal,' when it is recorded, and the blood-pressure is then taken. The differences are calculated and reference is made to the scale.

"For example-Case XX: L. V., age seventeen years, said to be in good condition at 11:20 A.M.

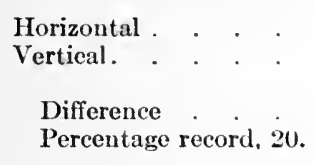

\begin{tabular}{cc} 
Pulse rate. & Blood pressure. \\
68 & 100 \\
104 & 94 \\
\hline+36 & -- \\
\hline
\end{tabular}

"This is a very poor record taken from an apparently normal, strong young football player of exceptional ability who had previously given records above 80 .

"I was at a loss to accoumt for this, for questioning failed to bring out any history of loss of sleep, dissipation, or illness. He looked quite as 'fit' as usual. He was absent next day, and remained home for a week with a 'cold and fever.' It is evident that the test revealed a weakened vasotone, the begimning of actual illness before any other symptom could be noted. Others who have used this test have noted similar cases."

If the vasomotor tone is deficient, the heart must make up for it by an increase in rate-the greater the increase, the less efficient the vasomotor tone.

"The, usual range of the systolic pressure is from +10 to -10 of the heart rate increase from 0 to 44 , as observed from records of a large number of cases. Upon a statistical balaneing of these two

I Blood Ptosis, New York Med. Jour., Novenıer S, 1913. 
series of frequencies, and assigning equal percentages to equal ranges, the following scale is constructed:

\section{PERCENTAGE SCALE.}

VASOMOTOR TONE.

Blood-pressure.

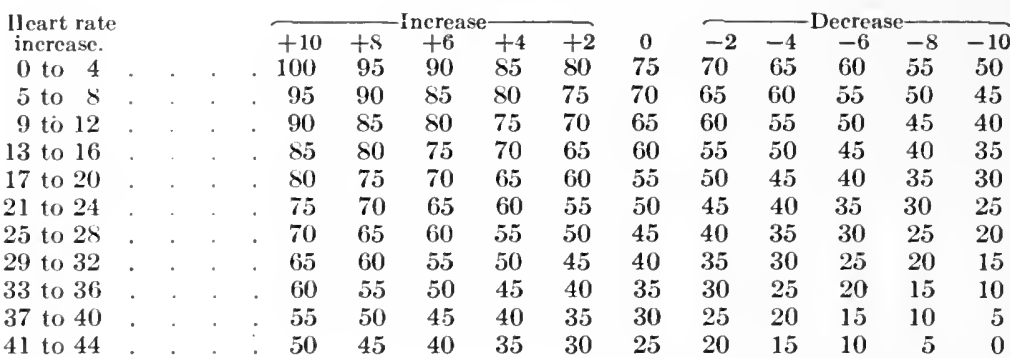

NoTE.-In case of inerease in pressure higher than +10 add 5 per cent. to the +10 column for each 2 millimeters in excess of 10 .

"This scale provides a convenient and intelligible method of recording and reporting cases and permits a numerical statement of the function in question. Its 100 mark indicates a perfectly efficient working of the rasomotor system under test, the zero is approximately the point where the average person is unable to maintain the erect posture."

Among 116 athletes R. Tait McKenzie found the postural change in pulse rate and systolic blood-pressure average as follows:

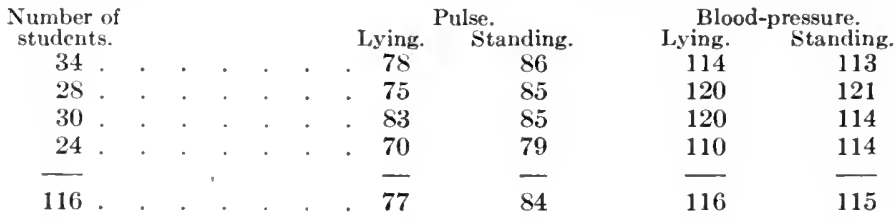

It will be noted that there was a very constant variation in the pulse rate amounting to about seven beats per minute between the two postures. The blood-pressure findings were much more variable and when an average was established they were practically identical in the two positions. Among 388 students with cardiac abnormalities-arhythmia, murmurs, etc., the systolic bloodpressure average was somewhat higher $(138+\mathrm{mm}$. Hg.) than among the athletes with normal hearts. 
Passive change of posture.

"When the element of muscular effort has been eliminated, change of bodily posture from the erect to the horizontal will cause an increase in the maximum pressure, a decrease in the minimum pressure, and an increase in the pulse pressure. After five minutes in the horizontal posture when the subject is retilted to the erect posture, the maximum pressure will diminish, the minimum pressure increase and the pulse pressure diminish. It will be noted that in both instances the pulse pressure follows the same trend as the maximum pressure. Change of posture from the erect to the horizontal causes a fall in the venous pressure. Change of posture from the horizontal to erect causes an increase of the venous pressure."'1

During muscular exercise we see an increased respiratory gas interchange, the amount of which may exceed that occurring during rest over twentyfold. Associated with this there is a marked increase in the rate of blood-flow, so that the normal circulation, the time of which is fifty-five seconds, may be reduced to five seconds during exercise. Such changes must, of course, produce enormous alterations in blood-pressure values.

Muscular exertion may be accompanied either by a fall or by a rise of pressure, and it has been found that the more fit and welltrained the animal the less the tendency for the pressure to rise. Thus in horses and in well-trained athletes there is a fall. 'The average man shows a primary rise, followed by a fall upon cessation of work. The more strenuous the work and the less trained the man, the greater and the earlier will be the fall. This may result from cardiac weakness or may be a result of Nature's safeguard against cardiac overstrain, the fall of pressure being brought about reflexly through the agency of the depressor nerve. During youth both pulse and blood-pressure are more labile during exercise and tend to keep pace with each other. In adults there is less tendency for the pulse and more for the pressure to rise. In health both pulse and pressure return promptly to the normal upon the cessation of work. With a diseased cardiovascular system this is not so promptly accomplished. The heart which has been weakened by infectious processes shows a wide range and a prolonged duration of pulse and blood-pressure variations. The amount of psychic

\footnotetext{
I Barach, Jos. H., and W. L. Marks, Effect of Change of Posture, without Aetive Iuscular Exertion, on the Arterial and Venous Pressures, Archiv. Int. Med., May 15,1913 , ii, No. 5 .
} 
effort required is of the greatest importance. Work which is performed passively, automatically, without any particular psychic concentration, is not accompanied by a rise in tension. The primary fall of pressure which occurs inder training is largely the result of eliminating the volitional psychic factor. An increase of pressure under muscular work depends more on the cerebral stimuli required than on the amount of work done. In the hypnotized subject, imaginary work produced an elevation of pressure to $200 \mathrm{~mm}$. $\mathrm{Hg}$. (Klemperer). These causes explain many of the failures of testing functional capacity by measured amounts of work. Although we can measure the work performed in kilogrammeters we have no means of estimating the amount of nervous force and psychic energy which may be expended upon the same task by different classes of trained and untrained, nervous and phlegmatic, energetic and lazy individuals (see p. 367).

The increase of pressure which is associated with muscular effort affects chiefly the systolic pressure; the diastolic element tends to lag behind, thus increasing the amplitude of the pulse. On assuming the erect posture the minute volume decreases slightly, the systolic output greatly ( 20 per cent.). In the recumbent posture the minute volume in women increases 17.2 per cent., and the systolic output 31 per cent. In men this change was not observed owing probably to a more efficient vasomotor system. ${ }^{1}$ Lowsley ${ }^{2}$ found that physical activity caused a rapid rise in bloodpressure which precedes the increase of the pulse rate. During continued exercise these curves show but little change. After cessation from work the fall in the systolic pressure again precedes the decrease in the pulse rate. A secondary rise of the pulse rate occurs which is a "reflex effort due to low blood-pressure of the subnormal stage."

Muscular effort sufficient to increase the pulse rate provokes a rise of the systolic, diastolic and the venous pressures. Inasmuch as the systolic pressure rises more than the diastolic, the pulse pressure is increased.

Following the primary maximal rise of systolic pressure a gradual decline occurs, but while exercise is being continued the pressure never reaches the normal level. The diastolic pressure generally

I Lindhard, J., Effect of Posture on the Output of the Heart, Skand. Arch. f. Physiol., 1913, xxx, 395.

2 The Effects of Various Forms of Exereise on Systolic, Diastolic, and Pulse Pressures and the Pulse Rate, Amer. Jour. Physiol., 1911, xxvii, 446. 
follows the systolic curve but its maximum is reached later and upon cessation from work it invariably falls below its normal level. According to Lowsley the longer the subnormal diastolic phase, the greater the circulatory fatigue. The venous pressure shows often a sudden drop to normal after cessation from exercise. Exercise associated with strain requiring a closed glottis and a rigid chest, disproportionately increases blood-pressure in relation to the pulse rate and very markedly increases venous pressure.

Graupner's Test.-Graupner ${ }^{1}$ endeavored to test cardiac functionation by noting the effects of measured work upon the pulse, the blood-pressure and the size of the heart, the blood-pressure being measured with a Gärtner tonometer and taken when the pulse had reached the same rate, after the exercise, which it had before this was commenced. His investigations led him to the following conclusions: (1) Blood-pressure remains constantcardiac sufficiency. (2) Blood-pressure falls-cardiac insufficiency. (3). Blood-pressure rises, but returns to normal-compensatory capacity. (4) Blood-pressure rises, falls rapidly, without a tendency to rise again-fatigue.

He insists that the work must be done by the same muscle groups, preferably the thighs; and must not be carried to the degree of cxhaustion, as this necessitates the introduction of other cardioaccelerator impulses, such as psychical effort, excess of metabolic waste products in the blood, etc. There must be no muscular strain, thoracic encumberment or unnecessary mental excitement. He prefers the Zuntz ergometer, ${ }^{2}$ built on the principle of the bicycle, to the Gärtner ergostats, ${ }^{3}$ on account of its greater accuracy.

The work consisted in having the patient turn a wheel supplied with a brake with which work could be measured. The tests were made at the same hour on successive days, the relation to mealtime being constant. He fully recognized the fact that apparent incapacity of the heart may be due to lack of training, owing to the fact that definite kinds of work may be performed by the skeletal muscles with much less cerebral and muscular fatigue after the individual has become habituated to the exercise, and the centres in the medulla have become used to increased quantities of carbon dioxide in the blood. Furthermore, even the diseased heart, unless compensation is extremely bad, may increase in power upon excrcise.

${ }^{1}$ Graupner, Die Mechanische Prüfung und Beurtheilung der Herzleistung, Berlin. Klin., 1902, xv, No. 174.

2 Zuntz, Centralbl. f. Physiol., 1898, p. 502.

${ }^{3}$ Gäirtner, Allg. Wien, med. Zeit., 1887, Nos. 49 and 50. 
Graupner believes, as the result of his investigations, that both in functional and organic incapacity of the heart, accelerated pulse rate and dyspnea are caused by the performance of work equivalent to from 45 to $300 \mathrm{mkg}$. per minute. In myocarditis less than the last-named figure is invariably sufficient to produce these symptoms, whereas, in merely functional incapacity more is often required. The same method of differentiation holds good between angina pectoris of sclerotic and of nervous origin.

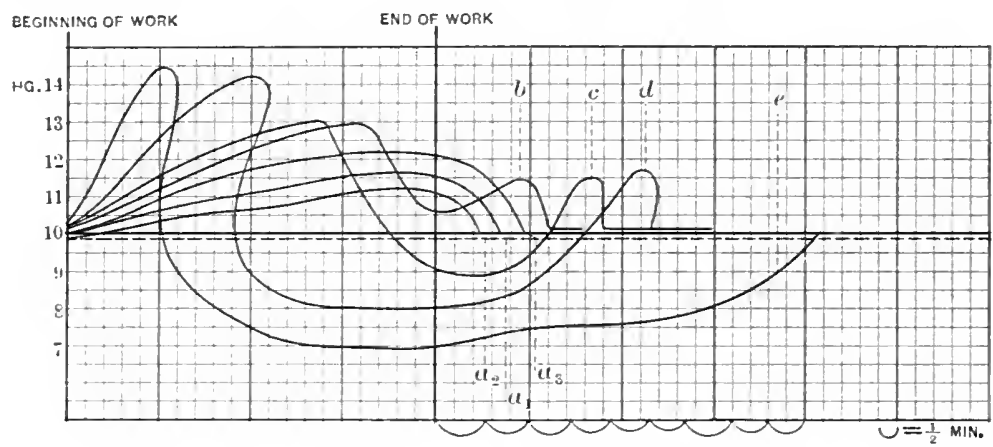

FIG. 68.-1. When functional eapacity is good the blood-pressure undergoes practically no change during exercise, or if severe effort is required rises slightly. Fluctuation begins thirty seconds after cessation from work.

2. Inmediately upon ressation from more strenuous work, blood-pressure is slightly elevated, but soon falls to the normal. The rapidity of this fall may be increased by training. If insufficiency exists, the elevation is greater, persists longer, and the normal is more slowly reached $\left(a_{1}, a_{2}, a_{3}\right)$.

3. More severe effort may produce a fall of pressure while work is going on, in which case it promptly rises to the normal after cessation from work (b),

4. Still more work nay eause a lower fall, which is later followed by a rise above the normal $(c, d)$.

5 . The absence of this seeondary rise even after repeated or more prolonged work bespeaks myocardial fatigue. In such cases we will usually find clinical signs of insufficiency $(e)$.

6. The onset of dyspnea may reflexly produce increased blood-pressure, but in such an event we soon find a fall far below the normal.

7. Conclusions must not be drawn from one set of observations alone, but if evidences of insufficiency are persistently encountered, we may feel reasonably sure that we are dealing with heart weakness; for if these findings were due to vasomotor influenees they would disappear under "training."

The glaring faults of Graupner's method are (1) that we cannot determine the amount or eliminate the effects of psychical and visceral influences upon the pulse and blood-pressure, although the author claims that by this joint observation the one controls the other; (2) it is necessary to make each test twice to see if the heart will respond the second time as it did the first, and, furthermore, we have no means of estimating the effects of the peripheral 
vessels. Finally, that all tests which are based upon an estimation of the pulse rate or blood-pressure do not take into account the normal rhythmic periodic fluctuation which these as well as other factors of the economy are subject to.

Graupner and others who have investigated the question have found that a fall in tension after exercise indicates insufficiency, or at least fatigue. Butermann also found that well-compensated valvular lesions and neurotic hearts responded to exercise by a rise of blood-pressure averaging $13 \mathrm{~mm}$., whereas myocardial cases showed a lessening of tension varying from 3 to $13 \mathrm{~mm}$.

Since the appearance of his original paper Graupner ${ }^{1}$. has been continuing his researches, and in a more recent article he reiterates his convictions, laying special stress upon the value of bloodpressure measurements. Regular changes in this indicate that the myocardium is able to adjust itself to the existing conditions. According to his observations normal hearts can perform arm-muscle work on the ergometer equivalent to from 3000 to $20,000 \mathrm{mkg}$. per hour. If the figures fall below $1000 \mathrm{mkg}$. absolute myocardial weakness exists, while a capacity of less than from 200 to $500 \mathrm{mkg}$. offers a bad prognosis as to life. When a certain group of muscles is exercised the blood is drawn toward them at the expense of the other vascular areas. To restore the balance later, the blood rushes away and pressure falls, to be followed by a return wave which in its turn abates. When measurements are made every halfminute after exercise it has been found that the amount of variability in the blood-pressure corresponds directly with the insufficiency of the myocardium. In other words, the greater the lieart weakness the wider the pressure fluctuations, and the longer is the time required for the restoration of balance (Fig. 67).

Some other observers have found that pressure falls in the case of well-trained athletes which, according to Graupner's contention, would indicate cardiac insufficiency, whereas a rise of pressure occurs in some cases of patients with diseased hearts as a result of high-pressure stasis. As Hoffmann has shown, the test often fails us when most needed-in the differentiation of neuroses from organic lesions.

Katzenstein's Test.-'This test for determining the degree of functional capacity of the heart is based upon the manner in which this organ responds to compression of both iliac arteries. ${ }^{2}$

${ }^{1}$ Deut. med. Woch., 1906, xxxii, 1029.

${ }^{2}$ Katzenstein, Berlin. klin. Woch., 1907, xliv, No. 16. 
Experimentally in animals, if some of the large vessels are obliterated, blool-pressure rises while the pulse rate remains stationary. In human beings it was found that if the heart was functionally potent, bilateral obliteration of the iliac arteries, maintained for from two to five minutes, caused a rise in blood-pressure of from 5 to $15 \mathrm{~mm}$. of mercury; the pulse rate remained stationary or even diminished. In the case of an hypertrophied heart, with good compensation, the bloorl-pressure rises perhaps to $40 \mathrm{~mm}$.,

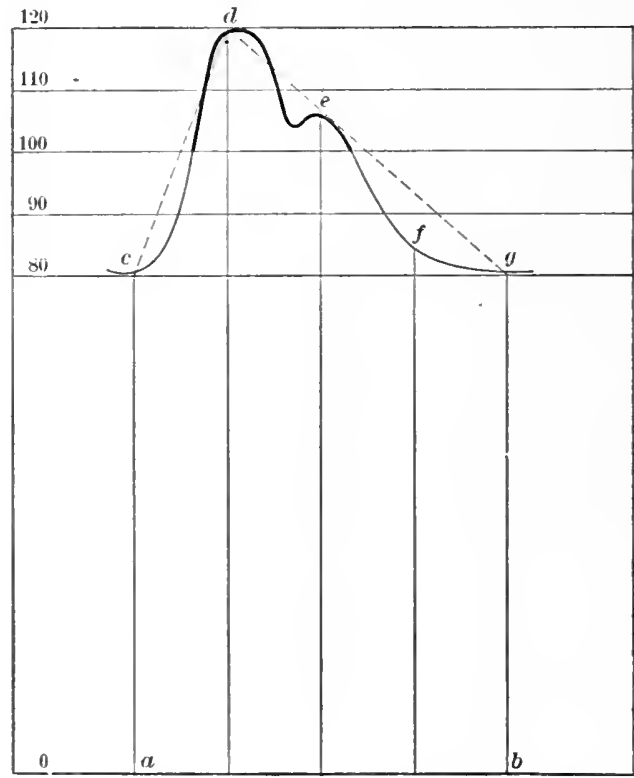

Fig. 69.-The absolute sphygmogram. If the cuff pressure be raised to $100 \mathrm{~mm}$. $\mathrm{Hg}$. only that part of the pulse wave which is in excess of this pressure will be traced (see page 146).

the rate being still unaffected. If there is slight insufficiency, the pressure does not rise, whereas the pulse does become accelerated. In severe insufficiency the pressure falls and the pulse becomes more rapid. These tests are made with the subject in the recumbent posture. The sources of error in this method are manifestly mental excitement, and the possibility of producing pain during the compression of the arteries, either of which might seriously militate against the accuracy of the results; but with due care both of these factors may be eliminated. 
With a view toward investigating Katzenstein's test, the author ${ }^{1}$ studied a number of cases both among healthy individuals and among those in whom cardiac weakness or disease was assured. As the result of these investigations it was found that, generally speaking, the cases responded to the test as has been contended by the deviser of it. There were, however, notable exceptions in a number of instances. Although the majority of cases with manifest cardiac weakness responded positively to the test, yet-and here lies the main ground for objection-many of them did so in such equivocal terms, that is, by such small changes in the pulse rate or blood-pressure, that the final decision was left largely to the personal equation of the observer. Morelli² suggests that compression be exercised by means of inflated rubber stockings. In a recent communication based upon three thousand cases, during the last ten years, Katzenstein ${ }^{3}$ reiterates his belief in the efficacy of his test. He has found it of especial value in discovering cardiac weakness in operative cases in which chloroform was subsequently used as an anesthetic.

This test has been investigated by numerous writers. It may at times have some corroborative value, but much dependence should not be placed upon it. A test based on the same general principle has been suggested by Mosler, ${ }^{4}$ who estimates the bloodpressure after a deep forced inspiration at the conclusion of which the breath is held.

Janowski, ${ }^{5}$ who tested his patients by (1) walking one thousand steps in twelve minutes; (2) the rapid climbing of three flights of stairs; (3) knee flexion while standing, twelve times at a given tempo; meanwhile observing the blood-pressure and pulse rate at intervals up to ten minutes after the completion of the task, failed to find differences which corresponded to the individuals studied. Neither pulse rate, blood-pressure, pulse pressure, nor blood-pressure quotient yielded the differences which he was led to expect.

The Venous Pressure Test (Schott's ${ }^{6}$ Test). - It appears that in health if the patient raises his arm to an angle of 60 degrees while

\footnotetext{
${ }^{1}$ Norris, G. W., The Functional Capacity of the Heart, Internat. Clinies, vol. i, Series 17.

${ }^{2}$ Della Capacita Funzionale del cuore e dei Vasi Sanguini, Boll. Soc. Med. Chir. di Pavia, March 12, 1910.

${ }^{3}$ Deut. med. Wehnsehr., 1915, vol. xli, No. 16.

"Der Atemstillstand in tiefer Inspirationsstellung: Ein Versuch z. Beurteilung der Kreislauffunktion, Zeit. f. klin. Med., 1913, lxxviii, 133.

${ }^{5}$ Die Funktionelle Herzdiagnostik, Berlin, 1910.

${ }^{6}$ Die Erhöhung des Druekes im venösen System hei Anstrengung als Mass für die Funktionstüchtigkeit des menschliehen Herzens, Deutseh. Areh. f. klin. Med., 1912, eviii, $537-553$.
} 
lying quietly without any other muscular effort, the venous pressure shows on an average an increased pressure of $0.5 \mathrm{~cm} . \mathrm{H}_{2} \mathrm{O}$ (sometimes no increase, nor even a fall). In well-compensated heart lesions the increased pressure averages $2.3 \mathrm{~cm}$.; in broken compensation, 4 to $7 \mathrm{~cm}$.; in conditions of anasarca, etc., $7.3 \mathrm{~cm}$. He therefore considers figures above $3 \mathrm{~cm}$. as pathological. Passive elevation of the limb produces no increase of venous pressure.
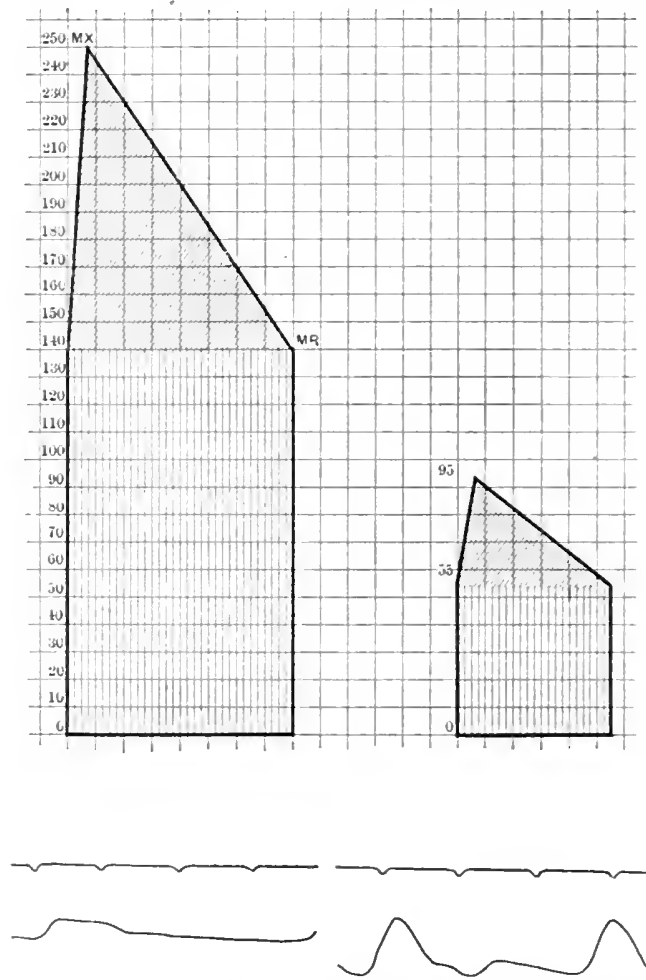

Fic. 70.-Absolute sphrgmograms and pulse tracings from a case of marked hypertension and from a case of marked hypotension. Showing that the pulse tracing gives a very inaccurate representation of the actual height of the arterial pressure. (After Gallavardin.)

The Absolute Sphygmogram.-The information obtainable from the pulse tracing alone, so far as blood-pressure is concerned, is slight because: (1) we do not know the actual value of the abscissa and (2) because the actual length of the waves depends largely on the arljustment of the sphygmograph (amount of pressure exerted on the underlying artery). 
In 1904 Sahli suggested a method for plotting the sphygmogram in absolute pressure values. His method depends upon the fact that if sphygmograms are taken from the radial artery while a blood-pressure cuff is applied to the brachial artery above with increasing pressures, the sphygmogram will be traced of only that portion of the pulse curve corresponding to intra-arterial pressures higher than the pressure in the cuff. Thus, if Fig. 69 represents the actual pressure curve of the pulse in the artery, if the pressure
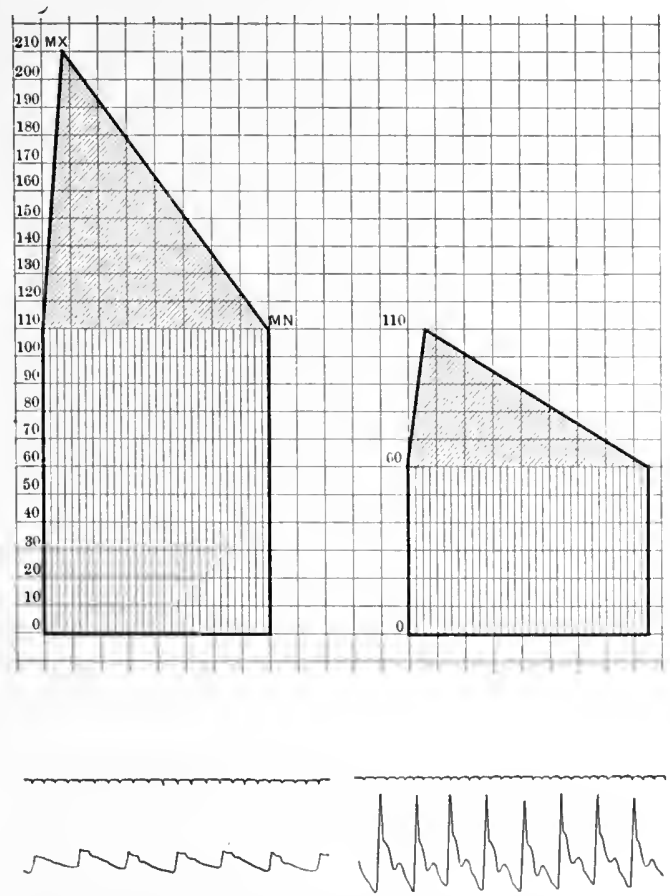

Fig. 71.-Absolute sphygmogram and pulse tracing from a case of hypertension and from a case of hypotension. (After Gallavardin.)

in the cuff be raised to 100 only that portion of the pulse curve drawn heavy and corresponding to pressures above 100 will be traced. It is therefore possible, by altering the pressures in the cuff and noting the amount of the sphygmogram traced at each pressure, to determine the absolute pressure of any point on the pulse curve.

The time relations of these points may be determined from the sphygmogram. The curve may then be plotted on cross-section 
paper, allowing the vertical lines to represent time units and the horizontal lines units of blood-pressure. It is only from such a curve that the true mean pressure can be determined. Thus, if the length of a sufficient number of equally spaced vertical lines from the base line $a-b$ to the pressure curve $c, d, e, f, g$, be measured and their average taken, such average will be the true mean pressure. For clinical purposes Sahli constructs a simplified form by plotting only the systolic pressure or crest of the pulse curve $(d)$, and the
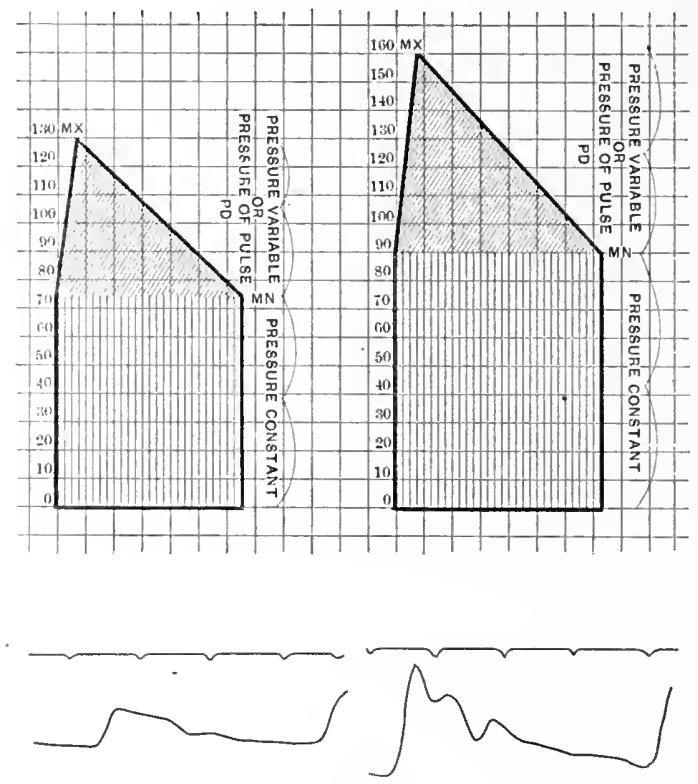

Fic: 72.- Showing absolute sphygmogram and pulse tracing from a subject with normal blood-pressure, and that of a case of nephritic hypertension. (After Gallavardin.)

diastolic pressure or lowest point of the pulse curve ( $c$ and $g$ ), thus obtaining the dotted figure $a, c, d, g, b$. From such a diagram one can judge accurately as to the quickness or slowness of the pulse (pulsus celer, or pulsus tardus).

The Blood-pressure Quotient.-Let us assume that the data obtained regarding systolic and diastolic pressures are correct. What conclusions are we warranted in drawing regarding the amount of work performed by the heart and the amount of its 
systolic output? The question has recently been ably summarized by $\mathrm{O}$. Müller, ${ }^{1}$ from whose article I have freely drawn.

Strassburger has shown that the pulse pressure divided by the systolic pressure is of value in estimating the part played by vascular resistance and cardiac work respectively, in changing circulatory conditions.

The rationale of the method is as follows: With increased cardiac work, the pulse pressure as well as the systolic pressure increases. If, on the other hand, peripheral tonus alone increases, the systolic pressure rises, while the pulse pressure falls. Vasodilatation produces a fall of systolic pressure and an increase of pulsẻ pressure. Therefore if the systolic pressure and the pulse pressure move in the same direction and proportionately, the cause lies in the heart; if in the reverse direction, or not proportionately, the cause lies at least partly in changes in vascular tonus. The cardiac element is also indicated by the number of systoles.

$$
\text { B. P. Q. }=\frac{\text { Pulse pressure }}{\text { Systolic pressure }}=(\text { normally about } 0.3) \text {. }
$$

1. A changed systolic pressure while the quotient remains the same points to a change in the work of the heart. If both systolic and pulse pressure rise, an increased; and if both fall, a diminished cardiac activity may be assumed.

2. When systolic pressure and blood-pressure quotient move in approximately equal but opposite directions, indications point to an alteration of vascular tone. Thus an increase in systolic pressure with a fall of quotient $=$ increased tonus and vice versa.

3 . When systolic pressure and quotient move unequally in the same or in opposite directions, both the heart and the vascular system contribute to the effect. With the limitations imposed by the response of the pulse pressure to factors other than the systolic output (already considered on page 50), and if our estimation of the systolic and diastolic pressures be accurate, these assumptions are warranted. 'The variations of aortic elasticity for different degrees of pressure are clinically a negligible quantity. But a far more serious source of error lies in the fact that the smaller arteries and arterioles possess a distinct tonus, the amount of which we are as yet unable to measure. If the arterial system merely consisted of rubber tubing our figures would certainly be at least approximately correct, and our deductions correspondingly aceurate;

${ }^{1}$ Die unblutige Blutdruckmessung u. ihre Bedeutung f. d. prakt. Med., Med. Klinik., January 12,1908 , p. 47 , et seq. 
in illustration of this it has been demonstrated that in cases of arteriosclerosis the pulse pressure is often twice as large as in a normal individual, but we have no right to conclude from this that in such a case the systolic output is correspondingly great. It is far more likely in such an instance that the difference is due to the rigidity of the arterial wall. The pulsatory reaction of a contracted artery is entirely different from that of a relaxed artery of the same caliber. Again, as F. Klemperer has shown, if we dip one arm into hot and the other into cold water, the pulse pressure will be found to decrease in the former and to increase in the latter; and yet we know that cold produces vascular contraction. Which of the two sides shall we choose as the index of systolic cardiac output?

It may be argued in reply that such extremes as the hot and cold water experiment do not occur under normal conditions, but notwithstanding we must bear in mind the fact that plethysmographic studies have indubitably demonstrated the fact that frequent and often extensive vasomotor changes are going on more or less constantly in the normal human economy, especially as the result of pain, psychic influence or temperature change, all of which are apt to enter into an ordinary blood-pressure estimation.

Fuerst and Soetbeer ${ }^{1}$ suggest that the Strassburger formula be modified to read:

Pulse pressure

Diastolic pressure $+\frac{1}{3}$ pulse pressure

believing that the pressure variations in the aorta are thus more nearly portrayed.

Applying a table originally devised by Beanus, Erlanger and Hooker indicate the deductions to be drawn as to cardiac activity and vascular resistance from blood-pressure, pulse pressure, and cardiac rate as follows. Since the mean pressure follows the diastolic more closely than the systolic they recommend the former as the indicator of blood-pressure in the table.

\begin{tabular}{l}
\multicolumn{2}{c}{$\begin{array}{c}\text { Determinable factors. } \\
\text { Pulse pressure } \\
\text { Diastolic } \\
\text { pressure. } \\
\text { Constse rate } \\
\text { (velocity of flow). }\end{array}$} \\
Diminished
\end{tabular}

\begin{tabular}{cc}
\multicolumn{2}{c}{ Causative factors. } \\
$\begin{array}{c}\text { Energy from } \\
\text { heart. }\end{array}$ & $\begin{array}{c}\text { Peripheral } \\
\text { resistance. }\end{array}$ \\
Increased & Diminished \\
Diminished & Increased \\
Increased & Increased \\
Increased & Unchanged \\
Unchanged & Increased \\
Diminished & Diminished \\
Unchanged & Diminished \\
Diminished & Unchanged
\end{tabular}

${ }^{1}$ Untersuchungen u. d. Bezichungen zw. Fuellung u. Druck. in d. Aorta, Deut. Arch. f. klin. Med., 1907, vol. xe. 
The table, however, is too diagrammatic to be of value except as an illustration of the general principles involved. For example, cases of nephritic hypertension with increased peripheral resistance and a compensatory increase of cardiac activity show commonly an increase both of diastolic pressure and of pulse pressure $\times$ pulse rate. The table would interpret such an effect upon the blood-pressure, pulse pressure and pulse rate as indicating increased cardiac activity, but unchanged peripheral resistance-an entirely erroneous interpretation. This limitation in the table is of course due to the fact that it deals with changes in the various factors only as to the direction of the change (whether incrcased or diminished), and not as to the degree of the change.

Velden found that if the two foregoing formulas-Strassburger's and that of Erlanger and Hooker-were applied to the same case they often led to diametrically opposite conclusions.

The Energy Index.-Barach ${ }^{\mathrm{i}}$ found that what he terms the energy index (S. D. R. Index) is useful as a clinical estimation of the amount of cardiovascular energy expended, $e . g$.:

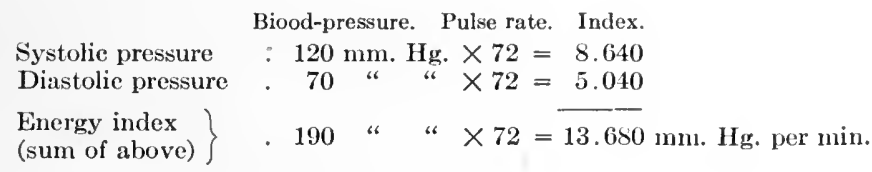

The highest energy index in normal cases is about $20,000 \mathrm{~mm}$. Hg. per minute. In pathologic cases figures as high as 50,000 were encountered. A considerable number of the cases with high indices died of apoplexy.

The Amplitude-frequency Product. - Von Recklinghausen ${ }^{2}$ approaches the question from a different direction, ret reaches conclusions somewhat similar to those of Strassburger. For him the vascular system consists of two reservoirs; the smaller pulsating (arterial) and the larger continuously flowing (venous). The circulation between the two by means of the capillaries is not directly affected by cardiac activity. He assumes that to a certain extent at least the diastolic blood-flow occurs as in a series of standpipes, according to Poiseuille's law. He believes that to a certain degree the diastolic limb of the pulse wave can be shown to be

1 The Energy Index, Jour. Amer. Med. Assn., 1914, lxii, 525.

2 Was wir durch d. Pulsdruckkurve u. d. Pulsdruckamplitude iiber d. grossen Kreislauf erfahren, Arch. f. exp. Patl. 1. Phar., 1906, tvi. 
identical with an ordinary outflow curve. He assumes that the arteries themselves are not actively concerned in the propulsion of the blood-stream. ${ }^{1}$

Now, if we register the pulse wave from a brachial cuff with a tambour manometer at a certain pressure, we shall find the curve indicating a certain relationship between the amount of blood propelled in a given time and the elasticity of the artery. Since it is not always feasible to take a tracing and lay off an absolute sphygmogram, he suggests as a practical method the amplitude (pulse pressure) multiplied by frequency (pulse rate) $=$ seconds volume $\div$ arterial dilatability; in other words amplitude multiplied by rate is proportionate to systolic output divided by distensibility. Thus, if we can be sure that distensibility remains unchanged, then amplitude is a relative measure of systolic output. But how are we to gauge this factor of distensibility? Von Recklinghausen suggests that the following axioms may throw some light on the question:

1. With a constant pressure and with increasing tonus the distensibility of the artery diminishes. With a decrease of tonus, it increases.

2. Increased arterial tension generally goes hand-in-hand with increased vascular tonus. We thus in all probability have a doubly decreased distensibility.

3. In sclerotic arteries the relative distensibility of bloodvessels is diminished.

We must not use the rules of Strassburger's blood quotient too absolutely. It is evident that we can draw no mathematically rigid conclusions from changes of pulse pressure even in the same individual, and much less can we hope to use the data thus obtained in comparison with other individuals. We cannot by any rule convert millimeters of pulse pressure into cubic centimeters of systolic output. It has been shown that in the case of the diseased heart which is manifesting fatigue, the amplitude-frequency product increases less rapidly than in health; it may indeed be entirely lacking. Tiedemann, who compared the amplitude and the amplitude-frequency product in healthy and diseased hearts after the lifting of weights, found that in the former case both of these factors increased, while in the latter they did so to a lessened extent or not

\footnotetext{
1 This belief has been mueh questioned of late, based on the observation of Gruetzner that with an equal amount of pressure applied to an artery, more blood can be foreed out in the direction of normal flow than in the opposite direction.
} 
at all. 'These results were indubitable, but what construction should be put upon them was less clear. Without a knowledge of the state of the vascular tonus no actual figures could be obtained.

\section{METHODS OF ESTIMATING THE RATE OF BLOOD-FLOW. ${ }^{1}$}

While a knowledge of the state of the blood-pressure is often of great value, it gives us no clew as to the rapidity of the circulation - the mass movement of the blood. A high vascular tension may be associated with a small blood-flow and vice versa; for the rapidity of the circulation depends not only upon arterial pressure but also upon the caliber of the vessels between artery and vein, and under some circumstances upon venous pressure. It has been shown that the circulation rate increases in proportion to the oxygen consumption in a manner corresponding to the increase in total ventilation; and it has been suggested that the chief controlling factor of the circulation rate is the hydrogen ion concentration of the arterial blood. ${ }^{2}$

G. N. Stewart ${ }^{3}$ has devised a method of determining the rate of flow which depends on the fact that "the amount of heat produced by" a part like the hand during rest is negligible in comparison with the heat conveyed to it by the arterial blood. If, then, we determine the amount of heat given off by the hand to a calorimeter in a given time, and know the temperature of the incoming (arterial) and of the outgoing (venous) blood, we can calculate how much blood must have passed through the hand in order that it might give off this amount of heat. The quantity of heat given off is estimated by putting the hand into a calorimeter."

"It consists of an inner copper vessel containing a known amount of water (in the experiments usually about three liters), into which the hand is inserted through an orifice of appropriate size and shape in the lid, heat-tight closure being made by the collar of thick felt on the top of the calorimeter. The inner vessel is packed in broken cork in a larger outer vessel and the lid is covered with sheet cork to reduce as far as possible the loss of heat, and to protect the calorimeter against irregular cooling when exposed to draughts. The actual loss is estimated by separate control experi-

\footnotetext{
1 See page 35 .

${ }^{2}$ Boothby, Walter M., A Determination of the Circulation Rate in Man at Rest and at Work, Am. Jour. Physiol., 1915, xxxvii, 383.

${ }^{3}$ Measurement of the Rate of Flow of the Blood in Man, Cleveland Med. Jour., April, 1911, x, 385; Studies on the Circulation in Man, Heart, October, 1911, iii, 33.
} 
ments, and added to the amount of heat given off by the hand as indicated by the rise of temperature in the water. The hand is prepared for insertion into the calorimeter by a preliminary immersion for not less than ten minutes in. a large bath containing water at approximately the same temperature as that in the calorimeter. The temperature of the water in the calorimeter is read on a thermometer permitting hundredths of degrees to be accurately estimated. The calorimeter is mounted on a stand which can be raised or lowered by a screw to permit its use either for a patient sitting in bed or for an ambulatory patient who is able to sit in a high chair. As a general rule the flow in the two hands is simultaneously measured. The quantity of blood in grams flowing through the hand in the time of the experiment is given by the formula:

$$
Q=\frac{H}{T-T^{1}} \times \frac{1}{s}
$$

"Here $Q$ is the quantity of blood, $H$ the heat given off by the hand, $T$ the temperature of the arterial blood, $T^{1}$ the temperature of the renous blood, and $s$ the specific heat of blood. In estimating $H$ the water equivalent of the hand itself (obtained by multiplying its volume by 0.8 ) and the water equivalent of the calorimeter ( 80 grams) must be added to the quantity of heat corresponding to the actually observed rise of temperature. The specific heat of blood is taken at 0.9. Knowing the volume of the hand, we can express the flow in grams per minute per 100 c.c. of hand substance. The volume of the hand is easily estimated by the amount of water which it displaces when immersed in a glass douche-can to the level to which it was inserted in the calorimeter. The douche-can is connected by the tubulure to a burette, on the scale of which the vertical displacement of the water is read off. The amount of water which must be added to that in the can in order to give the same level in the burette is clearly the volume of the hand. The distance to which the hand is to be inserted into the calorimeter is fixed by making a horizontal mark with a pencil at the level of the lower border of the styloid process of the ulna. A parallel mark is drawn above this at a distance equal to the combined thickness of the felt collar and the lid of the calorimeter, and this second mark is just kept in view above the collar during the experiment. The lower mark must then define the limit up to which the hand is 
enclosed in the calorimeter. 'The collar is supported by a flange around the orifice. The temperature of the arterial blood at the wrist is taken as $0.5^{\circ}$ below the rectal temperature, since this was the difference actually found in a normal person. It can be measured by determining that temperature of the calorimeter at which the hand neither gains nor loses heat. Where the rectal temperature cannot be conveniently obtained the mouth temperature is taken as that of the arterial blood at the wrist. The temperature of the venous blood is taken as the average temperature of the calorimeter during the experiment, since direct estimations of the temperature of blood collected by puncture of hand veins, during immersion of the hand in baths at known temperatures, showed that the excess of the temperature of the venous blood over that of the bath was so small as to be negligible for such bath temperatures as are used in the experiments."

The normal average flow as determined by Stewart ranged between 3.5 and 14 grams of blood per 100 c.c. of hand per minute at ordinary room temperature, and with an immersion of ten or fifteen minutes. Individual variations on different days were more or less constant. Elevation of the room temperature increased the flow. Muscular activity of one hand produced an increase of flow in that member over its fellow in proportion of nearly three to one. Individuals with habitually cold hands exhibited a lower rate of flow than normal people. A diminished blood-flow also results from venous stasis produced by moderate constriction of the wrist. The local application of heat increases the rate of flow.

Even a moderate amount of forced breathing decreases blood-flow, in explanation of which Stewart suggests "acapnia caused by the washing out of the carbon dioxid as a possible influence. Mechanical changes in the thorax, and particularly those affecting the filling and discharge of the heart, must also be taken into account. One moral to be drawn is that while the respiratory pump has a part of some consequence in the normal movement of the blood, and may even become the preponderant factor when the heart and the vasomotor mechanisms are crippled, the idea of the "deepbreathing" fanatics that voluntary interference with the delicately regulated respiratory mechanics must be good receives no support, at least so far as the circulation in the periphery is concerned."

Further findings regarding the rate of flow showed a diminished rate in cases of arteriosclerosis of long standing; of brachial neuritis 
with muscular atrophy; and of hemiplegia (associated with complete abolition of vasomotor reflexes on the affected side).

An increased flow was found in early peripheral neuritis on the affected side, probably owing to a paresis of the vasoconstrictor

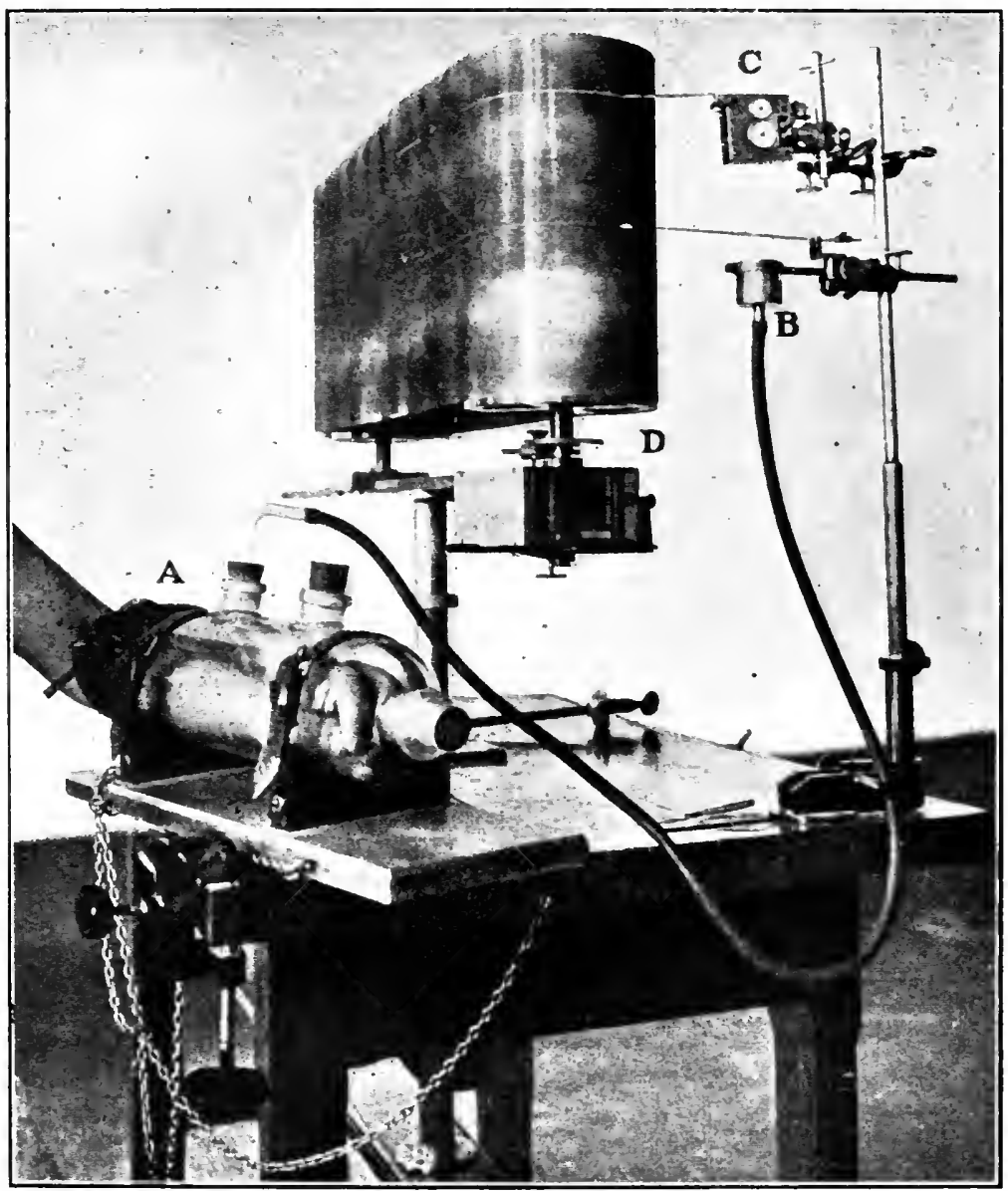

FIG. 73.-The plethysmograph: $A$, cuff; $B$, recording tambour; $C$, chronograph; $D$, drum. (After Hoffmann.)

fibers; in a case of occupation neuralgia; in case of an infected finger (with partial obliteration of vasomotor reflexes); in exophthalmic goitre.

The Plethysmograph.-This instrument gives us information regarding the volume pulse. The volume of all organs varies with 
the different phases of the cardiac cycle with those of the respiration and with vascular tonus, as well as with certain psychic influences, etc.

The Method.-The extremity to be investigated, generally the forearm, is introduced into a glass chamber which is filled with water and made water-tight by means of a rubber cuff. The water contained in the cylinder communicates by means of rubber tubing with a recording piston, the upward or downward displacement of which imparts its fluctuations to a tambour which records its movement on a kymograph. By this means the variations in volume may be studied.

The technique is somewhat complicated, and hence the plethysmograph is hardly likely ever to become a popular instrument for clinical work, but for purposes of scientific investigation, therapeutic effects, etc.,. it possesses a distinct field of usefulness (Fig. 73).

The actual amount of blood in the arm can be estimated as suggested by $O$. Müller, ${ }^{1}$ by rendering the limb bloodless by immersion in mercury, and then applying an Esmarch bandage to the upper arm, the constriction of which is not released until the plethysmographic adjustment has been made. The method is not infallible, however, as the primary anemia tends to the production of a secondary hyperemia. Some interesting results were obtained with the plethysmograph by Weber, who was able to show the effect of psychic stimuli on blood distribution. For instance a concentration of attention upon the arm-the subject being told to imagine he was performing muscular actions with it-is attended by an increased flow to the part.

Müller has constructed the following table showing the effects of different stimuli on blood distribution:

\begin{tabular}{|c|c|c|c|c|c|c|}
\hline & & & Brain. & $\begin{array}{l}\text { Periphery } \\
\text { of head. }\end{array}$ & $\begin{array}{l}\text { Abdominal } \\
\text { organs. }\end{array}$ & $\begin{array}{l}\text { Extremities, } \\
\text { etc. }\end{array}$ \\
\hline Cold & . & . & + & - & + & - \\
\hline Warmth & . & . & - & + & - & + \\
\hline Chloroform & . & . & + & - & + & - \\
\hline Adrenalin & . & . & + & - & - & - \\
\hline Desire . . & . & . & + & + & - & + \\
\hline Disgust & . & . & - & - & + & - \\
\hline Fright. & . & . & . + & - & t & - \\
\hline Mental effort & & . & + & - & + & - \\
\hline Sleep . . & & . & $t$ & + & - & + \\
\hline Imaginary ex & reises & 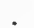 & + & - & - & + \\
\hline
\end{tabular}

${ }^{1}$ Exper. u. kritische Beiträge zur modernen Kreislauf Diagnostik u. ihr weiterer Ausbau durch Einführung des absoluten Plethysphygmogrammes, Verhandl. d. Kong. f. inn. Med. Wiesb., 1907, xxiv, 384. 
A small finger plethysmograph has been devised by Fleischer. ${ }^{1}$ It must be borne in mind that deductions drawn from plethysmographic readings can be applied only to the limb in question, not the system as a whole. Particularly useful data may be obtained if such observations are made simultaneously with blood-pressure observations (Fig. 74).

The plethysmograph gives us data regarding the peripheral circulation but tells us nothing as to the underlying causes which produce them, as to whether variations are due to an abnormal elasticity, or a small arterial bed, and as to whether these changes are normal for the individual or pathologic manifestations. How great a part is taken by vasomotor tone, and whether the stimuli causing these changes arise in the spinal cord or in the peripheral

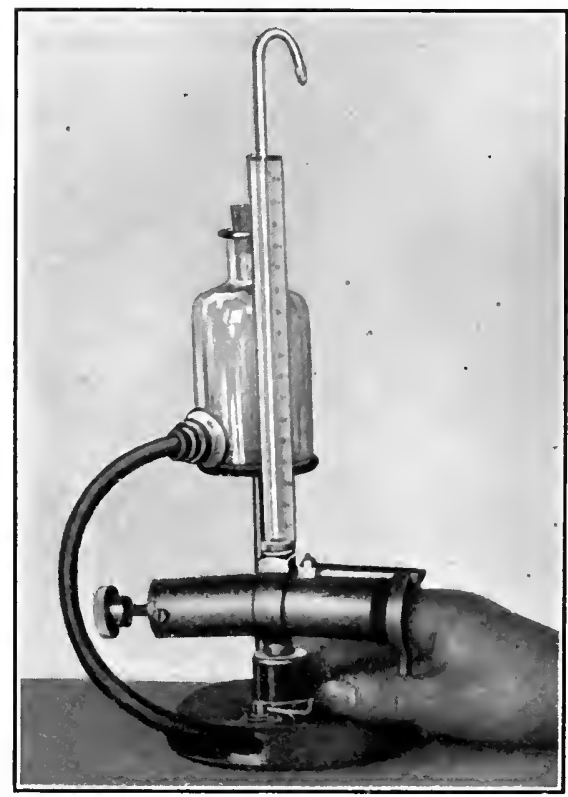

Fig. 74.-The finger plethysmograph. (Fleischer.)

ganglia, or whether the fault lies in the eapillaries or veins, upon these questions we vainly seek for information.

A. Müller ${ }^{2}$ has suggested that systolic output might be ganged by the determination of the venous pressure.

1 Berlin. klin. Woeh., 1908.

${ }^{2}$ Ueber Sehlagvolumen u. Herzarbeit des Menschen, Dent. Arch. f. klin. Med., 1909, xevi, 127. 
The Method.-The circulation in the arm is arrested by the sudden inflation of the brachial cuff from a large bottle containing air under pressure. The venous pressure is now measured by the Basch-Recklinghausen method, which gives us the resistance $(W)$ which the succeeding arterial pressure must overcome. The arm is next placed in a plethysmograph above which another cuff is applied and inflated to about $50 \mathrm{~mm}$. Hg., a pressure well below the minimum pressure and which therefore does not interfere with

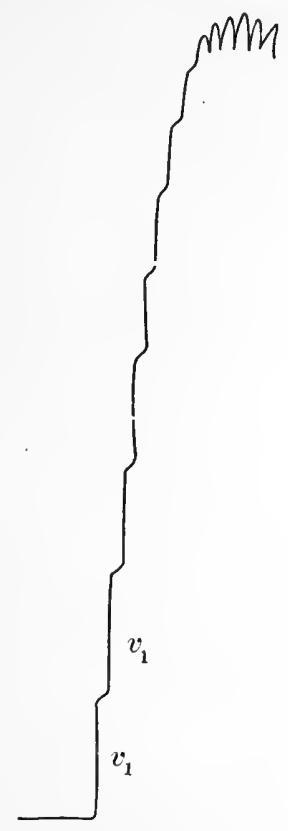

F1G. 75.-Plethysmographic curve used to determine $V_{1}$ (the arterial flow into the arm, against the resistance $W$ ).

arterial inflow, although it does with venous outflow. The pressure in the upper cuff is now suddenly dropped to $O$, the arterial blood flows into the arm but eannot flow out of its vein until the venous pressure rises above that in the cuff $(50 \mathrm{~mm}$.). Until this occurs the arm is in a hydrostatic condition and the resultant sphygmographic curve will be a curve of arterial outflow $(V)$ (Fig. 75).

Under these conditions the pressure in the occluded region will be a measure of the resistance $(W)$ against which the outflowing blood-strcam operates when the obstruction is removed. The 
second cuff which measures the venous pressure does not interfere with the inflow but prevents for a time the onward flow of venous blood. The height of the first rolume pulse indicates the value of $V$. If bodily weight and mean pressure are known, we can calculate $V^{V}$ the systolic output, and $V D$, the cardiac work.

Lsing this method Müller found that the systolic output areraged S.5 c.c. for men and 2.5 c.c. for women. An increase was found in nephritis, cardiovascular disease, exophthalmic goitre, and Addison's disease.

This method has been criticised as theoretically faulty by Christen and the practical results have varied considerably from those of Plesch. ${ }^{1}$

A somewhat similar method of procedure, based on the method of Brodie," has been proposed by. Hewlett and Van Zwaluwenburg, ${ }^{3}$ to determine the rate of blood-flow (Fig. 76).

The arterial blood enters the organ with undiminished speed at first, but soon the flow is retarded by the rise of pressure in the veins and capillaries. The organ therefore swells rapidly at first and progressively more slowly. The earliest portion of this curve represents the rate at which the blood enters under normal conditions. Brodie has shown that this method gives as reliable results as the Stromuhr.

The following method of testing the circulation time has been suggesterl by Bornstein. The patient is made to breathe $\mathrm{CO}_{z}$ and the time is noted at which the respirations become more forcible. This indicates the time taken by the blood to pass through the lung and left heart and to reach the respiratory centre; it is assumed to be equivalent to half the entire circuit. In health the halfcircuit is completed in from twelve to sixteen seconds, much less time being required after muscular effort, deep breathing and the inhalation of nitrite of amyl.

Fellner ${ }^{3}$ believes that the rapidity of flow may be estimated by measuring with a stop-watch the length of time required for the

${ }^{1}$ Hemodynamische Studien, Berlin, 1909.

2 The Determination of the Rate of Blood-flow Through an Organ. Reported at the Seventh International Physiological Congress, August, 1907. (Brodie estimated the blood-fiow in an organ by suddenly oceluding its peripheral vein and measuring the change of volume by means of an oncometer.)

${ }^{3}$ The Rate of Blood-flow in the Arm, Heart, $i, 87$.

- Eine blinische Methode z. Schātzung d. Kreislaufzeit, Münch. med. Woch., 1912, lix, 1456.

${ }^{5}$ Das Pulsometer, ein prakt. Instrument z. Bestimmung d. Stromgeschwindigkeit des Blutes am lebenden Menschen, Deut. med. Woch., 1907, No. 15. 
blood to reach the fingers-travel $50 \mathrm{~cm}$.- -after the release of tension in a pneumatic cuff applied to an arm previously rendered bloodless by bandaging. This method is open to many criticisms. (1) The air escapes from the cuff too gradually: (2) The ressels have been previously emptied and therefore do not offer the natural resistance to the flow. (3) The blood-path from the brachial to the digital arteries is not direct, owing to the branching and steadydiminution in caliber of the arterial tree.

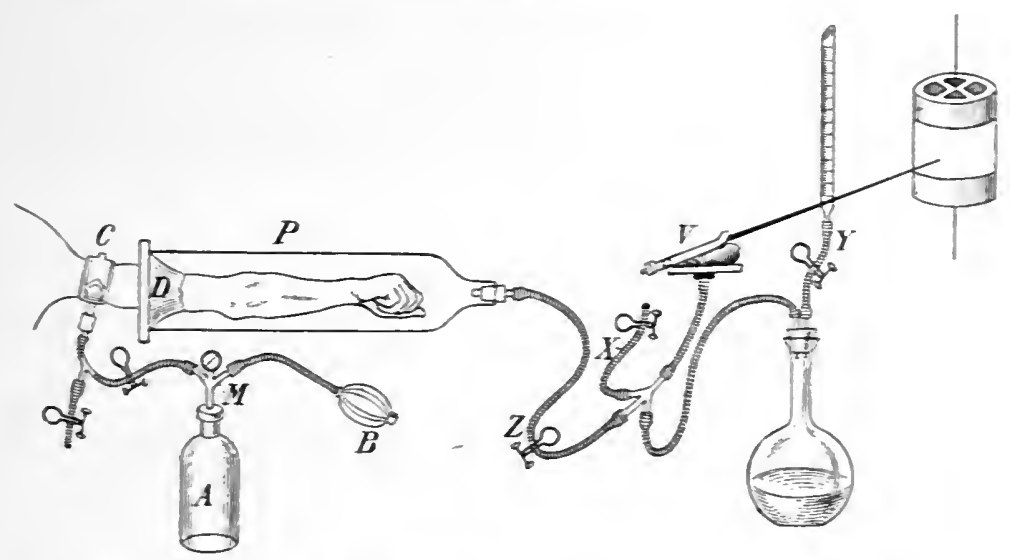

FIG. 76.-Hewlett and Tan Zwaluwenburg's method for estimating the rate of blood-flow. Diagram of apparatus for determining the rate of blood-flow through the arm. The arm is placed in the plethysmograph $P$. the opening of which is closed by a piece of rubber dam $D$, and the connection with the skin made tight with soapsuds. The narrow pressure cuff $C$ is placed around the arm about $3 \mathrm{~cm}$. above the opening into the plethysmograph. The pressure cuff is inflated by opening the stopcock connecting it with the large bottle $A$, in which the pressure has previously been raised by the rubber bulb, $B$. Pressures are read by the spring manometer $\mathbf{M}$. The plethysmograph is connected with the volume recorder $\mathrm{V}$. which writes upon a moving drum. Air can be let out of the system by the stopcock $\mathbf{X}$, and water can be introduced from the burette $Y$, so that the writing-point of the volume recorder can be adjusted at will. The stopcock $Z$ serves to disconnect the plethysmograph from the recording apparatus during adjustments of the former. The recarding apparatus is graduated by allowing 5 c.c. of fluid at a time to flow in from the burette, and marking the elevation of the volume reconder thus produced.

The Tachograph. - The blood-flor in the arm may also be estimated by means of a sensitive flame. Yon Kries's instrument, deviserl for this purpose, is known as the tachograph. " "The forearm is inserted into an air-tight glass crlinder, but in this case no water is introduced. With each șistole of the heart a certain amount of

${ }^{1}$ Ein Neues Verfahren zur Beobachtung der Wellenbewegung des Blutes, Berlin. klin. Woch., 1ssi, p. 559. 
air is forced out of the eylinder through the rubber tubing into a specially devised gasoline burner. The expelled air causes the

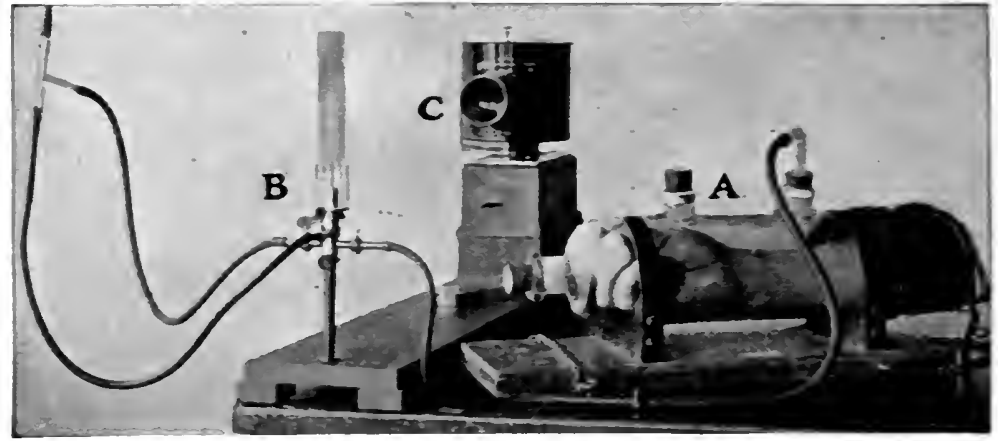

Frg. 77.-The tachograph of von Kries: $A$, cuff; $B$, sensitive flame; $C$, camera.

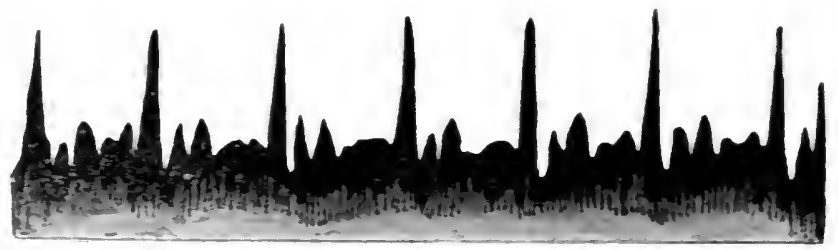

FIG. ス.-Tachogram after von Kries.

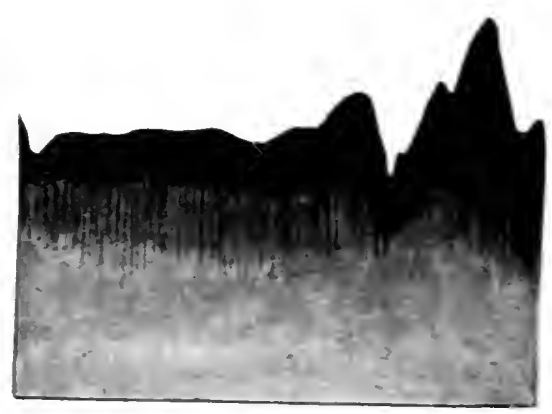

$a$

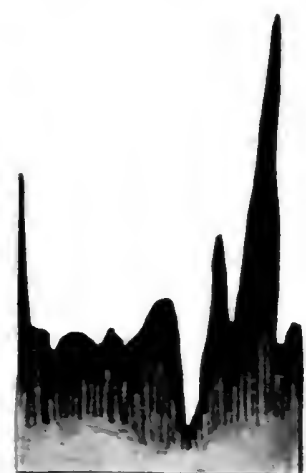

$b$

Fig. 79.- $a$, central tachogram, reading from right to left. $a$, at rest; $b$. after exercise. (Mûller and Veiel.)

gasoline flame to flare upward to a height proportional to the amount of air displaced. This flame is in turn photographed upon a revolving strip of paper sensitized with bromide of silver. When the 
tracing is developed it presents a succession of zigzags at first glance resembling the results of the electrocardiogram" (Fig. 77).

When the arm increases in volume during the ventricular systole, the enclosed air, meeting with no resistance to outflow through the tubing will emerge the more rapidly and suddenly the more quickly the blood is forced into the arm (Figs. 78, 79, and 80).

The Estimation of Resistance in the Large Vessels.-Bröcking ${ }^{1}$ made brachial blood-pressure observations in the following postures: $A$, recumbent; $B$, sitting in bed with the legs extended; $C$, sitting with the legs dependent; $D$, standing.

Normally, the pressure is highest in $B$, which he explains as due to a compensatory peripheral contraction instituted to prevent

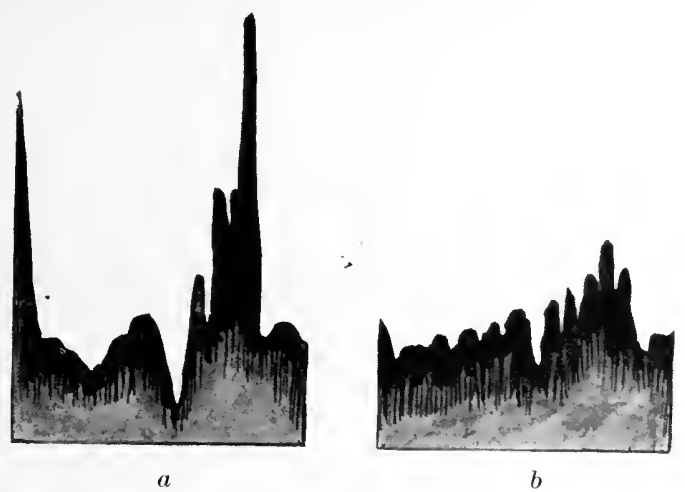

FiG. 80.- $a$, central tachogram in aortic insufficiency; $b$, peripheral tachogram in aortic obstruction.

splanchnic anemia. It is further increased by pressing the legs against the abdomen. If there be circulatory weakness, the maximum pressure in $B$ is lower than in $A$, showing a loss of peripheral arterial power.

The Estimation of Arterial Functionation.-O. Müller employs the following method: The arm is introduced into a plethysmograph and ice is applied to the arm above the instrument. Normal arteries show a distinct diminution, and, upon withdrawal of the cold, an increase in volume. In sclerotic arteries these changes are in part or entirely lacking. This test, although for the most part corroborated by other investigators, has also been criticised.

1 Ein Beitrag zur Funktionsprüfung der Arterien, Zeit. f, exp. path. u. Ther., 1907, iv, Pt. I. 
Cold contracts and heat dilates the arteries of healthy people but the psychic state also plays a considerable part. It is therefore necessary to arrange plethysmographic examinations in such a manner that thermic and psychic influences shall not be separated. Even if after repeated trials the vessels do not react one cannot on that account diagnosticate an anatomic lesion. One cannot conclude that a man is arteriosclerotic because his vessels do not react to ice, because many organic cardiac maladies do not give this response, but merely that his arterial functionation is not normal. In the majority of individuals hardened arteries are hyporeactional, or even areactional to ice, although some of them are hyperreactional. ${ }^{1}$

The Estimation of Vascular Tonus.-De Vries-Reilingh² suggests the following method for determining the degree of arterial tonus:

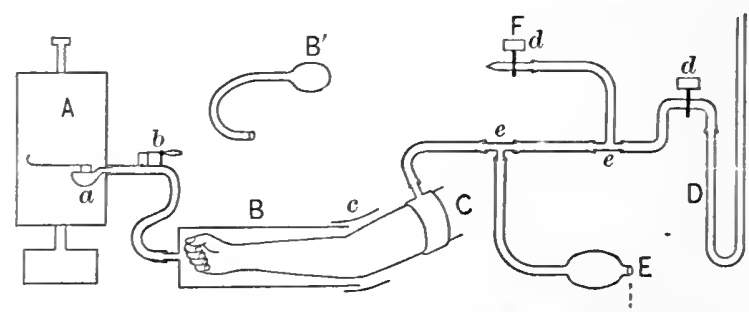

FIG. $81 .-A$, kymograph; $B$, plethysmograph of Mosso; $B^{\prime}$, plethysmograph of Wiersma; $C$, cuff; $D$, manometer; $E$, pump; $F$, escapement; $a$, tambour with pen; $b$, escapement; $c$, occlusive rubber cuff; $d$, stopeock; $e$, three-way glass tubing.

A small cuff is applied to the arm while the forearm is placed in a plethysmograph. Pressure is suddenly raised above the systolic pressure in the upper cuff. The tambour of the plethysmograph now registers a straight line; but as soon as pressure in the cuft falls sufficiently to allow blood to pass through, the tambour will rise. The pressure at this point is the maximum arterial pressure plus the arterial tonus. If pressure is gradually lowered a further rise of the tambour due to venous stagnation will occur. But in time a point will be reached at which the tambour falls because the cuff pressure has become less than the venous pressure. The difference in pressure between the first elevation and the first fall of the tambour is taken as the index of vascular resistance. This

${ }^{1}$ Romberg, V., and Müller, O., Ueber d. Bedeutung u. Technik d. Plethysphygmographischen Funktionsprüfung gesunder u. kranken Arterien, Zeitschr. f. klin. Med., 1912, $\mathrm{kxv}, 92$.

${ }^{2}$ Zur Blutdruckmessung, Zeit. f. klin. Med., 1913, lxxvii, 67. 
factor was found to range between 17 and $32 \mathrm{~mm}$. IIg., the normal average being $18 \mathrm{~mm}$. A source of error lies in the fact that during this prolonged procedure for some reason a general increase of pressure occurs. This rise of pressure renders an. estimation of the minimal pressure unreliable, and while such a rise may be obviated by placing the cuff at the distal end of the forearm, a new source of error is thus introduced by the protected position of the arteries at this point. The results would probably also vary according to the rate at which the cuff pressure was lowered (Fig. 81).

The Estimation of Capillary Blood-pressure.-Various and for the most part unsatisfactory methods of measuring capillary pressure have been suggested (see page 140). We have had no personal experience with the determination of the capillary tension, but it is manifest that if a method can be devised which is both accurate and clinically applicable much valuable information will be obtained. Simultaneous observations on the relationship between the arterial and the venous pressures yield more important data regarding the state of the circulation than either alone, since the arterial represents the beginning and the venous the end of the greater circulation.

Combined Observations.-A more accurate though clinically inapplicable method of functional diagnosis originally described by Marey and lately rejuvenated by $\mathrm{O}$. Müller, Romberg, and F. Kraus, consists of:

1. Prolonged continuous observation of the systolic and diastolic pressures.

2. Continuous tachographic observation undertaken simultaneously with the foregoing.

If both pressure and rate increase it is assumed that we are dealing with an increased systolic output; if the two values move in opposite directions, that an alteration of peripheral resistance in the arm under observation has occurred.

3. Plethysmographic observations to determine vascular changes in the arm.

But even this method does not permit us to draw deductions which can be expressed in actual figures.

The Sphygmobolometer.-This instrument devised by Sahli ${ }^{1}$ is used to estimate the energy of individual pulse waves, and thus indirectly the energy of cardiac systole. 
The Principle.- The pulse wave is made to transmit its force to a measured quantity of air enclosed in a cuff $(A)$ encircling the forearm and communicating with a manometer. The pressure in the cuff is raised until the maximum oscillation occurs in the oscillator $(d)$, this point being known as the optimum pressure.

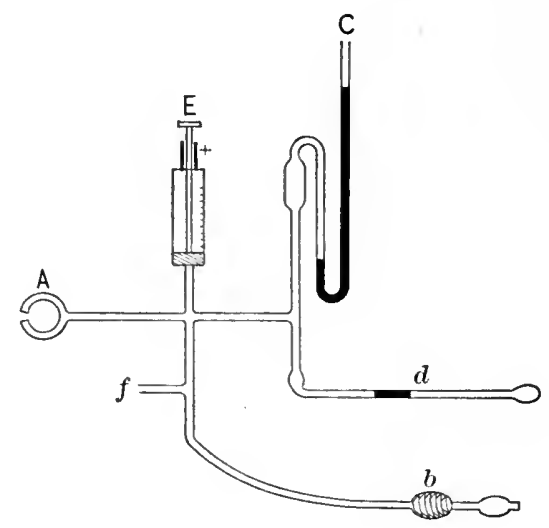

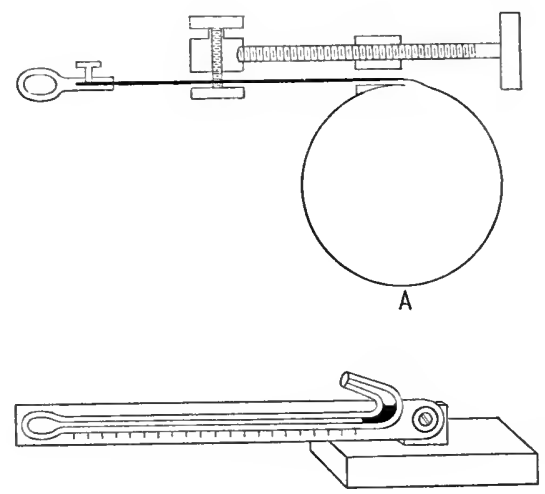

E

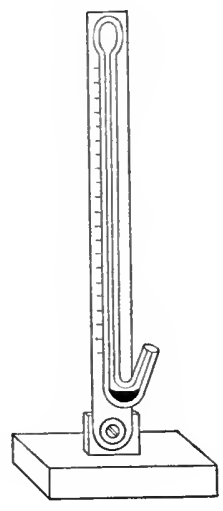

E

Fig. 82.-Schematic diagram of Sahli's sphygmobolometer: $A$, cuff; $b$, inflating bulb; $C$, mercurial manometer; $E$, graduated piston pump; $d$, index manometer. Lower diagrans: $A$, lateral view of the stecl cuff; $E$, index manometer in horizontal and erect position.

This is accomplished through the use of a steel outer cuff, with which tension can be very exactly regulated and the precise point noted at which the maximum oscillation is overstepped.

The energy of that portion of the aortic wave which reaches the brachial artery is expended in three directions: (1) That which 
passes the cuff. (2) That which is reflected backward toward the heart. (3) That which compresses the cuff. Manifestly only the latter portion can be measured, thus ever leaving two undeterminable factors.

By observing the actual height of the manometric pressure whieh corresponds to the optimum pressure, by noting the extent of oscillation of the latter, and knowing the volume of the enclosed air we are enabled to construct a formula which represents the amount of energy expended. The volume of air is measured by means of a graduated piston syringe $(E)$ with a capacity of 200 c.e. The cubic capacity of the index manometer is determined for each individual instrument, the lumen being calibrated in such a manner that each centimeter of length is equivalent to 0.02 c.c. The instrument is based upon Boyle's (Mariotte's) law that if temperature remains constant the volume of gas will vary inversely as the pressure, whence follows the formula:

$$
W=V\left(\begin{array}{c}
B \\
B+P
\end{array}\right) \quad\left(\frac{P}{B+P}\right)(\triangle \mathrm{p}) 13.6
$$

$\mathrm{W}=$ work performed by each pulse wave.

$V=$ volume of air.

$(\triangle p)=$ maximum oscillation reduced to $\mathrm{cm} . \mathrm{Hg}$.

$B=$ mean barometric pressure $=76.4$ (New York).

$P=$ pressure in $\mathrm{cm} . \mathrm{Hg}$. at maximum excursion of index manometer.

$$
F=\left(\frac{B}{B+P}\right)\left(\frac{P}{B+P}\right) 13.6
$$

P. F.

50.78

$6 \quad 0.92$

$7 \quad 1.04$

$8 \quad 1.16$

$9 \quad 1.28$

$10 \quad 1.39$

$11 \quad 1.49$

$12 \quad 1.59$

$13 \quad 1.69$

$14 \quad 1.78$

$15 \quad 1.87$

$16 \quad 1.95$

$17 \quad 2.02$

$18 \quad 2.10$

$19 \quad 2.17$

$20 \quad 2.23$

$21 \quad 2.30$

$22 \quad 2.36$

$23 \quad 2.42$ 
A table can be worked out so that each estimation requires but one multiplication: $(\triangle p) \times(F \times V)$.

The accuracy of any single determination depends mainly on the reading of $(\Delta p)$, errors in the other terms being negligible in comparison. Thi sinvolves a reading of the excursions of the kerosene index and a calibration of these excursions in terms of mercury. Computations show that under normal conditions results obtained by an experienced observer can be relied on to better than 10 per cent. (Potter). ${ }^{1}$

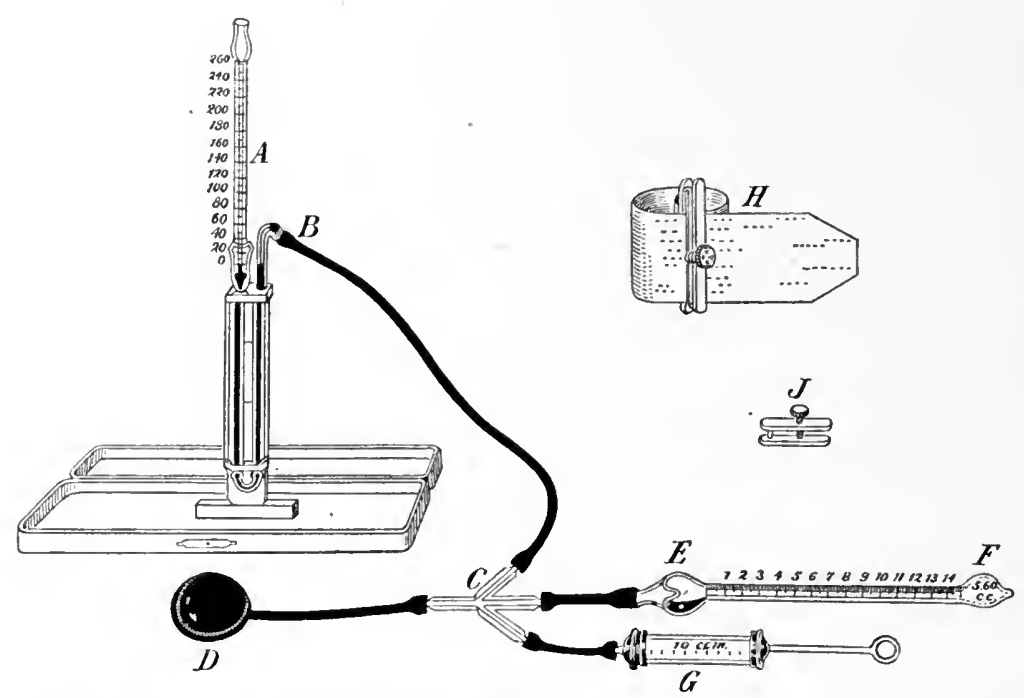

Fic. 83. - The sphygmobolometer. The rubber bag $D$ is applied over the radial artery by the cuff $H$ and conneets by means of tubing with the (1) 10 e.e. syringe $G$ and (2) with the index manometer $E F$, which contains colored kerosene and (3) with the vertical mercury manometer $A$. (Sahli.)

"The value of $B$ in a given locality may be assumed to be constant, as may also the volume $r$ for a given instrument. A similar table would have to be worked out for each individual instrument.

$$
W=F \times V \times(\triangle p)
$$

"The total work of the pulse per minute is obtained by multiplying the work of a single beat by the pulse rate. We are of course not measuring the entire energy of the heart but only that fraction

\footnotetext{
1 Jour. Amer. Med. Assn., A pril 19, 1913, p. 1211.
} 
of it which is consumed in furnishing the pulse wave in the brachial cuff-this Sahli believes to be a constant fraction."

The most recent modification of sphygmobolometry ${ }^{2}$ consists in the substitution of a Jacquet sphygmograph equipped with a spiral spring in place of a pneumatic cuff. The coefficient of elasticity of the spring is practically constant for different pressures, hence it is possible to take the arithmetic mean of the beginning and end tension of the pressure. In order to translate the spring tension into grams the apparatus is equipped with a device to draw upon the smoked paper nine parallel abscissæ. For each of these abscissæ, the pressure value of the spring is established in grams and tabulated. These abscissæ indicate the height of the pelotte plus the magnification due to the tambour. Thus it is possible to take tracings of different degrees of spring pressure, choosing those as our criteria which yield the largest work product. Needless to say, a sphygmograph must be applied with accuracy and care.

Dynamic Diagrams.-T. F. Christen suggests the use of "dynamic diagrams"3 as an index of cardiac work, a conception which he bases on the following hypothesis:

The Theory.-The old-fashioned palpation of the pulse tells us much more about the condition of the circulation than does the sphygmogram, in spite of the latter's seemingly higher scientific character. In the clinical sphygmogram there is no exact relation between the ordinates and the pressures. We do not even know where to trace the level of the pressure zero. Even if there were such a relation, sphygmography would still not be a dynamic method, as in dynamics we do not have to study alone the temporal variations of the forces, but essentially the effect of these forces. These two statements sufficiently explain the failure of the clinical sphygmogram. There are two dynamic diagrams of the pulse that may be determined in a mathematically exact manner. They are the

\footnotetext{
${ }^{1}$ For more detailed discussion see Sahli, Deutsch. med. Woch., 1907, No. 16, p. 628; No. 17, p. 665. Sahli, Diagnostic Methods, edited by Potter, W. B. Saunders Company, Philadelphia, 2d Ed. Sahli, Deutsch. med. Woch., 1910, No. 47, p. 2181. Christen, Zeitsch. f. klin. Med., 1910, lxxi, 390. Sahli, Zeitsch. f. klin. Med., 1911, lxxii, 1. Sahli, Zeitsch. f. klin. Med., 1911, lxxii, 214. Christen, Zeitsch. f. klin. Med., 1911, lxxiii, 55. Christen, Deutsch. med. Woch., 1911, No. 14, p. 644. Christen, Cor. Bl. f. sehweiz. Aerzte, 1911, p. 562. Sahli, Zeitsch. f. klin. Med., 1912, lxxiv, 230. Christen, Zeitsch. f. klin. Med., 1912, lxxiv, 447.

${ }^{2}$ Lipowetsky, L., Sphygmobolometrische Untersuchungen an Gesunden und Kranken mittels des Sahlischen sphygmobolographischen Verfahrens, Deutsch. Arch. f. klin. Med., 1913, cix, S. 498-514.

${ }^{3}$ Die neuen Methoden d. Dynamisehen Pulsdiaguostik, Zeit. f. klin. Med., 1911, Ixxviii, 55.
} 
graphic expression of clinical experiments based upon the following two questions: (a) What is the systolic increase of volume of the arteries covered by a pneumatic cuff by a given pressure? What is the amount of mechanical energy required for this movement? These dynamic diagrams, called stasis curves, are characteristic of the behavior of the pulse against a stasis pressure. They depend on the volume of air within the cuff as little as they do on the elasticity of the cuff and the soft parts. They depend only on the breadth of the cuff. We, therefore, have to compare stasis curves derived from experiments with cuffs of the same breadth. The exclusive consideration of pressure and its temporal variations will never permit us to solve the question of diagnosis of the pulse, for the mechanics of the pulse contain essentially dynamic problems more than any other province of physiology. The stasis curves (dynamic diagrams) replace the palpation of the pulse by an exact method, because they answer the same questions that palpation does, informing us concerning the "filling" and the "intensity" of the pulse. The method depends on the estimation of the force necessary to produce the oscillations which are observed in the determination of the pulse-pressure. This variation in the volume of the artery does not depend on the volume of air within the cuff, nor on the elasticity of the system. It can depend only on pressure exerted by the inflated cuff and on the dynamic qualities of the pulse.

The Method.-In order to find the value of the systolic increase of volume, $i$. $e$., the volume of blood the pushing forth of which against the pressure of the cuff produces the oscillations observed on the manometer, we make use of a syringe whose piston has to be pushed in up to the point where the oscillations have been displaced about once their own amplitude.

Example.-We observe on the manometer oscillations between the limits 170 and 176. (The unit of the manometer is $\frac{\text { gr. }}{\mathrm{CM}^{2}}$.) Pushing in the piston of the syringe we increase the pressure within the cuff, elevating both limits of these oscillations. We do this in such a manner as just to reach the point where 176 has become the lower limit of the oscillations, having been heretofore its upper limit. Thus we are sure that the volume of the piston, which is read off on its own scale, must be that incompressible volume which brought under the cuff increases the pressure within it from 170 to 176 . Two incompressible volumes, which, forced into the 
same air-chamber produce the same increase of pressure within it, must be equal. 'Therefore, the volume read off and the piston must be equal to the systolic increase of volume of the artery (or arteries) that is covered by the inflated cuff. Suppose that we

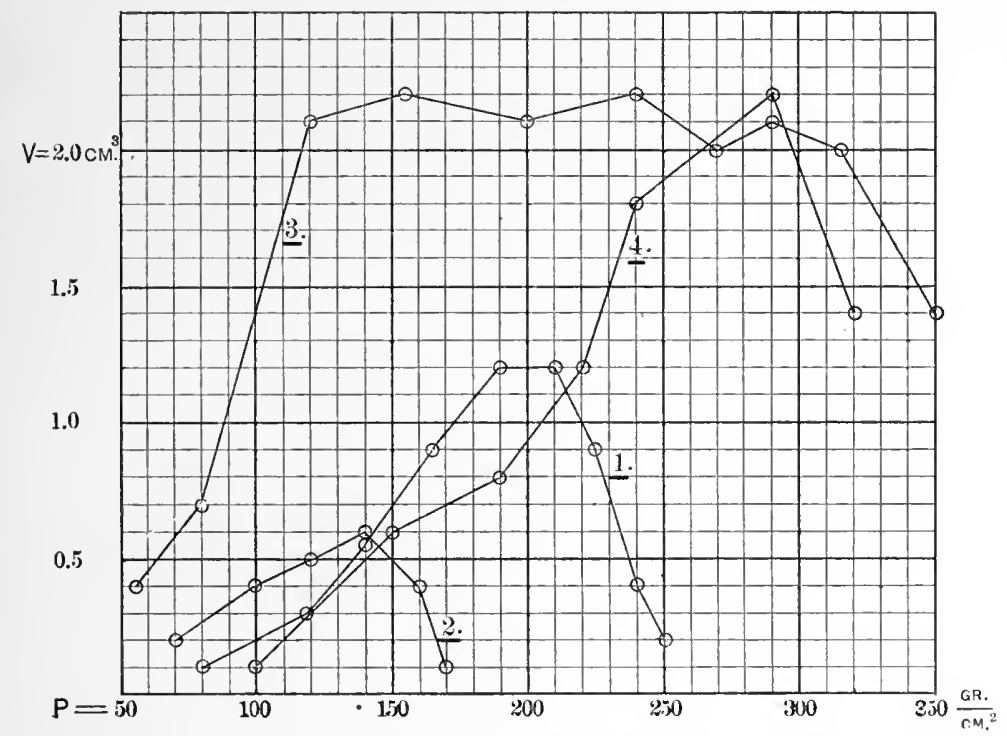

Fia. 84.-Dynamie diagrams. Curve of volume: 1, that of health; 2, of myocarditis; 3 , of aortic insufficiency; 4, of arteriosclerosis. In cases of forcille pulsation-arteriosclerosis, aortic insufficieney-the volumes may exceed $2 \mathrm{~cm}$. and the energy $600 \mathrm{gr}$. $\mathrm{cm}$. Furthermore, the character of the summit is quite different from that of arteriosclerosis.

read a volume of $0.7 \mathrm{~cm}$., then the mechanical energy required for the same increase of volume-the main pressure being:

$173 \frac{\mathrm{gr} .}{\mathrm{CM}^{2}}-$ must have the value $173 \frac{\mathrm{gr} .}{\mathrm{CM}^{2}} \times 0.7 \mathrm{~cm}^{3}=121 \mathrm{gr.} \mathrm{cm}$.

In this way we find for every pressure $(P)$ a volume $(V)$ and an energy $(E)$, the relation between which is $E=P V$. Repeating this experiment at different pressures we get a series of pressure volumes and energies which we arrange in the following manner: 


\section{EXAMPLE.}

$\begin{array}{ccc}\text { P'ressure. } & \text { Volume. } & \text { Enerky. } \\ 80 & 0.1 & 8 \\ 120 & 0.3 & 36 \\ 140 & 0.55 & 77 \\ 165 & 0.9 & 149 \\ 190 & 1.2 & 228 \\ 210 & 1.2 & 252 \\ 225 & 0.9 & 203 \\ 240 & 0.4 & 96 \\ 250 & 0.2 & 50\end{array}$

Diagrams.-In order to give a clearer idea of the relation between these quantities, viz., pressures, volume, and energy, we plot two curves which represent the volumes or the energies as functions

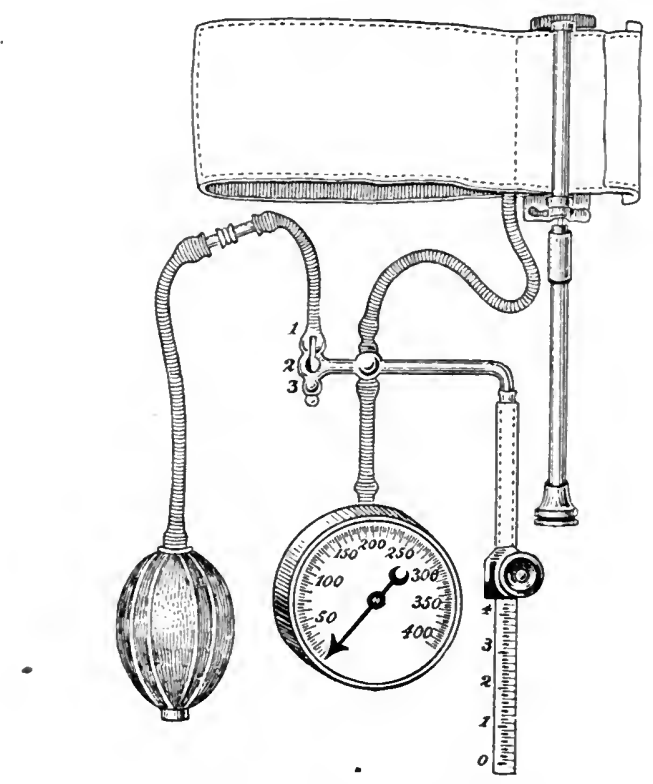

FIG. 85.-Christen's energometer.

of the pressure. Therefore, in our graphic method we have to plot the pressure as abscissæe and the volumes or the energies as ordinates. The author employed the leg instead of the arm for these determinations, because he found that in cases of hypertension disagreeable sensations and even pain may be produced, an occurrenee which it is said can be avoided when the euff is fastened around the calf (Fig. 8t). 
We have had no experience with Christen's method. 'The author claims that the results obtained by it when applied in studying the effect of work, rest, medication, etc., are satisfactory. It is essential that the variations of the summit-a rise or a fall, a shifting to a higher or lower level, a sharpening or flattening of the curve (Fig. 84) be expressed in absolute values-the pressure in $\frac{\mathrm{gr}}{\mathrm{CM}^{2}}$, the volume in $\mathrm{cm}$. and the energy in $\mathrm{gr} . \mathrm{cm} .{ }^{1}$

Extreme care in technique is absolutely essential. The readings must be taken with the patient as well as the patient's arm in the same position. The cuff must be applied to exactly the same region of the arm, which should be marked, and needless to say the same arm must be used for successive observations (Dunkan). Leg readings are inaccurate. The energy of the pulse wave even in

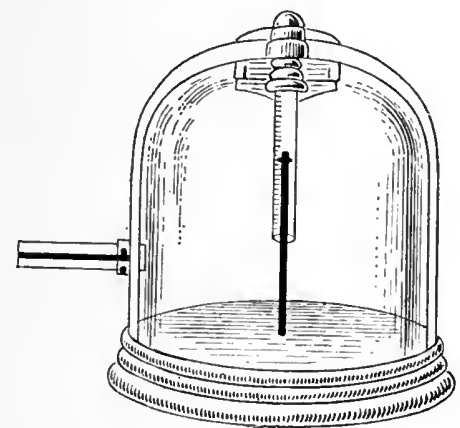

FIG. 86.-Engelen's instrument for the determination of intra-abdominal pressure.

normal subjects is variable, but there are limits which are not normally exceeded. Muscular exercise, and cardiac hypertrophy with good compensation show high readings. Adrenalin injections cause high values independently of any effect on blood-pressure (Drouven).

The Determination of Intra-abdominal Pressure.-Blood-pressure may be considerably influenced by intra-abdominal pressure, on which account it is sometimes desirable to know what the height of the latter pressure is. This may be determined (1), as suggested by Moritz, by introducing into the stomach a tube into which a thin rubber window has been inserted, the tube being connected

\footnotetext{
${ }^{1}$ Christeu's energometer, manufactured by Hausmann Instrument Company, St. Gallen, Switzerland.
} 
with a monometer. (2) By means of a special instrument devised by Engelen ${ }^{1}$ which consists of a large glass bell, the bottom of which is sealed with thin rubber dam. The latter will project either inward or outward, as the pressure relations on either side of the membrane may vary.

The instrument is placed upon the abdominal wall, connected with a manometer, and sufficient air is pumped into the bell to equalize the pressure on each side of the membrane in the interior of the bell and the outer abdominal wall-this being shown by an indicator.

Deep insiration may cause a pressure of $-57 \mathrm{~mm}$. Hg.; forced expiration, $+87 \mathrm{~mm}$. During normal parturition a pressure of 40 $\mathrm{mm}$. is usual which with dystocia may rise as high as $+125 \mathrm{~mm}$.

(3) Variations in abdominal pressure may also be determined by inserting a rubber bag (cylinder in casts $7 \mathrm{~cm}$. by $16 \mathrm{~cm}$.) into the rectum, which when inflated with air is connected with a Marey tambour. ${ }^{2}$ The normal intra-abdominal pressure is above that of the atmospheric pressure. It fluctuates with the respiratory excursions of the thorax and the diaphragm. Anything which diminishes the tone of these structures causes a lowering of abdominal pressure. Distention of the abdomen by fluid or gas increases the pressure and this is in turn followed by a fall of blood-pressure due to a diminished venous inflow, and consequently a decreased left ventricular sustolic output ${ }^{3}$ (see page 41 ).

\footnotetext{
${ }^{1}$ Ueber Messung u. Bedeutung des Abdominaldruckes, Deut. med. Woch., 1911, No. 19.

2 Weber, E., Ueber eine neue Methode zur Untersuchung der Druckschwankungen in der Bauch Höhle, Zentralbl. f. Physiol., Leipsic u. Wien, 1906, xx, 329-336.

${ }^{3}$ Emerson, H., Intra-abdominal Pressure, Arch. Int. Med., 1911, vii, 354.
} 


\section{AR'TERIAL HYPOTENSION.}

\section{ESSENTIAL HYPOTENSION (CONSTITUTIONAL LOW ARTERIAL PRESSURE).}

Arterial hypotension, by which we understand a systolic pressure of or below $115 \mathrm{~mm}$. Hg., generally in association with a diastolic pressure below $80 \mathrm{~mm}$., is a very common condition. Hypotension may occur after hemorrhage, in acute infectious diseases, in conditions of malnutrition or cachexia, such as tuberculosis, carcinoma, Addison's disease. It may also occur in anemia, in paresis, in osteo-arthritis, in cardiac dilatation, tachycardia and in cardiac decompensation. Two renal conditions-cyclic and orthostatic albuminuria; and amyloid disease of the kidney are often associated with low blood-pressure. It may also occur as a result of intoxication-anaphylaxis, nicotinism, alcoholism. Hypotension also occurs in surgical shock; as a result of some forms of anesthesia, especially that of chloroform, and as a terminal event during the last ebbing time of life.

Relative hypotension often follows hypertension when vascular and cardiac resources are overtaxed.

Arterial hypotension may occur in: (1) Acute infectious disease. (2) Chronic malnutrition, with or without toxemia. (3) Certain individuals in whom the condition or a marked tendency toward it appears to be a part of their constitutional make up. (4) In association with abnormalities of the endocrine functions of the male gonads.

Symptomatology.-Essential hypotension is a very common condition, the importance of which has been signally emphasized by L. F. Bishop. Many apparently healthy individuals, although not robust, often in youth and early adult life normally have a systolic and a diastolic blood-pressure ranging between 115 and 100, and between 80 and $70 \mathrm{~mm}$. respectively. Such individuals lack stamina, tire easily, complain of cold extremities and of the inability to do prolonged mental or physical work. They often suffer from 
dyspepsia, which upon investigation proves to be of the "nervous" variety, not infrequently due to hypomotility and superimposed on an anatomical background of partial or total splanchnoptosis. 'They are not actually ill, yet they are rarely well; they complain of all sorts of symptoms, headache, lassitude, mental depression, "gouty" pains, constipation, etc. Their bodily temperature is generally subnormal in the morning and they suffer from cold extremities due to a sluggish peripheral circulation. They are apt to be filed away in that much overloaded diagnostic pigeon-hole labeled "neurasthenia." They are often of the high-strung nervous temperament, "go on their nerves," are moody and emotional, and sensitive to caffein and nicotine, as well as to atmospheric and barometric fluctuations. Staehelin ${ }^{1}$ found that a fall of barometric pressure produces a lowering of the arterial tension in many individuals. In extreme cases they often fall asleep when sitting upright and suffer from insomnia when they go to bed. Perhaps enough has been said to illustrate the picture, although pages might be written upon the symptomatology.

The inability of the subjects of constitutional hypotension to stand the demands of prolonged physical exertion is the direct result of their low blood-pressure. Recent experimental evidence has shown that an increased arterial pressure such as occurs normally during exercise increases the height of muscular contraction when the pressure is below a certain critical level, whereas a decreased pressure below this point has the opposite effect. An increased flow flushes out and neutralizes the fatigue products. ${ }^{2}$

Observations over a period of two years in a case of constitutional low tension by Edgecombe ${ }^{3}$ showed that pressure was higher in the evenings than in the mornings. No relationship could be established between pressure and pulse rate. Exercise, mental effort, grief, altitude, tea, coffee, and tobacco had a pressure-increasing effect. Constipation lowered the tension. Therapeutically, caffein and pituitary substance raised, whereas alcohol lowered the pressure. Strychnin, digitalis, calcium, and glycogen were without effect.

While it is unsafe to generalize from a single case, the results reported correspond quite closely to what is usually observed in this class of cases.

${ }^{1}$ Ueber d. Einfluss d. täglichen Luftdruckschwankungen auf den Blutdruck, Med. Klinik, 1913, ix, 862.

${ }^{2}$ Gruber, C. M., Studies in Fatigue, Amer. Jour. Physsiol., 1913, xxxii, 221.

${ }^{3}$ Royal Soc. Med., Med. Sect., February 2S, 1911. 
Patients with essential hypotension not only have an habitually low blood-pressure, but their response to different stimuli which normally affect blood-pressure cither locally or generally is either quantitatively or qualitatively abnormal. The effect of a change of posture from the recumbent to the erect position is normally a variable one, but the maximum and minimum pressures invariably approach each other (Erlanger). The pulse rate decreases, the pulse pressure falls. These changes are due to the effect of gravity. The pressure in the femoral artery is higher than that in the carotid in proportion to the difference of the hydrostatic pressure of the column of blood which separates the two points of measurement.

$\begin{array}{lccccc}\text { Posture. } & & \text { Brachial. } & \text { Tibial. } & \text { Difference. } & \begin{array}{c}\text { Difference in height } \\ \text { of column separating } \\ \text { armlets. }\end{array} \\ \text { Horizontal . . } & . & 140 & 138 & 2 & 0 \\ \text { Standing . . } & 136 & 204 & 68 & 68.5 \\ \text { L. post., legs up . } & . & 122 & 76 & 46 & 46.1 \\ \text { Vertical, head down } & 148 & 70 & 78 & 77.7\end{array}$

If normal postural change in pulse rate ( 6 to 10 beats) does not occur, it shows too much; if exaggerated, too little splanchnic tone. If the response is reversed there is apt to be extensive disease of the cardiovascular system-generally hypertension. In healthy individuals the transition from the recumbent to the erect posture generally produces a slight fall of the systolic and a rise of diastolic pressure, thus a diminution of pulse pressure. In people with relaxed abdominal walls and enteroptosis a marked falling of the systolic pressure $(25 \mathrm{~mm}$.) and a slight lowering of the diastolic pressure have been noted. ${ }^{1}$ (See Crampton's test, p. 146). A lowered splanchnic tone leads to more or less cerebral anemia, and a sudden assumption of the erect posture when the individual has been recumbent or stooping to the ground with the knees flexed often causes a temporary vertigo. Syncopal attacks from relatively insufficient causes are in case of the female sex, at least, by no means rare. An engorged condition of the viscera is often evident on inspection during abdominal operations. The vascular supply especially in the veins is excessive. Ptosis of the right kidney not only tends to constrict its own vascular supply, but by dragging upon the inferior vena eava and the aorta may seriously disturb the lumen of these vessels, contributing in no small

${ }^{3}$ Birteh, Fayette W., and Inman, Thos. G., Blood-pressure Observations on Patients with Relaxed Abdominal Musculature, Jour. Amer. Med. Assoc., 1912, Iviii, S. 265-268. 
measure to vascular stasis. ${ }^{1}$ 'These results are noted clinically by the large gush of urine which often follows a short period of recumbency. (See Arterial Functionation, p. 173.)

Another frequent symptom resulting from low blood-pressure is headache, relief from which is too often sought in headache powders, which of course eventually aggravate the condition. Elevation of blood-pressure is followed by improvement.

Etiology.- "In the upright posture the blood tends to settle in the capacious channels of the splanchnic vascular bed. The 'head-up' position in four-footed animals may lead to extraordinary lowering of blood-pressure in the carotid arteries and presumably to critical anemia of the brain. But in man there has been developed a compensatory reaction, undoubtedly chiefly vasomotor, by means of which the vessels of the dependent parts of the body, probably the arteries of the splanchnic area, contract under stimulus of the upright posture and maintain a fairly uniform distribution of the blood-mass.

"No physiologic coördination can be conceived that is more important than this to the welfare of the organism as a whole. It is to be expected, therefore, on a priori grounds, that minor compensations having the same general purpose should here and there disclose themselves. When the radial pulse fails in a wrist which is raised above the level of the head, the result may be due either to passive drainage of the blood from the artery, followed by a purely loeal contraction of the arterial wall, or to active vasomotor contraction of the vessel under the stimulus of gravity. As touching the former view, Bayliss has shown that peripheral arteries freed from their nervous connections respond to variations of internal pressure by contracting as the pressure rises and dilating as it falls. Sifting the great number of observations bearing on this subject, the latter explanation seems to be the only possible one. In other words, according to this view pulse failure in the elevated radial artery is a sign of vasomotor activity and belongs to the series of physiological compensations. It is comprehensible as such, if we presume that contraction of the radial arteries helps to maintain blood-pressure in more vital tissues by diverting the blood-stream from less sensitive organs. It has often been observed that pulse failure which is obvious in the elevated wrist at the beginning of a series of observations is not evident later on. That

${ }^{1}$ Maekenzie, K. A. J., The Role of Movable Kidney in Intestinal and Vascular Stasis, Jour. Amer. Med. Assn., 1912, lix, 33s. 
is, the pulse failure mechanism seems subject to fatigue. Excitement or emotion which increases the total outflow of nerve energy tends to inhibit the pulse failure. In general, in perfectly normal individuals who are in vigorous physical condition, the radial pulse is maintained when the arms are raised. In the same class of persons, when more or less debilitated by confining occupations or subnutrition, the pulse often fails. In an acute febrile disorder the pulse usually remains when the arms are raised; during convalescence, especially with the establishment of compensation in cardiac disease, the pulse often fails. Numerous observations seem to show that these differences in the reaction of the pulse do not depend on the amount of blood volume or the degree of maximal arterial pressure. ' It is not only conceivable, but probable, that signs of physiologic compensation which are absent during robust health or active disease should become manifest when the normal vital energy is reduced or when, as a result of disease, the efforts of physiologic adjustment must become more strenuous to maintain the needful outflow and normal distribution of the blood-mass. The older writers in this field were prone to attribute the pulsus paradoxus to mechanical interference with the normal blood-flow, basing their position on the frequency with which the phenomenon attends pathologic adhesions between the mediastinal tissues. The facts that have been rehearsed seem to indicate that mechanical abnormalities within the chest may only indirectly cause pulse failure, by exaggerating certain reflexes which under perfectly normal conditions are masked.

"As already stated, failure of the elevated pulse does not seem to occur in persons showing signs of general arteriosclerosis. The thought may not be far amiss that this constant organic tendency of later life may in itself be a compensation which, by diminishing the distensibility of the vessels under the hydrostatic pressure of the blood, may more or less substitute that constant and necessary redistribution of the blood-mass which has become impossible to a senile vasomotor apparatus" (L. Hill).

Goldthwaite ${ }^{1}$ classifies the essential hypotensive individual as the "carnivorous type" of man and suggests that the low blood-pressure, weak pulse, subnormal matutinal temperature, etc., are due to mechanical causes. According to this conception the absence of retroperitoneal fat exposes the sympathetic ganglia and the large

${ }_{1}$ Shattuck Lecture, An Anatomic and Mechanistic Conception of Disease, Boston Med. and Surg. Jour., 1915, clxxii, 881. 
bloodvessels which lie unprotected on the anterior surface of the spine, to irritation and pressure except when the individual lies on his face or his side. This perhaps also applies to the adrenals. A long mesentery by dragging downward in the erect posture favors splanchnic stasis; and the low diaphragm with its small respiratory excursion does not efficiently aid the propulsion of blood from the abdominal to the thoracic cavity. While these anatomical factors perhaps cannot alone account for the symptoms, they are quite sufficient to do so when we add the faulty standing and sitting postures which are habitually assumed by individuals of this type.

The most constant finding in the class of individuals under consideration is arterial hypotension. They often have varying degrees of gastroptosis but so do many apparently healthy people whose blood-pressure is normal and who have no gastric symptoms so long as their nerve tone is up to par. It would seem, therefore, that the splanchnoptosis is not the underlying causative factor, although it unquestionably acts an important part in some cases. We further ask ourselves whether the hypotension is the cause of the nervous manifestations or whether a lack of proper nervous stimuli is the cause of the hypotension? This question cannot be positively answered. Undoubtedly there is a complex interaction of these causes by virtue of which the one may deleteriously affect the other, from which we could readily construct a beautiful and typical example of the "vicious circle" did it seem worth while to do so.

Chronic low arterial pressure has also been attributed to toxemia, and cholin salts have been suggested as the active substance. ${ }^{1}$ It has been claimed by Popielski and Modrokowski that chemically pure cholin would produce a rise, not a fall of pressure, but Mendel, Underhill and Renshaw ${ }^{2}$ have shown that the injection of chemically pure cholin did produce a short fall of pressure.

Functional or constitutional physiologic vasomotor unfitness, owing mainly to improper nerve balance, seems to be the most satisfactory explanation for the majority of cases of afebrile hypotension.

\footnotetext{
1 Morel, Albert, Recherches sur l'origine clinique de l'hypotension, Livre jubilaire du Pr. Teissier, Lyon, 1910.

2 Action of Salts of Cholin on Arterial Blood-pressure, Jour. Phar. and Exp. Therap., 1912 , iii, 649 .
} 


\section{ORTHOSTATIC ALBUMINURIA.}

In health the assumption of the erect posture produces an increased urinary flow, and a relatively increased sodium chloride output. In the subjects of circulatory disease the conditions are reversed. In orthostatic albuminuria there is a qualitative change which points to a less efficient renal circulation in the standing posture. ${ }^{1}$

Numerous observers have commented upon the frequent association of orthrostatic albuminuria and cardiovascular weakness. This form of albuminuria is often associated with arterial hypotension. The thought naturally suggests itself that the albuminuria is brought about by the fact that the splanchnic vasomotor apparatus only insufficiently compensates the blood-pressure changes induced by a change of posture, thus leading to vascular changes in the kidney and the albuminuria while the patient is erect. ${ }^{2}$ The exact nature and mechanism of these vascular changes is still in doubt. Renal anemia or venous congestion, either as the result of increased intra-abdominal pressure or from kinking of the vesscls in a more or less movable kidney, have been suggested.

Neither of these explanations is universally applicable. The kidneys are by no means always movable, and increase of intraabdominal pressure on standing occurs in normal individuals. Further, why should the albuminuria only appear on rising in the morning and not in the afternoon? According to Porges and Pribram, ${ }^{3}$ the cause lies in a spastic contraction of the renal arteries.

This type of albuminuria is sometimes seen in convalescence, either from fevers or from trauma, which has necessitated a long stay in bed, under which conditions the vasomotor response to changes of posture are soon lost, so that the individual on assuming the erect posture promptly becomes weak and dizzy.

Hooker found that with a constant mean pressure the rate of blood-flow and of urinary secretion bear a direct relation to the pulse pressure, whereas the albumin secreted by the kidney varied inversely, experimental evidence which is in accord with the findings of. Erlanger and Hooker in two cases of orthostatic albuminuria. ${ }^{4}$

I Loeb, H., Klin. Untersuch. ü. d. Einfluss von Kreislaufsaenderungen auf d. Urinzusammensetzung, Deut. Areh. f. klin. Med., 1905, Ixxxiv, 579.

${ }^{2}$ Müntzer, E., Zur Lehre v. d. vaskulären Hypotonien, Wien. klin. Woch., 1910, xxiii, 1341 .

${ }^{3}$ Zur Kentniss d. orthostatisehen Albuminurie, Deut. Arch. f. klin. Med., 1907, $\mathrm{xc}, 367$.

${ }^{4}$ Jour. Physiol., November 1, 1910. 
Recent researches of Bass and Wessler ${ }^{1}$ have, however, controverted a number of the previously described results. They found that blood-pressures in children suffering from orthostatic albuminuria differ but little from normal findings.

"Functional" albuminuria is not infrequently met with at the period of adolescence, often in association with anemia and low blood-pressure. Dukes, who has observed many of these cases classifies them in three categories.

1. Cases with cold extremities, clammy hands, low blood-pressure, and deficient vasomotor reactions, as shown by syncopal attacks, and by a fall of blood-pressure upon rising from a reclining to an erect posture.

2. Cases with very labile but generally high blood-pressures. This is the commonest type.

3. Cases occurring in spare, neurotic, high strung and generally hyperesthetic individuals.

It is of course well known that posture may affect albuminuria even in organic cases, especially those associated with cardiac insufficiency.

The significance of albuminuria may often be more correctly interpreted by means of blood-pressure observations. This symptom may occur in perfectly healthy young men after forcible exercise. In association with slight hematuria it is especially frequent after boxing bouts, in which "kidney blows" abet its appearance (Stanton). The occurrence of albuminuria after strenuous exercise is generally attributed to a secondary splanchnic vasodilatation which follows the primary vasoconstriction, and which produces a temporary renal congestion. Paroxysmal hemoglobinuria and hematuria have also been attributed to loss of vasomotor equilibrium. Ilemoglobinuria is sometimes brought on by walking, especially if this is done in a lordotic posture. This type of hemoglobinuria has been explained as resulting from vasomotor instability causing an abnormal circulatory state in the spleen. ${ }^{2}$

\section{ARTHRITIC AND RHEUMATOID CONDITIONS.}

Excluding lesions of a true gouty nature which are usually associated with hypertension, there is a large group of so-called

1 Blood-pressure in Children Showing Orthostatic Albuminuria, Arch. Int. Med., 1913 , xiii, 39 .

2 Porges and Strisower, Deutsch. Arch. f. klin. Med., January 7, 1915, No. 2. 
"rheumatic" ailments whose pathogenesis is still unknown, which often occurs in hypotensive individuals and which tends to disappear when, as the result of a more efficient circulation, better nutrition of the muscles, joints and fibrous structures has been obtained. To this class belong certain cases of lumbago, sciatica, neuritis, stiff neck, and "myalgia." Rheumatoid arthritis and "phosphaturia" also generally occur in association with low arterial pressure.

Low blood-pressure is also met with in cases of status lymphaticus in which condition, according to Wiesel $^{1}$ and Hedinger, ${ }^{2}$ it is associated with hypoplasia of the chromaffin system.

Treatment of Essential Hypotension.-The curvative results of therapy in arterial hypotension are never brilliant and often disappointing, and yet in the majority of cases, especially if the subject be not too far advanced in life, much can be accomplished. 'The trouble lies in the fact that we are combating an inbred constitutional weakness and that an arrest of symptoms often only lasts as long as the treatment is continued.

The treatment consists mainly in a proper regulation of the patient's daily life, the details of which must be supervised with the idea of diminishing the factors which lead to a lowered nerve tone. That mental overwork is often largely contributory in increasing hypotension is corroborated by Bonser's ${ }^{3}$ studies, which showed that intellectual fatigue among twelve students observed was capable of diminishing vasomotor reactions to emotional stimulation. A complete rest cure in extreme cases may be advisable at the beginning. After this a general routine mode of life as is gencrally advised for neurasthenic patients is in order. A few points, however, deserve to be emphasized: (1) Requisite rest and sleep (an eight-hour allowance of the latter being a minimum). (2) Relaxation-the avoidance of too prolonged mental or physical work. The necessity of a small but regular amount of physical exercise, preferably outdoor. (3) The avoidance of excessesdietary or sexual. (4) Tobacco-these patients are very sensitive to tobacco, an excessive use of which is often directly accountable for their symptoms.

IIydrotherapy.-Aside from the foregoing, hydrotherapy is by far the most successful method of treatment. The Nauheim bath,

'Zur Path. d. chromaffinen System, Virchow's Archiv., 1904, elxxvi.

2 Ueber d. Kombination von Morbus Addisonii mit Status Lymphaticus, Frankfurter Zeitsch. f. Path., 1907, i, 527.

${ }^{3}$ Psych. Rev., March, 1903. 
the Vichy douche ${ }^{1}$ or the needle bath may be recommended, but almost equally good results may be obtained with the ordinary home shower bath.

The patient is instructed to take a hot tub bath each morning on rising, practising active autofriction with rough linen tape wash rags or mittens. When thoroughly warmed he is to turn on the cold shower, this to be followed by active friction with a rough towel. The length of the stay under the cold spray is to be gradually increased and after a short trial will be attended by a good vigorous reaction, the skin becoming pink and accompanied by a pleasant tingling glow. This treatment must be kept up indefinitely. Where no shower bath is available, cold water may be poured over the head and shoulders from pitchers.

In patients with enteroptosis and with relaxed abdominal muscles sistematic cxercises, calculated to restore muscular tone, or some form of abdominal support, are of distinct utility. Massage is also beneficial.

Goodman ${ }^{2}$ has emphasized the importance of daily morning calisthenics, especially those which tend to compress the abdominal viscera, increase respiratory excursion and strengthen the muscles which maintain a correct standing posture. Such exercises must not be carried to the point of breathlessness, much less exhaustion, but should be gradually increased in severity and duration as the individual is trained up to his task.

Drugs.-Strychin as an adjuvant to increase nerve tone is the most generally useful drug, though it has no direct effect on bloodpressure. Digitalis is useless unless there be a definite cardiac lesion.

Although we possess numerous drugs which may be used to lower blood-pressure, few if any fulfil the purpose of raising blood-pressure. Watson ${ }^{3}$ found that atropin, camphor, cotarnin, digitoxin and strychnin were valueless for this purpose. Physostigmin, which may raise pressure, cannot be used in adequate dosage on account of nausea and vomiting. Tyramin (said to be the most important active constituent of the watery extracts of ergot) gave better results. Musser, Jr., has reported good results following the administration of pituitrin (see p. 340).

I The Vichy douche consists of a needle douche projerted downward on the patient throughout the duration of the bath, while general massage is administered by an attendant.

2 Some Cases of Arterial Hypotension Associated with a Definite Symptomatology, Am. Jour. Med. Sci., 1914, cxlvii, 503.

${ }^{3}$ The Value of Drugs as Blood-pressure Elevators, Practitioner, 1915, xcir, No. 4. 
T'ermination.-The ultimate outcome of cases of essential hypotension has, so far as we are aware, not been studied. Individuals belonging to this class are of necessity seriously handicapped in their career. They cannot, as a rule, lead the strenuous life which seems, in this country at least, to be fast becoming the "normal." But whether this enforced moderation leads to a longer life, with a diminished tendency toward the development of arteriosclerotic changes, is an interesting question. If our interpretation of the pathology of this condition is correct, the splanchnic vessels are habitually relaxed and overloaded with blood. Does such a condition predispose to vascular disease of the intra-abdominal vessels and organs, or does the passive overloading with venous stasis exert a less deleterious effect upon the vascular wall than an active distention due to a powerful heart and a high vascular tonus? Do these individuals later in life develop arterial hypertension as the result of splanchnic arteriosclerosis with its attendant dangers and discomfitures, or does Nature mitigate these tendencies as a compensation of earlier disabilities? These questions must await further studies before an answer can be given. The case of the puny dyspeptic "weakling" who weathers the storm of an acute infection to which the robust "full-blooded" athlete succumbs is well known, as is also the lanky dyspeptic who outlives his plethoric friends. "Causa latet, res ipse notissima." 


\section{BLOOD-PRESSURE IN ACUTE INFECTIOUS DISEASE.}

Practically all infectious fevers are accompanied by a fall of blood-pressure, although in some, this feature is specially marked. The personal equation of the patient of course greatly influences the result. In the so-called sthenic fevers blood-pressure is not much affected. In asthenic fevers the fall is very pronounced. Many of the ill effects of fever are directly due to low arterial pressure and it is now. generally admitted that death in many infections, is quite as much due to vasomotor as to cardiac failure. A few microbic toxins (Staphylococcus, pyocyaneus, mallein) seem to have hypertensive qualities (Bosc and Vedel).

Broadly speaking, the extent of pressure fall tends to vary directly with the fever, but there are many exceptions to this rule, for the reason that the pressure is influenced more by the toxemia than by the pyrexia. In severe infections with a weakening circulation the systolic pressure falls and pulse pressure decreases. A wellsustained pulse pressure is to be construed as a favorable sign.

Degeneration of the adrenal glands in infections, especially diphtheria, and the experimental production of similar lesions by toxin injections have led some authors to recommend adrenalin as a therapeutic medium, both on account of its effect in raising blood-pressure, and on account of an hypothetic protective power in the defense of the organism.

In 11 cases of infectious disease, Marx ${ }^{1}$ found no lack of epinephrin in the blood, nor was the blood-pressure especially low. The amoint of epinephrin recoverable from the suprarenal glands after death bears no relation to the character of the fatal disease. The height of blood-pressure bears no relation to the epinephrin content of the glands. The variable results are perhaps due to the gradual death of different sets of the glandular cells. It seems likely, therefore, that the beneficial effect of epinephrin in shock and allied states is due to its vasoconstrictor action. It is therefore

1 Ueber den Adrenalingehalt der Nebennierne, Med. Klinik d. Städt. Krankenanst, Mannheim, Dissert. Heidelberg, 1912, xxiv. 
merely a symptomatic and not a specific means of therapeusis. Elliot, ${ }^{1}$ on the other hand, found that the store of epinephrin in the adrenal glands is diminished by fright, anesthesia, cerebral injury and bacterial intoxications. The residual epinephrin has been found especially low after death from pneumonia, although diminished amounts were also found after measles. scarlatina, acute febrile tuberculosis, malignant endocarditis. Since the adrenal glands are not considered responsible for the normal maintenance of blood-pressure, but are regarded as emergency organs, this would indicate that in order to combat the hypotension entailed by these infectious processes the adrenals had been called upon for a reserve supply, and showed a corresponding exhaustion. The decrease of residual epinephrin encountered was never sufficient to account for circulatory failure.

During the height of continuous fevers, blood-flow at the periphery is somewhat slower than in health. The conditions are quite different than when bodily temperature is artificially raised by the external application of heat. In the latter case, as was shown by Hewlett ${ }^{2}$ and Van Zwaluwenburg, the peripheral circulation is enormously increased, just as it is when a person sweats owing to the high temperature of a room. In fevers the vessels still dilate under the influence of external heat, but the heightened temperature of the body does not influence the heat regulatory mechanism in such a way as to. cause their dilatation. The experimental researches of Newburgh and Lawrence ${ }^{3}$ indicate that, in lower animals degrees of hyperthermia not greater than those encountered in infections are sufficient to cause marked hypotension. The increased body temperature of infection is a potent factor in the production of the lowered blood-pressure which occurs in such conditions. The hyperthermia may be the entire cause of such hypotension. A temporary rise of pressure sometimes accompanies the outbreak of the rash in the exanthemata-scarlatina, morbili, variola, etc. ${ }^{4}$ The work of the heart is increased during fever if the blood-pressure remains constant. ${ }^{5}$

1 Pathologie Changes in the Adrenal Glands, Quart. Jour. Med., 1914, viii, No. 29,

2 The Effeet of Room Temperature upon the Blood-flow in the Arm, etc., Heart. ii, 230.

3 The Effect of Heat on Blood-pressure, Areh. Int. Med., 1914, xiii, 287.

${ }^{4}$ Weigert, K., Verhalten d. art. Blutdrueks bei akuten Infektionskrankheiten, Volkmann's Samml. klin. Vortr., 1907, No. 459; Inn. Med., exxxviii, 65.

${ }_{5}^{5}$ Wolf, H. F., The Influence of Temperature on the Output of the Heart, Arch. Int. Med., 1911, viii, 463. 
Convalescence from prolonged fevers is attended by a loss of splanchnic tone, in part due to depressed nervous influence and in part to weakening of the arterial musculature. When the patient assumes the erect posture there is a marked falling of the maximal pressure, even when the minimal pressure remains unchanged. This diminishes the pulse pressure, as a result of which the deficiency of cerebral blood-supply must be compensated for by an increased pulse rate, which throws an unnecessary strain on the heart. 'This condition is often associated with cardiac murmurs, accentuations, reduplications or arhythmia. Minor degrees of physical exertion may cause a fall of 30 to $40 \mathrm{~mm}$. Hg. Under such conditions the patient should not be allowed to leave his bed so long as there is a marked difference in pulse rate between the erect and the recumbent postures. Cardiac erythism and vasomotor instability are often encountered.

As elsewhere actual figures are of little value. It is the course of the pressure, upward or downward, or marked lability, which is of importance. For this reason pressure readings should be made and charted simultaneously with the temperature. Marked fluctuations in the height of the systolic pressure are of serious import. Many of the older reports on blood-pressure in infectious disease are practically useless because they record only the systolic pressures, and eren these were often made with unreliable instruments.

The fact is generally recognized that practically any infection may produce cardiovascular damage. Thaver found that individuals who had passed through attacks of typhoid fever showed a disproportionately higher pressure in later life than those who have not had this disease. The lesson is obvious: that convalescents should be spared all umnecessary activity for prolonged periods of time. Schwartzmann's ${ }^{1}$ recent studies lead him to conclude that in infectious diseases a high diastolic pressure indicates a tendency to splanchnic stasis - a severe infection. A fall of both systolic and diastolic pressure points to vasomotor weakness, whereas a fall of the systolic, associated with a rise of the diastolic pressure shows cardiac failure.

The venous pressure tends to fall with the arterial pressure in infectious disease, but when cardiac failure begins venous pressure rises.

${ }^{1}$ Klinische Bedeutung der Feststellung d. systolischen u. diastolischen Blutdruck bei Infektionskrankheiten, Zeitsch. f. inn. Med., 1914, xxxv, 745. 


\section{ACUTE INFECTIONS.}

Cholera.-In this disease the specific gravity of the blood may rise to 1060 to 1070 , due to loss of fluid, and systolic blood-pressures as low as $70 \mathrm{~mm} . \mathrm{Hg}$ are not rare.

In the algid stage, according to Lang, ${ }^{1}$ the pulse pressure decreases owing to a fall of the maximum and a rise of the minimum pressures. Diastolic pressure diminishes only in severe cases. These changes are due to loss of the liquid constituents of the blood and to consequent vasoconstriction. Saline transfusions averaging 2 liters are generally sufficient to restore the total volume of bloor and bring the pressure relations back to the normal. Larger transfusions often lead to supernormal values as the hypertonicity of the vascular system tends to continue for some time and the pulse rate to rise. Following the algid stage and during the "typhoid" stage an increased blood-pressure is the rule.

The blood-pressure is therefore a satisfactory criterion of the amount of saline infusion to be administered. When given in too large quantities an unnecessary amount of work is thrown upon the heart. The judicious employment of saline infusion with epinephrin has in one epidemic at least greatly lowered the mortality of cholera. ${ }^{2}$ During the recent epidemic in Serbia vaccine was administered in large doses of physiologic salt solution with definitely beneficial results.

Diphtheria.-Cardiovascular disturbances occur in about 10 per cent. of all diphtheria cases. Death is due to (1) the effect of the toxin on the vasomotor centre, heart, and adrenals; (2) to myocardial lesions (chiefly parenchymatous; (3) to involvement of the stimulus conducting system; (4) to bronchopneumonic manifestations. $^{3}$

Experimental Data.--The experimental injection of diphtheria toxin does not always cause an immediate fall of blood-pressure. Such a fall occurs some time after the injection of cultures and appears suddenly. ${ }^{4}$ Romberg and Paessler showed that in the

${ }^{1}$ Ueber den arteriellen Druck bei Chotera asiatiea u. s. Veraeudrungen unter d. Einfluss grosser Kochsaltzinfusionen, Deut. Arch. f. kliu. Med., 1912, eviii, 236.

${ }^{2}$ Rogers, L., A Second Season's Experience of Hypertonic Transfusions in Cholera, Controlled by Observations on the Blood Changes, Therap. Gazette, November, 1909, xxxiii, 761 .

${ }^{3}$ Leede, W. H., Beitr. z. Diphth. mit besonderer Berucksichtigung d. path. anat., Organ $u$. baeteriologisehen Leiehenblutbefunde und ihrem Verhalten zum klinisehen Bilde. (3671 eases), Zeit. f. klin. Merł., 1913, lxxvii, 297.

${ }^{4}$ Beek and Slapa, Ueber d. Finfluss d. Diphtheriegiftes a. d. Kireislauf, Wien. klin. Woch., 1895, xviii. 
early stages at least the hypotension was vasomotor in origin, but von Slejskall was able to show a direct toxic action upon the heart. Lethal doses of diphtheria toxin after a latent period of twenty-four hours produce a marked fall of pressure up to the point at which death occurs. The timely injection of antitoxin delays this fall of pressure and in sufficient dosage prevents death, but even in excessive dosage fails to restore a pressure which has already fallen dangerously low. ${ }^{1}$ MacCallum's ${ }^{2}$ experiments indicate that the death which occurs at the height of an attack of diphtheria is not exclusively the result of direct cardiac injury, although that may have a part in the process. Porter and Pratt $^{3}$ found that even in the late stages of lethal diphtheria intoxication the vasomotor centre still responds to both pressor and depressor stimuli, showing that death is not primarily due to vasomotor paralysis. Myers and Wallacet have shown that although the larger bloodvessels in intoxicated animals responded normally, the arterioles and capillaries in the splanchnic domain fail to react normally to epinephrin and are found in an engorged condition. It appears, therefore, that peripheral splanchnic paralysis accounts for the hypotension of diphtheria.

Clinical Data.-Diphtheria is accompanied by low arterial tension, the degree of which often stands in proportion to the severity of the attack. The highest readings occur in the first and the lowest in the second week of the disease, normal tension being reëstablished by the seventh week. Laryngeal cases, especially those requiring operation, show a higher pressure as a result of asphyxia. 'Tracheotomy is often followed by a sudden fall (20 to $40 \mathrm{~mm}$.). Pressure in the early stages is ordinarily not much affected by serotherapy; in the latter stages it may rise 40 per cent. In cases manifesting anaphylactic phenomena there is a great fall of pressure (see page 210 ).

Circulatory weakness is a prominent feature of the disease, and the normal postural blood-pressure changes are often absent or reversed even during convalescence. ${ }^{5}$ The onset of neplritis is not always accompanied by an increase in blood-pressure.

\footnotetext{
${ }^{1}$ Meyer, Fr., Beitr. z. Kenntnis d. Diphtherievergiftung, etc., Arch. f. exp. Phar., 1909 , No. 60.

2 The Mechanism of Circulatory Failure in Diphtheria, Amer. Jour. Med. Sci., 1914, cxlvii, 37.

3 The State of the Vasomotor Centre in Diphtheria Intoxication, Amer. Jour. Physiol., xxxiii, 431.

4 'The Vascular Response in Poisoning from Diphtheria Toxin, Proc. Soc. Exper. Biol. and Med., 1914, xii, 43.

' Rolleston, J. D., Blood-pressure in Diphtheria, British Jour. Children's Dis., October, 1911, viii, 28.
} 
There is no fixed relation between fever and blood-pressure. The fall of the latter is due to the absorption of toxins, as shown by animal experiments. The most marked fall in pressure occurs in those cases in which antitoxin treatment has been delayed. When the fall of pressure is marked there is usually more or less cardiac involvement. Intravenous saline injection sometimes produces temporary inprovement, probably by diluting the toxins. ${ }^{1}$ A fall of pressure after an amelioration of acute symptoms is often the first sign of persistent toxemia. ${ }^{2}$

Fatal cases sometimes show a marked fall of pressure several days before the lethal termination. A progressive fall of tension is of bad prognostic import, as is also a unilateral pressure difference, but high pressure does not of necessity presage a recovery.

As a means of differential diagnosis between true diphtheria and simulating infections, blood-pressure readings are valueless. The occurrence of paralysis is sometimes preceded by a fall of pressure (Kolossova).

Malaria.-According to Federn, blood-pressure rises during the chill and falls during the period of sudation. In chronic malarial cachexia, hypotension is the rule.

If some of the recent hypotheses regarding malaria, which are based upon facts discovered since the artificial cultivation of the plasmodium has become possible, should prove true, blood-pressure may have a more important bearing upon malaria than has been supposed. It has been suggested that after attaining a certain size the plasmodium reaches and lodges in the capillaries and that in this situation the inoculation of new corpuscles normally takes place. The debris and pigment which result from their destruction may, if the infection be severe, "plug" the brain capillaries and produce "cerebral malaria." Further, the height of the bloodpressure and the state of capillary dilatation or constriction would influence the stage at which the organisms would recede from the peripheral blood-stream into the capillaries to undergo segmentation, or destruction, or to invade new blood-cells. At this point, too, the organisms would be immune to the effects of quinine which, according to this view, is not directly toxic but merely increases the permeability of the red cell to the normal destructive serum. ${ }^{3}$

\footnotetext{
1 Cobliner, W., Blutdruckmessungen bei erwachsenen Diphtheriekranken, Dissert., Berlin, 1912, p. 45.

2 Schoen, C., Deut. med. Woch., March 27, 1913.

3 .Jour. Amer. Med. Assoc., 1914, lxii, 1330.
} 
Meningitis.-Epidemic cerebrospinal meningitis is almost constantly associated with high intracranial tension. Morderately increased blood-pressure is not infrequntly seen in the early acute stage, during exacerbations of symptoms late in the disease, or where the malady assumes a chronic aspect. Often the higher the pressure the more severe the case. The withdrawal of cerebrospinal fluid by lumbar puncture, while usually attended with a fall, has no constant effect on blood-pressure. It seems, therefore, that increased intracranial tension in meningitis does not necessarily cause a rise of blood-pressure unless it be late in the disease, when internal hydrocephalus may develop as a result of blocking the foramina of the fourth rentricle. ${ }^{1}$

Tuberculous Meningitis.-In tuberculous meningitis arterial pressure is but slightly elevated, the readings obtained are about normal; whereas, in tuberculous disease they are generally subnormal. In other forms of meningitis very high pressures are sometimes encountered $(230 \mathrm{~mm}$.) which sometimes tend to run a parallel course with the increased pressure of the cerebrospinal fluid. ${ }^{2}$

Since meningitis is sometimes treated by intraspinal injections it is important to know how high the intraspinal pressure may be raised without danger. It has been found experimentally that although there is a marked difference in sensitiveness in different individuals, a sudden increase of pressure is always more dangerous than a gradual one. The first mechanical effect is upon the respiratory centre, followed quickly by profound cardiac inhibition which causes a sudden and tremendous fall of blood-pressure. Inasmuch as this is not a vasomotor disturbance, epinephrin is not indieated. In fact it would probably do harm on account of its cardiac inhibitive effect. ${ }^{3}$ The best results are obtained through the use of cocain and atropin, the former stimulating the respiratory centre and the latter lessening cardiac inhibition. (See Lumbar Puncture.)

Pneumonia.-The view that death from pneumonia frequently results from peripheral vasomotor paralysis has been quite generally hell, despite the fact that clinical observations are not at all unani-

\footnotetext{
${ }^{1}$ Robinson, G. C., Blood-preșsure in Cerebrospinal Meningitis, Areh. Int. Med., May, 1910, p. 452.

2 Parisot, J., La pression arterielle dans les meningites, Soc. Med. de Naney, Decenber S, 1909 ; Rev. Med. de l'Est, 1910, p. 48.

${ }^{3}$ Carter, W. S., The Effect of Iutraspinal Injections of Ringer's Solution in Different Amounts under Varying Pressures, Arch. Int. Ned., 1912, x, 425.
} 
mous in supporting this view. Among 19 fatal cases, and 26 cases which recovered, Newburgh and Minot found that the systolic pressure in the fatal cases was continuously above that in the cases which recovered. In 1899 Romberg and his associates endeavored to test the state of the vasomotor mechanism by the use of stimuli to the skin and mucous membranes. After producing a fatal pneumococcus septicemia in rabbits, blood-pressure was observed in the animals after electrical stimulation applied to the nasal and anal mucous membranes. In the early stages of the disease sensory stimuli always caused a marked elevation of bloodpressure. At a later period, when the appearance of the animáls suggested the approach of death and the temperature was falling, the reflexes were still present but did not attain the normal height. Finally, when the animal was in a state of collapse and death was imminent no rise of blood-pressure followed peripheral stimulation. As a result of their observations, Romberg and his co-workers believed that they had proved that death in acute infectious diseases was the direct outcome of paralysis of the vasomotor centre in the medulla.

This, it is to be observed, is an experiment of a negative sort, since it rests entirely upon failure to obtain certain physiological responses.

Porter and Newburgh ${ }^{1}$ have recently completed experiments from which they obtained positive information regarding this problem.

They produced not only pneumococcic septicemia in rabbits, but also acute fatal pneumonia in rabbits, cats and dogs. In order to judge of the condition of the vasomotor apparatus, they measured the vasomotor reflex. But instead of using precarious reflexes from the mucous membranes, Porter and Newburgh exposed and cut the depressor and the sciatic nerves and stimulaterl the central ends.

The data obtained are probably more reliable than those first mentioned, since the depressor nerve is composed entirely of fibers which affect the vasomotor centres, whereas other nerves contain fibers of widely different function. As a result of these symptoms they found that the vasomotor centre was not impaired in any of the examples of fatal pneumonia studied.

It is not surprising, therefore, that low blood-pressure in pneu-

1 The State of the Vasomotor Apparatus in Pneumonia, Am. Jour. Physiol., 1914, $\mathrm{xxx}, 1$. 
monia is not invariably of evil omen, indeed the systolic pressure is often higher in fatal than in non-fatal cases, and hence Gibson's rule is far from infallible. The rate of the pulse and not the bloodpressure level, is the chief factor in deciding whether the pressure will or will not fall below the pulse. ${ }^{1}$

On the other hand, as opposing the view that pneumonic death is primarily a cardiac failure, it has been shown that: (1) The heart muscle is not functionally impaired in pneumonia since the pneumonic ventricle beats normally as soon as its food is normal; (2) pneumonic blood, suddenly fed to a normal heart muscle, lowers its efficiency, lessening the duration and the area of contraction; (3) the heart muscle in pneumonia gradually exposed to the action of the poison, largely adjusts itself to its poisoned food. ${ }^{2}$ Death in pneumonia is often attended with the same symptoms as those produced by surgical "shock." There is often no dilatation or engorgement of the right heart. In lobar pneumonia there is, in addition to hypotension, the added mechanical obstruction in the lungs with which the right heart has to cope. Pulmonary involvement is often extensive, but experiments have demonstrated that one-sixth of the total hung eapacity is sufficient to maintain life, ${ }^{3}$ and in the advanced stages of tuberculosis an individual often lives for weeks or months with very little lung tissue intact. It is not, therefore, the extent of the pulmonary lesion which is of primary importance in pneumonia but the degree of toxemia, and this is often reflected in blood-pressure estimations. Such readings should be made routinely in all cases.

As first discovered by Gibson, the ratio of pulse rate and bloodpressure may be of some prognostic value. "A pressure appreciably below the normal in pneumonia is invariably of evil omen, and any considerably fall bodes disaster. When the arterial pressure, expressed in millimeters of mercury, does not fall below the pulse rate expressed in beats per minute, the fact may be taken as of excellent augury, while the converse is equally true." This statement has found corroboration from many sources."

The pulse-pressure ratio must not be accepted too literally;

${ }^{1}$ Newburgh and Minot, Arch. Int. Med., 1914, xiv, 4S; Newburgh, Am. Jour. Med. Sci., February, 1915, p. 204.

${ }_{2}$ Newburgh, L. H., and Porter, W. T., The Heart Muscle in Pneunonia, Jour. Exper. Med., 1915, xxii, 123.

${ }^{3}$ Bernard, L., Le Play, Mantoux, C., Capacité pulmonaire minima compatible avee la vie, Jour. de physiol. Expér., 1913, xv, 16.

+ Goodman and Pitman, Therap. Gazette, July 15, 1911. 
for individuals who have habitually a high pressure may, when critically ill, show a more favorable ratio than their condition justifies. On the other hand, patients with essential hypotension may show a lower pressure while their actual condition may be quite satisfactory. Howell ${ }^{1}$ has found that in doubtful cases observation of the relative intensity and duration of the first four auscultatory phases may be distinctly helpful. Strong, clearcut sounds over an artery are indications of circulatory strength. This applies specially to the third phase, in pneumonia.

"More is to be gained from watching the changes in a succession of sequences than from isolated observations, for, indeed, one patient may be quite comfortable with a sequence which in another promises the worst." In pneumonia the persistence of the second phase, which is most readily lost, is of favorable import. All clear tapping sounds are of good augury, whether heard in that part of the sequence usually assigned to the third phase or not, whereas feeble, muffled sounds must be considered as unfavorable. "When the clear tap is lost and the sequence appears as a succession of dull, muffled sounds from systole downward, a high grade of peripheral relaxation and secondary cardiac exhaustion can be inferred. When to this arhythmia is added, the worst picture is drawn."

Venous pressure is low, but rises as cardiac weakness increases.

The beneficial effect of cold fresh air are unquestionable, but they cannot be explained as the result of any direct effeet upon blood-pressure. A slight rise of systolic pressure of variable duration is produced in normal adults by exposure of the face to cold air, but is often followed by a fall to the original level even while the exposure is continued. ${ }^{2}$ Van Ordt, ${ }^{3}$ it is true, found a slight rise of blood-pressure following exposure to cold, but not greater in amount than the normal physiologic range. Barringer obtained practically negative results, regardless of whether the entire body or only the face was exposed.

Scarlet Fever.-The fall of pressure, which occurs in about 25 per cent. of the cases ${ }^{4}$ is less than in typhoid fever, and stands

\footnotetext{
${ }^{1}$ Possibilities in the Use of the Auscultatory Method of Determining Bloodpressure in Pneumonia, Jour. Amer. Med. Assn., 1914, Ixii, 1230.

${ }^{2}$ Barringer, T. B., The Effect of Cold Air upon the Circulation in Healthy and Sick Individuals, Amer. Jour. Med. Sci., 1912, exliv, 233.

${ }^{3}$ Zeit. f. Dial. u. physik. Therap., 1905, ix, 338.

${ }^{4}$ Rolleston, J. D., The Blood-pressure in Scarlet Fever, British Jour. Child. Dis., 1912 , ix, 444.
} 
in direct relation to the severity of the attack. It appears during the period of eruption and is pronounced during febrile defervescence. In mild cases pressure is but slightly affected. In grave cases a marked fall is associated with a high temperature and a rapid pulse. The normal is gradually reëstablished during convalescence, more slowly after severe cases. Slight albuminuria may not be accompanied by a rise of pressure. ${ }^{1}$ In scarlatinal nephritis, blood-pressure sometimes rises before albumin has appeared in the uriné. Cardiac involvement is sometimes associated with a slight rise and marked lability of arterial pressure (Weigert). Dietary modifications-milk, salt, salt-free food-and minor complications have but little effect on blood-pressure. ${ }^{2}$

Smallpox.-The pustulation.stage is associated with a fall of arterial pressure which compares in degree to the toxemia. A return to the normal during convalescence occurs very slowly. ${ }^{3}$

Typhoid and Paratyphoid Fever.-The blood-pressure in typhoid fever falls below the normal after the patient takes to his bed and remains low until convalescence is established. This is the result of toxic vasomotor depression. The fall of pressure bears no constant relation to the pulse or temperature curve or the severity of the attack, but a rapid or progressive fall of pressure is often of serious augury. The value of blood-pressure readings as a means of differential diagnosis or prognosis is not of much value.

Both systolic and diastolic pressure fall during the later febrile period, and rise coincidently at the beginning of the afebrile period. Later on in convalescence the systolic and diastolic levels move farther and farther apart, owing either to a systolic rise and a diastolic fall or because the one pressure remains stationary while the other recedes from it. Finally, sometimes suddenly and at times coincidently with leaving bed, both pressures attain a higher level. Studies of the "pulse-pressure quotient" and the "amplitude frequency product" indicate that during the febrile period the heart performs increased work despite the lowered pressure, and that circulatory weakness is in part cardiac and in part vasomotor in origin. Further, that in one case which came to autopsy,

\footnotetext{
1 Teissier and Tanon, Le Pression artérielle dans la Searlatine de l'Adulte, Jour. Physiol. et. Path. Générale, 1908, x, 481.

2 Nobéeourt and Teissier, La Pression artérielle dans la Searlatine de l'Adulte, Jour. Physiol. et. Path. Générale, 190S, x, 481.

${ }^{3}$ Davidson, Blood-pressure in Fevers, Lancet, Oetober 19, 1907.

4 Weigert, K., Ueber d. Verhalten d. arteriellen Blutdrucks bei d. akuten Infektionskrankheiten, Samml. klin. Vortrïge, 1907, xvi, No. 9.
} 
toxemia was accountable for the condition, since no microscopic myocardial lesions were encountered (Fig. 87). ${ }^{1}$

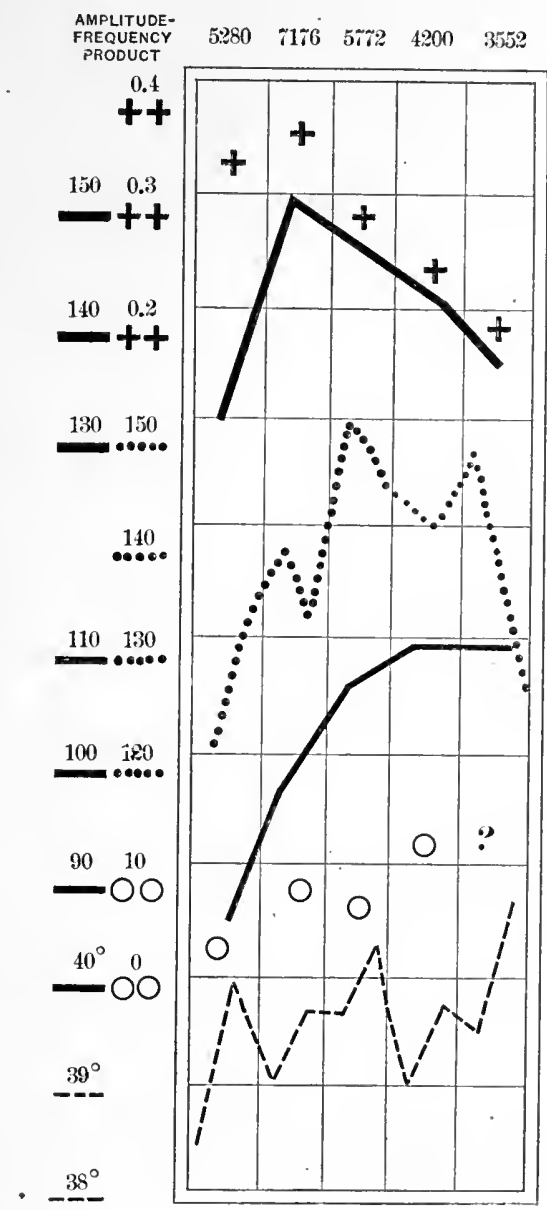

FIG. 87.-Record of a rapidly fatal case of typhoid fever in whieh death apparently resulted from cardiac weakness. The systolic pressure falls, the diastolie pressure rises, the quotient and the amplitude frequency product steadily decline, while the venous pressure and the pulse rate rise. Microscopie examination of the heart being negative death was aseribed to toxic factors. (Dietsehy and Hössli.)

In 115 cases the average pressure was found to be: First week, $115 \mathrm{~mm}$.; second, $106 \mathrm{~mm}$; third, $102 \mathrm{~mm}$.; fourth, $96 \mathrm{~mm}$.; fifth,

${ }^{1}$ Dietschy, R., and Hössli, H., Beiträge z. Beurteilung d. Kreislaufsverhaeltnisse bei Infektionskrankheiten, etc., Deut. med. Woch., April 24, 1908, xciii. 
$98 \mathrm{~mm}$. The occurrence of perforation is often followed within two to four hours by a rise of from 20 to $70 \mathrm{~mm}$. It is caused by (a) pain, and $(b)$ beginning peritonitis, in the early stages of which condition a marked pressure increase is often seen. ${ }^{1}$ In the later stages a toxic paralysis of the vasomotor and respiratory centres in the medulla occurs (Romberg and Heinéke).

In cases of relapse, the pressure which may have risen during the afebrile period undergoes a second drop, often to a still lower level. These changes may precede the rise in temperature.

During convalescence there is a slow, gradual rise of pressure (sometimes interrupted by temporary drops), which increases

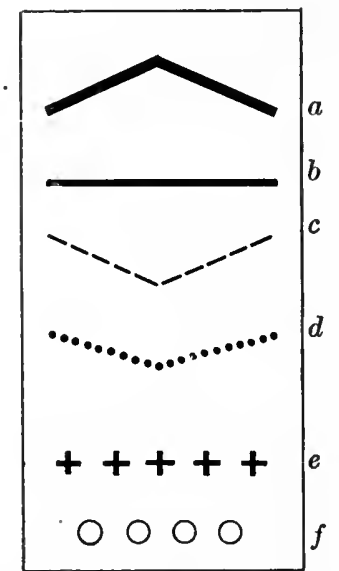

FIG. 88. $-a$, maximun pressure; $b$, minimum pressure; $c$, temperature; $d$, pulse rate; $e$, blood-pressure quotient; $f$, pressure of the jugular vein.

more rapidly when the patient is again up and about. IIemorrhage is usually followed by a fall, sometimes a marked fall, of pressure (20 to $40 \mathrm{~mm}$.). In cardiac weakness, marked diurnal variations may occur.

Cold sponging raises the pressure 10 to $15 \mathrm{~mm}$. in cases which respond favorably to the treatment; in the cases which become cyanotic, this rise does not occur. A complicating pneumonia may temporarily raise the pressure, as may also the psychic effect of too many visitors. ${ }^{2}$ To be of any value in typhoid fever bloodpressure observations should be made twice daily. The normal

${ }^{1}$ Crile, G. W., The Diagnostic Value of Blood-pressure Observations in the Diagnosis of Typhoid Perforation, Jour. Amer. Med. Assn., 1903, xl, 1292.

${ }_{2}$ Barach, Blood-pressure in Typhoid Fever, New York Med. Jour., August 24, 1907. 
individual range will then be reëstablished and changes definitely demonstrated. If perforation is anticipated readings should be made at half-hourly intervals.

Treatment.-Hypotension per se is not a symptom calling for treatment any more than is hypertension. The resting body can do with a pressure well below the normal without deleterious effects; indeed, this may be a method of conserving energy. Only when pressure falls progressively or is accompanied by other signs of cardiac or vasomotor failure is interference warranted. Many a case of typhoid fever or pneumonia weathers the attack satisfactorily, having a systolic pressure of about $100 \mathrm{~mm}$. Hg., without stimulation.

When, however, a falling pressure is associated with an increased pulse rate, pallor, sweating, cold extremities, and other signs of collapse, direct treatment, calculated to restore vasomotor tone, is indicated. It is generally easier to keep up pressure by early treatment-sponging, etc.- than to restore it once it has fallen to the danger limit. The best results will usually be obtained by fresh air, cold sponging, saline enteroclysis, or intravenous infusion. The addition of from 0.5 to 1 per cent. of sodium bicarbonate to the 0.8 per cent. sodium chloride solution is advisable. The addition of the former salt produces a greater rise of the diastolic pressure and seems to exert a definitely stimulating effect upon the heart ${ }^{1}$ (see page 382). In the course of fever the peripheral circulation, as indicated by the flow of blood in the foot, is distinctly subnormal. This apparently results from vasoconstriction, an effort on the part of Nature to secure an increased blood-flow for the organs chiefly involved. According to this hypothesis, fever is due to more rapid flow, and therefore increased metabolism of the internal organs. Hence, cold sponging would seem to be the most valuable form of treatment, since it tends to abet the natural process; whereas antipyretic and other vasodilator drugs are directly contraindicated, since they tend to interfere with the natural mechanism of healing. ${ }^{2}$ Since febrile hypotension is essentially toxic in nature, all available means must be employed to counteract or minimize toxemia. Febrile hypotension is often associated with tympanites,

\footnotetext{
${ }^{1}$ Dawson, The Changes in the Heart Rate and Blood-pressures Resulting from Severe Hemorrhages and Subsequent Infusion of Sodium Bicarbonate, Jour. Exp. Med., 1905, vii, 1.

2 Stewart, G. N., Blood-flow in the Feet, with Special Reference to Fever, Jour. Exp. Med., 1913, xviii, 354.
} 
which sImptom should be zealously guarded against, since it indicates indigestion and malnutrition, and because it seriously embarrasses both respiration and heart action.

Treatment therefore should consist of (1) Combating Toxemia. This is to be accomplished by increasing elimination by attention to the emunctories. Proper evacuation of the bowels and increasing the urinary output are useful measures. The latter is often best accomplished by increasing the fluid intake by mouth, by means of continuous enterocylsis (drip method), by intravenous saline infusion or hypodermoclysis. Fresh air plays an important role.

2. I'asomotor stimulation, by cold sponging or tubbing, or by the use of drugs which act upon the central or peripheral vasomotor sistem.

The drugs most commonly employed are strychnin, atropin, caffein, camphor, digitalis, strophanthus, pituitrin, epinephrin. Although they may do good, improvement cannot, except in case of the last two, be attributed to direct blood-pressure raising qualities (see under Therapeutics).

Medical or Toxic Shock.-A condition of shock practically identical with that seen after surgical trauma may occur as the result of anaphylaxis. The injection of foreign proteids, horse serum for instance, is sometimes followed by surlden collapse or even death from this cause. A distinction is often made between shock and collapse. In the former we are dealing with a lowering of vasomotor tone due to insufficient reflex splanchnic stimulation; in the latter the vasomotor paresis is due to depression resulting from toxemia. Pearce has shown experimentally that in both anaphylactic shock and in peptone intoxication in dogs there is a great fall of blood-pressure (20 to $30 \mathrm{~mm}$. Hg.) due to splanchnic dilatation and resulting in medullary anemia. Cardiac and respiratory abnormalities are entirely secondary manifestations.

Physiologic studies having for their object the determination of the mechanism by which the low pressure is caused, demonstrate that the condition is essentially a peripheral vasomotor paralysis. Pharmacologic studies indicate that the effect is on the nerve endings rather than on the muscle.

With independent cerebral transfusion the recovery from low pressure is more rapid than in the intact animal. This is true also when an animal is transfused by carotid anastomosis, and recovery is especially satisfactory when the transfusion is accompanied by simultaneous bleeding from the femoral vein. 
The researches of Auer and Robinson ${ }^{1}$ indicate that in animals at least the fall of pressure seen in anaphylactic shock may be partially cardiac in origin. The electrocardiogram shows variable abnormalities, ranging from an increased depression of the S-wave to complete dissociation of stimulus conduction.

Henderson and Barringer state that "in both hemorrhage and circulatory shock the decrease in the venous supply to the right heart is the critical factor. In this they differ from vasomotor failure, in which the peripheral resistance of the arterial system is decreased."

"The indications for treatment, therefore, appear to be (1) relief of splanchnic congestion, and (2) increase of volume of bloorl to the heart and medulla. Cardiac stimulants alone, or salt solution and adrenalin alone, cannot bring about a permanent improvement. A combination, however, of the slow injection of adrenalin in salt solution (1 to 40,000) intravenously with the addition of a pure cardiac stimulant, as digitoxin, leads to relatively rapid and permanent improvement, by promoting a determination of the blood to the right heart and increasing the eirculation in the brain" (Pearce). ${ }^{2}$

As a prophylactic measure in anaphylactie shock, the utility of atropin has been demonstrated by Auer. ${ }^{3}$ In guinea-pigs without atropin, 75 per cent. died; with this drug only 28 per cent. (see Surgical Shock, page 378).

\section{THE EFFECT OF DRUGS ON THE VASOMOTOR SYSTEM.}

Strychnin, while it may be useful in other ways, does not stimulate the vasomotor centre except in toxic doses.

Epinephrin has no direct effect on the centre, but may be useful in emergeney for its sympathetic stimulation.

Camphor is probably without effect on the eentre, as is also spartein. Ergot increases pressure by its stimulation of the sympathetic nerve.

The vasomotor centre is depressed by chloroform; ether

${ }^{1}$ An Electrocardiographic Study of the Anaphylactic Rabbit, Jour. Exp. Med., 1913, xviii, 450 .

2 A Study of Experimental Conditions of Low Blood-pressure of Non-traumatic Origin, Archives of Internal Medicine, August, 1910, vi, 218; also, A Study of the Action of the Heart in Anaphylactic Shock in a Dog, Jour. Phar. and Exp. Therap., 1912, iv, No. 1.

${ }^{3}$ Amer. Jour. Physiol., September, 1910. 
stimulates moderately, if it has any effect. Pituitrin has but a slight action on the centre. It is a useful emergency measure which raises pressure by its action on the peripheral arterial muscle. ${ }^{1}$ Alcohol is deleterious if increase of pressure is the desideratum.

If the hypotension results from cardiac weakness digitalis, or strophanthin may be tried, although their effects are often disappointing in febrile toxemia. An ice-bag applied to the precordium is often the most satisfactory method of steadying the heart.

1 Pileher and Sollmann, Studies on the Vasomotor Centre, Jour. Phar. and Exp. Therap., 1915 , vi, 323. 


\section{BLOOD-PRESSURE IN CHRONIC INFECTIOUS DISEASE.}

Syphilis.-Owing to the great frequency of aortitis as a lesion of secondary syphilis, and since it so often involves the mouths of the coronary arteries, it is not surprising that blood-pressure variations and other cardiac symptoms should frequently be encountered. 'Two-thirds of the 228 cases studied by Grassmann' showed cardiac disturbances-arhythmia, bradycardia, tachyeardia, murmurs, dilatation, etc. He found that the blood-pressure, which is normal at first, soon gives place to hypotension and instability. As soon as aortic valvular leakage is established, the pressure changes take on the characteristic manifestations of this lesion (see page 235). In 80 per cent. of Grassmann's cases the pressure (von Basch sphygmomanometer) was below $80 \mathrm{~mm}$. in the temporal artery. Some of these cases showed a further reduction of both pressure and hemoglobin on the institution of the mereurial treatment.

Based upon a study of 50 hypertensive eases, 90 per cent. of which yielded either a positive Wassermann or luetin test, Stoll ${ }^{2}$ has advanced the hypothesis that hereditary syphilis is one of the most common causes of hypertensive disease. He lays stress on the importance of performing a luetin test, especially if activated by a week's mixed treatment in hypertensive cases which have yielded a negative Wassermann test. He reports favorable results from specific treatment especially in patients with pressures below 200 $\mathrm{mm} . \mathrm{Hg}$. without any apparent deleterious effects upon the kidney, even in cases in which the phthalein exeretion was eonsiderably reduced. The best results were obtained by administering mereury by inunction, and potassium iodid by mouth (see salvarsan, p. 341).

Tuberculosis.- The fact that tuberculosis is generally associated with hypotension has long been recognized and is generally

${ }^{1}$ Klin. Untersuch, a. d. Kreislauforganen in Frühstadium d. Syphilis, Dent. Arch. f. klin. Med., 1901, lxix, 281.

2 The Role of Syphilis in Hypertensive Cardiovascular Discase, Am. Jour. Med. Sei. $1915, \mathrm{rl}, 178$. 
accepted. 'This applies to both the systolic and the diastolic pressures. The hypotension is secondary to the tuberculous process.

Thayer's figures are as follows:

$\begin{array}{cccc}\text { Age. } & \text { Normal. } & \text { Tuberculosis. } & \begin{array}{c}\text { Peters' cases. } \\ \text { (Altitude } 6000 \mathrm{ft} . \text { ) }\end{array} \\ 10 \text { to } 20 & 128.7 & 100.33 & 122 \\ 20 \text { to } 30 & 136.9 & 101.0 & 123 \\ 30 \text { to } 40 & 140.8 & 94.0 & 124 \\ 40 \text { to } 50 & 142.2 & 105.5 & 134 \\ 50 \text { to } 60 & 154.8 & 105.0 & 138 \\ 60+ & 180.0 & 114.0 & \end{array}$

This table shows hypotension at all ages in tuberculous subjects. Peters explains his higher readings as due to the higher altitude (6000 feet) at Silver City, New Mexico, and construes the results as indicating the advantages of such an elevation in regard to treatment, but F. C. Smith ${ }^{1}$ was unable to substantiate these findings.

As in health, the pressures are lower in women than in men. Blood-pressure bears no definite relation to the-stage of the disease, although it generally does to the degree of toxemia. Thus the pulse rate and the degree of temperature sometimes, although by no means alway's, bear an inverse relation to blood-pressure. Pressure tends to rise as the pulmonary condition improves. Arterial tension bears no definite relation to the hemoglobin content of the corpuseles nor to an antecedent gonorrhea, syphilis, alcoholism, or pneumonia. Stantion was able to corroborate in tuberculous subjects Thayer's statement that individuals who have had typhoid fever show disproportionately higher pressures later in life. A complicating nephritis may cause a rise of pressure, but rarely, if ever, to the degree attained in non-tuberculous cases. In the literature on the subject there is, however, much room for uncertainty", since the term "nephritis" as used in connection with tuberculosis may mean a tuberculous lesion engrafted on an old nephritis, amyloid disease, or a true tuberculous nephritis. In the latter condition, Reitter ${ }^{2}$ found actual hypotonia as contrasted with other forms of pyelitis and pyelonephritis in which the pressure was at least normal if not distinetly increased.

The vasomotor instability of tuberculosis is shown by the fact that at least a large proportion of the advanced cases show a fall of pressure and a considerable increase in pulse rate, on changing from the recumbent to the erect position.

1 The Effect of Altitude on Blood-pressure, Jour. Am. Med. Assn., 1915, Ixiv, 1 s12.

2 Nierentuberkulose u. arterielle Hypotension, Zeit. f. klin. Med., 1907, Ixii, 358. 
Bouchard, Arloing, Rhorlet, and Courmount believe that the toxin of the tuberele bacillus possesses distinct vasomotor influences. These results have been recently corroborated and much more satisfactorily demonstrated by Emerson. Man is apparently much less sensitive to this effect than animals, since a relatively larger and more concentrated dose is required to produce much fall of pressure. Whether this toxin is the sole cause of the hypotension is still undecided. For instance, it may result simply from tachycardia, for beyond a certain point systolic output falls with an increasing pulse rate, owing to insufficient diastolic inflow.

"Fever will cause rapid heart action by its effect on the accelerans nerve endings in the heart. Diminished general pressure results in lower cerebral pressure, which of itself stimulates the cerebral origin of cardiac sympathetic nerves, especially when the low pressure is due to vasoparesis" (Krehl).

Laryngeal and intestinal complications are often associated with low pressures. Arrested cases generally show a rise and relapsing cases a fall of tension. Ambulant patients yield higher readings than bed-fast cases. The cardiac hypertrophy seen in some cases in the early stages of tuberculosis is perhaps due to the effort of the heart to maintain an efficient cireulation, despite the vascular relaxation. The late atrophy probably results from inanition and myocardial degeneration.

There are three theories which have been used to explain the toxic hypotension of tuberculosis:

1. That it is due directly and chiefly to the heart muscle, which is pathologically altered, either as the result of the toxins of the bacillus or as the result of malnutrition.

2. That the bloodvessels are primarily the cause of the vasodilatation, which is the result of the tuberculous toxins.

3. That the nervous control of both heart and bloodvessels is essentially influenced by the toxemia, both the vascular dilatation and the tachycardia being of nervous origin.

Two practical facts present themselves:

First. Is the study of pulse and blood-pressure of prognostic or therapeutic value, and is it of diagnostic value previous to the time when a diagnosis may be made from physical signs?

Second. To what is the hypotension due, and to what extent can it be remedied by treatment?

There are wide diversities of opinion on these subjects. Broadly speaking, the French writers maintain that hypotension is a valuable 
early sign of tuberculosis, which may often be suspected on this account, even when latent; and further, that it is a valuable differential point in deciding between chlorosis, etc., and incipient tuberculosis, also in diagnosticating between tuberculous effusions in the pleura and in the peritoneum and non-tuberculous effusions. On the other hand, a large number of observers, of whom many are Germans, find that hypotension is inconstant, and when present only a late phenomenon. We agree essentially with the latter proposition, feeling that the stethoscope is a far better early diagnostic instrument than the sphygmomanometer, believing, however, that blood-pressure observations are capable of rendering useful data so far as the corroboration of diagnosis, the gauging of toxemia, the permitting of exercise, and the recognition of relapses are concerned. It is to be borne in mind, however, that there is a common type of constitutional hypotensive individual who has a low pressure without either active or latent tuberculous disease (see page 185 ).

As to the actual causes of tuberculous hypotension, Haven Emerson, ${ }^{1}$ who has published an excellent clinical and experimental study on the subject, arrives at the following conclusions:

"The causes of low blood-pressure in tuberculosis are probably primarily a toxic action on the vasomotor centre in the medulla, allowing of a vasoparesis or stimulating an active vasodilatation, and, secondarily, progressive cardiac atrophy or degeneration. Toxic action on the vasomotor nerves or their motor terminals or on the nervous mechanism of the heart cannot be positively denied, although there is no proof of it at present. Toxic action of tubercle products has not been demonstrated on the muscle of the vessel wall or heart, although with regard to the latter, the degenerated heart muscle found in advanced cases may have an influence in causing hypotension. Rapid heart action is a usual and necessary sequel to low blood-pressure, and will, if extreme, aggravate the hypotension by the very act of its shortened diastole. It has been suggested, but not proved, that lack of vagus inhibition, owing to pressure by enlarged bronchial lymph nodes and the presence of sympathetic excitation from similar or reflex causes, such as pulmonary or gastric irritation, are responsible for the tachycardia and hypotension.

"Hypotension or subnormal blood-pressure is universally found

1 Bloorl-pressure in Tuberculosis, Arch. Int. Merl., 1911, vii (bibliography). 
in advanced pulmonary tuberculosis, in which condition emaciation may be responsible for its causation. Hypotension is found in almost all cases of moderately advanced tuberculosis, or in early cases in which the toxemia is marked, except when arteriosclerosis, the so-called arthritic or gouty diathesis, chronic nephritis, or diabetes complicate the tuberculosis and bring about a normal pressure or a hypertension. Occasionally the period just preceding or during hemoptysis may show hypertension in a patient whose usual condition is that of hypotension.

"Hypotension has been found by so many observers in early, doubtful, or suspected cases, with or before physical signs of the disease in the lungs, and is considered by competent clinicians so useful a differential sign between various conditions and tuberculosis, that it should be sought for as carefully as it is the custom at present to search for pulmonary signs.

"Hypotension when found persistently in individuals or families or classes living under certain unhygienic conditions should put us on our guard against at least a predisposition to tuberculosis. Most unhygienic conditions, overwork, undernourishment, and insufficient air are of themselves causes of a diminished resistance, and it seems likely that a failure of normal cardiovascular response to exercise or change of position may be found to indicate this stage of susceptibility, especially to tuberculous infection.

"The difficulty of proving subnormal pressure is so much greater than that of determining an increase of pulse rate, or of temperature, that some means such as the one I suggest in the tests I have made personally should be tried before considering a patient normal just because a single systolic reading shows a pressure within normal limits. ${ }^{1}$

"Hypotension, when it is present in tuberculosis, increases with an extension of the process. Recovery from hypotension accompanies arrest or improvement. Return to a normal pressure is commonly found in those who are cured. Continuation of hypotension seems never to accompany improvement. Prognosis can as safely be based on the alteration in the blood-pressure as on changes in the pulse or temperature.

"The secondary anemia, with its diminished albumin, salts, and hemoglobin content, serves to add to the hypotension from purely physical causes in the blood-stream.

1 The test consisted in pulse and pressure olsservations after change of posture. 
"Diminished area of lung tissue, resulting in an increased carbon dioxid content of the blood, is suggested as a contributory cause of hypotension.

"Displacement of the heart, which is so common a result of retracted lung tissue, or loss of expansion due to cavity formation or adhesive pleurisy, is spoken of as a cause of tachycardia accompanying hypotension, and it certainly seems to be a contributory cause in some cases.

"Marked vasomotor irritability is recognized as a frequent phenomenon in pulmonary tuberculosis, and is supposed to result in tachycardia and hypotension; but it is not easy to see exactly what is meant by this term unless it is a certain functional instability for which we have no proof of any definite local cause.

"Disease of the adrenal bodies is to be mentioned as a possible factor, only to be set aside for lack of any consistent pathological proof.

"Primary cardiac atrophy, congenital or acquired, can no longer be considered as a factor in the presence of modern orthographic $x$-ray examinations, and in the absence of agreement among pathologists.

"The result of hypotension in tuberculosis or in any other condition is insufficient capillary pressure, more or less venous stagnation of the essential organs of the body.

"The treatment of tuberculosis in all its stages should take into consideration the need of assisting in every way the return to normal pressure, (1) by relieving the relaxed vessels of the load put on them during the vertical position or exercise, and (2) by assisting the heart by abundant nutrition, moderate exercise, and the stimulating effect on cardiac and rascular tone of cold fresh air, to meet the extra work put on it by the loss of vascular tone. Thus will the heart be able to maintain its normal bulk and strength, or even to gain from the atrophy or small size of the early disease to a normal size, so that brain, kidneys, lungs, and body at large may be properly nourished until the disease is arrested and the toxic products of the tubercle bacillus are no longer distributed from the site of the lesion."

The question whether hemoptysis is associated with increased blood-pressure has received both affirmation and denial. Nor is this surprising when we remember that the pressures in the pulmonary and in the srstemic circulation vary independently of each other. A progressive diminution of the pulse pressure indicates 
continued hemorrhage. At the time of actual hemorrhage the excitement incidental thereto tends to increase blood-pressure. There appears to be no ground to support the contention that hemorrhage occurs most frequently in cases with a high systolic pressure. The establishment of an artificial pneumothorax is likewise without any constant influence on arterial tension. ${ }^{1}$ In one of Smith's cases there was a marked unilateral pressure difference associated with incomplete collapse of the lung due to pleural adhesions.

Blood-pressure estimations have been used as a gauge whereby to control exercise in the tuberculous. Peters ${ }^{2}$ decreases the amount of exercise if the patient shows a drop of $6 \mathrm{~mm}$. or more after one hour's rest, or if exertion is immediately followed by a marked fall $(10$ to $20 \mathrm{~mm}$.) of pressure.

The Treatment of Pulmonary Hemorrhage.-Both clinically and experimentally, hemorrhages from the pulmonary vessels tend to cease spontaneously, owing to the low blood-pressure in the pulmonary circuit and to the reticulated nature of the lung parenchyma. In animals it is difficult to produce a fatal result, even when the main branch of a lobe is cut. The protective mechanism in man seems to be less efficient. The great discrepancy which exists regarding methods of treatment is due to the fact that. a distinction is not made between the early and late stages of hemorrhage in which the physiologic principles in therapeusis are radically different. Digitalis, atropin, the nitrites, aconite, chloral, chloroform, ergot, adrenalin, pituitrin, and other substances, have been recommended.

We have to consider (1) the effect of the drug on the pulmonary and (2) on the general arterial blood-pressures; (3) its effect on the small pulmonary arterioles; (4) its effect on heart rate and systolic output; (5) its effect on the respiratory centre. Generally speaking, a reduction of pulmonary pressure should reduce hemorrhage, but if this is done at the cost of an increased systolic output it will, of course, not do so. Constriction of the small pulmonary vessels tends to increase pressure.in the pulmonary arteries and thus to increase bleeding. It is evident, therefore, that the ability to constrict the pulmonary vessels is not a criterion of its hemostatic value. The maintenance of a normal mean pressure in the pulmonary circuit

${ }^{1}$ Smith, F. C., The Effect of Altitude on Blood-pressure, Jour. Am. Med. Assn., 1915, Ixiv, 1812.

2 Blood-pressure Control of Exercise in Tuberculosis, Colorado Medicine, 1915, xii. 
is a function which Nature tends to accomplish in spite of interference, just as it does in the case of cerebral anemia. Because we lower the systemic pressure, it of course does not follow that we lower the pulmonary pressure. Furthermore, in the late stages of bleeding cerebral anemia is prevented by splanchnic vasoconstriction. To thwart this result might produce death, which is certainly a strong argument against the use of the nitrites. What we are attempting to accomplish, is to reduce pressure in or reflux from the pulmonary vessels without embarrassing the respiratory or vasomotor centres or the heart in their efforts to maintain the circulation.

The physiologic effect of certain drugs upon the pulmonary and systemic circulations.

Digitalis (without alcohol) produces a rise of pulmonary pressure, slight at first, but increasing as its action becomes more pronounced. It therefore increases hemorrhage by augmenting the systolic output of the right ventricle and constricting the pulmonary vessels.

Ergot primarily decreases pulmonary arterial pressure by depressing the heart. It is followed, especially in normal animals, by an inerease due to a secondary augmentation of the beat. Pressure in hemorrhage in the pulmonary veins is permanently increased at the constriction of the small vessels. The action of the drug wanes as the loss of blood continues.

Pituitrin.-C'This drug has been highly recommended by Wiggers, since it elevates the systemic arterial pressure through vasoconstriction in spite of weakening of the left heart. The decrease in amplitude of contraction of the right ventricle causes a fall of pulmonary pressure, which neither the weak constricting influence of the blood on these vessels nor the backing up of the blood from the left heart counteracts. It does not affect the respiratory centre. These results occur both in normal and in bleeding animals.

Chloroform produces a fall of pressure in the pulmonary circuit due to depression of the heart and aided by diminished respiration. During dyspnea in hemorrhage small doses lessen the respiratory movements and so decrease pulmonary pressure and hemorrhage, while large doses act similarly through cardiac depression.

The Nitrites.-In normal-breathing animals a general increase in pulmonary pressure is noted, due to increased rate and systolic output of the heart. In animals breathing rapidly from hemorrhage, nitrites increase both pressure and hemorrhage from pulmonary arteries and veins. In the stage in which the nitrites reduce the 
amplitude of respiration a lowering of the pulmonary pressure and a reduction of hemorrhage occurs.

It is essential in the treatment of hemoptysis, as was shown by Wiggers ${ }^{1}$ (from whose articles many of the foregoing data were taken), to differentiate between the early and the late stages of the hemorrhagic process.

Effect of Various Agents on Systemic Pressure, Respiration, Pulmonary Artertal Pressure and Hemorrhage, and Pulmonary Venous

Pressure and Hemorrhage. (Wiggers.)

\section{Digitalis:}

Drugs. Systemic

Nomormal : $\cdot \cdot \cdot+$

Strophanthin:

Normal

Hemorrhage $\} \cdot \cdot \cdot+$

Ergotoxin:

Normal

Hemorrhage

Pituitary:

Normal Hemorrhage . . . +

Chloroform:

Normal

Hemorrhage

Nitrites:

Normal . . . . . -

Hemorrhages early . . -

Late

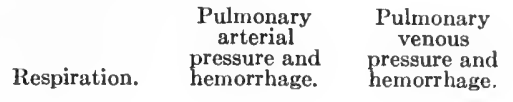

0

0

0

$-$

-

0

$-$

$-$

0

$+$

$$
\begin{aligned}
& + \\
& +
\end{aligned}
$$$$
+
$$$$
-+
$$$$
- \text { or }(+)-
$$$$
-
$$

$\stackrel{+}{(-)+}$

$\stackrel{+}{(-)+}$

Summary.-In the early stage of hemorrhage, in a practically normal subject with only occasional coughing, pulmonary pressure should be reduced by means of cardiac depressants, chloroform, or pituitary extract. The nitrites are contra-indicated. In the late stages, with tachycardia and tachypnea, the heart and bloodvessels no longer react in a typical manner to certain drugs. Cerebral anemia manifests itself. 'The blood-supply of the brain must be maintained. According to Wiggers, the only drug which can elevate the systemic pressure and simultaneously lower the pulmonary pressure is pituitary extract.

All clinical and most experimental evidence indicates that morphin is the most valuable drug we possess in the treatment of hemoptysis. Fear and excitement are among the most potent causes

1 A Physiological Investigation of the Treatment of Hemoptysis, Areh. Int. Med. July 15, 1911; Studies in Inaceessible Internal Hemorrhages, Ibid., March, 1909. 
of increased arterial tension in general, and probably in the pulmonary circulation, and under these conditions nervous sedatives are the most rational form of therapy.

Recent experiments, however, show that morphin may be harmful when death is threatened by a hemorrhagic fall of pressure. There are two factors to be considered: (1) Augmented breathing acts favorably from a mechanical point of view in maintaining bloodpressure. (2) Excessive breathing may produce acapnia and cause a fall of blood-pressure. Wiggers ${ }^{1}$ maintains that the former condition outweighs the latter in importance, and that morphin must be employed with caution. This statement, of course, applies only in the case of large hemorrhages in which death is to be feared, and the evidence furnished cannot be used to negative the wellattested clinical results which have shown the good effects of morphill.

Pleural Effusions. - The existence of pleural and peritoneal effusions, especially if large, tend to increase blood-pressure, and their removal is attended with a fall of arterial tension. The pressure, therefore, of such serous effusions may increase a pressure already high as the result of nephritis, and a fall of pressure following aspiration may not indicate any improvement in the basic condition from which the patient suffers. On the other hand, a cardiac hydrothorax may cause a pressure sufficiently high to make one suspect a coincident nephritis when no such complication exists.

The emotional disturbance entailed by a prospective operation as well as the actual pain of puncture produce a temporary elevation of blood-pressure. Aspiration of a pleural exudate is attended by a gradual fall of blood-pressure which occurs pari passu with the withdrawal of fluid. According to Capps, ${ }^{2}$ a fall of $20 \mathrm{~mm}$. is usual. The systolic pressure is chiefly affected. This fall of pressure is probably not merely the result of the mechanical pressure effects, but is in part due to vasodilator and cardio-inhibitory reflexes.

After withdrawal there is a gradual rise of pressure, but the initial limit may not be reached for some time. The rate of withdrawal is no less important than the amount of fluid removed in its effect on the immediate fall of pressure. The more long standing, the effusion the greater the fall of pressure, probably due to imperfect

${ }^{1}$ C. J. Wiggers, K. C. Eberly, and H. L. Wenner, The Pressor Influence of Augmented Breathing, Jour. Exp. Med., 1912, p. 174.

2 Observations on the Effect on the Blood-pressure of Withdrawal of Fluid from the Thorax and Abdomen, Jour. Amer. Med. Assn., January 5, 1907, xlviii. 
pulmonary expansion. Marked reactions and a slow recovery are seen in arteriosclerotic cases.

By observing the blood-pressure during thoracentesis, untoward effects such as weakness, vertigo or collapse, symptoms which often occur suddenly and without warning, may be anticipated and obviated. (See Aspiration of the Pleura, page 386).

Pneumothorax.-Pneumothorax and penetrating wounds of the chest, in addition to well-known symptoms, are often associated with bradycardia and a rise of blood-pressure (presenting somctimes an analogy to cerebral injuries), from which signs too optimistic conclusions may easily be drawn.

Experimental Data.-The reports to be found in literature on blood-pressure in artificial pneumothorax are very divergent. Kakowski ${ }^{1}$ and others report a rise in open pneumothoraces, other investigators no change, and still others a fall of bloodpressure.

According to Walther experimental pneumothorax in the normally breathing rabbit is associated with a slowing and an increase in the volume of the pulse. Elimination of vagus control does not check the rise of pressure which is apparently due to $\mathrm{CO}_{2}$ stimulation of the vasomotor centre. The change in the pulse rate, on the other hand, is believed to result from vagus stimulation. The difference in response between an open and a closed pneumothorax, which is only one of degree, has been explained as due to the fact that in the latter, in addition to stimuli which result from pleural irritation, those arising in the bronchial mucous membrane (since respiratory excursions are still present) are superadded. The irritation of the pleura is conducted by the sensory fibers of the vagus to the nucleus and produces increased vagus tone. ${ }^{2}$

Clinical Data.-Spontaneous pneumothorax may come on suddenly with acute pain and shock, especially if the onset occurs during exercise in apparently healthy individuals. Frequently, however, pneumothorax occurs insidiously ${ }^{3}$ during the course of tuberculosis. In the former instance a primary fall of bloodpressure is succeeded by a rise to above the normal, which is in part at least the result of anxiety, pain, and asphyxia. In the latter

1 Zur Frage d. Künstlichen Pneumothorax, P'flüger's Arch., 1910, cxxxiv, 31.

2 Walther, H. E., Zur Kentniss der Puls u. Blutdruckveränderungen beim Pneumothorax, Deut. Zeitsch. f. Chir., 1912, exix, 253.

${ }^{3}$ Among 500 cases of pneumothorax the onset was sudden in 77 and insidious in 23 per cent. of the cases. The latter class was evidently underestimated. Pepper, O. H. P., Amer. Jour. Med. Sci., October, 1911. 
instance blood-pressure changes are neither constant nor marked, especially if only a partial pneumothorax is present.

There is considerable reason to believe that the occurrence of pneumothorax in the course of tuberculosis may have beneficial effects. Certainly the artificial production of this condition is often attended with marked symptomatic improvement. Just to what extent this is due to changes in the pulmonary circulation is as yet uncertain. "It is disputable as to whether the contracted lung contains more or less venous blood. In fact, there is experimental evidence that the pulmonary circulation adapts itself promptly to the change and is therefore not particularly disturbed."

Symptoms of shock and occasionally sudden death have followed the therapeutic establishment of pneumothorax. Such events are generally due to a pleural reflex which consists of afferent medullary impulses by way of the vagus nerve from its terminal filaments, which have been rendered unduly susceptible to stimuli as a result of compression or inflammation. Death is apparently due to vasoconstriction of the cardiac or cerebral vessels. Artificial pneumothorax should never be employed in the presence of severe cardiac complications. ${ }^{2}$

1 Robinson, S., and Floyd, C., Artificial Pneumothorax as a Treatment of Pulmonary Tuberculosis, Arch. Int. Med., 1912, ix, 452.

${ }^{2}$ Sachs, T. B., Artificial Pneumothorax in the Treatment of Pulmonary Tuberculosis, Jour. Amer. Med. Assn., 1915, lxv, 1861. 


\section{CHAPTER IX.}

\section{EXOGENOUS INTOXICATIONS.}

Lead Poisoning.-The most important exogenous intoxication, so far as blood-pressure is concerned, is lead poisoning. Hypertension is produced directly as the result of plumbism and indirectly as a result of gout, nephritis, or arteriosclerosis, ${ }^{1}$ with each of which conditions plumbism is closely allied. The onset of both lead colic and encephalopathy is associated with marked exaccrbations of pressure even in the absence of nephritis or gross vascular lesions. This increase in pressure is believed to be the result of vascular spasm, since it disappears after the paroxysm. The arterial constriction is evident on examination of the retinal vessels; and Pal has reported a case of lead amaurosis which appeared with the onset and disappeared with the abeyance of hypertension. The systolic pressure is chiefly affected, and large as well as sudden variations of pressure are often encountered. Extended observations have shown that 80 per cent. of all lead-workers exhibit high arterial pressure over years of time even when free from symptoms of poisoning, a fact which might be expected if the contention of Schmidt, ${ }^{2}$ be correct that no real elimination of lead ever occurs, once it has entered the body, but merely a transference of it to different tissues. Most but not all cases of colic show an exacerbation of tension coincidently with the colic. The actual pressure does not generally exceed $200 \mathrm{~mm}$. Hg. except in cases of encephalopathy. It seems eminently likely that the pain markedly augments the pressure. But that pain is not the essential cause is shown by the fact that lowering of pressure relieves it (Riegel, Frank, Bardenhauer), while relief of the pain with morphia does not lower the tension (Riegel, Borgen), nor does pressure always fall with a spontaneous relief from colic. It has, on the other hand, been maintained that the pressure is compensatory, and

\footnotetext{
${ }^{1}$ The vaseular ehanges differ from those of ordinary arteriosclerosis. The vessels do not eollapse and exhibit a normal intima with marked hypertrophy of the media. Cardiae hypertrophy is not constant.

${ }^{2}$ Untersuchungen bei experimenteller Bleivergiftung, Deut. Areh. f. klin. Med., 1909, xevi, 587.
} 
when sufficiently high prevents the colic. The latter view does not commend itself. The hypertension has also been attributed to the effect of lead on the central nervous system. It has also been suggested that the hypertension is due to increased adrenal secretion. Heubel ${ }^{1}$ maintains that the rise of pressure is purely secondary nephritis, etc.; while Broadbent attributes it to the chemical formation of lead albuminates, which, being with difficulty broken down, complicate metabolic changes and render the elimination of metabolites difficult. Ménétrier and others believe that arterial hypertension is a primary and initial toxic phenomenon.

Lead colic is generally regarded as due to involvement of the solar plexus. Thus, Laignel-Lavastine speaks of the pain, constipation, and hypertension as symptoms of "solar stimulation." Lesions of the ganglia of the plexus have been demonstrated postmortem (Tanquerel, Kussmaul, Maier) and experimentally (Mosse).

Experimental Data.-The intravenous injection of lead salts produces arterial hypertension. Of course, it does not follow because acute intoxication produces this effect that therefore chronic poisoning will have similar results. The chief physiological action of lead is exerted on striated muscle, in which it produces a diminished functionation (Nothnagel and Rossbach). This would lead to vasomotor relaxation, and unless it were counterbalanced by compensatory vasoconstriction, to a fall of pressure. According to Jores, ${ }^{2}$ such a relaxation occurs in the smaller arteries of rabbits poisoned with lead.

Clinical Data.-Hypertension has long been observed clinically (Riegel and Frank). The presence of hypertension, especially if nephritis can be eliminated, should certainly suggest the possibility of plumbism even if the gingival blue line or basophilic degeneration of the erythrocytes camnot be demonstrated. Arterial stasis alone will not increase pressure, and since there is a marked hypertension during attacks of colic, we must assume a vasoconstriction, either as the result of a toxic effect or owing to some compensatory antagonistic physiologic action (central vasoconstriction?).

Furthermore, as shown by Hasebroek, the sphygmograph of a lead pulse is more like that of a normal pulse than that of an arterial hypertension. He uses this and some of the foregoing arguments to establish the existence of an active arterial diastole, and believes

${ }^{1}$ Path. u. Sympt. der chr. Bleivergiftung, Berlin.

2 Ueber die path. Anat. d. chronisehen Bleivergiftung des Kaninchens, Müneh. med. Woch., 1902, p. 713 . 
that plumbic hypertension results not from vascular spasm but from an "increased activity of the pressor components of physiologic vascular functionation." Stewart in his studies on blood-flow found in lead poisoning without paralysis a conspicuous tendency to reflex vasoconstriction.

Well-marked bradycardia is quite common. In 1179 cases Tanquerel found the pulse rate between 20 and 60 in 678 , between 65 and 70 in 376, and between 80 and 100 in 125 cases. Such cases are a true bradycardia due, it appears, to a toxic effect upon the vagus nerve or its terminations. The bradycardia and the arterial hypertension are independent phenomena. ${ }^{1}$

Encephalopathy.-In this condition very high pressures are often encountered. Ménétrier ${ }^{2}$ attributes the symptoms to the high pressure.

In a man, aged twenty-one years, during one of many attacks of colic, a pressure of $260 \mathrm{~mm}$. was found which soon reached 300 min. and was then associated with marked cerebral symptoms. At autopsy the kidneys were normal, as had been the urinary findings during life. The brain showed marked signs of pressure, edema, distended membranes, flattened convolutions, etc.

It appears, therefore, that plumbism may be accompanied by numerous clinical manifestations which strongly suggest vascular spasm as the basis of their production. Thus the abdominal colic, anginoid attacks, encephalopathy, including amaurosis, hemianopia, aphasia, deafness, etc., have been attributed to local vascular contraction.

Although generally associated there is no absolute parallelism between pain and vascular spasm; either may exist without the other. It is not yet proved that lead colic is due (1) to spasm of intestines or to (2) spasin of mesenteric arteries. It is apparently associated with a general vascular spasm in which, of course, the splanchnics have a part. Such a general contraction may begin suddenly or gradually, hence it is often difficult to say what the individual's normal is. The fact that pain is absent is no proof that the vessels are not contracted. Hypotension $(55$ to $75 \mathrm{~mm}$. Hg.) may follow colicky attacks. Relief from constipation often goes hand-in-hand with the disappearance of pain and with a fall of pressure (Riegel).

With so many divergent theories and sneh unequivocal experi-

${ }^{1}$ Lion and Marcorelles, Presse Méd., 1913, No. 12, p. 109.

${ }^{2}$ Soc. méd. des Hôpitaux, February 12, 1904. 
mental and clinical findings, we must therefore conclude by saying that although in lead poisoning hypertension is constant, and exacerbations of tension frequent, we are still unable to make positive statement as to the exact mechanism by virtue of which these vascular phenomena are produced, or whether they are primary or secondary manifestations. Persistent marked hypertension is of bad augury in plumbism. Borgen has observed the following phenomena in cases of lead colic: (1) period of rising pressure, of variable duration; (2) period of high pressure, one to four days; either of the above may be associated with colic; (3) period of decrease, two to four days, with disappearance of symptoms; (4) period of subnormal pressure $(95 \mathrm{~mm}$.).

Therapeutics.-If the conception of the pathologic process as a local (or general) vascular spasm is correct then purgation with salines and belladonna is a rational procedure. The administration of morphin is purely palliative. Pal endorses the custom sanctioned -administration of the iodides in large doses-saying that symptoms of lead colic cease as soon as those of iodism appear. Some researches have thrown doubt upon the efficacy of the iodides, at least so far as elimination is concerned, and have suggested the employment of bile salts. The administration of the nitrites sometimes relieves pain.

Phosphorus.-Acute phosphorus poisoning so far as the circulation is concerned, produces a degeneration of the heart muscle, and while this is doubtless a contributing factor in the cause of death, it appears from Pal's investigations that the primary cause lies in a fall of blood-pressure due to loss of vasomotor tone.

Chlorin Gas-The inhalation of chlorin gas which has been used. in trench warfare causes a marked fall in blood-pressure, a slowing and irregularity of respiration and pulmonary symptoms which are apparently due to obstruction of the pulmonary circulation rather than to spasm occlusion of the bronchioles. ${ }^{1}$

Arsenic.-Acute arsenical poisoning causes a fall of blood-pressure due to depression of the heart and the vasomotor centre.

Tobacco.- There has been much discussion as to whether the constitutional effects which follow the use of tobacco are due to nicotin or to the other substances. 'The evidence at hand indicates that nicotin is by far the most important, if not the sole factor,

ISchäfer, E., On the Immediate Effects of the Inhalation of Chlorin Gas, British Med. Jour., August 14, 1915, p. 245. 
but other substances, such as carbon monoxid, hydrocyanic acid, furfural and other aldehyds, have also to be reckoned with.

Lehman has shown that the slower the rate of smoking the smaller the amount of hydrocyanic acid formed. 'This substance, however, seems to be present in too small amounts to account for physiologic symptoms. The ordinary cheap cigarettes (Virginia tobacco) contain only a negligible quantity of nicotin. They do, however, contain a large quantity of furfural to which the harmful effects may be due, yet this substance is practically absent from Turkish cigarettes. ${ }^{1}$

Experimental Data.-With moderate quantities of nicotin the pulse rate is slowed; the heart may stop for a few seconds in diastole, then gradually assume an accelerated rhythm. The slowing is due to stimulation of the vagus ganglia. "It is not affected by section of the cervical pneumogastric, as the path from the ganglia to the cardiac ganglia is still intact, but, on the other hand, it is prevented by atropin, which acts on the extreme terminations of the inhibitory fibers, and therefore blocks the passages of impulses from the ganglia to the muscle."

Blood-pressure is increased owing partly to stimulation of the vasoconstrictor centre in the medulla, but chiefly to peripheral influences, for it occurs even after extirpation of the spinal cord. "The vasoconstrictor nerves pass through ganglia on their way to the vessels, and the rise of blood-pressure seems to be mainly caused by a stimulation of these ganglia." (Cushny.) Hemorrhage causes a marked increase in the vasomotor reaction to nicotin. ${ }^{2}$

The fact that on analysis a certain variety of tobacco is shown to contain a greater or a smaller quantity of nicotin by no means proves that when smoked it will yield an equivalent amount. In fact the very opposite result may be obtained. The nicotin content of the smoke depends largely on the completeness and quickness of combustion. Ordinarily, half of the nicotin is destroyed by combustion. Of the remainder variable quantities reach the mouth, being carried there by the hot smoke, which, in passing through the condensation area, volatilizes certain substances in the tobacco. Hence, the larger the condensation area (long, thick cigars) the greater the amount of nicotin, etc., which comes through.

The smoking of tobacco in the form of cigarettes is, for an equal amount of this substance consumed, less harmful than when used

${ }^{1}$ See editorial, Jour. Amer. Med. Assn., 1912, lix, 1798.

${ }^{2}$ Hoskins, Rowley and Rosser, Arch. Int. Med., 1915, xvi, 456. 
in the form of cigars or a pipe, assuming of course that inhalation is practised in each instance, because of the more complete combustion which ensues. Long cigars are relatively more injurious than short ones because they furnish a large condensation area in which the unconsumed nicotin accumulates. The last third of the cigar is, therefore, more toxic than the first two-thirds. For the same reason a cigar which has ceased to burn should not be relighted. ${ }^{1}$ The habit of holding an extinguished cigar between the lips should not be practised. Long-stemmed pipes are better than short ones.

Tobacco and Arteriosclerosis. - The importance of tobacco as an etiologic factor in arteriosclerosis has been much discussed and the question still remains unsettled. The tobacco habit is usually coupled with the use of alcohol and frequently with faulty methods of living. It has yet to be shown that the career of smokers is shorter than that of non-smokers. It is admitted that tobacco is a cardiovascular poison which is at first an excitant to the neuromuscular apparatus, later a motor nerve depressant, and finally a paralyzant of the cardiac nerves. But whether it produces arteriosclerosis, either by its direct toxic action on the vessels or indirectly by its effect on blood-pressure, is a very different question. Lee apparently succeeded in producing definite rascular lesions in rabbits which were made to inhale tobacco smoke over prolonged periods of time. Tobacco does no one any good, but it harms some less than others. The moderate smoking of tobacco in one who is accustomed to its use has but little effect on blood-pressure, and from a practical stand-point as a noxious factor is often negligible.

Clinical Data.-There are of course individual differences, but generally tobacco tends to raise both the pulse rate and the bloodpressure, the former being in part the cause of the latter. These effects often appear after the first inhalation of smoke, and may be accompanied by a sensation of nervousness and sometimes precordial fulness or palpitation. The secretion of the adrenals is controlled by the sympathetic nerve, which is at first stimulated and later depressed by the administration of nicotin. ${ }^{2}$

The elevation of blood-pressure ranges between 3 and $25 \mathrm{~mm}$., the systolic pressure being chiefly affected, indicating that the heart

${ }^{1}$ Lee, W. E., The Action of Tobaceo Smoke, with Special Reference to Arterial Pressure and Degeneration, Quart. Jour. Physiol., 1908, p. 335.

2 Cannon, Aub, and Binger, A Note on the Effect of Nicotin Injertion on Adrenal Secretion, Jour. Phar. and Exp. Therap., 1912, iii, 379. 
is more affected than the bloodvessels. Pressure generally falls to or slightly below the original level within twenty minutes: $\mathrm{Hesse}^{\mathrm{l}}$ found these results more marked in smokers than in non-smokers. The greatest rise in the pulse rate was twenty-five beats per minute. A return to the normal occurs in from twenty to forty minutes. Excessive quantities of nicotin cause a fall of pressure from depression of the vasomotor centre and may in this way actually cause collapse. Strong tobacco causes more marked effects than the weaker varieties and in strongly reacting individuals the pressure may remain high for two hours. ${ }^{2}$ Acute tobacco poisoning causes a fall of blood-pressure, associated with nausea, vomiting, vertigo, and sudation.

Tobacco, then, should be forbidden, or its consumption limited when (1) we wish to spare the heart-cardiac, renal, pulmonary disease, etc.; (2) in arterial hypertension; (3) in arteriosclerosis.

Chronic Alcoholism.-Chronic alcoholism is associated with variable blood-pressure, depending upon the extent to which renal and cardiovascular changes have occurred. But alcoholics in whom these organs are sound, show, when abstinence has been enforced, a marked rise of the systolic pressure followed after a few days by a gradual fall. The diastolic pressure is constantly high. Raff ${ }^{3}$ finds these results so constantly as to be of diagnostic value in differentiating between alcoholism and functional neuroses.

Delirium Tremens. - In the asthenic type of case the systolic pressure is uniformly low and the pulse pressure small. A reëstablishment of normal relations goes hand-in-hand with the subsidence of delirium. This would indicate that the height of blood-pressure, through its effect on the cerebral circulation, bears a causal relation to the delirium, a statement which is further borne out by the fact that if more normal values can be temporarily established by whatever means, symptomatic improvement occurs (Pettey). ${ }^{4}$ The treatment of delirium tremens by means of lumbar puncture has been attended by excellent results. It appears that the delirium is in part due to increased cerebrospinal pressure, and in part to toxicity of the spinal fluid. As pointed out elsewhere there is no

1 Deut. Arch. f. klin. Med., March 15, 1907.

2 John, M., Ueber d. Beeinflussung des systolischen u. diastolisehen Blutdrucks durch Tabakrauchen, Zeitsch. f. exp. Path. u. Therap., 1913, xiv, 352.

${ }^{3}$ Blutdruckmessungen bei Alkoholikern u. funktionellen neurosen unter Ausseliluss von Kreislaufstörungen, Deut. Arch. f. klin. Med., 1913, exii, 209.

${ }^{4}$ The Narcotic Drug Diseases and Allied Ailments, Philadelphia, 1913, p. 99. 
constant relationship between arterial and cerebrospinal pressure. (See Meningitis, and Lumbar Puncture.)

Morphinism.-According to Pettey, morphin habitués generally show a high pressure due largely, it seems, to portal congestion, since evacuation of the bowels often causes a fall of from 30 to 60 $\mathrm{mm}$. Hg. "This reduction of arterial tension by the preparatory treatment, now universally verified, is an essential factor in preventing collapse and other dangerous complications during the withdrawal period."

Patients admitted with a blood-pressure of 180 to $200 \mathrm{~mm}$. were usually found to have a blood-pressure of 140 to 150 after the sy'stem had been cleansed of toxic matter and the drug withdrawn. The lowered record is maintained throughout convalescence, showing that it was the individual's normal pressure.

The experience of the writer with this class of patients has been limited, but he nevertheless feels that the preceding statements must be accepted with reserve. Certainly, patients with the opium habit as seen in our hospitals are generally admitted in a condition of semicollapse, due to starvation, intoxication, and cachexia, with blood-pressure distinctly below the normal.

Valenti ${ }^{1}$ found that if morphin were withdrawn from dogs which had become accustomed to its use, marked circulatory disturbances such as arterial hypotension, arhythmia and tachycardia occurred. These symptoms abated if the use of the drug was renewed. Furthermore, the serum of dogs suddenly deprived of morphin caused similar symptoms when injected into other dogs, whereas the serum of unmorphinized dogs has no such effect. It seems evident, therefore, that during the withdrawal stage, circulation stimulation may be necessary.

\footnotetext{
${ }^{1}$ Experimentelle Untersuchungen ü. d. ehronisehen Morphinismus, etc., Areh. f. exper. Path. u. Pharmakol., 1914, lxxv, 437.
} 


\section{CHAPTER X :}

\section{BLOOD-PRESSURE IN CARDIAC DISEASE, ETC.}

Mechanical Hypotension. - When the total volume of blood is diminished from whatever cause (hemorrhage, diarrhea, sweating, polyuria) a fall of blood-pressure may result. For a time, of course, this may be counterbalanced by arterial constriction. Such a hypotension is seen typically in postpartum hemorrhages. Whether the hypotension seen in severe anemias, often with a diminished viscosity, has a similar genesis cannot be positively asserted.

Factors which hinder the flow of blood to or from the right heart (a fall of venous pressure, adhesive or effusive pericarditis, obstruction in the pulmonary circuit, portal or caval obstruction, etc.) produce arterial hypotension. The removal of serous effusions from the peritoneum or pleura tends to produce a similar effect, as do also cardiac and valvular lesions in the stage of broken compensation.

Functional Hypotension.-This form of hypotension occurs when vascular tone, especially in the splanchnic vessels, is below the normal or when a local vasodilation is insufficiently compensated by local constriction elsewhere. It is seen chiefy in fevers (toxemia), in constitutional low arterial tension, and in medical or surgical shock.

Terminal Hypotension.-Terminal hypotension may result either from mechanical or functional causes. By this term we mean the ultimate fall of pressure which occurs as a result of cardiovaseular failure. It is seen in the agonal and preagonal periods. It is common as the terminal stage of hypertension, in which the pressure may be only relatively, not actually, below the normal. Occasionally life may be maintained for several hours or even day's with a maximum pressure of $60 \mathrm{~mm}$. Hg.; indeed, figures as low as $45 \mathrm{~mm}$. (associated with unconsciousness and a subnormal temperature) have been reported by Neu.

Diseases of the Heart.-Despite the great abnormalities of the circulation, which are manifest during the course of valvular and 
myocardial disease, pressure observations often show relatively insignificant changes. Increased blood-pressure is essentially a vascular and myocardial, not a valvular phenomenon. Excluding the conspicuous findings of aortic insufficiency and cardiorenal hypertension, sphygmomanometric readings often are of discouragingly little clinical value, although a normal sustolic, associated with a high diastolic, pressure is very suggestive of cardiovascular disease. This is due to the fact that although the general systemic pressure may be normal, the rapidity of blood-flow, the mass movement of the blood, and especially the normal pressure relations between the arteries, arterioles, capillaries and veins have been either locally or generally disturbed. Regarding these conditions ordinary sphygmomanometric observations teach us but little by inference and nothing directly.

Frequently in valvular disease one finds diminished pressure, 100 to $110 \mathrm{~mm}$., but such a hypotension is more often attributable to coincident conditions, such as fever, etc., than to a direct cardiac effect. In acute rheumatic endocarditis the pressure is not different from that in old valvular lesions. Rarely blood-pressure and temperature rise and fall together. Only exceptionally does the pressure in afebrile cases fall below 90 . Not infrequently pressure values remain the same in broken compensation and during subsequent improvement. Digitalis produces practically no rise of pressure in valvular cases. (D. Gerhardt.)

We may have a stasis of blood due to increased resistance in the peripheral arteries without elevation of either the systolic or the diastolic pressure. 'The condition which Sahli has described as high pressure stasis ("hochdruckstauung") occurs when the arterioles are tonically contracted or sclerotic so that outflow is prevented. Under such circumstances eren an insufficient heart may, by forcing gradually more blood into the arterial reservoir, increase the pressure. This is further abetted by the accumulation of $\mathrm{CO}_{2}$ in the blood, which, by stimulating the vasomotor centre, leads to still further arterial contraction.

In these cases a plethoric, cyanotic face is often associated with edema and other evidences of cardiac failure, together with high arterial tension, which falls when circulatory improvement occurs. A fall of pressure is of serious import chiefly when associated with a rise of renous pressure, the latter being manifested by hepatic and pulmonary congestion. Vascular reflexes are sometimes abolished 
in advanced cardiac disease. Cases of cardiac decompensation often show a fall of pressure coincident with subjective and objective improvement. This is attributable to better renal elimination, to diminution of the $\mathrm{CO}_{2}$ content of the blood, and to a diminution of hydremic plethora (see page 268).

Aortic Lesions. - Experimental Data.-The establishment of experimental aortic insufficiency in animals, so far as concerns the mean arterial pressure, has shown divergent results. Some investigators have reported a fall of the mean pressure; others but little change. The results seem to depend, at least in part, upon the cardiac strength of the animal in question-dogs bearing the lesion better than rabbits. As a rule, there is some fall of the mean pressure, the amount of which tends to vary with the duration and severity of the lesion.

The arterial pressure is perhaps maintained in suddenly established aortic lesions by the vasomotor and cardiac reflexes which are engendered by stimulation of the endocardium, but the chief factor of compensation lies in the heart muscle itself. The mean pressure is maintained chiefly as the result of enlargement and hypertrophy of the left ventricle, which lead to an increased systolic output. ${ }^{1}$

The experiments of Stewart ${ }^{2}$ indicate that much less blood actually regurgitates into the heart during diastole than is generally supposed. This is owing to the increased tonicity which the left ventricle assumes. The great difference between the systolic and the diastolic pressure which occurs has been attributed to a reflex lowering of the minimum pressure. The main fall of pressure is systolic in time, and is due to increased capillary flow. This is borne out by the fact that even in case of good compensation the diastolic pressure may range between 40 and $60 \mathrm{~mm}$. Hg., while the systolic pressure is about $180 \mathrm{~mm}$. The tremendous pressure fluctuations to which the arteries are subjected is an important factor in the arteriosclerotic changes which the vessels undergo in these cases.

Wiggers's ${ }^{3}$ studies with optical manometers, however, indicate that the rapid systolic fall of pressure is not due to vasodilatation, since (1) the change occurs too rapidly (within a single beat), (2)

1 Krehl, L., Pathologische Physiologie, 1910, p. 18.

2 Experimental and Clinical Investigations of the Pulse and Blood-pressure changes in Aortic Insufficiency, Arch Int. Med., 1908, i, 102.

${ }_{3}$ The Dynamies of Aortic Insufficiency, Arch. Int. Med., 1915, xvi, 132. 
it occurs even when the vessels have been previously dilated by means of nitroglycerin, and (3) the condition is enhanced rather than abated by epinephrin.

He attributes the dynamic changes of aortic insufficiency to the fact that the initial interventricular tension is increased "owing to a regurgitation of pressure during diastole; this in turn causes a more vigorous ejection of a larger blood volume in the early portion of the next systole. This may be accompanied by an actual decreased ejection during the latter portion of sy'stole, thus, at once, accounting for the facts $(a)$ that the systolic decline becomes steeper and $(b)$ that the total systole output may not increase appreciably' beyond the normal."

Clinical Data.-The enormous pulse pressure which is frequently seen in aortic insufficiency with good compensation is often sufficient to alone establish a diagnosis. This finding is analogous to the other pulsatory phenomena which are so characteristic of this lesion.

Landolfi, Roch, and others have reported circulatory hippus as a sign.. It is generally manifest when the pulse is slow and the systole powerful (digitalis). Myosis corresponds with systole or increased pressure.

A high syrstolic (180 to $200 \mathrm{~mm}$.) associated with a low diastolic (60 to $30 \mathrm{~mm}$.) pressure is strong presumptive evidence in favor of aortic regurgitation. In estimating the diastolic pressure by the auscultatory method the fourth phase must be chosen as the criterion, since the fifth phase often persists down to $0 \mathrm{~mm}$. This phenomenon is suggestive but not pathognomonic of aortic insufficiency. A fall of pressure often indicates a failing ventricle. We should not speak of hypertension in cases of aortic insufficiency unless the srstolic pressure exceeds $200 \mathrm{~mm}$.

The intensity of the diastolic aortic murmur in some cases increases with a rise of systolie pressure. A fall of pressure may cause a feeble murmur to become inaudible. The arterial sound heard in some cases in the femoral artery without aortic leakage depends to a considerable extent upon the pulse pressure, but especially upon the existence of an abnormally low diastolic pressure (Dehio).

Differences in the Blood-pressure in the Arm and Leg.- Under normal conditions the systolic pressures in the arm and leg of an individual lying quietly in a horizontal position are equal. 
When the erect or an inverted posture is assumed the pressures in the arm and leg "differ by the hydrostatic pressure of the column of blood which separates the points of the measurements. In these postures the pressure in the arteries of the leg varies greatly, while in the arm the pressure is kept about the same by the mechanism which compensates for the influence of gravity." In aortic insufficiency a great difference between the arm and leg pressures in the recumbent posture has been found $(150 \mathrm{~mm}$. Hg. higher in the leg) which is of diagnostic significance. It is most marked in uncomplicated and compensated cases and diminishes as soon as mitral insufficiency and dilatation of the right heart occur. Both fever and the application of hot water lessen the difference by relaxing arterial tone in the lower extremities, ${ }^{1}$ in which the arteries are assumed to be in a contracted state in an effort to prevent cercbral anemia. ${ }^{2}$ Another explanation of the higher leg pressure is offered by Hill and Wells ${ }^{3}$ who believe that it is due to a better conduction by the pulse wave in contracted and more rigid arteries. Hill ${ }^{4}$ has recently stated that in addition to the just mentioned factors the resonating effect (periodic vibration) of the abdominal cavity also plays a part. According to the latter conception "Resonation of the tissues must be held to play an important part in the transmission of the pulse, and thereby to save the work of the heart. The work of the heart we know is largely conserved by the elastic recoil of the arteries. But this elastic recoil of the arteries is aided by the resonance of the tissues. Every artery is in intimate relationship with its immediate neighbor. 'The pulse of one individual artery is aided by the pulses of the other arteries. The vigor of the circulation depends on the tone of the tissues, on the tautness of skin and muscle, and particularly of the abdominal wall. The hardened body of the trained athlete swings in full resonance with the pulse of his heart; the soft, flabby, ill-conditioned body of the sedentary worker offers a poor slack drum for his heart to thump."

${ }^{1}$ Rolleston, H. D., On the Systolic Blood-pressure in the Arm and Leg in Aortic Incompetence, Heart, 1912, iv, 83.

${ }^{2}$ Hill, Flack, and Holzman, The Measurement of Systolic Blood-pressure in Man, Heart, 1909, i, 73; Hill and Rowlands, Systolie Blood-pressure, Heart, 1912, iii, 219.

${ }^{3}$ Wells, Russell, and Hill, Roy. Soe. Proe,, 1913, B. lxxxvi, 180; Hill and Flack, ibid., 365.

${ }^{4}$ Hill, McQueen, and Ingram, The Resonance of the Tissues as a Factor in the Transmission of the Pulse and in Blood-pressure, Proc. Royal Soc., London, 1914, Ixxxvii, 255. 
Cases Illustratixg the Differences in Arm and Leg Pressures in Aortic Insufficiency as Compared with Other Cardiac Lesions.

\begin{tabular}{|c|c|c|c|c|c|}
\hline \multirow{2}{*}{ No. } & \multirow[b]{2}{*}{ Diagnosis. } & \multicolumn{4}{|c|}{ Blood-pressure. } \\
\hline & & $\begin{array}{r}B \\
\text { Systolic }\end{array}$ & $\begin{array}{l}\text { hial. } \\
\text { Diastolic. }\end{array}$ & $\begin{array}{l}\text { Ext. } m \\
\text { Systolic. }\end{array}$ & $\begin{array}{l}\text { Uleolar. } \\
\text { Diasto }\end{array}$ \\
\hline 1 & $\begin{array}{l}\text { Arteriosclerosis, chronic nephritis, car- } \\
\text { diae hypertrophy and dilatation, em- }\end{array}$ & & & & \\
\hline 2 & $\begin{array}{l}\text { physema of lungs, portal congestion } \\
\text { Mitral and tricuspid insufficiency, car- }\end{array}$ & 171 & 106 & 167 & 107 \\
\hline & $\begin{array}{l}\text { diac dilatation, passive congestion of } \\
\text { lungs, liver, kidney's, portal system }\end{array}$ & 113 & 75 & 112 & 80 \\
\hline 3 & $\begin{array}{l}\text { Aortic obstruction and insufficiency, } \\
\text { arteriosclerosis, mitral insufficiency, } \\
\text { cardiac hypertrophy, etc. }\end{array}$ & 151 & 69 & 191 & 122 \\
\hline 4 & $\begin{array}{l}\text { Aortic obstruction and insufficiency, } \\
\text { mitral insufficiency, arteriosclerosis, }\end{array}$ & 101 & 03 & 101 & \\
\hline & Cardiac hypertrophy and dilatation. & 173 & 71 & 190 & 95 \\
\hline & aortic insufficiency & 222 & 127 & 300 & 190 \\
\hline
\end{tabular}

In Cases 1 and 2 there was no aortic leakage. 'The arm and leg pressures in the recumbent position were approximately equal. In Cases 3 and 4 (definite aortic insufficiency) there were marked differences in pressure. In Case 5, aortic regurgitation was doubtful, but the large pulse pressure, as well as the arm and leg difference, were strong evidence in favor of the existence of the lesion.

Traube's Sign.-a double tone heard over the femoral vesselsoccurs chiefly in aortic insufficiency, as does also Dutrozier's signa double murmur heard as the result of stethoscopic compression of the femoral artery. Both of these phenomena are most marked when the pulse pressure is large. The former is caused by sudden distention of the artery by the large systolic output and by the sudden distention of the femoral vein due to tricuspid insufficiency. ${ }^{1}$

Iuchard found that in aortic insufficiency of rheumatic origin the systolic pressure was increased only moderately; the diastolic pressure was subnormal; while in cases of arteriosclerotic origin both phases were markedly elevated. These findings he attributed to the greater valvular damage in Group 1 and the coincident presence of renal lesions in Group 2. ${ }^{2}$

In pure aortic obstiuction the systolic, the diastolic and the pulse pressures are generally slightly elevated, due in part to left ventricular hypertrophy and in part to general arteriosclerotic changes. The pulse pressure is small, and the pulse feels hard and small, being gradual both in onset and in disappearance.

${ }^{1}$ Schultz, W., Ueber d. Doppeltonbildung a. d. Cruralgefaessen, Deut. med. Woch., 1905 , xxxi, 2, 1381; Tice, T., Clinical Significance of Some Peripheral Signs of Aortic Insufficiency, Illinois Med. Jour., September, 1911.

${ }^{2}$ Huchard, H., and Amblard, La tension artérielle dans les insuffisances aortiques, Jour. de Practicicus, May 29, 1909. 
Aortic Aneurysm.-Many cases of aortic aneurysm have a normal arterial pressure. According to Williamson, ${ }^{1}$ blood-pressure tends to be higher in cases of simple aortic dilatation than in cases in which a distinct aneurysm is present. A pressure difference (5 to $20 \mathrm{~mm}$.) in the two arms is very commonly found, and oceurs in about the same proportion of cases whether the innominate artery is actually involved or not. Differences amounting to $30 \mathrm{~mm}$. or more occur in about one-third of the cases and indicate aneurysm rather than simple dilatation or mediastinal tumor, which latter causes inequality of tension somewhat less frequently than does aneurysm. Small inequalities of pressure are of little practical diagnostic value. Unilateral differences of $10 \mathrm{~mm}$. in the two arms without any constancy as to the side occurred in 20 per cent. of 36 cases studied by Phipps. ${ }^{2}$ They are common in arteriosclerosis. The administration of potassium iodid or the injection of sterilized gelatin, which often relieves pain, has no effect on arterial tension. ${ }^{3}$

Unilateral pressure differences may also be encountered in arteriosclerosis, in hemiplegia, and in cases of cervical rib. In the last-named condition lowering of the arm sometimes produces a demonstrable decrease in the pulse pressure.

Increased blood-pressure, especially that which is due to sudden muscular strain is, next to disease of the elastic fibers of the arterial media, the most important factor in the production of an aneurism.

Mitral Lesions.-In compensated mitral insufficiency arterial pressure is practically normal. The reëstablishment of functional efficiency after an attack of broken compensation is, as was first pointed out by Sahli, not rarely associated with a diminution of blood-pressure. In mitral lesions this rule applies to both maximum and minimum pressures. 'The same phenomena occur to an even greater extent in the broken compensation which occurs in pulmonary emphysema. In aortic and arteriosclerotic lesions the fall of pressure is much less marked. 'These apparently paradoxical phenomena are due to the fact that during the period of insufficiency the resistance to arterial outflow is increased as the result of (1) $\mathrm{CO}_{2}$ accumulation in the blood which produces peripheral vasoconstriction, and (2) owing to increased venous pressure. The physics of this phenomena are illustrated in the following diagram:

The reservoir $(I I)$ is.filled with water which escapes through

${ }^{1}$ Lancet, November 30, 1907. ${ }^{2}$ Boston Med. and Surg. Jour., 1915, clxxiii, 476.

${ }^{3}$ Mackinnon, M., Arterial Pressure in Thoracic Aneurysms, British Med. Jour., October 4, 1913 . 
$P$ (the capillaries) by means of $A$ (arteries). If the stopcock be wide open, water will rise in the standpipe $M$ (pressure) to the point $n$. If the stopcock be partially closed (increased peripheral resistance) it will rise to $m$, and even with a diminished head in $I I$ (lessened cardiac power) it will still rise to $o$.

A rise in the pulse pressure during symptomatic improvement is less constant, and when present it is more frequent in mitral lesions and in emphysema. This has been explained as due to the fact that under these circumstances the loss of compensation is the result of right heart weakness, the left heart being unaffected and being opposed by a greater peripheral tonus. With a constant blood-flow, aortic pressure and pulse pressure increase coincidently. ${ }^{1}$ The reëstablishment of compensation must be associated with better cardiac filling and more forcible contraction. As soon as

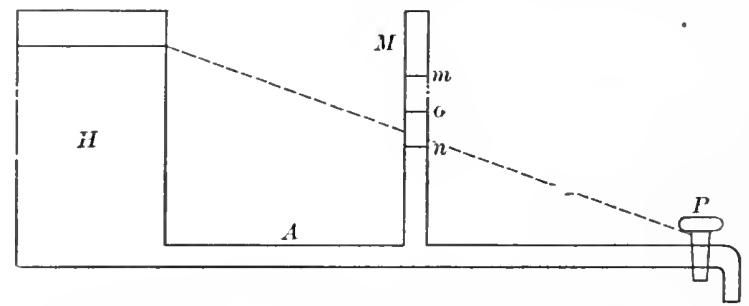

FiG. 89.-Diagram illustrating the rise of arterial pressure which results from inereased peripheral resistance. (After Lang and Manswetowa.)

an improved circulation occurs, oxygenation of the blood is increased and peripheral spasm relaxes. ${ }^{2}$ Several observers have constantly found that a temporary increase of pulse pressure occurs at the beginning of compensatory reëstablishment.

In compensated mitral obstruction pressure is, owing to peripheral vasoconstriction, more often above than below the normal. The pulse pressure is small. Hypotension may therefore be of some value in diagnosticating between hemoptysis due to tuberculosis and that due to mitral obstruction. Marked persistent high pressure without edema generally indicates renal involvement.

${ }^{1}$ Fürst and Soetbeer, Untersuchungen 1. d. Beziehungen zw. Füllung u. Druck m. d. Aorta, Deut. Arch. f. klin. Med., 1907, xc, 190; Strassburger, Ueber d. Einfluss d. Aortenelastizitit a. d. Verhältniss zw. Pulsdruck u. Schlagvolumen d. Herzens, 1907, Ibid., xei, 378.

${ }^{2}$ Lang, G., and Manswetowa, S., Zur Frage d. Veränderung des arteriellen Blutdruckes bei Herzkrankheiten während d. Kompensationsstörung, Deut. Areh. f. klin. Med., 1908, xciv, 455. (See Bibliography and numerous statements in the foregoing discussion.) 
Lagrange $^{1}$ found the arteriocapillary pressure (as indicated by the sphygmomanometer of Bouloumie) in mitral stenosis higher than normal, the arterial pressure about normal. In cases associated with arteriosclerosis, pressure was distinctly elevated (180 $\mathrm{mm}$.), and in such cases a sudden fall was prognostically grave.

Edema and Blood-pressure.- - It has been suggested that edema increases blood-pressure by mechanically compressing the peripheral arteries, and that this plays a part in the increased pressure which is seen in broken compensation. If it ever plays any such part it must be a very insignifieant one, since ordinarily edema and vascular tension bear no constant relation to each other. Pressure may either rise or fall while dropsy increases or diminishes. The occurrence of edema in cardiac no less than in renal disease is not, as formerly supposed, merely a question of hemodynamies but is closely related to certain metabolic abnormalities, notably the retention of sodium chloride in the body. Experimentally pulse pressure bears a definite relation to edema and infarction. It has been shown that edema, which occurs readily in perfused organs, may be largely prevented if an intermittent-pulsating-pressure be employed. ${ }^{2}$ Erythrocytic diapedesis occurs at the point at which pulsation of the arteries can no longer be seen. ${ }^{3}$

In uncomplicated valvular disease in which an increased pressure exists no effort should be made to lower tension by means of vasodilator remedies. Vasoconstriction is in these eases an effort to correct myocardial insufficiency by inciting the ventricles to restore the proper output (Starling). An increased pressure during compensation may be necessary to insure the requisite speed of capillary flow. ${ }^{4}$ (See p. 285.)

Myocardial Disease.-Much of what we have stated regarding arteriosclerosis and arterial hypertension applies to myocardial disease, because the latter generally is very frequently the terminal stage of the former. Since it is impossible to differentiate clinically between different forms of myocardial degeneration, they may be considered as a group.

As in valvular lesions, blood-pressure in chronic myocardial

1 Essai de Sphygmotonometrie clinique appliquee au diagnostie du retrécissement mitral, Arch. gén. de Méd., 1908, excix, 293.

${ }^{2}$ Hamel, Die Bedeutung des Pulses f. d. Blutstrom, Zeitseh. f. Biol., 1889, xxv, 474.

3 Mall and Weleh, Thrombosis and Embolism, Albutt's System of Medicine, 1899 , p. 254.

${ }^{4}$ Korke, V. T., Systolic Blood-pressure in Disenses of the Heart, Lancet, December 2,1911, p. 1547 . 
disease is variable, although with the exception of the terminal stages, an increase will usually be found. The pulse pressure is often small. Such a finding is common even when functional capacity is far from good. In these cases exertion produces oppression, dyspnea, etc., and a fall of pressure (see page 152). Stewart found a slow blood-flow as low as $0.2 \mathrm{gm}$. per 100 c.c. per minute as compared with a normal of 3 to 5 to $14 \mathrm{gm}$. Not infrequently myocardial cases show a normal systolic but a high diastolic pressure. Rosenfeld ${ }^{1}$ believes that a very low bloodpressure associated with mild anginal attacks, dilatation of the left ventricle and aorta, and auricular fibrillation especially if associated with a positive Wassermann reaction points strongly to syphilitic myocarditis. Salvarsan must be administered with caution in these cases owing to its tendency to lower blood-pressure and bring on tachycardia. 'The absence of hypertension and cardiac hypertrophy' in syphilitic arterial disease was pointed out by Cautley. ${ }^{2}$

Extrasystolic Arhythmia.-The genesis of this form of cardiac irregularity may be closely associated with arterial tension. Both experimentally and clinically it is often induced by increasing arterial pressure and abater when tension is diminished. When due to such a cause it may be interpreted as an indication that the heart is beginning to stagger under its burden. The extrasystoles themselves are less forcible than the regular contractions, and the ventricle is less well filled. Hence their systole pressure level is lower than that of the regular contraction. The postextrasystolic pressure is often higher-prolonged diastole. The existence of hypotension in association with extrasystoles, other things being equal, points to a purely functional origin of the arhythmia.

Tachycardia.-Low blood-pressure is generally associated with tachycardia, especially in fevers. In such cases the hypotension may in part be responsible for the rapid pulse, although both symptoms are in the main result of toxemia. In paroxysmal tachycardia, there is, during the paroxysm, often a fall of the arterial and a rise of the venous pressure due to insufficient diastolic filling of the ventricles.

Bradycardia is often accompanied by relatively slight bloodpressure changes. With a very slow pulse, however, the diastolic pressure is generally considerably below the normal. Gibson has reported heart block associated with a pressure of $270-80 \mathrm{~mm}$.

${ }^{1}$ Ueber Syphilitische Myocarditis, Deut. med. Woch., 1914, xl, 1044.

2 Lancet, April 6, 1901. 
Bradycardia is sometimes due to increased arterial tension. The latter condition produces central stimulation of the vagus, and this in turn a slowing of the pulse. When the hypertension is gradual in onset, an increased pulse rate is the rule, but when it is sudden, as occurs experimentally and in cases of acute nephritis, bradycardia is more apt to result (Krehl).

Pulsus Alternans.-A marked increase in pressure is the rule; in fact, this form of arhythmia, which is generally attributed to failure of ventricular contractility, may disappear with a fall and recur with .a rise of arterial pressure. Herrick ${ }^{1}$ finds the sphygmomanometer useful not only in bringing out latent cases of pulsus alternans, but also in increasing the tactile perception of the alternation. The euff is inflated to the point at which the pulse rate becomes halved. If the cuff pressure is allowed to fall somewhat lower all the beats will come through, but the alternation in their size becomes more noticeable than when the arterial lumen is uncompromised.

Auricular Fibrillation.-In auricular fibrillation successive pulse waves are so extremely variable in both size and tension that it is not possible to draw any accurate conclusions from ordinary blood-pressure readings. The largest pulse waves are often 40 or $50 \mathrm{~mm}$. higher than the smallest. An approximate average pressure may be obtained, as suggested by Janeway, by noting the pressure of the largest and the smallest pulse waves separately, a method which emphasizes the number of waves which fail to come through at a certain pressure. Silberberg ${ }^{2}$ employs a Mackenzie polygraph, upon which he records the pulsations of both radial arteries, one of them under increasing degrees of euff pressure, thus obtaining a graphic record of the relative pressure of different systoles (Figs. 89,90 , and 91 .

A more satisfactory method of estimating blood-pressure in auricular fibrillation has been suggested, based upon what is known as the relative pulse deficit. ${ }^{3}$

'The pressure in the cuff which has been raised above the highest systolic pressure is allowed to fall $10 \mathrm{~mm}$. at a time and interruptedly' checked. A count is now made of the number of pulse waves which pass the cuff at a given pressure, in relation to the number of cardiac contractions as counted per minute over the precordium by an

1 Jour. Am. Med. Assu., February 27, 1915.

2 British Med. Jour., April 6, 1912.

James, W. B., and Hart, T. S., Auricular Fibrillation; Clinical Olsservations on Pulse Deficit, Digitalis, and Blood-pressure, Amer. Jour. Med. Sci., 1914, exlvii, 63. 


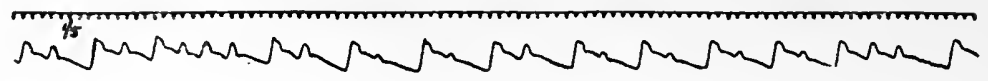

FIG. 90
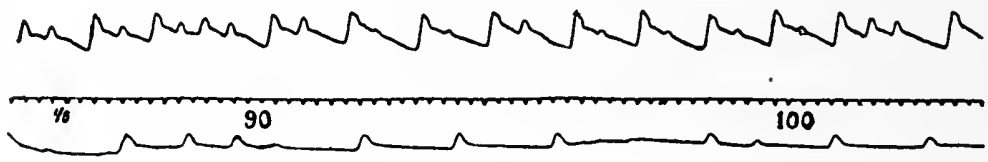

Frg. 91

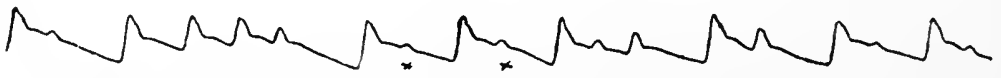

$120^{130} \quad 140$

Fia. 92

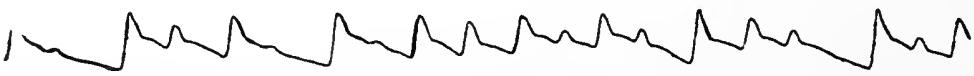

FIGs. 90, 91, and 92.-Three strips of tracing (right and left radial) taken from the same patient. Fig. 89, before brachial compression; all the beats come through. Fig. 90, the smallest beats $x x$ disappear at a pressure between 90 and $100 \mathrm{~mm}$. $\mathrm{Hg}$. Fig. 91, the largest beats fail to record at a pressure of $140 \mathrm{~mm}$. Hg.

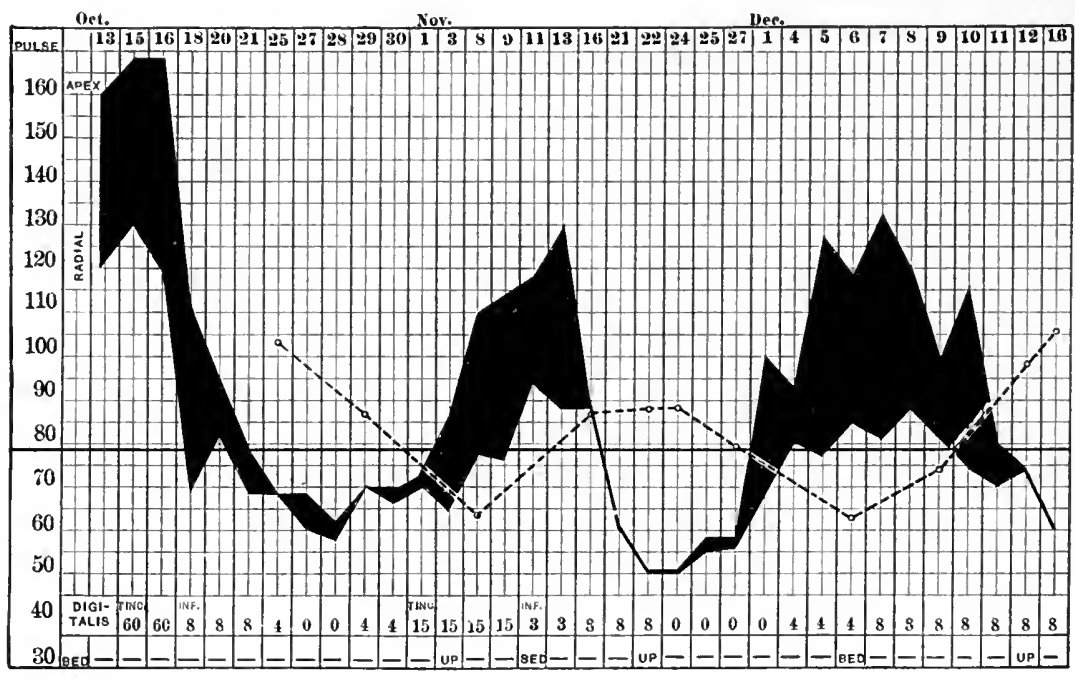

FIG. 93.-The shaded area represents the pulse deficit; the upper edge is the apex rate; the lower edge is the radial rate. The broken line indicates the range of the "average systolic blood-pressure." Digitalis figures indicate minims of the tincture and dram of the infusion. October 13, admitted to hospital. November 3, up in chair half an hour; November 9, up in chair two hours. December 4, up in chair four hours. At this time she had a crop of external hemorrhoids which caused much distress. (James and Hart.) 
assistant. 'The excess of the latter over the former constitutes the "deficit." The process is repeated until all the beats traverse the cuff-until there is no deficit.

Brachial pressure.
$140 \mathrm{~mm}$.
130 "
120 "
110 "
100 "

Radial count.
0
50
58
62
62

Apex count.
64
64
64
64
64

In this cases there is no actual deficit, because below $100 \mathrm{~mm}$. all the beats reach the periphery. But there is a relative deficit which occurs as soon as pressure is raised above $100 \mathrm{~mm}$.

The "average systolic pressure" is determined as follows:

"The apex and radial are counted for one minute, then a bloodpressure cuft is applied to the arm, and the pressure raised until the radial pulse is completely obliterated; the pressure is then lowered $10 \mathrm{~mm}$. and held at this point for one minute while the radial pulse is counted; the pressure is again lowered $10 \mathrm{~mm}$. and a second radial count is made; this count is repeated at intervals to $10 \mathrm{~mm}$. lowered pressure until the cuff pressure is insufficient to cut off any of the radial waves (between each estimation the pressure on the arm should be lowered to 0). From the figures thus obtained the average systolic blood-pressure is calculated by multiplying the number of radial beats by the pressures under which they came through, adding together these products and dividing their sum by the number of apex beats per minute. The resulting figure is what we have called the 'average systolic bloodpressure." "The following observation made on a patient will indicate the method of computation:

B. S., April 29, 1910. Apex, 131; radial, 101; deficit, 30.

Rrachial pressure.
$100 \mathrm{~mm}$.
90
80 "'
70 “"
60 “.
50

$$
\begin{aligned}
& \text { Radial count. } \\
& \begin{array}{r}
0 \\
13
\end{array} \quad 13 \times 90=1170 \\
& 47-13=34 \times 80=2720 \\
& 75-47=28 \times 70=1960 \\
& 82-75=7 \times 60=420 \\
& 101-82=19 \times 50=950 \\
& \text { Apex }=131) 7220 \\
& \text { Average systolic blood-pressure } \quad 55+
\end{aligned}
$$

Kilgore $^{1}$ has suggested the following method which is more accurate than that just described.

1 The Fractional Method of Blood-pressure Determination, Arch. Int. Med., 1915 , xli, 939. 
The Fractional Method.-Whis method differs from that of James and Hart in the fact (1) that diastolic readings are made by determining the fifth auscultatory phase and, (2) by a different interpretation of the systolic readings.

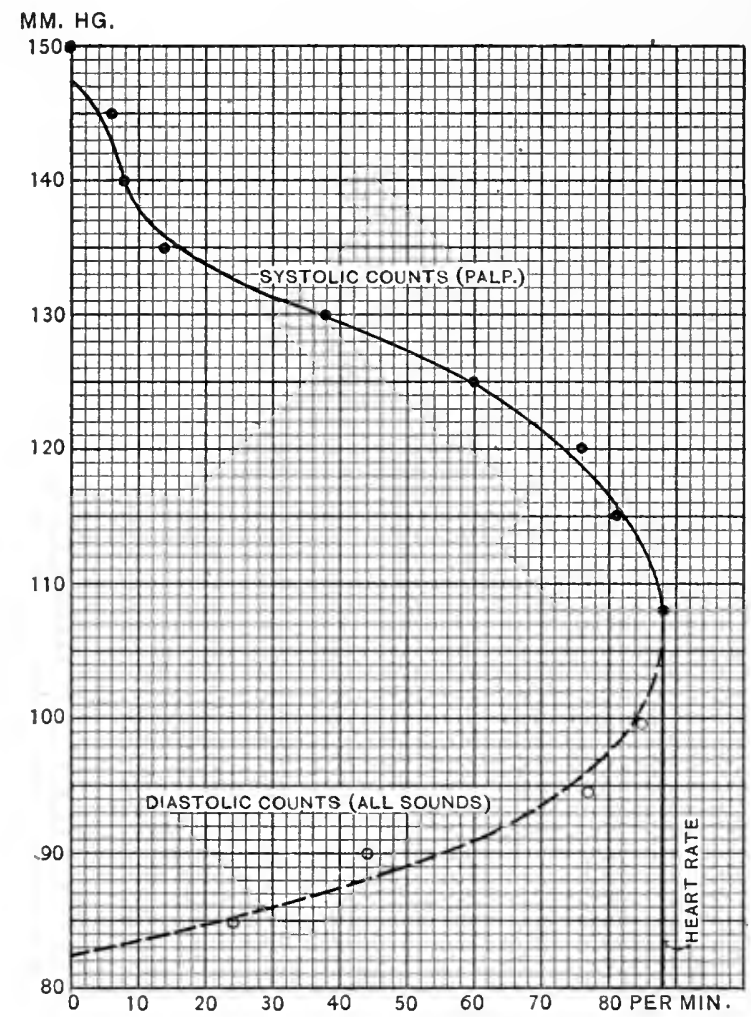

Fig. 94.- Showing the relation of systolic and diastolic pressures in a typical case of auricular fibrillation in which the diastolic pressure of one cycle never exceeds the systolic pressure of another. The numbers at the left indicate millimeters of mercury pressure in the pneumatic cuff; those at the bottom the rate per minute of beats counted in various ways. The upper line here represents the results of palpatory systolic counts, the lower line fifth phase diastolie counts. •

These readings are made at 5 instead of $10 \mathrm{~mm}$. Hg. intervals, and are tabulated as having an average value of $7.5 \mathrm{~mm}$. Thus in a given case with $S S$ pulse beats per minute and a pressure ranging between 110 and $145 \mathrm{~mm}$. the following chart and figures were obtained. 


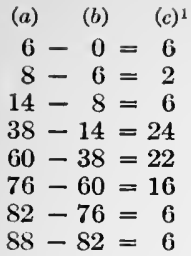

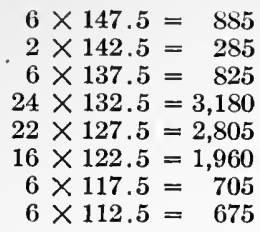
systolic pressure.

Lewis, ${ }^{2}$ who experimentally investigated the effect of auricular fibrillation upon the circulation as a whole, found that bloodpressure, while it might rise or fall or remain unchanged, generally fell, soon to regain its original level. The volume of the intestines behaved in a similar manner; the venous pressure in just the opposite manner. From these experiments it appears that auricular fibrillation does not necessarily entail any marked changes on the circulation as a whole. In human disease, however, we are dealing not only with an arhythmia but with a diseased myocardium as well, so that marked differences are readily accounted for.

Doubtless part of the blood-pressure variations which occur in association with various forms of aryhthmia are the result of mechanical respiratory influences. While Henderson has emphasized that heart rate changes in man have an important part in blood-pressure variations, and while in some cases they are the only determining influence, yet this is, according to Wiggers, ${ }^{3}$ not the only nor even the main factor in the majority of cases.

Practical Considerations.-Blood-pressure observations are often distinctly useful in the diagnosis of valvular lesions. A high systolic and a low diastolic pressure suggests aortic insufficiency, the murmur of which is often low pitched and easily overlooked. If

\footnotetext{
In the figures shown herewith the numbers on the base line correspond to the figures in column $a$ in the above tabulation; $i$. e., they represent total numbers of waves felt or sounds heard at the corresponding cuff pressures. For some purposes it would be an advantage to construct curves in which the base line figures represented the quantities in column $c, i . e_{\text {, }}$, the number of beats which have a certain pressure. In such a figure taken from a normal case there would be two narrow curves separated by the amount of the pulse pressure, while if it were from a very irregular case the diastolic and systolic curves might overlap. The work of constructing these additional curves is unnecessary if it is remembered that their lateral extent would depend on the slope of the curves here employed. In the figures herewith, the more uniform the pressure of the beats, the more nearly horizontal the lines, and the more variable the pressure the more sloping the lines.

${ }^{2}$ Fibrillation of the Auricles, its Effect upon the Circulation, Jour. Exp. Med., 1912 , xvi, 395.

${ }^{3}$ Does Cardiac Rhythm alone Determine Blood-pressure Variations? Jour. Fxp. Med., 1914, xix, 1.
} 
a double aortic murmur is heard, blood-pressure readings will often help us in deciding whether the obstruction or the insufficiency is the preponderant lesion. In aortic obstruction the pulse pressure is small. Again, in case of a presystolic murmur associated with an aortic lesion the sphygmomanometer may help us in deciding whether we are dealing with a Flint murmur or with an additional mitral obstruction. In the latter case the pulse pressure is small and the systolic pressure relatively normal.

In cases presenting the sumptoms of angina pectoris a high bloor-pressure is evidence in favor of an organic lesion, although the occurrence of a normal pressure by no means excludes it. Wellmarked hypotension in patients complaining of weakness and vertigo with dyspnea on exertion, points to a loss of vasomotor tone rather than to a myocardial lesion in which pressures are generally normal or slightly increased.

Many cardiac murmurs in advanced life are due to roughening and sclerosis of the valves, although their actual functional capacity may be but little impaired. Here, again, the absence of significant pressure abnormalities may help in solving the problem. Some light may also be thrown upon the strength of the heart muscle by the duration of the different auscultatory phases (see page 68 ). A certain amount of the increased tension seen in cardiac patients is due to their psychic state (discomfort, anxiety, fear, etc.). Janeway has suggested that a dose of morphin, which is often followed by marked and often lasting improvement in such cases, may also show how much of the increased pressure is due to the mental condition.

Pericardial Effusions.-It has been known ever since the experiments of Cohnheim that a rise of intrapericardial pressure is followed by a decreased aortic pressure, and vice versa.

Experimental Data.- Under normal circumstances the pericardium-which is in health indistensible and capable of withstanding a pressure sufficient to rupture the myocardium-acts as a protective covering to the heart under conditions of strain. It prevents cardiac dilatation beyond a certain point and in so doing adds measurably to the efficiency of the tricuspid valve. ${ }^{1}$ It prevents the further inflow of venous blood when a certain stage of dilatation has been reached, which, did it not occur, would lead to interstitial hemorrhage and myocardial rupture. ${ }^{2}$ It has been

1 Barnard, Proe. Jour. Physiol., 1898, xxii, p. 43.

${ }^{2}$ Kuno, Y., The Significance of the Pericardium, ibid., 1915. p. 1. 
shown that when the heart of a curarized animal (cat) is enclosed in a glass cardiometer very small changes of pressurc in the cardiometer system produce comparatively wide changes in the carotid pressure. Thus, an increase of $1 \mathrm{~mm}$. Hg. of extracardial pressure caused a fall of $8 \mathrm{~mm}$. in the systolic pressure. These changes occur within the space of time occupied by two heart beats. If intrapericardial pressure is raised with the heart in situ essentially similar results are obtained ${ }^{1}$ (see page 41 ).

Clinical Data. - In pericardial effusions we therefore find a lowered arterial tension and a decreased pulse pressure. Owing to the increased intracardial pressure, less blood reaches the right auricle, the blood-flow into which is normally abetted by the fact that extrathoracic pressure is higher than that in the venæ cavæ. Hence a smaller volume of blood reaches the left ventricle; and since the systolic output is decreased, arterial pressure must fall unless these effects are counterbalanced by a peripheral vasoconstriction. These same causes produce an increased venous pressure which is compensatory in nature; for if venous pressure were to fall below intracardial pressure no blood would reach the right auricle. Intrathoracic pressure being lower during inspiration and higher cluring expiration, it is evident that when venous and intrapericardial pressures are nearly equal the act of breathing will have an alternately inhibiting and accelerating effect upon the blood-flow to the heart, which results in the pulsus paradoxus-decreased volume during inspiration. For the reasons just stated, this phenomenon only occurs at a certain stage (broken compensation), and in certain cases of pericardial effusion. ${ }^{2}$

Bronchial Asthma.-Asthmatic attacks are nearly always accompanied by hypertension. 'This may result from (1) asphyxia or (2) complications, especially nephritis. The act of coughing produces a temporary marked increase of tension which is coincident with the expiratory movement.

${ }^{1}$ Lewis, Th., The Influence of Intrapericardial Pressure upon the Inspiratory Rise of Blond-pressure in Vagotomized Cats, Jour. Physiol., March 21, 1908, xxxvii.

${ }^{2}$ Calvert, W. J., Pulsus Paradoxus in Pericarditis with Effusion, Jour. Amer. Med. Assn., 1907, xlviii, 1168. 


\section{CHAPTER XI.}

\section{BLOOD-PRESSURE IN ARTERIOSCLEROSIS-VASCULAR CRISES.}

AвоUт one-third of all cases of well-marked peripheral arteriosclerosis have normal or subnormal pressures. In the remaining two-thirds the pressure is variably increased, depending mainly upon the degree of renal involvement. Arteriosclerosis is selective in its location; thus syphilis attacks the ascending aorta. Individuals who perform severe physical labor often develop peripheral vascular lesions of extreme degree, yet the pipe-stem radial artery is often a relatively benign type of the disease. Savill's ${ }^{1}$ investigations based on 400 autopsies on individuals of and over sixty years of age, showed that extensive patchy atheroma is consistent with extreme longevity, with an entire absence of symptoms or vascular complications. This same statement is applicable to marked generalized intimal and adventitial sclerosis so long as the medial arterial wall is relatively uninvolved. The foregoing statements are borne out by Ophül's studies, ${ }^{2}$ who failed to find cardiac hypertrophy in 35 per cent. of all cases of marked arteriosclerosis.

The hypothesis advanced by Hasenfeld and Hirsch that arteriosclerosis is unaccompanied by increased blood-pressure unless the arterioles in the subdiaphragmatic and splanchnic domain are the seat of disease has caused much discussion and is now not generally accepted. Sclerosis of either the large or the small splanchnic ressels is not a common finding. A spastic condition of these vessels may exist, however, which will increase tension, although this is not apt to occur once the vessels are definitely sclerotic.

Longcope and McClintock ${ }^{3}$ found that compression of the superior mesenteric artery and the coeliac axis gives rise constantly to an elevation of blood-pressure which may last for at least an hour. This rise of pressure is mechanical, due not to a reflex action

\footnotetext{
${ }^{1}$ Lancet, 1904, p. 506.

2 Subacute and Chronic Nephritis as Found in One Thousand Unselected Autopsies, Arch. Int. Med., 1912, ix, 158.

${ }^{3}$ The Effeet of Diminished Blood Supply to the Intestines upon the General Circulation, Johns Hopkins Med. Bull., 1910, xxi, No. 234.
} 
but to an increased amount of blood in the general circulation. The pressure remains increased until the excess of blood accumulates in the ramifications of the splanchnic vessels by way of collateral anastomosis. But even if this constriction is maintained for several months in dogs neither hypertension nor cardiac hypertrophy develop. ${ }^{1}$ 'This may be due to the compensatory effect of an efficient collateral circulation. Furthermore, Marchand ${ }^{2}$ was unable to establish any definite relationship between cardiac hypertrophy and the degree of arteriosclerosis of the abdominal aorta or the splanchnic vessels.

The evidence now at hand points strongly to the view that hypertension when present in arteriosclerosis is due to sclerosis of the systemic arterioles. While, therefore, the dictum that a man is as old as his arteries still holds good, yet it appears that the condition of the smallest arteries is more important than the largest ones; and, further, we are not justified in assuming that because the radial artery shows extensive disease, the more vital arteries and arterioles are therefore correspondingly involved.

The causes and pathology of vascular disease cannot be discussed here, and only the phases of the problem which have a distinct bearing on blood-pressure will be considered.

Osler states that (1) high blood-pressure is one of the causes of arteriosclerosis - the others being (2) wear and tear, (3) infections and (4) intoxications. The following classification of arteriosclerosis has been suggested by Allbutt: (1) Toxic: plumbism, diabetes, infectious diseases, notably syphilis; (2) involutionary, from senile degeneration; (3) secondary, following hypertension. Clinically, such changes are manifested by arterial hypertension, cardiac hypertrophy, albuminuria, and varying subjective phenomena; the first of these resulting from tonic contraction of the arterioles and capillaries. With prolonged increase of pressure the systemic arteries at first become thickened and hypertrophied. 'The cerebral vessels and the aorta being less liberally endowed with muscular tissue dilate, and, either with or without aneurysmal change, not infrequently rupture. The vascular damage in these cases is largely mechanical, as in occupations associated with hard labor; whereas arterial degeneration in the organs, espccially the kidneys, results mainly from toxic irritation. As a rule arteriosclerosis is unasso-

I The Effect of Permanent Constriction of the Splanichnic Arteries and the Assoeiation of Cardiae Hypertrophy with Arteriosclerosis, Arch. Int. Med., 1910, vi, 439.

2 Verhandl. Kong. f. inn. Med., 1904, xxi, 60. 
ciated with arterial hypertension. The blood-pressure in arteriosclerosis is of course higher than in youth, but the degree and the constancy of the elevation depend chiefly upon the location and character of the arterial changes, and sometimes upon the extent to which the splanchnic and renal arterioles are affected. Extensive, spontaneous and often unexplainable variations of pressure are of frequent occurrence.

Blood-pressure in Old Age.-In a study of 250 blood-pressures in the aged, Wildt ${ }^{1}$ measured the systolic pressure according to Riva-Rocci; the diastolic according to Ehret. After sixty and up to ninety years of age there is a rise of pressure (averaging 137 and $162 \mathrm{~mm}$. respectively). After ninety years the systolic pressure gradually falls. Higher values were found in women than in men. Only after eighty years are equal values found in the two sexes. Factors of hypertension are arteriosclerotic processes, or minor grades of interstitial nephritis; occasionally high-grade interstitial nephritis. The diastolic pressure is relatively low and does not keep pace with the increase in the systolic element, thus differing from the readings obtained in hypertensive cardiovascular disease. The pulse pressure is apparently dependent on the amount of sclerosis. of the aorta, as well as upon the degree of cardiac weakness.

The findings illustrate what has been stated, $i . e$., that arteriosclerosis per se does not, as a rule, produce much elevation of pressure, and that pressures exceeding $160 \mathrm{~mm}$. Hg. over prolonged intervals of time are usually attributable to other causes, especially chronic nephritis.

The great discrepancy in blood-pressure findings in arteriosclerotic cases is largely due to the different classes of patients from which statistics have been drawn. In public hospitals the cases are generally not seen until symptoms have become urgent, and often not until a terminal fall of tension has occurred; whereas cases observed in private or consultation practice among the well-to-do classes are seen during and at the beginning of the high-pressure period, the "presclerotic" stage of Huchard.

Vascular Reactions in Arteriosclerosis.-The circulation in arteriosclerosis differs in many ways from that in health. The normal arm, according to $O$. Müller's plethy'smographic studies, contains about 7 per cent. of blood; in arteriosclerosis this amount is much decreased. Abnormal local variations in blood-pressure,

\footnotetext{
1 ['eher Blutdruck im Greisenalter, Zentralbl. f. Herz. u. Gefässkrankh., 1912, iv, S. 4I-49.
} 
either unilateral or bilateral, in corresponding or different regions of the body are not uncommon in arteriosclerosis.

In the case of normal arteries the fall of pressure in passing from the aorta to the periphery is gradual. The first important fall occurs in the capillaries. In sclerotic vessels, however, vascular contraction of the arterioles and small arteries is capable of producing well demonstrable pressure differences in homologous peripheral arteries. Findlay ${ }^{1}$ found that as the age of the subject increased there was constantly increasing tendency for marked pressure variations to occur between the central and peripheral arteries, $e . g$. , brachial and digital. In the majority (80 per cent.) of the cases, the proximal vessel showed the higher pressure. These findings were especially noticeable in cases of arterial hypertension and are apparently due to arterial contraction.

This fact has been emphasized by 'Teissier, ${ }^{2}$ who believes that these localized inequalities of pressure indicate the site of the arteriosclerotic process. Thus, if the splanchnic vessels are chiefly affected, hypertension is said to occur in the dorsalis pedis artery, while increased tension in the temporal or the radial arteries points respectively to involvement of the intracranial or the intrathoracic vessels. This he explains as due to a segmental phenomenon to which the peripheral and the deep-seated arteries react similarly. In one case increased pressure in the temporal artery was soon followed by the appearance of glaucoma on the same side. In another case a similar pressure increase preceded attacks of facial paralysis, vertigo, and apoplectiform manifestations.

These observations are of great interest but must be accepted with considerable reserve on aceount of the possibility of instrumental error. The normal pressures as obtained with the Potain apparatus, which Teissier used, are 16 to $18 \mathrm{~cm}$. in the radial, 13 to $15 \mathrm{~cm}$. in the dorsalis pedis, and 8 to $12 \mathrm{~cm}$. in the temporal arteries. Even if careful allowances are made for postural variations, the unavoidable margin of error would often be greater than the actual supposedly abnormal pressure variations. (See also under Functional Tests.)

Inequality of the pressure of the pulse on the two sides of the body (5 to $15 \mathrm{~mm}$.), if constantly present and not attributable to

I The Systolie Pressure at Different Points of the Circulation in the Child and the Adult, Quart. Jour. Med., 1910, iv, 489. See also Pesei, Riforma Medica, June 7, 1909.

2 Rôle des Hypertensions partielles dans les detérminations symptomatiques de l'arteriosclerose, Bull. d. l'Acad. de Méd., February 25, 1908, Ixxii. 
any local abnormality, often points to arteriosclerotic changes. Engel $^{1}$ believes that this sign may be useful in diagnosticating between primary nephritis and arteriosclerosis. (See Aortic Aneurysm.)

Disproportionate hypertension of the arteries of the lower extremities has been described by Teissier as a constant phenomenon in abdominal aortitis. Recent observations of Pierret made with the Pachon apparatus on the pressures of the radial and tibial arteries have shown in 17 out of 20 cases higher readings in the lower limb (30 mm. systolic, $10 \mathrm{~mm}$. diastolic). Heitz ${ }^{2}$ found that the arm and leg readings differ very considerably, depending on the size of the cuff employed and whether the Pachon or the RivaRocci instruments are used.

The rapidity of transmission of the pulse wave depends upon both the condition of the vascular wall and to a less extent upon the height of blood-pressure. The normal rate is 8.3 to $12 \mathrm{~m}$. per second. In arteriosclerosis the rate has been found increased to $10.1 \mathrm{~m}$. (Friberger, $\left.{ }^{3}\right), 23 \mathrm{~m}$. (Münzer). ${ }^{4}$ In dicrotic pulses the transmission is delayed. This rapid transmission of the pulse wave, associated with a relatively low diastolic pressure often leads one to overestimate the vigor of the pulse. The rapidity of pulse transmission in different valvular defects depends far more upon the degree of tension and of arteriosclerosis than upon the character of the lesion. ${ }^{5} \quad$ (See Aortic Insufficiency, p. 235).

The Nitrite Test.--Rzentkowski ${ }^{6}$ found in healthy subjects only a slight and brief diastolic fall after the inhalation of amyl nitrite. In arteriosclerosis, on the other hand, much greater and more prolonged lowering occurred despite increased cardiac activity. He interprets these facts as indicating that the splanchnic vessels have lost their compensatory contractility, which in normal individuals prevents much disturbance of tension. Arteriosclerotic hypertension may therefore (since the peripheral vessels still retain

1 Berlin. klin. Woch., September 20, 1909, xlvi.

${ }^{2}$ Des Mensurations de Pression dans les Artères des Membres Inférieures, Arehiv. des Maladies du Cœur des Vaisseaux et du Sang, April, 1913, p. 285.

${ }^{3}$ Pulswellengesehwindigkeit bei Arterien m. fühlbarer Wandverdickung, Deut. Arch. f. klin. Med., 1912, evii, 280.

' Die Fortpflanzungsgesehwindigkeit der Pulswellen in Gesunden u. krankhaft veränderten Blutgefüssen, Kong. inn. Med., 1912, xxix, 431.

${ }^{5}$ Beiträg z. Lehre v. d. Fortpflanzungsgesehwindigkeit d. Pulswellen b. gesunden u. kranken Individuen, Samml. wiss. Arb. 1, Langensalze, Wendt and Klauwell, 1912 , p. 39.

'Untersuchungen u. d. Wirkung des Amylum nitrosum auf d. gesunde u. sklerotisehe Arteriensystem, Zeit. f. klin. Med., 1909, xlviii, 111. 
their power of dilating) be regarded as due to prolonged organic splanchnic contraction. Whether this explanation be correct or not the practical procedure may be of some value as a test of arterial functionation. F. Franck states that permanent hypertension cannot be due to vasoconstriction, a muscular spasm which he believes cannot persist indefinitely. He calls attention to the fact that vasoconstriction in one locality is sooner or later always counterbalanced by vasodilatation elsewhere.

The Stasis Reaction.-The blood-pressure is taken in the recumbent posture by the auscultatory method. Following this, the circulation in three extremities is occluded by inflation of rubber cuffs. This procedure in normal individuals when maintained for five minutes usually produces a rise of pressure of from 5 to 10 $\mathrm{mm}$. Hg. In arteriosclerotics the usual average reaction is 27 $\mathrm{mm}$., the maximum 60. An increase of pressure under these circumstances, therefore, points to arteriosclerosis. Its absence does not exclude this condition (Hertzell). ${ }^{1}$

In cases of hypertension due to fibrosis of the arterioles a marked rise of pressure (50 to $60 \mathrm{~mm}$. Hg.) occurs; if the increased pressure is due to spasm of the arterioles the rise of pressure is less (15 to $20 \mathrm{~mm}$. $\mathrm{Hg}$.) as well as more transient in character (Hare).

The Ice Reaction.--Romberg and his pupils have shown that the application of ice to the arm normally produces a diminished local blood-flow. In arteriosclerosis this reflex tends to be abolished. A merely hypertrophic artery may, however, show a normal or even an increased response. (See under Function Tests, page 173.)

The Arteriocapillary Pressure Index.-Fink ${ }^{2}$ maintains that there is a definite and characteristic relationship between arterial and capillary pressure in arteriosclerosis. If an index is established thus:

$$
\underset{\text { capillary pressure }}{\text { arterial pressure }}=\frac{17-18 \mathrm{~cm} . \mathrm{Hg} .}{9-10 \mathrm{~cm} .} \quad \begin{aligned}
& (\text { Potain) } \\
& \text { (Gaertner) }
\end{aligned}=1.80-2
$$

the quotient will lie normally between 1.5 and 2 . According to Fink we obtain in arteriosclerosis an index below 1.5, sometimes below 1. The smaller the quotient the more marked the evidences of circulatory insufficiency-dyspnea, edema, etc. But he specifically asserts that this is an index of circulatory efficiency and not

1 Die Stauungsreaktion bei Arteriosklerose, Berlin. klin. Woch. ,1913, 1, S. 535-538.

2 Nouvelles recherehes sur la valeur, du rapport des tensions artérielles et eapillaires dans l'artériosclérose, Rev. de Méd., August, 1908, p. 747. 
one of anatomic integrity. These observations would have more value if the factor of instrumental error could be reduced.

In normal individuals the local application of heat to the hand produces a fall of the brachial and a rise of the digital pressures. In arteriosclerotics negative or atypical reactions are generally observed (Dobrymin).

\section{VASCULAR CRISES.}

The term vascular crises was coined by Collier. The subject has been elaborately studied by $\mathrm{Pal}^{1}$ and others, who class under this heading conditions which result from local or general (1) vasocontraction, (2) rasodilatation, which arise without demonstrable anatomic lesions, the symptoms thus produced disappearing when normal vascular relations are reëstablished.

It is a self-evident fact that vasomotor stability, control, and compensation are among the most fundamental attributes of the animal economy. Furthermore, these intervascular relations must be adjusted and balanced to an extreme point of sensitiveness and delicacy. It is not surprising when one considers these facts that vasomotor incoördination should occur, especially under certain conditions which appear to predispose to it.

It has been clearly 'established that certain vascular domains are controlled by definite vasomotor centres, as the mesenteric vessels by the splanchnic nerves. It furthermore appears likely that the enervation of such a system may be disturbed in part after the manner of segmental distribution, and also that the disturbances may be limited to the vasomotor element alone. There exists also a definite reciprocal relationship between certain vascular areas, by virtue of which a contraction in one produces a dilatation of the other. This phenomenon has been ascribed by some to active vasodilatation, by others to a purely mechanical displacement of blood. A vascular crisis may be brought about by an abnormal (1) contraction, or (2) dilatation, in a given vascular domain, which results in a certain train of direct or indirect symptoms which may manifest themselves in the immediate neighborhood or at a distance from the seat of the vascular abnormality, or in general vascular phenomena. Certain conditions appear to predispose to such vascular crises, notably arteriosclerosis, nephritis, pregnancy, 
tabes, and plumbism. The actual symptoms produced will depend upon (1) the vascular domain involved; (2) whether vascular spasm or dilatation exists; (3) whether these changes are compensated for in other vascular domains; (4) to what extent the function of the part or organ is affected, etc. The existence of local vascular crises has been indubitably demonstrated in spasmodic contraction of the central retinal artery (see page 409).

I. Crises due to Vasoconstriction. - (a) The pectoral type, (b) the abdominal type, $(c)$ the cerebral form, $(d)$ the crises of the extremities (peripheral form), (e) the general vascular type, $(f)$ paroxysmal dyspnea.

The exact cause and mechanism of these vascular abnormalities is not easy to determine. It cannot be sought primarily in the anatomical abnormalities of the arteries in question, because the spastic phenomena are often manifested in vascular areas which are not essentially diseased or primarily affected. It may be (1) that stimuli which arise in the diseased area exert their influence upon other domains; (2) that the sympathetic system is called into play by local abnormalities in the vaso vasorum; or (3) that some pressor substance gains entrance into the blood-stream.

Angina Pectoris.-Although there is still much doubt regarding the genesis of angina pectoris, therc can be no uncertainty that many if not all cases are closely allied to vascular spasm. Whether such a contraction is purely local-limited to the coronary arteries thus causing ischemia of the myocardium or whether it be of a more general nature, cannot be definitely stated. Angina pectoris is sometimes associated with Raynaud's disease and intermittent claudication, both of which are typical examples of local vascular spasm. The writer was on one occasion taking a blood-pressure reading during which an attack of angina occurred. 'The systolie pressure, previously $160 \mathrm{~mm}$. Hg., suddenly rose to $210 \mathrm{~mm}$. and remained at that point for the few minutes during which the pain lasted. In many cases of angina, however, the pressure during attacks is either normal or actually lowered. 'The latter occurs especially in the syphilitic cases and is what one should expect if coronary arterial spasm is accepted as the cause of the attacks. It is also to be remembered that both pain and anguish have potent pressor actions and that a rise of pressure when present may be the result and not the cause of the paroxysm.

Anginal attacks may be preceded by or associated with evidences of local peripheral vascular crises-pallor, coldness, cyanosis. "It 
is probable that by disease of the aorta its sensitive endowments, those which regulate blood-pressure, are disordered-exalted or impaired; and, during or near the attack, pain, dread or distress make all measurements of blood-pressures untrustworthy."' 1 Transient aphasia, paralysis, dead fingers," etc., which are also angiospastic phenomena, may also occur coincidently with the paroxysms. ${ }^{2}$ Furthermore, angina pectoris and abdominalis may be alternately present in the same patient, and the symptoms of the latter are closely analogous to the abdominal vascular crises of plumbism, locomotor ataxia, angioneurotic edema, and purpura. Curschmann ${ }^{3}$ has reported cases illustrating the development of angina pectoris upon a basis of or as an accompaniment to peripheral vasomotor cramps, emphasizing the fact that the heart is embryologically only a specialized artery, so that its connection with the arteries is both anatomic and nervous.

The subjects of angina pectoris are the subjects of arterial disease - aortic, coronary, or general-and sclerotic arteries readily exhibit spastic manifestations. Most of these patients have previously had arterial hypertension for variable periods. During the paroxysms the pressure suddenly, markedly, and rapidly increases. While the rise may be abetted by the pain it is certainly not primarily caused by it, and it is often relieved by amyl nitrite. "In many cases the attack begins directly as a peripheral rasoconstrictor storm, with cold hands and cold feet, pallor of the face, and sweating. Nor is this simply in the so-called functional type, but in the severest forms an emotional disturbance may initiate a wide-spread contraction of the arteries. During the paroxysm it is by no means uncommon to find the radial pulse on one side much smaller than on the other" (Osler).

Many but not all cases of angina pectoris show marked hypertension (190 to $220 \mathrm{~mm}$.). 'The presence of increased pressure is often the best indication of an organic lesion, but its absence (150 to $160 \mathrm{~mm}$.) cannot be accepted as evidence of a mere functional or digestive disturbance. The following table shows the bloodpressure findings in cases of angina pectoris seen by the writer in private and consultation practice:

\footnotetext{
338 .

${ }^{1}$ Allbutt, C., Diseases of the Arteries Including Angina Pectoris, London, 1915, ii,

${ }^{2}$ Osler, Sir William, Angina Pectoris, Lancet, March 12 and 26, and April 9, 1910.

${ }^{3}$ Deut. med. Woch., 1906, xxxii, 38 .
} 


\begin{tabular}{|c|c|c|c|c|}
\hline No. & Age. & \multicolumn{3}{|c|}{ Blood-pressure. } \\
\hline 1 & 72 & 185 & and & 75 \\
\hline 2 & 73 & 200 & “ & 115 \\
\hline 3 & 49 & 140 & $"$ & 100 \\
\hline 4 & 60 & 160 & " & 110 \\
\hline 5 & 66 & 200 & $"$ & 130 \\
\hline 6 & 68 & 138 & “ & 120 \\
\hline 7 & 42 & 200 & “ & 110 \\
\hline 8 & 58 & 200 & " & 165 \\
\hline 9 & 59 & 190 & " & 100 \\
\hline 10 & 53 & 150 & “ & 100 \\
\hline 11 & 70 & 195 & $"$ & 100 \\
\hline 12 & 68 & 195 & " & 85 \\
\hline 13 & 62 & 225 & $"$ & 170 \\
\hline 14 & 72 & 160 & $" 6$ & $?$ \\
\hline
\end{tabular}

\author{
Outcome. \\ Death in paroxysm. \\ Death in paroxysm. \\ Death from rupture of veutricle. \\ Unknown. \\ Unknown. \\ Unknown. \\ Unknown. \\ Death in paroxysm. \\ Unknown. \\ Living, free from symptouns. \\ Death in paroxysm. \\ Death in paroxysm. \\ Death from apoplexy. \\ Death sudden, third attack.
}

Angina abdominalis - the abdominal counterpart of angina pectoris-is more or less closely associated with the clinical picture of abdominal arteriosclerosis. It is based upon the presence of periarteritis, local thrombosis, or vascular spasm of the mesenteric artery, and has been regarded as an intermittent dysperistalsis (Schnitzler, Ortner). The elinical manifestations consist of local abdominal pain, tenderness, tympanites, and of hypertension. Spasm of the iliac or femoral arteries leads to intermittent claudication. Disproportionately increased femoral blood-pressure has been described by French authors in association with acute abdominal aortitis.

Paroxysmal epigastric pain in arteriosclerotic subjects is not uncommon and frequently results from organic changes in the gastro-intestinal tract. These attacks are sometimes brought on by overexertion, overeating, emotional disturbances, or by lying down.

Buch $^{1}$ distinguishes two classes of arteriosclerotic abdominal pain: (a) abdominal cramp; (b) angina abdominalis. The clinical manifestations of the former, tympanites, constipation and pain, are associated with autopsy findings indicative of intestinal paresis, due to ischemia. In angina abdominalis the pain may begin in the epigastrium and remain localized there.

Paroxysmal dilatation of the abdominal aorta is a condition which occurs chiefly in run-down, emaeiated, neurasthenic women with ptosis of some or all of the abdominal organs. 'The condition is characterized by an abrupt forcible pulsation of the abdominal aorta, which is objectively visible and of which the patient is subjectively conscious. Associated with this there may be vomiting,

1 St. Petersburger med. Woch., 1904, xxix, No. 27; Arch. f. Verdauungskrankh., $1904, \mathrm{x}, 6$. 
epigastric pain, and sometimes faintness. The condition may slightly simulate aneurysm. The attacks end as suddenly as they begin, the termination being associated with the disappearance of pain and empty feelings in the epigastrium. They may last hours or days and during the attack blood-pressure generally is elevated 30 or $40 \mathrm{~mm}$. Between paroxysms. pressure is normal. ${ }^{1}$

Tympanites.-The subjects of cardiovascular disease complain greatly of tympanites. Not only is flatulence a frequent symptom among them, but very slight degrees of distention cause a disproportionately great amount of discomfort. How often the subject of angina pectoris complains only of his "stomach"!

The subject is complex. Lndoubtedly vagus effects are accountable for both conditions, but it is further clear that abdominal distention mechanically interferes with heart action. Further, Stadler and Hirsch ${ }^{2}$ showed experimentally that inflation of the bowel from the rectum causes a marked rise in blood-pressure. This may be due to a hindrance of the abdominal renous circulation, but more probably results from dyspnea, as it occurs coincidently with the rise of the diaphragm and fails to appear if the animals are curarized or if artificial respiration is practised. Funder's investigations indicate that reflex rather than purely mechanical causes are responsible for the symptoms (see page 41). BurtonOpitz has shown that distention of the intestine alone may cause a great diminution of the blood-flow in the mesenteric ressels ${ }^{3}$ (see pp. 183 and 306).

Renal and Biliary Colic.-Attacks of renal and biliary colic may be associated with marked increase in arterial pressure, a phenomenon which, while partly due to pain, is probably the result of reflex vasomotor constriction. This sudden increase of tension may account for the production of cardiac murmurs which have been reported as occasionally coincident with such attacks. ${ }^{4}$

Cerebral Vascular Crises.- The nervous phenomena of hypertension include headache, vertigo, convulsive seizures, paralyses (monoplegia, hemiplegia), aphasia, dementia, etc. These symptoms may occur spontaneously and after lasting for a few hours disappear almost as suddenly as they appeared. The generally accepted belief

1 Schlesinger, Deut. med. Woch., August 22, 1912, No. 34, p. 1592.

2 Meteorismuss u. Kreislauf, Mitt. u. 'd. Grenz. d. Med. u. Chir., xv, 3 and 4.

${ }^{3}$ Burton-Opitz, R., Ueber d. Strömung des Blutes in dem Gebiete d. Pfortader, Arch. f. d. ges. Physiol., 1908, cxiv, 479.

4 Riesman, D., Cardiac Murmurs during Attacks of Biliary Colie, Amer. Jour. Med: Sci., November, 1911. 
at present is that they are due, as was first suggested by George Peabody, ${ }^{1}$ to local vascular spasm. Organic lesions, hemorrhages or edema may perhaps also produce only transient effects, but the duration in such cases is longer and the recovery more gradual. Intermittent closing and opening of the cerebral vessels is by far the most tenable hypothesis upon which to explain brief, temporary attacks of paralysis. Sir William Osler, ${ }^{2}$ who has recently reported two very dramatic cases, states that temporary aphasia is one of the commonest of these transient manifestations. "Inability to talk, the consciousness of it, no paralysis, emotional disturbance and, within a few hours, complete recovery," are characteristics. As high as twenty such attacks may occur before the patient succumbs to some of the eventualities of arteriosclerosis. With the disappearance of symptoms the pressure falls (often 30 to $50 \mathrm{~mm}$.) to the patient's normal level.

Vertigo is, according to Finkelnberg' $\mathrm{s}^{3}$ investigations, generally associated with an increase of the minimum pressure and a decrease of the blood-pressure quotient. This diminution of pulse volume which is often associated with pallor, weakness, and syncope apparently results from cerebral anemia. Vertigo may occur in either high or low tension cases as a result of cerebral anemia. It has been suggested that rotary vertigo of labrynthine origin always results from bilaterally unequal vasomotor influences along some portion of the coördinating tracts. ${ }^{4}$

Apoplexy.-Vascular rupture is naturally more apt to occur if blood-pressure changes in addition to being great are sudden. The marked pressure effects of violent muscular action, especially when associated with volitional effort or psychic stimulation have been alluded to. It is not surprising, therefore, to find that fits of anger, fright, sudden stooping or heavy lifting, especially after a full meal, straining at stool, coitus, etc., are often the precipitating causes of vascular rupture and anginal attacks. The history of a preceding transient hemiplegic attack is not infrequently obtained in cases that ultimately succumb to apoplexy (see Hemiplegia).

Peripheral Vascular Crises.-This group includes intermittent claudication, erythromelalgia, and Raynaud's disease. In each

1 Trans. Assoe. Amer. Phys., 1891, vi, 170.

2 Transient Attacks of Aphasia and Paralysis in States of High Blood-pressure and Arterioselerosis, Canadian Med. Assn. Jour., Oetober, 1911.

${ }^{3}$ Ueber Blutdruckmessung bei Sehwindel, Münch. med. Woch., 1906, lii, 238.

4 Fowler, E. P., The Origin of Labrynthine Rest-tone, Jour. Am. Med. Assn., 1915, Ixiv, 118. 
case there is believed to be a disturbed balance between the vasodilator and the vasoconstrictor nerves.

There is reason for believing that some of these cases owe their origin to an undue excitability or exhaustion of the sympathetic nervous system. In a case reported by $\mathrm{Zweig}^{1}$ there were acute hallucinations, recurring urticaria, and sudden symptoms of hyperthyroidism. These simptoms bore no direct relation to each other but were all, it seemed, different expressions of sympathetic lability, due perhaps to some abnormality of the internal secretions, thus differing from the true exophthalmic goitre cases in which the thyroid syndrome is primary. The results of studies in this class of cases reported by different investigators have been quite variable. Curschmann² found that psychic and thermic stimuli failed to produce plethysmographic reactions. Stewart ${ }^{3}$ found that the application of cold was followed by an increased instead of a diminished flow; and Simons ${ }^{4}$ has reported great variability and așmmetry of rascular response. In Raynaud's disease it is believed that the arterioles are so contracted that blood-flow is practically occluded. Experimental evidence proves that this is quite possible. Macrilliam and Kesson ${ }^{5}$ have shown that an ocelusive spasm capable of resisting a pressure of $440 \mathrm{~mm}$. Hg. may occur in diseased arteries as large as the metacarpal and metatarsal of the horse. Balaauw ${ }^{6}$ has commented on the fact that spasm of the retinal ressels in cases of Raynaud's disease has probably never been authentically reported.

II. Angioneurotic Manifestations.-Abnormal vascular responses to stimuli produce a number of different symptoms which have been designated by Cohen as vasomotor ataxia. To this group belong dermographism, urticaria, angioneurotic edema, chilblains, etc. A patient studied by Hewlett ${ }^{7}$ who had dermographism and chilblains reacted to the local application of moderate cold (which precipitated his simptoms) by a definitely increased blood-flow in the arm exposed in comparison to its fellow. The normal reaction

I Zur Kasuistik u. Aetiologie vasomotorischtrophiseher Störungen, Berlin. klin. Wreh., 1912, xlix, 2268.

${ }^{2}$ Untersuchungen ueber d. funktionelle Verhalten d. Gefässe bei tropisehen u. vasomotorischen Neurosen, Müneh. med. Woeh., 1907, liv, 2519.

${ }^{3}$ The Neasurement of Blood-flow in the Hands, Heart, 1911, iii, 33.

4 Plethysphygmographisehe Untersuchungen d. Gefässreflexe bei Nervenkranken, Arch. f. Anat. u. Physiol. Phys., Abteil. 1910, Supplement, Band ceecxix.

${ }^{5}$ Heart, 1913, iv, 298.

${ }^{6}$ Die Augen-symptome d. Raynaudschen Krankheit, Augenheilk,, 1913, ix, Heft 5.

'Active Hyperemia following Local Exposure to Cold, Arch. Int. Merl., 1913 xi, 507 . 
to cold is of course just the reverse of this. The exudation may be serous or sanguineous, but in either case a marked local vasomotor disturbance exists. Osler has suggested that this group of ailments are all due to different degrees of the same poison. The abdominal crises of angioneurotic edema and purpura, a number of which have been attacked by the surgeons as a result of a mistaken diagnosis, have shown only local visceral edema or hemorrhage.

Abnormal local vasomotor phenomena are also exemplified by (1) the tâche cérébrale - a red line with white margins, produced by irritating the skin by drawing the finger nail across it. This is seen in meningitis, typhoid fever, etc. (2) The white line of adrenal insufficiency; a localized blanching of the abdominal skin produced by means of the finger nail, seen in Addison's disease and other conditions associated with low blood-pressure. It is directly due to local reflex capillary spasm. Neither of these two conditions is constant nor diagnostically of much importance.

Vascular Crises in Children.-While vascular crises are chiefly seen in arteriosclerotic individuals, angiospastic manifestations have also been described in children. Thus Kirsch ${ }^{1}$ has reported attacks of localized gastric pain, associated with a heaving cardiac impulse, tortuous carotids, and a tense and pulsating abdominal aorta, which he attributes to vascular spasm. Hamburger also calls attention to certain subjective symptoms relating to the heart, and to objective vascular manifestations due to this cause. ${ }^{2}$

Under the former are found palpitation, unpleasant sensations in the precordium, such as a "stitch," and a feeling of oppression. Palpitation occurs after physical exertion and to a less degree after psychical disturbances. In marked cases more or less pain and dyspnea may occur, and even symptoms comparable to the angina pectoris of adult life. These symptoms are, however, rare in comparison to palpitation. Objectively there is found a heaving somewhat diffused apex beat, and slight epigastric pulsation. 'The boundaries of the heart are normal, or but slightly increased; the sounds are clear. 'The epigastric pulsation is, in Hamburger's opinion, due to the descending aorta and not to the right ventricle. The lability of the pulse is shown, on the one hand, by a rapid increase in the rate on sitting up, standing, and on slight physical exertion; and on the other hand, by a pulsus irregularis respiratorius,

${ }^{1}$ Gefässkrisen im Kindesalter, Mitteil. d. Gesellsch. f. inn. Med. u. Kinderheilk., Wien, 1912, ii, S. 190-194.

${ }^{2}$ Münch., med. Woch., 1911, lviii, 2201. 
increasing with inspiration and diminishing with expiration. 'This symptom is most marked in severe cases, but also occurs in normal children. Among the subjective circulatory symptoms are headache, similar in onset to migraine, but involving both sides of the head, usually in the occipital region. Sudden local anemias of the brain cause dizziness and unconsciousness, these symptoms and the headache being due not to a general anemia, as is often supposed, but to changes in the vasomotor system. A tendency to cold hands and feet is another symptom of this class, and also the above-mentioned cardiac pains due, according to Nothnagel, to an ischemia of the heart muscle. Among the objective circulatory signs are noted flushing or paling on psychical disturbance, coldness of the extremities, dermographia, visible pulsation of the carotids, and increased tension of the arterial walls in the radial and temporal arteries. Certain forms of bronchial asthma probably belong to this class. There are, besides, a large number of symptoms occurring in combination, all of which can be traced back to a nervous irritability of the whole circulatory apparatus. This "vasoneurosis" is by far the most prominent and important manifestation of nervousness in later childhood (seven to fourteen years), and occurs also in younger children.

The rigidity of the arterial walls in nervousness is marked in many cases, and generally all children with apparently "thickened" arteries are nerrous. Normally, under six years, the pulse only; and not the arterial wall, is felt by the fingers; and not until the tenth year are the arterial walls frequently palpable. Pallor, especially in school children, is often construed to be an anemia, whereas it is arterial contraction due to nervousness; such children commonly have dark rings under the eyes. Marked vasoneurotic symptoms do not usually begin until about the seventh year, when the child begins to attend school. As etiological factors Hamburger attributes an inherited disposition and certain psychical and physical irritating or stimulating influences, such as fright and joy and autosuggestion on the one hand, and toxins on the other. The prognosis of the vasoneurotic condition is good if psychical causes can be discovered and avoided, and the child's disposition modified by good hygiene and intelligent mental training.

A number of the symptoms and signs which Hamburger describes are also met with in constitutionally hypotensive individuals.

The Treatment of Peripheral Arterial Spasm.-Cramps in the Legs.--Nocturnal cramps in the lower extremities are of common 
occurrence in hypertensive disease. The patient is often suddenly aroused from his sleep by an intense, muscular cramp of great severity. These cramps, which are a result of vasomotor spasm may sometimes be relieved by tightly tying a string, tape or handkerchief around the extremity above the point of pain, or by rubbing the limb vigorously with stiff brushes. The inhalation of amyl nitrite has in my experience not been very satisfactory, and immersion of the extremity in hot water is usually not feasible because the cramp is generally over before the water can be obtained. As a remedy for chilblains, Raynaud's disease and other conditions due to vasomotor ataxia, Brunton advises the use of the salicylates either with or without the bromides. He has also seen good results follow the administration of thyroid extract in small doses. (See Treatment of Animal Hypertension, p. 299.) 


\section{CHAPTER XII.}

\section{AR'TERIAL HYPER'TENSIVE CARDIOVASCULAR}

DISEASE, NEPHRITIS, ETc.

Blood-PREssure variations of from 10 to $30 \mathrm{~mm}$. Hg., and perhaps more, may be purely physiologic in their nature. We can draw conclusions of the import of pressure variations only if they exceed these amounts, or if they are more or less regularly encountered in the same individual. A systolic pressure constantly above $160 \mathrm{~mm}$., or a diastolic pressure constantly above $100 \mathrm{~mm}$. IIg., is definitely pathologic al any age. The younger the subject with such a pressure the more abnormal must it be considered. Before middle life $145 \mathrm{~mm}$. should not be exceeded.

Arterial sclerosis is often very variable in its distribution. As an example, the tendency for syphilis to involve the ascending aorta may be mentioned. Extreme degrees of nodular peripheral sclerosis often occur without corresponding involvement of the splanchnic vessels, and, on the other hand, severe renal vascular lesions may be associated with only moderate peripheral thickening. Palpation of the radial artery is therefore not a procedure by which we can estimate the seriousness of arterial degeneration, nor is the absence of an accentuated aortic second sound-although this is usually present-an indication that arterial pressure is not increased. The view that peripheral arteriosclerosis and cardiac hypertrophy are secondary to hypertension is constantly receiving corroboration. It is therefore quite erroneous to speak of "hardening of the arteries" as the cause of increased blood-pressure, since it is the latter which is the cause of the former.

The height of the diastolic pressure is often more important than that of the systolic tension. The former is far less subject to temporary variation and it further indicates the resistance which the heart has to overcome. It is also more of an index of the mean pressure than is the systolic pressure. A constant diastolic pressure of or above $100 \mathrm{~mm}$. indicates hypertension regardless of whether the systolic pressure be 180 or $140 \mathrm{~mm}$. 


\section{THE CARDIAC OVERLOAD IN HYPERTENSION.}

Under normal conditions we often find the systolic pressure about 120 , the diastolic pressure about 80 , the pulse pressure, therefore, 40. "Thus the amount of energy expended in maintaining the circulation in excess of that required to open the aortic valves and overeome the resisting pressure of 80 , is 40 . The normal load may therefore be considered $\frac{40}{80}$, or 50 per cent. of the diastolic pressure" (Stone ${ }^{1}$ ). Applying this conception to hypertension it is apparent that a systolic and diastolie pressure of 170 and $100 \mathrm{~mm}$. respectively yielding a pulse pressure of 70 would furnish a heart load of $\frac{70}{100}$ ( 70 per cent.), or an overload of 20 per cent. Stone's studies indicate that while the clinical symptoms of hypertension generally do not appear until the overload exeeeds 25 per cent., and while with a modification of the habits of life 50 per cent. may be borne, yet with an overload of 50 per cent. myocardial exhaustion may be precipitated by any sudden strain. We have not found the pulse pressure percentage of the diastolic pressure, "the cardiac load," of any value in the estimation of eirculatory efficieney.

\section{THE SIGNIFICANCE OF HYPERTENSION.}

Given a patient with a well-marked hypertension $(190 \mathrm{~mm}$. systolic and $110 \mathrm{~mm}$. diastolic) we are de facto warranted in assuming that the patient is suffering from Bright's disease, at least until further investigation has shown that the hypertension is due to some other cause. Authenticated cases, substantiated by autopsies, are on record in which the kidneys of such cases have been entirely normal, but such instances are the exeeption. ${ }^{2}$ To what the rise of pressure in such cases is due has not been satisfactorily explained, but arteriosclerosis alone without some renal involvement rarely, if ever, produces hypertension. 'The absence of albumin and casts does not necessarily exclude disease of the kidneys; furthermore, repeated and careful urine examinations will often show characteristic findings in eases which, on a few examinations, may yield negative results. It is of vital importance that nephritic changes be discovered early, and therefore an arterial pressure disproportion-

1 The Clinical Significance of High and Low Pulse Pressures with Special Reference to Cardiac Load and Overload, Jour. Amer. Med. Assoc., 1913, lxi, 1256.

2 Krehl, Ueber die krankhafte Erhöhung des arteriellen Druekes, Dent. med. Woch., 1905, xxxi, 1872; Schlayer, Untersuchungen ueber d. Funktion kranken Nieren, Dent. Arch. f. klin. Merl., 1911, cii, 371. 
ately high in relation to the age of the individual always calls for careful investigation, not only by means of the routine urine examinations but also, if possible, by some of the functional renal tests, preferably phenolsulphonephthalein. It should be remembered that in the early stages increased blood-pressure may be only intermittently present. The normal physiologic causes which raise arterial tension in nephritic eases produce an exaggerated response. In the later stages of the disease, when the eardiovascular system is weakening, the pressure often falls, but such a fall is generally coincident with the appearance of untoward symptoms and physical signs-dyspnea, vertigo, palpitation, a diminished urinary output edema, etc. These occurrences may also be brought about if a high pressure be forcibly reduced by means of the nitrites; indeed, uremia may thus be precipitated.

No definite general rules can be laid down for the prognostic or therapeutic import of a fall or a rise of blood-pressure. The significance of such an event depends both on the nature of the disease and on the state of the patient. In patients seriously ill a rise may indicate improvement, whereas the same finding in a case of arteriosclerosis or nephritis may mean that uremic manifestations are impending. Again, in the case of cardiovascular disease, increase of pressure may indicate better compensation and elimination and be associated with general improvement. A fall of pressure, however, may also be a harbinger of good omen as indicating a lessening of toxic products and carbon dioxid accumulation, and peripheral rasoconstriction-the class of case which Sahli has described as "high-pressure stasis." The fall of pressure just alluded to may be merely a fall of the systolic pressure or such a fall associated with an inerease of the pulse pressure, which, were it always demonstrable, would afford an easily comprehensible explanation of the physical improvement. Such is, however, by no means always the case. Some eases show marked improvement coincident with a deerease of both maximum and pulse pressure. Capillary pressure, upon which nutrition depends, does not vary directly with arterial pressure, but is rather dependent upon the state of the arterioles and the veins. High pressure is not necessarily associated with a good, nor low pressure with a poor, circulation in the capillaries. If the arterioles are contracted the arterial pressure, however high, fails to reach the eapillaries.

Classification of Arterial Hypertension.-A satisfaetory classification of the cases of arterial hypertension cannot be made until our 
knowledge of the etiologic factors is more complete, but certain types are more or less well differentiated. Arterial hypertension is met with in association with:

1. Demonstrable nephritis, as evidenced by urinary findings, retinal lesions, cardiac hypertrophy and accentuation of the aortic second sound.

2. Demonstrable arteriosclerosis as indicated by radial thickening (leathery arteries), tortuosity of the temporal arteries, cardiace hypertrophy, retinal arteriosclerosis (front-piece): Syphilis is the common cause of this type.

3. A combined type in which both renal and arterial lesions are manifest.

4. Non-nephritic hypertension (essential hypertension).--Regarding this variety of the disease, if the hypertension of polyeythemia is excluded, there is great difference of opinion.

Some authorities hold that latent glomerulonephritis is the cause of the increased blood-pressure; others maintain that it is a distinct pathologic entity due to some as yet unknown and probably chemical cause, such as auto-intoxication or abnormality of endocrine secretion.

Riesman ${ }^{1}$ whose classification has just been given, emphasizes the importance of recognizing a variety of non-nephritic hypertension occurring often in women past middle life, who are obese, undersized, ruddy complexioned and possessed of considerable mental and physical energy, without demonstrable arterial or renal abnormalities. The men of this group are apt to be deep-chested, robust and great expendors of energy. Obesity is per se not infrequently associated with arterial hypertension. Among 59 cases without apparent renal or vascular disease, 16 showed pressures between 145 and $160 \mathrm{~mm}$., 9 between 165 and 180, and 4 between 185 and $250 \mathrm{~mm}$. Hg. ${ }^{2}$

This class represents a large and important group of hypertension cases but many of them die a nephritic death, although this may not occur for many years after the onset of the increased pressure. The writer is of the opinion that many of these cases are due to a latent nephritis, but that certainly all of them are not. In the essential hypertension cases the blood-pressure is generally lower in both its phases than in the nephritic group. It is also more

1 Are We Exaggerating the Dangers of Arterial Hypertension? Penn. Med. Jour., Deeember, 1914.

2 Faber, Ugeskrift f. Laeger., 1915, Ixxvii, No. 23. 
amenable to treatment. As a rule the dietetic treatment of these cases may be much more liberal than in the nephritic group.

The Symptoms of Arterial Hypertension.-Cardiovascular Symptoms.-Dyspnea, palpitation, vertigo, oppression-often on going to bed-cardiac erythism, angina pectoris, muscular cramps, spontaneous or on exertion, edema of lungs, arhythmia.

Renal Symptoms.-Polyuria, with nocturnal micturition-the urine having a low specific gravity and containing a few hyaline or granular casts and a trace of albumin.

Gastro-intestinal Symptoms.-Dyspeptic manifestations with eructations, flatulence, postprandial discomfort, hyperacidity, constipation, epigastric pain, tenderness, or oppression.

Nerrous Symptoms.-Matutinal headache, nervousness, restlessness, irritability, inability to concentrate, easy fatigue from mental exertion, insomnia, numbness, tingling, migraine, transitory aphasia, hemiplegia, apoplexy.

Ocular Symptoms.--(a) Spasmodic symptoms, without definite anatomical lesions: amblyopia, transient amaurosis, hemianopia.

(b) Constant symptoms, with increased pressure of cerebrospinal and cerebro-arachnoid fluid. They tend to be progressive and may lead to subconjunctival or retinal hemorrhages, glaucoma, etc. (see page $40 \overline{7}$ ).

Aural Symptoms.-Tinnitus aurium, either with or without vertigo. (See page 261).

It frequently happens that the subjects of arterial hypertension complain only of respiratory or gastro-intestinal symptoms. Unless blood-pressure elevation and cardiac hypertrophy are sought for by the examiner the true etiological factor is apt to be overlooked. Indigestion, bloating after meals, slight dysspnea on exertion, and necturnal micturition are very suggestive manifestations. Such individuals are usually overweight—overfed and underexercisedespecially in the case of men who have a plethoric, robust appearance. But hypertension also occurs in abstemious individuals, and in such seems to bear a very frequent relation to overwork, worry, and insufficient relaxation. According to Volhard, ${ }^{1}$ the Japanese are rarely the subjects of arterial hypertension, a fact which suggests that diet and mode of life may account for their relative immunity. Severe or recurrent epistaxis is sometimes associated with and due to arterial hypertension and in such cases may act beneficially in reducing the pressure.

\footnotetext{
${ }^{1}$ Ueber d. funktionelle Unterscheidung der Schrumpfnieren, Kong. f. inn. Med.
} xxviii, 735 . 
Physical Signs.-Heart.-Hypertrophy-chiefly left-sided, accentuation, splitting or reduplication of the aortic second sound; later, systolic murmurs at the mitral or aortic areas are often found. Cardiac or aortic dilatation, arhythmia - generally extrasystolic or when compensation fails, auricular fibrillation; bradycardia, rarely pulsus alternans.

Arteries.-Flushing and duskiness of the face and hands; a hard, relatively incompressible pulse. Cervical pulsation, prominent and tortuous superficial arteries (temporal, brachial), arterial sounds. Retinal vascular selerosis, rhythmic movements of the head (pulsatile), arcus senilis.

In most of the cases high blood-pressure is mainly the result of vascular spasm. Thus there may be, for example, relatively normal bloodvessels with marked vascular contraction, or distinct fibroid changes with moderate spasm. Marked lability of bloodpressure is common. We have seen that in health pressure variations are constantly brought about by diverse influences. In arterial hypertension all such responses are exaggerated, and although arteriolar pressure is high, eapillary pressure is low. Intercurrent infections generally cause a temporary fall of pressure. When high pressure has been long standing we frequently find a loss of vascular compensation which results in a lowering of pressure, simply beeause the arterial museulature is no longer able to maintain a high degree of tonieity. 'The arteries become relaxed, tortuous, stretched, and are prominent on inspection. Such a break in vascular compensation may temporarily give relief to an overburdened heart, but usually does so at the cost of general nutrition and sometimes precedes a progressively downward course from insufficient renal aetivity. Such a broken cardiovascular compensation accounts for the secondary fall of pressure often seen in the late stages of arteriosclerosis.

The exact mcchanism which leads to dilatation of the arteries is still uncertain. The transplantation of sections of a vein into an artery, after the method of Carrel, does not lead to a dilatation of the vein, despite the greater pressure to which it is, in its new situation, exposed; in fact, a thickening and decrease in caliber results. This faet would indieate that "increased bloorl-pressure cannot in itself produce a lasting progressive dilatation of the bloodvessel."'

Moderate pressures are naturally better borne than very high

${ }_{1}$ Fischer and Schmieden, Frankfurt Zeitsch. f. Path., 1909, iii, 8. 
ones, and as a general rule ambulant patients with constant pressures of $170 \mathrm{~mm}$. are in no immediate danger. A systolic tension of 200 $\mathrm{mm}$. and over, however, renders sudden catastrophies not unlikely, although such cardiovascular strains may be borne for eight or ten years (see p. 131).

Arterial hrpertension is more common in men than in women, but high pressures are generally better and longer endured by the latter, owing to their more ready adaptability to a restricted life and to their lessened exposure to sudden hypertensive influences.

In some cases of hypertension marked slowing of the pulse is a striking clinical feature which, in the absence of local cardiac disease of the conductive system, is due to the fact that hypertension stimulates the cardio-inhibitory centre. This inhibitory action is brought about either by the direct effect of increased pressure in the ressels supplying the cardio-inhibitory nerves, or reflexly by stimulation of the peripheral nerves in the vessels. Increased pressure in the left ventricle has little effect, but in the thoracic aorta the effect is more marked. This reflex stimulation may disappear while the effect of direct pressure on the bloodvessels of the cardio-inhibitory centre still remains. ${ }^{1}$

\section{NEPHRITIC HYPERTENSION.}

The increased blood-pressure which occurs in connection with renal disease, especially with that form which is clinically designated as chronic interstitial nephritis, is the most striking and diagnostically perhaps the most important abnormality of arterial tension which is met with in the entire domain of medicine. There seems to be a tendency to revert to the old concept of Gull and Sutton, that chronic interstitial nephritis results from general and not merely local disease of the arterioles-that it is primarily a rascular disease of which the renal ehanges are but secondary manifestations. Certainly it is a fact that the clinical pictures as well as the blood-pressure findings of chronic interstitial nephritis are quite different from those seen in the "parenchymatous" variety of renal disease. Whether the rise of blood-pressure occurs before structural vascular lesions exist is still in question. The belief that it does has been put forth by von Basch ("angiosclerosis"), by Huchard ("presclerosis"), and by Allbutt ("hyperpiesis").

\footnotetext{
${ }^{1}$ Eyster and Hooker, Slowing of the Pulse from Increased Blood-pressure, Amer. Jour. Phys., 1908, xxi, 373.
} 
Hyperpiesis.-The term hyperpiesis has been applied to hypertension not due to demonstrable renal, cardiac and arterial disease. It is suspected that if unchecked this condition may be an antecedent of organic hypertension. In arteriosclerotic conditions the increased blood-pressure, at least up to a certain point, subserves a necessary function; in hyperpiesis, so far as we can determine, the pressure increase in addition to being sudden and associated with unpleasant symptoms subserves no useful function.

Glomerulonephritis.-The recent studies of Volhard and Fahr. ${ }^{1}$ have emphasized the fact that blood-pressure is increased in diffuse glomerulonephritis regardless whether the condition is acute, chronic or in its terminal stage, whereas simple degenerative nephritis (parenchymatous nephritis) - disease of the epithelial tubules-is not associated with hypertension. In the interval between the acute and the terminal stage of glomerulonephritis, increased blood-pressure, together with slight albuminuria and occasional casts are the only signs of Bright's disease, other renal tests showing normal results.

Simple Renal Sclerosis.-The highest blood-pressures are often met with in renal arteriosclerosis, in cases in which the intima of the smallest renal arterioles is thickened and in which only occasional glomeruli or tubules show abnormalities. This condition has been termed simple or benign sclerosis, since it is compatible with many years of useful life. This type of renal disease corresponds to Gull and Sutton's primary vascular disease. Its elose connection with the heart both in its symptomatology and mode of termination (cardiac failure) has led Janeway to suggest the term cardiovascular hypertensive disease. Ten or fiftcen per cent. of these cases, however, insidiously develop renal symptoms such as albuminuric retinitis, decreased phthalein elimination, polyuria or increased non-protcin or urea nitrogen in the blood. Such eases elinically and sometimes histologically represent a combination form of the simple sclerotic and the glomerulonephritic types.

In this type of renal disease eardiac hypertrophy is marked, especially if the arterial changes in the kidney begin early in life. When developed later in life the frequently assoeiated coronary sclerosis renders cardiac hypertrophy less readily possible. Among

${ }^{1}$ Die Brightisehe Nierenkrankheit, Berlin, 1914. For an excellent resumé see Austin, J. H., Prog. Med., 1915. 
268 cases studied by Volhard and Fahr, 102 had a systolic pressure above $200 \mathrm{~mm}$.; 104, between 170 and $200 \mathrm{~mm}$., and only 61 pressures below $170 \mathrm{~mm}$.

Symptoms.-The symptoms of functional hypertension may be any of those seen in the organic variety. The pressure readings associated with such symptoms are characterized by their lability, the exacerbations of pressure being evidently spastic in origin. Hyperpiesis oceurs in men after middle life, and in women at the time of the menopause (see page 396). A disturbed balance between the organs of internal secretion, especially the thyroid, the adrenals and the sexual glands, has been suggested as a possible cause.

In the rast majority of cases a systolic blood-pressure of over $160 \mathrm{~mm}$. and a diastolic pressure of over $100 \mathrm{~mm}$., if constantly present, points indubitably to that symptom-eomplex which is designated as interstitial nephritis, especially if it be associated with polyuria and urinary abnormalities. It does not necessarily mean, of course, that the individual in question will die of uremia or dropsy, for many cases succumb to cardiac or vascular lesions. Occasionally eases are encountered in which practically normal kidneys are found at autopsy, but as a general rule the small red atrophic organ is found. Many forms of renal disease are associated with a slightly increased blood-pressure, the notable exceptions being degenerative (tubular) nephritis, amyloid disease, suppurative pyelonephrosis, and tuberculosis.

Arterial hypertension is generally a condition of gradual development extending over years of time, with a tendency to increase, and with periods of spontaneous intermission or exacerbation. These latter are often, but by no means always, traceable to hygienic or dietetic variations. They are very closely associated with psychic phenomena which are generally by far the most potent factors for good or ill.

The degree of tension which can be borne without subjective consciousness is mainly an individual question, but a pressure of $180 \mathrm{~mm}$. is not often exceeded without symptoms, and constant pressures of $200 \mathrm{~mm}$. or over are generally not long maintained before leading to some sudden catastrophe, such as angina pectoris, uremia, or apoplexy. The signifieance as well as the seriousness of high blood-pressure often receives useful elucidation as the result of an ophthalmoscopic examination.

Etiology.-- Yolumes have been written based upon clinical and pathologic findings, upon thcoretic considerations, and upon 
experimental evidence to account for nephritic hypertension, since 1836, when Richard Bright ${ }^{1}$ described the disease which now bears his name, and suggested as a cause of the cardiac hypertrophy which occurs in many cases, even in the absence of valvular disease: (1) "That the altered quality of the blood affords irregular and unwonted stimulus to the organ immediately," or (2) "that it so affects the minute and capillary circulation, as to render greater action necessary to force the blood through the distant subdivisions of the vascular system."'2 'The subject, which cannot be exhaustively discussed here, has recently been most ably reviewed by Janeway, from whose article I have freely drawn. ${ }^{3}$

It is now generally believed that the cardiac hypertrophy is in nearly all cases due to arterial hypertension, and to explain the cause of the latter a number of hypotheses have been offered.

I. Mechanical Theories.-The old mechanical theory of 'Traube, which attributed the hypertension to resistance in the kidney itself, and which had almost fallen into oblivion (since ligature of the renal vessels does not produce an increase of blood-pressure), has recently been rejuvenated. Katzenstein has reported that in a dog constriction of the renal arteries, without complete obliteration, leads to an increased pressure. These results were not corroborated by others. Thus Alwens, ${ }^{4}$ by placing the kidneys in an oncometer without touching the vessels, on increasing the pressure 2 or $3 \mathrm{~mm}$. $\mathrm{Hg}$. above the arterial tension, found that blood-pressure in the aorta rose and was maintained as long as renal compression continued. But in these experiments the flow from the renal vein was diminished, almost suppressed, a fact which renders the experiment incomparable to nephritis, in which no such obstruction to venous outflow exists. Embolization of the kidney with paraffin, as practised by Müller and Maas, did not increase blood-pressure. The blood flowing through the kidneys under normal and under increased pressure generally shows no increase in flow, "evell at the

1 Jores found that the absence of hypertrophy in advanced interstitial nephritis is usual in the form known as the secondary contraeted kidney. In the typiral red granular kidney hypertrophy is the rule, with, however, occasional exceptions. Verhandl. d. deutsch. path. Gesellsch., 1908, xii, 187.

${ }^{2}$ Bright, R., Cases and Observations Illustrative of Renal Disease Accompanied with the Secretion of Albuminous Urine, Guy's Hospital Reports, London, 1836, i, 338 .

${ }^{3}$ Janeway, 'T. C., Nephritic Hypertension, Clinical and Experimental Studies, Amer. Jour. Med. Sci., 1913, exlv, 625 (bibliography).

${ }^{4}$ Experimentelle Untersuchungen ü. d. Bedeutung d. mechanisehen Theorie d. nephritischen Blutdrucksteigerung, Deut. Arch. f. klin. Med., 1909, xeviii, Nos. 2 and 3 . 
time when very pronounced rises in arterial pressure had developed" (Burton-Opitz and Lucas). ${ }^{1}$

$\mathrm{Loeb}^{2}$ demonstrated the fact that the degree of arterial hypertension bore a more or less definite relation to the degree of glomerular renal involvement, and suggested that the increased pressure was a reflex compensatory effort of the system-an attempt to supply, by means of splanchnic constriction, an adequate bloodsupply which local vasodilatation alone was no longer capable of furnishing. This attractive hypothesis has, however, been proved incorrect by Jores and others, who have shown that glomerular changes were often conspicuously slight when blood-pressure was highest, and vice versa. Furthermore, as has been recognized by many, and recently emphasized by Janeway, "amyloid disease, which is par excellence a disease of the glomeruli, in its pure form is almost invariably without effect on the blood-pressure, and without an accompanying hypertrophy of the heart. This fact is one of the most difficult to be reconciled with any theory of nephritic hypertension."

II. Chemical Theories.-The chemical hypothesis attributes the increased blood-pressure to substances in the blood as resulting from either (1) insufficient renal elimination; (2) disturbances of intemal secretion; (3) toxic substances set free by the diseased kidney.

Following the suggestion of Bright, that an altered composition of the blood might account for the cardiovascular changes, it was for a time believed that hypertension was due to retained toxic products which the kidneys failed to eliminate, which produced first spastic hypertension and later arteriolar hypertrophy.

If after remoring one kidney from a dog, portions of the remaining kidney (not exceeding two-thirds of the total kidney substance) be resected, the animals after dereloping polyuria ultimately die of cachexia. Five among eighteen dogs upon whom these graduated nephrectomies were practised by Paessler and Heineke ${ }^{3}$ showed an average blood-pressure increase of $21.5 \mathrm{~mm}$., together with left ventricular hypertrophy. They found that after destruction of a certain amount of kidney substance the quantity of urine increased, but was poor in extractive substances, especially the

1 Jour. Exp. Med., 1911, xiii, 308.

${ }_{2}^{2}$ Ueber d. Blutdruek u. Herzhypertrophie bei Nephritikern, Deutseh. Arch. f. klin. Med., 1905, lxxxv, 348.

${ }^{3}$ Versuche z. Pathologie d. Morbus Brightii, Verhandl. d. deutsch. path. Gesellsch., $1905, \mathrm{ix}, 99$. 
nitrogenous elements which tended to accumulate in the blood and the tissues. The same phenomena perhaps occur in nephritis, but they do not manifest themselves for a long time. 'There is a polyuria at the begiming; later extractive substances are eliminated in diminished quantity; finally, nitrogen accumulation occurs in the blood and tissues, forming what is designated as the products of nitrogen retention.

In a recent summary Paessler states that (a) cardiac hypertrophy in nephritis is due to the renal disease; $(b)$ as a result of the latter there occurs an increased stimulability of the vasoconstrictors causing spastic contraction of the arterioles; $(c)$ that right-sided cardiac hypertrophy is secondary to left ventricular failure. These graduated nephrectomy experiments leading first to hypertension, polyuria, and albuminuria, and later to gastrointestinal disturbances, cachexia, hypotension, and death, have been corroborated by Pearce ${ }^{1}$ and Janeway. ${ }^{2}$ The exact mechanism by virtue of which this hypertension is produced is still uncertain, but it is in all probability due to increased arterial tonus. It has been found experimentally that in gradually nephrectomized animals, arterial spasm is brought about by stimuli which in normal animals are insufficient to engender similar results. It must be borne in mind, however, that "although the results of reduction experiments are striking, the procedures by which they are obtained are not such as to involve only a single factor, but bring several forms of kidney injury into play; that is, reduction of functional substance and productive atrophic and vascular changes, accompanied by the elimination of albumin and casts" (Pearce).

Ten cases of high-grade hypertension were studied at autopsy by Herxheimer. ${ }^{3}$ In no case was the parenchyma of the kidney microscopically normal, but in most cases the changes were small in comparison with the constant high-grade arteriosclerotic vascular degenerations, especially of the arterioles. In all ten cases extensive hyaline thickening and fatty degeneration of the intima occurred. Slight or complete obliteration of the lumen was found. In some cases especially the vasa afferentia of the glomeruli were involved,

1 The Influences of the Reduction of Kidney Substance upon Nitrogenous Metabolism, Jour. Exp. Med., 1908, x, 632; A Study of Experimental Reduction of Kidney Tissue with Special Reference to the Changes in that Remaining, Ibid., 1908, x, 745.

${ }^{2}$ Note on the Blood-pressure Changes Following Reduction of the Renal Arterial Circulation, Proe. Soc. Exp. Biol. and Med., 1909, vi, 109.

${ }^{3}$ Niere und Hypertonie, Verhandl. d. deutsch. path. Gesellsch., 1912, xv, S. $211-$ 216. 
and also some loops of the glomeruli showed the fatty and hyaline degeneration. In addition, atrophy of the tubules, increase of connective tissue, infiltration of round cells were noted (the vascular changes of the old Gull and Sutton arteriofibrosis); in the other organs, in opposition to Jores's findings, there were only occasional vascular changes (in the pancreas, testicles, liver, and always much less extensive than in the kidney). Only in the spleen were vascular changes constantly found, but among 410 spleens examined there was in 170 marked hyaline change in the intima of the small vessels, even in young people, so that the findings in the spleen have no particular weight.

It seems, therefore, that although general arteriosclerotic changes occur, they are more marked in the kidneys than elsewhere, and that the arterial changes are the cause, not the result, of the arterial hypertension.

Renin.-Retention of ordinary metabolites, such as urea, does not explain arterial hypertension, and many attempts have been made to identify pressor substances in the blood. Tigerstedt and Bergmann succeded in extracting from a rabbit's kidney a substance (renin) which experimentally increased arterial tension when injected into animals. It has been suggested that autolysis of the diseased kidney causes this substance to be thrown off into the general circulation. ${ }^{1}$ Ascoli found that nephrotoxic serum possessed hypertensive properties, while Riva-Rocci and Maragliano obtained a similar substance in increased quantity in the diseased kidney. While those findings have been widely quoted, Pearce ${ }^{2}$ has definitely shown that nephrotoxins are not truly specific, that "the production of autonephrolysin by injuring one kidney was doubtful, and that Ascoli's claim of a blood-pressure raising substance could not be confirmed."

Pearce $^{3}$ has shown experimentally that a dog's kidney, unlike that of a rabbit and certain other animals, does not contain a depressor substance. Of course it does not follow that the results in human nephritis run parallel with those of experimental nephritis, but a comparison is, to say the least, interesting.

\footnotetext{
1 Shaw, H. B., Auto-intoxication; its Relation to Certain Disturbances of Bloodpressure, Goulstonian Leetures, Laneet, 1906, i, 1295, 1375, 1455.

2 The Theory of Chemieal Correlation as Applied to the Pathology of the Kidney, Areh. Int. Med., August, 1908.

${ }^{3}$ An Experimental Study of the Influence of Kidney Extracts and of the Serum of Animals with Renal Lesion on the Blood-pressure, Jour. Exp. Med., 1909, xl, 430; The Influence of Kidney Extracts on Blood-pressure, Areh. Int. Med., 1912.
} 
Moreover, the injection not only of saline kidney extract but also that of other organs except the adrenal, pituitary, and spleen, has failed to increase blood-pressure, ${ }^{1}$ and last, although by no means least, "hypertension is most extreme in those intensely chronic types of nephritis in which breaking down of kidney substance must be at a minimum if there is any at all" (Janeway).

Epinephrin.-Although first suggested by Neusser, the hypothesis that nephritic hypertension is due to increased activity of the suprarenal glands was prominently brought to the fore by $\mathrm{Vaquez}^{2}$ and the main exponents of this doctrine are still to be found in France.

Marcuse believes that as the result of the resistance thus opposed by the kidneys to the circulation, an enlargement of the inferior suprarenal artery, a branch of the renal artery, occurs. The suprarenal gland being thus orersupplied with blood secretes an increased quantity of epinephrin. The objection to this explanation lies in the fact that the inferior suprarenal artery is often lacking or, when present, is not given off from the renal artery.

From an experimental standpoint it seems well established that there exists a definite physiologic antagonism between pancreas extract and adrenalin, regarding their effect on blood-pressure in normal animals. Zondek ${ }^{3}$ has recently shown that pancreas extraet exerts its usual hypotensive effects after the production of experimental nephritis. An injection of pancreas extract is capable of completely neutralizing increased blood-pressure in uranium and mercurial nephritis, but fails to do so in the case of chromium lesions.

Although the epinephrinemia hypothesis is an attractive one, with certain facts to commend it, accumulating evidence points strongly to its failaciousness. Thus it appears (1) that there is no constant relation between the degree or even the presence of hypertension and the anatomic strueture of the glands. ${ }^{4}$ (2) The results obtained by estimating the epinephrin content of the glands in different conditions are inconclusive. ${ }^{5}$ (3) Most of the studies

1 Miller, J. L. and E. M., The Effeet on Blood-pressure of Organ Extracts, Jour. Physiol., 1911, xliii, 242.

2 Hypertension, Proc. Congrès Française de Méd., 1904, p. 338.

${ }^{3}$ Beeinflussung des Blutdrucks d. akuten Experimentellen Nephritis d. Kanninchens durch Paukreas extrakt, Deut. Arch. f. klin. Med., 1914, exv, 1.

4 Pearce, R. M., The Relation of Lesions of the Adrenal Gland to Chronic Nephritis and to Arteriosclerosis; an Anatomical Study, Jour. Exp. Med., 1908, x, 735; Borberg, Das Chromaffine Gewebe. Nebennierenuntersuchungen, Skand. Arch. f. Phys., 1912, xxviii, 91. (Abstr. Zentralbl. f. d. Ges. inn. Med., 1913, iv, 3s3.)

s Ingier and Schmorl, Ueber d. Adrenalingehalt. d. Nebennieren, Deutsch. Arch. f. klin. Med., 1911, civ, 262. 
based upon the adrenalin content of the blood are faulty because of lack of controls - in order to be conclusive the test should respond to a frog's eye, coronary artery, and intestinal ring method, and, as Janeway suggests, show not only qualitatively typical but also quantitatively possible epinephrin effects. (4) Recent physiologic evidence indicates that the adrenal glands are normally only intermittently active. They are emergency organs, but not constantly concerned with the maintenance of normal blood-pressure. ${ }^{1}$ All deductions based clinically or experimentally upon qualitative or quantitative analysis of adrenalin in the serum must be accepted with reserve. Chemical tests are out of the question, and biologic tests such as the frog eye or muscle strips, are unreliable, since substances in the blood other than epinephrin may produce the reaction. ${ }^{2}$ (5) The injection of epinephrin into the blood causes an increased glycemia which, if sufficient in amount, appears as glycosuria, and yet investigations upon patients with high arterial tension from the standpoint of hyperglycemia have shown indefinite results.

From what has preceded it is sadly evident that we are still unable to satisfactorily explain either the cause or the mechanism of nephritic hypertension. The whole subject is so complex and the evidence at hand so contradictory that judgment is difficult. Janeway, than whom no one is better qualified to speak, draws the following conclusions:

1. Hypertension may arise through purely quantitative reduction of kidney substance below the factor of safety. It is difficult to conceive of this as other than a vascular hypertonus due to retained poisons of some kind. Its clinical paradigm is the hypertension accompanying bilateral ureteral obstruction or the unfortunate surgical removal of the only functionating kidney. Possibly it is one factor which helps to produce hypertension in the contracted kidney.

2. Hypertension may arise in connection with the unknown intoxication which causes disturbances of the central nervous system, and which we call uremia. This intoxication is not one of

1 Trendelenburg, W., Ueber d. Beziehungen d. Nebennieren z. normalen Blutdruckhöhe, Ztschr. f. Biol., 1914, xliii, 155; Hoskins and McClure, The Adrenals and Blood-pressure, Arch. Int. Med., October, 1912, p. 343.

${ }^{2}$ Stewart, G. N., So-called Biologic Tests for Adrenalin in the Blood, with Some Observations on Arterial Hypertonus, Jour. Exp. Med., 1911, xiv, 377; O'Conner, Ueber d. Adrenalingehalt des Blutes, Arch. f. exp. Path. u. Pharmakol., 1912, Ixvii, 195. For other references see Janeway's article, loc. cit. 
retention, in a strict sense, although it is most commonly present in those cases of advanced nephritis which manifest marked nitrogen retention. Clinically it is associated with severe acute nephritis, sometimes at its very onset, besides the subacute and chronic inflammatory affections of the kidney.

3. Hypertension may arise in primary irritability of the vasoconstricting mechanism from unknown, probably extrarenal, causes which lead eventually to arteriolarsclerosis. In this type the disease in the kidney is the sequence, not the cause, of the generalized vascular lesion. When it progresses to a condition of extreme atrophy, resulting in the true primary contracted kidney, a renal element may be added to the existing hypertension. In some cases arteriosclerosis of the larger vessels may spread peripherally and produce a similar form of disease. In these forms of primary vascular disease it is probable that eventually wide-spread narrowing of the arterial stream bed in some cases produces a permanent organic increase in peripheral resistance.

Very recently Voegtlin and Macht ${ }^{1}$ have succeeded in isolating from the blood and serum a crystalline pressor substance, whose pharmacologic action is different from epinephrin and from any other body hitherto obtained from the blood. It produces marked cardiac stimulation and a prolonged vasoconstrictor effect. They found that its physical and chemical properties seem "to point to its relation to cholesterin on the one hand and to the cortex of the adrenal gland on the other." Gubar ${ }^{2}$ has shown that the blood-serum of nephritics has a pressor effect and Cantieri ${ }^{3}$ found that there was no relation between high blood-pressure and the cholesterin content of the blood. It is quite possible that this new substance may prove to be of considerable clinical importance. Oceasionally hypertension is caused by focal infection, sueh as pyorrhea, sinusitis, chronic tonsillitis, suppuration in the antrum, ete., presumably as a result of toxin absorption and renal irritation. The relation of hypertensive cardiovascular disease to syphilis has been considered under the heading of the latter, p. 213.

Renal Circulation and Functionation.--Renal functionation is extremely sensitive to vascular changes in the kidney. 'The kidney differs from other organs of the body by functionating more or

1 The Isolation of a New Vasoconstrietor Substance from the Blood and the Adrenal Cortex, Presence of the Substance in the Blood and its Action on the Cardiovaseular Apparatus, Jour. Amer. Med. Assoe., 1913, Ixi, 2136.

${ }^{2}$ Russk. Vratch, 1913, xx, 725.

3 Wien. klin. Woch., Oetober 16, 1913. 
less constantly and by being frequently forced to do extra work by its possessor. According to Tigerstedt, from ten to nineteen times more blood passes through the kidneys, despite their small size, than through all the other organs combined. ${ }^{1}$ The kidney normally contains about 1.63 per cent. of the total blood (Ranke). During well-marked diuresis it may contain 5.6 per cent. ${ }^{2}$ The anatomic arrangement of the ressels insures the longest possible blood-path, together with a double system of capillaries. The kidney may show an increased rate of blood-flow without an increase either in volume or in pressure, a fact which Hasebroek uses to substantiate his belief in an active vascular diastole. Renal activity is independent of general systemic pressure. ${ }^{3}$ Vasoconstriction alone may cause a great reduction of blood-flow in the kidney (B. Opitz).

The secretion of urine varies directly with the pulse pressure. ${ }^{4}$ In fact, a marked increase of pressure may produce albuminous urine, and a decrease below a certain point causes anuria.

It has generally been believed that the circulation through the kidneyss, as regards vascular dilatation or contraction, depended upon the amount of urogenous material in the blood, and that the secretion of urine increased with an increased blood-pressure and blood-flow. The increased pressure is believed to especially cause an excretion of water, the concentration of the urine depending upon the activity of the renal epithelium. Hypertension only causes a polyuria if the blood contains a definite amount of urogenous material. In case of atrophied kidneys with a restricted capillary area it is assumed that an increased pressure is required to keep up elimination. This view has had very wide acceptance from clinicians who believed that a fall of pressure meant diminished urine and often led to uremia, and who therefore regarded the hypertension as compensatory and necessary. Experimental evidence, on the other hand, in a negative way indicates that (a) increased renal resistance is not the cause of hypertension; $(b)$ that the latter does not cause an increased blood-flow through the kidneys; $(c)$ that hypertension is not accountable for the polyuria.

The results of experimentation, however, cannot in the present state of our knowledge be accepted as equivalent to what occurs

\footnotetext{
'Lehrbuch d. Physiologie des Kreislaufes, Leipzig, 1893, p. 552.

2Landergren and Tigerstedt, Skand. Arch. f. Physiol., 1892, iv, 242.

3 Weber, Arch. f. exp. Path. u. Phar., 1905, vol. liv.

${ }^{4}$ Erlanger and Hooker, Johns Hopkins Hosp. Rep., 1904, xii, 145; Hooker, Amer. Jour. Phys., 1910, xxxvii, 24.
} 
in human disease, because the entire problem is extremely complex, and because the syndrome which constitutes chronic interstitial nephritis has not yet been produced experimentally.

Clinical Phenomena.-The rise of pressure which occurs in chronic interstitial nephritis is generally regarded as a compensatory effect. A certain amount of kidney substance has been destroyed, but renal elimination must be maintained at any cost, and the cost is always a high one. More blood must pass through the renal vessels in a given time, and this Nature accomplishes by raising the blood-pressure in the renal arteries, which entails an elevation of the general systemic pressure. The latter greatly increases vascular wear and tear and, when the heart is capable of it, brings about a cardiac-chiefly left ventricular-hypertrophy.

Lawrence, ${ }^{1}$ who has recently reviewed this question, finds, however, that clinical reports are by no means unanimous in declaring hypertension to be compensatory and protective, and quotes numerous investigators to the effect that a lowering of pressure is coincident with symptomatic improvement. Lawrence's own studies show that there is no definite relation between changes in either the systolic or the diastolic pressure per se and alterations in urinary secretion, but that when an increased pulse pressure results from a diastolic fall the urinary secretion is augmented. This occurred in only half of the cases in which the increased pulse pressure was due to a systolic rise. Thus "an increase in the caliber of the vascular system seems to be more efficient in promoting diuresis than does increased pressure in the aorta and its great branches."

It is the maximum pressure which is chiefly affected in nephritic hypertension; the minimum pressure lags behind, thus affording a larger amplitude. ${ }^{2}$ In nephritic hypertension both the minute volume and the systolic output remain practically unchanged (Bergmann and Plesch); indeed, they may even be subnormal in spite of the increased systolic and diastolic pressures. Nor is the rute of flow increased. Even pressures of $200 \mathrm{~mm}$. may be accompanied by only a normal rate of flow, while a fall of pressure may actually mean an increased rate (Stewart). As a rule the blood-pressure increases gradually over a period of years, the individual appearing

${ }^{1}$ The Relation of Hypertension to Urinary Exeretion, Amer. Jour. Med. Sei., September, 1912 , p. 330.

2 Musser, J. H., Jr., The Relation of High Systolic to Diastolic Pressure, Arch. Diagnosis, July, 1914. 
in perfect health, and is discovered perhaps accidentally while the patient consults the physician for some other ailment, or it may be that a palpitation of the heart, fulness of the head, gastro-intestinal disturbances, or dyspnea on exertion cause him to seek medical advice. But high blood-pressure sometimes appears very early in cases of acute nephritis. Buttermann in one case observed a rise of $50 \mathrm{~mm}$. within forty-eight hours of the onset of albuminuria.

Lee $^{1}$ found renal lesions in 71 per cent. of his autopsied cases of hypertension, and of these in 72 per cent. the lesion was atrophic. Arteriosclerosis was present in 69 per cent., but in only 1 case could all other hypertensive factors be eliminated. Among the 15 cases in which no renal disease was found, 7 presented cerebral lesions. No patient without either nephritis or arteriosclerosis shoued a blood-pressure of over $200 \mathrm{~mm}$., and all who had a pressure constantly or repeatedly abore this figure had some renal lesion.

During four years at the Heidelberg Clinic there were 450 cases with a pressure above $140 \mathrm{~mm}$. Among 148 cases a pressure over 200 occurred ( 1 case $272 \mathrm{~mm}$.); of these there were 24 with unquestioned and 106 with doubtful nephritic symptoms. There were 16 cases which showed no albumin among the above-mentioned 148. If we consider only the cases having a pressure over 200,87 per cent. shouced renal changes. Strikingly frequent were the arterial changes, both clinically and anatomically. Among 450 cases of hypertension 44 died; 43 were autopsied with the following results; contracted kidney, 10; contracted kidney with general arterial sclerosis, 14; sclerosis of the renal arteries, 3; secondary contracted kidney, 1; secondary contracted kidney with arterial sclerosis, 3; parenchymatous nephritis, 5; arterial sclerosis, 3; arterial sclerosis with myocarditis, 2; pyelonephritis, 1; hydronephritis, 1. In other words, arterial processes played a part in 58 per cent. of the cases. ${ }^{2}$

Among 550 patients with continuously high blood-pressure, 62 per cent. of those with readings above 140 showed signs of pronounced kidney disease, as did also 80 per cent. of those with pressures above $160 \mathrm{~mm}$. Of 42 examined postmortem, every one showed anatomic changes indicating progressive kidney disease, even those in which there had been no clinical evidence of it and

\footnotetext{
'Pathologic Findings in Hypertension, Jour. Amer. Med. Assn., 1911, Ivii, 1179.

2 Schönthaler, Bericht ueber in den letzten 4 Jahren an der Heidelberger Medizinischen Klinik beobachteten Hypertonien, Dissertation, Heidelberg, 1912, p. 39.
} 
in which congested kidney or idiopathic dilatation of the heart had been diagnosticated.'

Elevation of blood-pressure occurs chiefly in the atrophic kidney when the damage is mainly glomerular. It occurs experimentally in uranium nephritis, and in such cases cardiac hypertrophy has been reported. ${ }^{2}$ It may occur in exceptional cases of parenchymatous nephritis, hydronephrosis, etc., in explanation of which fact we have only to remember that the pathologic renal changes are often complex, and the lesions rarely limited exclusively to either the epithelial or endothelial structures.

The feeding of protein bears a direct relation to the amount of nitrogen retention in the blood of nephritics, especially in interstitial nephritis associated with hypertension. This does not occur in passive congestion. Nitrogen retention is often associated with a low phthalein output and increased blood-pressure. ${ }^{3}$

The relationship between the nitrogen content of the blood and the degree of blood-pressure is, however, not constant, although patients with a high index are more subject to edema, nausea, vomiting and uremia. ${ }^{4}$

IIyperglycemia of a slight degree was found by Hopkins ${ }^{5}$ to occur in many high pressure nephritics, often in association with a low phthalein output. There is, however, no definite relation between the degree of hyperglycemia and that of hypertension. Most of the cases of nephritis without high blood-pressure have a normal amount of sugar in the blood. Venous blood-pressure is not increased in nephritis until cardiac insufficieney begins.

Sodium Chloride Metabolism and Blood-pressure.-The elimination of sodium chloride from the diet of nephritics is sometimes attended by a marked increase of the urinary output. This substance is often eliminated with difficulty and its retention in the tissues favors the retention of fluid. The effect of sodium chloride on blood-pressure is also a variable one. In some cases the institution of a salt-free diet will definitely lower blood-pressure while chlorin-

i Fischer, J., Relations between Permanently High Blood-pressure and Kidney' Disease, Deutseh. Areh. f. klin. Med., 1913, eix, Nos. 5-6.

2 Siegel, W., Ueber experimentale nephritis, Kongr. f. inn. Med., 1907, xxiv, 217.

3 Hopkins, A. H., and Jonas L., Studies in Renal Function with Speeial Reference to Non-protein Nitrogen and Sugar Concentration in the Blood, Phenolsulphonephthalein Elimination and Blood-pressure, Areh. Int. Med., 1915, xv, 964.

4 Seymour, M., The Effeet of Nitrogenous Waste Produets in the Blood in Chronie Interstitial Nephritis, Boston Med. and Surg. Jour., 1913, elxix, 795.

5 The Concentration of Blood Sugar in Health and Disease as Determined by Bang's Micromethod, Am. Jour. Med. Sci., 1915, cxlix, 254. 
ization will produce an increase in pressure perhaps associated with general fluid retention and edema of the lungs. ${ }^{1}$ Indeed it has been claimed that hypotensive properties of different medicinal substances owe their effects directly to their influence on the urinary chloride elimination. ${ }^{2}$ This riew has been strenuously opposed by most investigators. Brodzki ${ }^{3}$ was unable to produce a rise of pressure in nephritic cases by the administration of sodium chloride or meat extracts. (See page 241.)

Blood-pressure and Urinary Secretion.-The relation between blood-pressure and urinary secretion is complex and not altogether understood. It has been established, however, that a certain minimum pressure is essential to secretion and also an intermittent pressure, but above the said minimum pressure the rate of flow is much more important than the actual height of pressure. The effect of combined high pressure upon the glomerular structure has been described by J. McCrae as follows:

The capillary coil which we call the glomerulus has a large afferent ressel in which blood-pressure is high, and a small efferent vessel in which it is low. The glomerulus is therefore much more subject to wear and tear due to increased arterial pressure than are the tubular capillaries. "The kidney thus resembles a compound engine, in that most, if not all, the blood goes to the high-pressure cylinder, thence much of it goes to the low-pressure tubular capillaries, just as the stream, deprived of much of its expansive force, goes to the low-pressure cylinder; in all this the mechanical advantage is evident." (J. McCrae.) When, however, the glomeruli have as a result of incessant wear and tear, undergone hyaline degeneration, the high pressure is transferred to the tubules which stand this abnormal strain badly, and may rapidly become functionally insufficient. Experimentally it has been shown that the amount of urine excreted varies directly with the magnitude of the pulse pressure. Exceptionally sudden vascular pressure changes have an augmenting influence. The chloride, urea and total nitrogen elimination generally vary with the pulse pressure. Reasoning from the foregoing facts, a drug which will increase pulse pressure without markedly lowering the general blood-pressure or

${ }^{1}$ Löwenstein, Ueber Beziehung zw. Kochsalzenthalt u. Blutdruck bei Nierenkranken, Areh. f. exp. Path., 1907 , lvii, 137.

${ }^{2}$ Reneau, A., Rapports de la Chlorure uninaire avec l'hypertension artérielle, etc., Thèse de Lyon, 1909.

${ }^{3}$ Exper. Untersuch. u. d. Verhalten d. Blutdrucks u. d. Einfluss der Nahrung auf denselben f. chroniseher Nephritis, Deut. Arch. f. klin. Med., May, 1908. 
unduly constricting the renal arterioles, should be a good diuretic. Digitalis and strophanthus fulfil these requirements and their diuretic effect appears to be due directly to their effect on pulse pressure (Gesell). ${ }^{1}$

In disease the relation between urinary output and blood-pressure is not constant. This may be due to nervous influence by virtue of which the supply of renal blood does not stand in proportion to the general blood-pressure. It cannot be due to exacerbations of local inflammation because, as Herringham" declares, the "apparent discrepancy alters from day to day in so inconstant a manner that no fresh access of inflammation can explain it, and in the second place we should expect, if that explanation were true, that the albumin should vary with the inflammation. A fresh attack would probably result in an increase of the albumin. But no such variation takes place constantly in the albumin. The decrease of urine is not always accompanied by an increase in the proportion of albumin."

As a general rule, however, low-pressure cases excrete less urine than high-pressure cases, and the most satisfactory diureties do not have a depressor action. 'The complexity of the problem and the likelihood that numerous factors have a variable effects on blood-pressure is shown by the following observations:

"In a case of nephritis with albuminuria and persistently high arterial pressure studied by Stewart ${ }^{3}$ the pressure was reduced by forced breathing. This effect was in part due to an increased elimination of carbon dioxid by the lungs and to mechanical interference with the circulation. The administration of large doses of sodium bicarbonate was also associated with a marked fall of pressure. In another case the withdrawal of cerebrospinal fluid reduced the arterial pressure apparently by lowering intracranial pressure. No pressor substance could be demonstrated in the cerebrospinal fluid."

The Complications of Hypertension.-I. Cremia.-Chronic uremia is associated with high, acute uremia with very high, blood-pressure. The latter is sufficiently marked to be of great diagnostic value. The inerement in pressure depends largely on the height of the preceding average pressure. When this has been low, increases of 100 per cent. are not unusual.

\footnotetext{
- I'The Relation of Pulse Pressure to Renal Secretion, Amer. Jour. Physiol., 1913, xxxii, 71 .

${ }^{2}$ Kidney Diseases, London, 1912, p. 220.

So-ealled Biological Tests for Adrenalin in Blood, with some Observations ou Arterial Hypertonus, Jour. Exp. Med., 1911, xiv, 4.
} 
The cause of these sudden increments of tension is not and camnot be known until the causes of hypertension in nephritis and the nature of uremia have been elucidated, but the hypothesis that many of the uremic symptoms are the direct result of abnormal local pressure relations is extremely plausible.

The symptoms of uremia are well known and require no special consideration. All of the transient nerrous phenomena described under Cerebral Vascular Crises (see page 260) may result from uremia. Vascular spasm may be the etiologic factor in either case.

II. Paroxysmal Dyspnea.-Paroxysmal attacks of increased tension and dyspnea are not uncommon in arteriosclerotic subjects, especially with renal involvement. It is quite likely that these phenomena are due to irritation or disease of the depressor nerve in the aorta, hence they are encountered especially in cases of syphilitic aortitis. Bittor $\mathrm{f}^{1}$ has reported degeneration of the depressor nerve in two patients with aortic sclerosis (aged forty-three and fifty-six years) associated with hypertension and cardiac hypertrophy.

"Haring still more important bearing on this subject are some old experiments of François Frank ${ }^{2}$ on aortic reflexes. By irritating the inner surface of the aorta of dogs he was able to produce quite constantly certain respiratory phenomena. These consisted of three types: (1) Sudden apnea with the respiratory muscles in spasm either during the inspiratory or expiratory phase, or apnea with general inhibition of all respiratory movements; (2) tachypnea, without serere constitutional symptoms, and (3) a slow dyspnea of severe and grave form. The cause of this dyspnea he showed quite plainly was spasm of the bronchial musculature. He believed, too, that there was coincident contraction of the pulmonary artery. Associated with this type of dyspnea was a contraction of the peripheral ressels and rise of blood-pressure. Occasionally a spasm of the laryngeal muscles occurred. In other cases he was able to produce all the signs of aortic insufficiency (the capillary and collapsing pulse) save a diastolic murmur through irritation of the root of the aorta and without injury to the sigmoid valves. Stewart ${ }^{3}$ has observed this last phenomenon and considers it as a reflex from the root of the aorta, and in some experiments in which aortic insufficiency was performed on $\operatorname{dogs}$ I have repeatedly

${ }^{1}$ Deut. med. Woch., 1910, No. 46.

${ }^{2}$ Arch. de Physiol., 1890, series 5, ii, 508 and 547; Jour. de l'anat., 1877, xiii, 545.

${ }^{3}$ Archiv, Int. Med., 1908, i, 102. 
confirmed this observation. That Frank's respiratory phenomena have not been noted since is almost certainly due to the method of experimentation, for in Stewart's experiment, and in those which others have performed, full ether anesthesia or artificial respiration was employed.

"It is thus evident that disturbing reflexes may be set up experimentally in animals by irritation of the root of the aorta, and there is no reason to suppose that the same thing should not be true for man. The dyspnea caused by bronchiospasm, and the contraction of the peripheral arteries producing heightened blood-pressure in the experimental animal is a close reproduction of the paroxysmal dyspnea as it occurs in syphilitic aortitis, and it seems quite justifiable to suggest that the two conditions are the same. It would be difficult to explain the increase in blood-pressure which occurs during these attacks in man on the presence of pain or of cyanosis, for pain is frequently absent and dyspnea may continue for some time (fifteen to thirty minutes) after the sudden drop in bloodpressure which comes with the relief of acute symptoms. Our observations, therefore, seem to lend strong support to the idea that these symptoms are dependent on a reflex generated at the root of the aorta by the syphilitic inflammatory process. ${ }^{1}$ 'The attacks have been found to consist of three phases: (1) A very high systolic and diastolic pressure 210-190; (2) falling pressures in which the diastolic may remain disproportionately high (a bad sign); (3) a gradual return to the normal. ${ }^{2}$

Paroxysmal dyspnea may also occur when the cerebrospinal pressure becomes too high. Such attacks may be associated with cerebral symptoms-headache, vomiting, vertigo, and with subjective oppression and respiratory acceleration, but without preëxisting asthmatic symptoms. It has been suggested that although certain forms of non-cyanotic dyspnea frequently described as "renal" or "cardiac" are associated with high blood-pressure, they are basically due to renal defects and are directly dependent upon an acid intoxication. ${ }^{3}$ Peabody's ${ }^{4}$ studies showed that when acidosis, as indicated by the alveolar carbon dioxid tension, occurs in cardiac and cardiorenal disease, it is associated with an increased stimula-

${ }^{1}$ Longeope, Arch. Int. Med., January, 1913.

2 Amblard, L. A., La Tension Artérielle dans l'oedem aigu du Poumon, Presse Médicale, 1911 , xix, 657 .

${ }^{3}$ Lewis, Ryffel, Wolf, Cotton, and Bareroft, Observations Relating to Dyspnea in Cardiac and Renal Patients, 1913, Heart, v, 45.

4 The Effect of Carbon Dioxid in the Inspired Air on Patients with Cardiae Disease, Arch. Int. Med., 1915, xvi, 846. 
bility to carbon dioxid in the inspired air. In patients of this class dyspnea occurs more readily than in normal subjects or in patients without acidosis. He was led to conclude that while acidosis is probably not the only factor in cardiorenal dyspnea it may play a considerable role. A lowering of the blood-pressure often brings relief.

When dyspnea (asphyxia) is the cause of the hypertension, bleeding, digitalis and general measures are indicated. In the Cherne-Stokes trpe of breathing, lumbar puncture, which relieves cerebrospinal pressure, is in order, although it has not much effect upon the general pressure. Morphin is useful in either type. ${ }^{1}$

III. Cheyne-Stolies Respiration.-Cheyne-Stokes breathing is frequently encountered in association with arterial hypertension. This symptom when occurring in association with experimentally increased intracranial tension has been shown by Cushing ${ }^{2}$ to be accompanied by high pressure during hyperpnea and low pressure during apnea. The importance of this fact from a clinical standpoint in order to differentiate between Cheyne-Stokes respiration with and without increased intracranial pressure was demonstrated by Eyster; ${ }^{3}$ the findings of the latter were corroborated by Pollock, ${ }^{4}$ who explains the exact mechanism of the symptoms as follows:

"The intracranial tension being higher than the general bloodpressure, cerebral anemia exists and apnea is present. The vasomotor centres are automatically stimulated to raise the general blood-pressure in an effort to produce an equilibrium between it and the intracranial tension. As the general blood-pressure rises, respiratory movements recommence; at their height the vasomotor centres are no longer stimulated, the general blood-pressure again falls, and the respiratory movements diminish and finally cease."

Hyperpnea is associated with increased amplitude of the apex beat, and the pulse rate increases with the rise of blood-pressure. Arhythmia and prolongation of the $a-c$ interval may also occur. Symptomatic relief often follows lumbar puncture, which temporarily reduces intracranial tension.

From Eyster's investigations it would seem that the stimulability of the respiratory centre is at a permanently low level, which requires an abnormally intense stimulus to produce a response, the stimulus being supplied by the toxic products which have accu-

\footnotetext{
${ }^{1}$ Pal, J., Paroxysmale Hochspannungsdyspnea, Vienna, 1907.

2 Amer. Jour. Med. Sei., 1902, exxiv, 375; 1903, exxr, 1017.

3 Johns Hopkins Hosp. Bull., 1906, xvii, 296.

4 Blood-pressure in Cheyne-Stokes Respiration, Areh. Int. Med., 1912, ix, 406.
} 
mulated in the blood through apnea and low pressure. 'The administration of $\mathrm{CO}_{2}$ toward the end of the hyperpneic period has in one instance prevented the recurrence of the apneic periods. ${ }^{1}$

IV. Acute Pulmonary Edema.-Pulmonary edema may oceur in patients with chronic vascular hypertension. Attacks are preceded by a rise of both systolic and diastolic pressures. During the attack the maximum pressure, which has been very high (240 to $280 \mathrm{~mm}$.), falls greatly, the minimum slightly. At such times venesection, although often beneficial, exerts but little effect on the pressure. After the attack, in cases which recover, pressure rises gradually to the normal. These symptoms, as was first shown by Welch and Cohnheim, ${ }^{2}$ find their explanation in the fact that a temporary left ventricular failure, while causing a fall in the systemie circulation, produces a great stasis rise in the pulmonary vessels which the right ventricle is no longer able to overcome, and which either in isolation or in association with toxins leads to serious oozing or vascular rupture into the pulmonary parenchyma. Experimentally, epinephrin may produce pulmonary edema through vascular degeneration, alveolar inflammation; and increase of pressure. It may also be produced by clamping the aorta or eompressing the left ventricle. The primary disturbance, therefore, seems to be some influence on the nervous system which interferes with the normal regulation of the correlated vaseular tension, heart action, and respiration in the case of an already diseased heart. ${ }^{3}$. It has been suggested that attacks of pulmonary edema in nephrities are the result of an effort on the part of the system to rid itself of irea and chlorides. ${ }^{4}$ Among 405 cases of pulmonary edema postmortem, Coplin found renal lesions in 333.3. An early and marked fall of pressure may result from cardiac exhaustion.

Prognosis.-Chronic arterial hypertension is a serious condition which generally spells chronic interstitial nephritis. The degree of hypertension bears no constant relation to the severity of the nephritis as determined by the routine urine examination, the phthalein elimination or the amount of nitrogen retention. The mildest eases may show the highest pressures.

\footnotetext{
1 Observations on Two Cases of Cheyne-Stokes Respiration, Jour. Physiol., 1906, xxxiv, 6 .

2 Gesammte Abhandl., Berlin, 1885, p. 594.

${ }^{3}$ Amblard, A., Presse Médicale, August 12, 1912; Petren, Berlin. klin. Woch., December 27, xlvi.

${ }^{4}$ Lesieur, Froment, and Roehaix, Oedemes aigus du poumon. Comparison du taux de l'uree et de chlorures dans le serum sanguin et dans l'expectoration, Soc. méd. des Hôp., Paris, November 19, 1909.
} 
The absence of hypertension in a case which presents the other clinical evidences of chronic interstitial nephritis suggests the existence of a tuberculous, syphilitie or amyloid lesion of the kidney. When associated with severe cardiac symptoms and retinal hemorrhages a lethal termination may be expected within a few years. The teaching was formerly current that nephritic retinal hemorrhages were invariably followed by death within a period ranging from six months to two years. While this is doubtless true where hemorrhages are severe and recurrent, yet in the case of well-to-do patients who can and will modify their manner of life, there can be no question that such a prognosis is unnecessarily austere. Many calses of retinal hemorrhages are not seen by the ophthalmologist in their early stages. Plenty of cases are on record in which patients have lived ten years or more after the onset of nephritie retinal hemorrhages. It is a common observation that patients having a pressure ranging around $180 \mathrm{~mm}$. (systolic) and $110 \mathrm{~mm}$. (diastolic) without marked urinary findings, or of ocular or cardiae symptoms, may live for many years. Strauss ${ }^{1}$ has reported the case of a patient who lived five years with a systolic pressure ranging between 260 and $270 \mathrm{~mm}$. Hg. (See page 131.) Hypertensive eases are apt to have exacerbations of pressure $(200 \mathrm{~mm}$. and over) associated with more or less troublesome symptoms-insomnia, nervousness, irritability, weakness, fatigue, inability to concentrate attention, mental depression, dyspnea, precordial oppression, ete.-which will under appropriate treatment subside. Such cases are regarded as bad insurance risks, and justly so, and yet with an intelligent comprehension of their condition and a willingness to curtail the expenditnre of chergy, they may leal lives of usefulness and comparative comfort for many years. 'These statements are borne out by Paessler's" graduated nephrectomy experiments, in which he showed that rears of comfort and activity were compatible with a pressure of 200 to $220 \mathrm{~mm}$. The prognosis, therefore, of ten hinges quite as much on the individual as on the disease. The oceurrence of wellmarked edema in hypertensive cases is more serious than in cases of ralvular disease. The following rules, as laid down by Lichty, ${ }^{3}$ indieate the greneral lines upon which a prognosis may be hased:

"1. Where hypertension exists with but little or no recognizable disturbed function of other organs the outlook is most favorable.

'Deut, med. Woch., 1915, xli, No. 16.

samml. klin. Vortringe, No. $40 s$.

"Hypertension: A Report of Cases under Prolonged Observation, and a Protest Agninst some Ldens, Am. Jour. Med. Sei., May, 1913. 
(2) Where it is associated with marked disturbance of any one other organ alone it is more serious. (3) Where it is associated with grcat defect of several other organs, such as kidney insufficiency, cardiac insufficiency, cirrhosis of the liver, and lesions of the gastrointestinal tract, the prognosis is most serious. (4) When the hypertension is associated with symptoms and physical signs which disappear after a more or less active and prolonged treatment which, however, fails to lower the tension, a favorable prognosis is not unwarranted. Such cases are rather frequent, and I believe it is just as unreasonable to take an unfavorable view of them as it is to predict a speedy fatality in all cases of pulmonary tuberculosis where the bacilli are found in the sputum. (5) When the tension is found in connection with changes in organs producing such extreme symptoms as dropsy, ascites, cyanosis, and orthopnea, and when these symptoms cannot be relieved, whether the tension is modified or not modified by heroic treatment covering five to eight weeks, then defeat is sure."

As to the cause of death in hypertension, this may result from apoplexy, uremia, angina pectoris, broken cardiac and vascular compensation, or from intercurrent disease. Among 565 cases of renal disease at St. Bartholomew's Hospital 209 died from renal discase, as follows:

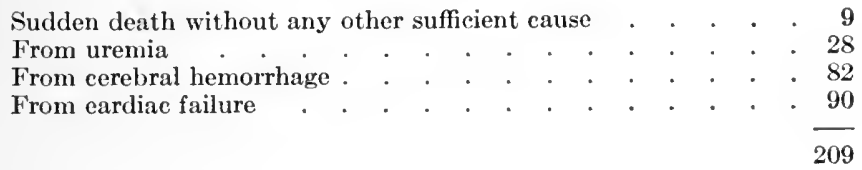

The following tables are taken from Janeway's article based on a study of 100 cases with a blood-pressure of or above $170 \mathrm{~mm}$. $\mathrm{Hg}$.

The Age of the Patients.

Below 20 years

20 to 29 years

30 to 39 years

40 to 49 years

50 to 59 years

60 to 69 years

70 and over
2 cases, about 2 per cent.

0 cases, about 0 per cent.

3 cases, about 3 per cent. 24 cases, about 21 per cent. 39 cases, about 34 per ccut. 32 cases, about 28 per cent. 14 cases, about 12 per cent. 
The Duration of the Illness in Relation to the Causes of Death.

\begin{tabular}{|c|c|c|c|c|c|c|c|}
\hline \multirow{2}{*}{ Causes of death. } & & \multicolumn{6}{|c|}{ Duration. } \\
\hline & & & $\begin{array}{l}\text { Ave } \\
\text { Years }\end{array}$ & age. & $\begin{array}{l}\text { Longest. } \\
\text { Years. }\end{array}$ & $\begin{array}{l}\text { Sho } \\
\text { Years. }\end{array}$ & st. \\
\hline Cardiac insufficiency . & & 26 & 3. & 10 & 10 & .. & 4 \\
\hline Acute & & 14 & 3 & & 8 & .. & 5 \\
\hline Chronic uremia & & 21 & 3 & 1 & 7 & .. & 4 . \\
\hline Cerebral apoplexy . & & . 14 & 4 & 6 & 11 & . & 11 \\
\hline Angina pectoris & & 3 & 4 & 3 & 6 & 3 & 6 \\
\hline Acute edema of lungs & & 4 & 3 & . & 4 & 1 & 8 \\
\hline
\end{tabular}

The Immediate Cause of Death.

\begin{tabular}{|c|c|c|c|c|c|c|c|c|c|c|c|c|}
\hline Gradual cardiac insu & ufficient & & & & • & $\cdot$ & & & & & & \\
\hline Uremic convulsions & or sudd & den & coma & . & . & . & • & & . & & . & \\
\hline Chronic uremia & . . & . & . & . & . & . & ${ }^{\circ}$ & . & . & . & . & 20 \\
\hline Uremic psychosis & . . & . & . & . & . & . & 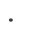 & & . & & & \\
\hline Cerebral apoplexy & & . & . . & . & . & . & . & · & . & . & . & \\
\hline Acute edema of the & lungs & . & .. & . & . & . & . & • & . & & . & \\
\hline Angina pectoris . & & & . & . & . & . & . & . & - & & & \\
\hline Sudden death (uncla & assified) & & . & $\theta^{\circ}$ & . & . & . & • & . & & . & \\
\hline Progressive anemia & . & . & . & . & . & . & - & . & . & . & . & \\
\hline Acute pneumonia & . & . & . & . & . & . & . & . & . & . & . & \\
\hline Unrelated diseases & . & . & . & . & & . & • & & & & & \\
\hline
\end{tabular}

Stone ${ }^{1}$ has called attention to the fact that the cardiorascular type of hypertension can be differentiated from the nephritic, or as he prefers to designate it, the cerebral type, by the pulse pressure and the cardiac load (see page 267). Thus in arterial hypertension associated with valvular or myocardial lesions, the diastolic pressure is persistently lower than in the cerebral type. Hence with an equal systolic pressure the overload is increased-in 24 patients to an average of 46 per cent. Such a differentiation has a good deal more than an academic importance because both prognosis, therapy and mode of death vary very distinctly in the two types.

Hypertensive disease as seen in hospitals presents a very different picture from that seen in private practice. In the former instance we see chiefly the terminal stages. Since the alleviation of symptoms as well as the prolongation of life depends mainly upon an early recognition of the condition, it is important to know the early symptoms from which patients have suffered. These are shown in the following table from Janeway's recent article. ${ }^{2}$

1 The Differentiation of Cerebral and Cardiac Types of Hyperarterial Tension in Vascular Disease, Arch. Int. Med., 1915, xvi, 775.

${ }^{2}$ A Clinical Study of Hypertensive Cardiovascular Disease, Arch. Int. Med., 1913, xii, 755 . 
The Relation of Prominent Early Symptoms with High Blood-Pressure to Causes of Death.

\begin{tabular}{|c|c|c|c|c|c|c|c|c|c|c|c|c|}
\hline & & & & & tom & & & & & & & \\
\hline 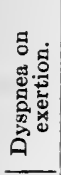 & 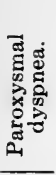 & 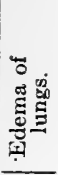 & 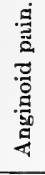 & 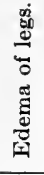 & 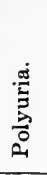 & 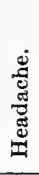 & 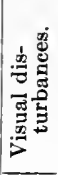 & 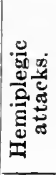 & 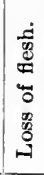 & 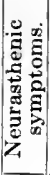 & Causes of death. & 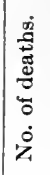 \\
\hline 24 & 13 & 1 & 5 & 7 & 8 & 1 & 1 & 2 & 2 & 1 & $\begin{array}{c}\text { Gradual cardiac insuffi- } \\
\text { ciency } . . \\
\text {. }\end{array}$ & \\
\hline 7 & 2 & 0 & 1 & 0 & 7 & 2 & 1 & 2 & 3 & 3 & $\begin{array}{l}\text { Uremic convulsions or } \\
\text { sudden coma. }\end{array}$ & 15 \\
\hline 5 & 1 & 2 & 3 & 0 & 11 & 8 & 5 & 4 & 5 & 1 & Gradual uremia & 20 \\
\hline 6 & 2 & 0 & 2 & 2 & 2 & 2 & 1 & 4 & 0 & 4 & $\begin{array}{l}\text { Cerebral apoplexy or its } \\
\text { results }\end{array}$ & \\
\hline 1 & 1 & 0 & 2 & 0 & 0 & 0 & 0 & 0 & 0 & 0 & Angina pectoris & 3 \\
\hline 2 & 2 & 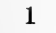 & 1 & 1 & - & 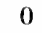 & 0 & 0 & 1 & 1 & Edema of the lungs & 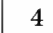 \\
\hline 3 & 3 & 0 & 1 & 1 & 3 & 2 & 0 & 1 & 3 & 6 & Other causes & 15 \\
\hline 48 & 24 & 4 & 15 & 11 & 32 & 15 & 8 & 13 & 14 & 16 & & 100 \\
\hline
\end{tabular}

It is evident from the foregoing tables that cardiovascular failure is the commonest cause of death, while uremia and apoplexy are frequent, and angina pectoris is relatively rare. Early ocular changes are often followed by uremic death. A certain number of cases die as the result of a progressive anemia which has its basis in the renal lesion, and a small number from acute pulmonary edema.

Janeway's recent careful analysis of cases of hypertensive cardiovascular disease showed that the average duration of life in these cases after the onset of hypertension was four years for men and five for women. During the first five years one-half the cases died, one-quarter lived between five and ten years, and the remainder over ten years. He further found that definite prognostic conclusions, based upon the actual height of the pressure found, were unwarranted.

Certain conclusions may generally be drawn as to the ultimate manner of death from the history and the physical signs. Thus the early onset and predominance of dyspnea or other symptoms of cardiac weakness indicates "a more than 50 per cent. probability of an eventual death by cardiac insufficiency." The occurrence of anginoid pain may be similarly interpreted, although only onethird of these cases actually die in a paroxy'sm of angina pectoris. In contrast to these cases patients with polyuria, especially if 
nocturnal, and those with headaches present on waking and gradually disappearing, will in about 50 per cent. of instances die of uremia. Progressive or marked loss of weight is a bad symptom (Janeway).

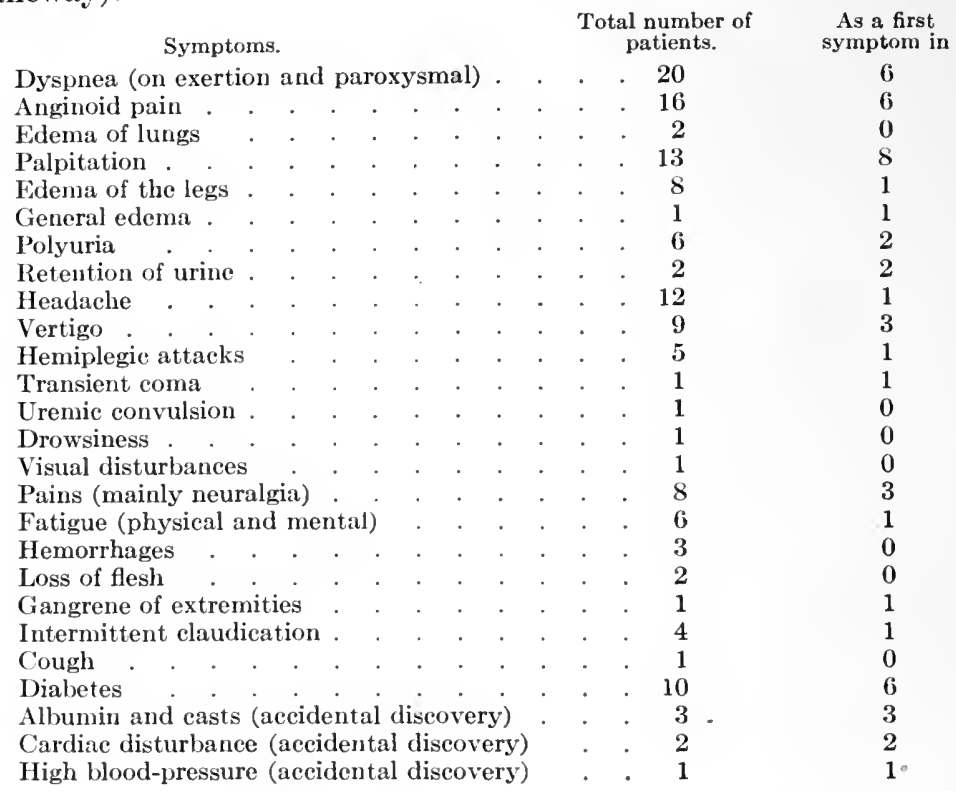

Acute Nephritis.-Even in children acute nephritis often produces a marked elevation of blood-pressure which is of distinct diagnostic value, but the rise of pressure does not always occur. The pressure may vary according as the glomeruli or tubules are chiefly involved. In some cases the amount of blood or albumin in the urine seems to bear a relation to the height of the pressure. The mean average pressure is not so high as in chronic nephritis, and during the height of some infectious fevers the blood-pressure rise entailed by the nephritis may be more or less counterbalanced by the hypotensive effect of the febrile toxemia. Cardiac hypertrophy may be noticeable in ehildren and young adults four weeks after the onset of scarlatinal nephritis. ${ }^{1}$ Rolleston found a temporary rise of pressure in 12 out of 33 such cases.

Mercurial Poisoning.-Poisoning with the mercurial salts produces renal necrosis associated with anuria. Janeway and

1 Friedländer, Arch. f. Physiol., 1881, p. 168. 
Müller have reported cases of bichloride poisoning with increased blood-pressure $\left(170 \mathrm{~mm}\right.$.) until the terminal fall occurred. ${ }^{1}$

Polycythemia.-Arterial hypertension occurs in some cases of polycythemia; indeed, a special type of this disease is often alluded to as polycythemia hypertonica. The fact that increased bloodpressure is not a constant finding shows that the origin of this symptom cannot lie in increased hemic viscosity, which is present in all cases, and which is doubtless often counterbalanced by reflex vasodilatation. Ordinary hypertension has no direct or constant relation to the erythrocyte count. ${ }^{2}$

In Lucas's ${ }^{3}$ collection of 179 cases the blood-pressure was reported in 66 instances as follows:

\begin{tabular}{|c|c|c|c|c|c|c|c|c|c|c|c|c|}
\hline & & & & & & & & & & & & Cases. \\
\hline Bloo & ssure & 145 to 170 & . & . & . & . & & . & . & & & 23 \\
\hline & “ & 180 to 200 & . & & & & & . & . & & & 13 \\
\hline “ & “ & $210,235,240$ & , and & 310 & ) (one & & ch) & . & . & & & 4 \\
\hline “ & “ & 220 & . & . . & . . & . & . & . & . & & & 3 \\
\hline “ & “ & 200 & . & . & . & . & . & . & . & & & 2 \\
\hline
\end{tabular}

"The blood-pressure is usually above normal in cases showing no splenomegaly (Geisbock's polycythemia hypertonica)." Münzer suggests that polycythemia may result from arteriosclerotic changes in the hemopoietic organs, which reflexly bring about an increased blood-pressure and increased functional activity.

Arterial Hypertension and Hemic Viscosity.-Martinet ${ }^{4}$ has studied the pressure-viscosity relation in a large series of cases, and based upon this investigation classifies them as follows:

1. Eusystolic type, in which the relation between maximum pressure and blood viscosity yields a quotient close to 4 (3.8 to 4.5). These cases even if the pressure is abnormal possess no serious renal nor cardiac lesion.

2. Hypersystolic type, with a sphygmoviscometric index greater than 4.5, an increased tension with a lowered viscosity. These cases have sclerotic kidneys, left ventricular enlargement, polyuria, hydruria, and a tendency to vascular rupture.

3. Hyposystolic type, index less than 3.5, low tension and high

1 Janeway, T. C.. Nephritic Hypertension, Amer. Jour. Med. Sei., May, 1913, 651. Müller, F., quoted by C. Thorel, Path. d. Kreislaufsorganc, Ergebn. d. allg. Pathol. u. path. Anat., 1910, xiv, 133.

2 Moller, Deut. med. Woch., October 29, 1908, xxxiv.

3 Erythremia or Polycythemia with Chronic Cyanosis and Splenomegaly, Areh. Int. Med., 1912, x, 597.

${ }_{4}^{4}$ Pressions artérielles et visensité sanguine (eirenlation, nutrition, diurése), Paris (Masson), 1912. 
viscosity. Venous stasis: emphysema, tuberculosis, mitral lesions, cyanosis, dyspnea, enlargement of right heart.

He believes that in the second type the hypertension begins as an increased viscosity (from overnutrition), which falls when the kidnevs begin to fail and the blood becomes more dilute. $\mathrm{He}$ therefore distinguishes two classes of hypertension:

(a) Simple hypertension (hyperviscosity).

(b) Cardiorenal hypertension (hypoviscosity), and considers that both prognosis and treatment are quite different in each case. Thus in two cases the iodides increased pressure, dropsy, and albuminuria, Class $(b)$, while the iodides proved beneficial in Class (a). He further suggests that much light may be thrown upon the action of certain drugs (purgatives, diureties) and other therapeutic measures (hydrotherapy, etc.) by a study of the sphygmoviscosity index.

Pellissier's ${ }^{1}$ studies lead him to believe that in cardiac disease and in gestational toxemia a fluctuating blood-pressure associated with an increased hemic viscosity is of very serious import and in pregnancy often an indication for immediate active treatment.

'Arch. Mens. d'Obstet. et de Gyn., 1915, iv, No. 5. 


\section{CHAP'TER XIII}

\section{THE TREATMENT OF ARTERIAL IYYPERTENSION.}

HYPERTENSION per se is not an indication for treatment except along preventive lines any more than is the presence of a heart murmur. Hypertension is one of Nature's methods of compensating circulatory or visceral deficiency, and in the carrying out of her purpose she can fortunately not often be thwarted. Reduce the pressure in a case of Bright's disease by violent means, such as the nitrites, if you will. After a very brief space of time the pressure will rise to its former height. The direct reduction of blood-pressure by means of drugs or otherwise is a procedure which should only be undertaken after the most careful consideration of the case from all its aspects. We must never thoughtlessly or ruthlessly interfere with Nature's delicately balanced mechanism of compensation. Only in the face of impending circulatory failure or vascular rupture or temporary pain is direct treatment indicated. On the other hand, in arterial hypertension, as Langdon Brown has aptly put it, "the patient is living too close to the limits of his cardiac reserve," and often a very high pressure can be more or less reduced without producing any deleterious results and with marked symptomatic amelioration, to say nothing of the saving of cardiovascular wear and tear. Just how much pressure lowering is possible and desirable must be determined for each individual case, and in practically no instance is such an effect to be sought merely by the administration of drugs. Treatment is generally much more satisfactory and its effects more lasting in cases showing only moderately increased pressure. This has been well expressed by Brooks, in saying that hypertension is not pathologic but is usually, if not always, physiologic in import; that it tends to prolong life rather than to shorten it, and that when its causes cannot be removed it should not be treated but maintained.

The reduction of vascular hypertension relieves the heart of an immense amount of work-entirely unnecessary work in cases in which the high arterial pressure results from the toxic factors attendant upon a faulty mode of living. 
"The heart, beating at the rate of 70 times a minute, $i$. e., 4200 times an hour, 100,800 times daily, and 36,792,000 times annually, would pump on an average $2 \frac{1}{2}$ ounces of blood at each contraction, 175 ounces a minute, 6564 pounds an hour, or $7 \frac{1}{2}$ tons a day," which "is equivalent to lifting one ton 122 feet high."'

Since about 10 pounds of blood are pumped by the heart per minute it is self-evident that an increase of pressure ranging between 10 and $50 \mathrm{~mm}$., not to mention higher figures, must call for the expenditure of an enormous amount of cardiac work. Furthermore, the heart which is called upon to meet such demands is often already affected by arteriosclerotic changes. The wear and tear on the rascular system is of course a no less important factor. Dilatation of the aortic arch, even in non-syphilitics under fifty years of age is of frequent occurrence in cases of nephritic hypertension. ${ }^{2}$ The entire hydrodynamic system is working under a continuous condition of forced draught. Brunton has suggested that hypertension does harm not only by interfering with tissue nutrition but also by hindering the vascular supply of the arteries themselves; the rasa rasorum being compressed between the intima and the adventitia of the arterial wall, whereas the alternate contraction and expansion which occurs in health, exercises an effect like massage which favors the nutritional flow.

The Direct Reduction of Blood-pressure.-Blood-pressure should very rarely be directly reduced by means of drugs or venesection except in case of emergency. The term emergency in this connection includes:

1. Cases with or without excessively high pressure $(200 \mathrm{~mm} .+)$ in which owing to the presence of symptoms such as vertigo, headache, numbness, tingling, loss of power, mental confusion or actual palsy, an apoplexy is feared. Venesection is the method of choice.

2. Cases with threatened or actual angina pectoris. Here the nitrites, especially nitrite of amyl, are useful.

3. Cases with high pressures in which a cataract extraction is to be performed. Sodium nitrite or erythrol tetranitrate will lower blood-pressure and diminish the risk of intra-ocular hemorrhage.

In addition to the foregoing types, Nicholson ${ }^{3}$ has suggested the use of the nitrites in hypertension cases about to be anesthetized.

1 Pope, C., Month. Cyclop. and Med. Bull., January, 1910.

${ }^{2}$ Smith, W. F., and Kilgore, A. R., Dilatation of the Arch of the Aorta in Chronic Nephritis with Hypertension, Am. Jour. Med. Sci., 1915, cxlix, 503.

${ }^{3}$ The Clinical Significance of Blood-pressure, Med. Record, March 20, 1915. 
This would, however, seem of questionable advisability owing to the danger of increasing "shoek" (see page 378).

There are often two factors concerned in the production of arterial hypertension: (1) A basic or essential factor, the point to which pressure must be raised to maintain metabolism, and (2) a superadded or toxic factor which results from faulty habits of life. It is the latter only which we are justified in treating. Ordinarily a pressure of $190 \mathrm{~mm}$. or over is an absolute indication for entire cessation from work, and generally rest in bed, at least until the case has been carefully studied.

The following history is illustrative of what may be accomplished in cases of hypertension:

Mr. B., aged sixty-one years, high-strung, intellectual, always a hard worker, president of a large manufacturing company, presented himself in 1908 complaining of fatigue, nocturnal mieturition, headaches and palpitation, loss of weight, and anginoid attacks. Blood-pressure: systolic, 200; diastolic, $120 \mathrm{~mm}$.

The arteries were very sclerotic, hard, and tense, the temporal vessels prominent, the eyes injected, the heart enlarged to the left with aortic accentuation, a systolic mitral murmur, and a reduplicated first sound. Urine: trace of albumin with hyaline casts.

He has intelligently coöperated in carrying out the treatment, which consisted in lessening his working hours, avoiding hurry and worry as much as possible, relegating all. unessential work and needless telephone calls to others. Moderation in diet and rest in the recumbent posture late each afternoon. Occasional sweat baths, with spinal massage.

In 1915 he is comfortable, puts in a long day's work, but realizes his limitations and submits to them. Blood-pressure, 165 to $75 \mathrm{~mm}$.

He occasionally has hypertensive attacks brought on by molue strenuosity which yield to full doses of nitrites, purgation, rest in bed, and a milk diet, but, on the whole, feels vastly better and accomplishes essentially as mueh as he did six years ago.

The treatment of nephritic hypertension is fraught with difficulties and requires the best of judgment. High blood-pressure leads to cerebral hemorrhage and cardiovascular failure, but high pressure is compensating and necessary; if reduced too much the patient is apt to suffer from insufficient elimination, which may lead to uremia, and from nutritional failure due to loss of eapillary pressure.

Generally speaking, the first step is to secure rest and place the 
patient upon a milk and farinaceous diet. Attention to the intestinal tract is extremely important. Regular and free evacuation of the bowels is essential. This can generally be accomplished by a judicious regulation of diet, fluid intake, and exercise. When drugs are required agar-agar, cascara, and other mild laxatives are often useful. The occasional employment of blue mass or calomel followed by a saline is often attended with excellent results. We cannot subscribe with enthusiasm to the laudatory results which are said to follow the use of the so-called intestinal antiseptics. The use of some of the various forms of fermented milk seems to be beneficial, chiefly, however, if other forms of dietary indulgence be limiter. As has been pointed out there are two main groups of hypertensive cases: (1) those presenting chiefly cardiac symptoms and usually dving a death from cardiac decompensation, and, (2) those with renal sumptoms who die with uremic manifestations. The treatment of these two types is somewhat different. In the former dietary restrictions and eliminative treatment are less important, while digitalis and Nauheim baths are more indicated. In the latter diet, purgation, sweat baths, etc., are more essential.

Some but by no means all the headaches of nephritis are due to high blood-pressure, and such cases as are, do not yield readily to therapy: Certainly direct treatment of the blood-pressure is neither justifiable nor successful, unless there is a distinct element of toxic vascular spasm which should be relieved by purgation or other eliminative means. The urgent symptoms of uremia are sometimes satisfactorily relieved by lumbar puncture and the withdrawal of cerebrospinal fluid or by renesection.

Purgation.-Although drastic purgation has long been used for conditions associated with marked edema, ascites, and anasarca, the advisability of such therapeusis in cases of cardiac weakness has been questioned. With a view to investigation of this problem, Yeilson and Hyland ${ }^{1}$ have carried out experiments with the bloodpressure, etc., after the use of different cathartics.

After two doses of a purgative given in the morning, before food, the sustolic pressure, which was chiefly aflected, showed an average lowering of 17 per cent. (the diastolic 8 per cent., the pulse pressure 24 per cent.), while the pulse rate increased 14 per cent. Symptoms such as vertigo, chilliness, cold extremities, dyspnea, clammy skin, etc., were observed. Hypertensive cases

1 The Effect of Strong Purging on Blood-pressure and the Heart, Jour. Amer. Med. Assoc., 1913. 1x, 436. 
showed the most marked results, and in these arhythmia occasionally occurred.

The results are presumably due to (1) loss of fluid from the blood; (2) unequal distribution of fluid, the splanchnic area being chiefly affected, and a condition comparable to the experimental section of the splanchnic nerves having been induced; (3) eardiac weakness due to the small quantity of blood which the heart has to handle, increased viscosity; (4) the absorption of mineral salts from salines may have a direct action on the heart or its nervous mechanism. The fact that the pulse rate is sometimes decreased after such purgation despite the lowered blood-pressure tends to support this hypothesis. ${ }^{1}$

It was found that compound jalap powder produced a more marked, constant, and prolonged fall of pressure than the salines, a reduction of 5 to 15 per cent. being generally demonstrable at the end of twenty-four hours.

The milder laxatives are often useful in the reduction of arterial tension. They act by depleting the portal system and by removing toxie materials whieh have irritant effects on the vaseular walls and on the kidneys. The administration of ten grains of blue mass followed in six or eight hours by brisk saline purge is generally the most satisfactory method of treating the usual symptoms of hypertension sueh as headaehe, irritability, insomnia, palpitation, etc.

Enteroclysis is often beneficial for similar reasons, especially in cases of colonic stasis.

Sleep.-In many if not all cases blood-pressure falls during sleep (often 30 to $50 \mathrm{~mm}$.) and is associated with a diminished peripheral pulse wave.

Brooks and Carroll2 found that night pressures were lower than day pressures in those who worked by day, whereas the eondition was reversed in night workers. Pressure variations of from 7 to $44 \mathrm{~mm}$. were observed. The least amount of fall occurred in those with already low pressures. The maximum fall oceurred about two hours after sleep began, the time at which insensibility is the greatest, and was followed by a gradual rise which attained its maxinum in the afternoon. Getting out of bed entailed only a

1 The intravenous injection of magnesium sulphate in cats leacls to a sudden fall of pressure, and if the dosage is sufficiently large, to death. Ritter, Ueber d. Einfluss v. Salzlösungen auf den Blutdruck, ete., Deut. Arch. f. klin Med. Chir., 1910, 11. Sce also MeNider and Mathews, Amer. Jour. Physiol., 1907, xx, 323.

2 Proc. Assoc. Amer. Phys., 1912. 
slight temporary rise without materially influencing the regular cycle. Frequent interruption of sleep tends to prevent the full measure and suddenness of the primary fall of tension. If the primary drop occurs before the patient wakes it does not recur the same night no matter how sound the sleep. The amount of nocturnal urinary secretion is apparently independent of the fall of pressure. Prolongation of the sleep interval does not produce lower tension. Nocturnal sleep, or even absolute rest, causes an average slowing of the pulse rate of twenty per minute as long as compensation is good. ${ }^{1}$

Some cases of insomnia show a reversal of the normal pressure relations, $i$. e., a higher pressure by night than by day. When sleep is produced by paraldehyde the pressure is reduced to $10 \mathrm{~mm}$. below the normal individual sleep level. ${ }^{2}$

The nitrites are sometimes efficient somnifacients in cases of arterial hypertension, which is eridence in favor of the fact that the fall of pressure is a factor in the production rather than the result of sleep. Most somnifacients owe their efficiency more to general nervous sedation than to depression of blood-pressure. The administration of bromides or chloral even in large doses does not increase the fall of pressure during sleep. Sleep may often be induced by warm baths, moist abdominal compresses, hot mustard foot baths, or gentle general massage administered two hours after the evening meal, which should be light in character.

Insomnia is a frequent and troublesome symptom in hypertensive cases. Often it is the only symptom of which the patient complains. Successful results can only be obtained by a careful study of the individual case, but as a general rule the following factors should be eliminated or avoided: Concentrated mental work or strenuous physical exercise during the evening. Excitement of any kind whether by playing "Bridge" or otherwise. Excessive smoking or any coffee drinking after dinner. If strychnin or nux vomica is administered for therapeutic reasons the evening doses must be omitted. Business worries and household cares must be laid aside.

Rest.--Rest in bed for a week, with a milk diet is often the best remedy for reducing blood-pressure. It is likely that the mental relaxation thus brought about is more important than the physical repose. Gumprecht called attention to the fall of pressure observed

${ }^{1}$ Klewitz, F., Der Puls im Schlaf, Deut. Arch. f. klin. Med., 1913, cxii, 38.

${ }^{2}$ Bruce, Edin. Medico-Chir. Soc., June 6, 1900, p. 156. 
in laborers on entering the hospital, a result which he attributed to the unwonted rest. But it is also a fact that patients who take their worries and anxieties to bed with them show less improvement than do those who submit to the ordeal and succeed for the time being in throwing all responsibilities overboard, leaving their intellectual realm bordering on a temporary nirvana. This is also strikingly exemplified by the patient whose symptoms all disappear the minute he leaves home for a vacation.

Exercise.-In ambulant cases of chronic arterial hypertension with good compensation the milder forms of exercise are of great benefit. Walking or golf are to be recommended, but with the warning that such exercise must be deliberate and that sudden, violent, or too prolonged efforts are harmful. The benefieial effects are attributable to a greater distribution of blood to the periphery, sudation, improved digestion and metabolism, as well as to the distraction and psychic relaxation thus induced. Severe or sudden exercise such as heavy lifting, rumning or stooping must be avoided. Patients should be cautioned against straining at stool. 'The writer has seen disastrous retinal hemorrhages result from lack of this precaution. Severe physical exertion in man may raise the blood-pressure from 110 to $180 \mathrm{~mm}$., chiefly owing to the rise of intra-abdominal and intrathoracic pressure, ${ }^{1}$ and it is more than likely that where hypertension exists, even greater increments of pressure may occur.

Diet.-Overeating.-The eating of food which has not been earned by physical exercise, is one of the greatest causes of arterial hypertension; hence the regulation of diet plays an important part in treatment. The chief indications are to restrict proteid food, especially meats, salt, and substances capable of causing renal irritation (condiments, alcohol) or digestive disturbance. The diet should be largely lactovegetarian, and limited in quantity so as to just supply the nutrient and caloric requirements. It is surprising on how small a ration metabolic equilibrium can be maintained in people past middle life. A high proteid ration, it is true, even with an increased amount of non-protein nitrogen in the blood, does not appear to directly influence blood-pressure, but there are certain types of nephritis in which it is harmful and certainly it must throw additional work on the kidneys. ${ }^{2}$ Prolonged

1 McCurdy, J. H., The Effect of Maximum Muscular Effect on Blood-pressure, Am. Jour. Physiol., 1901, v, 98.

2 Frothingham and Smillie, "Different Nitrogenous Diets in Nephitis," Arch. Int. Med., 1915, xv, No. 2. 
fasting produces a gradual fall of blood-pressure which in Charteris's case amounted to 25 per cent. of the normal reading. The pressure returns rapidly to the normal after the resumption of food.

In the case studied by Higgins in Benedict's ${ }^{1}$ Laboratory, the general trend of both the systolic and the diastolic curves in both the sitting and in the prone posture was to decrease during the first fifteen day's of the fast, systolic 134 to 94 , diastolic 100 to $72 \mathrm{~mm}$. $\mathrm{Hg}$. This was followed by either a constant value or a slight tendency to rise during the last part of the fast.

The decline of the blood-pressure is apparently due to: (1) a decreased contractibility of the heart muscle, and to (2) decreased vasomotor tone. The heart becomes smaller during the fast. Three day's after the end of the fast, pressure was again normal.

Thorough mastication and slow eating are essential. Neither mental nor physical exertion should be allowed immediately after food. Rest before eating often materially assists the subsequent digestion. $^{2}$

Blood-pressure rises after the ingestion of food, probably as the result of gastric distention; this is followed by hypotension, which corresponds to the time of active gastric secretion, and finally there occurs a second rise which is probably duc to intestinal distention and plethora, and which is proportional to these factors. It is evident, therefore, that large, bulky, stimulating meals should be avoided in arterial hypertension.

In an uncooked state certain foods may exert a direct effect upon blood-pressure, perhaps due to their effect on the thyroid gland. Liver substance fed to rabbits causes a 50 per cent. increase in pressure, and, according to Lubarsch, vascular lesions; while the administration of taurocholate or glycocholate of sodium causes a fall. ${ }^{3}$

The question as to whether cardiac symptoms result from purely mechanical disturbances, such as alterations in blood-pressure, upward displacement of the diaphragm or heart, or from reflex action on the part of the ragus receives some elucidation from recent experiments.

The abdomen is encircled by an inelastic bandage, and pressure

${ }_{1}$ Study of Prolonged Fasting, Pub. Carnegie Inst. of Washington, 1915, p. 119.

2 The question of Diet has been reviewed and ably diseussed by Heeht, Ueber d. diatetische Beeinflussung pathologischer Blutdrucksteigerung, Zeit. f. klin. Med. 1912, Lxxvi, 87 .

${ }^{3}$ Van Leersum, E. C., Alimentäre Blutdruckerhohung, Zeit. f. exp. Path. u. Ther., 1912, xi, 408. 
(110 mm. Hg.) is exerted upon it by the inflation of a large rubber bag, observations on blood-pressure, together with radiographic studies of the diaphragm, being made. In health such a procedure produces a slight increase in the pulse and respiratory rate with a trifling fall of blood-pressure. In cardiopaths occasionally marked fluctuations of blood-pressure occur, but usually the results are closely akin to those produced in healthy people, showing that mere elevation of the diaphragm is of but little importance. Inflation of the stomach with air causes a slight fall, and with carbon dioxid, a rise of the diaphragm, chiefly of the left side. In health a slight rise of blood-pressure results from this procedure, in cardiopaths-especially in mitral disease-a marked fall of pressure (20 $\mathrm{mm}$.) occurs in about half of the cases, a fall which bears no relation to diaphragmatic displacement. ${ }^{1}$

It would seem, therefore, that gastric disturbances cause cardiac embarrassment chiefly through reflex and only seccndarily through direct mechanical causes (see Tympanites, page 260).

The main dietary rule is moderation. Meat in quantities up to 100 or $150 \mathrm{gm}$. a day may generally be allowed. The prejudice against red meat and eggs is unfounded. There is chemically but little difference between red and white meats. If the amount be within the allowable limit it makes but little difference which the patient takes (see Nitrogen Retention, page 285). It is often necessary to restrict the use of sodium chloride, especially if the patient is edematous; for while there is no invariable absolute parallelism between salt retention and blood-pressure, the ingestion of salt in quantities greater than is demanded for physiologic equilibrium places unnecessary work on the kidney's. As a general rule, both the number and the bulk of meals is to be reduced, and extractires, soups, gravies, atc., are to be avoided. The use of fruits and regetables, especially if cooked, and if they do not cause flatulence or other simptoms of indigestion, is to be encouraged. An absolutely salt-free diet is difficult to secure and excessively irksome to the patient, while the benefits derived from the same do not justify its adoption. Recent experiments indicate that the average individual consumes much more salt than is necessary for physiologic equilibrium. This combined with the use of condiments leads to greater consumption of both food and fluid than is required for nutrition, and thus throws an unnecessary work on the eliminative

\footnotetext{
1 Funder, Ueber d. Einfluss intraabdominaler Drucksteigerung u. des Füllungszustandes des Magens auf d. Blutdruck, Deutsch. med. Woch., 1913, xxxix, 646.
} 
organs. Freshly made cheeses may be taken, but those which have been ripened must be avoided, since they contain oxyphenylethylamin, a bacterial decomposition product with marked pressor potency and identical with one of the toxic bases to which ergot owes its effects. ${ }^{1}$

Conservation of Energy.- In the early stages of increased bloodpressure the proper regulation of the daily life and diet is the only rational or efficacious method of treatment. The avoidance of hurry and worry are of prime importance, and are often the most difficult matters to correct. The patient should understand that the "doing of things against time," the doing of several things at once, the ceaseless mental concentration upon different problems, the assuming of unnecessary tasks and responsibilities, the frequent interruption of a train of thought by telephone calls, etc., are unwarranted drains upon vital energy, and that he who leads the high-pressure life is a physiologic spendthrift who is bound to end in early bankruptcy. This applies with special emphasis to that class of high-strung individuals who "go on their nerves," and who, as Holmes has said, "put energy out at interest and receive nervous derangement in return." "Worry, suspense, anxious anticipation, disappointment, consciousness of failure or of failing health, the hunted feeling that comes of overwork, and arrears, regret, sorrow, despair"- "wear out not only the nervous, but also the cardiovascular system." (Bruce.)

Fluid.- Too much fluid throws an unnecessary burden on the kidneys. The old idea of flushing out the system, of diluting toxic products, etc., in patients already dropsical has been shown to be erroneous. The total intake in whatever form should in nondropsical patients not exceed or fall below two liters per diem. A tumblerful of hot water flavored with lemon juice or rind, or with the addition of citrate of potassium, between meals and at bedtime is often beneficial. It is sometimes, although rarely, advisable to diminish the fluid intake even in cases free from dropsy. This should not be done if there is nitrogen retention in the blood.

$\operatorname{Hogan}^{2}$ has administered intravenously, saline solution containing sodium carbonate (20 gm. cryst., 2000 c.c.) in cases of high bloodpressure associated with uremia and believes that he produced a

${ }^{1}$ For literature see Edit. Am. Jour. Med. Sci., October 17, 1914.

2 Alleged Danger of Intravenous Injections in High Blood-pressure, LancetClinic, January 2, 1915. 
marked fall of pressure, with sudation and symptomatic amelioration. He explains this as due to the fact that if the concentration of the infusion is right, "the tissues and blood colloids are robbed of their water, and while tissue edema and high blood-pressure are decreased, free water is made available for excretion as urine, sweat and intestinal fluid."

Alcohol is contra-indicated. In small well-diluted doses it may do no great harm, but when possible it should be eliminated entirely. The use of tobacco should be restricted (see Tobacco and Bloodpressure, page 228).

Hydrotherapy.-Warm baths are valuable depressor remedies, and an occasional Turkish or electric-light bath is often useful, although these procedures must never be prescribed without due consideration of the possible deleterious effects, $i$. $e$., marked temporary increase in blood-pressure before sweating occurs, vascular rupture, cardiac strain, and reduction of nervous tone. Saline baths (one pound of washing soda or sodium chloride to the tub) are more stimulating and produce more marked sudation. Nauheim baths are especially useful if there be cardiac dilatation or weakness. A good sweat often causes a 10 to $20 \mathrm{~mm}$. fall in pressure, which tends to last throughout the day, especially if the procedure be repeated daily.

Occasionally, for reasons not yet understood, a series of sweats will reduce the pressure for weeks or months. This method of treatment is of course specially indicated if there be renal insufficiency or threatened uremia, but the occasional employment of sweat-producing hydrotherapy is often advisable in the absence of these conditions. Just how it does good in uremia is uncertain. 'The amount of urea eliminated through the skin even with a copious sweat cannot exceed 10 or 15 grains, and it is improbable that other toxic products are thrown off in any greater proportions. 'The beneficial effects are probably brought about by a change in the mass movement and a redistribution of the blood, especially that in the splanchnic domain, the peripheral arterioles and capillaries being temporarily dilated and flushed with blood. According to Amblard, electric-light baths produce less primary rise in pressure, and are therefore to be preferred.

After the sweat the patient should be allowed to cool off gradually, being first wrapped in blankets, and after a time sponged or sprayed with cool water or alcohol. A sudden plunge in a cold pool or immersion under a cold shower bath, especially while the 
patient is still actively perspiring, may produce an increase of 50 $\mathrm{mm}$. in blood-pressure, an occurrence which throws a tremendous strain on the cardiovascular system.

It has been shown that the relative concentrating power of the skin and kidney for urea and ammonia are very different, though in each instance the values are higher than in the blood. ${ }^{1}$ Further investigations may teach us that, depending upon the character of the toxin to be eliminated, sweat baths may be definitely indicated or contra-indicated in a given case.

Hot Baths. - Baths of a temperature of $104^{\circ}$ to $106^{\circ} \mathrm{F}$. $\left(40^{\circ}\right.$ to $41^{\circ}$ C.) produce a primary rise of blood-pressure (vasoconstriction) with an increased pulse rate, followed by a temporary fall (vasodilatation) and finally a second rise (increased systolic output with increased pulse rate) which persists until exhaustion occurs. On terminating the bath the pressure falls below the normal. 'The systolic pressure is chiefly affected. Hot air, steam, and electriclight baths exercise effects proportional to their temperature. Hot baths must be emploved with caution in the treatment of hypertension in diabetes. It has been shown that if the bodily temperature be raised either by fever or by artificial means the sugar content of the blood increases. ${ }^{2}$

Warm Baths.-Baths of a temperature between $99^{\circ}$ and $101^{\circ} \mathrm{F}$. $\left(37^{\circ}\right.$ to $38^{\circ} \mathrm{C}$.) generally lower blood-pressure, owing to a gradual primary vasodilatation, which is not, as in the case of hot baths, overbalanced by increased cardiac activity.

Foot Baths.- Hot foot baths to which mustard may be advantageously added are often a satisfactory treatment for the headache and insomnia of hypertension. If the patient is to sleep the feet must be kept warm, and for this purpose a hot-water bag in bed is often desirable.

Cold Baths.-The application of cold, especially in the form of forcible sprays and douches (mechanical stimuli), increases bloodpressure. 'The lower the temperature and the more sudden the application, the greater the rise. A sudden plunge into a cold pool, accompanied by the additional muscular efforts of swimming, produces a great increase in pressure, quite sufficient in arteriosclerotic cases to cause vertigo and precordial oppression, to pre-

${ }^{1}$ Plaggemeyer, H. W., and Marshall, E. K., A Comparison of the Exeretory Power of the Skin with that of the Kidney through a Study of Human Sweat, Arch. Int. Med., 1914, xiii, 159.

${ }^{2}$ Rolly, F., and Oppermann, Das Verhalten des Blutzuckers b. Gesunden u. Kranken, Biochem. Zeitseh., 1913, xlvii, 187. 
cipitate an apoplexy, an attack of angina pectoris, or of pulmonary edema.

In normal individuals the vascular reaction to heat and cold are general reactions, $i$. e., a cold bath reduces peripheral blood-flow and temperature, not only in the parts immersed but elsewhere. Even sitz baths have a similar effect, although the idea still persists

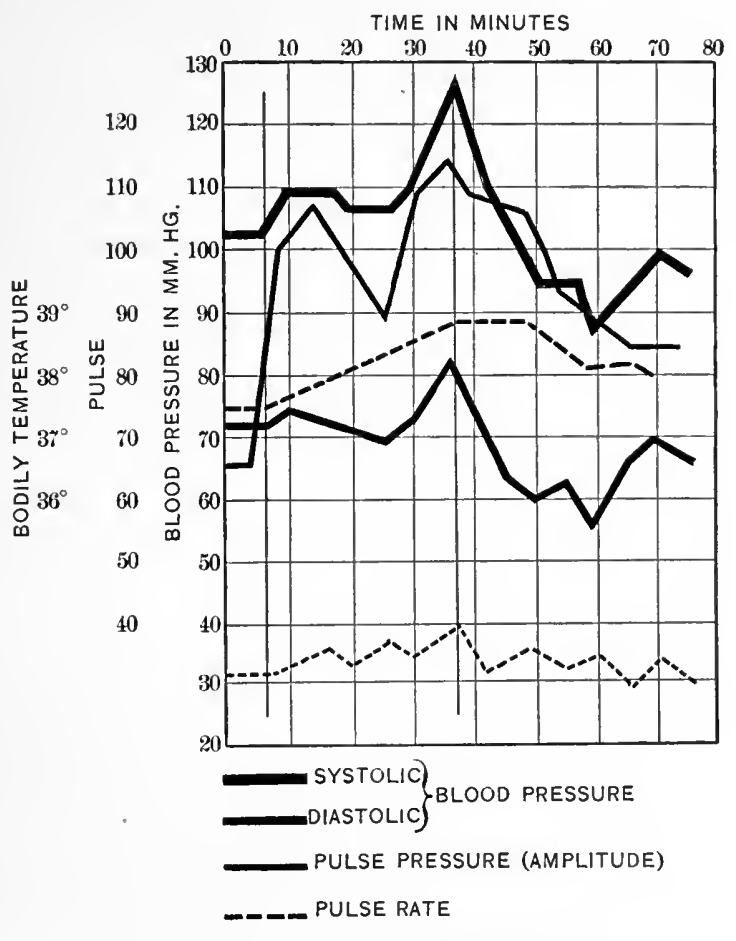

BODILY TEMPERATURE C.

Fig. 95.-Chart showing the effect of a tub bath of $41^{\circ} \mathrm{C}$. $\left(105.8^{\circ} \mathrm{F}\right.$.) upon the blood-pressure, pulse rate, and bodily temperature. The vertical lines indieate the beginning and end of the bath. (After Strassburger.)

in some minds that blood is simply crowded out of the area to which the cold is applied and forced into other peripheral areas. This has been shown to be incorrect. In abnormal conditions, however, whether functional or organic in origin, local peripheral variations may occur. The peripheral application of cold produces cerebral hypercmia, whereas heat has the opposite effect. It also decreases systolic output, whereas hot baths increase the 
latter. It should be remembered, however, that where sensory stimuli (friction, $\mathrm{CO}_{2}$, etc.) are added to the cold bath the systolic output may be increased despite the low temperature of the bath. Following a cold bath the reaction becomes reversed, the systolic output is increased and peripheral vascular dilatation occurs. When the circulatory apparatus is efficient or if the cold bath has been preceded by sweating, etc., the reversed reaction may set in even while the subject is still in the cold bath. The changes in blood-pressure which result from sensory or thermic stimuli are dependent both upon the state of the splanchnic veins and upon that of the peripheral arterioles. Blood-pressure may increase despite splanchnic dilatation as a result of peripheral contraction (cold baths, etc.), so long as the splanchnic vessels retain their tone.

A cold bath is by far the most potent remedy which we possess for altering blood distribution. It adds, however, a distinct burden upon the heart. Spray and shower baths still further increase the burden by the sensory stimulation which they provoke. If the heart is weak, instead of a rise in pressure cold baths may actually cause a fall, because the heart weakens instead of responds to the call for increased effort. Indeed, syncope and sudden death may thus be precipitated. Nature, it appears, often wards off the latter events by rendering vasomotor nerves reactionless.

O. Müller has shown that not infrequently the peripheral arteries of cardiopaths are plethysmographically irresponsive to the local application of ice. It must be remembered, however, that although this may be true for minor reactions, such as those just mentioned, nevertheless a major reaction such as that produced by a cold bath may be suddenly fatal. With diseased arteries the sudden elevation of pressure which follows a cold bath may produce vascular rupture. ${ }^{1}$

T'oo frequent or too vigorous hydrotherapeutic measures tend to lower nerve tone and may do actual harm. In the thin, high-strung, hypertensive patient they must be used with caution. Too violent forcing down of the arterial pressure by means of baths, etc., is just as reprehensible as when the same end is attained by means of the nitrites. If the patient feels listless, weak, or exhausted, as a result of the baths, they are doing him harm, despitc the fact that his pressure nay be more nearly normal. Cold baths, although

1 This subject has been admirably summarized by O. Müller and F. Veiel, Samml. klin. Vorträge, 1909-10, N. F., 167-196 (imere medizin), p. 64l. 
increasing the arterial, lower the capillary blood-pressure. The effect of hot baths upon the capillary pressure is less constant (Landerer).

Carbonated Brine Baths.-Despite the fact that Müller and Weiland have furnished plethysmographic evidences of peripheral vasoconstriction, and Liwschutz tachographic indications of an increased systolic output, it must be confessed that in a large number of cases treated with carbonated brine baths no constant pressure effects can be demonstrated.

The pressure at the end of the bath or upon the completion of the treatment, in cases either of hypertension or of hypotension, is variable. On the whole, pressure is more frequently raised than lowered, but it is impossible to know beforehand in which way a given case may respond. ${ }^{1}$ We do not mean to derogate the usefulness of this form of treatment, which is often followed by excellent results, especially in cases of cardiac weakness, but merely to point out that neither the subjective nor objective improvement rests on a basis of blood-pressure change. It is evident from the foregoing that such treatment must never be advised without due consideration. An increased pulse or systolic blood-pressure may be distinctly harmful in a case of myocardial degeneration. Even less marked and less constant results are observed after the use of alternating current baths. ${ }^{2}$

The organism reacts to electric stimulation (faradic, galvanic or alternating currents) by a negative volume ehange as shown by the plethysmograph. The effect upon the pulse rate and the bloodpressure is inconstant both in the same and in different individuals. Whether the benefits which sometimes follow electric baths in cardiac disease are due to cutaneous reflexes or to muscular contraction is uncertain. ${ }^{3}$

Of course the effects of the baths depend largely on the temperature. If below $33^{\circ}$ to $35^{\circ} \mathrm{C}$. $\left(92-95^{\circ} \mathrm{F}\right.$.) the pressure will be more apt to rise, if below this point, to fall. But with temperatures above $40^{\circ} \mathrm{C} .\left(104^{\circ} \mathrm{F}\right.$.) the pressure first rises and later falls. Hot and cold douches elevate and lukewarm douches lower tension. The effect on the pulse rate is equally important, heat accelerating

I Swan, J. M., The Influence of Carbonated Brine (Nauheim) Baths on Bloodpressure, Arch. Int: Med., 1912, x, 73.

2 Laquer, A., Ueber d. Verhalten des Blutdruckes nach Kohlensüure 1 . Wechselstrombädern, Zeit. f. exp. Path. u. Therap., 1909, vi, 855.

3 Geissler, Der Einfluss Elektrischer Reize auf d. Blutvertheilung in Menschlichen Körper, Münch. med. Woch., 1908, Iv, 92. 
and cold retarding it. These effects are generally transient, not lasting more than an hour or two.

Exception is often taken to the fact that carbon dioxid baths are used both in the treatment of arterial hypotension and hypertension. Their beneficial effects, which are unquestionable, are to be explained thus: The baths have essentially a cardiovascular tonic effect. Ordinarily they are followed by a rise in blood-pressure, an increased systolic output, and a slowing of the pulse. This leads to a generally improved circulation, nutrition, and excretion, and may indirectly lower blood-pressure, especially in cases of high-pressure stasis. Their action is thus comparable to that of digitalis. Those most competent of judging, state that these baths are harmful in cases of advanced renal lesions. 'This is probably. due to the temperature $\left(83^{\circ}\right.$ to $90^{\circ} \mathrm{F}$.) to which the baths are lowered, for certainly the secretion of sweat and urine is distinctly increased in the average case to which this treatment is given.

Cool carbon dioxid baths slow the heart and cause contraction of the peripheral arterioles. The thick layer of bubbles which surrounds the body in part prevents the loss of heat which occurs in ordinary cool baths, thus causing a pleasant sensation of warmth. Warm carbon dioxid baths increase the pulse volume, but have the opposite effect from cool baths upon the arterioles. Since these baths make considerable demands upon the circulation they must only be given under medical supervision.

Oxygen Baths.-Oxygen baths have been extensively employed as a remedial measure in cases of arterial hypertension, and are said by numerous observers to be attended with very satisfactory results. A lowering of tension is more constant than in case of carbon dioxid baths. These baths are contra-indicated in the terminal hypotension of arteriosclerosis (Winternitz), ${ }^{1}$ especially if associated with anemia (Baedeker). ${ }^{2}$

'The baths which may be administered at home ${ }^{3}$ are given at a temperature of $90^{\circ}$ to $95^{\circ} \mathrm{F}$., for from ten to twenty-five minutes. A course of baths (twenty-four) should be given on successive days, with occasional intermissions, either in the morning or at night (for insomina), but not too soon after a meal.

Phlebotomy.- Tenesection is one of the most prompt and efficacious methods of lowering blood-pressure and relieving a distended

1 Blätter f. kiin. hydrother., 1907, p. 1.

2 Therap. d. Gegenwart, 1910, p. 2.

3 "Perogen" haths. Morgenstern \& Co., New York. The oxygen is liberated by the admixture in the bath of two powders (sodium perborate and magnesium borate). 
right heart. Its beneficial effects in uremia are generally attributed to the foregoing effects and not to the amount of toxic material which is removed from the blood-stream. Phlebotomy is chiefly indicated in conditions of venous stasis, whether due to right or left ventricular weakness. It of ten yields excellent results in the treatment of acute pulmonary edema. In order to obtain definite results 300 to 500 c.c. of blood must be withdrawn. ${ }^{1}$ A rapid venesection amounting to from 300 to 500 c.c. will generally reduce blood-pressure from 5 to $30 \mathrm{~mm}$. As a rule the more rapid the withdrawal the more pronounced the fall of pressure.

Bandaging of the Extremities.-The application of tourniquets to the extremities with sufficient constriction to prevent venous outflow, although allowing arterial inflow, has temporarily much the same effect upon the system as a phlebotomy. It may remove a 14-liter quantity of blood from the general circulation, and has been recommended as a method of treating heart weakness, but has not found much favor. If too much pressure is exerted, inhibition of arterial inflow and marked increase of general blood-pressure will result. The method must, of course, not be applied in the presence of edema or varicose veins of the extremities. Relaxation of the bandages must always be gradual so as not to suddenly increase cardiac work. By bandaging all four extremities, 20 per cent. of blood can be removed from the general circulation and the heart spared a corresponding amount of energy. Subjective symptoms are said to show a distinct amelioration. ${ }^{2}$ This procedure has been useful in cases of threatened pulmonary edema and other conditions associated with increased venous pressure. Pronounced passive congestion in the extremities may cause a fall of $20 \mathrm{~mm}$. Hg., sometimes rather suddenly, with signs of collapse.

High-frequency Currents. ${ }^{3}$ - Although there is as yet no unanimity of opinion on the subject, some very favorable reports bave been published regarding the efficacy of high-frequency currents for the reduction of high arterial pressure. The current is said to act by lowering peripheral resistance and also by acting as a cardiac tonic.

Moritz and Tabora, Verhandl. d. Kong. f. inn. Med., 1909, xxxvi, 378.

2 Lillienstein, Der unblutige Aderfass, Phlebostase, Med. Klinik, 1912, vii, 316.

3 Also spoken of as Arsonvalization after d'Arsonval, to whose physiologie studies so mueh of our present knowledge on the subject is due. For further information regarding the history, electric data, detailed description, and literature on this subject see Allen, W. C., Radiotherapy and Phototherapy, Philadelphia, 1904. Mann, L., Krause and Garre's Lehrbuch d. 'Therap. Inmeren Krankheiten, 191 1, i, 4\$8, 509. 
Nagelschmidt, ${ }^{1}$ who reports good results in a series of 120 cases, believes that the discrepancies in the results attained by different observers with the high-frequency current are due to the fact that an insufficient amperage has generally been employed.

The exact means by which high-frequency currents reduce bloodpressure are not thoroughly understood. 'The belief at present is that the effect is mainly thermal (local heating, rise of bodily temperature, and pulse rate, sudation and peripheral vasodilation) and that it is not due to cardiac depression.

"If two electrodes from a galvanic or a faradic current are placed in a bowl of water, decomposition occurs with bubbles at either pole. Even if quite weak, a hand placed between them experiences painful electrolytic sensations. If these electrodes are replaced by high-frequency ones, hardly any electrolysis occurs. The water merely gets warm, and a hand placed between them experiences no sensation whatever excepting that of warmth, even with currents so strong that were they galvanic or faradic they would cause chemical and electrolytic changes capable of destroying the tissues" (Sayer). ${ }^{2}$

"With regard to alterations in the blood itself, we may look upon the action of high frequency in reducing blood-pressure as being in some way analogous to the action of a rise of temperature, when, unless there is dyspnea and cyanosis, the rapid metabolism caused by the onset of any fever is always accompanied by a fall in blood-pressure, an increased excretion of uric acid, and a quick capillary reflux, exactly what happens on a small scale with a dose of high frequency."

The effect on the nervous system is definite, but the sensory and motor stimuli are too rapid to permit of a response, "for all electric vibrations beyond ten thousand per second lie beyond the limits of the range of frequencies to which these nerves can respond. Although no direct effects can be demonstrated, yet their application to any muscle or nerve does in some way affect it, for it is found afterward that its excitability to all ordinary electric (galvanic and faradic) stimulation is lessened" (Sayer). The highfrequency currents act specially upon the nerves of the vasomotor system, the splanchnics and the large sympathetic trunks, and

\footnotetext{
1 Diathermic Treatment of Circulatory Disorders, Arch. Röntgen Ray, February, 1912.

${ }^{2}$ The Effeets of Electrical Currents upon Blood-pressure, British Med. Jour., Octoher 8, 1910, p. 1052 .
} 
the greatest effects are produced if the current is passed through these tissues. In nerves containing both constrictor and dilator fibers, ordinary electric stimulation produces a more marked effect, especially if weak currents are employed (Crile).

Method of Application.-I. Induction.-The patient is placed in the centre of a wire cage, through the wires of which the current is passed. An induction current is thus generated in the patient.

The presence of such a current can be demonstrated by means of an incandescent globe, held in the hand of the patient, which without any direct connection with the solenoid will become luminous (Mann).

II. Condensation.-When the patient rests on an insulated couch or chair cushion, a high-frequency current derived from a static machine, or preferably from a specially designed apparatus, is passed through his body, using either a monopolar or a bipolar method (the patient being connected with the circuit by one or two pole electrodes, and acting as a current condenser). The strength of the current passing through the coil is ganged by an ampèremeter, while the amount received by the patient can be gauged with a milliampèremeter placed between the table and the couch. Burch ${ }^{1}$ has reported untoward results in some cases. He found that if patients who scarcely responded to from 20 to 50 milliampères were subjected to 200 to 800 milliampères a very marked fall of pressure with signs of collapse sometimes occurred. Patients who readily respond to the smaller dose are, he believes, instances of spastic hypertension which can safely be treated with the heavier dosage. The cases that show no response to the milder current show the best results under the larger dosage. These cases are supposedly due to both a vasomotor spasm and a vascular degeneration with a relatively strong heart.

III. Local Application. (Diathermic. Method).-By means of electrodes of different kinds the current is conducted from a small solenoid to the patient. In order to insure greater penetration and to minimize the local effect which this mode of application chiefly favors, the current is taken from an alternating current generator and then interrupted by means of a jump-spark attachment. 'The action of this (diathermic) current is somewhat different from that of d'Arsonval (Mann). There is no cutaneous resistance. The current passes from one electrode to another by the shortest

${ }^{1}$ Electrical Treatment of Arterial Hypertension, Med. Record, 1911, Ixxx, 866. 
route, permeating the intervening tissues with practically equal intensity. It is claimed that blood-pressure can be (1) raised, by precordiodorsal application, as the result of cardiac stimulation and cutaneous irritation, and (2) lowered, through peripheral dilatation, by application to the central nervous system (medulla). When one desires to heat the heart muscle itself, the large indifferent electrode is placed over the dorsal region, the smaller, movable one over the precordium. The latter must be so constructed that it is possible to pass 1000 to 1200 milliampères for at least five minutes without undue heating of the skin (Nagelschmidt).

Indications.-High-frequency currents yield the best effects in cases of spastic hypertension with a relatively good heart muscle and kidneys. 'The effects produced are said to be more lasting than those resulting from the use of vasodilator drugs. They can lead to permanent benefit only when coupled with hygienic and dietetic correction. Too great a fall of pressure as the result of such therapeusis may, according to Moutier and Challamal, ${ }^{1}$ be counteracted by the application of this current to the vertebral column. According to Fontana ${ }^{2}$ the variable results obtained by high-frequency currents upon blood-pressure depend upon the permeability of the kidneys. The vascular dilation and increased metabolism which the current produces throws extra work on the kidneys which, if these organs are functionally active, causes a fall of blood-pressure, whereas insufficient kidneys fail to react and blood-pressure remains high.

Massage.-Although the primary effect of massage, as the result of cutaneous stimulation, tends to raise blood-pressure, this effect is more than counterbalanced by its effect on lymphatic and venous flow. Good general massage, especially in association with Swedish movements, is one of the most useful measures which can be employed in the treatment of arterial hypertension. It supplies many of the benefits of exercise without the attendant expenditure of energy:

Abdominal Massage.-Some authorities hold that this form of massage is contra-indicated in cases of hypertension and cardiac disease. Experimentally in animals it increases blood-pressure, but some clinical observations show that this is by no means always

\footnotetext{
${ }^{1}$ De l'abaissement de la pression arterielle au dessous de la normale par la d'arsonvalization, Compt. rend. Acad. d. sci., Paris, 1905, cxl, 742.

${ }^{2}$ Dell'azione delle correnti ad alta frequenza sulla pussione arteriosa in rapporto alla permeabilita renale, Gazz. d. Ospedali e. d. Clin., 1914, xxxv, 523.
} 
the case. Indeed, a fall of pressure may be observed. Certainly compression of the large abdominal arteries tends to raise the general pressure at least temporarily, but generally (with judicious massage) this rise is slight and is soon more than counterbalanced by the good effect which the manipulations have upon digestion and intestinal peristalsis.

When skilfully performed it has a generally sedative effect accompanied by a slowing of the pulse and often a fall of blood-pressure, probably by its influence on local stasis in the mesenteric vessels, although vagus action may have a part in the results. ${ }^{1}$

Vibratory Massage.-This form of massage yields good results, requires less time, and does not necessitate undressing, but, as a therapeutic measure, it is less satisfactory than good general massage with passive movements. A valuable factor of the latter procedure lies in the hour's rest and relaxation which the patient should be instructed to take after its completion. Even prolonged vibration of the precordium is without effect upon the pulse rate, the blood-pressure, or the systolic output. ${ }^{2}$ It has been claimed that definite blood-pressure-lowering effects can be obtained by concussion of the seventh cervical vertebra for a period of about five minutes, and that vibration of the sixth and seventh dorsal vertebræ tends, although less constantly, to increase vascular tension. ${ }^{3}$

Cutaneous Irritation.-Local irritation of the skin (mustard, capsicum, etc.) produces redness by dilatation of the capillaries and venules without a corresponding dilatation of the underlying arterioles. ${ }^{4}$ Such methods of counterirritation find a distinct field of usefulness when applied to the feet, neck, or precordium in helping to relieve some symptoms of hypertension (insomnia, headache, precordial and epigastric oppression) as well as in mitigating the restlessness and delirium of acute infections, notably attacks of pneumonia.

Passive Exercises.-This form of gymnastics administered either by an attendant or by machinery (Zander apparatus, etc.), since it supplies the beneficial effects of muscular exertion without an attendant expenditure of volitional nervous energy, tends to lower

1 Ekgren, E., Ueber den Einfluss d. Abdom. Massage auf Blutdruck, Herzthätigkeit u. Puls. etc., Zeit. f. diätet. u physik. Therap., 1902, v, 191.

${ }_{2}$ Plate and Bornstein, Ueber d. Einfluss d. Herzvibration mit hoher frequenz auf d. Kreislauf, Ibid., 1913, xvii, 65.

${ }^{3}$ Abrams, A., Spondylotherapy, 1910, p. 248.

4 Wood and Weisman, Arch. Int. Med., Septenber, 1912. 
blood-pressure in a satisfactory manner, and is a useful adjunct to other forms of treatment.

Climate.-A warm, equable climate is desirable. Winters may be spent in Egypt, Jamaica, Bermuda, Southern Califormia, etc. Moderate warmth and sunshine conduce to outdoor exercise which, when accompanied by gentle perspiration, is most desirable, but sudden chilling must be sedulously guarded against (see Altitude).

Psychic Treatment.-Certain types of high-pressure individuals are extremely susceptible to suggestion, and tend to become introspective. Cautionary advice in such instances must be tempered with encouragement. The additional element of self-worry must not be added to their other burdens. For this reason it is often inadvisable to tell the patient anything about pressure changes, particularly if these should happen to be for the worse. The beneficial effects of light exercise and diversions of different kinds owe much of their good effect to psychic impressions.

Respiratory Gymnastics. - In certain cases of hypertension both symptomatic improvement and lowering of tension may be induced by breathing exercises. Space will not allow discussion in detail of the mechanism by which this is brought about; but such results are not difficult to understand when we consider that better aëration of the blood diminishes its centrally pressure-raising $\mathrm{CO}_{2}$ content. Proper action of the respiratory muscles, especially of the diaphragm, facilitates blood-flow to and from the heart, and increases venous and lymphatic flow in the digestive organs and elsewhere. Thus tissue metabolism and elimination are directly favored.

That the question is not, howerer, a perfectly simple one is shown by Stewart's investigations, which proved that peripheral blood-flow (in the hands) was diminished by deep breathing.

"The cause of the diminution in blood-flow produced by forced respiration is without question in part a mechanical effect on the action of the heart due to the changes in the intrathoracic pressure. Hill and Flack have stated that the left ventricle becomes smaller, as shown by Röntgen-ray pictures, the radial artery emptier, and the arterial blood-pressure lower with each forced thoracic inspiration. That a chemical change may also be concerned is indicated by experiments in the case of cyanosis already mentioned, in which oxygen inhalation distinctly increased the blood-flow in the hand without affecting the respiratory movements or the total pulmonary ventilation, while in normal persons it had no such effect. It may be that the washing out of the carbon dioxid 
by the forced respiration causes, even in such short experiments and with such moderate exaggeration of the respiratory movements, those changes in the distribution of the blood, particularly its accumulation in the great veins, which Henderson associates with 'acapnia,' and which, according to him, are so important a factor in surgical shock. Other vascular changes - for example, a diminution of the flow in the coronary circulation due to the fall of pressure in the aorta, which aid the mechanical changes in the thorax in decreasing the average output of the heart-may occur. Changes produced through the vasomotor centre on the peripheral vessels may also play a more direct part. This is at any rate suggested by the fact that the perceptible change in the flow caused by forced breathing is not the same for the two hands, as it might be expected to be were the whole effect a cardiac one. However, it is not intended to lay much stress on this suggestion, for the initial differences in the flow. in the two hands are not sufficiently great to permit without hesitation the application of this criterion. The beneficial influence of oxygen on the flow in the case of cyanosis may be explained as the result of the oxygen action in diminishing the excitability to carbon dioxid of the vasomotor centre and other mechanisms affected by hypercapnia."

Differential pressure in the lungs, breathing into rarefied air, has been used as a method of promoting blood-circulation. Bruns found that when the pressure of the air into which the patient breatlres has been reduced to 8 to 10 e.c. $\mathrm{H}_{2} \mathrm{O}$, both pulse and respiratory rate increase, peripheral flow as shown by the plethysmograph, is increased and venous pressure falls. ${ }^{2}$

Radio-active Substances.-Our knowledge of the physiologic effects of radio-active substances is very meager. 'The employment of these remedies is still in its experimental stage, and there is considerable evidence to show that the efficient therapeutic dose and the toxic dose are separated by but a narrow margin.

The drinking of water charged with radium emanations appears to increase tissue metabolism, the activity of ferments and urinary flow. Good results attending its administration in cases of gout and of arterial hypertension have been reported. It is now generally believed that the beneficial effects obtained at certain health

1 Stewart G. N. Studies on the Circulation in Man, III. The Influence of Forced Breathing on the Blood-flow in the Hands, Amer. Jour. Physiol., 1911, xxviii, No. 3 , p. 196.

2 Bruns, O., Die künstliche Luftdruckerniedrigung u. d. Lungen, etc., Münch. med. Woch., 1910, Ivii, 2169. 
resorts such as Bad Gastein in the Austrian 'Tyrol, and Hot Springs, Arkansas, are due to the radio-active waters there present.

Water charged with radium emanations, however, quickly loses its potency and hence bottled waters are inert. The artificial charging of water with radium emanations is now, however, possible, and farorable results may be obtained with home-charged water having a potency of 5000 to 20,000 Maché units when taken over prolonged periods of time.

Solutions of thorium- $X$ have been administered intravenously. Plesch, who emploved this method of treatment in pernicious anemia, found a considerable lowering of blood-pressure associated with increased metabolism, oxygen consumption, and loss of weight.

The action of ranadium on different organs and tissues is but little understood. It appears that the intravenous injection of this substance in mammals is but slightly toxic to the heart. It causes a rise of blood-pressure due to peripheral vasoconstriction, but repeated injections cause a fall of pressure due to fatigue of the vasomotor centre and the heart. The action of moderate doses upon the peripheral ressels is more marked than in the case of barium, and upon the splanchnic vessels, more marked than adrenalin. ${ }^{1}$

Prolonged application of the $x$-ray to the adrenals of dogs produces well-marked structural degenerative changes in the glands. ${ }^{2}$ Zimmern and Cottenot $^{3}$ obtained a prolonged clinical fall of pressure $(20-80 \mathrm{~mm}$.), associated with subjective improvement, in sixteen cases of chronic hypertension. Quadrone reports somewhat similar results, but Groedel was unable to obtain any reduction in cases of hypertension by this means. ${ }^{4}$ There is no rational basis for this form of treatment. The evidence at hand is not in favor of adrenalinemia as a cause of chronic hypertension, and too prolonged exposure may do serious structural damage to the glands.

The usual result of exposure in a radium emanatorium is a fall of the systolic blood-pressure amounting to 20 or $25 \mathrm{~mm}$. $\mathrm{Hg}$. Together with this there generally occurs a decrease in cardiac

1 Jackson, D. E., The Pharmacological Action of Vanadium, Jour. Pharm. and Exper. Therap., 1912, iii, 477.

${ }^{2}$ Cottenot, Mulow et Zimmern, Aetion des vagous sur la corticale sur renali, Compt. rend. hebdom. des sciences Soc. biol., 1912, lxxiii, 717. Similar results by Galansino and Decostello.

${ }^{3}$ Zimmern et Cottenot, La radiotherapie des glandes sur renales, ses resultats, ses effects hypotenseurs, Arch. d'electr., 1912, xx, 500.

${ }^{4}$ Groedel, F., Strahlentherapie II, 1913, p. 224. 
work and a lowering of the diastolic pressure. 'These changes are apparently due to vascular dilatation. ${ }^{1}$

Surgical Treatment.-Renal decapsulation has been performed in some cases of chronic nephritis. Goodman reports such a case in which both the systolic and the diastolie pressures were very definitely reduced for about two weeks following decapsulation, although the patient ultimately died. ${ }^{2}$

1 Loewy and Plesch, quoted by Rowntree and Baetjer, Radium in Internal Medieine, Jour. Amer. Med. Assoc., 1913, 1xi, 1438.

2 Goodman, E. H., Effect on Blood-pressure of Decapsulation of the Kidney, New York Med. Jour, October 3, 1914. 


\section{CHAP'TER XIV.}

\section{EFFECTS OF DRLGS AND GLANDULAR EXTRACTS ON BLOOD-PRESSLRE (ARRANGED ALPHABETICALLY).}

Aconite.-The effect of aconitin upon the mammalian heart may be divided into two phases: (1) It produces slowing of the rate with a weakening of contraction. The pulse rate may (experimentally) be reduced one-half and blood-pressure will fall proportionately. These phenomena are due to central stimulation of the pneumogastric nerve. (2) If the administration of the drug is pushed still further, tachycardia and arhythmia appear, apparently as a result of direct action upon the myocardium.

Aconite has been used for the reduction of arterial hypertension, but its use has not been satisfactory. The tincture which is marketed without physiologic standardization varies greatly in potency, and in the doses usually employed is often inert. Robinson's ${ }^{1}$ investigations indicate that in maximal dosage aconite increased rentricular irritability.

Alcohol.-There is much difference of opinion as to the clinical effects of alcohol on the circulation, and equally discordant findings have resulted from its employment experimentally. Most of the evidence shows that alcohol is only a momentary cardiac stimulant which acts reflexly through its irritation of the mouth, esophagus, and stomach. Its main effect is vasodilatation, and if pushed, nervous and muscular cardiac debility. If, therefore, temporary stimulation is desired it should be given in concentrated form; if vascular relaxation, diluted. Although it may briefly raise the sirstolic pressure it raises the diastolic pressure even more, and thus lowers the pulse pressure. ${ }^{2}$ In chilliness due to peripheral vasoconstriction it does good, but if the patient be cold as the result of collapse, with a low blood-pressure, it is directly contra-indicated. It may act in part as a food by sparing the proteids or assist in

\footnotetext{
${ }^{1}$ An Investigation of the Potency of Tincture of Aconite, Arch. Int. Med., 1915, xv, Part I, 645 .

2 Lieb, C. C., Reflex Effects of Alcohol on the Circulation, Jour. Am. Med. Assn., 1915, Ixiv, 89S.
} 
preventing acidosis, but in the vascular failure of infectious disease or in surgical shock it is harmful. It is irritant to the kidneys and delays the elimination of certain substances (uric acid, etc.). Both clinical and experimental evidence are against its use as a stimulant in conditions of low blood-pressure.

It produces an unstable vascular tonus. Luzzato, on administering 30 to $50 \mathrm{gm}$. of alcohol to students, found no constant relation between psychic phenomena, pulse or respiration and blood pressure. ${ }^{1}$ Splyggmobolometric studies in fever cases generally show a diminished amplitude, a lowered pressure, and decrease in cardiac work. ${ }^{2}$ The onset of drunkenness is associated with a fall of the systolic and the pulse pressure, together with an increased pulse rate. ${ }^{3}$

The syndrome of cardiac dilatation with hypertension which is seen in excessive beer drinkers is in part due to the alcohol, in part to the increased volume of the blood. For obvious reasons alcohol should be taken sparingly if at all in cases of nephritic hypertension.

Alkalies.-This class of drugs may indirectly assist in lowering tension by counteracting acid irritants, by combating dyspeptic tendencies or by their diuretic effect.

Ammonium.-Ammonium in the form of the aromatic spirit is a promptly acting though temporary cardiac stimulant. It is useful in transient cardiac and vasomotor weakness, and extremely valuable in the relief of gaseous gastric distention in arteriosclerotic cases with precordial oppression. 'The following formula has often proved most satisfactory:

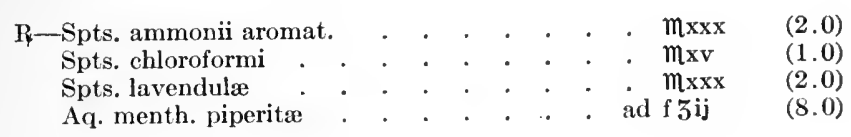

To this prescription spirit of glonoin may sometimes be advantageously added.

Atropin.-Atropin increases the pulse rate by depression of the peripheral ends of the vagus nerve. It has thus in part an antagonistic action to digitalis. Experimentally it increases blood-pressure by a slight stimulation of the vasoconstrictor centre and an increased

${ }^{1}$ Luzzato, Accad. dei Fisiocritici di Siena, January 31, 1909.

2 Dennig, Hindelang, and Grünbaum, Ueber d. Einfluss des alkohols auf d. Blutdruek u. d. Herzarbeit in path. Zuständen nanent lich beim Fieber, Deut. Areh. f. klin. Med., 1909, xevi, 153.

${ }^{3}$ Holzmann, Blutdruek bei Alkoholberauschten, Arch. f. Psychiatrie, 1909, xlv, 92. 
capable of increasing urinary secretion even if a volumetric increase is prevented, probably as the result of increased blood-flow. ${ }^{1}$

Clinical Data.-The effect of caffein is more prompt and more brief than that of the digitalis group. It may be administered either in its pure form or as the citrate or in the form of tea or coffee. Medicinally it rarely produces a demonstrable rise of bloodpressure. It is useful as a cardiovascular and renal stimulant in both acute and chronic disease, but to be effectual must often be given in larger dosage than is customary. Better results are obtained by using theobromin-sodium salicylate, since the untoward effects of caffein are avoided. ${ }^{2}$

Camphor.-Camphor is said to raise blood-pressure by medullary and cardiac stimulation. The drug has long been used specially as a cardiac stimulant. Both clinically and experimentally it produces at least a temporary improvement of the pulse even in the agonal stage of infecticus disease. It is normally neutralized in the body and thus rendered inert by glycuronic acid, and may produce toxic symptoms in cases in which the latter substance is diminished (starvation, cachexia, sepsis, eclampsia, $\mathrm{CO}_{2}$ poisoning). Nothing short of a toxic dose has any effect on blood-pressure in normal animals in health because compensatory vascular changes readily neutralize abnormal stimuli. Head and Brooks ${ }^{3}$ failed to get any definite circulatory results either clinically or experimentally. The administration of the drug in emulsion has been commended as being a more certain and satisfactory method of obtaining the physiologic effects. ${ }^{4}$

The Digitalis Group.-Based purely upon experimental evidence digitalis should be the ideal drug to employ in case of arterial hypotension, since it increases the force of cardiac contraction as well as vascular tonus. Clinical data, however, show that in therapeutic dose digitalis often does not increase blood-pressure; in fact, it frequently lowers it, especially in heart diseases with highpressure stasis and in hypertension due to toxic renal retention. Furthermore, its action is not sufficiently prompt for emergencies, and in febrile toxemias it often has very little if any effect, $e . g$., tuberculosis. So much experimental and clinical evidence has been amassed bearing on the subject of digitalis therapy as to forbid

1 Weber, Arch. f. exp. Path. u. Phar., 1906, liv, 1.

2 Taylor, I., Clinical Studies in Caffein, Arch. Int. Med., 1914, xiv, 769

3 Aner. Jour. Med. Sci., 1913, exlv, 238 (bibliography).

4 Doctoriwitch, Therap. 1. Gegenwrt., xlvii, 343. 
even a satisfactory summary. The good effects of this group of drugs cannot be ascribed essentially to an increased blood-pressure, and the belief that danger may attend its administration in conditions of high blood-pressure is equally unwarranted. For emergency use digitalis may be administered subcutaneously or intravenously. ${ }^{1}$ H. C. Wood, Jr., believes that although medicinal doses of digitalis do not raise blood-pressure, yet they probably do have some slight effect on the vasomotor system. This view is based upon the fact that: (1) if slowing of the pulse is abolished by atropin, strophanthus causes a marked increase of pressure in normal man; (2) the diuretic effects of the drug, although independent of sistemic pressure changes, may result from a relative splanchnic contraction at a time when the renal vessels are dilated by the drug. ${ }^{2}$

Marvin, ${ }^{3}$ in experiments on healthy students, found an increase in blood-pressure (average $13 \mathrm{~mm}$.) which reached its maximum in five hours and gradually returned to the normal in fifty hours.

Digitalis is often the most useful drug in cases of hypertension when the heart is beginning to fail. In such cases its use need not be feared on account of increasing vascular pressure. This effect is generally not in evidence, whereas it often lowers tension, especially the diastolic pressure, by securing better elimination and a more efficient circulation. In auricular fibrillation digitalis does raise the arerage systolic pressure (see page 243). The action of digitalis and digitoxin is more prolonged than that of strophanthus and digitalin. ${ }^{*}$

In nephritic hypertension, as the renal lesion progresses, pressure continues to rise, and unless some other lethal termination occurs, either the heart or the vascular system must in time give way.

Heart failure may manifest itself by (1) a fall of blood-pressure, edema, drspnea, oliguria, and cardiac dilatation, or (2) high pressure stasis, a condition in which blood-pressure remains high or even rises (increased toxemia) and is associated with d̦̣spnea, cardiac arhythmia and visceral stasis.

In both these conditions digitalis is indicated. Lnder its administration renal elimination is increased and an actual lowering

1 Digipuratum (extract of leaves), $0.1 \mathrm{gm}$. in twenty-four hours: Digalen, $\mathrm{mxv}$ (1 c.c.), digitalin (German) gr. $\frac{1}{30}$ to $\frac{1}{4}(0.002$ to $0.015 \mathrm{gm}$.).

2 Wood, H. C., Jr., Newer Ideas Concerning Digitalis, Therap. Gaz., June 15, 1915.

${ }^{3}$ Arch. Int. Med., 1913, xi, 418.

${ }^{4}$ Hatcher, R. A., The Persistence of Action of the Digitalins, Arch. Int. Med., $1912, \mathrm{x}, 268$. 
of arterial pressure not infrequently seen. The final break can of ten be delayed by appropriate stimulation, for which purpose digitalis is by far the most satisfactory remedy.

Digitalis exerts its effects on the kidney indirectly by its-local effect on blood-flow; it also produces a direct local dilatation of the renal vessels. Its chief use in renal disease consists in its improvement of the local circulation by its effect upon the heart. It is the drug par excellence in cardiac insufficiency if the myocardium is still capable of response. Experimentally, ligation of the renal vein causes suppression of urine as well as ligation of the renal artery, showing that secretion is a question of blood-flow, not merely of blood-pressure, and digitalis not only increases arterial flow but diminishes venous stasis. When too freely administered, digitalis may cause centric vomiting. It has long been taught that emeties should not be given to arteriosclerotics lest the increased blood-pressure produced eause vascular rupture.

From recent researches ${ }^{1}$ it appears that vomiting is generally associated with a sudden and very great fall of pressure, due to cardiac inhibition but always associated with very great pressure variations. Great and sudden pressure oscillations may cause vascular damage at actual pressure heights which, had the rise been gradual would have been without serious effect. It is just as important, therefore, to avoid emesis in arteriosclerosis as was early taught, though for a somewhat different reason.

Failing cardiac compensation is frequently associated with an increase of venous blood-pressure to or above $20 \mathrm{em}$. Such a rise is therefore prognostically unfavorable, especially if it continucs or increases despite the administration of digitalis or its eongeners. ${ }^{2}$

Strophanthus and squills act in the same manner, but are less satisfactory than digitalis because of the variability of absorption of the former and because of the variability of activity and irritant (gastro-intestinal) qualities of the latter. In therapeutic dose neither strophanthin nor digalen affect the volume of the arm nor its normal reaction to the application of cold, although injections of these substances are said to affect the tonus of the larger arteries. ${ }^{3}$

1 Brooks, C., and Luckhardt, A. B., Blood-pressure during Vomiting, Am. Jour. Physiol., 1915, xxxvi, 104.

2 Clark, A. H., The Diagnostic and Prognostic Significance of Venous l'ressure Observations in Cardiac Disease, Areh. Int. Med., 1915, xvi, 587.

${ }^{3}$ Hewlett, A. W., The Circulation in the Arm of Man, Amer. Jour. Med. Sci., 1913, exlv, 656. 
Depressants.-Chloral, the coal-tar group, bromides, etc. will, in sufficient dose lower blood-pressure. 'They should, however, never be used for this purpose, although they may on occasion be useful in relieving pain or promoting sleep. Chloral may produce degenerative changes in heart and liver similar to those following the administration of chloroform.

Emetin.-This drug is sometimes administered intravenously. This is not a safe procedure, since the drug is a cardiac depressant. If this method of administration must be employed, coincident blood-pressure readings are desirable with a view to detecting evidences of circulatory failure (R. L. Levy).

Epinephrin.-(See under Physiology, page 33). Epinephrin may be administered by mouth without any demonstrable cardiorascular action. Prolonged use in this way leads to dyspepsia associated with colicky pains. Large doses given to rabbits produce arterial degeneration without increasing blood-pressure.

Experimental Data.-Epinephrin produces upon the cardiovascular system the same effect as would electric stimulation of the sympathetic-a marked rise of blood-pressure due to vasoconstriction and increased cardiac action (increased systolic output). This rise is of brief duration, due to fatigue. Venous pressure rises slightly. The different arteries are contracted in proportion to their sympathetic innervation. The coronary arteries are said to be unaffected (Schafer), owing to the absence of sympathetic fibers. In experimental animals epinephrin causes coronary dilatation, but in man (isolated arteries) and in monkey (perfused hearts) it causes coronary constriction, presumably because in the latter species the arteries are supplied with constrictor nerves of true sympathetic (thoracico-lumbar) origin. ${ }^{1}$ Epinephrin diminishes the pulse rate through vagus stimulation. ${ }^{2}$ In small dose it increases the rapidity of the pulmonary circulation; in excessive dose it may cause pulmonary edema in men (Bennett) as it does in animals. Large doses and ragus stimulation diminish it. Apnea during artificial respiration has no effect on pulmonary circulation. ${ }^{3}$ The intraspinal injection of adrenalin causes a slower but more

1 Barbour and Prince, Influence of Epinephrin on the Coronary Circulation of the Monkey, Jour. Exp. Med., 1915, xxi, 300.

2 Meek and Eyster, Effect of Epinephrin on the Heart Rate, Am. Jour. Physiol., 1915, xxxviii, No. 1.

${ }^{3}$ Langlois, P., and G. Desbouis, Sur la vitesse de la cireulation pulmonaire, Adrénaline, Digitaline, Asphyxie. Respiration Artificielle, Mém. 2, Journal dé physiol. et de Pathol. gén., 1912, xiv, S. 1113-1123. 
prolonged rise of pressure (40 $\mathrm{mm}$. for half an hour or longer), although occasionally preceded by a primary fall $(9$ to $50 \mathrm{~mm}$. for a half to four minutes). ${ }^{1}$

In using intraspinal injections care must be taken to avoid sudden increase of pressure. It is safer to allow inflow only by the force of gravity. 'Too high an intraspinal pressure is first manifested by cessation of respiration, soon followed by cardiac inhibition and an enormous fall of blood-pressure. To combat cardiac failure under these circumstances, atropin is the most useful drug, whereas cocain is the best respiratory stimulant. Since the fall of pressure is not due to vasomotor failure, epinephrin is not indicated. The lowering of the intraspinal pressure after symptoms have arisen is unproductive of benefit. It has been suggested that a local hypodermic prophylactic injection of cocain and atropin be given at the site of the spinal injection, especially if the patient be under the effects of chloroform, since it not only minimizes the danger of sudden death but allows a larger injection to be given (Carter). ${ }^{2}$ (See page 390.) Experimentally, epinephrin has by some investigators been found to be beneficial in hemorrhage, chloroform poisoning, pneumococcus septicemia, and diphtheria intoxication. On the other hand, Gottleib has shown that in rabbits receiving diphtheria toxin daily until cardiovascular symptoms appear, the injection of adrenalin only produces a brief and deceptive rise of arterial tension which soon leads to a fatal termination. ${ }^{3}$

Clinical Data.-A marked fall of blood-pressure due to vasomotor depression without cardiac weakness is the chief indication of the use of epinephrin. Thus in shock, chloral poisoning, and collapse occurring in ether or chloroform narcosis it is decidedly useful. It should be administered intravenously in 5- to 10-minim doses ( 1 to 1000 solution) or, better still, in the proportions of 1 to 50,000 as a continuous saline infusion. If a slower and more prolonged action is desired it may be given subcutaneously, in which event its effects are usually manifest in from five to fifteen minutes.

One naturally thinks of epinephrin in the vasomotor pareses of infections, but its use is under these circumstances disappointing. The condition here is not a sudden emergency with a good cardiac

1 Auer and Meltzer, Proe. Soe. Exp. Biol. and Med., 1912, ix, 79. (Experiments on deeply nareotized apes).

2 The Effect of Intraspinal Injections of Ringer's Solution in Different Amounts under Varying Pressures, Arch. Int. Med., 1912, x, 425.

${ }^{3}$ Gottleib, Arch. f. exp. Pharm., xxxviii and xliii. 
muscle, but a gradual failure of vasomotor and often cardiac strengtl. We must not forget that if the heart weakness is present a sudden increase of arterial pressure may be more of a load than the staggering organ can bear. The best and most lasting effects are obtained by slow intravenous administration in dilution with normal salt solution. Epinephrin has proved useful in vasomotor failure due to diphtheria, pneumonia, plague, peritonitis, and in surgical shock.

Iypodermic epinephrin injections are variable in their effects. They do not always increase blood-pressure, but when they do so the rise generally lasts about one hour. The systolic phase is influenced more than the diastolic, indeed the latter may actually fall. Glycosuria may occur, but this bears no constant relation to pressure changes. A preliminary dose of atropin may increase the reaction eren without increasing the heart rate. Marked reactions, both objective and subjective are often produced in cases of essential hypotension, whereas low pressure due to diarrhea and cachexia is but little influenced by epinephrin. In arterial hypertension marked reactions are sometimes obtained and caution must be observed. Epinephrin increases the demarcation of the sound phases as heard in the auscultatory estimation of bloodpressure (Clough).

Ergot.- Experimental Data. - "The action of ergot may be shortly defined as a primary stimulation followed by paralysis (if given in large dose) of the motor terminations of the sympathetic nerve, which arises from the thoracic and lumbar spinal cord. The point at which ergot acts is thus analogous to that affected by adrenalin, but the range of ergot is more limited, for adrenalin affects not only the motor or positive nerve ends but also the inhibitory or negative fibers. In addition the effects of ergot are not so transitory as those of adrenalin, while, on the other hand, the latter does not exercise any subsequent paralyzing action of consequence" (Cushny).

An intravenous injection of ergotoxin is followed by a prompt, abrupt rise of blood-pressure; this occurs after section of the splanchnic nerres, showing it to be a peripheral effect. The ressels of the abdomen and the extremities become contracted. The pulse rate is often increased at first, then diminished partly from vagus stimulation from high blood-pressure and partly from direct action upon the heart muscle. The rise of pressure varies greatly in different species (Cushny). The administration of a large dose 
produces a secondary fall of arterial tension due to paralysis of the sympathetic fibers, which even epinephrin fails to overcome. ${ }^{1}$

Para-oxyphenylethylamin (isolated from ergot).-Experimentally this substance causes a rise of pressure due to capillary contraction associated with bradycardia and an increased pulse amplitude and diminution of blood in the veins. Its action is transient and followed by normal readings. ${ }^{2}$

Clinical Data.-Ergot is of very little if any use as a drug with which to restore vasomotor tone, its effects being very fugacious. Its action upon the pulmonary circulation has been discussed under Hemoptysis (see page 219). It is highly important that all ergot preparations used in medicine be physiologically standardized, not only because some preparations are inert but also because it has been shown that ergot sometimes contains small amounts of acetylcholin which has been pronounced to be the most powerful circulatory depressant known, as small an amount as one-millionth part of a milligram causing a fall of blood-pressure in rabbits. ${ }^{3}$

Hydrastis. - Although rarely employed in medicine, hydrastis is occasionally recommended in the treatment of hemoptysis owing to a supposed vasoconstrictor aetion. Experimental evidence, however, indicates that it has no such effect. W. W. Williams, ${ }^{4}$ from a careful experimental research, comes to the following conclusions:

The most constant and conspicuous effect of the intravenous injection of hydrastis is a prompt fall of blood-pressure. With small doses the pressure promptly returns to normal, and there may be a slight rise above normal. With larger doses (from 0.07 c.c. to 1 c.c. per kilogram of body weight) there is only partial recovery from the fall of blood-pressure, or it may remain low. The pressure phenomena are attributable to depression of the cardiae muscle, causing the fall, and to stimulation of the muscle, causing the rise. Very large doses depress and paralyze the vagus and vasomotor system; otherwise there is no evidence deduced from the myocardiograms and oncometer that the vasomotor system has any important part in the blood-pressure changes.

The two principal alkaloids of hydrastis, hydrastin and berberin,

1 Dale, Jour. Physiol., 1906, xxxiv, 163.

2 Bickel, A., and Pawlow, M., Untersuchungen z. pharm. Wirkung des p. Oxyphenylaethylamins, Biochem. Zeitsch., 1912, xlvii, 345.

3 Editorial, Jour. Am. Med. Assn., 1914, lxiii, 2136. Diseussion and literature.

4 The Effects of Hydrastis and its Alkaloids on Blood-pressure, Jour. Am. Med. Assn., January 4, 1908, p. 26 (bibliography). 
cause qualitatively the same blood-pressure changes, although berberin is the more active and is responsible for about 85 per cent. of the effect of hydrastis-hydrastin causing the remaining 15 per cent. Hydrastis given by mouth or hypodermically causes no change in the blood-pressure, heart rate, or respiration.

Hydrastinin, an artificial alkaloid, derived from hydrastin, causes a rise of blood-pressure above normal, which is usually preceded by a slight fall when injected intravenously. The rise is well sustained and is principally caused by stimulation of the cardiac muscle.

"The results of this investigation do not support the clinical theories concerning hydrastis. It is possible that conditions in man and in disease may modify the actions observed by me, but this is rather improbable. At least the uniform contradiction of the experimental results and clinical opinions demands that the latter be examined critically before they are accepted."

Hormonal.--This drug has been used as a remedy against intestinal atony and paralysis. Mohr states that his experimental research on hormonal has established beyond question its pronounced effect in reducing blood-pressure even with doses proportionately far below those used in the clinic to date. He had an experience with a threatened collapse in a man, aged twentyeight years, after intravenous injection of 14 c.c. of hormonal, and since then, he says, four similar cases have been reported. Madlener has recently reported the death of a patient after injection of 20 c.c. hormonal for paralytic ileus following a myoma operation. Sabatowski's research on dogs has further confirmed the sudden pronounced drop in the blood-pressure after intravenous injection of hormonal.

The Iodids.- Regarding the effect of the iodids on bloodpressure there is a difference of opinion. Certainly they are not active vasodilators in the sense that the nitrites are. On the other hand, cases are occasionally encountered in which a lowering of tension occurs, a response which naturally suggests that the hypertension was reflexly due to syphilitic aortitis or directly to endarteritis. The former may exert an effect on the depressor nerve, the latter may cause loss of vascular elasticity from cellular infiltration.

Numerous explanations for the physiologic effect of the iodids have been offered: (1) germicidal action; (2) increase of opsonins; (3) leukocytosis and increased lymphatic flow; (4) specific action of free iodin on gummatous tissues; (5) increased vascular perme- 
ability; (6) stimulation of the thyroid gland; (7) the formation of a proteolytic ferment (Dick); (8) decreased hemic viscosity. Of these the last three chiefly merit consideration.

Decreased viscosity undoubtedly occurs, but, according to Determann, ${ }^{1}$ not in excess of normal physiologie variation. Capps $^{2}$ has in a recent excellent review favored the theory of proteolysis, due to a ferment which has a selective although not specific action "on diseased granulomatous tissue of the arteries in a certain group of diseases," there being evidence to show that the iodids may exert such an effect in tuberculosis, actinomycosis, and leprosy. Such a ferment is assumed to result from the union of the iodid with the antibodies of the infected invididual.

Experimentally, potassium iodid, if administered intravenously causes a fall of blood-pressure, but it has been shown that this effect is solely due to the effect of the kation (potassium) while the action of the ion (iodid) is actually one of stimulation, both of heart and bloodvessels. ${ }^{3}$ A number of organic iodin preparations have been placed on the market with the claim of lessened gastro-intestinal irritation, absence of iodism, ready absorption, etc. Excepting preparations of the thyroid gland, they have no speeific action other than that of iodin. The iodized proteins cause less gastric irritation; the more stable compounds are not entirely split in the body, and, therefore, not well utilized, while the less stable ones have no advantage over the alkaline iodids. Iodized fats and fatty acids are more slowly and evenly split, so that the amount of available iodin in the blood varies less than when the alkaline iodids are used. The diminished frequency of iodism seems to result from the difference in the available iodin present in the body at a given time. ${ }^{4}$

The absorption of iodids from the intestinal tract is rapid up to a certain point, beyond which a stoppage oceurs which apparently' results from some local action. It is unaffected by blood-pressure except when this is very low, when absorption is somewhat slower. ${ }^{5}$

Summary.-So far as hypotensive effects are concerned the iodids may be employed in syphilitic arteritis and in lead poisoning, $q \cdot v$.

I Das Verhalten d. Blutviskosität bei Ioddarreichung, Deut. med. Woch., 1908, No. 20 .

${ }_{2}$ Effects of Iodids on the Circulation and Bloodvessels in Arteriosclerosis, Jour. Am. Med. Assn., October 12, 1912, p. 1350.

3 Macht, D. I., Action of Potassium and Sodium Iodids and of Iodin Ion on the Heart and Bloodvessels, Bull. Johns Hopkins Hosp., September, 1914.

${ }^{4}$ MeLean, F. C., Organic Iodin Preparations, Their Pharmacologic and Therapeutic Value, Arch. Int. Med., 1912, x, 505.

${ }^{5}$ Hanzlik, P. J., Quantitative Studies on the Gastro-intestinal Alsorption of Drugs, Jour. Pharm, and Exp. Therap., 1912, iii, No. 4. 
The eustom of giving small doses over prolonged periods of time in other varieties of hypertension, while very common, is probably useless. The iodids have no direct effect upon blood-pressure.

Mistletoe (I'iscum Album).-Mistletoe has been used, chiefly in Europe, for the purpose of lowering blood-pressure. Experimentally (in a dog) intravenous injection promptly and progressively lowers blood-pressure 20 or $30 \mathrm{~mm}$. $\mathrm{Hg}$. This is associated with acceleration of the pulse, with diminished amplitude, and after a stationary period, a gradual rise of pressure to the normal level (one to two hours). This action is the result of central depression of the vasomotor centre. ${ }^{1}$ Mistletoe has been given in doses of 30 to $60 \mathrm{~m}$. of the fluidextract.

The Nitrite Group.-The most potent, as well as the most maladministered and abused of the blood-pressure-lowering remedies are the nitrites. Their action is rapid and their effect is for the most part fugacious: Furthermore, a tolerance for this class of drugs is rapidly established (Fig. 96). They should therefore be given frequently and in sufficient dose to produce their physiologic effect. 'The trpical action is not only vasodilatation but also an increased systolic output which occasions a more rapid flow. ${ }^{2}$ The following table shows the effect of these drugs upon normal individuals:

Table 1.-Average Blood-pressure Results from Administration of Nitroglycerin, Sodium Nitrite, and Erythrol Tetranitrate to Normal Persons. (Wallace and Ringer.)

\begin{tabular}{|c|c|c|c|c|}
\hline Drug. & $\begin{array}{l}\text { Time of } \\
\text { beginning } \\
\text { action. } \\
\text { IIin. }\end{array}$ & $\begin{array}{l}\text { Time of } \\
\text { maximum } \\
\text { effect. } \\
\text { Min. }\end{array}$ & $\begin{array}{l}\text { Time of } \\
\text { duration of } \\
\text { action. } \\
\text { Min. }\end{array}$ & $\begin{array}{c}\text { Maximum } \\
\text { extent of } \\
\text { action. } \\
\mathrm{Mm} . \mathrm{Hg} .\end{array}$ \\
\hline
\end{tabular}

Amyl nitrite, 3 minims . Nitroglycerin, $1 \frac{1}{2}$ minims,

1 per cent. sol. . . . 2

Sodium nitrite, $1 \mathrm{gr}$. . . 10

Ery"throl tetranitrate, $\frac{1}{2}$ gr. 15

13

$3 \quad 7$

15

11

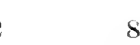

$8 \quad 30 \quad 15$

$32 \quad 120$ to $240 \quad 16 \quad 14$

It will be noted that promptness of action and duration of the effect tend to vary inversely. The therapentic effects are obtained about as promptly when the drug is given by mouth as when it is administered hypodcrmically. The percentage of fall is markedly uniform, the higher the initial pressure the greater the drop.

Physiologic Action.-Different members of the nitrite group of drugs have an essentially similar action. Their effects vary only in promptness, intensity, and duration. The chief action of the

\footnotetext{
I Gaulthier, R., Etudes physiologiques sur le qui, Arch. Internat. d. pharmacodynamie, 1910 , Nos. 1 and 2.

2 Cameron and Hewlett, Jour. Med. Research, December, 1906.
} 
nitrites is that of lowering blood-pressure (depression of the nerve endings and musculature of the arterioles) and acceleration of the pulse rate, thus causing an increased rate of blood-flow. 'The veins are also dilated. The vessels most affected are those of the splanchnic system and of the head.

Mode of Action.-Nitroglycerin is represented by the formula:

$$
\prod_{\mathrm{CH}_{2} \mathrm{ONO}_{2}}^{\mathrm{CH}_{2} \mathrm{ONO}_{2}}
$$

"It is probable, however, that in the presence of water and hydrochloric acid in the stomach it is decomposed, and that its decomposition products $\mathrm{NO}_{2}$ and $\mathrm{NO}$ are disengaged in the form of brown vapors which are irritants and strong oxidizers. These ultimately produce an impression on the centripetal nerve endings in the gastric mucosa, which impulse is carried to the vasomotor centre and by the centrifugal nerves conveyed from the centre of the vascular wall, thereby causing the expansion of the vessels. This dilatation is due either to inhibition of the vasoconstrictors or to stimulation of the vasodilators" (W. H. Porter). ${ }^{1}$

Not infrequently patients exhibit marked idiosyncrasies to the nitrites: some bear them very badly and are much more affected by one member of the nitrite group than by another; others can take enormous doses (one grain of nitroglycerin daily has been given with apparent benefit). The administration of the nitrites coincidently with digitalis is a therapeutic blunder. The effect of the former will have passed off long before that of the latter has begun to act. Nitroglycerin has a retarding effect on renal secretion (Loeb). Nitrite of anyl acts primarily upon the cerebral vesselsmore so than the other nitrites.

Table II.-Average Blood-pressure Results firom Abministization of Nitroglycerin, Sodium Nitrite, and Erythrol Temtranitrate to Patients with Arteriosclerosis. (Wallace and Ringer.)2

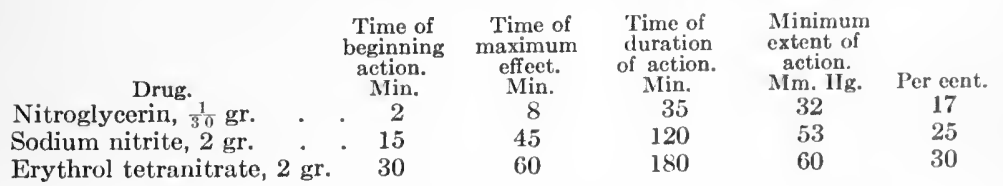

1 Jour. Am. Med. Assn., August 3, 1912.

2 The Lowering of Blood-pressure by the Nitrite Group. Jour. Am. Med. Assn., 1909, liii, 1630 . 
In hypertensive cases the response is sometimes less prompt (delaved absorption) and the action more prolonged (delayed excretion). The percentage fall is much the same in both tables. Headache is less frequent in high-pressure cases; indeed, it is often relieved by these drugs.

The employment of the nitrites in hypertension is purely symptomatic medication. It temporarily relieves high pressure as certain forms of headache are relieved by acetanilid. It does not affect the underlying cause, and if used indiscriminately may do actual harm. The nitrites are often given in too small doses and too infrequently, especially since toleration is rapidly established; $\frac{1}{50}$ grain of nitroglycerin is often a better dose than $\frac{1}{100}$, and as high as $\frac{1}{10}$ may be given in emergencies.

The nitrites are essentially emergency drugs to be given in case of a threatened apoplexy or cardiac failure from hypertension, etc. The splanchnic dilatation relieves the systemic, especially the intracranial pressure, but, of course, after intracerebral vascular rupture has occurred the nitrites are contra-indicated. Many preparations on the market are inert. The dosage must be based on the physiologic effect, flushing, headache, and a fall of pressure. Nitroglycerin is also used as a therapeutic test agent to determine whether certain symptoms are due to spastic vascular contraction. It is contra-indicated in arterial hypotension, and should therefore not be used as a primary cardiac stimulant in acute febrile disease or shock.

Form of Administration.- Nitrite of amyl, in glass capsules (to be kept in the dark), to be crushed in a handkerchief and inhaled. The primary fall of pressure is followed by a rise to above the original level (Hewlett). In cases of cardiac weakness, instead of a secondary rise of 6 to $10 \mathrm{~mm}$., a fall of pressure occurs (Abrams).

Nitroglycerin, preferably as a 1 per cent. solution (spiritus glycerylis nitratis), to be swallowed, or as tablets to be dissolved on the tongue. Dose $\frac{1}{100}$ to $\frac{1}{10}$ grain.

Sodium Nitrite.-Tablets to be swallowed or dissolved on the tongue. Dose: gr. ss to gr. ij (solutions deteriorate rapidly).

Erythrol Tetranitrate.-Tablets. Dose: gr. $\frac{1}{4}$ to gr. ss. Erythrol tetranitrate produces the most severe headaches.

Mannitol nitrate (gr. j); effect even more prolonged than erythrol.

On the whole the spiritus glycerylis nitratis is the best preparation, both because of its reliability and because of the ease with which the dose can be increased. 
If a prolonged and gradual effect is desired the nitrites should be given after meals. If taken between meals the drug should be well diluted. Sudden effects are generally as useless as they are undesirable.

Summary.-1. "The general indications for the use of nitroglycerin are $(a)$ to relieve symptoms of localized arterioselerosis or arterial spasm in vitally important regions of the body and when there is pain due to contracted or diseased arteries in other regions; $(b)$ to reduce general high blood-pressure in selected cases, if its continuance threatens accidents to the cardiovascular apparatus; and (c) to clear the diagnosis (see p. 300).

2. The chief contra-indications to the use of nitroglycerin are (a) low or relatively low blood-pressure; (b) advanced chronie nephritis with very high blood-pressure and toxemic conditions producing high blood-pressure, as a rule; and $(c)$ the presence of an idiosyncrasy in regard to its action."'

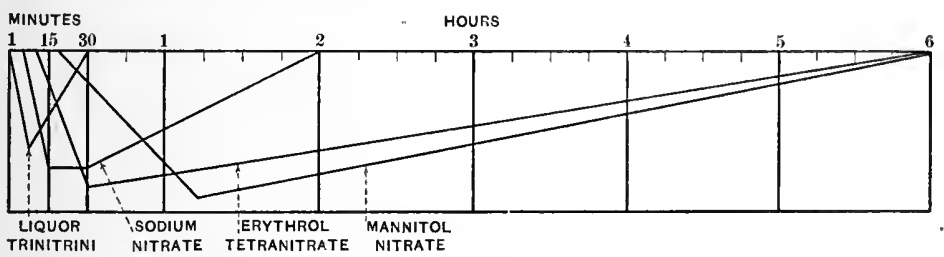

F1G. 96.-Diagram showing the comparative promptness of action and duration of effect of different blood-pressure-lowering drugs. (After Mathews.)

Opium.-In some cases of hypertension (angina pectoris, broken compensation, etc.) morphin is an indispensable drug. It often produces a most satisfactory fall of pressure as well as relief from symptoms, the former being doubtless largely due to its general sedative influence on the nervous system and its stimulation of the vagus nerve. Under its influence the cyanotie skin is often replaced by a pink flush indicating a dilatation of the peripheral circulation. The danger of this drug in nephritis has been greatly overestimated. The beneficial effects of opium are also in part due to its effect on the respiration. Edsall has shown that superficial rapid respirations such as are seen in eardiae dyspnea, etc., are funetionally less efficient than slower labored movements. ${ }^{2}$ It tends to lower

1 Cornwell, E. E., When and How to Use Nitroglycerin, Jour. Am. Med. Assn., 1913, Ixi, 118.

${ }_{2}$ Edsall, D. L., The Efficiency and Siguificance of Different Forms of Respiration, Trans. Assoc. Amer. Phys., 1912, xxvii, 560. 
blood-pressure by central vagal slowing of the pulse. ${ }^{1}$ In the case of normal hearts during nocturnal sleep the pulse rate is slowed about twenty beats per minute, and although the slowing thus produced becomes progressively less, the worse the cardiac compensation, yet notwithstanding the establishment of sleep, induces very considerable cardiac rest. ${ }^{2}$ Morphin produces a mild, codein only a slight, dilatation of the coronary arteries. Narcotin and papaverin cause marked dilatation. But morphin and narcotin combined have less effect than either separately. A combination of caffein (which of itself produces coronary dilatation) with papaverin causes dilatation of the coronary ring. ${ }^{3}$

Pilocarpin.-Is a powerful diaphoretic, the physiologic action of which is directly opposite to that of atropin. Full doses slow the cardiac rate and diminish contraction through vagus stimulation. Still larger doses depress the heart muscle directly. Toxic doses cause peripheral vasodilatation by depressing the vasoconstrictor centre. It therefore tends to lower blood-pressure.

It has long been used as a diaphoretic in renal disease, especially in conjunction with hot packs or hot-air baths if the patient does not sweat sufficiently from the latter procedures alone. It must, however, be given with caution in cases of heart weakness, since it may produce pulmonary edema. This accident can, however, be promptly corrected, if treated in time by full doses of atropin (gr. $\frac{1}{50}$ hypodermically). The usual dose of pilocarpin for adults subcutaneously is $\frac{1}{10}$ grain $(0.006)$.

It has been recommended in small doses (gr. $\frac{1}{30}$ ) thrice daily in water after meals to relieve arterial hypertension and its associated symptoms, especially headache. ${ }^{4}$

Pituitary Extract.- This substance has a more prolonged though less marked blood-pressure raising effect than epinephrin. 'Too frequently repeated it depresses respiration. It also has a diuretic effect due to direct stimulation of the renal cells, usually aided probably by a concomitant vasodilatation, because there is no constant relation between pituitrin diuresis and either systolic or pulse pressure or the ratio between them. As a general rule, however,

\footnotetext{
1 Van Egmond, Die Wirkung des Morphins auf d. Herz, Arch. f. exp. Path., $1911,1 \times 197$.

2 Klewitz, F., Der Puls im Schlaf, Deut. Arch. f. klin. Med., 1913, cxii, 38.

3 Macht, D. I., Action of the Opium Alkaloids, Jour. Am. Med. Assn., 1915, lxiv, 1489.

${ }^{4}$ Robinson, W. D., Pilocarpin in High Blood-pressure, Trans. Am. Climat. Assn., $1914, \mathrm{xxx}, 290$.
} 
it is accompanied by a decreased pulse pressure. ${ }^{1}$ Furthermore, there is no constant relationship between renal volume and pituitary diuresis, ${ }^{2}$ with local renal vasodilatation. ${ }^{3}$ It stimulates the heart. It may be given hypodermically, intravenously, or by mouth. It may be used instead of epinephrin in cases of temporary hypotension or in cases of pulmonary hemorrhage, $q . v$. It causes eontraction of the peripheral arterioles, increases cadiac contraction, and slows the pulse. These results are due to a direct effect upon the cardiac and arterial musculature. It has been recommended in all cases in which lowered blood-pressure is due to loss of splanchnic vascular tone, and in hypotension due to shock or toxemia, especially in combination with saline infusion. ${ }^{4}$ Musser $^{5} \mathrm{Jr}$., who administered pituitary extract by mouth $(0.065$ dried gland, q. d.) obtained very constantly a distinct elevation of blood-pressure (up to $28 \mathrm{~mm}$. Hg.) which often persisted for a time after discontinuance of the drug. Diarrhea was sometimes produced. Commercial preparations of the posterior lobe vary greatly in potency, and should be standardized, Roth suggests, by their action on the isolated uterus of the virgin guinea-pig.

Spartein.-Spartein sulphate stimulates the vagal ganglia and. depresses the heart muscle. It slightly stimulates the ganglia of the vasoconstrictor nerves but clinically produces no demonstrable rise of blood-pressure.

Strophanthus.-The action of this drug is essentially that of digitalis. Its effects are somewhat less lasting, perhaps because, as suggested by Eggleston, it is less firmly fixed in the tissues and more promptly eliminated. If used, it should be given intravenously in the form of strophanthin. ${ }^{6}$ Its absorption from the gastrointestinal tract is variable and uncertain (Hatcher).

Salvarsan.-Experimentally, small doses produee but slight and temporary effects on blood-pressure. Moderate doses cause a slight fall at the end of two minutes, a secondary rise and a tertiary decline (two to six minutes). Large doses lower pressure at once.

I Hoskins and Means, Relation of Vascular Conditions to Pituitrin Diuresis, Jour. Phar. and Exper. Therap., 1913, iv, No. 5.

2 Schaeffer and Herring, Philos. Trans. Royal Soe., Sect. 13, 1908, exeix, 1.

3 King and Stoland, Am. Jour. Physiol., 1913, xxxii, 405.

${ }_{4}$ Klotz, Internat. Congr. Physiotherapy, Berlin, 1913.

5 Effects of Continuous Administration of the Pituitary Gland, An. Jour. Med. Sei., 1913, cxlvi, 208.

${ }^{6}$ Subcutaneous dose: cryst. strophanthin, gr. $T^{\frac{1}{2}}{ }_{0}(0.0005$ gram), once in twentyfour hours. Intravenously in 1 to 6000 saline solution of strophanthin (Bochringer), gr. $\frac{1}{120}$ to $\frac{1}{60}(0.0005$ to $0.001 \mathrm{gram})$. 
Both alkaline and acid solutions of salvarsan experimentally cause a fall of blood-pressure. If an excess of alkali is added bloodpressure may rise but this is purely due to the alkali which has physiologically overantagonized the salvarsan. Cardiac dilatation may also be directly produced by salvarsan and in such an event the heart shows changes in rate and volume before the bloodpressure falls. Sudden death following its administration is therefore generally a cardiac death (Luithlen). ${ }^{1}$ The fatal termination has also been ascribed (Hoke and Rihl) to a central vasomotor depression. The administration of sodium arsenate produces a marked fall in blood-pressure without any direct effect upon the heart, showing that the action of salvarsan is not identical with that of arsenic. In vascular disease, doses which are not toxic to the heart may cause a sudden fatal termination through their effect on the bloodvessels. Salvarsan causes contraction of the coronary arteries and a decreased pulse rate. ${ }^{2}$ Rindfleisch has emphasized the danger of salvarsan injection in status thymolymphaticus, and has reported two cases in which a summation of depression, salvarsan and thymus extract caused death. ${ }^{3}$

The depression of blood-pressure caused by salvarsan in medicinal dosage is never sufficiently great to endanger a normal circulatory system, but this form of medication may be definitely dangerous in cases of marked hypotension. ${ }^{4}$

Neosalvarsan.-During the administration of neosalvarsan both systolic and diastolic pressures, although variable, are usually increased apparently as a result of excitement. After the injection (seven hours) both pressures are decreased and remain so for several days. At first the systolic, later the diastolic, pressure is most affected. ${ }^{5}$

Strychnin.-Strychnin is one of the most useful drugs in the treatment of symptoms associated with low blood-pressure, especially if the vasomotor depression is central in origin. It acts reflexly and its beneficial effects are indirect. It is capable of increasing the stimulability of the vasomotor system. ${ }^{6}$ Like

1 Die Exper. Analyse der Salvarsanwirkung, Ztsch. f. exp. Path. u. Therap., 1913, xiii, 495 .

${ }^{2}$ Cznbalski, F., Abstr. Centralbl. f. d. ges. inn. Med., 1913, iv, 149.

${ }^{3}$ Rindfleisch, W., Status Thymolymphaticus u. Salvarsan, Berlin. klin. Woch., $1913,1,542$.

'Sieskind, Das Verhalten des Blutdruckes bei intravenösen Salvarsaninjektionen, München. med. Woch., 1911, No. 11.

${ }^{5}$ Rolleston, Influence of Neosalvarsan on Blood-pressure, British Med. Jour., 1915, ii, 285.

${ }^{6}$ Sollmann and Pilcher, Am. Jour. Phys., 1912, xxx, 369. 
caffein, its effects are more pronounced if the blood-pressure is subnormal. Marvin ${ }^{1}$ found a marked increase in pressure after doses of gr. $\frac{1}{30}$ to $\frac{1}{20}$ in healthy students, but practically no results from smaller doses. The pulse rate was invariably slowed. The recent investigations of $\mathrm{Newburgh}^{2}$ have shown, however, that in infectious diseases in medicinal doses, strychnin does not increase the cardiac output, slow the pulse, or materially increase bloorlpressure. Nor is strychnin of value as a heart stimulant in acute or chronic cardiac failure. ${ }^{3}$

Thyroid Extract.-Injected intravenously, thyroid extract produces, as do the extracts of most tissues and organs, a fall of bloodpressure. It has been suggested that the hypotensive effect of thyroid substance is due to cholin, but Vincent believes that the depressor effect is due to other substances.

Since a diminished thyroid secretion or an increased adrenal secretion tends to raise blood-pressure, and since these secretions appear to neutralize each other, and as exophthalmic goitre paticnts often have relaxed peripheral bloodvessels, the administration of thyroid gland seems justified as a therapeutic measure in hypertension. It stimulates thyroid secretion and cutaneous activity. It is best given in small doses, one to three grains daily. Such treatment is sometimes attended by beneficial results which not infrequently outlast its administration. The experiments of Reid Hunt indicate that some of the effects of diet are due to its action on the thyroid gland (see Exophthalmic Goitre, page 352). ${ }^{4}$

Vasotonin (Yohimbin and Urethane).-The results reported upon the use of this drug are contradictory. Favorable action has been claimed both experimentally and clinically by some observers. A lowering of pressure without depression of the heart or vasomotor or respiratory centres has been reported. It is given in 1 c.c. doses daily or on alternate days, and may be administered hypodermically. It is said to cause less headache than the nitrites, ${ }^{5}$ and to produce dilatation of the vessels in the extremities especially. It has been recommended for angina pectoris and arterial hypertension by

1 Arch. Int. Med., 1913, xi, 418.

2 Newburgh, L. H., Strychnin and Caffein as Cardiovaseular Stimulants in Acute Infectious Disease, Areh. Int. Med., 1915, xv, 458.

3 Newburgh, L. H., On the Use of Strychnin in Broken Cardiac Compensation, Am. Jour. Med. Sci., 1915, cxlix, 696.

${ }^{4}$ Hunt, Jour. Am. Med. Assn., October 19, 1907, p. 1323; September 23, 1911 , p. 1032 .

5 Mueller and Fellner, Ueber Vasotonin, ein neues druckherabsetzendes Gefaessmittel, Therap. Monatshefte, 1910, xxiv, 285. 
Fellner $^{1}$ who reports favorable subjective and objective results. He states that it does not produce the aphrodisiac effects of yohimbin, nor does it produce cardiac depression.

Veratrum Viride.-Is chiefly employed by some physicians to lower blood-pressure in eclampsia. Just why is difficult to understand, since the drug is not a vasodilator. In small doses it "exercises its effect upon blood-pressure neither peripherally through its action on the vessel walls, or on the vasomotor nerve endings, nor through a direct action on the vasomotor centre or on the heart, but reflexely through the afferent vagus fibers."' Clinically this drug has never been widely used because of its danger, uncertainty of action, and because its pharmacological status is still unsatisfactory. It is usually employed in the form of the tincture in doses of 10 to 20 minims. The effective therapeutic close of the tincture of veratrum album, according to Collins, ${ }^{3}$ ranges between 30 and $75 \mathrm{~m}$. Such doses reduce the pulse rate from 12 to 42 beat per minute, and lower blood-pressure in both its phases about $30 \mathrm{smm}$. Hg. In hypertensive cases the systolic pressure may be even more reduced, but the diastolic pressure is much less affected.

Yohimbin.--Pongs ${ }^{4}$ reports in twenty-two cases after doses of 0.5 to $2 \mathrm{~cm}$. a slight fall of pressure followed by a secondary rise up to 35 per cent., reaching its high point in forty-five minutes and lasting one to five hours. No hypotensive effects were demonstrable even with larger dosage; no untoward results-uremia, angina pectoris-were noted. Genital manifestation occurred only twice.

\section{THE EFFECT OF DRUGS ON THE CORONARY ARTERIES.}

It seems evident, a priori, that the local effect of different forms of medication upon the pressure and blood-flow in the coronary arteries must be of extreme importance in the selection of our therapeutic remedies. The circulation in the coronary arteries is directly dependent upon blood-pressure.

This question has been studied experimentally by noting (1) the contraction or relaxation of arterial strips, and (2) the amount of blood-flow from an opened artery. Such investigations have,

1 Klinische Erfahrungen über Vasotonin, Kong. f. inn. Med., 1910, xxvii, 647.

${ }^{2}$ Cramer, W., The Action of Veratrum Viride, Jour. Pharm. and Exper. Therap., 1915 , vii, 64 .

${ }^{3}$ Collins, R. J., The Clinical Actions of Veratrum, Arch. Int. Med., 1915, xvi, 54.

${ }^{4} Z$ tschr. f. exp. Path. u. Therap., 1912, x, 479. 
however, not led to uniform results, because, as Rabe ${ }^{1}$ discovered, in either method the experimental heart is deprived of the effect of the central nervous system and is functionating under abnormal conditions.

Voegtlin and Macht ${ }^{2}$ found that digitoxin, digitalin, strophanthin, and buffagin produced contraction, and that the nitrites and digitonin, digalen, dilatation, of the coronary artery. If these findings hold good under normal conditions they would in a measure justify the coincident use of the nitrites with digitalis or strophanthus. Rabe noted contraction from strophanthin, digitalein, epinephrin, and slight contraction from sodium nitrite and caffein. Myer's experiments (dogs and cats) corroborate the belief that epinephrin dilates the coronary arteries and increases blood-flow through them. ${ }^{3}$ An increase of the $\mathrm{CO}_{2}$ content of the blood causes dilatation of the coronary arteries, together with cardiac dilatation and a fall of blood-pressure. Experimental asphyxia causes an even greater coronary dilatation, which increases in proportion to increased demands upon the heart muscle. ${ }^{4}$

\section{THE EFFECT OF MEDICATION ON VENOUS BLOOD- PRESSURE.}

The experiments of Capps and Mathews ${ }^{5}$ carried out upon dogs under light ether anesthesia yielded the following results:

The Digitalis Group.-The venous pressure was not materially altered.

Epinephrin.-Small doses produced no effect. Large doses caused a rise of from 10 to $80 \mathrm{~mm}$. The rise in venous pressure was coincident and coextensive with halting irregular heart action, and presumably the result of it rather than dependent upon venomotor stimulation.

Pituitrin.-Acted similarly to, but more feebly than epinephrin. Caffein.-This drug had no appreciable effect.

1 Die Reaetion der Kranzgefässe auf Arzneimittel, Ztschr. f. exp. Patl. u. Ther., 1912, xi (bibliography).

2 Jour. Pharm. and Exper. Therap., September, 1913.

${ }^{3}$ Myer, Zur Frage d. Adrenalin Wirkung auf d. Coronarkreislauf, Berlin klin. Woch., 1913, 1, No. 20.

${ }_{4}$ Markwalder and Starling, A Note on Some Factors which Determine the Bloodflow through the Coronary Cireulation, Jour. Physiol., 1913, xlvii, 275.

5 Venous Blood-pressure as Influenced by the Drugs Employed in Cardiovascular Therapy, Jour. Ain. Med. Assn., 1913, lxi, 385. 
Strychnin.-Had no effect on venous pressure except in toxic doses, in which it caused a rapid increase of pressure.

The Vitrites.-Both inhalation and injection of these substances caused a decided fall in renous pressure, due apprently to depression of the peripheral venous nerve endings.

Morphin.-This drug in small doses had little effect; in large doses it lowered venous pressure, but not nearly to the extent that did the nitrites.

Alcohol.--Large doses increased venous pressure in proportion to the degree of cardiac and arterial depression produced. 


\section{CHAPTER XV:}

\section{METABOLIC DISEASES AND MISCELLANEOLS}

CONDITIONS.

Diabetes.-Experimental Evidence--Recent researches indicate that glycosuria is in some way related to the secretion of the adrenal glands. Sugar excretion can be induced by the injection of epinephrin. The latter also produces a marked rise of blood-pressure through vasoconstriction. Neubauer's ${ }^{1}$ investigations have shown that other substances which raise blood-pressure (e.g., barium chloride) may also induce glycosuria, whereas narcotic drugs, opium, chloral, alcohol, etc., which tend to lower blood-pressure, may diminish or prevent the occurrence of glycosuria after puncture of the roof of the fourth ventricle. It has further been shown that when sugar excretion is brought about by vasoconstrictor drugs it is associated with marked hepatic hyperemia. A local stasis of blood in the liver will, it seems, bring about this liberation of glycogen in the form of glucose, with an attendant hyperglycemia and a glycosuria. It appears that transient glycosuria may be brought about by the same emotional states-pain, rage, fear, excitement-as those which cause an increased outpouring of epinephrin into the blood-stream. Until the pathogenesis of diabetes has been more thoroughly established no definite conclusions regarding the relationship of arterial hypertension to this disease can be drawn. The evidence at hand, however, suggests that a general vasoconstriction, when accompanied by a local vasodilatation of the hepatic vessels, may account for some cases of glycosuria. Hagelberg found the sugar content of the blood abnormally high in twenty-six cases of nephritis or arteriosclerosis, and suggested that an increased adrenalin content of the blood accounts for both the hyperglycemia and the hypertension. ${ }^{2}$ It is more likely, however, that the relationship between hypertension and glycosuria is not a causal one. There is no constant relation between blood

${ }^{1}$ Ueber d. Wirkung antiglyeosurischer Mittel u. üher Leberglucosurie, Biochem. Ztschr., 1912, xliii, 335.

2 Hagelberg, Berlin klin. Woch., Octoler 7, 1912. 
sugar and blood-pressure, nor is there a sufficient reason for assuming that these two conditions are dependent upon a common etiologic factor (epinephrin). (See Hyperglycemia, page 285.)

Clinical Consideration.-Diabetes bears no constant relation to blood-pressure. Cases occurring in advanced life often show hypertension as the result of renal and cardiovascular complications, which are commonly associated lesions. Janeway found high blood-pressure in 50 among 220 diabetics. Diabetes in the young is accompanied by normal pressures. In the later stages hypotension may occur. Blood-pressure estimations may be of diagnostic value in differentiating between diabetic and uremic coma. In the latter condition hypertension is the rule until the terminal fall of pressure occurs, whereas diabetic coma is associated with hypotension. ${ }^{1}$ Although this occurs less constantly than does hypertension in uremia, it is sometimes an early manifestation. The socalled "diabetic collapse" which has been believed to occur independently of coma, appears to be the result of a rapid fall in bloodpressure due to cardiac and vasomotor weakness, resulting from acidosis. Experimentally the injection of B-oxybutyric acid causes a fall of blood-pressure.

The pulse rate is increased in diabetes, and, as has been emphasized by Benedict and Joslin, stands in intimate relation with increased metabolism. ${ }^{2}$ Preceding the onset of coma, bloodpressure falls sometimes 40 to $50 \mathrm{~mm}$. $\mathrm{Hg}$. Decrease of intra-ocular tension, which occurs simultaneously, is well known and results, in part at least, from the lowered arterial tension. Blood-pressure readings_are therefore of value in detecting the onset of coma as well as in indicating treatment. It has been found that in coma cases with low blood-pressure better results are obtained when cardiovascular stimulants are combined with the usual alkaline therapy. The administration of thyroid substance to diabetics often causes a rise of blood-pressure (Biedl).

Gout.-Until the pathogenesis of gout is more definitely elucidated it is impossible to make positive statements in regard to the relation of this disease to arterial hypertension. It seems that the products of protein metabolism, if increased or abnormal in character owing to overeating, suboxidation, or faulty excretion, may produce hypertension. Occasionally metabolic intoxication

\footnotetext{
${ }^{1}$ Ehrmann, R., Ueber cardiovasculare Symptome u. d. Therap. bei diabetisehem Coma u. Pracema, Berlin klin. Woch., 1913, 1, 11, 1423.

${ }^{2}$ Metabolism in Severe Diabetes, Carnegie Institute of Washington, 1912, Pub. 176,85 .
} 
seems to lead to hypotension. High pressure is, of course, the rule, with added increments of tension during the acute attacks (pain), but gout is very commonly associated with arteriosclerosis and chronic nephritis. The latter certainly accounts for a measure, if not all, of the increased blood-pressure. Hypotension is sometimes seen in the later stages in association with cachexia, acidosis, and cardiac weakness. It has also been described as occurring at the onset of an acute attack. The presence of hypertension may be of some value in diagnosticating between lesions of the joints, skin, eye, etc., as to whether they be tuberculous or gouty in origin.

Experimental Data.-Investigations point strongly to the conclusion that the occurrence of gouty symptoms is closely associated with abnormalities of protein metabolism, especially with that portion of it which relates to the breaking down and elimination of the amino-acids.

In animals the intravenous injection of guanin $(0.02$ to $0.03 \mathrm{gm}$. per kilo) causes a fall of blood-pressure of from 2.4 to 4 c.c. Hg. If other substances are employed which stand chemically near to guanin but have no animo group, e.g., xanthin, uric acid, an increase of pressure results $(0.7$ to $2.6 \mathrm{~cm}$. $\mathrm{Hg}$.). In the transition from guanin to uric acid there is a splitting off of the animo group, to which guanin probably owes its pressor effect. ${ }^{1}$

Jaundice.-Jaundice is often associated with hypotension. According to Federn, the latter can often be demonstrated before the bile appears in the urine.

Addison's Disease.-Arterial hypotension is frequently but not invariably found in Addison's disease. It bears no relation to the degree of pigmentation, but is believed to oceur when the medullary portion of the adrenal gland is diseased. This tends to corroboratc the belief generally held at present that the adrenal secretion may raise blood-pressure, but that it is not under normal conditions the sole cause of its maintenance, ${ }^{2}$ and further, that Addison's disease results not merely from degeneration of the adrenal glands but from involvement of the entire chromaffin system.

Grunbaum found the oral administration of epinephrin, while

1 Desgres and Dorleans, Influenee de la constitution des corps purques sur leur action vis-a-vis de la pression artérielle, Compt. rend. Acad. des Seiences, Paris, 1913, elvi, 93.

2 Hoskins, R. G, and Rowley, IV. N., The Effeets of Epinephrin Infusion on Vasomotor Irritability, Am. Jour. Physiol., 1915, xxxvii, 471; also Cannon, W. B., Bodily Changes in Pain, Hunger, Fear, Rage, New York, 1913. 
it did not raise blood-pressure in health, did so in case of adrenal insufficiency. He suggests the use of this substance as a diagnostic test. Together with careful blood-pressure readings, three grains of adrenalin are administered thrice daily. Any distinct subsequent rise of pressure in the absence of a valvular lesion is, he believes, suggestive of Addison's disease even in the absence of asthenia and pigmentation. Too much reliance must not be placed upon this test.

On the other hand, recent experiments have shown that a fall of pressure occurs after the injection of dilute solutions of epinephrin, and that hypertension occurs only when abnormally large doses are used. In amounts sufficient to raise blood-pressure, intestinal peristalsis is completely inhibited. If these observations are correct the low pressure seen in adrenal insufficiency and in Addison's disease are probably due rather to an interference with muscular metabolism, including that of the heart and the arteries, than to failure of a normal tonic stimulation of the sympathetic nervous system. ${ }^{1}$

Auto-intoxication.-Much has been written and but little is known regarding gastro-intestinal auto-intoxication. The literature on the subject is enormous and concerns itself mainly in nebulous hypothecation. It has been shown that two pressor bases may be extracted from putrid meat, and the assumption that they are generated in the intestine through bacterial action seems probable. Granger was unable to confirm the observation of Abelous and Bain that the normal urine contains pressor bases, and there is as yet no definite clinical proof that these urinary pressor substances are identical with those isolated from decaying proteids. $^{2}$

Hydroxyphenylethylamin is a substance which can be prepared from tyrosin, a derivative of protein cleavage, by splitting off carbon dioxid from the tyrosin molecule. This reaction may be caused by putrefactive bacteria. Hydroxyphenylethylamin has been isolated by Barger $^{3}$ from putrefying proteids and shown to have a pressor action. The group of aromatic amins to which

${ }^{1}$ Hoskins and McClure, Am. Jour. Phys., 1912, xxxi, 59; Hoskins and McPeek, The Effects of Adrenal Massage in Blood-pressure, Jour. Am. Med. Assn., 1913, $\mathrm{Ix}, 1777$.

${ }^{2}$ Granger, A. S., Concerning the Presence in the Urine of Certain Pressor Bases, Arch. Int. Med., 1912, x, 202.

${ }^{3}$ The Isolation of Pressor Principles of Putrid Meat, Jour. Physiol., 1908, xxxviii, 
it belongs is related chemically to epinephrin. It is an interesting fact that this aromatic amin (p-hydroxyethylamin) with its bloodpressure-raising qualities is found in epinephrin, in ergot, and as the result of bacterial putrefaction. "Our poisons and our drugs are in many instances the elose relatives of harmful eompounds that represent the intermediary steps in the daily routine of metabolism." As Abel" has recently stated, "It would appear that we have at last got onto the right road for the ehemical investigation of alimentary toxemia and its alleged consequences, such as arterioselerosis and ehronic renal disease. Phenylalanine, tyrosin, tryptophane and histidine, the harmless precursors of toxic amins are always present in the intestine, and when they are acted upon by an excessive number of certain microörganisms the resulting toxic bases will surely be formed in excess. If they are then taken up into the blood in quantities too large for transformation by the liver or other defensive organs, into harmless derivations, they must inevitably manifest their pharmacologieal and toxicologieal properties."

Not only arterial hypertension but also hypotension has been elinically attributed to alimentary toxemia. That the latter no less than the former may have basis in fact is shown by the observation of Barger and Dale ${ }^{2}$ who were able to obtain a highly toxic depressor base-B-amino-azolylethylamin from the intestinal mucosa.

This substance appears to be formed from histidine through the ageney of the B-aminophilus intestinalis. ${ }^{3}$ It has further been suggested that the toxic substance which causes anaphylactic shock bears an allied chemical composition.

The Effects of Glandular and Tissue Extracts.-The extracts of most animal tissues when injeeted intravenously lower bloodpressure. It has been suggested that in many instances this depressor action is due to cholin, a substance abundantly found in brain-tissue extraets and in tissues in which proteolytie changes, which oecur very early, have taken place. 'The action of cholin is characterized by the fact that the first injeetion fails to establish tolerance to a second injection, and by the fact that the reaction may be counteraeted by atropin. The depressor effects of peptone

1 The Blood and the Specific Secretory Products of the Organs of Internal Secretion, Science, 1915, N. S., xlii, 165.

2 Jour. Physiol., 1910, xl, 38.

3 Berthelot and Bertrand, Compt. rend. de l'Acad. d. Seicnees, 1820 , cliv, 1643. 
have long been known, but in this case tolerance is established by the first dose $^{1}$ (see Toxic Shock, page 210).

Certain glandular extracts have also been shown to possess a specific action on blood-pressure. Thus extract of the adrenals and hypophysis possess well-marked pressor effects, while pineal and thyroid extracts cause a fall of pressure.

Acromegaly. - In acromegaly pressure is variable (see Pituitary Extract, page 340). Chronic hypopituitarism, both clinical and experimental, is characterized by a deposition of fat, a lowering of body temperature, a retardation of the pulse and respiratory rates, somnolence and lowered blood-pressure. These symptoms, as has been pointed out by Cushing and Goetch, ${ }^{2}$ bear a striking resemblance to the state of hibernation as it occurs in some animals. IV. Landon Brown ${ }^{3}$ states that in the early stages of acromegaly in association with hemianopia, lowered sugar tolerance and osseous hypertrophy, blood-pressure is high, whereas in the later stages with a high sugar tolerance blood-pressure is low. In Frölich's syndrome (primary hypopituitarism) smooth, dry skin, scant pubic and axillary hair, small, thin finger-nails-blood-pressure is low.

Exophthalmic Goitre.-Experimental Data.-In 1894 Oliver and Schïfer reported that the intravenous injection of aqueous or glycerinized extracts of the thyroid gland produced a fall of bloodpressure in which the heart took no part. These observations have been widely corroborated. A few observers found an increased pressure and local vascular constriction after thyroid injections. The pulse rate has been reported both as accelerated and retarded.

Von Cyon and Oswald found lowering of pressure and slowing of the pulse after the intravenous injection of iodothyroid even after atropinization and section of the vagi. Later experiments have shown that the effect of this substance upon cardiac inhibition is questionable, and that the fall of pressure is not a specific action, but occurs after the injection of many organic extracts which contain cholin, a substance which is associated with the metabolism of lethicin and other lipoids, and to which the fall of blood-pressure is due. On the other hand, it has been maintained that the fall of pressure is not due to a cholin but to a specific substance, vasodilatonin (Popielski), which acts even after atropinization. This

\footnotetext{
1 Sanford and Blackford, Comparative Study of the Effects on Blood-pressure of the Extracts and Serums of Exophthalmic Goitre and other Substances, Jour. Am. Med. Assn., 1914, lxii, 117 (bibliography).

2 Jour. exp. Med., 1915, xxii. No. 1.

${ }^{3}$ Physiological Principles in Treatment, Loudon, 1914, p. 34.
} 
substance seems not to be a specific thyroid product. In contradistinction to the equivocal results in $\operatorname{dogs}$ and rabbits, in cats iodothyroid produces a marked lowering of blood-pressure which is due to both vascular and cardiac action; but here again it seems that similar effects may be produced by other iodized albumins. Therefore the contention of von Cyon that iodothyroid possesses a fundamental cardiovascular regulatory function has not been substantiated, nor has the hypothesis that the cerebral circulation is controlled by an interaction between the thyroid, hypophysis, and pineal glands any better foundation. The intravenous injection of thyroid extract in cats and rabbits increases the stimulability of the depressor function and the rise of pressure produced by adrenalin (Biedl). ${ }^{1}$

The oral administration of thyroid substance in man produces an increased pulse rate and often a fall of blood-pressure; in diabetics a rise of pressure occurs which outlasts the employment of the medication by several days. The prolonged use of thyroid usually produces similar effects, although both experimentally and therapeutically the results are inconstant and have again been explained as not due to specific thyroidal action (Biedl). Blackford and Sanford found that a powerful depressor substance exists in exophthalmic goitre cases and that a primary injection establishes tolerance to the action of any further administration. Atropin does not inhibit its action, but heating to $70^{\circ} \mathrm{C}$. does, hence the substance does not behave physiologically like cholin. The action is chiefly the result of peripheral vasodilatation associated with some diminution of the systolic output. Irritability of the vagus is not decreased. The existence of a crossed tolerance between the depressor action of extract of exophthalmic goitre and of serum from patients suffering from this disease suggests that the two substances are identical. ${ }^{2}$

Clinical Data.-Clinical reports of blood-pressure findings in exophthalmic goitre are divergent. Some observers report hypertension and some hypotension, as a characteristic finding. Arterial tension is often variable and labile. The very high readings of some cases are not part and parcel of the disease, but result from arteriosclerotic changes which occur early in thcse cases as the result of prolonged wear and tear (Muenzer). 'The question as to whether exophthalmic goitre results from a mere quantitative

1 Innere Sekretion, 1913, i, 205.

? Blackford, J. M., and Sanford, M. D., Med. Record, 1913, Ixxxiv, 379. 
increase or from a qualitatively abnormal thyroid secretion is still unsettled. Plummer ${ }^{1}$ has put forth the suggestion that the (systolic) hypertension which he usually found in exophthalmic goitre, when long continued, not infrequently leads to chronic arterial hypertension.

As might be expected from the symptoms of this disease, flushing, warmth, perspiration, and a rapid pulse, blood-flow is rapid. Stewart found in a case studied by him that immersion of one hand in cold water reduced the flow in its fellow from 14 to $7 \mathrm{gm}$. per 100 c.c. per minute. Weber found that in exophthalmic goitre and in neurasthenia there was a tendency for the normal vasomotor reflexes associated with attention and the suggestion of movement to be readily lessened or even reversed. In other words, fatigue phenomena occurred more readily than in normal cases.

IcCrea has ealled attention to a special type of exophthalmic goitre associated with a gain in weight, an increased blood-pressure (200), a high lymphocytosis and drowsiness. Improvement occurs under the use of thymus extract.

Kaess ${ }^{2}$ found the viscosity of the blood in exophthalmic goitre normal in 19 per cent., decreased in 50 per cent., and increased in 31 per cent. of his eases. The latter occurred in the vagotonic eases and probably resulted from inereased perspiration, diarrhea, etc. Diminished viscosity occurred in the sympathicotonic eases and was perhaps due to abnormality of the serum. Hemic viscosity, hcwever, bears no constant relation to blood-pressure. 'There is no constant relation between the sererity of the disease and vascular tension, although, as a general rule, the worst eases show a low pressure. ${ }^{3}$ Pressure changes are probably due to both the heart and the vasomotor system.

Periarteritis Nodosa.-Although Meyer, in 1878, believed that this disease was due to high blood-pressure which caused a rupture of the arterial media, recent investigations point to an infectious origin. In 5 of the 38 cases of this disease collected by Lamb ${ }^{4}$ the blood-pressure readings were reported as $140,215,77-100,130-165$, 104 , showing therefore no constant relationship.

Myxedema.-In myxedema blood-pressure is generally increased.

Adiposity.-Adiposity is not associated with any constant blood-pressure abnormalities. When the circulatory system is

1 Trans. Assn. Am. Phys., 1915.

${ }^{2}$ Bruns, Beitr. z. klin. Chir., 1912, Ixxxii, 253.

${ }^{3}$ Spiethoff, D., Centralbl. f. inn. Med., August 23, 1902.

${ }^{4}$ Periarteritis Nodosa, Arch. Int. Med., 1914, xiv, 481. 
normal a loss of weight entails no fall of pressure, but if hypertension is associated with adiposity a reduction of weight produces a fall of pressure, ${ }^{1}$ largely, it would seem, owing to a diminution of metabolic waste products which result from overeating. (See p. 269).

Scurvy.-The Capillary Resistance Test.--In the early stages of scorbutus, even before other clinical manifestations have oceurred, a cuff applied to the arm and kept inflated for three minutes will cause the appearance of petechial spots. ${ }^{2}$ The test is of course not specific for scurvy but merely indicates the resistance of the capillaries to pressure.

Status Lymphaticus.-In status lymphaticus we have to do with an individual who is constitutionally subnormal. The muscular physique is poor and the physical strength and constitutional resistance below the normal standard. The blood-pressure is apt to be somewhat lower than the subject's age and weight would lead us to expect. According to Muenzer, hypotension is found associated with lymphocytosis. While this may hold good for extreme cases, certainly lymphocytosis has not been shown to be a constant phenomenon in adults, nor, so far as we are aware, in children. Operative procedures in this class of cases are dangerous, especially if chloroform is used as an anesthetic. 'The toxemia of acute infectious disease is also badly borne by these patients. Sudden and unexpected death during anesthesia, infections, while swimming, or in children while quietly resting in bed, appears to bear a relation to enlargement of the thymus gland. The experimental intravenous injeetion of thymus substance lowers bloodpressure, but whether this results from vasomotor depression or from purely mechanical causes (embolism, thrombosis), as sugrgested by Popper, ${ }^{3}$ is uncertain. It may also be simply the non-specific fall of pressure which attends the intravenous introduction of different foreign proteids.

Anemia.-Experimental Data.-Loss of blood tends to cause a fall of blood-pressure. When hemorrhage las been moderate this tendency is compensated by reflex vasomotor constriction and an increased pulse rate; when excessive, death occurs witl a progressive fall of pressure, owing to cerebral anemia.

1 Dumin, Th., Der Blutdruck in Verlaufe d. Arterioselerose, Ztseh. f. klin. Med., 1904 , liv.

2 Hess and Fish, Infantile Scurvy, Am. Jour. Child. Dis., 1914, viii, No. 6.

3 Ueber d. Wirkung d. Thymus extrakts, Sitzungsber. d. k. Akad. d. Wissensch., 1905 , cxiv. 
The quality of the blood exerts an influence on vascular tonus and blood-pressure. Lesser found that section of the cord between the second and fourth cervical vertebræ caused a fall of pressure and a diminution of hemoglobin in the large vessels, whereas stimulation of the sectioned cord increased the hemoglobin. Section above the splanchnic centre diminishes the erythrocytes (Cohnheim and Zuntz). Experimental epinephrin hypertension (in dogs) increases the red cells in the capillaries and reins but not in the large arteries. According to Erb, the arterial and venous hemic concentration increases with a rise and decreases with a fall of pressure. Holobuts attributes the variations of the number of erythrocytes to changes in the volume of the corpuscles. The dried residue and the plasma show but little change with pressure variations (Horner). ${ }^{1}$

Clinical Data.-Blood-pressure observations in the different forms of anemia are of little value, except in the detection of nephritic complications. If the anemia is marked, pressure will be low in proportion to the cachexia and general weakness. Chlorosis is said to be accompanied by slight hypertension in the early stages, followed later, if its course be prolonged or severe, by hypotension. Blood-pressure bears no constant relation to either the percentage of hemoglobin or to the total number of corpuscles. According to Plesch the minute volume of the heart is increased in the anemias. Stewart found peripheral blood-flow in anemia less than normal. This peripheral constriction has been construed as a necessary compensatory arrangement for increasing blood-flow through the lungs. The deficiency in the hand flow is less in chlorotic anemia than in the other forms. Muenzer ${ }^{2}$ found lymphocytosis frequently associated with arterial hypotension. In pernicious anemia very low readings are often observed. Cabot states that systolic pressures of 80 and even $60 \mathrm{~mm}$. are not unusual.

Cachexia.-Nutritional failure from whatever cause is often associated with low arterial pressure, but this is by no means invariably the case. The high pressures of nephritis may be somewhat reduced by the coincident presence of cachexia. A remarkable case of persistent hypotension has been reported by Rolleston: ${ }^{3}$ that of a man with carcinoma of the tongue and amyloid disease

1 Der Blutdruck d. Menschen, Vienna, 1913, p. 101.

2 Blutdruck u. Blutbild, Med. Klinik, 1913, ix, 2028, 2074.

${ }^{3}$ Low Blood-pressure in Carcinoma of the Tongue with Amyloid Disease, Lancet, September 12, 1914, p. 692. 
who managed to live for several weeks with systolic and diastolic pressures of 70 and 35 respectively.

The Effects of High Altitudes on Blood-pressure.-The disturbances of physiologic function which result from high altitudes are mainly due to a deficiency of oxygen. The vertigo, confusion of the senses, and fatigue can be abated by the inhalation of oxygen. At altitudes of from 4000 to 8000 meters (13,000-26,000 feet) no special precautions are necessary, provided the partial pressure of the oxygen in the lungs is raised. Beyond this, special devices insuring adequate oxygenation must be employed. Mountain sickness results from a combination of diminished oxygen supply associated with muscular exertion, producing syncope, a weak pulse, and other symptoms of cerebral anemia. Circulatory symptoms are especially apt to occur in aviators if very rapid ascents or descents are made.

Henderson's studies made on Pike's Peak (14,000 feet) showed that there was a slight fall of arterial pressure. It seems to be generally agreed that altitude of a considerable degree does tend to lower blood-pressure and to increase the pulse rate, and that these changes are more marked at first, $i$. e., before "acclimatization" has taken place. Such a fall of pressure may range between 1 and $22 \mathrm{~mm}$., and the effects are naturally more marked in hypotensive individuals. There is, however, a great difference in individual susceptibility. The systolic pressure is chiefly and more constantly affected, and diastolic pressure may even rise. Ordinarily slight clevations produce but little change in blood-pressure. It is quite impossible either from a man's general physique or from any other criterion to forecast how much his pressure may be affected. ${ }^{1}$ 'The rate of blood-flow was increased from 30 to 76 per cent., by residence at Pike's Peak. This was associated in part with an increased pulse rate and a fall of venous pressure. The arterial pressure was not clearly altered either by altitude or by oxygen inhalations. The latter did slow the pulse rate 14 per cent. at 14,109 feet, and 54 per cent. at 6000 feet. $^{2}$ The readings obtained by Clough at an elevation of over 5000 feet were practically identical with those taken at the sea level. Miners in suddenly descending or ascending 1700 feet to and from their work show

\footnotetext{
1 Schneider and Hedblom, Blood-pressure, with Special Reference to High Altitudes, Amer. Jour. Physiol., 1908, xxiii, 90.

2 Schneider, E. E., and Sisco, D. L., Cireulation of the Blood at High Altitudes, Am. Jour. Physiol., 1914, xxxiv, 1 (literature).
} 
variations hardly exceeding $5 \mathrm{~mm}$. Hg. ${ }^{1}$ Hess ${ }^{2}$ has attempted to explain the changes in blood-pressure at high altitudes by the fact that the normal balance between the concentration of the arterial and the renous blood (which he believes is normally regulated by the lungs) is upset.

Dcubtless many of the contradictory statements regarding the effects of altitude on blood-pressure are due to the fact that normal and abnormal subjects have been classed together. Thus among tuberculous patients an elevation of 6000 feet appears to increase blood-pressure, which on the whole should have a distinctly beneficial effect. ${ }^{3}$ The rate of blood-flow and of the pulse is markedly increased at high altitudes $(14,000$ feet $)$. This is associated with a fall of renous pressure and with arteriolar dilatation. These changes are apparently due to a deficiency of oxyen, since inhalation of this substance affects both pulse rate and rate of flow. ${ }^{4}$ Altitude has a more pronounced effect upon hypertension due to general arteriosclerosis than upon that associated with well-marked nephritic lesions ${ }^{5}$ (see page 50 ).

The question as to the effect of altitude upon the individual is an important and practical one. The physician is constantly consulted regarding the effects of high altitude in tuberculosis, heart disease, emphysema, etc. It is generally agreed that residence at a high elevation increases the number of erythrocytes and the percentage of hemoglobin. There is also reason to believe that the cells of the pulmonary alveoli possess and may take on an increased capacity to secrete oxygen into the blood. These changes are of a compensatory nature. Although the whole question of the physiologic effect of altitude is still unsettled, it seems evident that the simptoms from which balloonists, aviators, and mountain climbers suffer cannot be explained on a basis of blood-pressure alterations.

Plungian's investigations indicate that barometric changes at a given altitude exercise an effect upon individual blood-pressure,

${ }^{1}$ Clough, F. E., Blood-pressure Variations as Influenced by Rapid Changes in Altitude, Arch. Int. Med., 1913, xi, 590.

${ }^{2}$ Die Beeinflussung d. Flüssigkeits anstauches zw. Blut. u. Geweben durch Schwankungen d. Blutdrucks, Deutsch. Arch. f. klin. Med., 1904, lxxix, 128.

${ }^{3}$ Peters and Bullock, Blood-pressure Studies in Tuberculosis at a High Altitude, Arch. Int. Med., 1913, xii, 458.

"Schneider and Sisco, The Circulation of Blood in Man at High Altitudes, Am. Jour. Physiol., April, 1914, xxxir.

${ }^{5}$ Schrumpf, P., Blutdruckuntersuchungen U. Energometerstudien im Hochgehirge bei Herz- u. Kreislaufstörungen, Deut. Arch. f. Klin. Med., 1914, xeiii, 466. 
although this is inconstant, both quantitatively and qualitatively. The most marked changes generally oceur in tuberculous arod arteriosclerotie subjects after a sudden fall of the barometer. ${ }^{1}$

The Effects of Tropical Climate.-Much has been writtern osn the effects of tropical sunlight on white men. 'T"s whatever the deleterious effects are due, they are apparently not the result of blood-pressure changes. 'The average blood-pressure of Arnericanss in the Philippines is quite similar to that when at home. The lowest readings are obtained during the first three months of tropical residence and during the hottest months, but the fall of pressure is quite inconsequential $(3 \mathrm{~mm}$.). No difference has been noted among individuals with light or dark complexions, nor when different types of underwear are worn. ${ }^{2}$ According to McCay the pressure of the natives of India is lower than that which obtains in western Europe, and yet arteriosclerosis is very common among them. Possibly the low protein diet and more constant perspiration may have a bearing upon the low pressure. Oliver's investigation showed that during prolonged hot weather blowd-pressure was lowered in the brachial artery, but was increased in the phalangeal vessels. ${ }^{3}$

Life Insurance.-The importance of blood-pressure examinations as a means of sifting out undesirable "risks" has now become generally recognized by life insurance companies and is routinely required by many of them. Statistical studies have shown that blood-pressure values, abnormal in relation to the age and sex of the individual, are definitely associated with a shortenerl expectancy of life. It would seem that pressures of $20 \mathrm{~mm}$. either below or above the normal standard require an explanation. As has been stated elsewhere, high pressures often indicate renal, and low pressures, tuberculous disease.

Fisher ${ }^{4}$ has pointed out that a pressure of 150 gives a higher mortality than an average risk, and an increase of 15 per cent. above the normal pressure for a given age should always excite suspicion. Among 2,661 risks with an average pressure of 142.43, at the ages of forty to sixty, the mortality was about the same as

1 Plungian, M., Ueber d. Wirkung athmosphaerischer Einflusse auf d. Blutdruck. Dissert. Basel, 1913, p. 43.

2 Chamberlain, W. P., A Study of the Systolic Blood-pressure and the Pulse, Rate of Healthy Adult Males in the Philippines. Philippine Jour. Sci., 1912, vi, 467.

\& Quoted, Allbutt, Diseases of the Arteries, Including Angina Pectoris, London, $1915, \mathrm{i}, 181$.

4 The Diagnostic Value of the Sphygmomanometer in Exaninations for Life Insurance, Jour. Am. Med. Assı., 1914, Ixiii, 1753. 
the average general mortality of the company, but with an average pressure of 152.58 among 525 risks the mortality increased 30 per cent. in excess of the average. Among the rejected risks with an average pressure of 161.44 (1970 applicants) the mortality was 190 per cent. of the medico-actuarial table and more than double the average mortality of the company. During the interval of from 1907 to August 1, 1913, 1970 applicants were refused insurance solely on the basis of a pressure of $160 \mathrm{~mm}$. Hg. These cases but for the pressure readings would have been accepted. The continued accumulation of life insurance statistics will throw much light not only upon the diastolic pressure and upon hypotension, but also on a large and important class of individuals who have only slightly increased pressure, who consider themselves in perfect health and do not, therefore, come under the observation of the physician.

As a ready means of calculating what a given individual's bloodpressure should be, Faught has suggested the following rule: Assume that the normal systolic pressure of a person aged twenty years is $120 \mathrm{~mm}$., and add $1 \mathrm{~mm}$. Hg. for each additional two years of life.

The different phases of blood-pressure as applying generally to insurance have been discussed in different chapters of this book. The reader is specially referred to Instrumental Technique, page 125; Sources of Error, page 122; Physiologic Variations with Special Reference to Age, page 53; Hypertension in Nephritis, page 267; Tuberculous Hypotension, page 213.

Infants and Children.-Blood-pressure estimations in infancy and early childhood are made with difficulty and fraught with inaccuracy. The psychic effects produced by the procedure, which often result in crying or struggling, absolutely vitiate the accuracy of the readings. The arm is too small to permit satisfactory application of the cuff, hence the readings should be taken on the thigh and a small cuff $(6.5 \mathrm{~cm}$.) employed. As a temporary makeshift the usual $12 \mathrm{~cm}$. cuff may be folded in half. The child will pay less attention to the cuff when it is applied to the lower extremity. The diaper must be loosened. Auscultation is more satisfactory than palpation. The diastolic pressure which is determinable with difficulty owing to the small size of the artery and of the pulse pressure, must generally be estimated either by auscultation or by means of a Feddé oscillometer or some similar device. In childhood and youth the transmission of the pressure wave from 
the centre to the periphery behaves differently than in the adult. In the former the systolic pressure in the digital artery equals that registered in the brachial, whereas in the adult, especially in cases of nephritis and arteriosclerosis, the arterioles are tonically contracted as well as less elastic, and the systolic brachial pressure is much higher than that in the digital arteries. ${ }^{1}$

The diastolic pressure is relatively higher than in adults. ${ }^{2}$ On the whole, blood-pressure estimations are of much less value in children than in adults.

Normal Pressure.-Before birth blood-pressure is higher in the pulmonic than in the systemic circulation. The expansion of the lungs promptly lowers pulmonary blood-pressure. Before this occurrence most of the blood passes from the pulmonary artery through the ductus arteriosus into the aorta, but afterward, the lowering of blood-pressure which occurs in the systemic circulation when the placental circulation is cut off, causes a collapse of the ductus and thus favors its closure. Additional causative factors may be the equalization of pulmonary and aortic pressures and the muscularity of the ductus. (Abbott. ${ }^{3}$ ) At birth systolic bloodpressure is said to range between 35 and $55 \mathrm{~mm}$. Waking and suckling cause a rise of about $15 \mathrm{~mm} .^{4}$

Preceding the onset of puberty there is a tendency toward a fall of pressure, during pubescence there is a period of increased pressure which is in turn followed by a slight decrease after puberty has been established (Albutt). (See Gonads.) The attempts which have generally been made to establish normal standards in relation to age have led to more or less confusion. Pressure increases with age, and during the first month of life the normal range lies between 60 and $68 \mathrm{~mm} . \mathrm{Hg}$. Some recent investigations have shown that blood-pressure increases with height and weight, and that these criteria are more definitely fixed than the mere age factor. ${ }^{5}$ Further, the variations which have been attributed to the influence of sex are really dependent upon the effect of height and weight.

1 Findlay, L., The Systolic Pressure at Different Points of the Circulation in the Child and the Adult, Quar. Jour. Med., 1910-11, iv, 489.

2 Melvin and Murray, Blood-pressure Estimation in Children, British Med. Jour., April 17, 1915, p. 2833.

3 Congenital Cardiac Disease, Osler and MeCrae's Modern Med., 1915, iv, 411.

${ }^{4}$ Balard, P., Le Pouls et la Tension Artérielle de l'Enfant et du Nouveau-né, Gaz. des Hôp., 1913, Ixxxvi, 837.

Nos. 4-6.

5 Wolfensohn-Kriss, Ueber Blutdruck im Kindesalter, Arch. f. Kinderheilk., liii, Nos. 4-6. 
Table 1.-Comparison of Height and Systolic Blood-pressure. ${ }^{1}$

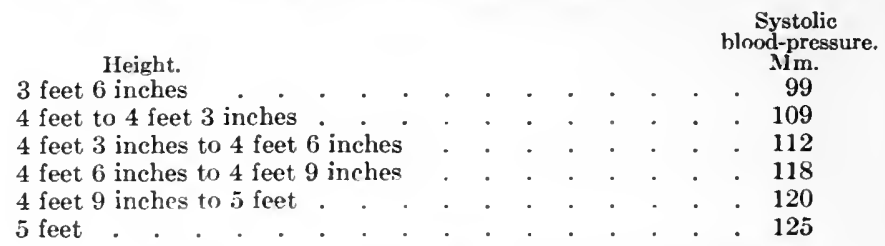

Table II.-Comparison of Weight and Blood-pressure.

\begin{tabular}{|c|c|c|c|c|c|c|c|c|c|c|c|c|c|c|}
\hline & Weight. & & & & & & & & & & & & & $\begin{array}{l}\text { Plood-pressure. } \\
\text { Mm. }\end{array}$ \\
\hline 30 to 4 & $40 \mathrm{lbs}$. & & . & $\cdot$ & & & & & & & & & & \\
\hline 0 to 5 & 50 “ & . & . & . & . & . & . & . & . & . & . & . & . & . 100 \\
\hline 50 to 6 & 60 “ & . & . & . & . & . & . & . & . & . & . & . & . & . 107 \\
\hline 60 to 7 & 70 " & . & . & . & . & . & . & . & . & . & . & . & & 112 \\
\hline 70 to 8 & 80 “ & & . & . & . & . & . & . & . & . & . & . & & . 116 \\
\hline 0 to 9 & 90 " & & . & . & . & . & . & . & . & . & . & • & & . 122 \\
\hline 90 to 10 & 00 “ & & 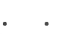 & . & . & & & . & . & . & . & . & . & . 126 \\
\hline
\end{tabular}

The foregoing facts explain the statement frequently made that "robust" children have higher pressures than delicate ones, and perhaps also that breast-fed infants give higher readings than those artificially nourished. Popoff ${ }^{2}$ found the systolic and diastolic pressures in the newborn infant to average 75 and $34 \mathrm{~mm}$. Hg. respectively. Prematurely born infants had lower pressures (see page 53). Laitao found that at the end of seven months the systolic pressure was generally $100 \mathrm{~mm}$., and that the pulse pressure which during the first month areraged $18 \mathrm{~mm}$., at the end of the first year had reached $28 \mathrm{~mm}^{3}{ }^{3}$ These values are higher than those obtained by Kaupe, ${ }^{4}$ who found an average pulse pressure of 8 to $10 \mathrm{~mm}$. Hg.

In infants after feeding the pressure is increased $\delta$ to $10 \mathrm{~mm}$., and during sleep pressure ranges between 60 and $70 \mathrm{~mm}$. In children an hour's exercise may elevate the pressure 5 to $10 \mathrm{~mm}$., at which point it may remain for half an hour (Trumpp) (see also Acute Infections, page 196).

Blood-pressure and Physical Efficiency.-Studied from the standpoint of the "Intercollegiate Standard," which consists of adding the strength of the back, plus that of the legs, plus that of the right and left forearms, plus the lung capacity divided by 20 , plus bodily weight divided by 10 and multiplying the last figure by

1 Michael, M., A Study of Blood-pressure in Normal Children, Amer. Jour. Dis. Children, 1911, i, 272.

${ }^{2}$ Beiträge z. Frage nach d. Blutdruck b. gesunden Kindern, Dissert. St. Petersburg, 1913 , p. 200.

${ }^{3}$ Laitao, M., Pression Artérielle chez l'enfant, Arch. d. Mal. d. Enfants, 1913, xri, 102.

${ }^{4}$ Der Blutdruck im Kindesalter, Monatsschr. f. Kinderheilk., 1910, ix, 257. 


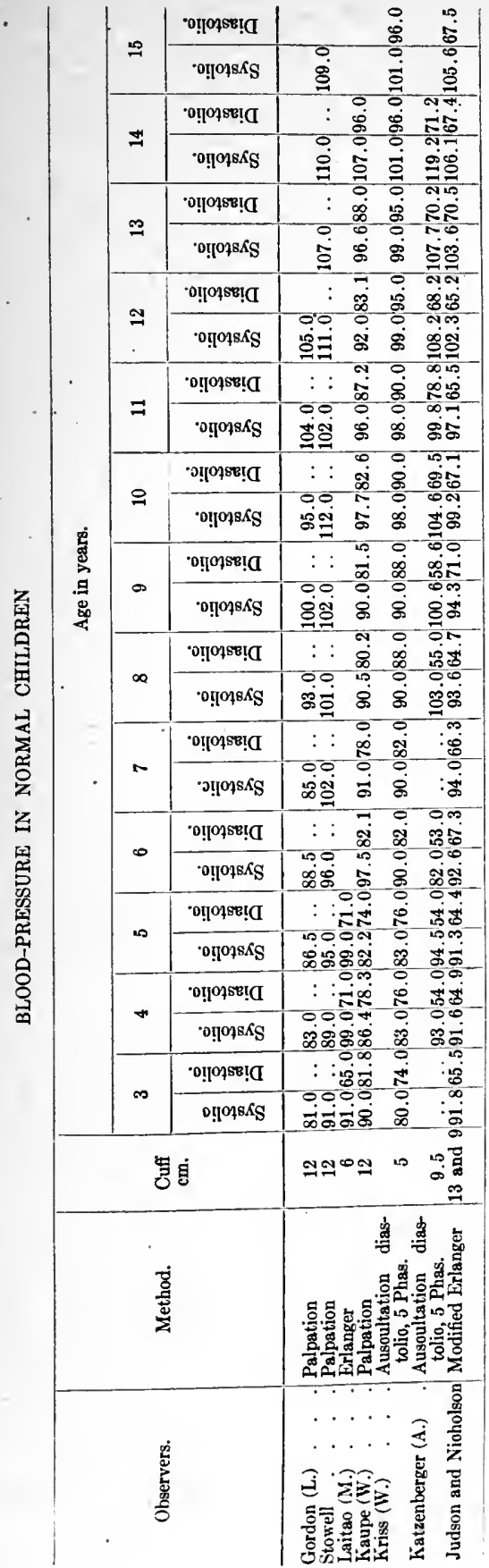

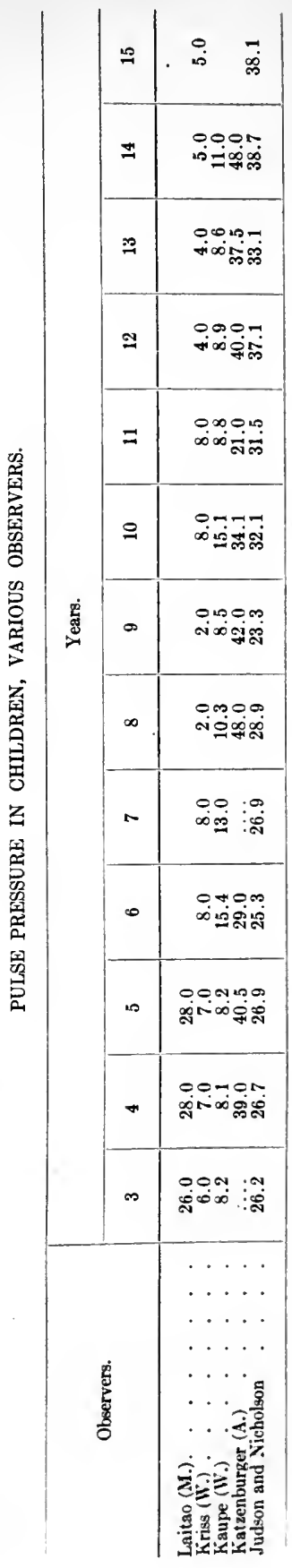


number of times the subject is able to dip his body by the strength of lis arms and shoulders, Barach and Marks found that there is no constant relation between physical efficiency (which also means circulatory efficiency), and either the pulse pressure, diastolic pressure ratio, nor between the pulse-pressure percentage of the diastolic pressure.

The following tables compiled by Barach and Marks ${ }^{1}$ show the actual systolic and diastolic pressures encountered among 742 male sturlents:

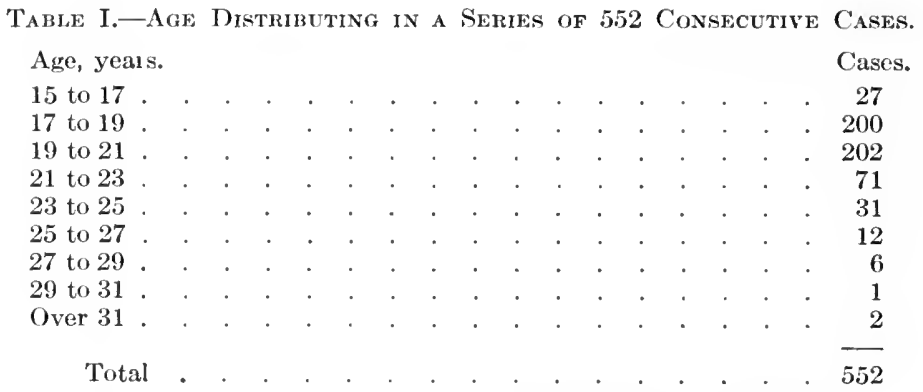

Table II.-Distribution of Maximem Pressure in 656 Cases.

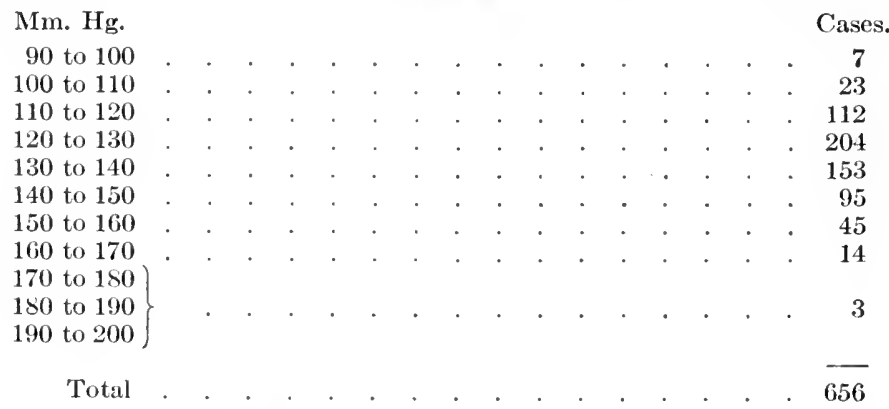

Table III.-Minimum Pressure Read at the Last Clear Sound Mm. Hg.

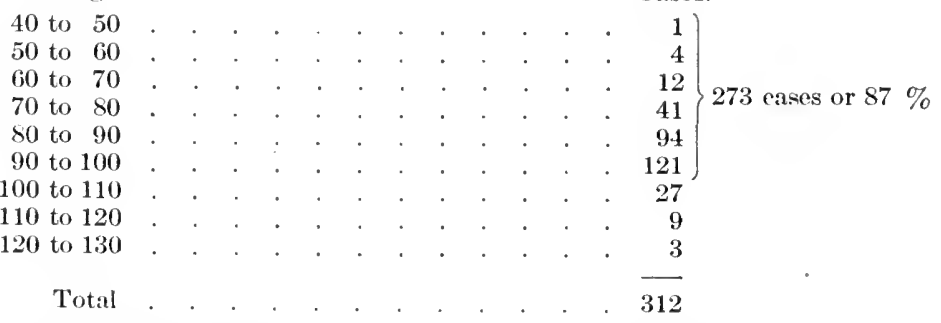

1 Blood-pressure, their Relation to Each Other and to Physieal Efficieney, Arch. Int. Med., 1914, xiii, 648 . 
The foregoing tables are taken from Judson and Nicholson, ${ }^{1}$ who include their own figures based upon a study of 2300 children. As these authors point out, "The widest variations occur from the tenth to the fourteenth year when there is a marked rise in the systolic pressure. From the fourth to the fourteenth year, inclusive, the variations do not exceed $5 \mathrm{~mm}$. in two-thirds of the cases." Their systolic readings were higher than those previously obtained by others. There is a gradual rise of pressure as age increases between the ages studied, amounting in all to $14 \mathrm{~mm}$. Hg. Up to eight years of age a $9 \mathrm{~cm}$. cuff gives accurate readings, but after eight years a $13 \mathrm{~cm}$. armlet should be employed. The onset of the fourth auscultatory phase (muffling) must be used as the criterion.

Athletics.-The physician is frequently consulted regarding the advisability of allowing certain individuals with functional or organic heart lesions to indulge in athletic exercises. The tendency at present is certainly to be more liberal in what may be allowed that was formerly the case. This is specially true of ehildren and young adults with heart murmurs. Before assenting to or advising such procedures, however, we must realize that although exercise is as necessary for the heart as for any other muscle, yet certain forms of exercise throw an enormous burden upon the circulatory apparatus (see pages 46 and 300 ).

The more sudden and violent the exercise (sprinting, hammer throwing, etc.) the greater the rise of blood-pressure $(200 \mathrm{~mm}$.) and the more rapid the pulse. Endurance exercises (long distance running, etc.) produce a more gradual and less extreme rise of pressure, but a pressure which remains high for a longer time. The sudden changes of pressure in the former group probably do no harm to young normal individuals with resilient arteries, but they should be avoided by elderly men in whom permanent cardiae dilatation may result. The prolonged strain of endurance exercises among young growing children should certainly not be encouraged (McKenzie).

The Effect of Marathon Races.-Before the Race.-Most of the men had trained for six to nine months preceding the race of twentyfour miles. Most of the men showed evidences of cardiae enlargement and had a slower pulse rate than normal. The cases which showed a more than average diurnal pulse variation also showed a cardiac hypertrophy and an increased blood-pressure. The highest

1 Blood-pressure in Normal Children, Am. Jour. Dis. Children, 1914, xii, 257. 
blood-pressures were found in coincidence with the largest hearts. The men who had heart murmurs had even larger hearts and higher pressures. As one would expect the men who were overweight, whose occupations were laborious, and whose periods of preliminary training were short, showed higher than average cardiac enlargement and blood-pressures.

After the Race.-The average fall of blood-pressure was $20 \mathrm{~mm}$. Cases with heart murmurs showed an even greater drop. Some cases showed marked cardiac dilatation ( $x$-ray), and in these blood-pressure was lower than in those whose heart dilated less. At the end of six months both heart and blood-pressure examinations rielded normal findings in most of the cases but some increased much more slowly than others. ${ }^{1}$

If there is any doubt whatever regarding the organic character of a murmur it is better to err on the safe side and advise against prolonged or severe forms of athletics.

Much has been written of late concerning the evil effects of overathleticism, and regarding this subject there is much difference of opinion.

Severe exercise of an hour has been shown to not infrequently cause the appearance of albumin, casts and blood cells in the urine of healthy subjects, phenomena which are doubtless due to circulatory changes in the kidney at a time when waste products in large amount must be eliminated. ${ }^{2}$

Prolonged and severe athletic training produces demonstrable cardiac hypertrophy with increased blood-pressure. These phenomena may be transient and ephemeral, but they are not always so.

It certainly does not seem reasonable to believe that Marathon races run by poorly developed and untrained individuals as we often see them in our city streets, can be beneficial. Nor does it seem logical to assume that four-mile boat races at the end of which even trained athletes go into a state of srncope, can be considered healthful exercise. It is believed by some that strenuous athletics use up an individual's reserve force-his vital reserve. Whether this be so or not cannot be proved, but certainly the picture of the husky giant who succumbs in the early stages of typhoid fever or pneumonia is an all too common one.

1 Savage, W. L., Physiological and Pathological Effects of Severe Exertion (The Marathon Race), Am. Phys. Educ. Rev., 1910, xv, No. 9; 1911 , xvi, Nos. 1-5.

${ }^{2}$ Barach, J. H., Against Overathleticism, Trans. IV Interuational Congress of School Hygiene, Buffalo, August, 1913. 
It is very questionable whether in the ordinary course of life, the "athletic heart" is an asset. Investigations among the athletes at the University of Wisconsin have shown cardiac abnormalities among a considerable proportion of the athletes. ${ }^{1}$ 'This has been attributed by some apologists to faulty methods of training. The report of the Surgeon-General of the United States Navy attributes a large excess of deaths attributable to cardiac overstrain among the cadets at Annapolis, to overathleticism. In cardiac cases the physician carefully questions the patient regarding antecedent infections, but rarely in regard to athletic overindulgence or mishaps.

It seems merely conservative, therefore, to insist that competitive athletics should be conducted only under competent medical supervision, and that growing boys should not be allowed to indulge in the prolonged and exhausting competitions, which greatly tax even a fully developed heart.

The Effect of Athletic Training.-Training renders an individual more efficient (1) by increasing cardiac and vasomotor responses to meet sudden or prolonged demands for physiological activity; (2) by educating the brain centres so that muscular actions become semi-automatic and hence less volition is required in their performance; (3) by accustoming the medullary centres to an increased quantity of carbon dioxid; and (4) by increasing the rate of excretion of waste products of metabolic activity.

Middleton ${ }^{2}$ has found that training during a football season produces fairly uniform results which may be expressed as follows:

1. A proportionate decrease in both systolic and diastolic pressure during the training period.

2. After the rest following the training season a rise of both pressures occurs which exceeds the ante-season records.

3. The blood-pressure reaction to a test of fifty stationary running steps is practically unaltered by the training.

The decrease in the blood-pressure mentioned may be due to peripheral vascular relaxation or to cardiac dilatation, or to a combination of both factors.

Blood-pressure in Gastro-enteritis.-The suggestion has been made that the collapse which occurs in the course of epidemic diarrheas is in part due to a fall of blood-pressure resulting from

\footnotetext{
I Schumacher, L., and Middleton, W. S., The Cardiac Effects of Immoderate College Athleties, Jour. Am. Med. Assn., 1914, lxii, 1136.

2 The Influence of Athletic Training on Blood-pressure, Am. Jour. Med. Sei., 1915 , cl, 426.
} 
loss of fluid in the tissues and producing anuria, and that saline infusion should be used in combating this symptom ${ }^{1}$ (see Cholera, page 199).

Tomiting causes a fall of blood-pressure which is in part due to the cardiac inhibition which occurs when the romiting centre is stimulated $^{2}$ not only a fall, however, but very sudden and extensive pressure variations are also met with. ${ }^{3}$

1 British Jour. Child. Dis., 1912, ix, 343.

2 Miller, Am. Jour. Physiol., May, 1915.

3 Brooks and Luckhardt, Blood-pressure during Vomiting, Am. Jour. Physiol., 1915, xxxvi, 104. 


\section{CHAPTER XVI.}

\section{DISEASES OF' 'THE NERVOUS SYSTEM.}

Neurasthenia.--When we consider what a large number of different organic and functional abnormalities go to make up the clinical picture of this condition it is not surprising to note that blood-pressure findings are inconstant. As has been deseribed elsewhere, many of these cases belong in the category of "constitutional low arterial pressure" $(q . v$.), but some cases have been reported as associated with hypertension. Symptomatic improvement is generally attended by the reëstablishment of a more normal pressure, whether this means a rise or fall." "Neurasthenia" associated with high blood-pressure generally calls for a vision of diagnosis, which will not infrequently be changed to nephritis or cardiovascular hypertensive disease.

"In true neurasthenia, in real exhaustion of the nervous centres from whatever cause arising, the blood-pressure is low, lower than in any other chronic malady, except the terminal phases of cancer tubercle and other wasting disease" (Leonard Williams). The effects of prolonged mental and physical strain and exhaustion as exemplified by soldiers returning from the battle front in France produces arterial hypotension. Montier reports that a well-marked fall in pressure occurs both in soldiers starting out with normal pressures and in those who do so with hypertension.

Neuroses, etc.-In the neuroses are often found unequal radial pressure on the two sides as well as a general lability of pressure. These signs may be of use in diagnosticating between neurosis and simulation. ${ }^{2}$ Pain produces a rise of blood-pressure and an increased pulse rate. Simulated pain does not. This fact has been turned to practical application in the detection of malingering in relation to industrial insurance. In persons with normal sensibility the blood-pressure under the application of faradic stimulation to the upper thigh rises from 8 to $15 \mathrm{~mm}$. In organic affections of the

1 Macnamara, Lancet, July 18, 1908.

${ }^{2}$ Sand, Les Anomalies de la Tension, Sanguine, ete., Arch. intern. de Méd. Legale, 1910 , p. 349. 
spinal cord, multiple sclerosis, hysteria, etc., it remains unaffected when stimulation is applied to analgesic regions. ${ }^{1}$

If the systolic pressure is taken in one arm and simultaneously the diastolic pressure in the other (for celerity of procedure), and the subject is emotionally disturbed by conversation, gesture, or the application of ice to the abdomen, (1) marked variations of the maximum pressure are said to indicate abnormal stimulability; (2) if the diastolic pressure meanwhile remains unaltered the vascular system is normal; (3) if the diastolic pressure shows sudden variations of over 15 per cent. of the systolic pressure we are dealing with arteriocapillary sclerosis. ${ }^{2}$ Landerer found abnormal arteriocapillary pressure relations in neuroses.

Peripheral Neuritis. - In the early stages of brachial neuritis, Stewart found the blood-flow greater than in the unaffected hand, a finding which he interpreted as indicating a partial paralysis of the vasoconstrictor fibers in the nerves involved.

In neuritis affecting chiefly the muscular nerves, disturbances of the blood-flow were less conspicuous than when the cutaneous nerves were involved. 'This is presumably due to the fact that a large amount of the total blood-supply of the extremities traverses the skin of the parts.

Cases of long-standing unilateral neuritis were found by Stewart to show a diminished blood-flow on the side affected, owing either to diminution of the arterial lumen following injury of the vasomotor nerves or perhaps to an adaptive correllation between diminished function and blood-supply.

Mental Disease.-The reports on blood-pressure in abnormal psychic states are so variable and contradictory, and the range of abnormality so slight, that one cannot but feel that in the majority of cases at least no definite relationship exists. Nor is this surprising when we consider how greatly blood-pressure is normally affected by emotional disturbances.

In simple, acute, passive, and demonstrative melancholia high pressures have been reported, whereas chronic melancholia is associated with hypotension. Hawley states that cases of mania show definite and typical blood-pressure changes-a large pulse amplitude with sudden pulsatory oscillations and an increased pressure. These phenomena disappear when the mental state again

1 Curschmann, Deut. med. Woch., October 15, 1907.

2 Schrumpf and Zabel, Die diagnostische Bedeutung der psychogenen Labilität des Blutdruckes, Münch. med. Woch., 1911, Iviii, 1952. 
becomes normal. In pure senile mania and melancholia the readings correspond to those registered in the same states in earlier life. Senilc dementia, characterized by restless fidgeting, etc., shows increased pressure. ${ }^{1}$ In depressed and stuporous mental states low pressures are found. ${ }^{2}$

Dementia Precox.-According to Perazzolo 74 per cent. of these cases have a low blood-pressure. Turner found no definite relation between blood-pressure and either exalted or depressed mental states. The height of the pressure seemed mainly dependent upon the age of the patient and upon the presence of renal or cardiovascular complications. ${ }^{4}$

Epilepsy.-Epileptics not infrequently have a slow pulse rate, a low blood-pressure, and evidences of peripheral stasis. Russell, ${ }^{5}$ in his Goulstonian lecture, enumerated many interesting and suggestive points in an analogy between epileptic seizures and vascular spasm. We cannot, however, assume that such conditions affect the cerebral vessels in the same manner as or simultaneously with the peripheral arteries. Furthermore, there is still some difference of opinion as to whether the cerebral vessels possess vasomotor nerves. But the clinical picture of epilepsy presents many features which fit well into a hypothesis of vasomotor crises (aura, pallor, syncope, cenvulsions, etc.).

Blood-pressure has been found to increase several hours before the attack begins. During the seizure it is said to rise still further (20 per cent.) and to fall after the attack; but often to remain somewhat elevated between paroxysms. ${ }^{6}$ It has been shown that in many cases rhythmic blood-pressure variations occur which are not due to respiratory movements (Traube-Hering waves). Preceding the seizure (twenty-six to sixty seconds) there is a rise of pressure (followed in some cases by a sudden fall), then apnea, and finally the convulsion." The pulse is variable. "A study of the changes in the respiratory and circulatory systems in some

${ }^{1}$ Alexander, Lancet, July 5, 1902.

${ }^{2}$ Hawley, Blood-pressure in States of Excitement and Depression, Arch. Int. Med., 1913 , xii, 526.

${ }^{3}$ Revista sperimentale di Freniatria, April 30, 1909.

${ }_{4}$ Turner, Observations on the Blood-pressure and Vascular Disease in the Female Insane, Jour. Ment. Sci., 1909, xlv, 419.

${ }^{5}$ Some Disorders of the Cerebral Circulation and Their Clinical Manifestations, Lancet, April 3, 1909, pp. 963, 1031, 1093.

${ }^{B}$ Lallemand and Rodiet, Des Modifications de la tension artérielle chez les epileptiques L'Encephale, iv, No. 11.

${ }^{7}$ Gibson, Good, and Penny, The Pulse Immediately Preceding the Epileptic Attack, Quarterly Jour. Med., 1910, iv, 1. The authors could find no alteration of the pulse amplitude up to the point of the seizure. 
of the cases of epilepsy suggests that the site of the discharge is in the medulla and pons (the 'lowest level of fits' of Hughlings Jackson). Likewise it points to the medulla as participating in the discharge in all cases of epilepsy whether this discharge originates them or not."'

Brain Tumors, Hemorrhages, Meningitis, etc.-The increased intracranial tension which occurs in cases of apoplexy and brain tumor may be closely simulated experimentally. The symptoms of the two conditions as well as their mechanism is essentially similar. Sudden increase of intracranial tension produces an acute anemia of the brain, which, being enclosed within osseous confines, is relatively incompressible. The only possible relief from such compression can come through absorption of the cerebrospinal fluid. If the rise in arterial tension is prevented death occurs from medullary anemia.

With increasing compression the following symptoms generally occur in the order mentioned: (1) unconsciousness; (2) respiratory abnormalities; (3) increased arterial tension with bradycardia; (4) hypotension, tachycardia, death. Associated with these symptoms rhythmic fluctuations of blood-pressure (Traube-Hering waves) and Cherne-Stokes respiration are apt to occur (see pages 41 and 290).

The attendant rise of sistemic blood-pressure is therefore compensatory; an effort to prevent medullary anemia, and if prevented causes death. The simptoms which arise are for the most part due not directly to pressure but to cerebral anemia. When intracranial pressure becomes sufficiently great certain symptoms and signs become apparent (headache, vertigo, romiting, choked disk, etc.). If pressure is increased still further, bulbar symptoms develop: bradycardia, hypertension. Finally, if anemia threatens the vital centres life ean only be maintained by a marked rise of general blood-pressure, and this at least intermittently usually occurs. Some of the highest blood-pressure readings on record (300 mm.+) have been encountered under these conditions. Eventually, unless the pressure is relieved, death occurs as a result of central and systemic exhaustion, preceded by a fall of tension and an increased pulse rate (see page 42).

Any increase of the cerebrospinal pressure, whether due to tumor, softening, hemorrhagie meningitis, cerebral or cerebrospinal meningitis (serous or purulent) tends to cause a rise of sy'stemic blood-pressure. Especially is this the case if the pressure is exerted

${ }^{1}$ Pollock and Treadway, A Study of the Respiration and Circulation in Epilepsy, Arch. Int. Med., 1913, xi, 445. 
in the posterior cerebral fossa, causing a stimulation of the vasoconstrietor centre, and if the rise of intracranial pressure be rapid. Brain tumors are usually slow in growth and arterial hypertension, bradycardia and respiratory disturbances are not marked unless a sudden increase of intracranial tension occurs-hemorrhage, edema or ventricular effusion (Cushing).

If the cerebrospinal pressure is relieved by lumbar puncture or trephining, blood-pressure falls, the bradycardia is replaced by a normal pulse rate and respiratory abnormalities disappear. There is often a distinct parallelism between the two pressures. ${ }^{1}$

From what has been stated it is evident that phlebotomy is generally contra-indicated in cases of cerebral hemorrhage. On the other hand, a progressively rising arterial pressure is often the most useful indication of an increase of hemorrhage and of consequent cerebral compression, and may therefore be used as a criterion as to whether an operation for decompression is or is not warranted.

Apoplexy.-Immediately after an apoplexy, bradycardia with very high blood-pressure is sometimes seen. These symptoms result from increased intracranial pressure. The usual range of pressure "after a stroke" is between 150 and $200 \mathrm{~mm}$., but normal pressures may be found, or higher pressures $(300 \mathrm{~mm} .+)$ than are encountered in almost any other clinical condition. The value of pressure readings depends mainly upon a knowledge of the pressure which existed preceding the attack. It is more important to know the general trend of the pressure-whether upward or downward than actual figures. Thus a steadily rising pressure indicates cerebral compression and may call for decompression, whereas a steady fall points to vasomotor failure, which often leads to death. Blood-pressure readings may be of value in diagnosticating between apoplexy and embolism. High pressure is evidence in favor of the former, and low pressure of the latter. It appears, however, that apoplexy, even when associated with hypertension and chronic nephritis, is more commonly due to thrombosis with cerebral softening than to actual vascular rupture. Certainly this has been the case at the Philadelphia General Hospital. The importance of pressure exacerbations as induced by exercise in precipitating apoplexy has perhaps been overestimated, a considerable number of "strokes" occurring while the patients are at rest if not actually asleep, although, theoretically, increased pressure would tend to favor apoplexy or embolism, and decreased pressure thrombosis.

1 Parisot, J., Hypertension cephalo-rachniclienne et pression artérielle, Compt. rend. Soc. biol., 1909, Ixvi, 939. 
The statement has been made that in cases of hemiplegia, due to thrombosis, the pulse is rapid, intermittent and of low tension, whereas hemorrhage produces hypertension and bradycardia. This appears to be true in typical cases. Often even rapidly fatal cases of hemorrhage fail to show either of the last-named symptoms (Collins).

Hemiplegia.-Pressure differences in corresponding extremities are not infrequently seen in cases of hemiplegia. Dana states that marked differences on the two sides of the body suggest involvement of the optic thalamus. ${ }^{1}$ Stewart found an abolition of vascular reflexes in an old case of hemiplegia on the affected side (see page 239). "In hemiplegia there is, in general, a marked deficiency in the blood-flow in the paralyzed members. Considerable differences, however, exist in different cases in this regard, and also in the extent to which the vasomotor reflexes from the normal to the paralyzed part are affected. Whether these differences depend at all on the position of the lesion or are associated with the duration and completeness of the paralysis has not been determined. There is some evidence that reflex vasoconstriction is more easily produced in the paralyzed parts than reflex vasodilatation."'2

It has been shown experimentally that in dogs and cats there is a limitable region of the cortex, irritation of which causes a bloorl-pressure increase that is not coextensive with the motor zone, but is in cats situated more anteriorly. Irritation of this zone is sure to cause splanchnic constriction. It has been suggested, but as yet not demonstrated, that the pressor effects of certain cortical regions are limited in their action to certain regions of the body. The occurrence of unilateral differences in temperature and edema in hemiplegia when the secondary effects of muscular paralysis can be excluded, indicate that similar cortical pressor centres exist in man. ${ }^{3}$

Locomotor Ataxia.-The crises of locomotor ataxia have long been recognized as clinical manifestations of the disease. The etiologic part taken by the sympathetic nerve was explained by Duchenne. Putnam suggested that lesions of the sympathetic nerve might, owing to their influence on the vasomotor system, account for certain secretory abnormalities. 'The importance of vascular spasm in the genesis of the crisis has been chiefly cham-

${ }^{1}$ Dana, Jour. Am. Jour. Med. Assn., December 19, 1909.

2 Stewart. G. X., Blood Flow in the Hands and Feet in certain Diseases of the Nervous System, Arch. Int. Med., 1915, xvi, 270.

${ }^{3}$ Lewandowsky, W., and Weber, E., Blutdruck u. Hirnrinde, Med. Klinik, 1906, ii, 385 . 
pioned by Pal, who reported a number of cases in which the crises were associated with hypertension (in one case $240 \mathrm{~mm}$. Hg.) and who regards the abdominal cramps as resulting from a spasm of the splanchnic arterioles, and a stretching of the nerve plexuses of the arterial wall. The attacks are sometimes relieved by nitrite of amyl. The rise of pressure occurs somewhat before the onset of pain, which begins when a certain variable high point is reached. The arteries become tense and the pulse rate increased. The pressure increase generally amounts to more than 50 per cent.

Heitz and Norero ${ }^{1}$ believe that the rise of pressure is the result of pain which they explain is due to a paroxysmal irritation of the posterior roots, and which is accompanied by local reflex phenomena in the corresponding segments; either (1) motor (vomiting, constipation) or (2) vasoconstrictor (hypertension). A rise of pressure is often absent in old cases owing to degeneration of the posterior roots, especially if chronie hypertension is associated. In opposition to Pal they failed to find arterial hypertension in cases of peripheral crises, although in most of their cases the disease was advanced. Stewart found the rate of blood-flow in both hands and feet diminished in tabes; together with feeble vasomotor reflexes.

Paresis. - The early observations of blood-pressure in paresis made by Pilcz and Alexander showed hypotension. Craig found the tension high during mental depression and low during exaltation. The more recent studies of Walton ${ }^{2}$ indicate that the average pressure in paresis is high, due doubtless to arteriosclerotic accompaniments. When cardiac and renal diseases are excluded the pressure is slightly lower than normal. Such hypotension is, however, neither sufficiently great nor constant to be of diagnostic or prognostic value. The excited states are associated with variable pressures, but depression is more often accompanied by high than by low pressures. 'Toward the termination of the disease very marked hypotension may occur.

Syringomyelia.-In a case of syringomyelia thermic stimulation of the affected arm produced no reaction in its fellow, whereas similar stimulation of the unaffected side arm produced a normal reaction on the diseased opposite side. These findings have been construed as showing that sensible stimuli occur in the "antidromal direction" after the manner of an axon reflex and produce a vasodilatation. ${ }^{3}$

1 De la pression artérielle chez les tabétiques, Areh. d. Mal. du Cœur, 1908; i, 505.

2 The Blood-pressure in Paresis, Jour. Am. Med. Assn., 1906, xlviii, 1341.

${ }^{3}$ Hess, L., and v. Bergmann, E., Ueber Gefaessreflexe, Wien. klin. Woch., 1913, xxvi, 1297. 


\section{CHAPTER X YII.}

\section{BLOOD-PRESSLRE IN SURGERY AND OBSTETRICS.}

The Effects of Hemorrhage.-When an artery of large size is torn or cut and the resultant hemorrhage is considerable, the diastolic pressure falls, as does also the systolic pressure, although to a lesser degree. The pulse pressure therefore increases and the left ventricle continues its normal output. Peripheral venous flow is, however, promptly reduced and soon the fall in venous pressure fails to supply an "effective" right auricular pressure. As a result of inadequate filling of the right heart, pressure falls in the pulmonary artery and in the left auricle, and systolic left ventricular output promptly falls. The medullary centres are stimulated by the decreased blood-flow, the pulse rate rises from depression of the cardio-inhibitory centre and vasoconstriction results from central stimulation. In addition to this mechanical factor chemical changes also produce their effects. The loss of erythrocytes from the blood-stream with its consequent reduction in the oxygen-carrying power of the blood, together with a sluggish circulation in the peripheral capillaries, leads to a decrease in the volume per cent. of oxygen in first the venous, and later, the arterial blood, while the carbon-dioxid content of each remains unchanged. This reduction in the hematogenous oxygen content causes hyperpnea, which causes for the time being a better average auricular pressure. In brief, "the fall of arterial pressures is counteracted by the augmented breathing, an accelerated heart, an accommodative contraction of the larger vessels and by peripheral constriction leading to an increased resistance and diminished flow in spite of the reduction of viscosity.".

When hemorrhage ceases the loss of the blood bulk is made up by the absorption of lymph from the tissues. The rise of bloodpressure which results may be abetted by intravenous saline or salo-alkaline infusion. There appears to be no experimental evidence supporting the view generally held that active secondary, posthemorrhagie vasoconstriction plays much of a part in this posthemorrhagic rise of the mean pressure. ${ }^{2}$

The consecutive changes occurring during hemorrhage are represented in the following schemas taken from Wiggers's artiele.

1 Wiggers, C. J., Modern Aspects of the Circulation in Health and Disease, Philadelphia, 1915 , p. 339.

2 Wiggers, C. J., Pathologie Physiology of the Circulation during Hemorrhage, Areh. Int. Med., 1914, xiv, 33. 


\section{SEQUENCE OF EVENTS DURING HEMORRHAGE. \\ Reduction in Total Volume of Blood.}

Reduetion in red cells and percentage of hemoglobin

\section{Venous blood- $\mathrm{CO}_{2}$ unehanged decreased}

Arterial blood- $\mathrm{CO}_{2}$ increased $\mathrm{O}_{2}$ deereased

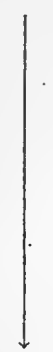

Stream slowed in tissues

Osmosis toward blood-stream

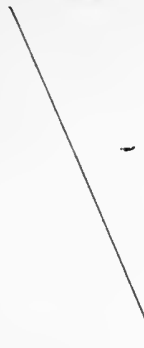

\section{$\downarrow$}

$\downarrow$

Inerease in volume

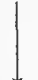

Reductions of red eells by dilution of blood

Decreased viseosity
Reduetion in total arterial resistance $\downarrow$

Decreased diastolic pressure

$$
\downarrow
$$

Inereased systolic discharge of heart $\downarrow$

Lower systolic, increased pulse-pressure $\downarrow$

Decreased flow in peripheral veins

$$
\downarrow
$$

Decreased right auricular pressure

$$
\downarrow
$$

Decreased output of right ventricle

$$
\downarrow
$$

Decreased pulmonary arterial pressure and left auricular pressure

$$
\downarrow
$$

Deereased output of left ventricle

Smaller pulse-pressure

Further fall in systolic and diastolie pressures

Deereased flow through medulla
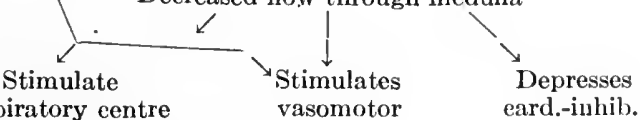
respiratory centre (deeper respirations) eentre centre

Tend to counteraet fall of pressure

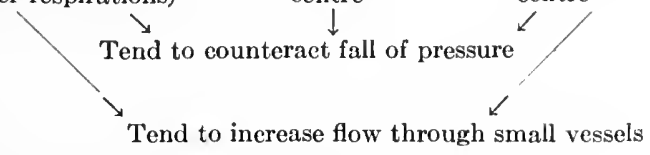

SEQUENCE OF EVENTS DURING TERMINAL STAGES.

Loss of Blood Volume Exceeds Resorption of Lymph.

Red eorpuseles reduced to minimum

Oxygen pereentage in arterial blood decreases

Progressive increase in $\mathrm{CO}_{2}$
Systolic diseharge diminishes due to lack of diastolic filling

Pulse-pressure progressively diminished

$\searrow$ $\downarrow$

Respiratory centre depressed

Heart depressed by anemia

Beats slower.

$\downarrow$

Feebler

$\downarrow$

Respiratory periodic gasps

Asphyxial conditions

$\downarrow$

Heart slow, large amplitude of contraction $\downarrow$

Ventricular fibrillation, eessation 
Blood-pressure readings should be made in all cases in which anesthetics or surgical procedures are employed before, if possible during, and after the operation. Arterial hypertension will often indicate the presence of renal disease when the routine urine examination fails to reveal it. A fall of pressure during the course of the operation is one of the surest methods of detecting the beginning of shock, whether this occurs during or after the conclusion of the operation. A fall of pressure to below $100 \mathrm{~mm}$. Hg., together with a rising pulse rate, is an indication for immediate treatment. Shock is at hand when the pulse pressure falls to 10 to $15 \mathrm{~mm}$., or when the diastolic pressure falls to or below $70 \mathrm{~mm}$. Hg. (McKeson). When possible the patient's normal pressure should be obtained the day before the operation, or at least not immediately preceding it, because fear and excitement both tend to increase the systolic pressure, and the pulse rate. A steady pressure is the best indication of a satisfactory condition on the part of the patient. Fluctuations of tension point to exhaustion. Patients with marked hypertension (180 to $210 \mathrm{~mm}$. Hg.) require special care. The possibility of apoplexy, uremia, or heart failure must be borne in mind, and yet such patients not inf requently make perfect recoveries. Blood-pressure readings alone camot be used as a criterion of safety; the patient's general condition must be carefully considered. Cases of arterial hypotension, especially if this condition is associated with lymphocytosis and other eridences of status lymphaticus, withstand anesthesia as badly as they do infectious disease.

"The practical surgeon should have knowledge of the following data: 'The effect of the general narcotic on blood-pressure and its relation to shock; whether in certain operations shock is less if the operation is carried on under spinal or local anesthesia, the effect of hemorrhage, the duration of the operation, exposure of the tissues to the air, effects of extreme heat or cold, the effect of manipulation or injury in various tissues or organs" (Bloodgood). While the manipulation of sensitive organs causes a primary rise of pressure, such a procedure may go far toward exhausting the vasomotor centre.

Surgical Shock. - Surgical shock has been described as "a state of general apathy, reduced sensibility, extreme motor weakness, great pallor, very small pulse, thready soft arteries, irregular gasping respirations, and subnormal temperature."' There is perhaps no

${ }^{1}$ Meltzer, S. J., The Nature of Shock, Arch. Int. Med., July, 1908, p. 571. 
subject in the whole domain of surgery regarding the pathology of which more radical differences of opinion exist than shock. This is at least in part due to the fact the term shock is applied to various conditions which may arise from trauma, intoxications, hemorrhage, etc. It has been suggested (Mann) that the term "shock" (similarly to the much abused and too inclusive term of "rheumatism" should be dropped or if used at all, be limited to a condition in which without demonstrable hemorrhage, the amount of circulating fluid has been greatly diminished owing to venous or capillary stasis, or to exudation of serum or the diapedesis of corpuscles. Since the publication of Crile's work on blood-pressure in relation to surgical shock, the view that this condition is the result of an exhaustion of the vasomotor centres has been widely accepted. Crile originally stated that "neither the heart muscle, nor the cardiac centres, nor the respiratory centre, are other than secondarily involved." Collapse is due to a suspension of the function of the cardiac or of the vasomotor mechanism or to hemorrhage. These statements are based upon investigations of a large amount of clinical and experimental material which showed that (1) the only constant finding in cases of shock was a fall of blood-pressure; (2) there were no constant demonstrable lesions in the circulatory apparatus; (3) the fall of pressure was not due to exhaustion of the peripheral nerve vascular mechanism; (4) that cocainization or destruction of the vasomotor centres produces a fall of pressure to the shock level; (5) that drugs or other methods of treatment which raised arterial pressure were beneficial, whereas other stimulants which were employed were either useless or definitely detrimental. According to this hypothesis, then shock is due to an exhaustion of the vasomotor centre resulting from centripetal sensory stimuli.

In shock we find the diastolic pressure decreased, the pulse rate and the pulse pressure increased. When cardiac exhaustion occurs the systolic pressure falls toward the diastolic level until the pulse pressure becomes so small that the cardiac and respiratory centres are no longer sufficiently supplied with blood and death occurs.

"A respiratory rate of more than 30 per minute is too rapid to assist in moving the blood, thus throwing the whole burden upon the heart" (McKeson).

The experiments of Porter $^{2}$ have shown conclusively that the

${ }^{1}$ Crile, G. W., Blood-pressure in Surgery, Philadelphia, 1903.

2 The Relation of Afferent Impulses to the Vasomotor Centres, Am. Jour. Physiol., 1910, xxvii, 276; and The Vasotonic and Vasoreflex Centre, ibid., 1915, xxxvi, 418. 
depression or fatigue of the vasomotor centre can no longer be considered as the primary cause of shock. Nor has it been demonstrated that the peripheral bloodvessels constantly or uniformly lose their tone. Mann ${ }^{1}$ found that no amount of sensory stimulation would produce shock in the anesthetized animals so long as the abdomen is not opened, and hemorrhage is prevented.

Based on more recent investigations, Crile ${ }^{2}$ believes that the essential lesions of shock are in the brain cells and are caused by the conversion of potential energy in the brain cells into kinetic energy at the expense of certain chemical compounds stored in the cells. As a result of certain noci impulses (fear, exhaustion, anesthesia, insomnia, etc.) the brain threshold is lowered and the trauma of opcration or injury may produce the shock. In order to avoid shock it is necessary to exclude these noci impulses both of the special senses and of common sensation. The technique of anoci-association consists briefly in excluding all psychic stimuli by proper preoperative care of the patient, protecting the brain against destructive psychic strain during the operation by complete anesthesia and preventing nerve trauma by blocking the nerves with local anesthetics. Crile lays the greatest stress on the psychic element, a conception which is not essentially at variance with Henderson's theory.

Yandell Henderson, ${ }^{3}$ on the other hand, believes that shock results from a lack of fluid in the circulation. According to this view there is a loss of $\mathrm{CO}_{2}$ from the blood (acapnia) which results from increased respiration due to pain, trauma, fever, acidosis, exposure of tissues, or maladministration of the anesthetic. This causes a diminution of hemic osmotic tension, increased fluid transudation to the tissues, and a decrease in the total volume of blood. Owing to a fall of venous pressure the heart is no longer arlequately filled with blood, and hence the systolic output diminishes, and a condition analogous to hemorrhage ensues. The experiments of Morison and Hooker ${ }^{4}$ indicate that loss of venous tome, causing a stagnation of blood in the veins and an insufficient filling of the right heart (as previously maintained by Henderson) plays a considerable part. Mann's investigations indicate that the loss

1 The Peripheral Origin of Shock, Bull. Johns Hopkins Hosp., 1914, xxv, 205.

2 The Kinetic Theory of Shock and its Prevention through Anoci-association (Shockless Operation), Lancet, July 5, 1913, p. 7.

3 Amer. Jour. Physiol., 1910, xxvii, 167.

${ }^{4}$ The Vascular Tone and Distribution of the Blond in Surgical Shock, Am. Jour. Physiol., 1915, xxxvii, 86. 
of serum and erythrocytes caused by handling of the delicate vascular area of the splanchnics plays an important role.

Corbett' $\mathrm{s}^{1}$ experiments lead him to conclude that shock is of a composite nature, in which epinephrin exhaustion and oligemia are predominating factors, while anesthesia, pain, fright and trauma are immediate agents in producing epinephrin exhaustion as well as shock. Not "that epinephrin loss is shock, but rather epinephrin is necessary to overcome shock, and anything that depletes epinephrin favors the development of that condition."

The foregoing hypotheses have aroused much discussion, and the true explanation must as yet be regarded as unsettled. Numerous other theories have been advanced and discussion has been inconclusive. It appears agreed that "low blood-pressure is a constant accompaniment of shock, so constant, indeed, that investigators practically always express the state of shock in terms of manometric pressure." ${ }^{2}$ For a more complete discussion the reader is referred to Parham's recent review of the subject. ${ }^{3}$

In the early stages of shock a marked rise of pressure often occurs as the result of stimuli which exert a vasoconstrictor influence, but owing to continuous stimulation of this kind a stage of depression occurs which may result in death.

Hemorrhage is an important contributing factor in the production of shock, and all avoidable bleeding should be promptly checked. Sudden or large hemorrhage manifests itself by a fall of blood-pressure. A rapid and persistent fall of pressure with an increased pulse rate often indicates a loss of blood too great for the organism to compensate for by peripheral vasoconstriction and by an additional expenditure of cardiac energy. 'The diastolic pressure is an index of far greater importance than the systolic, and a diastolic pressure of $50 \mathrm{~mm}$. seems to be the lowest pressure at which the circulation can be maintained. (See Hemorrhage, p. 376.$)^{4}$ Rough manipulation, tearing of the tissues, especially when these have a large afferent nerve supply, must be avoided. "During the operation the amount of anesthetic

1 The Suprarenal Gland in Shock, Jour. Am. Med. Assn., 1915, lxv, 380.

2 Gray and Parsons, Anis and Gale Lectures, Royal College of Surgeons of London, March, 1912, British Med. Jour., April and May, 1912.

${ }^{3}$ Parham, F. W., Shock, Its Nature and Management, South. Med. Jour., 1913, vi, 763 ; see also, The Present Status of Our Knowledge of Shock, J. M. Wainwright, Penn. Med. Jour., Deember, 1914, p. 180; and Anesthesia, Gwathmey, New York, 1914.

${ }^{4}$ Balard, B., La Tension Artérielle Minima Element de Prognostic des Hemorrhagies Graves de la Grossesse, Arch. Mens. d'Obst. et de Gyn., 1914, iii, 241. 
should be as small as possible, the operation should be performed as quickly as is compatible with the safety of the patient and the purpose of the intervention; tissues should be handled as gently as possible; if large nerves must be divided, as in amputations, they should be blocked with cocain injection; tissues should be exposed to air only when absolutely necessary; they should be protected with warm, moist gauze; the patient should not be exposed to extremes of heat or cold. The weaker the patient to be subjected to operation, the more attention must be given to these details which lessen shock."' 1 Complicating factors such as anemia, nephritis, diabetes, alcoholism, acute and chronic infections, metabolic disturbances, and psychic factors must also be borne in mind.

The handling of tissues and organs supplied with sensory nerves by the cerebrospinal system is more apt to induce shock than is the manipulation of those whose nerve supply is derived from the sympathetic nerves or the lower vagus. Furthermore, inflammation enhances the sensibility. Tumors are said to be insensible, but their connection with other tissues must be borne in mind.

The Treatment of Shock. - The keynote of treatment lies in prevention which has already been considered. The arterioles once they are relaxed, do not readily regain the tonus. Hence the pressure required to keep up the circulation in the medulla and in the coronary arteries must be produced by increased cardiac work. Drugs are for the most part unsatisfactory. The most rational - therapeusis, however, consists in the slow and prolonged intravenous administration of pituitrin or epinephrin in normal salt solution. The amount of infusion necessary may be gauged by blood-pressure readings. Recent researches have shown that the intravenous administration of a molecular (8.4 per cent.) solution of sodium bicarbonate produces a marked rise of blood-pressure, an increased cardiac amplitude, and depth of respiration. These effects are due mainly to alkalinity, much less to bulk and hypertonicity, and in part to some other undetermined factor acting chiefly upon the heart, but not to the increased carbon dioxid content of the blood. ${ }^{2}$ Investigations have shown that in shocked animals camphor, administered intravenously, causes an actual fall of blood-pressure. It is more apt to be efficacious when given

\footnotetext{
1 Bloodgood, J. C., Surgical Shock, American Practice of Surgery, Bryant and Buck, 1906, i, 469.

2 Seelig, Tierney, and Rodenbaugh, Jour. Am. Med. Assn., 1913, 1x, 238.
} 
subcutaneously. Strychnin likewise does not raise blood-pressure, although it may be useful on account of its stimulation of the nerve centres. Nitroglycerin is definitely contra-indicated, although it may temporarily give a better color to the skin. Strophanthin, intravenously increases pressure and is often useful. Caffein intravenously tends rather to lower than to raise blood-pressure. Atropin when the skin is clammy is often useful.

Inversion of the patient to increase blood-pressure in the brain may be tried, but not of course if the patient has already been in the Trendelenburg position. This posture may cause arhythmia and, according to Pope, ${ }^{1}$ even heart block, "due either to acute dilatation, to toxins, or fatigue, or sepsis, or coronary clots, or direct trauma to some area of cardiac reflex." "It is quite possible, however, that from this position slight edema or exudate into the fourth ventricle may occur and may disturb the respiratory and pneumogastric centres. In shock from this cause, Pope suggests venesection from the jugular vein, cardiac massage and epinephrin in Ringer's solution forced backward in the arterial stream, as giving the only possible chance for recovery." 2 Atropin, strychnin, or caffein in hypodermic administration are currently employed. The patient's head should be kept low, the feet elevated, the body warm and quiet. Blood-pressure may be elevated by bandaging the extremities. Fresh air should be supplied and carbon dioxid in 6 per cent. concentration may be tried.

According to Crile's anoci hypothesis, ${ }^{3}$ stimulants are contraindicated, while morphin and other sedatives may prove useful. Normal saline is beneficial temporarily and within limits, while human blood may be expected to yield the best results. All authorities are agreed that preventive measures are of the greatest importance. The elimination to the greatest possible extent, of fear and anxiety on the part of the patient before, and in preparation for an operation is of the utmost importance. For this purpose a hypodermic injection of morphin before the patient is anesthetized is often most beneficial. With the same object in view the patient should see and hear as little as possible of the operating room and of the preparations which are being made for the impending operation.

The Posture of the Patient.--The posture of the patient during operations of necessity entails marked changes in blood-pressure

1 California State Jour. Med., Deember, 1913, p. 499.

${ }^{2}$ Edit. Jour. Am. Med. Assn., 1914, Ixii, 776.

${ }^{3}$ Crile \& Lower, Anoci-association, Philadelphia, 1914. 
and blood distribution, with which the heart under the benumbing influence of anesthesia or if handicapped by disease, may be unable to cope. Thus in the morphinized animal carotid pressure falls owing to this effect of gravity in the head-up posture, and rises when the fect are uppermost. Compensation is established by cardiac and vasomotor reactions in the stronger animals, but rabbits die if the feet-down position is long maintained (fifteen minutes to two hours) from cerebral anemia, due to splanchnic dilatation which can no longer be overcome by respiratory movements (abdominal compression and diaphragmatic suction). The normal vasomotor tone is depressed by ether, and specially so by chloroform. Again, dogs may be killed by a prolonged Trendelenburg. position plus ether (not morphin) anesthesia, the death resulting from failure of respiration, the heart action remaining good and the blood-pressure high. While death may occur similarly in the horizontal position, partial or complete respiratory faliure is more common in the head-down posture.

Recent researches indicate that "the Trendelenbury position is harmless for patients with normal hearts, provided that respiration is free and unobstructed." This "position should be used only with extreme caution in cases of cardiac disease." The position should not be established or relinquished suddenly. Using this posture forces a large amount of venous blood toward the heart, and, if this organ is capable of handling it, produces temporarily a marked increase of brachial blood-pressure. If the position is suddenly abandoned the venous pressure, and consequently the arterial pressure, fall and bulbar anemia may result, especially if vascular tone is depressed.

Struggling, and pressure upon the abdomen by gauze pads, bandages, etc., may cause cardiac dilatation. The administration of morphin in moderate doses preceding the administration of ether is often advisable. It diminishes the amount of ether required, tends to prevent struggling, excitement, and acapnia, and does not in man impair vasomotor tone. Patients with heart disease, pneumonia, or empyema should be anesthetized gradually, in the sitting or semirecumbent posture (Gatch, Gann, and Mann). ${ }^{1}$

The Effect of Chilling during Anesthesia.-Under ether anesthesia the application of cold to dogs produces a marked rise of bloodpressure; under chloroform no such rise occurs. It seems, therefore,

1 The Danger and Prevention of Severe Cardiac Strain during Ether Anesthesia, Jour. Am. Med. Assn., 1913, lx, 1273. 
that the narcotic dose of ether does not abolish vasomotor reflexes as does chloroform. The rise of pressure in the former instance causes a congestion of the internal viscera, a condition comparable to what occurs during the act of "catching cold," and probably accounts for the greater frequency of respiratory infections after ether. ${ }^{1}$

Visceral Manipulation.-Blood-pressure observations have taught the importance of avoiding, or at least minimizing, certain surgical manipulations. Thus a marked fall of pressure is caused by irritation of the dura and of the subdiaphragmatic peritoneum, traction upon bloodvessels or nerves, renal or mesenteric vessels, the brachial plexus, or the testicles; by rough sponging, blunt dissection, exposure or rough handling of viscera, peritoneal flushing, scparating adhesions, delivering tumors, extirpation of the kidney, exposure of the spinal cord, section of large nerve trunks unless previously cocainized. The upper portion of the peritoneal cavity is more sensitive than the lower.

On the other hand, blood-pressure rises during divulsion of the sphincter ani and in stretching of the sciatic nerve.

The following procedures produce but slight blood-pressure changes: incision of the scalp, chiselling of bone, separation of the periosteum, hernia operations (without adhesions), the resection of ribs, appendectomy (simple), incision of the kidney. Dragging upon the mesentery produces a much greater fall of pressure than handling the omentum. Especial care should be exercised in the region of the duodenum, pylorus and gall-bladder, owing to the possibility of interfering with the circulation in the larger venous trunks. Trauma to the pelvic viscera, on the other hand, has much less tendency to produce shock (Gatch). Under normal circumstances the diaphragm materially aids the circulation by alternately increasing the pressure in the thoracic and in the abdominal cavities. But once the abdomen is opened, the diaphragm no longer increases intra-abdominal pressure during inspiration and hence less blood reaches the right heart.

Concussion of the brain produces a fall of blood-pressure, compression, a rise (see page 372), but severe experimental injury of the cerebral hemispheres under complete anesthesia does not cause a fall of pressure. "Collapse from interference with the medullary centres is of course not true surgical shock" (Crile).

1 Stursberg, Das Verhalten d. Blutdrucks unter d. Einwirk. v. Temperaturreizen in Aether u. Chloroform Narkose., Mitt. a. d. Grenzgeb. d. Med, u. Chir., 1910, $\mathrm{xxii}, 1$. 
Manipulations of the Thoracic Viscera.-The effects of various surgical manipulations upon the blood-pressure, pulse rate, and respiration have been made by Flint. ${ }^{1}$

The detailed results cannot be given here, but the conclusions reached show that "the reactions of the medullary centres to operative traumatism as shown in these experiments suggest that we should be cautious in the treatment of the parietal pleura, particularly in tearing it at the angles of the intercostal wound by injudicious application of the rib-spreader. The lungs may be handled freely, but manipulations that tend to transmit traction to the great ressels and bronchi at the root of the lung should be reduced to a minimum. In heart suture the Sauerbruch method of temporary hemostasis leads to too serious a fall in blood-pressure to be safe except where other means of hemostasis fail. Any mechanical stimulation of the heart, either directly or through the pericardium, during suture, should be avoided as far as possible. Furthermore, in packing off the lungs to obtain an exposure of other thoracic viscera it would be wise to avoid any unnecessary trauma which might tend to reduce the blood-pressure excessively. These are the stimuli which, in the present set of experiments, have produced the most serious reactions."

Manipulation of the Pelvic Viscera.-Manipulation of the pelvic organs during laparotomies may produce either a rise or a fall of pressure, depending upon the manner in which the viscera are handled. Experimental irritation of the genital mucous membrane (in dogs) produces a fall of pressure ranging between 5 and 50 per cent. The fall of pressure is more marked if the stimulation is applied in the lower vagina; in the upper portion a rise of pressure may result. Irritation of the rectal mucosa causes a fall of pressure. ${ }^{2}$

Schröder found that manipulations of the female generative organs by way of the ragina caused less lowering of pressure than when a laparotomy was performed; but Crile found that manipulation always produced a rise which was proportional to the traumatism.

Aspiration of the Pleura.-Vertigo, syncope, and even death may follow the withdrawal of fluid from the pleural cavity. It has been shown that these effects are not merely the result of altered pressure

\footnotetext{
${ }^{1}$ Flint, J. M., Physiologic Basis of Thoracic Surgery, Jour. Am. Med. Assn., 1912 , lix, 760 .

${ }^{2}$ Belfield, W. J., Ueber depressonsche Reflexe, erzengt durch Schleimhautreitzung, Arch. f. Anat. u. Phys., 1882, p. 298.
} 
relations in the thorax, but that they are due to a depressor reflex originating in the pleura, which may be: (1) A central reflex cardioinhibitory type, in which the heart is slow, intermittent, and there are great variations in the systolic and diastolic pressures, associated with slow respirations. (2) A vasomotor type showing a steady rapid decline of pressure without any great difference between the systolic and the diastolic readings. Respirations are shallow, sometimes rapid. This type, in contradistinction to the first, not infrequently terminates in death. The reflex may be either central or peripheral. To minimize the likelihood of these accidents care should be taken to avoid irritating the pleura with the trocar or drainage tube. ${ }^{1}$ In seven out of eight pleural aspirations Clark found a fall of venous pressure (see page 141).

Paracentesis Abdominalis. - This procedure is attended with much the same blood-pressure changes as those which occur when the pleura is aspirated. As might be expected a greater fall (32 mm. on the average) occurs.

"Up to a certain point the general arterial pressure increases with an increase in the pressure of intra-abdominal fluid; beyond this point the blood-pressure falls. Quirin attributes the early rise in blood-pressure to increased resistance by compression to the flow of blood through the abdominal arteries; the fall occurs when the heart, handicapped by a diminished supply of venous blood, is no longer able to overcome the resistance of the abdominal arteries. Ascitic fluids have an intra-abdominal pressure of 19 to $42 \mathrm{~mm}$. Hg., according to Quincke. Quirin found that the intra-abdominal pressure fell 10 to $14 \mathrm{~mm}$. after tapping, and that this corresponded with the simultaneous fall in arterial pressure of 5 to $10 \mathrm{~mm}$. Hg. after paracentesis in four cases. Cook and Briggs record one case of abdominal paracentesis in which the pressure fell $35 \mathrm{~mm}$. Hg. during the withdrawal.'2

The rate of withdrawal is apparently more important than the amount so far as the immediate pressure is concerned. The ultimate pressure depends more upon the quantity of fluid withdrawn. External abdominal pressure favors a rise of pressure, as does also the recumbent posture. "Improvement is most pronounced

\footnotetext{
${ }^{1}$ Capps and Lewis, Blood-pressure Lowering Reflexes, Amer. Jour. Med. Sei., 1907, exxxiv, 868 .

2 Capps, J. A., Some Observations on the Effect on the Blood-pressure of the Withdrawal of Fluid from the Thorax and Abdomen, Jour. Am. Med. Assu., January 5, 1907, xlviii.
} 
in those cases that undergo a marked fall in pressure during the operation" (Capps).

The emotional disturbance entailed by a prospective operation, as well as the actual pain of puncture, produce a temporary elevation of blood-pressure (see under Abdominal Distention and Bloodpressure, page 260).

The majority of surgeons scem to be impressed. with the importance of blood-pressure readings in brain surgery. Horsley states that 25 per cent. of the sudden deaths after prolonged operations may be avoided by frequent observations.

Drainage of the Bladder.-Drainage of the bladder, either by suprapubic incision or by a retained catheter as a preliminary procedure to prostatectomy, definitely lowers blood-pressure (average in 50 cases, 166 to $145 \mathrm{~mm}$.) and diminishes mortality. ${ }^{1}$

Anesthetics.-Anesthetics differ in their effect upon bloodpressure, and the degree to which a given drug lowers pressure is an index of the danger with which its administration is associated.

Ether.-'The early stages of ether anesthesia are associated with an increased blood-pressure due to psychic stimuli and muscular effort. This increased pressure is maintained through stimulation of the respiratory centre. Recent experimental evidence has shown that ether alone, even when employed in large amounts, rarely produces much depression of blood-pressure. ${ }^{2}$ When anesthesia is complete the pulse rate and the blood-pressure are practically normal. A slight postanesthetic rise is often observed, which the administration of oxygen generally increases. During ether-oxygen anesthesia blood-pressure is invariably increased. If ether is administered intravenously a preliminary use of pressure $(6-25 \mathrm{~mm}$.) occurs to be followed at the end of from one to three hours by a fall of like degree (Honan and Hassler). Since ether is excreted by the stomach and reabsorbed, it has been found that the introduction of olive oil into the stomach by means of a catheter before the patient has regained consciousness binds the ether and prevents or lessens reabsorption in the gastro-intestinal tract (E. Graham). A fall of pressure during anesthesia may result primarily from either vasomotor or from cardiac failure. Henderson ${ }^{3}$ believes that both conditions are fundamentally due to acapnia resulting from excessive

1 Balfour, D. C., Mayo Clinies, 1913, p. 73.

${ }^{2}$ Guy, W., Goodall, A., Reid, H. S., Blood-pressure in Anesthesia, Edinburgh Med. Jour., 1911, n. s., iii, 126.

${ }^{3}$ Primary Heart Failure in Normal Subjects under Ether, Surg., Gynec. and Obstet., 1911, p. 161. 
pulmonary ventilation in the stage of excitement. Prolonged ether-chloroform narcosis in rabbits is said to produce injury to the chromaffin system (Schur and Wiesel).

The effect of morphin and ether anesthesia combined differs from that of ether alone. In the latter instance sensory stimuli are not completely blocked, although the sensorium is abolished and surgical anesthesia may be sufficient.

Chloroform.-Blood-pressure is often reduced even in the earlier stages; under full anesthesia a marked fall is often present which in fatal cases continues progressively until death occurs, due to cardiac depression. Even in the earlier stages, however, the heart may temporarily cease contracting, owing apparently to reflex inhibitory stimuli originating in the air passages. In case of a diseased heart the arrest may be permanent. The depressant action of chloroform upon the heart is much greater than that of ether. Regarding the primary vascular effect of chloroform there is still divergence of opinion. Some investigations show an initial rise of pressure due to stimulation of the vasomotor centre. This stimulation does not increase blood-pressure, as it is more than counterbalanced by cardiac weakness (Cushny). Unlike ether, clloroform does not have any compensatory stimulating action to mask the effects of peripheral stimulation; it is this property which constitutes its danger (G. Müller). If oxygen is combined with chloroform blood-pressure is much less reduced than by chloroform alone.

Ethyl Chlorid.-The effect of ethyl chlorid upon the heart muscle, similarly to that of chloroform, is depressive, but nineteen times more of the former is required to produce similar results. Ethyl chlorid causes local peripheral dilatation. Vagus inhibition of the heart occurs readily. In concentration of from 10 to 20 per cent. a fall of blood-pressure results from vagus stimulation. If 30 per cent. or more is used, blood-pressure falls from cardiac muscular depression, although this is not so pronounced as after chloroform. ${ }^{1}$ Ethyl chlorid generally slows the pulse and produces a fall of bloodpressure. ${ }^{2}$ It is by many regarded as a dangerous anesthetic, far inferior to nitrous oxid.

Nitrous Oxid.-The administration of this drug is attended with a slowing of the pulse and a marked rise of arterial pressure. These

1 Embley, Proe. Royal Soc., 1906, Laneet, April 20, 1907, Pharm. Jour., xxiv, 650.

${ }^{2}$ MrCardie, The Position of and Mortality from Ethyl Chlorid as a General Anesthetie, British Med. Jour., Mareh 17, 1906, p. 616. 
effects have been attributed both to a specific action and to the asphyxial condition of the blood. In arteriosclerosis, especially if associated with hypertension, it may cause vascular rupture. Crile states that "under approximately equal trauma the changes in the brain cells were approximately three times as great under ether anesthesia as under nitrous oxid anesthesia; that the fall in the blood-pressure was on the average two and a half times greater under ether than under nitrous oxid; and finally, that the condition of the animal was better after trauma under nitrous oxid than after equal trauma under ether." Bloodgood's experience with nitrous oxid and oxygen anesthesia, even in cases of arterial hypertension, has been most favorable.

Cocain.-Cocain stimulates the heart muscle directly or as the result of accelerator influence. In the early stages of intoxication there is peripheral vasoconstriction (local action and central stimulation) causing a rise of blood-pressure. Later, pressure falls owing to peripheral action. Cocain has been almost entirely supplanted by the less toxic drugs, stovain and novocain, the former being used for spinal and the latter for local anesthesia.

The subject of anesthesia cannot, of course, receive prolonged consideration here, but a few points may be emphasized. There seems to be no valid reason for chloroform as a substitute for ether in major operations. It should never be administered after a preceding period of ether excitement. For minor operations nitrous oxid is preferable. In using ether the subject should be quickly narcotized and kept "well under." Struggling during the administration and incomplete anesthesia are dangerous. The beneficial effects of rebreathing $\left(\mathrm{CO}_{2}\right.$ stimulation) in preventing shock, as shown by Gatch, lend strong support to Henderson's explanation of this condition as resulting from acapnia. The preliminary administration of morphin is often desirable. Nitrous oxid with oxygen is for short operations an excellent anesthetic.

The Cerebrospinal Fluid and Blood-pressure.-The normal pressure of the cerebrospinal fluid ranges between 60 and $100 \mathrm{~mm}$. $\mathrm{H}_{2} \mathrm{O}$. It may be increased to $200-800 \mathrm{~mm}$. in meningitis or brain tumor. It should never be reduced below $60 \mathrm{~mm}$. $\mathrm{H}_{2} \mathrm{O}$. It varies and is practically identical with the pressure in the venous sinuses. ${ }^{1}$ A relatively small increase in cerebrospinal pressure stimulates the vasomotor, cardio-inhibitory and respiratory centres. When

' Frazier and Peet, Factors of Influence in the Origin and Circulation of the Cerehrospinal Fluid, Am. Jour. Physiol., 1914, p. 1018. 
pressure is increased considerably above the normal arterial pressure, respiration ceases, and there is an enormous rise of arterial pressure with a slow pulse. When the cardio-inhibitory centre becomes exhausted, blood-pressure rises still higher owing to the continued activity of the vasomotor centre, until the latter becomes paralyzed, ${ }^{1}$ whereupon blood-pressure falls.

Lumbar Puncture.-Blood-pressure is the most valuable guide during this procedure. A well-marked fall of tension indicates immediate cessation. Lumbar puncture is sometimes followed by sudden syncope, especially when medicinal substances are injected. This may result from (1) the puncture of the dura-a rare but possible reflex disturbance; (2) increased intraspinal pressure; (3) the introduction of chemicals used for purposes of medication or for the preservation of sera. Auer's researches indicate that tricresol, which is used for the last-named purpose, causes a greater fall of blood-pressure than chloroform or formalin.

" 1 . The puncture of the skin is accompanied by a rise in bloodpressure which varies in extent with the degree of consciousness, the pain, and the disturbance produced by the operation, but which is not accounted for entirely by these factors. Puncture of the dura causes a much larger rise, which is due not to pain or the disturbance produced but to a definite effect on the vasomotor centre. Similar rises occur in dogs under full anesthesia.

" 2 . Withdrawal of cerebrospinal fluid per se tends to lower the blood-pressure, but the net result of lumbar puncture is to raise it for at least twenty minutes afterward.

" 3 . The type of blood-pressure chart obtained in lumbar puncture, when the increased intracranial pressure is of subtentorial origin, is the same as that obtained in normal individuals; or as that obtained when the increased pressure is of supratentorial origin; but in the subtentorial types the variations are less pronounced. In subtentorial cases the rise is more sustained." ${ }_{2}$ Bloodpressure readings should always be made during intraspinous medication. Berghausen ${ }^{3}$ suggests that the needle be left in situ until blood-pressure returns to the same height as that at which it was preceding the spinal puncture. This seems hardly necessary if the serum has been given by the gravity method. According to

1 Dixon and Halliburton, The Cerebrospinal Fluid, Jour. Physiol., 1914, xlviii. 317.

2 Gray, H. T., and Parsons, L., Blood-pressure Variations Associated with Lumbar Puncture and the Induetion of Spinal Anesthesia, Quart. Jour. Med., 191 1-12, v, 339.

${ }^{3}$ Intraspinous Medication, New York Med. Jour., 1914, c, 1006. 
the last-named author, sudden respiratory changes follow only when blood-pressure change has been marked-usually after a fall of pressure (see Meningitis). If collapse symptoms occur, the head should be lowered, the feet elevated, and ether and strychnin should be given hypodermically.

Spinal Anesthesia.- "1. Blood-pressure records from cases of high spinal anesthesia show at the outset an abnormally high blood-pressure due to mental anxiety; then a rise following lumbar puncture; next a 'preliminary fall,' followed by a further more marked 'main fall' as the paralysis affects the thorax. Finally, as the paralysis passes off, the blood-pressure rises to its original values. The 'preliminary fall' is due to:

"(a) Flaccid paralysis of the abdominal and skeletal muscles.

"(b) Subsidence of the disturbance caused by lumbar puncture.

" $(c)$ Onset of mental calm, amounting possibly to sleep.

"The 'main fall' is due to the thoracic paralysis, which is not compensated for by overaction of the diaphragm, and consequently the aspiratory action of the thorax is diminished.

"2. Marlied deviations from this common type of chart are due to:

"( a) Volumtary inspirations by the accessory respiratory muscles, and

"(b) To a lesser extent by the activity of the higher centres.

"Variations in the pulse rate do not closely follow those of the blood-pressure, but a certain degree of resemblance is often seen owing to the consciousness of the patient.

"3. Vomiting during spinal anesthesia is due to thoracic paralysis, which induces either:

"(a) Anemia of the medulla.

"(b) Excessive action of the diaphragm, which interferes with the stomach.

"4. Blood-pressure records from cases of low spinal anesthesia show the 'preliminary fall' but not the 'main fall.' 'The 'preliminary fall' in these cases is due to:

"(a) Subsidence of the disturbance caused by lumbar puncture.

"(b) Onset of mental calm.

"(c) In some cases from the degree of flaccidity of the abdominal muscles.

"5. In the supine position there is no stagnation of blood in the great vessels, even when a very high spinal anesthesia is induced."

Inasmuch as the normal blood-pressure depends upon afferent 
and efferent pressor and depressor stimuli it is evident that serious results may follow the cutting off of such impulses. A cessation of pressor impulses, for instance, would leave the effect of depressor fibers in predominance.

The question of blood-pressure in spinal anesthesia is a complex one, owing to the physiologic action of the substances employed. In fifty cases studied by Mori the results were very variable, owing, it seemed, to the inconstant effects of cocain, epinephrin, and the patient's psychic state. ${ }^{1}$ George Müller, in Frazier's Clinic at the University of Pennsylvania, found that lumbar puncture caused a marked elevation of the blood-pressure not due to the pain, because it occurs even under anesthesia. In correct spinal anesthesia the complete block of peripheral impulses makes the pressure curve independent of the nature of the operation. If the anesthesia is allowed to extend upward it cuts off the pressor stimuli to the splanchnic area and may produce the effect of shock, or if it extends still farther upward, respiratory embarrassment or even paralysis may occur (see page 331). Smith ${ }^{2}$ states that blood-pressure can be as profoundly lowered by spinal anesthesia as by section of the cervical cord. The greatest fall occurs when injections are made in the thoracic region, at which point the splanchnic vasomotor fibers are given off. This part of the vasomotor mechanism is first affected when injections are made in the lumbar region. It appears that diffusion of the drug depends greatly upon the bulk of the injection-the more fluid injected, the greater the diffusion. The most pronounced effects on blood-pressure occur when epinephrin was used in conjunction with novocain. Efforts to raise the fallen pressure by the intravenous infusion of epinephrin and pituitrin result in only a transient rise.

Hemostasis by Belt Constriction (Momburg).-As a means of checking hemorrhage after severe trauma, in obstetrics, etc., with certainty, without an assistant and without the danger of local wound infection, Momburg has suggested winding a rubber tube tightly around the waist, one turn after another, until the femoral pulse disappears. This procedure, which causes a sudden rise of blood-pressure and which throws a corresponding burden upon the heart, should not be employed in case of cardiovascular disease.

${ }^{1}$ Mori, M., Das Verhalten des Blutdruckes bei Lumbalanästhesie, Deut. Ztschr. f. Chir., 1904, lxxiv, 173 .

2 Blood-pressure in Spinal Anesthesia, Boston Med. and Surg. Jour., 1915, cxxiii, 502 . 
The suddenness of the cardiac strain may be somewhat mitigated by preliminary constriction of the thighs. It is absolutely essential that the constriction be very gradually relieved so that vasomotor tone and the distribution of blood may be gradually reëstablished. It should never be applied in the case of elderly or obese women. Numerous accidents have occurred from defective technique. This procedure has not met with any practical acceptance, and is by many authorities condemned as a dangerous and unjustifiable procedure.

Abdominal Conditions.-Blood-pressure readings may be of diagnostic value in abdominal lesions. Thus unilateral suppurative renal disease causes a rise of pressure which falls after evacuation or extirpation. ${ }^{1}$ Readings are of value in the diagnosis in visceral rupture, ectopic pregnancy, gastric or intestinal perforation, etc., all of which conditions cause a sudden and often marked fall of pressure. As a rule the extent of the fall depends upon the patient, the size of the perforation, the coexistence of hemorrhage, the presence of localizing adhesions, and the portion of the peritoneum involved. The larger the perforation, the less it is walled off; the greater the associated hemorrhage and the higher its location, the more will arterial pressure fall. The pain which often precedes or is associated with perforation causes a preliminary rise of pressure. Pressure readings naturally have a much greater value if the patient's normal pressure is known. Hence the value of routine pressure records. It is impossible to give any rules based upon absolute figures, but a systolic pressure below $90 \mathrm{~mm}$. Hg. in association with other signs would point to hemorrhage or perforation even in toxic patients prostrated by typhoid fever (see page 206). Acute inflammatory lesions in the peritoneum, such as appendicitis in the early stages, may cause a slight increase in blood-pressure, whereas renal colic, plumbic cramps, and tabetic crisis generally show a marked rise in tension. The onset of peritonitis is accompanied by a distinct increase of pressure which gradually falls with increasing toxemia. The rise is due to local pressor, the fall to central depressor, impulses.

The custom now instituted in all up-to-date clinics of having the anesthetist or his assistant chart the pulse rate and blood-pressure during the course of anesthesia, with the addition of occasional notes dictated by the operator, is most commendable. It is not

\footnotetext{
'Kato and Kotzenberg, Ueber d. Verhalten d. arteriellen Blutdruekes bei chirurgischen Nierenerkrankungen u. Appendieitis, Beitr. z. klin. Chir., 1908, lviii, 404.
} 
only a signal assistance to the surgeon at the time of the operation, but it also leads to a more careful analysis of the effects of anesthesia and trauma, and furnishes permanent records for statistical study.

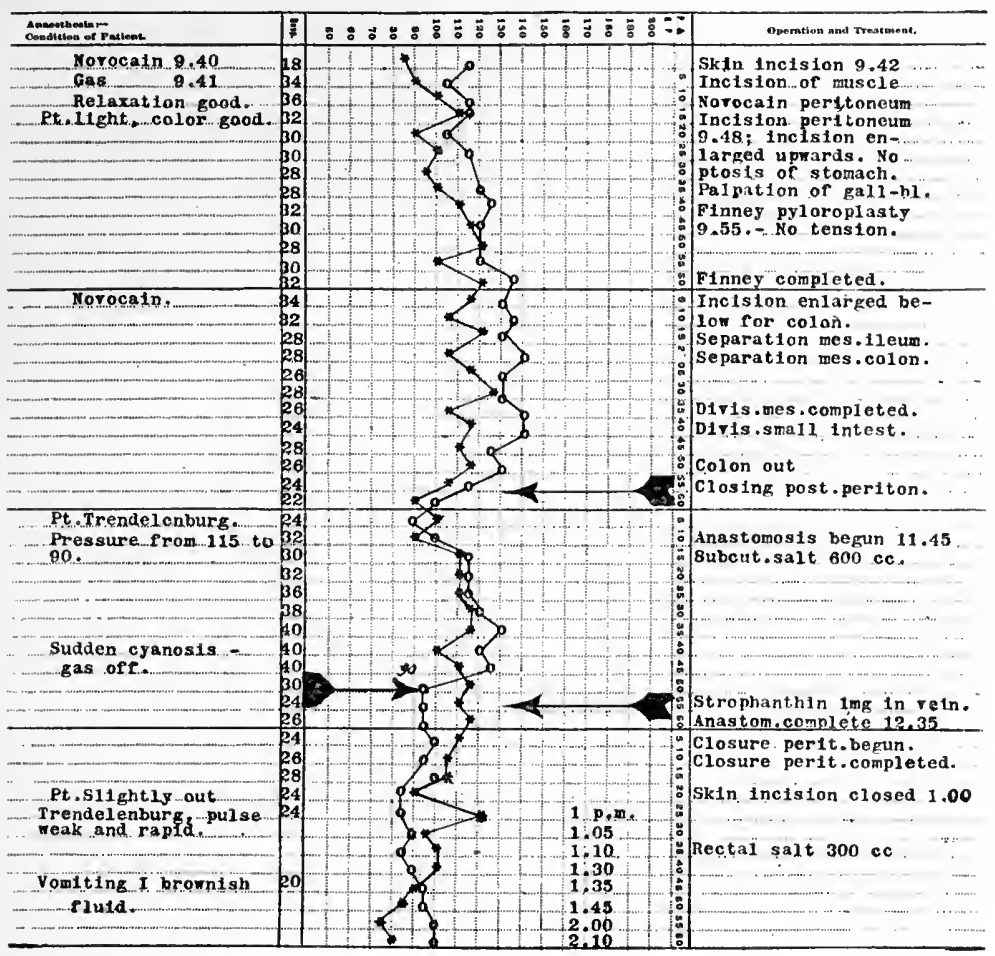

Frg. 97.-Chart from a case of gastromesenteric ileus, together with pyloric stenosis due to a healed ulcer, requiring a pyloroplasty in addition to resection of the right half of the colon. The operation lasted three hours and twenty minutes. No ether was used. A marked fall of pressure occurred while closing the peritoneum. This was remedied by temporarily placing the patient in the Trendelenburg position. Sudden cyanosis with a second fall of pressure occurred while the abdominal wound was being closed. The nitrous oxid was stopped and the operation finished under local anesthesia. Salt solution, strophanthin, and the inverted posture again restored the patient to a satisfactory condition. (After Bloodgood.)

Menstruation.-Blood-pressure frequently rises for a day or two preceding the onset of menstruation. There is a fall of bloodpressure during the period which occurs independently of the loss of blood, and which has been attributed to a slowing of the pulse and to psychic factors. ${ }^{1}$ It is lowest on the second day of the flow

1 Wiessner, M., Ueber das Verhalten des Blutdruckes während der Menstruation, etc., Leipsic, 1904. 
and is not much affected by the occurrence of pain at this time. ${ }^{1}$ Large doses of atropin ( $0.00075 \mathrm{gm}$. hypodermically) have sometimes been successfully used in the treatment of dysmenorrhea of the spasmodic type associated with cramp-like pain a day or two before the onset of flow, especially in vagotonic cases. Much stress has been laid by Stolper upon the importance of blood-pressure estimations in the proper selection of cases. He states that women with a normal or slightly increased pressure during the intermenstrual interval react most promptly to atropin, whereas those with a marked increase in blood-pressure show less response. Bogdanovics ${ }^{2}$ and others have reported a premenstrual rise of bloodpressure in normal women, followed by a gradual decline after the onset of flow. Based upon pulse rate, blood-pressure, temperature, and muscular strength tests, the statement has been made that a cyclic rhythm occurs in normal women which renders them subject to periodic intervals of inefficiency. ${ }^{3}$ The recent studies of King ${ }^{4}$ indicate that this tendency has been overemphasized and in so far as observations are concerned, made upon the systolic, diastolic and pulse pressures of eleven women, such irregular results were obtained that they do not support the wave theory.

The Menopause.-Circulatory abnormalities are of frequent occurrence during the menopause. Among these "flashes of heat;" formication, numbness, vertigo, cardiac palpitation, dyspnea on exertion, etc., may be encountered. A rise of arterial pressure is not uncommon after the cessation of menstruation. Whether it is merely a symptom or, as Potter ${ }^{5}$ suggests, the cause of the symptoms, is uncertain. If pressure changes are marked and prolonged the possibility of renal disease must be borne in mind.

Ovarian extirpation clinically often produces evidences of increased irritability of the sympathetic nervous system. This has been substantiated experimentally, since this operation in bitches results in from six to eight weeks in a markedly increased vasomotor reaction after one injection of nicotin. The reaction to epinephrin, however, is not increased. ${ }^{6}$

\footnotetext{
1 Tenji, T., Ueber d. Verhalten des Blutdruckes zw. d. Menstruellen u. nicht Menstruellen Ztschr., Arch. f. Gynäk., 1909, lxxxix, 517.

2 Zentralbl. f. Gynäk., 1910, xxxiv, 994.

${ }^{3} \mathrm{Jacobi}$, The Question of Rest for Women during Menstruation, Boylston Prize Essay, 1876.

'4 Concerning the Periodic Cardiovascular and Temperature Variations in Women, Am. Jour. Physiol., 1914, xxxiv, 203.

${ }^{5}$ Blood-pressure at the Climacteric, British Med. Jour., December 2, 1911, p. 1472.

${ }^{6}$ Hoskins and Wheelon, Ovarian Extirpation and Vasomotor Irritability, Am. Jour. Physiol., 1914, xxxv, 119.
} 
Extirpation of the testes is followed by a fall of blood-pressure, and the reaction to nicotin is constantly lowered. It would seem, therefore, that normally functionating testes bear a relationship to irritability of the sympathetic. ${ }^{1}$

Pregnancy.-Blood-pressure in a normal pregnancy rarely excceds $120 \mathrm{~mm}$. Hg., and readings of $140 \mathrm{~mm}$. or more should be regarded with suspicion and carefully watched for eclamptic manifestations. Even in the later months of gestation the average pressure ranges between 115 and $120 \mathrm{~mm}$.

What was previously described as the normal cardiac hypertrophy in pregnancy is really' only a change in the lateral percussion area, due to the more transverse position of the heart which the upward pressure upon the diaphragm entails (Stengel and Stanton). This transverse position tends to cause a kinking of the large vessels, which adds to the work expended by the heart, and in the case of a diseased myocardium this factor may become important. It also explains the occurrence of accidental pulmonary murmurs. In normal women under thirty years of age, according to J. C. Hirst, the systolic pressure during the first eight months of pregnancy will average 118 . In the last month it averages about 124 . A slight fall may follow the subsidence of the uterus.

In patients between thirty and forty years of age the pressure ranges between 126 and $128 \mathrm{~mm}$.

The normal relations between systolic and diastolic pressure should maintain. Cases showing a pulse pressure of or over $50 \mathrm{~mm}$. should be watched and are often benefited by digitalis. ${ }^{2}$

The normal blood-pressure during the second stage of labor, if taken between uterine contractions, ranges between 130 and 150 nmm. After delivery normal values are reëstablished. This fall is due to fatigue, cessation of excitement, and pain, as well as to vascular relaxation in the splanchnic domain. During the pains pressure estimations for obvious reasons cannot be made, but doubtless the pain as well as the abdominal compression eause much higher blood-pressure. Rupture of the membranes usually produces a marked but temporary fall in pressure. The birth of the child is again followed for a time by a fall of from 60 to 90 $\mathrm{mm}^{3} \quad$ These changes in blood-pressure are due to the sudden

1 Wheelon, H., Influence of Testes on Blood-pressure, Am. Jour. Physiol., 1914, xxxv.

2 Hirst, John C., Blood-pressure in Pregnancy, Penn. Med. Jour., May, 1915, p. 615.

${ }^{3}$ Heynemann, Th., Herz u. Zwerch fellstand währund Schwangerschaft, Ztsehr. f. Geburtsh. u. Gynäk., 1913, Ixxiv, 854. 
diminution of intra-abdominal tension, to which it takes some time for the rasomotor system to accommodate itself. Large hemorrhages tend to delay the return of pressure to the normal. A rising pressure during the latter part of pregnancy is the most constant symptom of gestational toxemia and is a frequent precursor of eclampsia (Hirst). ${ }^{1}$ It is very important to know what the woman's usual pressure has been, otherwise the existence of a moderately high pressure may be over- or undervalued. The hypertension increases with the severity of the attack. Many of Edgar's cases showed pressures of $200 \mathrm{~mm}$. or more. "A fall of blood-pressure with amelioration of the other symptoms is the most favorable prognostic sign, but with aggravation of other symptoms indicates impending death." Intermissions with lowered pressure are favorable omens. The other simptoms are generally an increased pulse rate, epigastric pain, headache, visual disturbances, edema, albuminuria. Cases with renal disease rarely pass through the period of gestation without some manifestations of toxemia. The absence of increased blood-pressure does not always exclude the possibility of eclampsia; the presence of high pressure between conrulsions does exclude epilepsy (Chirie).

In the early stages of toxemia, gastro-intestinal symptoms may be marked before blood-pressure changes are obvious, and fatal convulsions may not be attended by a pressure over $160 \mathrm{~mm}$. Hirst has reported a reading of $320 \mathrm{~mm}$. in eclampsia and $192 \mathrm{~mm}$. in a case of toxemia without eclampsia. He has seen recovery in two cases with pressures of 420 and $400 \mathrm{~mm}$. respectively, showing that the danger of death is not necessarily proportionate to the height of pressure. The average eclampsia pressure ranges between 190 and $200 \mathrm{~mm}$.

The administration of ergot does not appear to raise bloodpressure in the toxemia of pregnancy (see page 33.2 ), but the employment of pituitrin may cause a very marked rise (Hirst).

Summary.-During pregnancy a pressure of $125 \mathrm{~mm}$. is normal; a pressure between 125 and 150 is to be regarded with suspicion; while a pressure of over 150 if accompanied by other toxemic symptoms calls for prompt and energetic treatment; often the induction of premature labor (J. C. Hirst).

The Treatment of Eclampsia. - Some obstetricians of wide experience recommend the use of veratrum viride in the treatment

\footnotetext{
1 The Importance of Blood-pressure in the Toxemia of the Latter Half of Pregnancy. New York Med. Jour, 1910, xci, 1204.
} 
of eclampsia. Hirst states that "the most successful remedial measures are those which reduce blood-pressure most quickly and most effectually, such as puncture of the membranes, sweating, purgation, venesection, veratrum viride, and nitroglycerin," while Edgar writes: "I prefer ether, veratrum viride, glonoin, and chloral in the order named." Now clinical results are of primary importance, but in view of some definitely established facts it would appear that they are open to revision. We have seen under the discussion of nephritis that hypertension often appears to be Nature's defense against anuria and coma, and the most experienced clinicians no longer attempt to reduce blood-pressure by direct means except in cases of an emergency. Is eclampsia such an emergency? The reduction of pressure allays the convulsions, but is this due to the fact that the patient is too depressed to respond to stimuli? It has been established that collapse and shock are associated with and are in part the result of low bloodpressure. By arministering cardiovascular depressants are we not reducing our eclamptic patient to a condition of collapse? It has been shown that rapid emptying of the uterus in eclampsia frequently produces a fall of blood-pressure amounting to $100 \mathrm{~mm}$. $\mathrm{Hg}$., while a full dose of veratrum viride of itself may cause a depression of $145 \mathrm{~mm} .{ }^{1}$ It is unpleasant to contemplate the degree of blood-pressure fall which a combination of these two procedures might entail. Even if the eclamptic rise of pressure is not of a protective nature, and even if it should appear desirable to lower pressure by medicinal means, it would seem far preferable to do so by the administration of the nitrites-drugs which do not also depress the heart and the medullary centres. Chloroform is undesirable not only because of its effect upon the heart, but also on account of its tendency to produce necrosis of the hepatic cells. Eclampsia is more than a vascular crisis, and while much higher blood-pressure favors and doubtless precipitates visceral hemorrhages, such a rise of tension is only a symptom and not the basis of gestational intoxication. So far as the writer is aware, bloodpressure readings yield no clue as to the relative indication for immediate Cesarean section or conservative treatment.

IIyperemesis gravidarum is usually associated with hypotension, perhaps as a result of impoverished nutrition, ${ }^{2}$ and indicating that the toxin is different from that causing eclampsia.

\footnotetext{
${ }^{1}$ Bailey, A. C., Shock in Eclampsia, Am. Jour. Obstr., 1911, lxiv, No. 2.

2 Wallich, V., L'hypertension gravidique, Ann. d. Gynec. et d'Obstr., 1912, xxxix,
} 653. 
In extra-uterine pregnancy the occurrence of anemia with a fall of pressure is the most important indication of internal hemorrhage (Horner).

Attention has been called to the fact that shock associated with and probably due to low blood-pressure may, even in the absence of large hemorrhages, lead to sudden death during childbirth. Women with pressures not exceeding $90 \mathrm{~mm}$. may pass through labor normally, but such low readings, especially if associated with anemia, always indicate special care and the institution of such measures as tend to prevent shock. ${ }^{1}$ Poorly developed, neurasthenic women or women with contracted pelves often have low pressures.

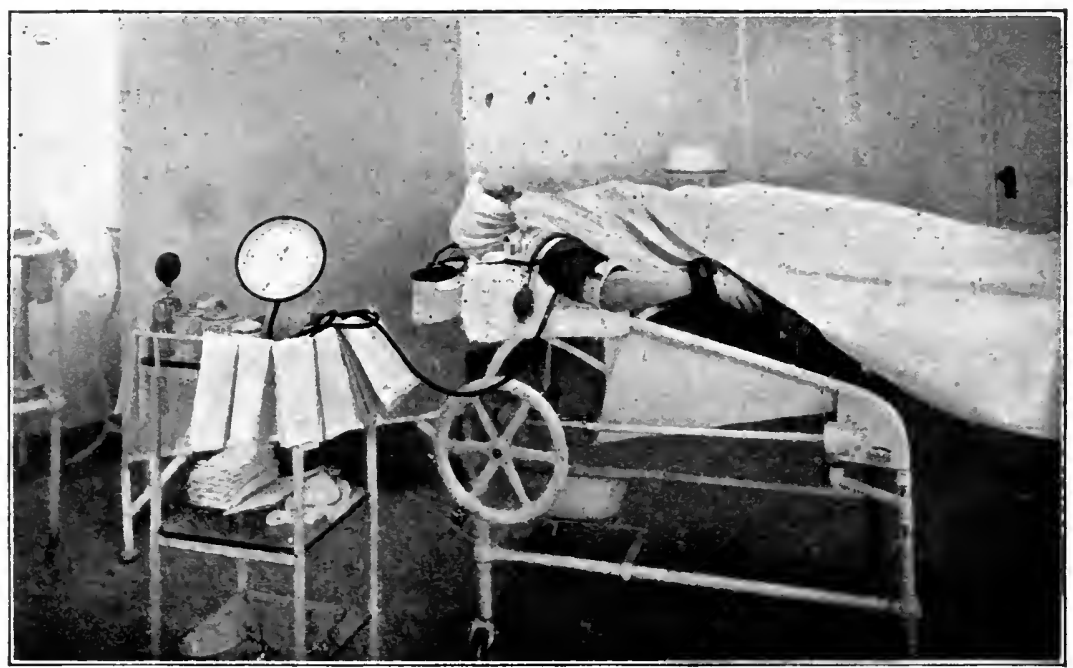

FiG. 95.-McKeson's sphygmomanometer for use during operations (Gwathmey.)

Blood-pressure Observations during Anesthesia.-With the intention of rendering blood-pressure readings much easier during anesthesia, Nicholson has fitted his new pocket sphygmomanometer with a Feddé indicator, in which a pith-ball plays up and down as long as pulsations of pressure are transmitted to it. This is attached to the left-hand side of the box and connected up with the manometer and two cuffs, one above and one below the elbow or knee-joints.

${ }^{1}$ Lynch, F. W., Blood-pressure during Pregnancy, Surg., Gynec. and Obstr., $1913, x v i i, 472$. 
An air pressure of about $80 \mathrm{~mm}$. may be maintained in the lower cuff throughout the operation, and the character, volume, and rate of the pulse noted continuously. 'The anesthetizer inflates the upper cuff with one hand whenever he desires to know the systolic pressure, simply noting the height of the mercury column when the pithball ceases oscillating. He then releases the air pressure in the upper cuff and continues the anesthesia. In perineal operations the cuffs are placed on the arm and forearm instead of the thigh and leg. 


\section{CHAP'TER XVIII.}

\section{OPHTHALMOLOGY.}

SINCE the ophthalmoscope is the only instrument which renders the naked arteries and veins visible during life it is not surprising that important data regarding blood-pressure or stasis, or vascular degeneration should be forthcoming from this source. The characteristic retinal lesions due to arteriosclerosis and arterial hypertension are not infrequently discovered by the ophthalmologist before other symptoms cause the patient to consult his physician. Ophthalmoscopic examinations are of value to the student of blood-pressure no less than are sphygmomanometric estimations to the ophthalmologist.

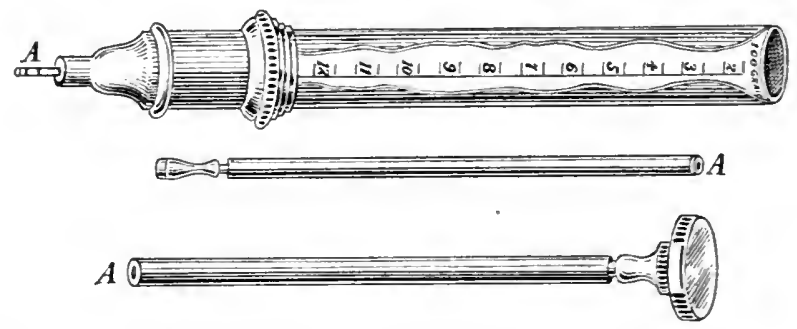

FIG. 99.-Rubino's modifieation of the Bloch-Verdin Sphygmometer.

Blood-pressure in the Retinal Arteries.-'The maximal pressure in healthy retinal arteries ranges between 80 and $112 \mathrm{~mm}$. $\mathrm{Hg}$. according to Rubino, who has modified the Bloch-Verdin apparatus for its measurement. The determination is based upon the principle that a certain pressure exerted upon the eyeball will arrest the circulation, this being indicated by the temporary loss of sight in the eve in question (Fig. 100).

The Relation of Blood-pressure to Ocular Tension.-For practical purposes the eye may be regarded as an inelastic capsule, having a tension greater than the atmosphere, which varies as the intraocular contents are increased or decreased. Such changes are brought about mainly by alterations in the blood-supply, the 
vitreous or the aqueous. "The circulatory conditions in the eye resemble those in the intracranial cavity, with the exception that the intra-ocular is much higher than the intracranial pressure, and is therefore not affected by changes in the general venous pressure" (Hill and Flack ${ }^{1}$ ). Intra-ocular tension generally varies

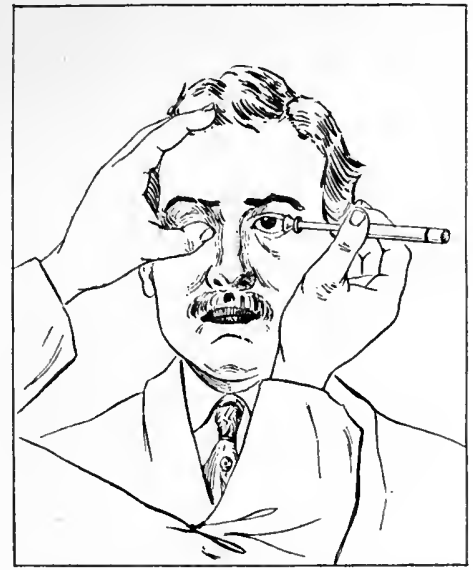

FIG. 100.-The Rubino instrument in use.

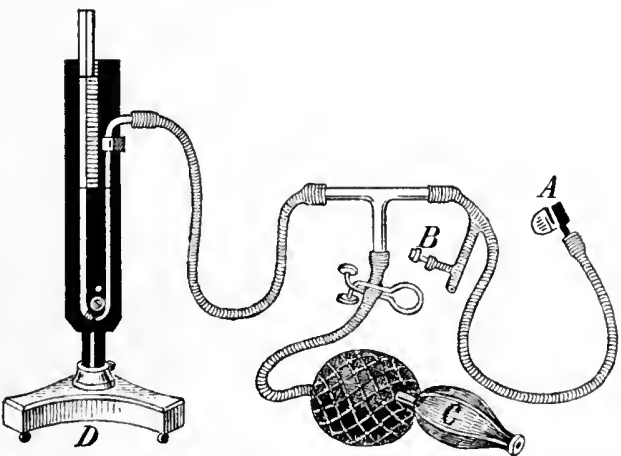

FIG. 101.-The Bajardi instrument for estimating retinal arterial pressure.2

with arterial pressure, but peripheral vasodilatation which lowers systemic pressure tends to increase intra-ocular pressure. Normally

1 I. The Relation between Capillary Pressure and Secretion. II. The Secretion of Aqueous and the Intra-ocular Pressure, Proc. Roy. Soc., Series 13, August 12 , $1912, \mathrm{xxxv}$.

${ }^{2}$ La pressione del sangue nell' artéria retinica e suoi rapporti eon la pressione nel cercolo del Willis, Riforma Med., 1911, xxvii, 1345. 
any change of tension is compensated by a reciprocal lymphatic flow. Excluding external causes (eyelids, ocular muscles, tumors of the eyeball or orbit), changes in tension mean loss of secretory compensation, which may come about "either by mechanically blocking the normal excretory channels or by so altering the normal ciliary secretion as to interfere with its ready interchange"- the "secretion" of the ciliary body is not truly a secretion in the physiologic sense of the term, but an osmotic process, the passage of fluid through a dialyzing membrane. There are no lymphatics in the eye, and the iris and vitreous have no secretory function." According to Starling and Henderson the difference between arterial and intra-ocular tension averages $84 \mathrm{~mm}$., and it is upon these pressure differences that the rate of secretion depends. The specific gravity of the secretion varies directly with blood-pressure and inversely with ocular tension. "Permanently increased tension is not due to high blood-pressure directly, but may coexist with it only in the absence of adequate compensation" (Ibershoff), (see page 43).

The foregoing statements are generally accepted. In opposition to them Henderson believes that intra-ocular and intracranial tension run closely parallel. The latter depends much more upon the general venous than upon the general arterial pressure. It varies directly and absolutely with the pressure in the vena cava, which makes itself felt at once by backward pressure on the cerebral venous capillaries. A rise of arterial pressure only produces a proportional rise in the intracranial pressure, because between the aorta and the cerebral veins lies the unknown and varying resistance of the arterioles.

Based upon experimental evidence, Henderson believes that what has just been stated regarding intracranial tension applies to intra-ocular pressure, except that response in the latter is less prompt owing to the ramifications of the ocular veins. 'Therefore "the intra-ocular pressure must be the same as the intra-ocular venous pressure." Since the corneoscleral envelope is an unyielding case with a fixed cubic capacity, analogous in every respect to the bony cranium, the intra-ocular pressure is not a question of volume of the intra-ocular contents but purely a question of pressure of a fixed volume, which is governed in turn by the intra-ocular and general venous pressure. The intra-ocular pressure therefore respresents "the pressure which remains in the eyeball after the force of the heart has been expended in driving the blood through 
the intra-ocular arterioles. On account of the elastic nature of the circulatory system of tubes a rise of arterial pressure can only produce an increase in intra-ocular pressure in proportion as the resistance in the arterioles is overcome and intravenous pressure. raised."1

The Estimation of Intra-ocular Tension.-The old method of gauging ocular tension, which for practical purposes may be considered synonymous with intra-ocular pressure, by means of the fingers, is for obvious reasons falling into disuse when anything

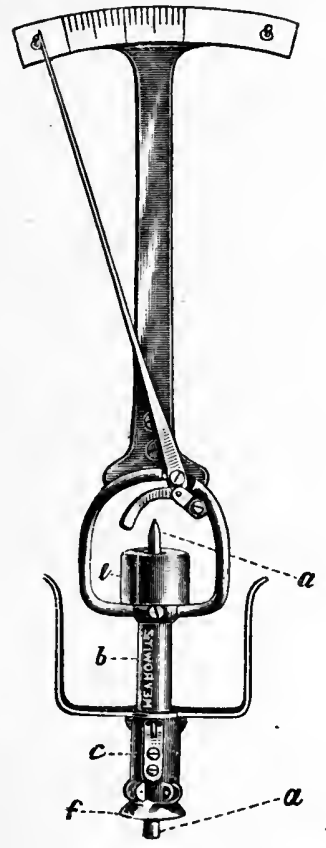

Frg. 102.-The Schiötz tonometer.

like accurate measurement is desired. The tactus eruditus cannot compete with instrumental precision. Several instruments are now available for the purpose. Among these the Schiötz tonometer has received wide-spread commendation (see Fig. 102).

Intra-ocular tension is rated with this instrument by measuring the depth of the depression produced in the anesthetized eornea by the weight of the shaft of the tonometer; the higher the tension, the less the depression. The data obtained are, however, based

${ }^{1}$ Henderson, 'T., Glaucoma, E. Arnold, London, 1910. 
upon (1) the elasticity of the sclera and cornea, (2) the intra-ocular tension, and (3) the condition of the ocular drainage system. ${ }^{1}$ Normal readings should not exceed $26 \mathrm{~mm}$. Hg.; the normal pressure is about $20 \mathrm{~mm}$.

As with other ocular tonometers, considerable practice is required before accurate readings can be obtained. Even with careful application considerable discomfort to the patient is entailed. No pressure should be made upon the eyeball except that exerted by the weights.

The Stephenson tonometer is applied over the closed upper lid. It is furnished with a handle for holding it in position on the patient's face and with a milled head for adjustment to varying depths. The end of the inner tube, which consists of a concave cup, contains a spiral spring actuated by a jointed lever. The instrument is adjusted to zero, the lever makes pressure upon the eye, and the moment at which indentation occurs is shown on the indicator. Since the tension of the orbicularis palpebrarum and the recession of the eyeball into the orbit are fairly constant in a given individual, temporal variations of pressure can be estimated. The technique with this instrument is easily acquired. No anesthetic is necessary and readings may be made in about one minute. Just what proportion of the pressure obtained is true intra-ocular tension and how much is pressure of the retrobulbar tissues is as yet not positively determined. ${ }^{2}$

Ocular Lesions due to Increased Arterial Pressure. - This subject cannot of course be discussed here in extenso. "In the later stages of intra-ocular vascular disease, hemorrhages frequently appear. If centrally placed, their very position interferes with vision, or they may invade the vitreous, may result in proliferating retinitis, may cause glaucoma, and, if extensive, detachment of the retina. Closely allied to vascular changes are also lenticular cataract, some varieties of optic atrophy, and some cases of retrobulbar neuritis" (de Schweinitz). According to William F. Norris, retinitis in some form occurs in not less than 25 per cent. of all cases of Bright's disease seen in general hospitals.

In a study of 104 cases of ocular disease, Peter $^{3}$ found bloodpressure as follows:

1 Schönberg, M. J., Experimental Study of Intra-ocular Pressure and Ocular Drainage, Jour. Am. Med. Assn., 1913, 1xi, 1098.

${ }^{2}$ Reber, W., A Clinieal Study of Ocular Tonometers, Pennsylvania Med. Jour., 1913 , xvii, 281 .

${ }^{3}$ Peter, L. C., Arterial Hypertension and its Relation to Morbid Changes in the Eye, Pennsylvania Med. Jour., 1911, xiv, 411. 
9 syphilitic neuroretinitis, average systolic blood-p essure $132 \mathrm{~mm}$.

3 chronic parenchymatous nephritis, average systolic blood-pressure 20 retinitis, average systolic blood-pressure.

59 neuroretinitis, average systolic blood-pressure

3 albuminuric retinitis, average systolic blood-pressure

6 hemorrhagic retinitis, average systolic blood-pressure

3 papillitis, average systolic blood-pressure .

Lenticular Cataract.-What has already been said regarding the effect of arterial hypertension in altering the osmotic balance of the ocular fluids may in a measure apply to lenticular cataract, if we assume that clouding of the lens is due to an abnormal dialysis between the anterior and the posterior chambers of this structure.

Glaucoma.-The general consensus of opinion appears to be that arterial hypertension bears only an indirect relation to glaucoma. This condition, which is predisposed to by a large lens and a small cornea, may be precipitated by a congestion or hemorrhage in the ciliary region which interferes with the compensating mechanism. "In the former the heightened blood-pressure causes increased secretion which in the presence of altered arterial walls is of a higher specific gravity than normal, so that the already impaired drainage is further embarrassed and compensation fails. In the case of hemorrhage, the lymph mixed with blood clogs the outflow channels and failure of excretion follows" (Ibershoff). If the foregoing facts are accepted, operative measures should not be attempted without first lowering arterial pressure, since the opening of the anterior chamber or of the sclera would be followed by "the rapid reformation of ocular fluids of a much higher specific gravity and osmotic coefficient." The danger of expulsive hemorrhage would also be diminished if systemic pressure were lowered. Craggs and Taylor, from several hundred comparisons, were unable to establish any relations between glaucoma and increased arterial tension. ${ }^{1}$ McRae's ${ }^{2}$ studues of 20 cases lead him to draw similar conclusions.

Löhlein, ${ }^{3}$ who studied some twenty cases of glaucoma by comparing the general blood-pressure, the intra-ocular pressure, and the adrenalin content of the blood, was unable to corroborate Kleczkowski's statement that a definite relationship existed. It is generally conceded, however, that no study of either acute or

${ }^{1}$ Craggs, H. C., and Taylor, C. G., A Researeh into the Relation between Systemic Blood-pressure and Raised Intra-ocular Tension, Ophthalmoseope, 1913, xi, 350 .

2 Ophthalmoscope, April, 1915 , p. 168.

${ }^{3}$ Ueber Blutuntersuchungen bei Glatcomkranken, Graefe's Arch. f. Ophthal., Ixxxiii, 547 . 
chronic glaucoma is complete without a record of the systemic arterial tension.

The belief that primary glaucoma is the product of retention of intra-ocular volume has been widely accepted. In opposition to this hypothesis, Thomas Henderson offers a different explanation. Every case of primary glaucoma depends upon (1) a constant predisposing sclerotic factor, and (2) on the variable vascular factor which is directly due to the general circulatory pressure. Since neither the eye nor the brain possesses any protective circulatory mechanism, "a rise in the vena cava pressure produces, millimeter for millimeter, a corresponding and equal rise in intra-ocular and intracranial pressures, while increase of pressure in the aorta causes a rise of pressure in the eye and brain whose exact amount depends on the varying resistance of the arterioles. Glaucoma therefore may be caused by a rise of arterial pressure, but is specially to be feared when there is a rise of the general venous pressure. This in. an eye with a sclerotic cribriform ligament, and therefore a diminished access of aqueous to the veins, precipitates the attack. ${ }^{1}$

Retinal Hemorrhages.-Retinal hemorrhages often show a more or less direct relation to increase of vascular tension, and ocular changes are most commonly noted in severe cases of nephritis at the time at which the pressure is highest. Thus hemorrhages into the retina are often precipitated by, and directly attributable to conditions which suddenly raise the intracranial arterial pressure, such as lifting, stooping, straining, etc.

The presence of retinal hemorrhages in a case of chronic arterial hypertension point almost indubitably to a severe nephritis. Retinal changes in nephritis have been reported as follows: Wagner in 6 per cent. of 157 cases, Frerichs in 15 per cent. of 41 cases, Lecorché in 22 per cent. of 286 cases, Eales in 28 per cent. of 100 cases of chronic kidney disease, Miles Miley in 31 per cent. of 164 cases of acute and chronic kidney disease, Galezowski in 31 per cent. of 154 cases.

The durution of life after the first retinal ehanges were noted: Bull reported 103 cases: 86 of the patients died, 57 within the first year, 18 in the second year, 6 in the third year, 4 in the fourth year, and 1 in the sixth year of observation, making 87 per cent. in the first two years. Miles Miley, in 164 patients with acute or chronic kidney disease, found 105 with healthy eyes and 51 with

${ }^{1}$ Henderson, Thomas, Glaueoma, London, 1910. 
retinitis albuminurica (the other 8 had affections of the eyes which are not pertinent to our subject); 27 per cent. of the former and 53 per cent. of the latter died. The mortality shown among those having retinitis albuminurica was twice as great as among patients with healthy eyes. Most of the patients lived about twelve months after the first indications of retinal changes, two lived nearly fourteen months, and one about eighteen months. In those cases in which the onset of the retinal disturbance could be definitely established the average duration of life did not exceed six months. ${ }^{1}$ (see page 292).

The existence of high systemic blood-pressure is of ophthalmologic interest in relation to postoperative hemorrhage after cataract extraction and in spontaneous subconjunctival hemorrhages, although the latter may for other reasons occur with normal or even low arterial tension. The higher the arterial tension, the less favorable is the case from an operative stand-point. In such cases vasodilators may be administered to reduce pressure during the operation. Morphin is also useful, since it helps to prevent the emotional disturbance with which a cataract extraction is of necessity associated, and which has such a potent pressor effect. ${ }^{2}$

Spasm of the retinal arteries of variable duration and of not infrequent recurrence is now a well-established ophthalmologic entity. The vascular spasm has been observed in its incipience and disappearance by means of the ophthalmoscope. There seems to be a growing conviction that a number of the cases which were previously attributed to embolism were really instances of spastic arterial contraction. The literature on the subject has been summarized by Zentmayer, ${ }^{3}$ who also reported a case in which he repeatedly observed a spasm of the central retinal artery. The condition is generally associated with arteriosclerosis and hypertension, and causes temporary blindness, usually of the hemianopic type (Peter) ${ }^{4}$ (see Vascular Crises, page 256).

Pulsation of the Retinal Vessels.-Blood-pressure in the ophthalmic artery of animals is only a few millimeters $\mathrm{Hg}$. below that of the carotid. While this relation may not hold good for man,

\footnotetext{
${ }^{1}$ Quoted by Posey, W. C., The Significance of Ocular Findings in Estimating Longevity, Jour. Am. Med. Assn., 1913, lx, 1867.

${ }^{2}$ Reber found a rise of pressure of $30 \mathrm{~mm}$. occasioned by the psychic stimulation incident to preparation for a cataract extraction.

${ }^{3}$ Some Unusual Ocular Manifestations of Arterioselerosis, Jour. Am. Med. Assn., 1906.

${ }^{4}$ Arterial Hypertension and its Relation to Morbid Changes in the Fye, Pennsylvania Med. Jour., 1911, xiv, 411.
} 
arterial pressure is well above the intra-ocular tension. Under normal conditions the vascular pulsation cannot be ophthalmoscopically demonstrated, probably because the pulsatile movement is damped by the intra-ocular tension, and because it is spread over the large area of the eyeball.

Under pathologic conditions, aortic insufficiency, aneurysm, exophthalmic goitre, etc., arterial pulsation can sometimes be seen either as (1) "a true pulse wave, accompanied by locomotion of the vessels, and (2) an intermittent flow of blood or pressure pulse. In the latter instance, the arteries fill with blood only with the heart beats, being empty between them; and pulsation is only visible upon the disk. This type of pulsation is a pure pressure phenomenon, and is caused by any considerable increase of intraocular tension with normal or lowered blood-pressure, as in glaucoma, or by any considerable diminution of blood-pressure with normal intra-ocular tension, as in syncope, orbital tumors, etc. The true arterial pulse occurs in cases of aortic regurgitation (Quincke) or aneurysm, in Graves's disease, etc.; it is not confined to the optic disk. It is equally a pressure phenomenon, but the differences of pressure are smaller."

"Capillary pulsation is seen only in aortic regurgitation as a systolic reddening and diastolic paling of the disk." Venous pulsation occurs in three forms: (1) the normal negative venous pulse; (2) the positive venous pulse (tricuspid insufficiency), and (3) the transmitted centripetal venous pulse (an exaggerated form of the normal type due to venous congestion). ${ }^{1}$

A careful ophthalmoscopic examination, in conjunction with blood-pressure studies, is therefore often of the greatest value. It should be made with a dilated pupil, as minor degrees of vascular changes are easily overlooked, but strong mydriatics, such as atropin, should not be used on account of the danger of precipitating an attack of glaucoma. De Schweinitz recommends euphthalmin (3 drops of a 5 to 10 per cent. solution) to be followed after the examination by the instillation of a few drops of a solution of pilocarpin, gr. $\mathrm{j}$ to the ounce.

Visual Accommodation.--Systemic blood-pressure also bears a relation to visual accommodation which is apt to be better late in the day when pressure is at its highest. Hypotensive individuals often have less accommodative power. The onset of presbyopia is 
often concomitant with the beginning of arterial hypertension (de Schweinitz). Attention has also been called by this eminent author to the fact that certain forms of asthenopia, especially those occurring in women in the late forties, owe their origin to angiosclerosis, and while rebellious to the usual methods of treatment, yield to dietetic and therapeutic measures which tend to lower arterial tension.

A large percentage of the early cases of increased blood-pressure are overlooked unless the sphygmomanometer is employed, and likewise many cases of incipient arteriosclerosis will not be detected if the ophthalmoscope is not called into requisition. This applies especially to those cases in which the arterial involvement is chiefly cerebral.

In 90 cases of general arteriosclerosis Rählmann ${ }^{1}$ found macroscopic lesions of the vessels of the retina in practically all. Wellmarked changes occurred in about 20 per cent.

The Eye-grounds in Arterial Hypertension.- "The eye-ground lesions of persistent high arterial tension, when this is a symptom of arteriosclerosis, may conveniently be divided into those which are suggestive and those which are pathognomonic. The suggestive signs include uneven caliber and undue tortuosity of the retinal arteries, increased distinctness of the central light streak, an unusually light color of the breadth of the artery, and alterations in the course and caliber of the veins."

The pathognomonic signs include changes in the size and breadth of the retinal arteries of such character that a beaded appearance is produced; distinct loss of translucency; decided lesions in the arterial walls, consisting of white stripes in the form of perivasculitis; alternate contractions and dilatations of the veins, and particularly -and this is the most important of the signs-indentation of the veins by the stiffened arteries in the same manner as a solid rod would indent a rubber tube when lying across it. Sometimes the vein is simply flattened slightly at the point of crossing, or merely pushed aside, or its caliber is contracted so that beyond the point of crossing there is an ampulliform dilatation. In addition to these clear signs there may be changes in the venous walls so that they are bordered with white stripes. The veins may be exceedingly tortuous and contain varicosities. Finally, there are edema of the retina in the form of gray opacity around the disk or following

\footnotetext{
1 Quoted by de Schweinitz, Intra-oeular Angiosclerosis and its Prognostic and Diagnostic Significance, Internat. Clinics, scries $17, \mathrm{i}, 177$.
} 
the course of the vessels; hemorrhages manifesting themselves as linear extravasations or roundish infiltrations, or sometimes assuming a drop-like form" (de Schweinitz). ${ }^{1}$

The Ocular Reflex. (Aschner).-If sufficient pressure is exerted upon the eyeball beneath the supra-orbital ridge, the eye being turned downward so as to avoid pressure upon the cornea, a reflex lowering of the pulse will normally occur. The reflex inhibition, as has been experimentally demonstrated, travels over the fifth to the tenth nerve. The reflex has been found exaggerated in epilepsy, and in exophthalmic goitre. It is not abolished by ether anesthesia. It is increased by the administration of pilocarpin and diminished by atropin. Considerable pressure is required to bring about the reflex, and this in turn entails a good deal of pain to the patient and perhaps danger to some eyes. It is not a procedure which can be readily or frequently applied, and should therefore be reserved for the study of exceptional cases. The pressure above mentioned will sometimes slow the pulse when direct pressure over the vagus in the neck fails to do so. It is interesting to remember that Robinson and Draper have sliown that the right vagus inhibits the heart more than does the left, and similarly pressure over the right eye seems to especially exert its effect upon the sinus region of the heart, whereas pressure over the left eye seems to exert more influence upon the conductive system (Levine).

The reflex is said to be positive when a slowing of the pulse occurs. Normally when it occurs the reflex is positive. The absence of pulse retardation or an actual increase in rate occurs in sympathicotonics. In vagotonics, on the other hand, marked slowing of the pulse occurs. Indeed, in them pressure maintenance for ten to twelve seconds may cause syncope from complete temporary asystole. These symptoms are due to an abnormally low nervous threshold in the vagus system, since they disappear under atropin. ${ }^{2}$ The claim that the reflex is of diagnostic value in differentiating cardiac weakness due to myocardial lesions from those due to nervous disturbances has not been substantiated. It may be of some value in differentiating between postfebrile bradycardia and auriculoventricular heart block. ${ }^{3}$ This reflex is generally absent in tabes dorsalis.

${ }^{1}$ Loc. cit.

2 Neugebauer, H., Beitr. z. Klinik d. Vagotonie, Wien. klin. Wchnschr., 1914, $\mathrm{xxvii,} 1023$.

${ }^{3}$ Gunson, E. B., The Oculocardiac Reflex, British Jour. Child. Dis., 1915, xii, No. 136, n. 97 . 


\section{IN D EX.}

A

Abdominal aorta, paroxysmal dilatation of, 259

Acromegaly, blood-pressure in, 352

Addison's disease, blood-pressure in, 349

Adiposity, blood-pressure in, 269, 354

Adrenal glands, physiology of, 33,280 , 330 in nephritis, 279

insufficiency, acute, 381 white line in, 263

Age, blood-pressure and, 53

Air, cold, effect on blood-pressure, 205

Albuminuria, blood-pressure and, 287 orthostatic, 191

Alcohol, blood-pressure effects of, 324

Alcoholism, blood-pressure in, 231

Alkalies, blood-pressure and, 325

Altitudes, blood-pressure and, 50, 35i

Ammonium, blood-pressure effect of, 325

Amyloid renal disease, 274

Anaphylaxis, 210

Anemia, blood-pressure in, 355

Aneroid sphygmomanometer, 108

Anesthesia, blood-pressure during, 400 chilling during blood-pressure and, 384

Anesthetics, blood-pressure and, 388 .

Aneurysm, aortic, blood-pressure in, 239

Angina abdominalis as vascular crisis, 259

pectoris as vascular crisis, $25 \%$

Angioneuroses, blood-flow in, 262

Angioneurotic edema, vascular crises and, 263

Aorta, abdominal, paroxysmal dilatation of, 259

Aortic aneurysm, blood-pressure in, 239 insufficiency, blood-pressure in arm in, 236

in leg in, 236

Durozier's sign of, 238

Traube's sign of, 238

lesions, blood-pressure in, 235

obstruction, blood-pressure in, $23 \mathrm{~S}$
Aortitis, syphilitic, blood-pressure in, $213,235,288$

Apoplexy, 261, 295, 300, 373

in vascular crises, 261

Arhythmia, extrasystolic, blood-pressure in, 242

Arm, blood-pressure in, in aortic insuffciency, 236

estimation of blood-flow in, 167

Arsenic poisoning, blood-pressure in, 228

Arterial blood-pressure, constitutionally low, 185

function, estimation of, 173

hypertension, 299

pressure, ocular lesions due to, 406

tonus, estimation of, 174

Vries-Reilingh's method, 174

Arteries in arteriosclerosis, 252

coronary, effect of epinephrin, 3.30

effects of alterations of blood-pressure on, 54

functional tests of, 255

physiology of, 28

retinal, blood-pressure in, 402 pulsation of, 409 spasm of, 409

in symptoms of arterial hypertension, 270

Arteriocapillary index in blood-pressure, 255

Arterioles, effects of alterations of blood-pressure on, 55

physiology of, 29

Arteriosclerosis, arteries in, 252

bilateral variations of pressure in, 253

blood-pressure in, 250

in tobacco poisoning, 228

ice reaction in, 2.55

nitrite test of, 254

stasis reaction in, 255

vascular reactions in, 252

Aspiration of pleura, blood-pressure effect of, 386

Asthma, bronchial, blood-pressure in, 249 
Athletics, blood-pressure and, 46, 149, Blood-pressure, alterations of, effects 305,365

Atropin, blood-pressure effect of, 325

Aural symptoms of arterial hypertension, 270

Auricular fibrillation, blood-pressure in, 243

Auto-intoxication, blood-pressure in, 350

\section{B}

BAJARDI's ocular sphygmomanometer, 403

Barach's formula of blood-pressure quotient, 161

Barometric pressure, 358

Bendick's sphygmomanometer, 82

Berberin, blood-pressure effect of, 333

Bichloride of mercury poisoning, 296

Biliary colic and vascular crises, 260

Bing's sphygmomanometer, 106

Birth, blood-pressure at, 361

Bishop's sphygmomanometer, 79

Bladder drainage, effect on blood-pressure, 388

Bleeding. See Hemorrhage, phlebotomy, $314,355,381$.

Blood and blood-pressure, 34

Blood-flow in angioneuroses, 262

blood-pressure and, 35

estimation of, 163

in arm, 167

Bornstein's method, 170

dynamic diagrams in, 179

Fellner's method, 170

Hewlett's method, 170

Stewart's method, 163

plethysmograph in, 166

tachograph in, 171

Van Zwaluwenburg's method, 170

von Kries's method, 171

in fevers, 197

renal function and, 281

systolic output, estimation of, by venous pressure, 164

Blood-pressure, absolute sphygmogram in, 156

in acromegaly, 352

in acute endocarditis, 234 nephritis, 296

in Addison's disease, 349

in adiposity, 269, 354

age and, 53

albuminuria and, 287

alcohol and, $32 t$

in alcoholism, 231

alkalies and, 325

alterations of, effects of, on arteries, 55

of, on arterioles, 55

on capillary pressure, 55

on heart, 54

on kidneys, 56

on organs of body, 54

on pulmonary pressure, 55 .

on venous pressure, 55

altitudes and, 50, 357

ammonium and, 325

in anemia, 355

anesthetics and, 400

in aortic aneurysm, 239

lesions, 235

obstruction, 238

in arm, in aortic insufficiency, 236

in arsenic poisoning, 228

arterial, constitutionally low, 185

arteriocapillary index in, 255

in arteriosclerosis, 250

in aspiration of pleura, 386

athletics and, 46, 149, 305, 365

atropin and, 325

in auricular fibrillation, 243

in auto-intoxication, 350

bandaging of extremities and, 315

and barometric pressure, 358

berberin and, 333

in bichloride of mereury poisoning, 296

at birth, 361

blood in, 34

blood-flow and, 35

in bradycardia, 242

bromides and, 330

in bronchial asthına, 249

in cachexia, 356

caffein and, 326

camphor and, 327

capillary, estimation of, 175

in carcinoma, 356

cardiac cycle in, 25

in cardiac disease, 233

in cerebral hemorrhages, 372

chemical regulation of, 33

in Cheyne-Stokes respiration, 290

in childhood, 53, 360

chilling during anesthesia and, 384

and climate, 359

chloral and, 330

after chlorin gas inhalation, 228

chloroform and, 389

in chlorosis, 356

in cholera, 199

cocain and, 390

in collapse, 210

counterirritation and, 319

cuff, 59

location of, 61

in delirium tremens, 231 
Blood-pressure in dementia precox, 371 in diabetes, 347

diastolic, 37, 130, 266

diet and, 305

digestion, effect of, 49

digitalis and, 327

in diphtheria, 199

in diseases of heart, 233 of myocardium, 241

of nervous system, 369

during anesthesia, 400

and dysmenorrhea, 396

in eclampsia, 398

edema and, 241

electricity and, 315

in encephalopathy, 227

treatment of, 228

in epilepsy, 371

epinephrin and, 330

ergot and, 332

erythrol tetranitrate and, 338

estimation of, 58

accuracy of, 130

auscultatory, 66

graphic method of, 83

instrumental, 58

oscillatory method of, 102

palpatory, 62

personal equation of examiner in, 127

possible accidents in, 129

precautions in, 125

sources of, error in, 122

subjective method of, 119

technique of, 125

value of, 130

visual method of, 102

ether and, 388

ethyl chlorid and, 389

exercise and $46,149,305,365$

in exogenous intoxications, 225

in exophthalmic goitre, 352

in extrasystolic arhythmia, 242

in extra-uterine pregnancy, 399

extremes, compatible with life, 131

factors maintaining, 24 regulating, 24

feeding and, 49

fluid intake and, 308

fresh air and, 205

in gastro-enteritis, 367

glandular extracts and, 351

in glaucoma, 407

gonads, effect of, 399

in gout, 348

hormonal and, 334

heart in, 24

in hemiplegia, 374

in hemorrhage, 355,381

in hemorrhages of brain, 372

hemic viscosity and, 297
Blood-pressure, hydrastinin and, 334

hydrastis and, 333

hydrotherapy and, 309

in hydrothorax, 222

in hyperemesis gravidarum, 399

hyperglycemia and, 285, 347

in infants, 360

in infectious diseases, 196 acute, 199

chronic, 213

treatment of, 209

instruments, choice of, 132 classification of, 133

different types of, 133

graphic registration, 87

intra-abdominal, 41 estimation of, 183

Moritz's method, 183

increased, 260

intracranial, 42

intra-ocular, 43

intrapericardial, 41

iodids and, 334

in jaundice, 349

kidneys and, 275, 286

in lead poisoning, 225

in leg, in aortic insufficiency, 236

in lenticular cataract, 407

life insurance and, 359

in locomotor ataxia, 374

in lumbar puncture, 391

lymph flow and, 34

mean, estimation of, 37

in malaria, 201

manipulations of pelvic viscera and, 386

of thoracic viscera and, 386

mannitol nitrate and, 338

massage and, 318

maximum, 22

measurement of, method of, 19

in meningitis, cerebrospinal, 202 tuberculous, 202

in menopause, 396

in menstruation, 395

in mental diseases, 370

in mercurial poisoning, 296

in metabolic diseases, 347

minimum, 22

mistletoe and, 336

in mitral insufficiency, 239

obstruction, 240

stenosis, 241

morphin and, 339

in morphinisin, 232

muscular exertion in, 46, 149, 305, 365

in myxedema, 354

Nauheim baths and, 309

neosalvarsan, 342

in nephritis, 272 
Blood-pressure in neurasthenia, 369 in neuroses, 369 nitrites and, 336 nitrogen retention and, 285 nitroglycerin and, 336, 337 nitrous oxid and, 389 normal, 53, 361 rule for estimating, 360 in obstetrics, 376 ocular tension and, 402 in old age, 252

in ophthalmology, 402 opium and, 339 orthostatic albuminuria and, 191 pancreas extract and, 279 in paracentesis abdominalis, 387 in paratyphoid fever, 206 in paresis, 375 para-oxyphenylethylamin and, 333 in paroxysmal dyspnea, 288 passive change of posture in, 149 pediatrics and, 360

in pericardial effusions, 248 phlebotomy and, 314 in phosphorus poisoning, 228 physical efficiency and, 362 pituitary extract and, 340 in pleural effusions, 222 in pneumonia, 202

in pneumothorax, 223

in polycythemia, 297

postural response in, 146.

posture and, 48 patient anfd, 384

in pregnancy, 396

at puberty, 361

in pulmonary edema, 291 hemorrhage, 219 pulse, 37

in pulsus alternans, 243

purgation and, 302

quotient, 158

Barach's formula, 161

Erlanger and Hooker's formula, 160

Fuerst and Soetbeer's formula, 160

Strassburger's formula, 159

von Recklinghausen's formula, 161

radio-active substances in, 321

renal decapsulation and, 323

respiration and, 38

in retinal arteries, 402

hemorrhages, 408

salvarsan and, 341

in scarlet fever, 205

secretion and, 46 of urine and, 286

in shock, 210

treatment of, 211
Blood-pressure, significance of, 132 sleep and, 50, 303

in smallpox, 206

sodium chloride metabolism and, 285

nitrite and, 338

in spinal anesthesia, 392

in status lymphaticus, 355

strophanthus and, 341

strychnin and, 342

in surgery, 376

in surgical hemorrhage, 381 shock, 378

in syphilis, 213

in syphilitic aortitis, 288

myocarditis, 242

in syringomyelia, 375

systolic, 37 .

in tachycardia, 242 paroxysmal, 242

testes and, 399

throughout vascular tree, 23

thyroid extract and, 343

tissue extracts and, 351

in tobacco poisoning, 228 arteriosclerosis and, 230

tropical climates and, 359

in tuberculosis, 213

in tumors of brain, 372

in typhoid fever, 206

in uremia, 287

urethane and, 343

vasotonin and, 343

venous, 23,27

in shock, 380

visceral manipulations and, 385

visual accommodation and, 410

yohimbin and, 344

Bloodvessels, resistance of, estimation of, 173

Bornstein's method of estimation of blood-flow, 170

Bouloumie's sphygmomanometer, 119

Bradycardia, blood-pressure in, 242

Brain, hemorrhages of, blood-pressure in, 372

tumors of, blood-pressure in, 372

Bromides, blood-pressure and, 330

Bronchial asthma, blood-pressure in, 249

Brugsch's sphygmomanometer, 97

Bussenius's sphygmomanometer, 102 .

\section{C}

Cachexia, blood-pressure in, 356

Caffein, blood-pressure and, 326

Camphor, blood-pressure and, 327

Capillaries, physiology of, 29 
Capillary blood-pressure, estimation of, 1 . 140,175

pressure, effects of alterations of arterial pressure on, 55

Carcinoma, blood-pressure in, 356

Cardiac action, venous pressure and, 143 cycle and blood-pressure, 25

disease, blood-pressure in, 233

load, 267

rate, 27

tone, 27

Cardiovascular disease, arterial hypertension in, 266

symptoms in arterial hypertension, 270

Cataract, lenticular, blood-pressure in, 407

Cerebral hemorrhages, blood-pressure in, 372

vascular crises, 260

Ccrebrospinal meningitis, blood-pressure in, 202

pressure in blood-pressure, 372, 392

Chemical regulation of blood-pressure, 33

Cheyne-Stokes respiration, blood-pressure in, 290

Chilblains, vascular crises and, 262

Childhood, blood-pressure in, 360

Chloral, blood-pressure effect of, 330

Chlorin gas poisoning, 228

Chloroform, blood-pressure effect of, 389

in pulmonary hemorrhage, 219

Chlorosis, 356

Cholera, blood-pressure in, 199

Christen's energometer, 179

Circulation, functional efficiency of, 145 amplitude frequeney product of, 161

Crampton's table of, 146 energy index in, 161 Graupner's test of, 151

Katzenstein's test of, 153

Claudication, intermittent, in vascular crises, 261

Climate, 359

Cocain, blood-pressure effect of, 390

Colic, biliary, in vascular crises, 260 renal, in vascular crises, 260

Collapse, blood-pressure in, 210

Conduction of pulse waves, 124

Coronary arteries, effect of drugs on, 344

Corpulence, 269, 354

Cramps in the legs, treatment of, 264

Crampton's table of functional effieiency of circulation, 146

Critical venous pressure, 26

Cuff, blood-pressure, 59

Decompensation and venous bloodpressure, 329

Delirium tremens, blood-pressure in, 231

Dementia precox, blood-pressure in, 371

Depressor nerve, physiology of, 31

Dermographism, vascular crises and, 262

Diabetes, blood-pressure in, 347

Diastole, time relations of, 25

Diastolic pressure, definition of, 17 estimation of, 64, 121

by palpation, 65

significance of, 130

by visualization, 65

Diet, blood-pressure and, 305 in hypertension, 305

Digitalis, blood-pressure effect of, 327

in failing compensation, 328

in high-pressure stasis, 328

in pulmonary hemorrhage, 220

Dilatation, paroxysmal, of abdominal aorta, 259

Diphtheria, blood-pressure in, 199

Dysmenorrhea and blood-pressure, 396

Dyspnea, paroxysmal, blood-pressure in, 288

Drugs, effects of, on blood-pressure, 324

on coronary arteries, 344

on vasomotor system, 211

on venous pressure, 345

Durozier's sign of aortic insufficiency, 238

Dynamic diagrams in estimation of blood-flow, 179

\section{$\mathbf{E}$}

Eclampsia, blood-pressure iil, 398

Edema, angioneurotic, vascular crises and, 263

blood-pressure and, 241

pulmonary, blood-pressure in, 291

sodium chloride and, 285

Ehret's phenomenon, 65

Electricity, blood-pressure and, 315

Encephalopathy, blood-pressure in, 227

End-pressure, definition of, 18

Endocarditis, acute, blood-pressure in, 234

Energometer, Christen's, 179

Energy index in functional efficiency of circulation, 161

Epilepsy, blood-pressure in, 371

Epinephrin, blood-pressure and, 33, 330

Ergometer, Zuntz's, 151

Ergostat, Gaertner's, 151 
Ergot, blood-pressure and, 332 in pulmonary hemorrhage, 220

Erlanger and Hooker's formula blood-pressure quotient, 160

Erlanger's sphygmomanometer, 91

Erythrol tetranitrate, blood-pressure and, 338

Ether, blood-pressure effect of, 388

Ethyl chlorid, blood-pressure effect of, 389

Exercise, blood-pressure and, 46, 149, 305,365

Exogenous intoxications, blood-pressure in, 225

Exophthalmic goitre, blood-pressure in, 352

Extrasystolic arhythmia, blood-pressure in, 242

Extra-uterine pregnancy, blood-pressure in, 399

Eye-grounds in arterial hypertension, 411

\section{F}

FAsTing, effect on blood-pressure, 306

Faught's sphygmomanometer, 73

Feddé's oscillometer, 105

Feeding, blood-pressure and, 49

Fellner's method of estimation of blood-flow, 170

Fevers, blood-flow in, 197

Fibrillation, auricular, blood-pressure in, 243

Finger plethysmograph, Fleischer's, 168 Fleischer's finger plethysmograph, 168 sphygmomanometer, 101

Fluid intake, blood-pressure and, 308

Francois Frank's sphygmomanometer, 118

Frank and Reh's method of estimation of venous pressure, 137

Frey's method of estimation of venous pressure, 136

Fuerst and Soetbeer's formula of bloodpressure quotient, 160

Function, arterial, estimation of, 173

Functional capacity of heart, 145 efficiency of circulation, 145 hypotension, 233

tests of arteries, 255

\section{G}

Gaer'Tner's ergostat, 151

method of estimation of venous pressure, 135

phenomenon, 135

sphygmomanometer, 76

Gastro-enteritis, blood-pressure in, 367
Gastro-intestinal symptoms of arterial hypertension, 270

Gibson's sphygmomanometer, 88

Glandular extracts, blood-pressure and, 351

Glaucoma, blood-pressure in, 407

Gonads, effect on blood-pressure, 34, 399

Gout, blood-pressure in, 348

Graphic method of estimation of bloodpressure, 83

Graupner's test of functional efficiency of circulation, 151

\section{H}

Heart and blood-pressure, 24

diseases of, blood-pressure in, 233

effects of alterations of blood-pressure on, 55

functional capacity of, 145

symptoms of arterial hypertension, 271

work done by, 299

Hemic viscosity, blood-pressure and, 297

Hemiplegia, blood-pressure in, 374

Hemoglobinuria, vasomotor, 192

Hemorrhage, blood-pressure in, 355, 381

Hemorrhages of brain, blood-pressure in, 372

pulmonary, blood-pressure in, 219

retinal, blood-pressure in, 408

surgical, blood-pressure in, 381

Hertz's sphygmomanometer, 81

Hewlett's method of estimation of blood-flow, 170

Hill's sphygmomanometer, 72

Hooker and Eyster's method of estimation of venous pressure, 137

Hormonal, blood-pressure and, 334

Howell's method of estimation of venous pressure, 137

Hürthle's manometer, 20

Hydrastinin, blood-pressure effects of, 334

Hydrastis, blood-pressure effects of, 333

Hydrotherapy, blood-pressure and, 309

Hydrothorax, blood-pressure in, 222

Hyperemesis gravidarum, blood-pressure in, 399

Hyperglycemia and hypertension, 285, 347

Hyperpiesis, 273

Hypertension, arterial, 299

bandaging of extremities in, 315

in cardiovascular disease, 299 climate in, 320 
Hypertension, arterial, complications of, 287

conservation of energy in, 308

counterirritation in, 319

diet in, 305

electricity in, 315

exercise in, 305

eye-grounds in, 411

fluid intake in, 308

hemic viscosity and, 297

hydrotherapy in, 309

massage in, 318

Nauheim baths in, 309

phlebotomy in, 314

prognosis of, 291

radio-active substances in, 321

respiratory gymnastics in, 320

retinal hemorrhages in, 408

specific treatment in, 213

surgical treatment in, 323

symptoms of, 270

arteries in, 271

aural, 270

cardiovascular, 270

gastro-intestinal, 270

heart in, 271

nervous, 270

ocular, 270

renal, 270

signs in, 271

treatment of, 299 psychic, 320

hyperglycemia and, 285,347

in nephritis, 272

etiology of, 274

symptoms of, 274

nitrogen retention and, 285

renal circulation and, 281

function and, 281

secretion of urine in, 282

viscosity and, 297

Hypnosis, 150

Hypotension, 185

causes of, 185

etiology of, 188

extreme, prolonged, 356

functional, 233

in lumbago, 193

mechanical, 233

in myalgia, 193

in neuritis, 193

in phosphaturia, 193

in rheumatoid arthritis, 193

treatinent of, 193

in sciatica, 193

in status lymphaticus, 193

symptoms of, 185

terminal, 233

treatment of infectious disease, 209
I

INFECTIOUs diseases, blood-pressure in, 196

venous pressure in, 198

Insufficiency, mitral, blood-pressure in, 239

Intoxications, exogenous, blood-pressure in, 225

Intra-abdominal pressure, 41 estimation of, 183 increased, 260

Intracranial blood-pressure, 42

Intra-ocular blood-pressure, 43

pressure, venous pressure and, 404

tension, estimation of, 405

Intrapericardial blood-pressure, 41, 248

Intravenous injection, venous pressure and, 144

Iodids, blood-pressure and, 334

\section{J}

JACGUET's sphygmotonograph, 87

Janeway's sphygmomanometer, 72

Jaundice, blood-pressure in, 349

\section{$\mathbf{K}$}

Katzenstein's test of functional efficiency of circulation, $\mathbf{1 5 3}$

Kidney, decapsulation and blood-pressure, 323

Kidneys, amyloid disease of, 274, 276

blood-pressure and, 275, 286

effects of alteration of blood-pressure on, 56, 282, 286

nephritis, 274,276

tuberculosis of, 274

I

LATERAL pressure, definition of, 18

Lead poisoning, blood-pressure in, 225

Leg, blood-pressure in, in aortic insufficiency, 236

Lenticular cataract, blood-pressure in, 407

Life insurance, blood-pressure and, 359

Locomotor ataxia, blood-pressure in, 374

Lumbago, hypotension in, 193

Lumbar puncture, blood-pressure in, 391

Lymph circulation and in blood-pressure, 34 
M

MALARIA, blood-pressure in, 201

Malingering, detection of, 369

Mannitol nitrate, blood-pressure effect of, 338

Manometer, Hürthle's, 20

maximum, 21

mercury, 19

minimum, 21

(see sphygmomanometer)

Manometers, spring, in estimation of venous pressure, 135

Massage, blood-pressure and, 318

Mean pressure, definition of, 17 rule for estimating, 37

Mechanical hypotension, 233

Meningitis, cerebrospinal, blood-pressure in, 202

tuberculous, blood-pressure in, 202

Menopause, blood-pressure in, 396

Menstruation, blood-pressure during, 395

Mental diseases, blood-pressure in, 370

work, physiology of, 31

Mercer's sphygmomanometer, 72

Mercurial poisoning, blood-pressure in, 296

Mercury manometer, 19

Metabolic diseases, blood-pressure in, 347

Metabolism, sodium chloride, bloodpressure and, 285

Mistletoe, blood-pressure and, 336

Mitral insufficiency, blood-pressure in, 239

obstruction, blood-pressure in, 240 stenosis, blood-pressure in, 241

Momburg's belt constriction, 393

Moritz and Tabora, intravenous ncedle of, in estimation of venous pressure, 138

Moritz's method of estimating intraabdominal pressure, 183

Morphin, blood-pressure and, 339

Morphinism, blood-pressure in, 232

Muenzer's sphygmomanometer, 99

Muscular exertion in blood-pressure, $46,149,305,365$

Myalgia, hypotension in, 193

Myocarditis, syphilitic, 242

Myocardium, blood-pressure in diseases of, 241

Myxedema, blood-pressure in, 354

\section{N}

Naunem baths, blood-pressure effects of, 309
Neosalvarsan, effect on blood-pressure, 342

Nephritis, acute, blood-pressure in, 296

chronic, blood-pressure in, 272

hypertension in, 272

Nerve, depressor, physiology of, 31

Nervous symptoms of arterial hypertension, 270

system, diseases of, blood-pressure in, 369

Neurasthenia, blood-pressure in, 369

Neuritis, hypotension in, 193

Neuroses, blood-pressure in, 369

Nicholson's sphygmomanometer, 71

Nitrite test of arteriosclerosis, 254

Nitrites, blood-pressure and, 336

in puimonary hemorrhage, 220

Nitrogen retention and blood-pressure, 285,286

Nitroglycerin, blood-pressure effects of, 336,337

Nitrous oxid, blood-pressure and, 389

Normal arterial blood-pressure, $\overline{5} 3,361$ venous blood-pressure, 141

OBstetrics, blood-pressure in, 376

Obstruction, aortic, blood-pressure in, 238

mitral, blood-pressure in, 240

Ocular sphygmomanometer, Bajardi's, 403

Rubino's, 403

symptoms of arterial hypertension, 270

Oliver's sphygmomanometer, 79

Oplithalmology, blood-pressure in, 402

Opium, blood-pressure and, 339

Orthostatic albuminuria, 191

blood-pressure and, 191

Oscillations of first order, 17

of second order, 17

of third order, 18

Oscillatory method of estimation of blood-pressure, 102

Oscillometer, Feddé's, 105

Ovaries and vasomotor reactions, 396

\section{$\mathbf{P}$}

PAChon's sphygmomanometer, $10 \mathrm{~S}$

Palpatory estimation of blood-pressure, 62

Pal's sphygmoscope, 107

Pancreas extract, effect on blood-pressure, 279 
Paracentesis abdominalis, blood-pressure effects of, 387

Paratyphoid fever, blood-pressure in, 206

Paresis, blood-pressure in, 375

Para-oxyphenylethylamin, blood-pressure and, 333

Paroxysmal dilatation of abdominal aorta, 259

dyspnea, blood-pressure in, 288

tachycardia, blood-pressure in, 242

Pediatrics, blood-pressure and, 360

Pelvic viscera, manipulations of, and blood-pressure, 386

Periarteritis nodosa, 354

Pericardial effusions, blood-pressure in, 41,248

Phenomenon, Gaertner's, 135

Phlebotomy, blood-pressure and, 219, 314,376

Phosphaturia, hypotension in, 193

Phosphorous poisoning, blood-pressure in, 228

Physical efficiency and blood-pressure, 362

Physiology of arteries, 28

of arterioles, 29

of capillaries, 29

of depressor nerve, 31

of mental work, 31

of vasoconstrictor system, 29

of vasodilator nerves, 32 system, 32

of vasomotor system, 29

of veins, 29

Pilocarpin, 340

Pituitary extract, blood-pressure and, 340

Pituitrin in pulmonary hemorrhage, 220

Plethysmograph in estimation of bloodflow, 166

Fleischer's finger, 168

Pleura, aspiration of, effect on bloodpressure, 386

Pleural effusions, blood-pressure in, 222

Pneumonia, blood-pressure in, 202

Pneumothorax, blood-pressure in, 223

Poisoning, arsenic, blood-pressure in, 228

lead, blood-pressure in, 225

mercurial, blood-pressure in, 296

phosphorus, blood-pressure in, 228 tobacco, blood-pressure in, 288

Polycythemia, blood-pressure in, 297

Postural response in blood-pressure, 146

Posture, blood-pressure and, 48

Potain's sphygmomanometer, 118
Pregnancy, blood-pressure and viscosity, 298

extra-uterine, blood-pressure in, 399

normal, blood-pressure in, 396

Pressure, diastolic, definition of, 17

lateral, definition of, 18

mean, definition of, 17 estimation of, 37

normal, 53, 361

pulse, definition of, 17

systolic, definition of, 17

venous, 135

Puberty and blood-pressure, 361

Pulmonary edema, blood-pressure in, 291

hemorrhage, blood-pressure in, 219 chloroform in, 220

digitalis in, 220

ergot in, 220

nitrites in, 220

pituitrin in, 220

treatment of, 219

pressure, effects of alterations of blood-pressure on, 55

Pulse, blood-pressure and, 37, 246

deficit, 243

pressure, definition of, 17

urinary secretion and, 282, 286

range of, 38

significance of, 132

waves, conduction of, 124

Pulsus alternans, blood-pressure in, 243 paradoxus, 189, 249

Purgation, blood-pressure and, 302

\section{$\mathbf{R}$}

RADIO-ACTIVE substances effect on blood-pressure, 321

Reflex, ocular, 412

Renal circulation, hypertension and, 281

colic in vascular crises, 260

function, blood-flow and, 281

hypertension and, 281

symptoms in arterial liypertension, 270

Respiration, blood-pressure and, 38

Cheyne-Stokes, blood-pressure in, 290

venous pressure and, 143

Retinal arteries, blood-pressure in, 402 pulsation of, 409

spasin of, 409

hemorrhages, blood-pressure in, 408

Rheumatoid artliritis, hypotension in, 193 
Riva-Rocci type of sphygmomanometer, 62

Rubino's ocular sphygmomanometer, 403

\section{$\mathbf{S}$}

SaHLI's sphygmobolometer, 178

Salvarsan, blood-pressure and, 341

Scarlet fever, blood-pressure in, 205

Schiötz's tonometer, 405

Schott's test by venous pressure, 155

Sciatica, hypotension in, 193

Scurvy, 355

Secretion, blood-pressure and, 46

Shock, blood-pressure in, 210, 378 medical or toxic, 210

Significance of blood-pressure changes, 130

Silbermann's sphygmomanometer, 96

Singer's sphygmomanometer, 91

Sleep, blood-pressure and, 50, 303

Simallpox, blood-pressure in, 206

Sodium chloride, edema and, 286 metabolism, blood-pressure and, 285

nitrite, blood-pressure and, 338

Spasm of retinal arteries, 409

Sphygmobolometer, 175

Sphygmogram, absolute, in blood-pressure, 156

Sphygmomanometer, aneroid, 108 standardization of, 115

classification of, 133

compressed-air, 79

Bendick's, 82

Bing's, 106

Bishop's, 79

Bouloumie's, 119

Brugsch's, 97

Bussenius's, 102

Erlanger's, 91

Faught's, 73

Fleischer's, 101

Francois Frank's, 118

Gaertner's, 76

Gibson's, 88

Hertz's, 81

Hill's, 72

Janeway's, 72

McKeson's, 400

Mercer's, 72

Muenzer's, 99

Nicholson's, 71

ocular, Bajardi's, 403 Rubino's, 403

Oliver's, 79

Pachon's, 108

Potain's, 118

Riva-Rocci type of, 62

Silbermann's, 96
Sphygmomanometer, Singer's, 91

Stanton's, 63

Tycos, 112

Uskoff's, 93

Van Westenrijk's, 79

Vaquez's, 108

von Recklinghausen's, 115

Widmer's, 106

Wybauw's, 102

Sphygmomanometers, comparative values of, 120

Sphygmoscope, Pal's, 107

Sphygmotonograph, Jacquet's, 87

Spinal anesthesia, blood-pressure in, 392

Standardization of aneroid sphygmomanometer, 115

Stanton's sphygmomanometer, 63

Stasis, high pressure, 234, 268, 314, 328

digitalis in, 328

Status lymphaticus, blood-pressure in, 355

hypotension in, 193

Stephenson's tonometer, 406

Stewart's method of estimation of blood-flow, 163

Strassburger's formula of blood-pressure quotient, 159

Strophanthus, blood-pressure effect of, 341

Strychnin, blood-pressure effect of, 342

Subjective method of estimation of blood-pressure, 119

Suckling, effect of, on blood-pressure, 361

Suprarenal glands, physiology, 33, 280, 330

Surgery, blood-pressure in, 376

Surgical hemorrhage, blood-pressure in, 381

shock, blood-pressure in, 378 treatment of, 382

Syphilis, blood-pressure in, 213

Syphilitic aortitis, blood-pressure in, 235, 288

Syringomyelia, blood-pressure in, 375

Systole, time relations of, 25

Systolic blood-pressure, 37

output, 26,130

pressure, definition of, 17 estimation of, 62

\section{T}

TACHe CÉrÉbrale, 263

Tachograph in estimation of bloodflow, 171

von Kries's, 172

Tachycardia, blood-pressure in, 242

Terminal hypotension, 233 
Testes, effect of, on blood-pressure, 399

Thoracentesis, effect of, on blood-pressure, 386

Thoracic viscera, manipulations of, and blood-pressure, 386

Thyroid extract, blood-pressure and, 343

Tissue extracts, blood-pressure and, 351

Tobacco poisoning, blood-pressure in, 228

Tonometer, Schiötz's, 405

Stephenson's, 406

Tonus, arterial, estimation of, 174

Toxemia, alimentary, 350

Training, effect of, on blood-pressure, 367

Traube-Herring waves, 41

Traube's sign in aortic insufficiency, 238

Tropical climates, blood-pressure and, 359

Tuberculosis, blood-pressure in, 213 of kidney, 274

Tuberculous meningitis, blood-pressure in, 202

Tumors of brain, blood-pressure in, 372

Tycos sphygmomanometer, 112

Tympanites in vascular crises, 260

Typhoid fever, blood-pressure in, 206

Tyramin, blood-pressure effect of, 194

\section{U}

URANIUM nephritis, blood-pressure in, 285

Uremia, blood-pressure in, 287

lumbar puncture in, 302

venesection in, 302

Urcthane, blood-pressure and, 343

Urine, secretion of, blood-pressure and, 286

in hypertension, 282

Urticaria, vascular crises and, 262

Uskoff's sphygmomanometer, 93

\section{V}

VAN WestenriJK's sphygmomanometer, 79

Van Zwaluwenburg's method of estimation of blood-flow, 170

Vaquez's sphygmomanometer, 108

Vascular crises, 256 angina abdominalis in, 259 pectoris in, 257

angioneurotic edema and, 263 apoplexy in, 261

biliary colic in, 260

cerebral, 260

chilblains and, 262
Vaseular crises in children, 263

compensation, 271

dermographism and, 262

epilepsy and, 260, 371

intermittent claudication in, 261

in locomotor ataxia, 374

peripheral, 261

renal colic in, 260

treatment of, 264

tympanites in, 260

urticaria and, 262

vasoconstriction and, 257

vertigo in, 261

reactions in arteriosclerosis, 252

tree, blood-pressure throughout, 23

Vasoconstriction, vascular crises and, 257

Vasoconstrictor system, pliysiology of, 29

Vasodilator nerves, physiology of, 32

system, physiology of, 32

therapeusis, 211

Vasomotor efficiency test, 146

system, physiology of, 29

Vasotonin, blood-pressure and, 343

Veins, physiology of, 29

Venesection, effect on blood-pressure, 314,376

in uremia, 302

Venous blood-pressure, 23, 27, 135

cardiac action and, 143

critical, 26

effect of drugs on, 345

effects of alterations of bloodpressure on, 55

estimation of blood-flow, systolic output by, 164

Frank and Reh's method, 137

Frey's method, 136

Gaertner's method, 135

Hooker and Eyster's method, 137

Howell's method, 137

intravenous needle of Moritz and Tabora in, 138

spring manometers in, 135

factors influencing, 141

in infectious diseases, 198

intra-ocular pressure and, 404 intravenous injection and, 144

in nephritis, 285

respiration and, 143

Schott's test of, 155

Veratrum viride, 344, 398

Vertigo in vascular crises, 261 
Visceral inanipulations and blood-press- Vomiting, dangers of, 329 ure, 385

Viscosity of blood, 35

in pregnancy, 298

and hypertension, 297

Visual accommodation, blood-pressure and, 410

method of estimation of bloodpressure, 102

Vries-Reilingh's method of estimation of arterial tonus, 174

Von Kries's tachograph, 172

- Von Recklinghausen's formula of bloodpressure quotient, 161

sphygmomanometer, 115

Vomiting, blood-pressure during, 329, 372

of pregnancy and blood-pressure, 399

W

White line of adrenal insufficiency, 263

Widmer's sphygmomanometer, 106

$\mathbf{Y}$

Yominвis, blood-pressure effect of, 344

$\mathbf{Z}$

ZסNTz's ergometer, 151 




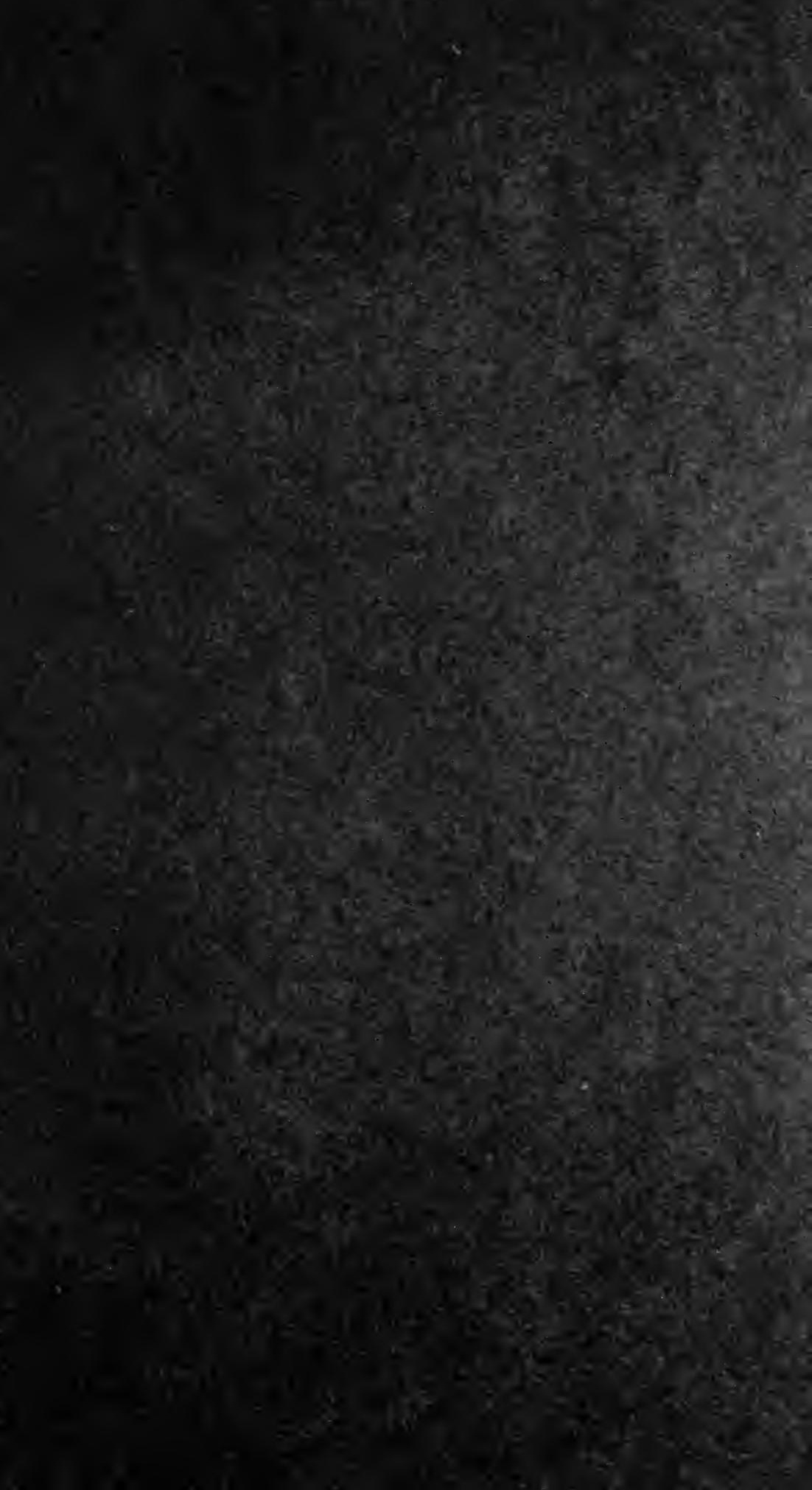




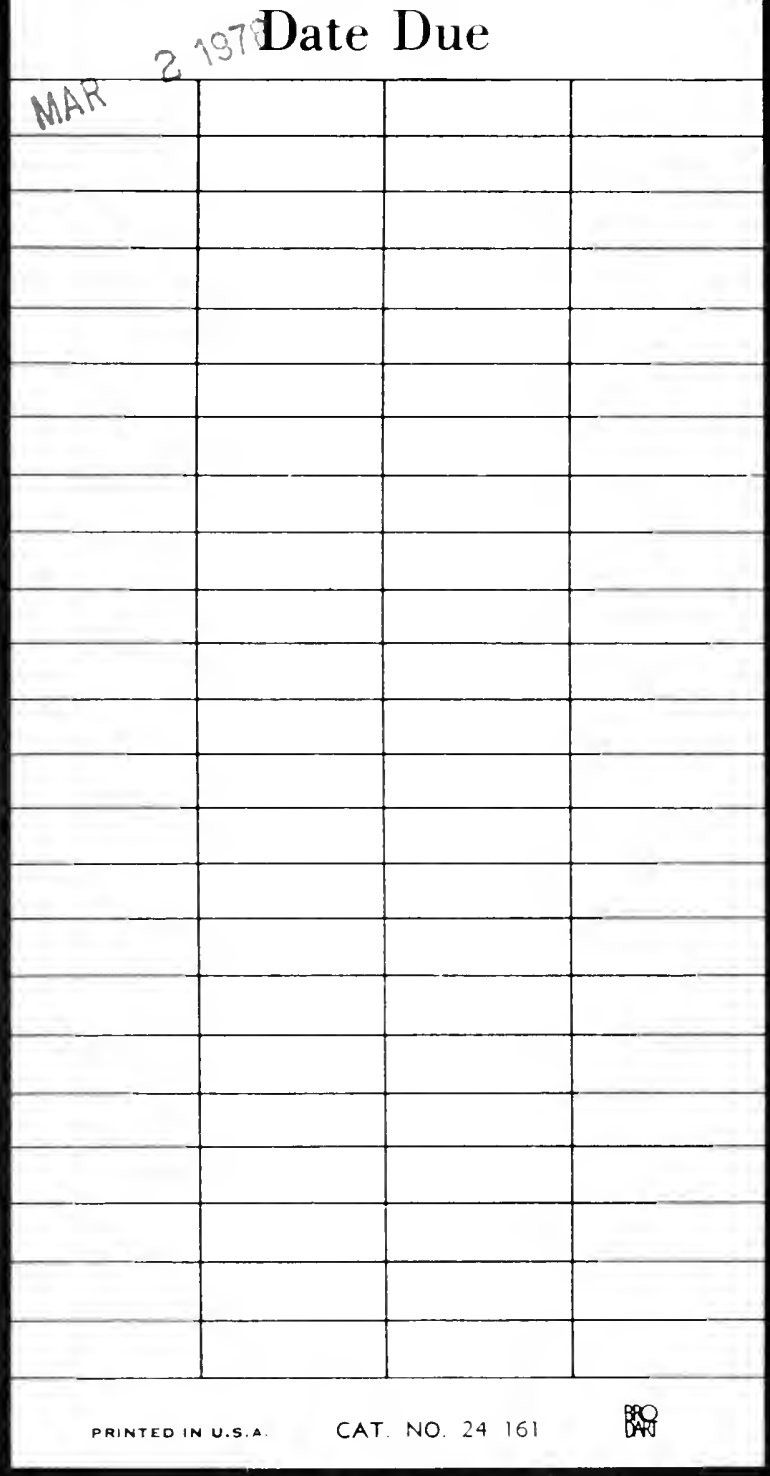


iorris, Gecrme Tililiam.

I.lood-nressure, its cliricai

armlications

Jorris, Georre tillidam.

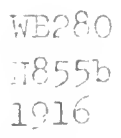

BIcoa-pressure, its clinical applic ticns

MEDICAL SCIENCES LIBRARY UNIVERSITY OF CALIFORNIA, IRVINE IRVINE, CALIFORNIA 92664 
